San Jose State University

SJSU ScholarWorks

Master's Projects

Master's Theses and Graduate Research

Fall 2011

\title{
A Machine Learning and Compiler-based Approach to Automatically Parallelize Serial Programs Using OpenMP
}

Nam Quang Lam

San Jose State University

Follow this and additional works at: https://scholarworks.sjsu.edu/etd_projects

Part of the Computer Sciences Commons

\section{Recommended Citation}

Lam, Nam Quang, "A Machine Learning and Compiler-based Approach to Automatically Parallelize Serial Programs Using OpenMP" (2011). Master's Projects. 210.

DOI: https://doi.org/10.31979/etd.9g3m-st77

https://scholarworks.sjsu.edu/etd_projects/210

This Master's Project is brought to you for free and open access by the Master's Theses and Graduate Research at SJSU ScholarWorks. It has been accepted for inclusion in Master's Projects by an authorized administrator of SJSU ScholarWorks. For more information, please contact scholarworks@sjsu.edu. 
A Machine Learning and Compiler-based Approach to Automatically Parallelize Serial Programs Using OpenMP

\author{
A Writing Project \\ Presented to \\ The Faculty of the Department of Computer Science
}

San Jose State University

In Partial Fulfillment

of the Requirements for the Degree

Master of Science in Computer Science

By

Nam Quang Lam

December 2011 
(C) 2011

Nam Quang Lam

ALL RIGHTS RESERVED 


\section{SAN JOSE STATE UNIVERSITY}

The Undersigned Project Committee Approves the Project Titled

A MACHINE LEARNING AND COMPILER-BASED APPROACH TO AUTOMATICALLY PARALLELIZE SERIAL PROGRAMS USING OPENMP

by

Nam Quang Lam

APPROVED FOR THE DEPARTMENT OF COMPUTER SCIENCE

$\begin{array}{lll}\text { Dr. Teng Moh, } & \text { Department of Computer Science Date }\end{array}$

\begin{tabular}{lll}
\hline Prof. Ronald Mak, Department of Computer Science & Date
\end{tabular}

\begin{tabular}{lll}
\hline Dr. Jon Pearce, & Department of Computer Science
\end{tabular}

APPROVED FOR THE UNIVERSITY

\begin{tabular}{lll}
\hline Associate Dean & Office of Graduate Studies and Research
\end{tabular} 


\begin{abstract}
Single core designs and architectures have reached their limits due to heat and power walls. In order to continue to increase hardware performance, hardware industries have moved forward to multi-core designs and implementations which introduces a new paradigm in parallel computing. As a result, software programmers must be able to explicitly write or produce parallel programs to fully exploit the potential computing power of parallel processing in the underlying multi-core architectures. Since the hardware solution directly exposes parallelism to software designers, different approaches have been investigated to help the programmers to implement software parallelism at different levels. One of the approaches is to dynamically parallelize serial programs at the binary level. Another approach is to use automatic parallelizing compilers. Yet another common approach is to manually insert parallel directives into serial codes to achieve parallelism.

This writing project presents a machine learning and compiler-based approach to design and implement a system to automatically parallelize serial C programs via OpenMP directives. The system is able to learn and analyze source code parallelization mechanisms from a training set containing pre-parallelized programs with OpenMP constructs. It then automatically applies the knowledge learned onto serial programs to achieve parallelism. This automatic parallelizing approach can be used to target certain common parallel constructs or directives, and its results when combined with a manual parallelizing technique can achieve maximum or better parallelism in complex serial programs. Furthermore, the approach can also be used as part of compiler design to help improve both the speed and performance of a parallel compiler.
\end{abstract}




\section{ACKNOWLEDGEMENTS}

I would like to thank my advisors Dr. Robert Chun and Dr. Teng Moh for their dedication, insight, and guidance throughout the period of this writing project. I also would like to express many thanks to committee member Prof. Ronald Mak for his valuable time to provide support and advice. The three of you are great assets to the university and to the students. Knowledge required to complete this project comes directly from all of your classes.

I also would like to take this opportunity to thank my parents for their encouragement and to especially thank my wife, Mai Nguyen, for her encouragement and continuous support for the completion of this project. 


\section{TABLE OF CONTENTS}

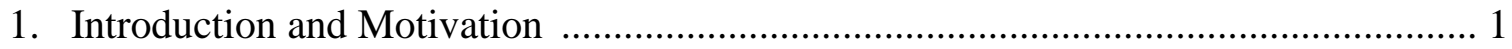

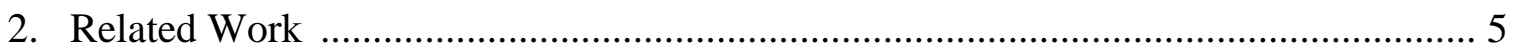

2.1. Adaptive Multi-versioning for OpenMP Parallelization

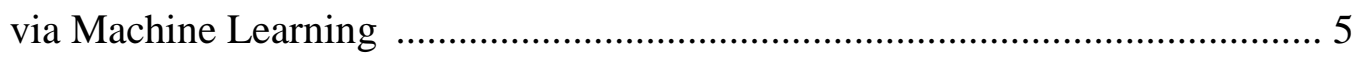

2.2. Automatic Parallelization of Sequential C Code ........................................ 9

2.3. The Algorithm of Parallel Recognition Based on Data Dependence ............... 11

2.4. Mapping Parallelism to Multi-cores:

A Machine Learning Based Approach ....................................................... 13

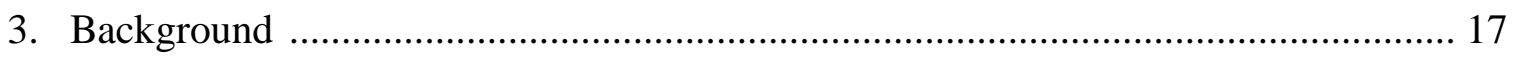

3.1. Multi-Core Programming and OpenMP ...................................................... 17

3.1.1. Parallel Programming Models ....................................................... 17

3.1.2. Parallel Programming Patterns ..................................................... 21

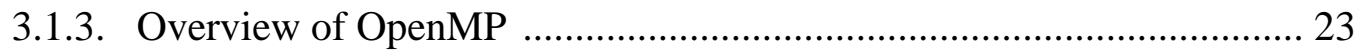

3.1.4. OpenMP Programming Model and Features ................................... 24

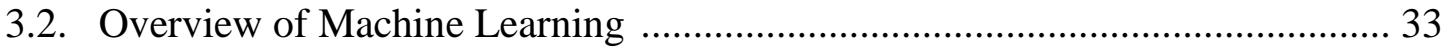

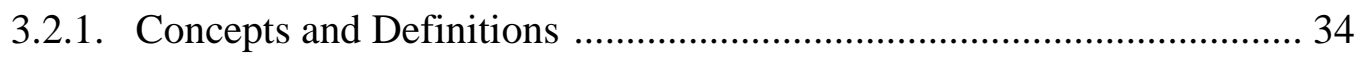

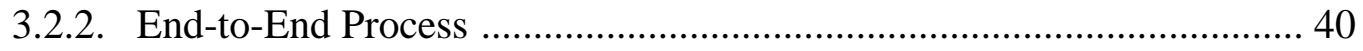

3.2.3. Selected Techniques and Algorithms ............................................ 42

3.3. Concept of Compiler Design .................................................................... 47

3.3.1. General Framework of a Typical Compiler ..................................... 48

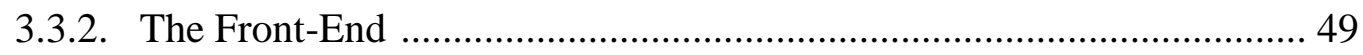

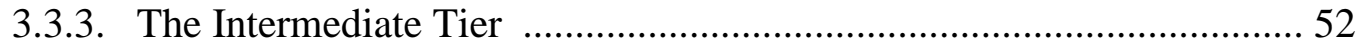




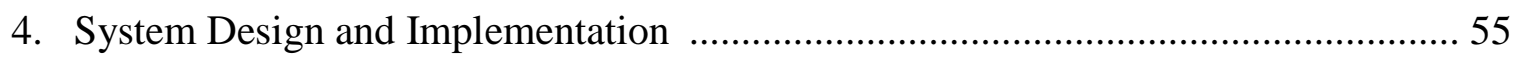

4.1. Purpose of the System and Design Goals ..................................................... 55

4.2. The Machine Learning and Compiler-based Approach …………………......... 56

4.2.1. Machine Learning Approach …………………………………….... 57

4.2.2. Compiler-based Design Approach ........................................................ 66

4.3. Development Environment and Tools ........................................................... 72

4.4. Software Architecture and Design Patterns ...................................................... 73

4.5. Implementation: Packages and Classes ………………………….............. 75

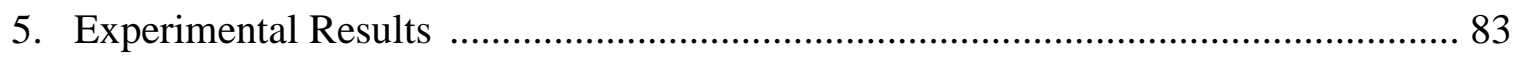

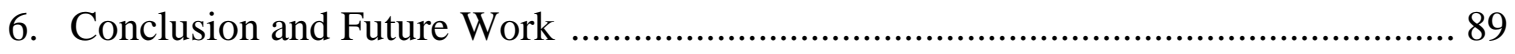

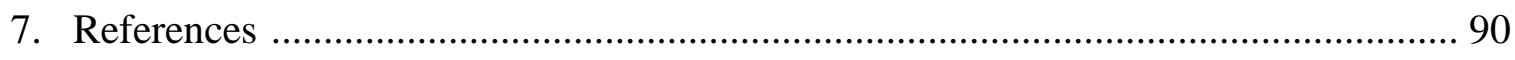

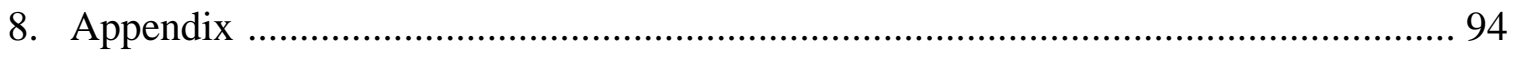

8.1. Appendix A: Training Instances in ARFF Format …….................................. 94

8.2. Appendix B: OpenMP Training Source Files …………………......................94

8.3. Appendix C: Serial Program Test Files …………………………………..... 97

8.4. Appendix D: Auto-Parallel Result Files ………………………………..... 102

8.5. Appendix E: C Grammar in BNF Format ................................................. 109

8.6. Appendix F: C Grammar for JavaCC ……………................................... 113

8.7. Appendix G: The Project Source Code ………………………………….... 126 


\section{LIST OF TABLES}

Table 1. Summary of Data Dependence Matrices ................................................... 12

Table 2. Two Major Types of Parallel Programming Patterns ................................... 21

Table 3. Syntax Format of Two Major OpenMP Work-sharing Constructs .................... 26

Table 4. Description of Two Major OpenMP Work-sharing constructs ....................... 27

Table 5. Commonly-used Clauses of the "parallel" and "for" Directives ..................... 31

Table 6. Summary of Four Different Attribute Types [3] …...................................... 35

Table 7. Sample Training Instances of Tax Defaulted Borrower Problem [3] ............... 36

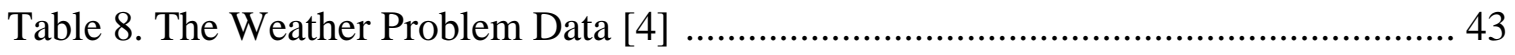

Table 9. A New Unknown Instance of the Weather Problem ..................................... 44

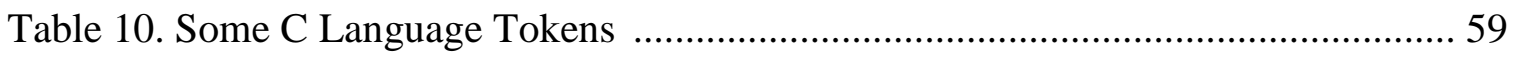

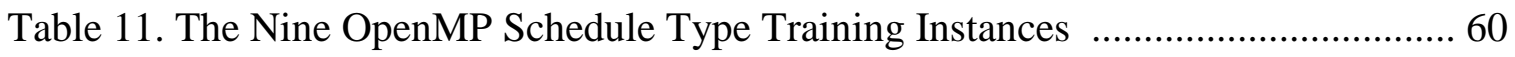




\section{LIST OF FIGURES}

Figure 1. Sequential version (A) and corresponding multiple OpenMP parallel versions

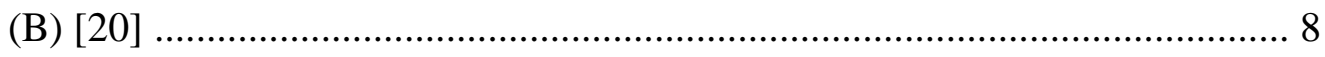

Figure 2. Pseudo-code of the Candidate Selection Algorithm [20] ................................. 9

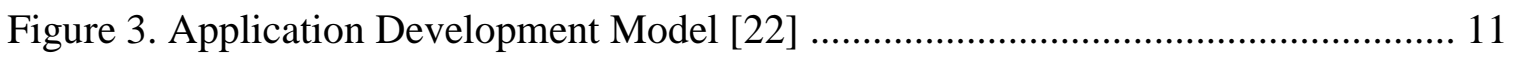

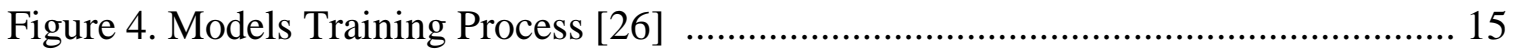

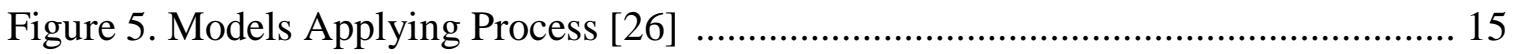

Figure 6. Shared-memory Hardware Architecture ............................................... 18

Figure 7. Distributed-memory Hardware Architecture ........................................... 19

Figure 8. Distributed-memory Message Passing Model .......................................... 20

Figure 9. A Typical Data-level Parallelism ............................................................. 22

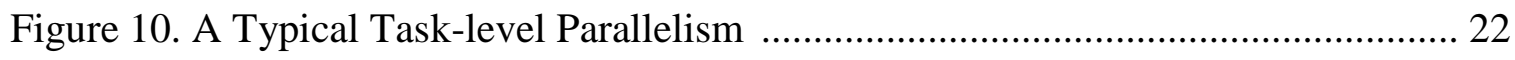

Figure 11. OpenMP Fork-Join Execution Model .................................................... 24

Figure 12. General OpenMP Syntax Structure ..................................................... 25

Figure 13. An Example of A Simple OpenMP Program ........................................... 32

Figure 14. Relationship Among Machine Learning and Data Mining .......................... 34

Figure 15. Sample Training Data of the Classification Problem Example ..................... 37

Figure 16. Sample Training Data of the Regression Problem Example ........................ 38

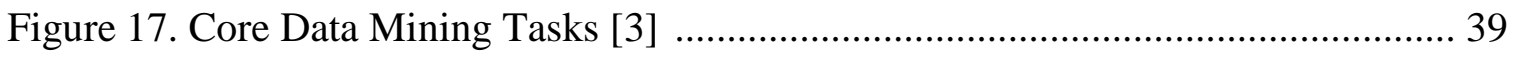

Figure 18. General Process of Data Mining ....................................................... 40

Figure 19. A Decision Tree for the Weather Classification Problem ............................ 44

Figure 20. The 1-, 2-, and 3-Nearest Neighbors of an Instance [3] .............................. 46

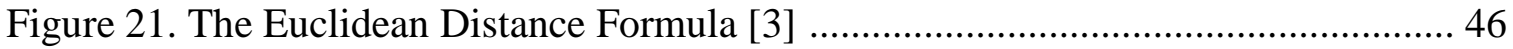


Figure 22. A High-level View of A Compiler ……………............................................ 47

Figure 23. General Framework of a Typical Compiler [5] ............................................. 48

Figure 24. Syntax Diagram and BNF of Digit Grammar ............................................ 51

Figure 25. The Symbol Table Structure [5] …………............................................... 52

Figure 26. An Expression and Its Corresponding Parse Tree (AST) ………………….. 53

Figure 27. End-to-End Process of the Machine Learning Approach .............................. 57

Figure 28. Sample Features Extracted from a for Loop .................................................. 60

Figure 29. An Example of Loop Imbalanced Workload Condition .................................. 61

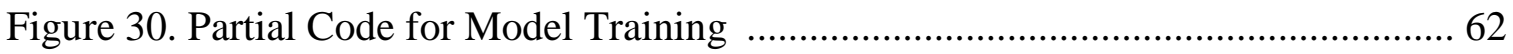

Figure 31. Partial Code for Model Applying …………….............................................. 64

Figure 32. Partial Code to Construct the "for" Directive ................................................. 66

Figure 33. Partial Project C Grammar for JavaCC …………………….................... 68

Figure 34. The Symbol Table …………………….................................................. 69

Figure 35. An Example of Loop-carried Dependence .................................................. 70

Figure 36. An Example of A Partial AST Generated from serial_matrix_product_1.c ...71

Figure 37. The Project System Software Architecture .................................................. 74

Figure 38. The Abstract Factory Design Pattern …………………........................... 75

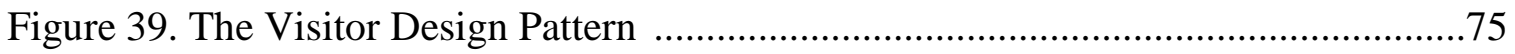

Figure 40. The Simple Scrum Backlog of the Project ..................................................... 79

Figure 41. Classes Under nql.ml.classifiers Package ……........................................... 80

Figure 42. Classes Under nql.cd.frontend Package ...................................................... 80

Figure 43. Classes Under nql.cd.intermediate Package ................................................ 81

Figure 44. Classes Under nql.cd.backend Package ........................................................ 81 
Figure 45. Program Usage for Command Line Version 82

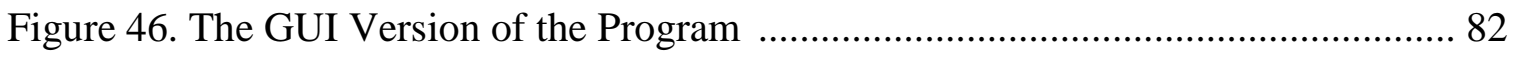

Figure 47. Real Time Chart of Test Cases $1 \& 3$ ………........................................... 87

Figure 48. Real Time Chart of Test Cases 2, 4, 5, \& 6 .............................................. 87 


\section{Introduction and Motivation}

Over the past decades, computer hardware designs and architectures have rapidly evolved in both macroscopic and microscopic directions [6]. Detailed descriptions of the evolutions of computer hardware architectures are beyond the scope of this writing project. In other words, the following macroscopic and microscopic views of hardware solutions give a brief introduction but adequate enough to show a big picture of hardware trends that lead to our current explicit parallel programming paradigm.

Macroscopically, in 1981, IBM introduced its first line of personal computers (PCs) to the consumer market $[7,13]$. This was a major breakthrough in personal computing industry; this was also the beginning of the era of single node computers or desktop workstations that are still being used today. A stand-alone computer is used mostly by an individual in an office or at home to carry out small daily tasks. Computing power of a single PC is limited and self-contained. In order to be able to run computeintensive or heavy workload programs, such as scientific applications, multiple computers are put together to share the computational workload of a large application; this is the basic idea of cluster computing. A cluster computer is group of tens to hundreds of workstations or nodes commonly interconnected via a local area network. All nodes are usually very highly coupled $[8,9,10]$. A cluster is an integration of computing resource that has a frontend node to provide access to various services performed by a collection of backend nodes. Thus, a cluster computer appears as a single supercomputing system to the end users. Another technological advancement that also makes use of multiple computer resources to create a virtual supercomputer to achieve high-performance computing environment to run complex and resource intensive 
applications is grid computing. A grid is similar to a cluster in a way that both integrate many computers together to work as one; however, a grid is composed of thousands of nodes that are more loosely-coupled and are geographically distributed, resulted in a suitable computing environment for a variety of distributed applications. Grids use special-purpose software libraries called middleware to coordinate the work and resources among the nodes [18]. In short, the grid architecture is reliable and scalable to integrate and utilize multiple resources including, but not limited to, clusters, mass repositories, specialized computers, software, etc. to serve a common goal or various computing purposes $[10,11,12]$. Evolved from the grid is cloud computing, a current hot research topic and also a future information technology for everyone. Cloud computing is a TCP/IP- or Internet-based computing that utilizes most or all aspects of grid computing, utility computing, virtualization, and service-oriented architecture (SOA) [12, 14, 17]. As a result, cloud computing is very scalable, loose coupling, highly secure, service-oriented, and has strong fault tolerance. High-level overview of the cloud architecture, which is transparent or abstracted from the end users, includes cloud service, cloud platform, cloud infrastructure, and cloud storage. With a single point of entry via a thin client, a user is able to access a variety of on-demand services provided by the cloud, including Infrastructure-as-a-Service (IaaS), Platform-as-a-Service (PaaS), and Software-as-aService (SaaS) $[15,16]$. A thin client accessing the cloud is analogous to a terminal accessing the mainframe; however, a subtle difference is that the mainframe provides concurrent data access concurrent programming whereas the cloud provides parallel access to on-demand services at larger scope. In short, macroscopic evolution of hardware architecture directly exposes parallel programming to the programmers. 
Microscopically, computer processors in the past decades have gone through a significant evolution in terms of clock frequency and number of transistors. In 1981, when IBM first PC was introduced to the market, it was featured with a single Intel 8088 microprocessor running at $4.77 \mathrm{MHz}$ and containing fewer than 30,000 transistors [13, 19]. However, in 2006, an Intel Core 2 Duo was shining at an amazing clock frequency of $2.9 \mathrm{GHz}$ and having around $291,000,000$ of transistors [6]. This correctly validated Moore's law, which stated that number of transistors would double approximately every 18 to 24 months $[1,6]$. Yet, everything has its limit. Single core designs and architectures have reached their limits due to heat and power walls. In order to continue to increase hardware performance, hardware industries have moved forward to multi-core designs and implementations, which introduces a new paradigm in parallel computing [1,6]. Just as macroscopic view, in short, microscopic evolution of hardware architecture also directly exposes parallel programming to the programmers.

Hardware solution has dragged software industries into a higher demand for parallelism [6]. As a result, software programmers must be able to explicitly write or produce parallel programs to fully exploit the potential computing power of parallel processing in the underlying multi-core architectures. Since the hardware solution directly exposes parallelism to software designers, different approaches have been investigated and/or implemented to help the programmers to implement software parallelism at different levels. One of the approaches is to dynamically parallelize serial programs at the binary level [33]; another approach is to use automatic parallelizing compilers $[24,31]$. Yet another common approach is to manually insert parallel directives into serial codes to achieve parallelism [1,2]. 
This writing project presents a machine learning and compiler-based approach to design and implement a system to automatically parallelize serial $\mathrm{C}$ programs via OpenMP directives. The source language for the system implementation is Java, and the target programs are written in the $\mathrm{C}$ language. The system is able to analyze and learn source code parallel mechanisms from a training set containing pre-parallelized programs with OpenMP constructs. It then automatically applies the knowledge learned onto serial programs to achieve parallelism. Practically, the project focuses on work-sharing at looplevel because loops take all or most of the execution time of a running program. This automatic parallelizing approach can be used to target certain common parallel constructs or loop directives, and results then combined with a manual parallelizing technique to achieve maximum or better parallelism in complex serial programs. Furthermore, the approach can also be used as part of compiler design; it helps to improve both speed and performance of a parallel compiler. 


\section{Related Work}

Over the years, many different approaches have been researched and implemented to help software developers to write parallel programs or to exploit parallelism for multi-core processors; however, not that many of the approaches are directly related to the work presented in this project. This project proposes a new way to achieve automatic parallelism for the underlying multi-core architectures via OpenMP using the combination of machine learning and compiler-based methods. As a result, it is not an easy task to actually compare the performance of the implementations of other approaches with this project's approach. This section describes some of previous works that are related to this project only on certain aspects but relevant enough to contribute to knowledge base for completing this writing project.

\section{1. “Adaptive Multi-versioning for OpenMP Parallelization via Machine Learning" [20]}

In general, OpenMP APIs allow programmers to produce parallel programs by manually inserting OpenMP directives or constructs into corresponding serial programs to guide the compiler about which regions of the codes can be executed in parallel [2]. Details of OpenMP are covered in the later Background section. However, OpenMP does not guarantee an optimal execution of the resulting programs. Motivated from this fact, the authors of the paper, Adaptive Multi-versioning for OpenMP Parallelization via Machine Learning [20], presented a mechanism to be integrated into a compiler to adaptively achieve parallelism in a cost-aware manner. The mechanism guarantees that the implemented compiler would be able to properly parallelize the workload of a program to 
produce a corresponding optimally performing OpenMP parallel version at runtime for a multi-core system.

Briefly, the proposed solution works as follows. When the compiler encounters a loop in a serial program, it generates multiple OpenMP parallel versions (i.e., multiversioning) of the loop with various numbers of threads for each version that it can evaluate with various inputs of different sizes, as demonstrated in Figure 1. The compiler then selects a proper number of representative versions using the algorithm shown in Figure 2, which will be embedded into the final executable. With proper mapping features, an appropriate version is selected to execute at runtime.

Actually, it is very difficult or almost impossible to select an appropriate number of threads at compile time for a specific program to be properly executed in a parallel environment that yields an optimal performance. Therefore, a technique called adaptive optimization [20] is recommended to provide best-possible solution for selecting a suitable number of threads at runtime. Adaptive optimization can be dynamic compilation or multi-versioning; yet the authors decided to choose multi-versioning technique because it virtually beats all adaptive static versions in terms of different runtime contexts while "dynamic compilation needs extra time for runtime recompilation of some code regions."

The authors made use of K-Nearest Neighbor machine learning algorithm [3, 4] for runtime version selection. The basic idea is that when a loop is encountered at runtime, its input sizes are computed and compared with a pre-calculated version's input size which results in a corresponding number of threads of the closest match embedded version to be chosen. The machine learning technique being used here is less similar to a 
switch programming structure with an extra capability to choose a closest match entity. It is not fully implemented as an end-to-end machine learning approach [3, 4] (or at least not mentioned in the paper). Details of machine learning are covered in the later Background section.

One can appreciate that the main contribution of the paper to parallelism is the pre-calculation process to obtain a best possible selection of representative OpenMP versions of loops which will be chosen at runtime to yield an optimal parallel workload allocation. This adaptive multi-versioning technique would definitely help compiler designers to implement a parallel cost-aware compiler for current and future multi-core architectures. 


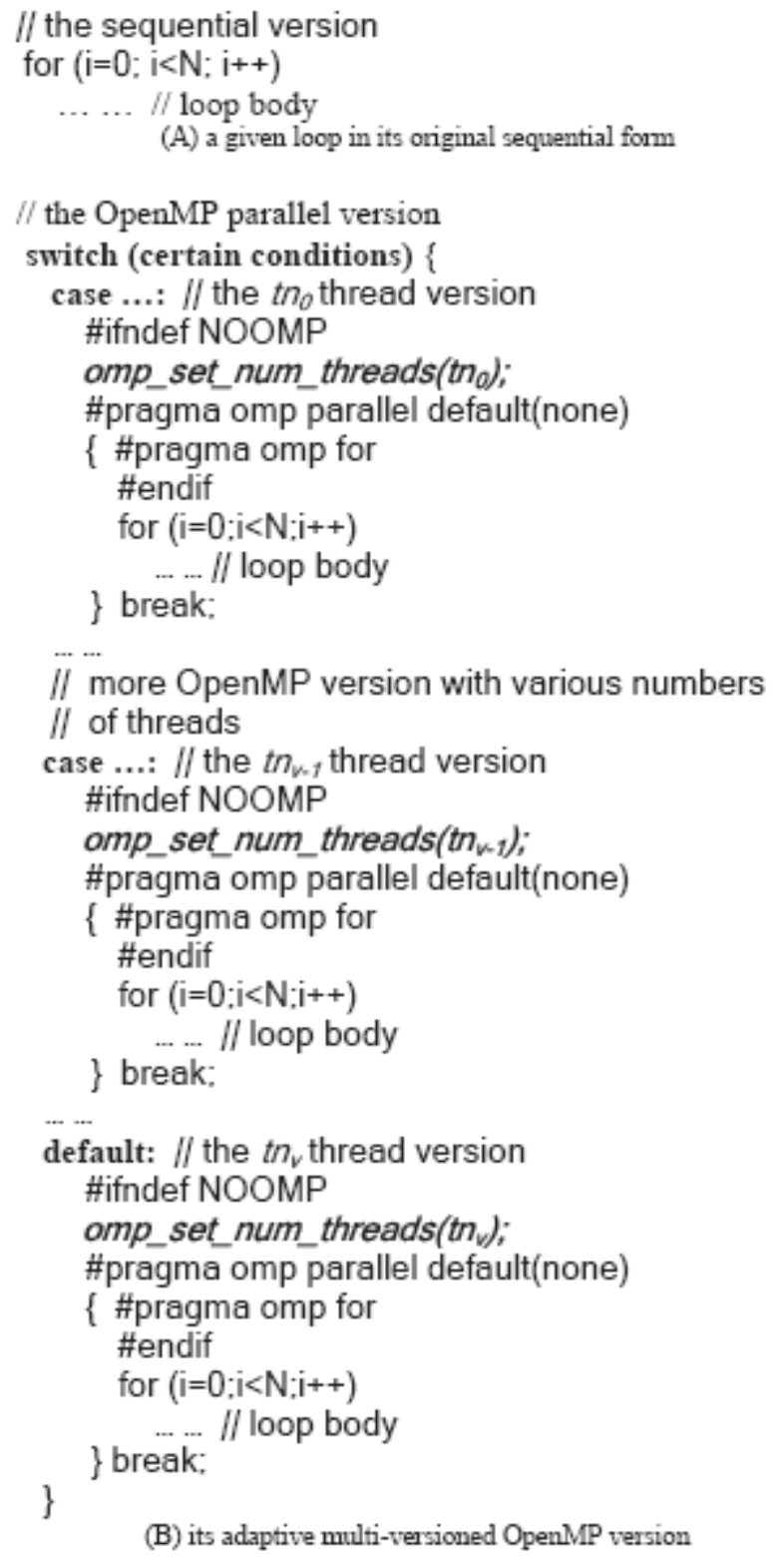

Figure 1. Sequential version (A) and corresponding multiple OpenMP parallel versions (B) [20] 


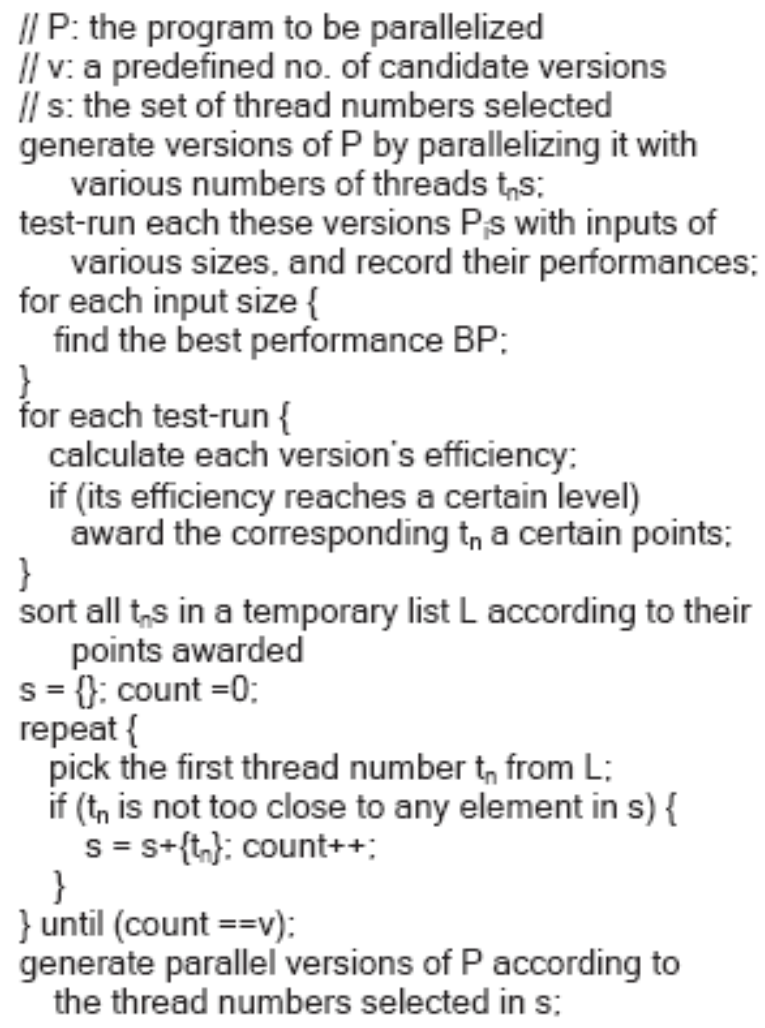

Figure 2. Pseudo-code of the Candidate Selection Algorithm [20]

\section{2. “Automatic Parallelization of Sequential C Code" [22]}

Motivated from the high demand for parallel applications in order to fully take advantages of computing power in cluster environments, the authors of the paper, "Automatic Parallelization of Sequential C Code" [22], presented an automatic parallelization approach to parallelize serial $\mathrm{C}$ programs to be executed under clusterbased architectures. This is an ongoing project that still needs help from user inputs to actually carry out the automatic parallelizing process.

The goal of the project is to provide a useful tool to help the users to comprehend the inside of a given program, mainly about loop structures, for parallelism. At a high level overview, the tool works as follows. It analyzes serial source codes and generates a 
graphical presentation of the program execution to the users. Based on the presented structure, the users are able to define parallelizable regions of codes to guide the automation process. The result is a Message Passing Interface (MPI) parallel program. MPI implementations allow programmers to achieve parallelism in distributed memory environments $[6,25,37]$ that are suitable for cluster computing architectures.

Derived from ideas of compiler design technique and framework (details of compiler design are covered in the later Background section), the project was implemented as shown in Figure 3. First, a programming language utility named ANTLR (ANother Tool for Language Recognition) was used to build the $\mathrm{C}$ language parser needed to parse and process source code of a $\mathrm{C}$ program and to build a corresponding AST (abstract syntax tree) $[22,40]$. Then, a data structure was implemented to classify the source code into basic-blocks in order to construct a graphical presentation of the program workflow, focusing on loop level. A graphical user interface was also designed for various user interactions. Since this is an ongoing project, the authors postponed the Dependency Analysis feature for later development. So, as the last stage of the development, an MPI Workpool model and code generator were designed and implemented to actually put together all the pieces, such as the flow graph, AST, various data structures, user inputs, etc., to properly generate MPI-based parallel programs for cluster computing architectures.

Although this project is ongoing and still relies on user inputs to actually carry out the automatic execution, it has made a great start for automatic parallel programming in cluster-based environments. Specifically, both distributed application developers and 
compiler designers can benefit from the ideas, techniques, and tool presented in this paper for current and future parallel program implementations.

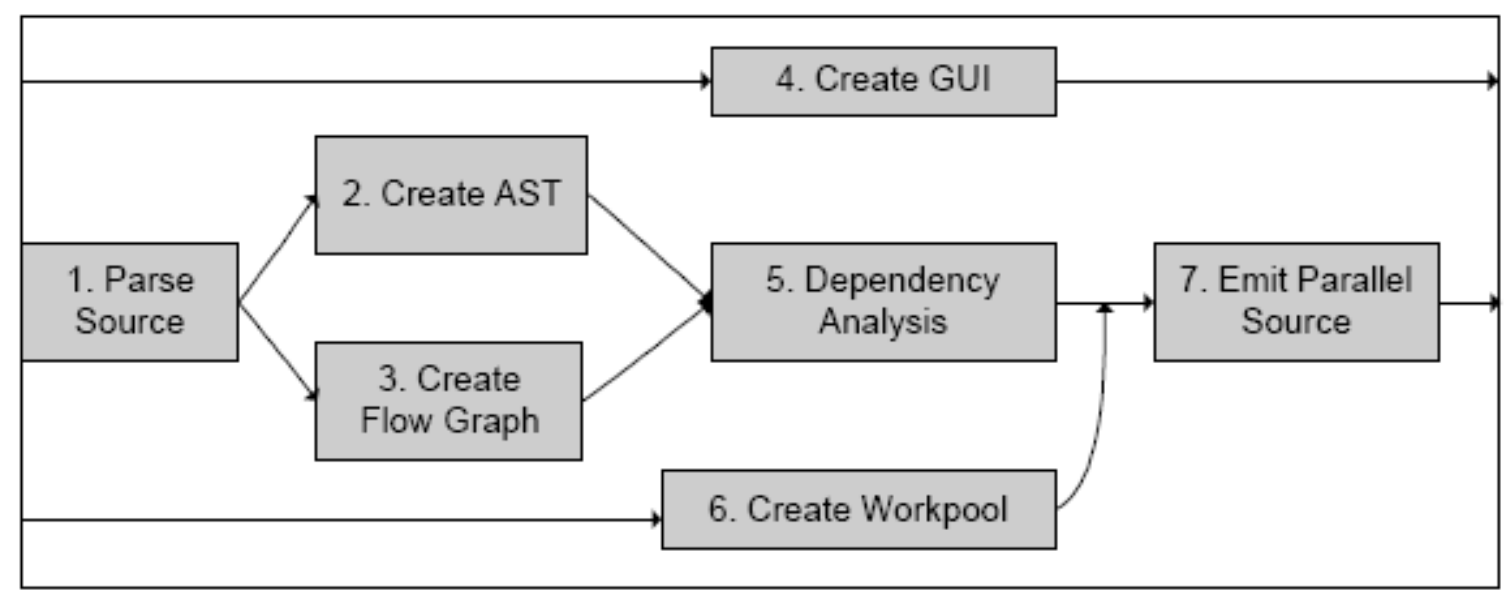

Figure 3. Application Development Model [22]

\section{3. "The Algorithm of Parallel Recognition Based on Data Dependence" [27]}

In this paper, the authors investigated and presented an algorithm that would be used to recognize parallelizable regions or blocks of code in serial programs based on data dependence. Motivated from the fact that it is difficult and takes time to manually parallelize large and complex serial programs, the goal of the algorithm is to provide a mechanism to be integrated into modern compilers to achieve automatic program parallelization or to design an automatic parallel compiler. The basic idea behind this paper is briefly described as follows.

In order to parallelize a serial program, various parts or blocks of the program are extracted and analyzed for data dependence among them. The authors define that a block "is the program element that there is only one entrance and only one exit. The character of the blocks is that the whole codes of blocks either must be executed or must not be 
executed." [27] Data dependence between blocks is recognized based on input and output variables that the blocks have access at the same time. If output variables of a block depend on input variables of another block and vice versa, these blocks have data dependence and cannot be executed or computed in parallel.

Mathematically, data structures that are used to analyze data dependence between blocks compose of various matrices, including input variable matrix $M_{\mathrm{i}}$, output variable matrix $M_{\mathrm{o}}$, and dependence matrix $M_{\mathrm{d}}$. These matrices can be summarized in Table 1 [27].

\begin{tabular}{|c|c|c|}
\hline$M_{i}(i, j)=\{$ & $\left.\begin{array}{ll}0, & a_{j} \notin B_{i I} \\
1, & a_{j} \in B_{i I}\end{array}\right\}$ & $\begin{array}{l}\text { Input variable matrix } M_{\mathrm{i}} \text { sized }(\mathrm{m} \times \mathrm{n}) \text { with blocks } B_{\mathrm{i}} \\
\text { (rows) and variables } \mathrm{a}_{\mathrm{j}} \text { (columns), where } \\
\mathrm{i}=1,2,3, \ldots \mathrm{m} \\
\mathrm{j}=1,2,3, \ldots \mathrm{n} \\
0: \mathrm{a}_{\mathrm{j}} \text { does not belong to input variables of } B_{\mathrm{i}} \\
1: \mathrm{a}_{\mathrm{j}} \text { belongs to input variables of } B_{\mathrm{i}}\end{array}$ \\
\hline$M_{o}(i, j)=$ & $\left\{\begin{array}{ll}0, & a_{j} \notin B_{i O} \\
1, & a_{j} \in B_{i O}\end{array}\right\}$ & $\begin{array}{l}\text { Output variable matrix } M_{\mathrm{i}} \text { sized }(\mathrm{m} \times \mathrm{n}) \text { with blocks } B_{\mathrm{i}} \\
\text { (rows) and variables } \mathrm{a}_{\mathrm{j}} \text { (columns), where } \\
\mathrm{i}=1,2,3, \ldots \mathrm{m} \\
\mathrm{j}=1,2,3, \ldots \mathrm{n} \\
0: \mathrm{a}_{\mathrm{j}} \text { does not belong to output variables of } B_{\mathrm{i}} \\
1: \mathrm{a}_{\mathrm{j}} \text { belongs to out variables of } B_{\mathrm{i}}\end{array}$ \\
\hline$M_{d}(i, j)=$ & $\left\{\begin{array}{c}0 \\
n(n>0)\end{array}\right\}$ & $\begin{array}{l}\text { Dependence matrix } M_{\mathrm{d}} \text { with rows and columns are } \\
\text { composed of blocks to describe dependency between } \\
B_{\mathrm{i}} \text { and } B_{\mathrm{j}} \text {. } \\
0 \text { : no dependence } \\
\text { n: dependence }\end{array}$ \\
\hline
\end{tabular}

Table 1. Summary of Data Dependence Matrices 
Basically, the goal of the algorithm is to look for dependency status between blocks in serial programs in order to determine whether the blocks can be executed in parallel or not. Major steps of the algorithm can be briefly described as below.

- Step 1: Identify and label the blocks in the source code of a serial program.

- Step 2: Process the variables of the program and build corresponding matrices.

- Step 3: From resulting matrices, determine execution order of the blocks.

This paper has presented a simple but practical algorithm that can be useful to the design of a parallel compiler which can produce parallel programs to be executed on multi-processors environment. However, the authors also indicated that under certain conditions, the algorithm needed to be properly optimized in order to truly achieve parallel performance due to thread scheduling overheads, etc.

\section{4. "Mapping Parallelism to Multi-cores: A Machine Learning Based Approach" [26]}

As seen in previous sections, there were a variety of methods and technologies that could be used to design and implement parallel programs to fully take advantage of multi-core platform performance. In fact, programmers can write parallel programs from scratch for a particular multi-core platform, or they can manually apply a parallel mechanism to legacy serial programs to run on multi-core systems. In this paper, the authors have introduced a different approach to multi-core programming. In stead of applying parallelization directly to a program, the authors proposed an approach that takes a program that has been already parallelized and maps it to a multi-core processor via machine learning technique. 
The paper pointed out that parallelizing a program at source code level is one thing, but efficiently mapping the code to the underlying hardware architecture is another thing. Mapping parallelism to underlying hardware is a very difficult task that is highly platform dependent. Furthermore, hardware architecture is rapidly changing and varies from one generation to the next. Therefore, finding an efficient or best mapping is time consuming and highly depends on various factors and decisions, including tasks or threads scheduling, number of processors, the right amount of parallelism to be exploited, and so on. As a result, it motivated the authors to use machine learning to design an automatic mapping approach for parallel compilers. Main goal of this approach is to automatically predict program scalability to select the correct number of threads and to automatically classify program scheduling policies, which would result in an automatic efficient mapping of parallelism to multi-core processors.

At a high level of overview, the authors made use of two different machine learning techniques called Artificial Neural Network (ANN) $[3,26]$ and Support Vector Machine (SVM) [3, 26] to build two different learning models to predict program scalability and to classify scheduling policies, respectively. The models were trained using a set of training data that consisted of pre-parallelized programs with selected features and desired mapping decisions. After the models were trained, they would be used to predict and classify a new or unseen parallel program, which resulted in correct mapping decisions for a parallel compiler. Details of machine learning will be covered later in Background section of this paper.

The two figures below are extracted directly from the paper to describe training and predicting processes of the machine learning models. 


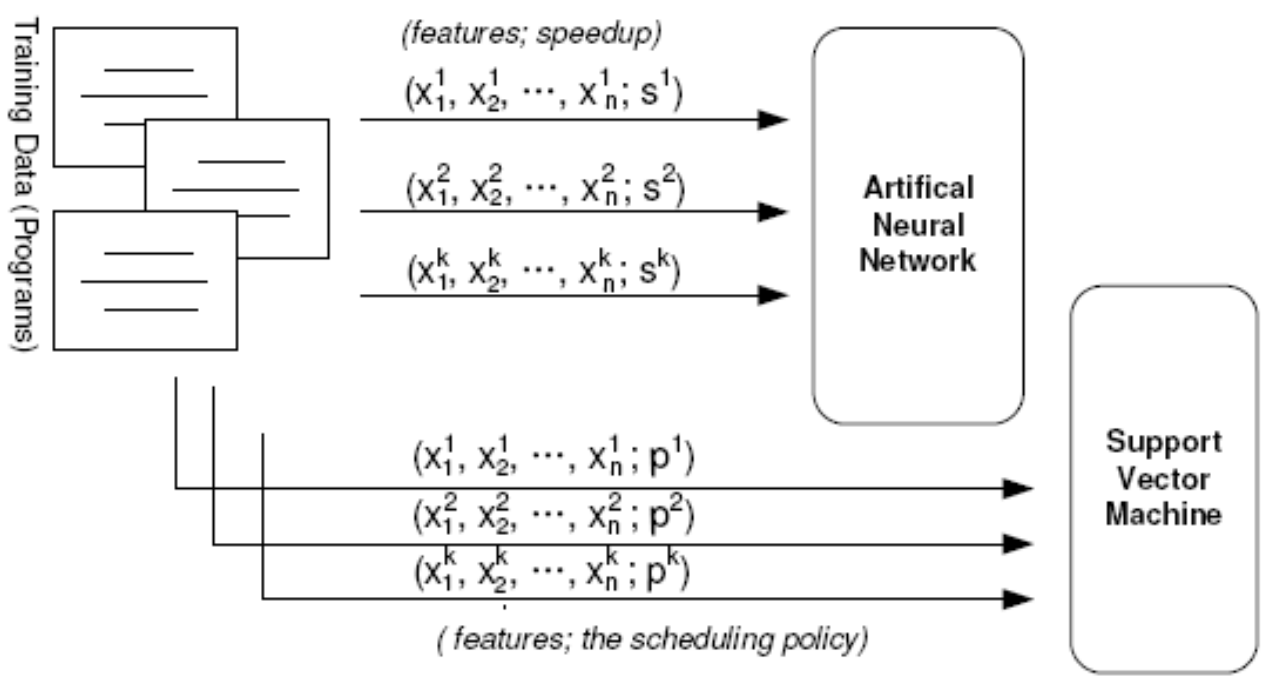

Figure 4. Models Training Process [26]

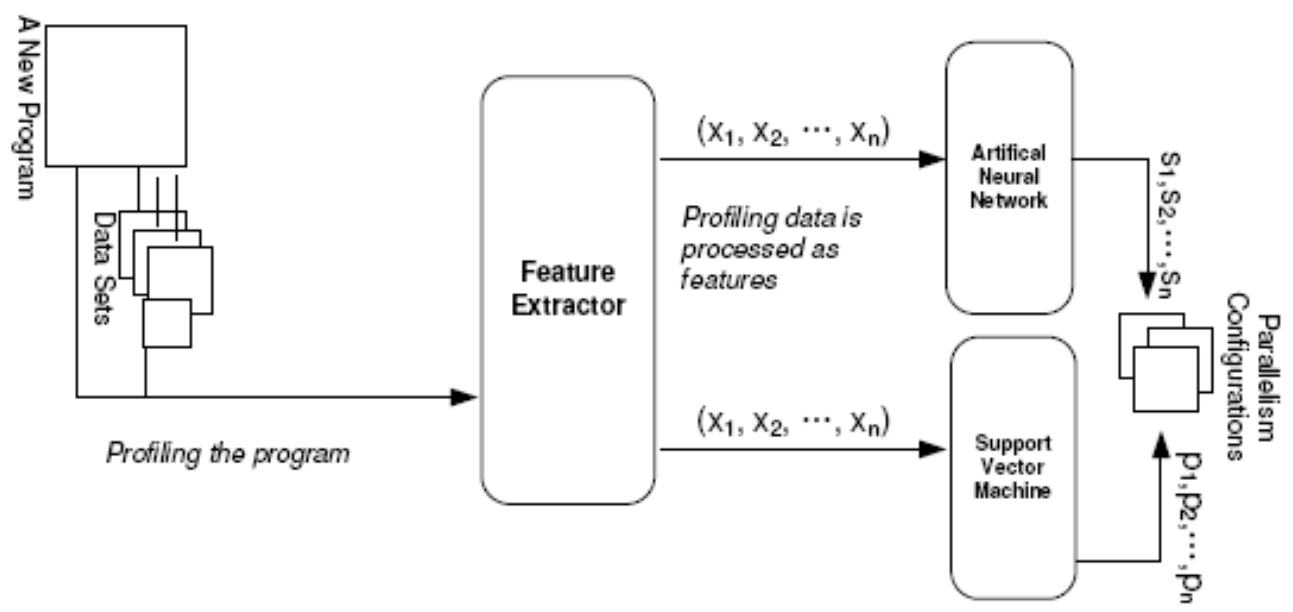

Figure 5. Models Applying Process [26]

The authors did the experiments of the models using different sets of data as well as different types of multi-core platforms featuring both shared-memory and distributedmemory parallel programming models. Their results showed that their approach "has the 
best performance on average" and the performance "is stable across programs, data sets, and architectures." [26]

In summary, this article presented two machine learning techniques to build automatic mapping models that are portable and efficient to be integrated into the design of a parallel compiler. 


\section{Background}

\subsection{Multi-Core Programming and OpenMP}

\subsubsection{Parallel Programming Models}

Parallel programming is not new. For decades, it has always been a great topic for researchers, computer scientists, or software developers due to its nature in providing exceptional performance gains. In 1989, there was already an investigation in implementing a compiler using an input partitioning technique to exploit parallelism for multiple and independent processors systems [30]. Then in 1990, a functional programming language compiler to generate parallel programs to exploit data-parallelism for massive parallel computers was designed [29]. These are the two examples among many other examples of long history parallel programming. However, it is worth noting that the studies and implementations of high-level parallelism were mainly for commercial multi-processors or distributed systems, not home user computers. One key reason was that single-core processor performance could still be improved by increasing number of transistors and, importantly, by implementing low-level hardware parallelisms, such as Instruction-Level Parallelism (ILP) and hardware pipelining, which are transparent to the software programmers [6].

Nevertheless, low-level implicit hardware parallelizations have reached their limits due to heat and power walls [6], which ended the single-core processors era. In 2005, Intel rolled out a new line of multi-core processors to the consumer market [1]. This has signaled a beginning of high-level explicit parallel programming. As a result, now both system-level and application-level software programmers have to learn to 
explicitly write parallel programs in order to exploit the computing power of parallel processing in the underlying multi-core architectures. Generally from hardware design perspective, there are two types of memory architecture [6, 34]: shared-memory architecture and distributed-memory architecture.

Under shared-memory architecture, global memory space is available and shared by all processors; whereas under distributed-memory architecture, each processor has its own memory space. Each design has its own advantages and disadvantages in terms of costs, scalability, complexity, usability, and communication management. Since details of these two hardware designs are beyond the scope of this report, the Figures 6 and 7 show the differences between these two memory models.

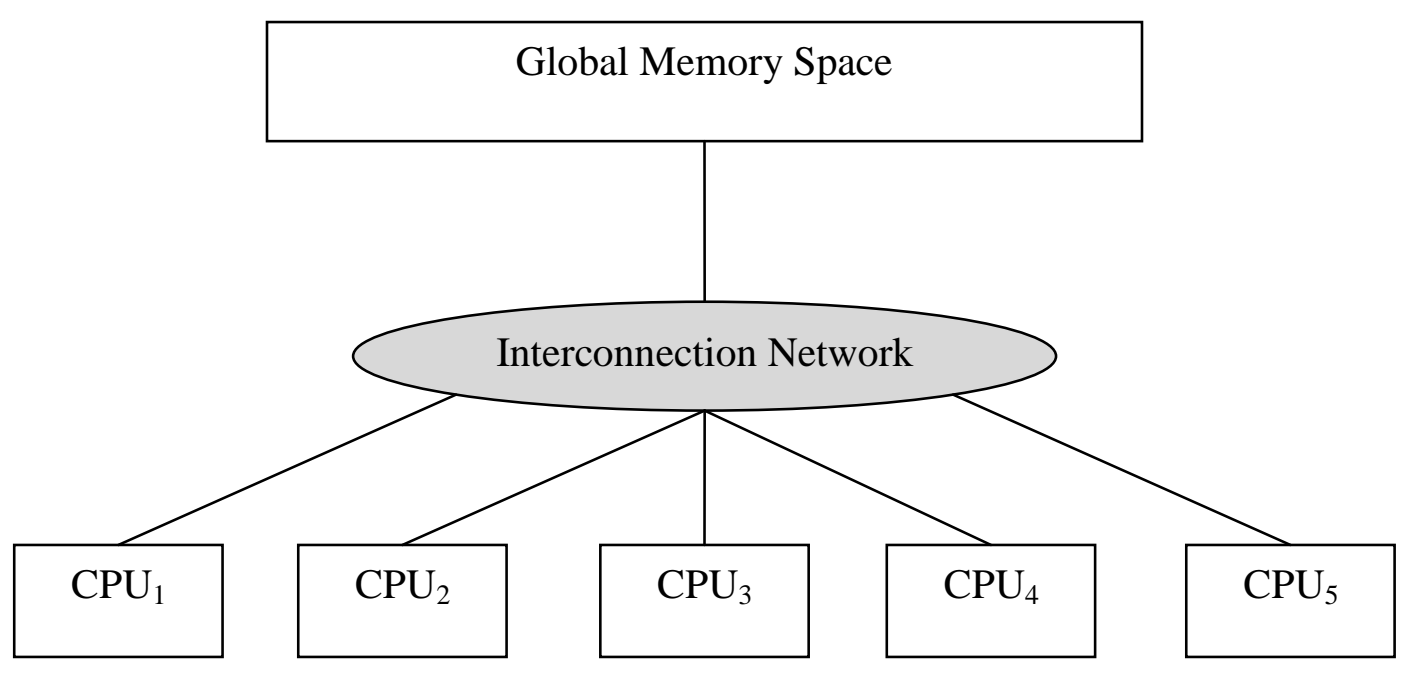

Figure 6. Shared-memory Hardware Architecture 


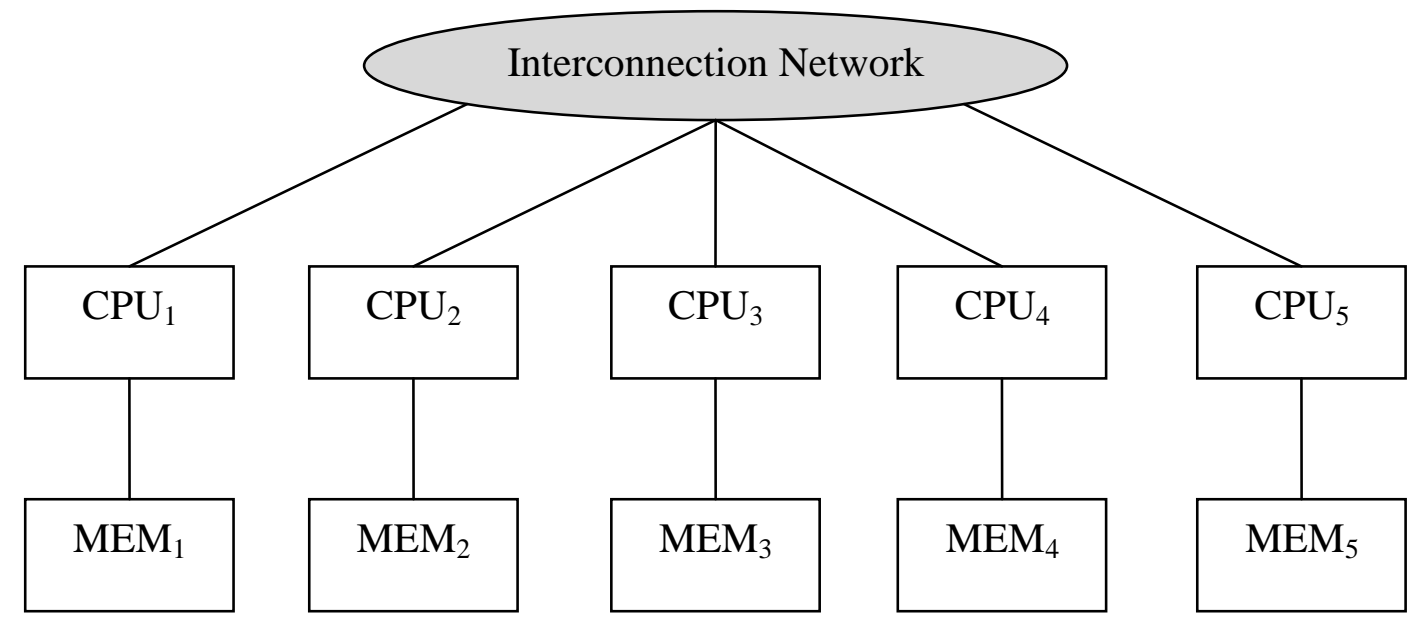

Figure 7. Distributed-memory Hardware Architecture

The two memory architectures have led to two major parallel programming models [6]: shared-memory programming model and distributed-memory (or message passing) programming model. They are briefly described in subsequent sections below according to $[2,6,34]$.

\section{Shared-memory parallel programming model:}

In this model, a parallel program is executed via multiple independent threads that all have access to their same program address space. These threads are utilized on multicore processors in a shared-memory architecture. Since data is shared among the threads, proper thread scheduling and synchronization mechanism must be implemented in order to ensure correctness of the program. Specifically, when software programmers write multi-threaded applications, they must be able to handle common multi-threaded problems, such as deadlocks, race conditions, mutual exclusion, and so on. Sharedmemory parallel programs can be implemented using OpenMP, a parallel programming API that practically realizes this model. OpenMP is described in depth in its own dedicated subsequent section. 


\section{Distributed-memory parallel programming model:}

In this model, a parallel program is executed via multiple independent processes each of which has its own address space. These processes are utilized on multi-core processors in a distributed-memory architecture. This distributed-memory model is also called message passing parallel programming model. Since each process operates on its own private address space, the processes communicate data with each other in distributed fashion by explicitly exchanging messages. This implies that data communication requires cooperation between senders and receivers. As a result, software programmers are required to have solid background in distributed computing; specifically, they are responsible for details and correctness of data communication implemented using message passing mechanism. The Message Passing Interface (MPI) standard provides a rich set of library routines that enable software programmers to write efficient distributed-memory parallel code. See Figure 8.

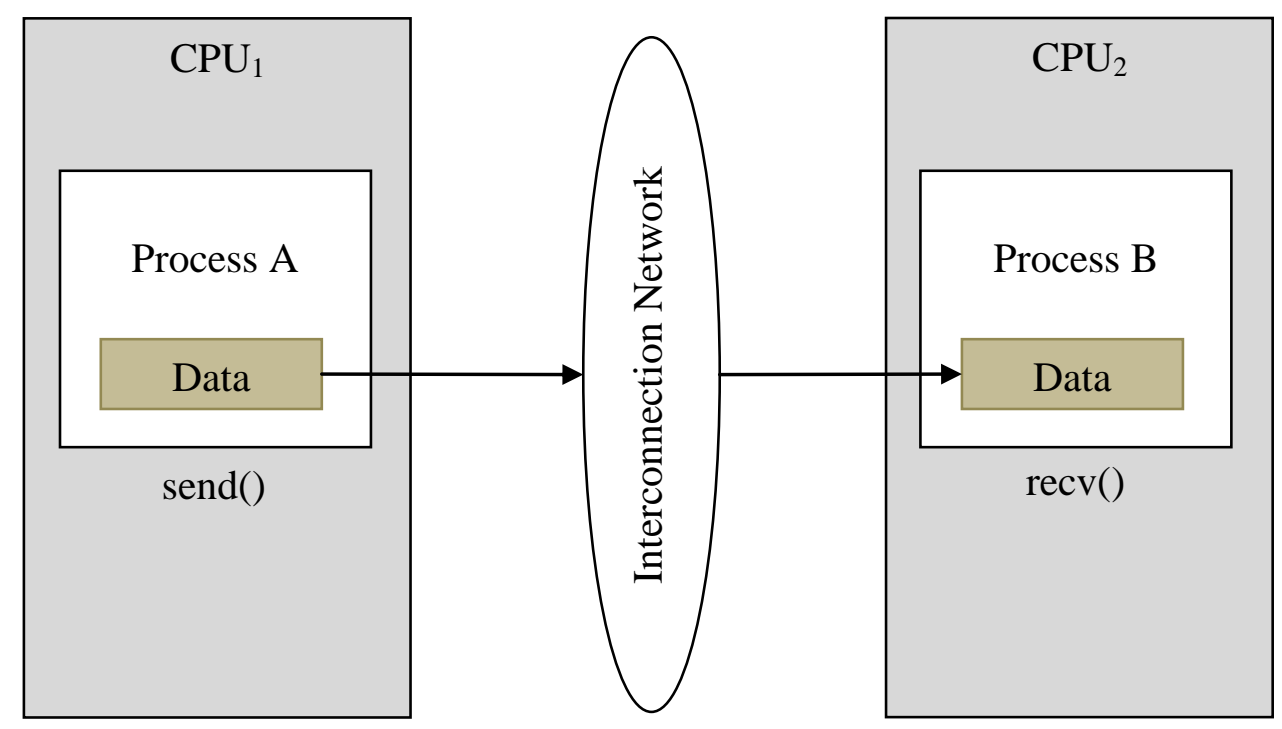

Figure 8. Distributed-memory Message Passing Model 


\subsubsection{Parallel Programming Patterns}

Designing a parallel program requires a programmer first to understand the problem that he or she is trying to solve in parallel. Then, the programmer needs to identify which part of the problem is potentially a good spot to apply parallelization. The programmer has to partition or decompose the problem into smaller chunks or individual tasks that can be executed in parallel $[1,2]$. This results in two major parallel programming patterns, which are summarized in the Table 2 and Figures 9 and 10.

\begin{tabular}{|l|l|}
\hline Parallel Patterns & \multicolumn{1}{|c|}{ Description } \\
\hline Data-level parallelism & $\begin{array}{l}\text { This parallel programming pattern realizes parallelism by } \\
\text { focusing on the data set associated with a problem. It means } \\
\text { that the data set is partitioned into smaller blocks of data, } \\
\text { where each block is operated on by an individual thread or } \\
\text { process to accomplish a specific task. }\end{array}$ \\
\hline Task-level parallelism & $\begin{array}{l}\text { Also being called functional-level parallelism, this parallel } \\
\text { programming pattern realizes parallelization by focusing on } \\
\text { nature of tasks to solve a problem. If the tasks can be } \\
\text { performed in parallel, i.e., there has no dependency between } \\
\text { them, then they are accordingly scheduled and distributed } \\
\text { among threads or processes in the program to be } \\
\text { independently executed. }\end{array}$ \\
\hline
\end{tabular}

Table 2. Two Major Types of Parallel Programming Patterns 


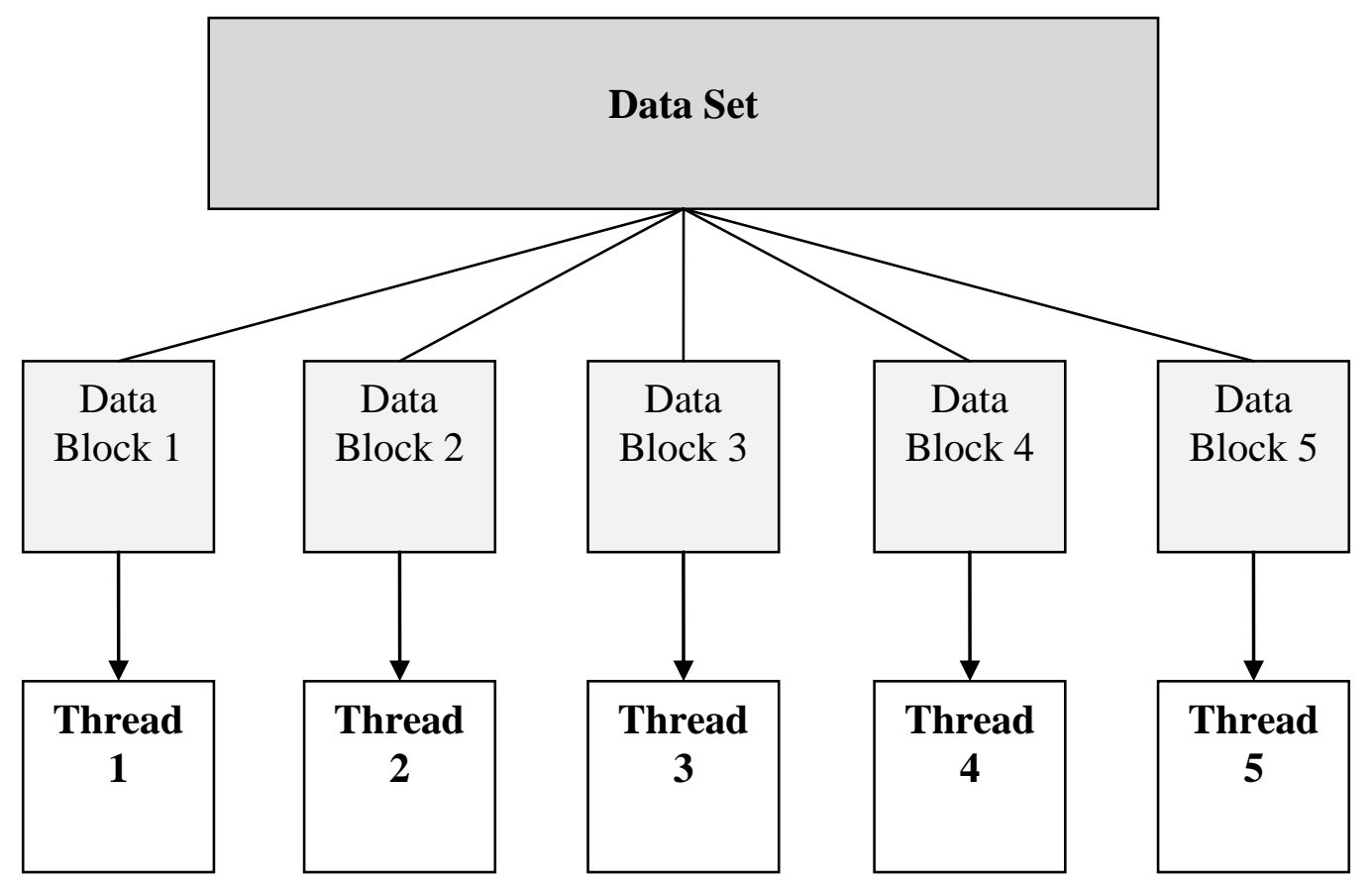

Figure 9. A Typical Data-level Parallelism

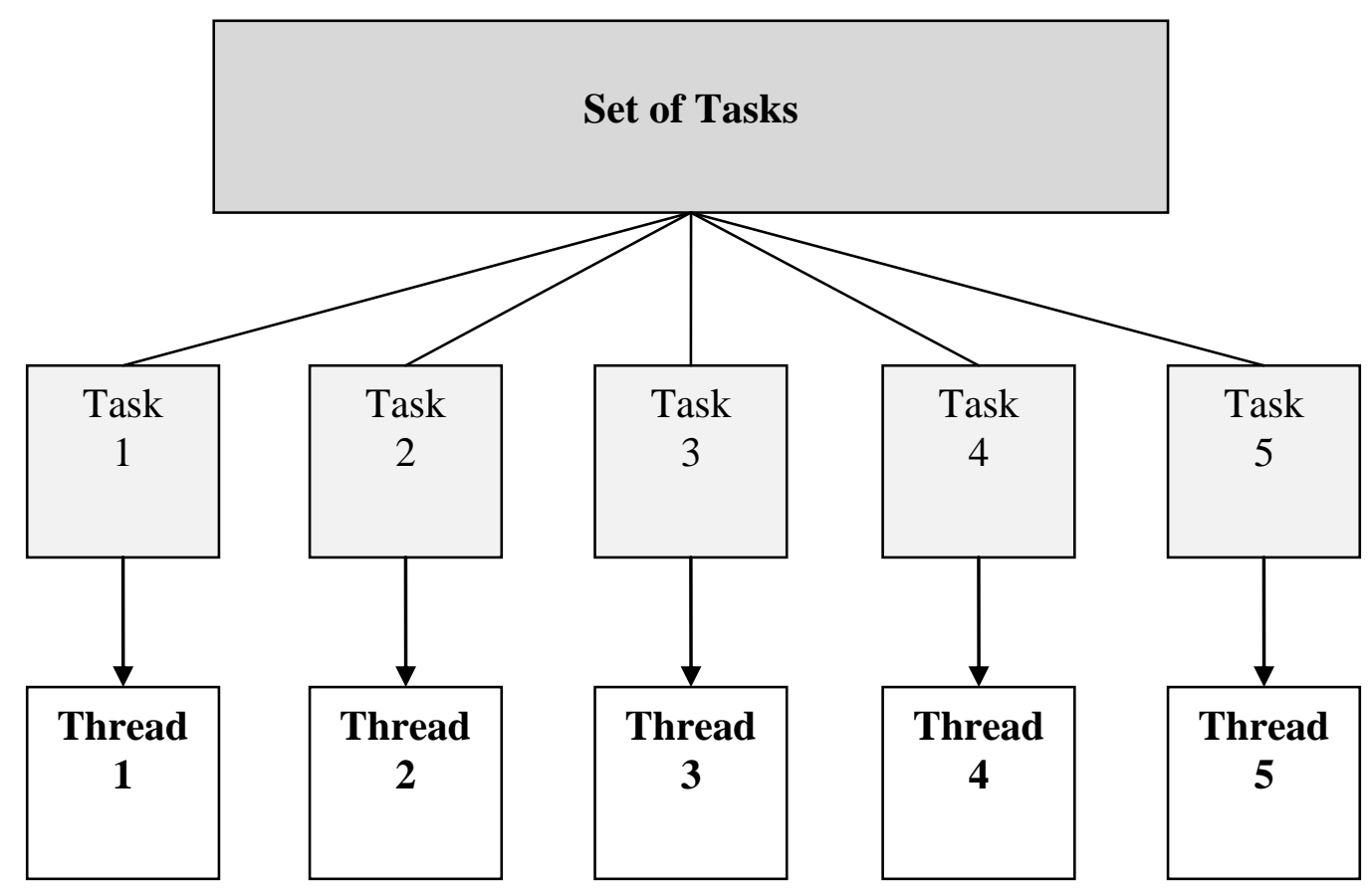

Figure 10. A Typical Task-level Parallelism 


\subsubsection{Overview of OpenMP}

Due to the nonstop increase in the number of shared-memory parallel computers, specially multi-core architectures, OpenMP was developed to implement the sharedmemory model. It enables the software programmers the ability to explicitly write parallel software applications that fully exploit and utilize parallel processing power of multi-core processors $[1,2]$.

Recall that OpenMP (Open Multi-Processing) is a shared-memory Application Programming Interface (API) that implements the shared-memory parallel programming model. OpenMP is not a programming language; it is rather a programming specification that provides application developers a powerful set of compiler directives that are inserted into a serial program written in $\mathrm{C}, \mathrm{C}++$, or Fortran to guide a target compiler to generate a corresponding parallel program to run on multi-core systems with minimal changes to the serial code. The OpenMP specification has been created and standardized $[2,42]$ as a result of mutual agreement among the members of Architectures Review Board (ARB) which now includes "almost all the major computer manufactures, major compiler companies, several government laboratories, and groups of researchers belong to the ARB.” [2]

OpenMP is portable, scalable, and easy-to-use. It hides most multi-threaded program development complexities from software programmers so that the programmers can resourcefully concentrate their design and problem analysis for program parallelization. The following section will give a high-level overview of the OpenMP programming or execution model, focus on discussion of certain important features that 
are associated with system design and implementation of this writing project, and present some practical OpenMP sample codes.

\subsubsection{OpenMP Programming Model and Features}

OpenMP provides an explicit, multi-threaded programming model for shared-memory multi-core platforms. In order to manage parallel execution of threads, OpenMP implements the fork-join thread execution model $[2,42]$. In this execution model, as illustrated in Figure 11, a program process starts with a single initial thread, called the master thread, which serially executes until it encounters a parallel region. A team of threads is created or forked to share the workload in the parallel region. After the parallel workload execution is completed, this team of threads is released or joined, which leaves only master thread to continue its serial program execution. The entire process repeats when a parallel region is encountered again.

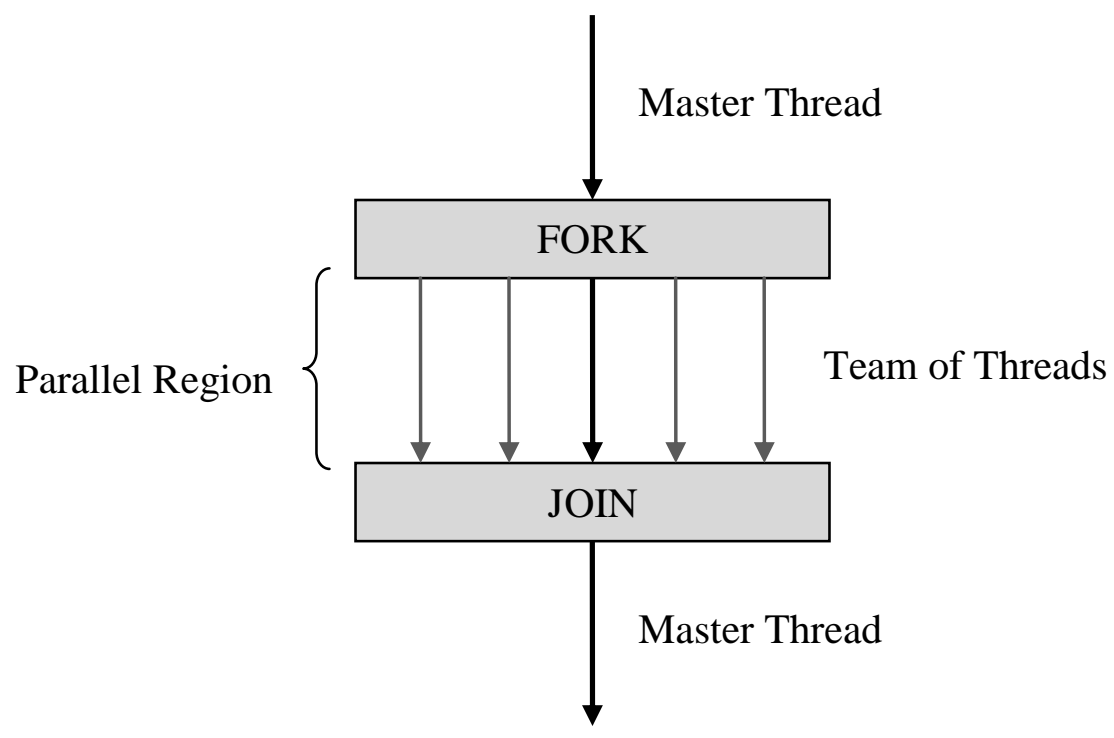

Figure 11. OpenMP Fork-Join Execution Model 
Parallel execution in the parallel region can be task-level parallelism or data-level parallelism. General OpenMP syntax structure to initiate a parallel region or parallel construct uses the "parallel" directive in a C source file, as shown in Figure 12:

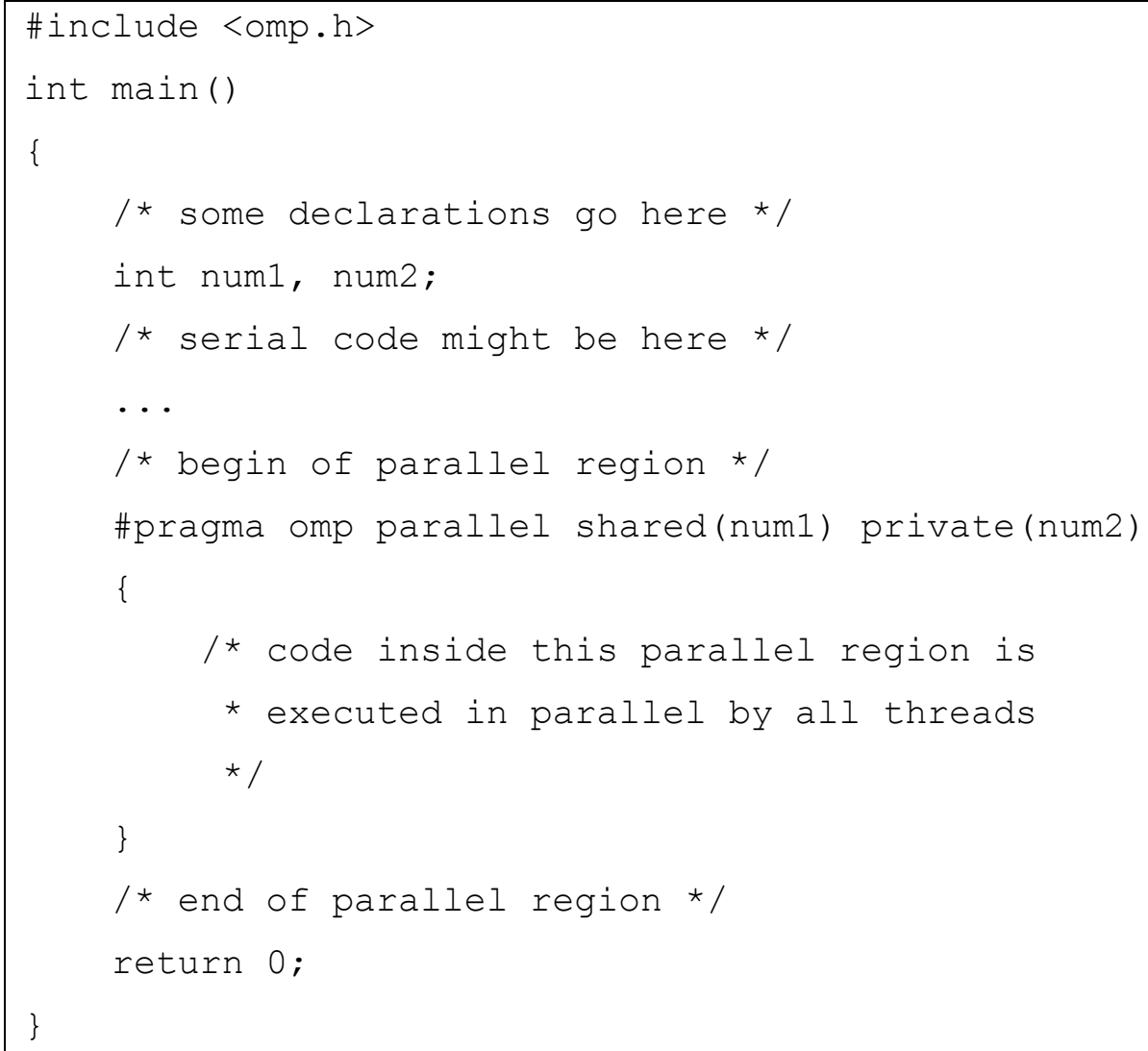

Figure 12. General OpenMP Syntax Structure

Main features of OpenMP come from its set of compiler directives to create worksharing constructs. General syntax format of an OpenMP directive is:

\section{\#pragma omp directive-name [clause,...] newline}

There are two major types of work-sharing constructs, FOR and SECTIONS, which represent data-level parallelism and task-level parallelism, respectively. These two 
constructs are created using the "for" and "sections" directives, which are summarized in Tables 3 and 4 [2, 42].

\begin{tabular}{|c|c|}
\hline $\begin{array}{l}\text { Work-sharing } \\
\text { Construct }\end{array}$ & Combined Syntax Format in $\mathrm{C} / \mathrm{C}++$ \\
\hline FOR & $\begin{array}{l}\text { \#pragma omp parallel for [clause...] newline } \\
\qquad \begin{array}{l}\text { schedule (type [, chunk]) } \\
\text { private (list) } \\
\text { shared(list) } \\
\text { reduction(operator: list) } \\
\\
\ldots \\
\text { for_loop }\end{array}\end{array}$ \\
\hline SECTIONS & 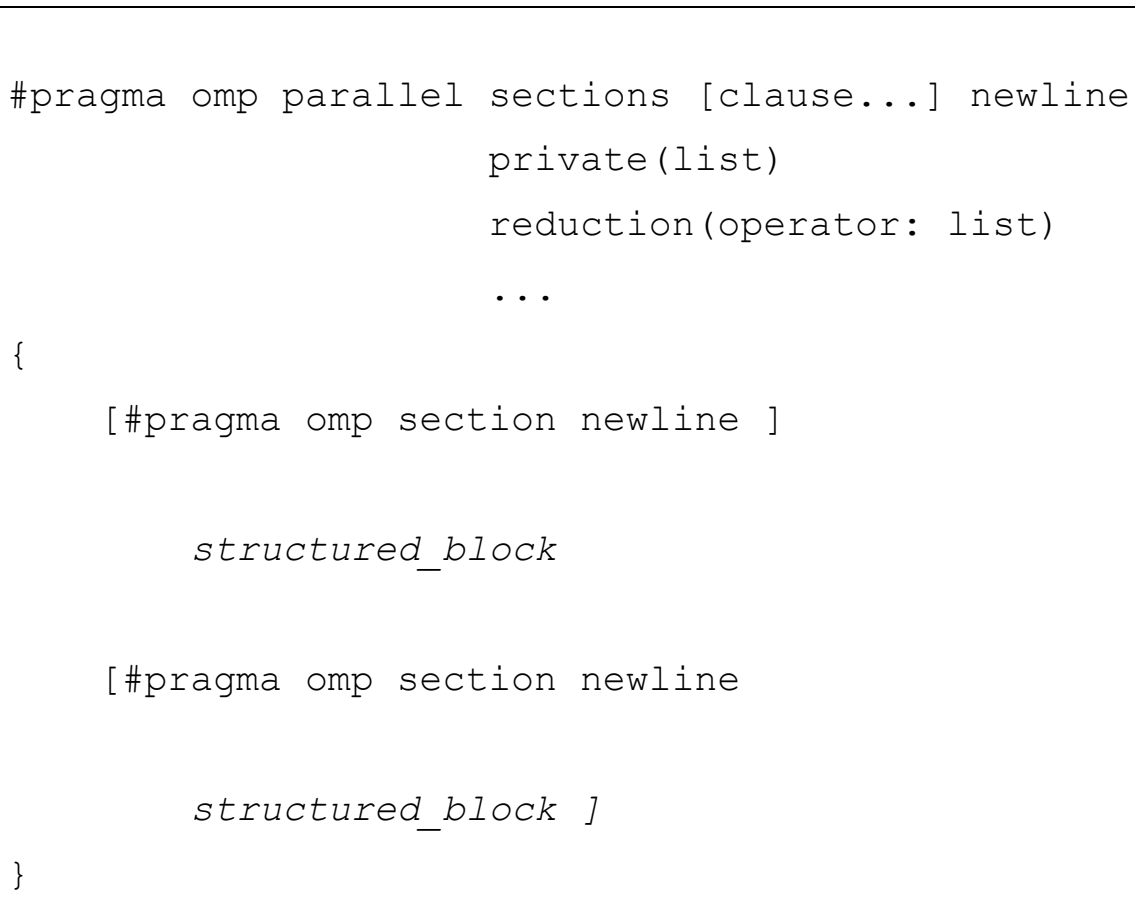 \\
\hline
\end{tabular}

Table 3. Syntax Format of Two Major OpenMP Work-sharing Constructs 


\begin{tabular}{|l|l|}
\hline $\begin{array}{l}\text { Work-sharing } \\
\text { Construct }\end{array}$ & $\begin{array}{l}\text { This work-sharing construct is initiated using the "for" directive. } \\
\text { As the name implies, this construct is to apply to loop-level } \\
\text { parallelization, specifically, representing data-level parallelism. The } \\
\text { construct must be enclosed within a parallel region that has been } \\
\text { initiated using the "parallel" directive. The "for" directive bears a } \\
\text { certain number of clauses to control the parallel execution with the } \\
\text { parallel region. OpenMP specification indicates that iterations of a } \\
\text { for loop that immediately follow this "for" directive would be } \\
\text { executed in parallel by corresponding team of threads in the region. } \\
\text { Distribution behavior of loop iterations depends on the "schedule" } \\
\text { clause, which will be described, along with other related clauses, in } \\
\text { the subsequent paragraphs below. }\end{array}$ \\
\hline SECTIONS & $\begin{array}{l}\text { This work-sharing construct is initiated using the "sections" } \\
\text { directive. This construct represents a type of functional- or task- } \\
\text { level parallelism. As with the FOR construct, this construct must be } \\
\text { enclosed within a parallel region that has been initiated using the } \\
\text { "parallel" directive. Within a "sections" directive, there are nested } \\
\text { "section" directives to specify different portions of code to be } \\
\text { executed in parallel by corresponding team of threads in the region. } \\
\text { Details of SECTIONS end here because this project is interested }\end{array}$ \\
\hline
\end{tabular}

Table 4. Description of Two Major OpenMP Work-sharing constructs 
Since the scope of this writing project is to focus on the FOR work-sharing construct, corresponding selective clauses of the OpenMP "parallel" and "for" directives are described in details in Table 5. These clauses are necessary and important enough to parallelize most common serial C programs.

\begin{tabular}{|c|c|}
\hline $\begin{array}{l}\text { Commonly-used } \\
\text { Clauses }\end{array}$ & Description \\
\hline num_threads & $\begin{array}{l}\text { num_threads (integer-expression) } \\
\text { This clause is used to specify number of threads to be created to share } \\
\text { workload in parallel region. A common problem associated with this } \\
\text { clause is threading overhead when it is wrongly used to create more } \\
\text { threads than needed. Program performance associated with the } \\
\text { number of threads also depends on the number of cores available in } \\
\text { the underlying system. Ideally, the number of threads should equal } \\
\text { number of cores available. }\end{array}$ \\
\hline shared & $\begin{array}{l}\text { shared (list) } \\
\text { This clause is used to specify that those variables enclosed in the list } \\
\text { are shared among the threads in parallel region. All threads in the } \\
\text { team can asynchronously access, such as read or write, the variables. } \\
\text { Users have the responsibility to correctly specify these variables in } \\
\text { order to avoid various multi-threaded problems, such as race } \\
\text { conditions, deadlocks, etc. }\end{array}$ \\
\hline
\end{tabular}




\begin{tabular}{|c|c|}
\hline private & $\begin{array}{l}\text { private (list) } \\
\text { This clause is used to specify that those variables enclosed in the list } \\
\text { are not shared or are private to each thread in parallel region. That is, } \\
\text { each thread in the team is given a unique local copy of each variable } \\
\text { so that the thread can safely modify or update the variable. Common } \\
\text { usage for this clause is to specify a list of variables whose values are } \\
\text { being modified or updated in the parallel region. }\end{array}$ \\
\hline default & $\begin{array}{l}\text { default (none|shared) } \\
\text { This clause is used to specify default scope for the variables in } \\
\text { parallel region. The default sharing scope for the variable can be } \\
\text { either none or shared. Note that private is not an option. }\end{array}$ \\
\hline reduction & $\begin{array}{l}\text { reduction (operator:list) } \\
\text { There are some cases where software programmers want to } \\
\text { incrementally update and store values of a variable, such as } \\
\text { summation problems. In some sense, the variable needs to be shared } \\
\text { because all threads in the team need to have access to the value; at the } \\
\text { same time, the variable also needs to be private because its value is } \\
\text { being modified which might result in race conditions. OpenMP } \\
\text { provides the users an easy way to implement this kind of recurrence } \\
\text { calculations via the use of reduction clause, where operator can be an } \\
\text { associative operator or a commutative operator that operates on the } \\
\text { variables in the list. There is no need to specify these variables in a } \\
\text { shared clause. }\end{array}$ \\
\hline
\end{tabular}




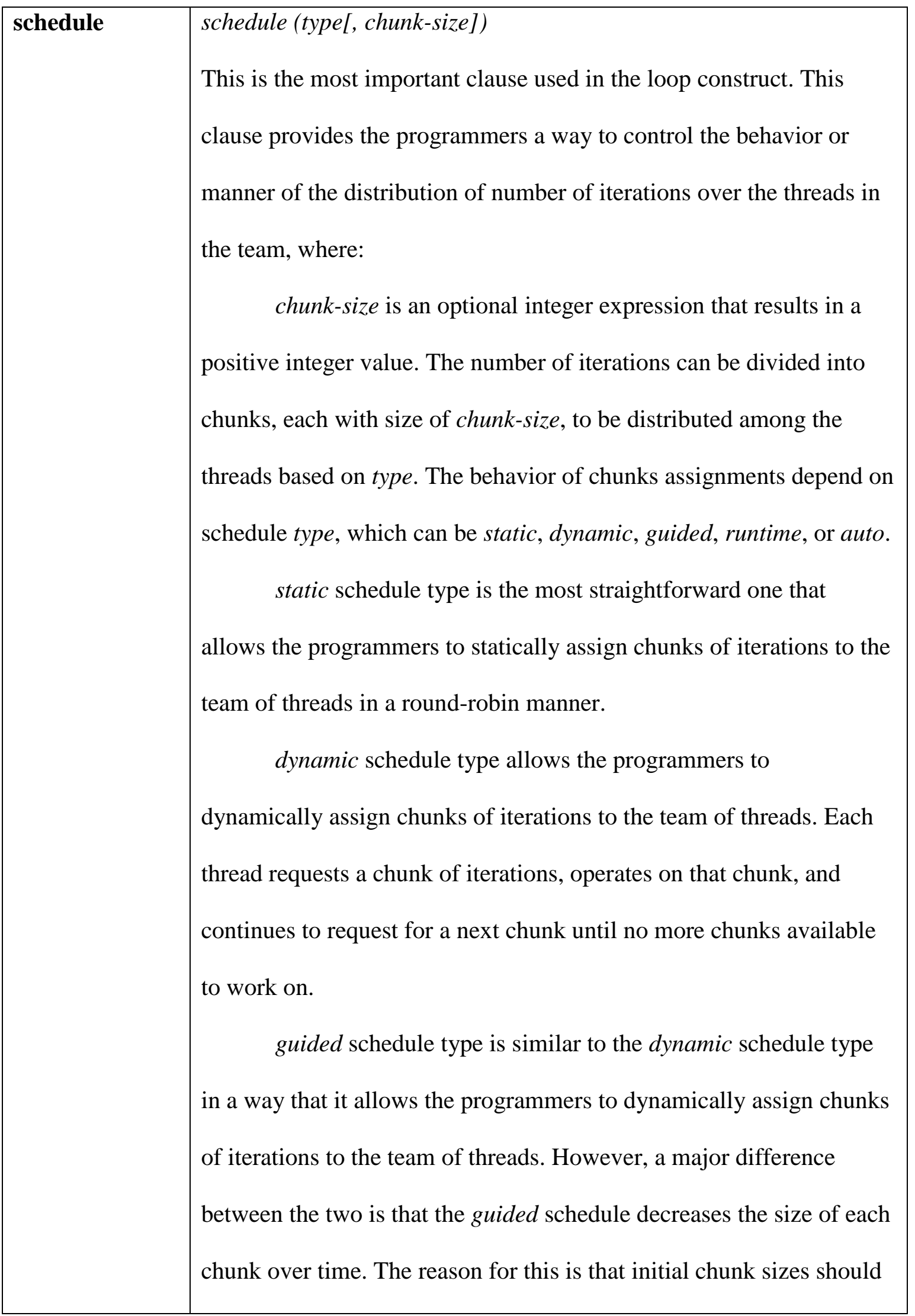




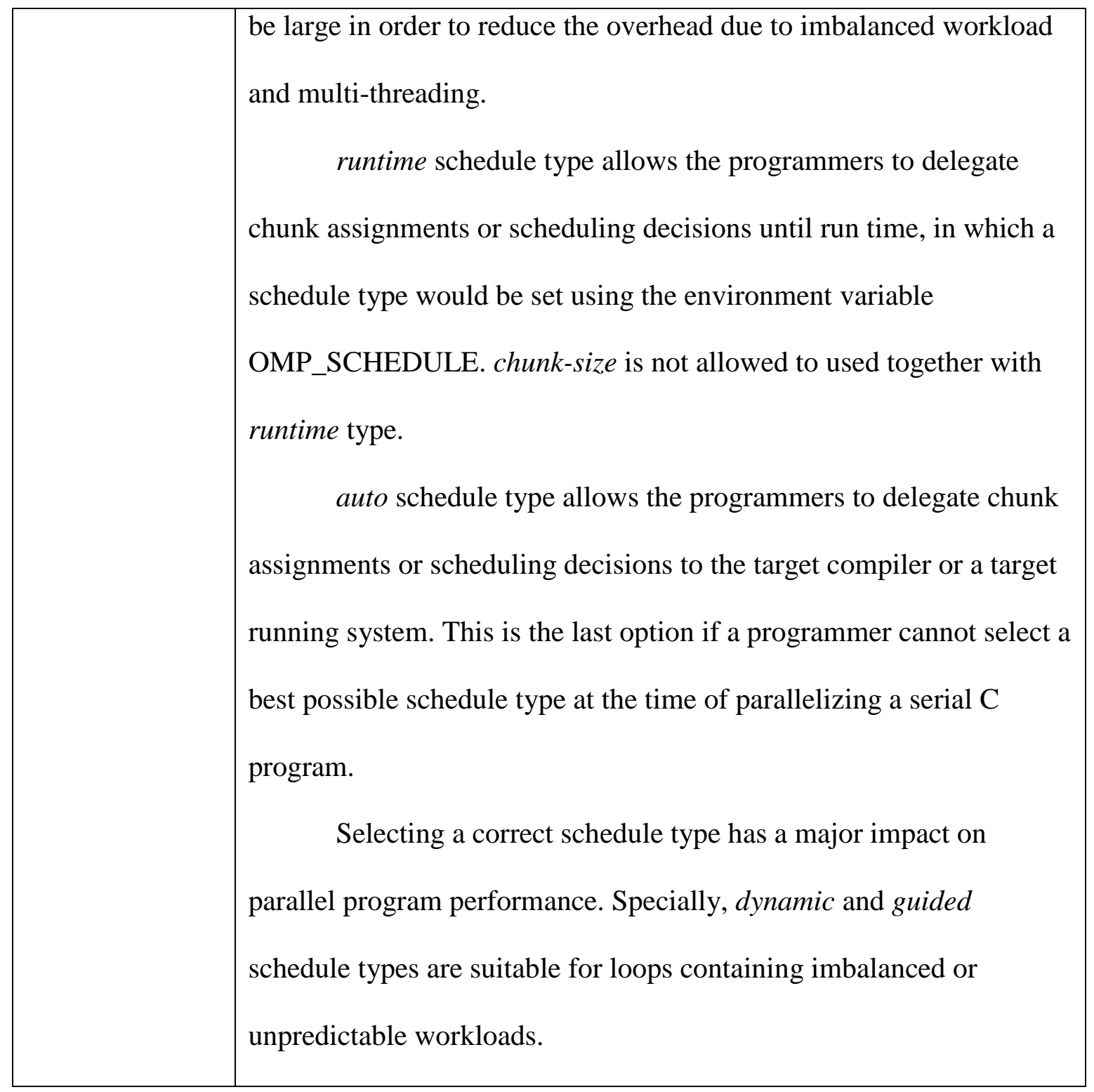

Table 5. Commonly-used Clauses of the "parallel" and "for" Directives

By hiding all complexities of underlying parallel problem analysis for serial program parallelization, creating an OpenMP program requires software programmers to follow two basic steps. In the first step, the programmers need to be able to analyze and identify portions of serial code that are potential "hot spots" for program parallelizations, which can be task-level or data-level parallelisms. In the second step, the programmers need to properly select and apply OpenMP compiler directives to achieve parallelism. 
The programmers have to do this parallel work incrementally, that is, repeatedly apply OpenMP directives and perform tests, until achieving a best possible or acceptable parallel program performance.

Figure 13 is an example of a simple OpenMP program, called dot-product that illustrates the important concepts of OpenMP.

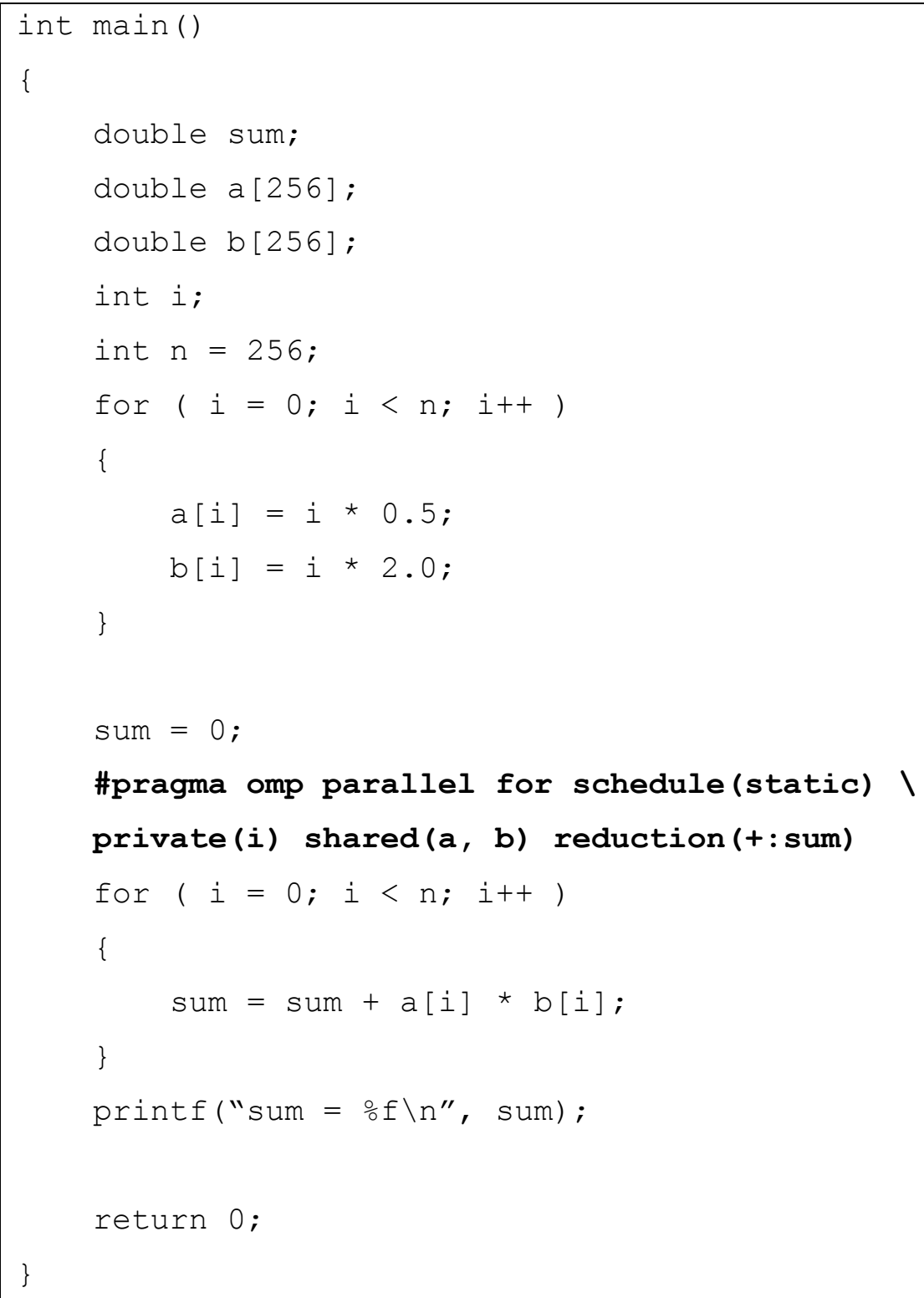

Figure 13. An Example of A Simple OpenMP Program 


\subsection{Overview of Machine Learning}

A short but precise definition of machine learning from the book titled Introduction to Machine Learning by Ethem Alpaydin [23] is: "Machine learning is programming computers to optimize a performance criterion using example data or past experience." In other words, machine learning is a scientific method to program a computer to solve a given problem from existing sets of data or sample records. Machine learning has been applied to various fields of study, including, but not limited to, artificial intelligence and data mining, resulted in many practical and intelligent machine learning applications found today, such as weather forecasting, Web data filtering, bioinformatics data pattern recognition, robot behavior optimization, sales target prediction, digital image processing, and so on $[3,4,23,21]$.

Machine learning and data mining are overlapping or inter-related to each other. Data mining is a process of exploring data to discover useful information for various technological and scientific decisions [3, 24], in which machine learning is one of major techniques used in the data discovery process. In fact, this writing project is a combination of machine learning and data mining; where prior knowledge or sets of data are OpenMP C program source files. The terms machine learning and data mining are used interchangeably throughout this report. Figure 14 shows the relationship among machine learning and data mining. [3] 


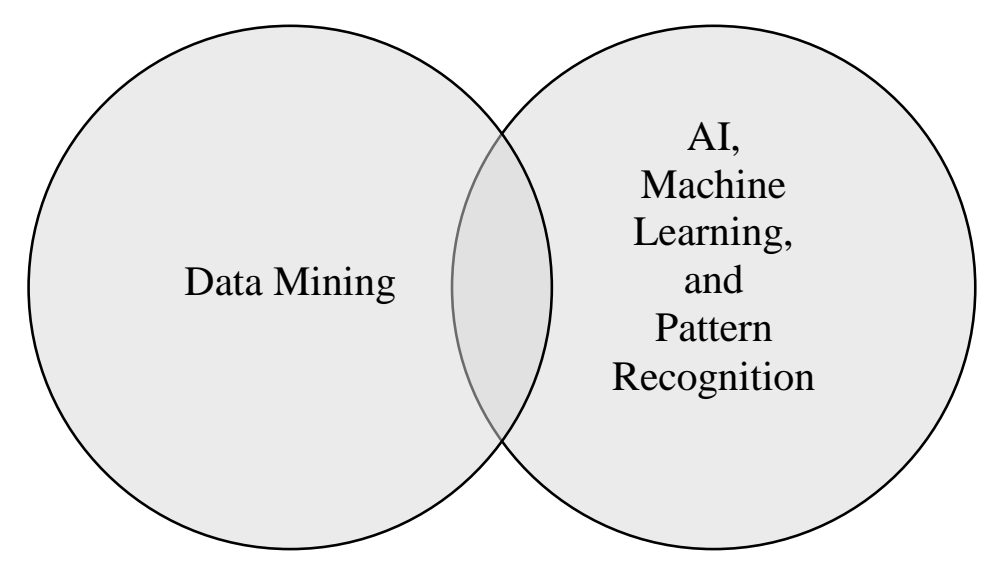

Figure 14. Relationship Among Machine Learning and Data Mining

\subsubsection{Concepts and Definitions}

Data is everything in machine learning and data mining. An existing set of data represent prior knowledge that is analyzed and learned by a learning algorithm in order to train and build an engine or a model for a specific machine learning task. A sample data set could be a collection of images of a road which would be used to train a robotic vehicle model; another example of a data set could be a collection of Web pages which would be used for target marketing prediction; and many more [21].

Data originally collected in raw format is not so useful to machine learning. In other words, collected data should be preprocessed to filter out noises and/or to extract useful information in order to produce a data set that has direct benefit to a learning algorithm. From this point on, the term "data set" conventionally refers to a ready-to-use set of data, which should have been preprocessed.

A data set contains a collection of data objects, which are usually referred to as data records or data instances $[3,4]$. Data sets are usually stored either in flat files or in a database system. Each data record is composed of a number of fields where each field is 
an attribute representing a property of that record. There are four different attribute types: nominal, ordinal, interval, and ratio. Direct definitions of these attribute types are extracted from the book titled Introduction to Data Mining by Pang-Ning Tan [3] and are summarized in Table 6.

\begin{tabular}{|c|c|c|}
\hline \multicolumn{2}{|c|}{ Attribute type } & Description \\
\hline \multirow{2}{*}{ 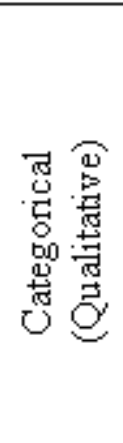 } & Nominal & $\begin{array}{l}\text { The values of a nominal attribute are just different names; i.e., } \\
\text { nominal values provide only enough information to distinguish } \\
\text { one object from another. Comparison operations: }(=, \neq)\end{array}$ \\
\hline & Ordinal & $\begin{array}{l}\text { The values of an ordinal attribute provide enough information to } \\
\text { order objects. Comparison operations: }(<,>)\end{array}$ \\
\hline \multirow{2}{*}{ 兽 } & Interval & $\begin{array}{l}\text { For interval attributes, the differences between values are } \\
\text { meaningful, i.e., a unit of measurement exists. Comparison } \\
\text { operations: }(+,-)\end{array}$ \\
\hline & Ratio & $\begin{array}{l}\text { For ratio variables, both differences and ratios are meaningful. } \\
\text { Comparison operations: }(*, n)\end{array}$ \\
\hline
\end{tabular}

Table 6. Summary of Four Different Attribute Types [3]

Data records that are used to train a machine learning model for a specific data mining task are called training records or training instances. Table 7 contains an example training set for a tax defaulted borrower detection problem. 


\begin{tabular}{|l|l|l|l|l|}
\hline ID & Refund & $\begin{array}{l}\text { Marital } \\
\text { Status }\end{array}$ & $\begin{array}{l}\text { Taxable } \\
\text { Income }\end{array}$ & $\begin{array}{l}\text { Defaulted } \\
\text { Borrower }\end{array}$ \\
\hline 1 & Yes & Single & $125 \mathrm{~K}$ & No \\
2 & No & Married & $100 \mathrm{~K}$ & No \\
3 & No & Single & $70 \mathrm{~K}$ & No \\
4 & Yes & Married & $120 \mathrm{~K}$ & No \\
5 & No & Divorced & $95 \mathrm{~K}$ & Yes \\
6 & No & Married & $60 \mathrm{~K}$ & No \\
7 & Yes & Divorced & $220 \mathrm{~K}$ & No \\
8 & No & Single & $85 \mathrm{~K}$ & Yes \\
9 & No & Married & $75 \mathrm{~K}$ & No \\
10 & No & Single & $90 \mathrm{~K}$ & Yes \\
\hline
\end{tabular}

Table 7. Sample Training Instances of Tax Defaulted Borrower Problem [3]

Machine learning tasks are categorized into two groups: predictive tasks and descriptive tasks $[3,21]$. Predictive tasks include classification and regression, whereas descriptive tasks include association and clustering. The objective of predictive tasks is to predict the value of a specific attribute of a new data instance based on the availability of other attribute values in the data instance. On the other hand, the objective of descriptive tasks is to discover interesting patterns or relationships in the data. Each task is briefly described below, according to [3, 23, 21], to present enough information to better understand the machine learning task selected for this writing project.

Classification model - The task of this model is to classify a new data record into one of several predefined categorical values of an attribute. This attribute is called the output or target attribute, and its nominal values are called classes. An example of a classification problem could be a prediction of risk levels of banking customers. The model, often called the classifier, would learn all provided past information about the 
customers, including three known classes of risk levels, such as low-risk, medium-risk, and high-risk. The classifier is now able to predict or classify a new customer into one of the three classes of risk levels based on corresponding information obtained from the new customer. The training data set of this example can be visualized in Figure 15, where each square corresponds to a training instance that belongs to each of the three predefined classes, low-risk ('-`), medium-risk ('o’), and high-risk ('+’). For simplicity, only two input attributes, debt and income, are presented.

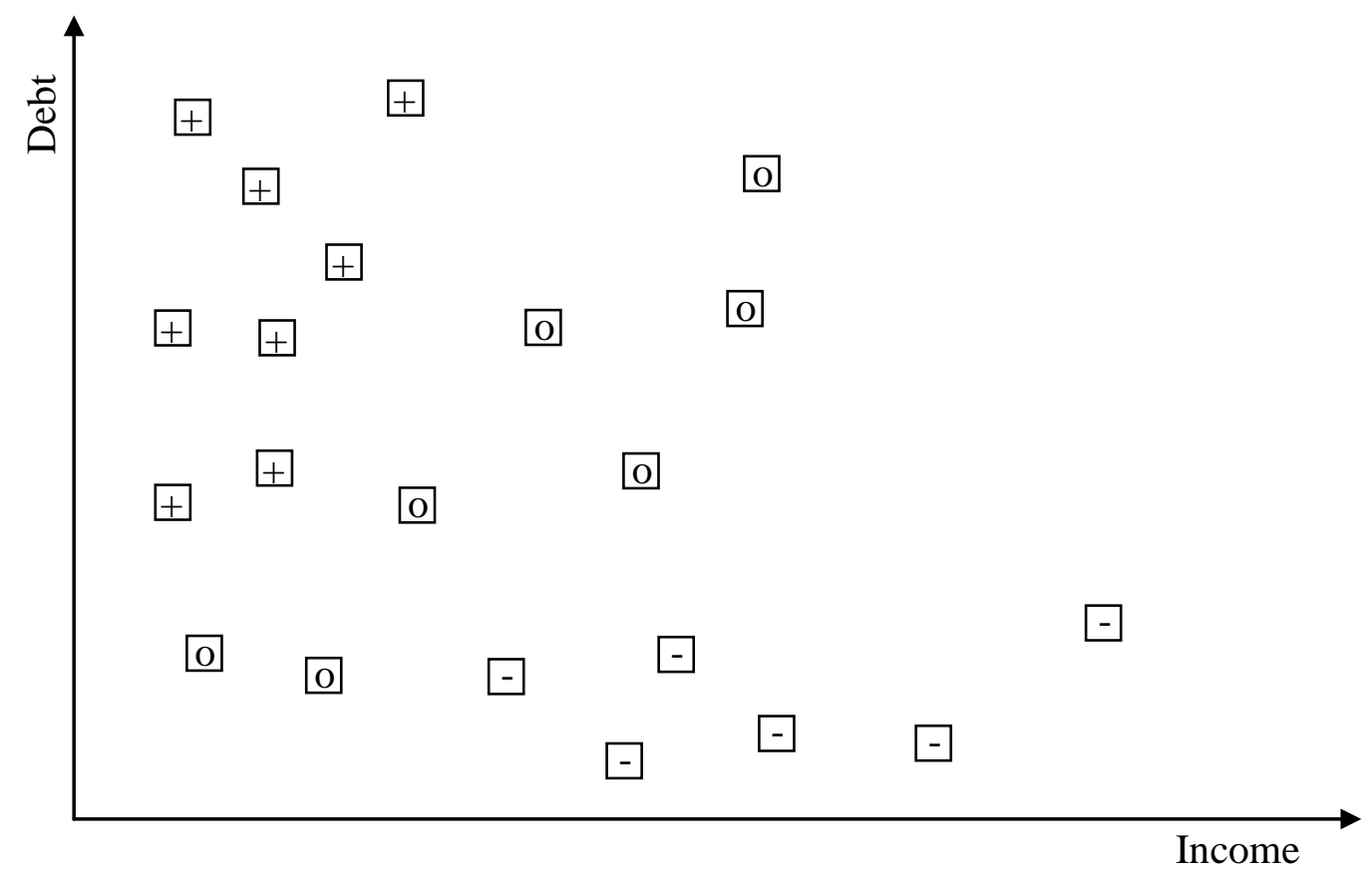

Figure 15. Sample Training Data of the Classification Problem Example

Regression model - The objective of this model is to predict a real value of the target attribute of a new unknown data instance. Since the output attribute value is a number, a continuous value, it is called the regression model. An example of a regression problem could be a prediction of used car prices. Each training data instance for the 
model might have a corresponding set of attributes about a used car, such as make, model, year, mileage, and a known price. For simplicity, let $\mathrm{X}$ be the input attribute, "Mileage" and Y be the output or target attribute "Price." The training data set of this regression example can be visualized in Figure 16.

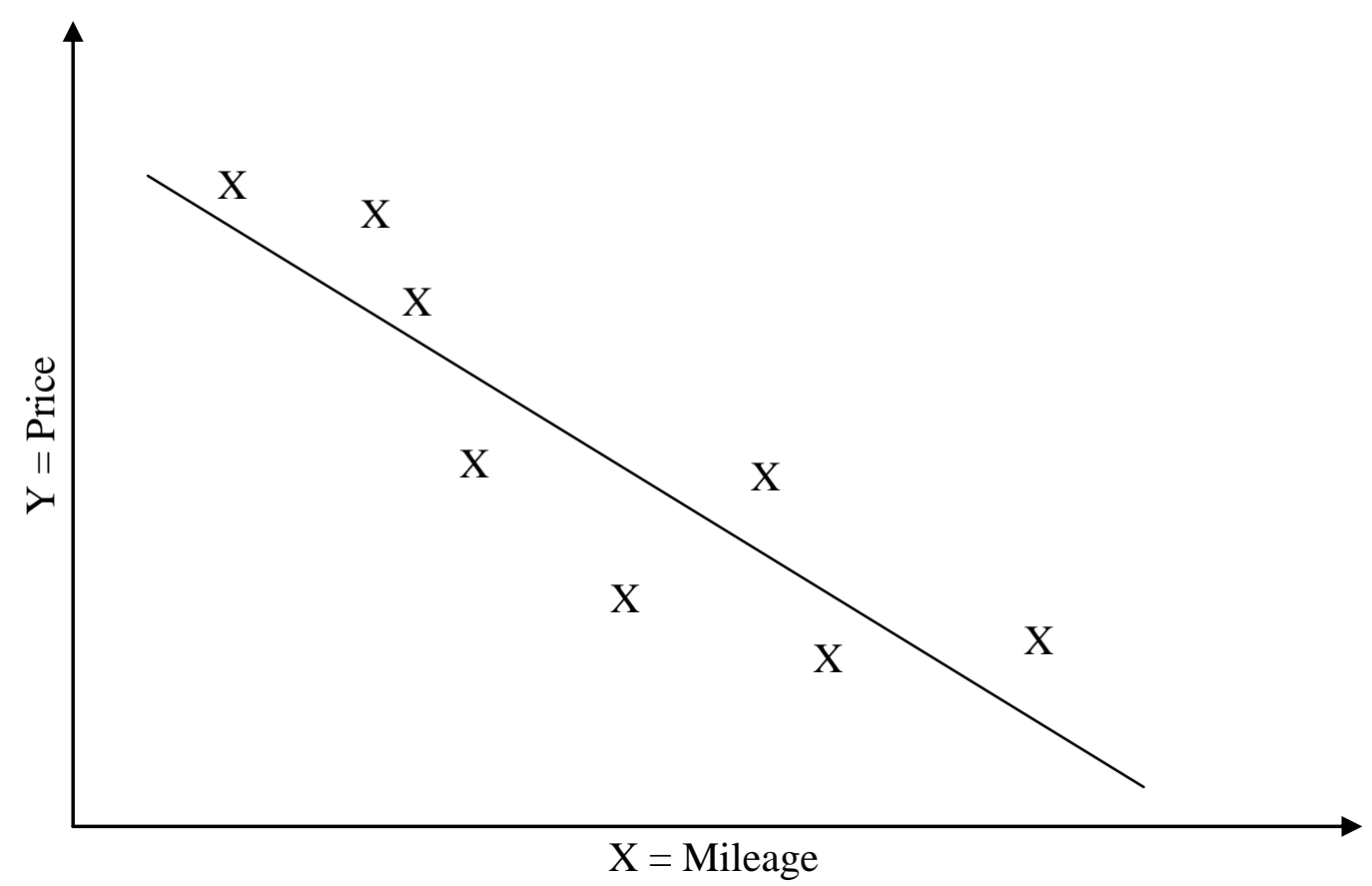

Figure 16. Sample Training Data of the Regression Problem Example

Association and clustering models - These two descriptive tasks are not the scope of this project, so they are briefly described here. An association task aims to discover or to extract certain patterns of associated features in provided data sets, whereas a clustering task is to group related or similar data items into clusters for various information analyses. Figure 17, a snapshot from [3], efficiently visualizes the difference between these two descriptive data mining tasks. 


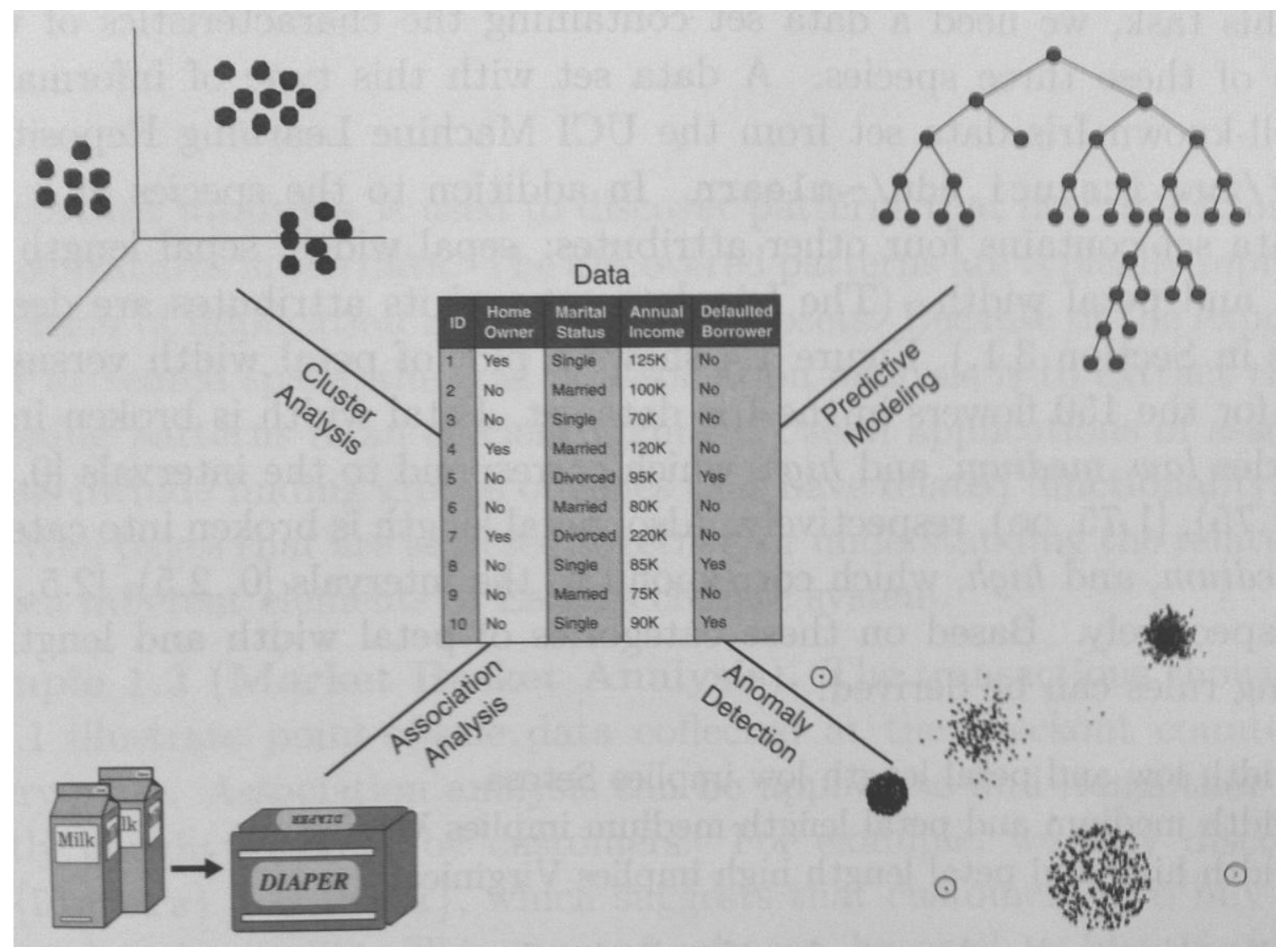

Figure 17. Core Data Mining Tasks [3]

Another important concept in machine learning is the difference between supervised learning and unsupervised learning. In supervised learning, both input attributes and the correct corresponding output or target attribute of each record are given in a data set. Thus, the purpose of supervised learning is to train a machine learning model using data sets with known correct outputs, the model being supervised, so that the resulting model can be applied to a new record to derive a best possible output or result. In unsupervised learning, the data contains only input attributes, or we have only input data. The task of a machine learning model is to find or discover patterns or relationships among the input data items for various purposes in different fields of study. 


\subsubsection{End-to-End Process}

This section describes a high-level overview of an end-to-end process in data mining. Details of the entire process are dedicated to the Design and Implementation section which presents the process with actual data and explanation. A data mining process consists of a sequence of steps, which can be generalized in Figure 18. The process is similar to Knowledge Discovery in Database (KDD) [3].

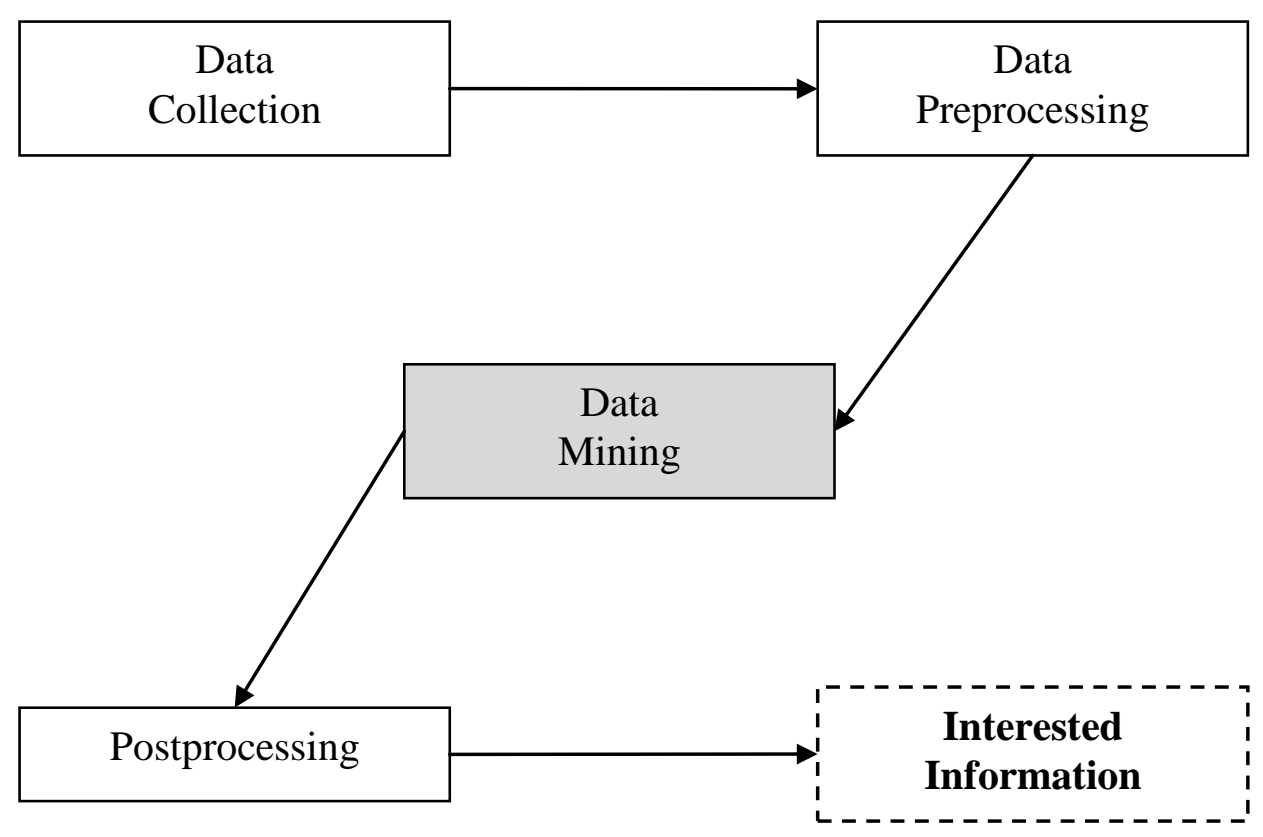

Figure 18. General Process of Data Mining

Data collection is the first important step in data mining or machine learning process. At this step, interested data is collected to solve a given problem. Quality of collected data is important because it is everything that a computing system has or is based upon to build a best possible corresponding machine learning model to solve the problem. Depending on the nature of the problem being solved, the data could be 
systematically constructed from natural information saved over time in a database or from various statistical data sources. In general, the various algorithms that are used should tolerate quality problems, which are unavoidable in naturally collected data [3]. However, this is not the case in this writing project because interested data is systematically analyzed and constructed, resulted in high quality data being used for the project.

Data preprocessing is the next important step that involves one or more preprocessing phases, such as data transformation, data filtering, and/or data features extraction. Collected data in the first step is usually called raw data, which might not be suitable to train and build a target machine learning model. Therefore, preprocessing phases are needed to build proper training data sets that are ready for model training and system building.

Data mining is obviously a major step in the entire process where all the intelligence is made. Ready-to-use data sets provided from the previous step are used to train and build a machine learning model for a particular data mining task to solve the problem. A data mining task can be carried out using one of the machine learning techniques, such as decision tree classifiers, rule-based classifiers, instance-based classifiers, support vector machines, and many more [3]. Each technique can be implemented via a variety of machine learning algorithms, including, but not limited to, Hunt's algorithm, sequential recovering algorithm using learn-one-rule function, knearest neighbor algorithm, and so on. After the model is built and tested, it is ready to carry out the data mining task. 
Postprocessing is the step where the results produced from applying the model in previous step are processed, if it is necessary, to generate meaningful or interested information. This concludes a general data mining process. Interested information now can be used for various scientific and/or technological decisions.

\subsubsection{Selected Techniques and Algorithms}

There are numerous machine learning techniques. This section covers only two important classification techniques whose resulting models are usually called decision tree classifier and instance-based classifier. The decision tree classifier is presented here as an introduction to the classification task to describe the basic concepts about classification in machine learning and data mining. The instance-based classifier is the learning model chosen for this writing project due to nature of its benefits.

\section{The decision tree classifier:}

This classification technique is simple but widely used to solve various data mining problems. In order to explain how a decision tree works, for simplicity, let us consider a weather problem with the sample data set presented in Table 8, where the target attribute is "Play." This weather data set is directly extracted from [4] and contains a small number of training records, which is good for illustration purposes.

\begin{tabular}{|l|l|l|l|}
\hline Outlook & Humidity & Windy & Play \\
\hline sunny & high & false & no \\
sunny & high & true & no \\
overcast & high & false & yes \\
rainy & high & false & yes \\
rainy & normal & false & yes \\
\hline
\end{tabular}




\begin{tabular}{|l|l|l|l|}
\hline rainy & normal & true & no \\
overcast & normal & true & yes \\
sunny & high & false & no \\
sunny & normal & false & yes \\
rainy & normal & false & yes \\
sunny & normal & true & yes \\
overcast & high & true & yes \\
overcast & normal & false & yes \\
rainy & high & true & no \\
\hline
\end{tabular}

Table 8. The Weather Problem Data [4]

From the sample weather training data set, the goal of a decision tree classifier is to learn and build a corresponding decision tree representation or abstraction. To classify a new unknown instance, the classifier walks its decision tree with the input attributes until it reaches a leaf node, which is the target class that the instance belongs to. So, the only question left is how to construct a decision tree with a given training data set. There are "many existing decision tree induction algorithms, including ID3, C4.5, and CART." [3] This paper describes a simple divide-and-conquer [4] algorithm called Hunt's algorithm [3].

The algorithm can be expressed verbally and recursively as follows: start with an attribute $\boldsymbol{t}$ as a root node. If all the records under $\boldsymbol{t}$ belong to the same class $\boldsymbol{y}_{\boldsymbol{t}}$, then $\boldsymbol{t}$ becomes a leaf node labeled class $\boldsymbol{y}_{\boldsymbol{t}}$. If all the records under $\boldsymbol{t}$ belong to more than one classes, then split the records into subsets based on attribute values of $t$; this is also called attribute test condition. This process now can be repeated and recursively applied to each subset of the records. 
By applying the algorithm to the weather problem data set, a resulting decision tree is presented in Figure 19.

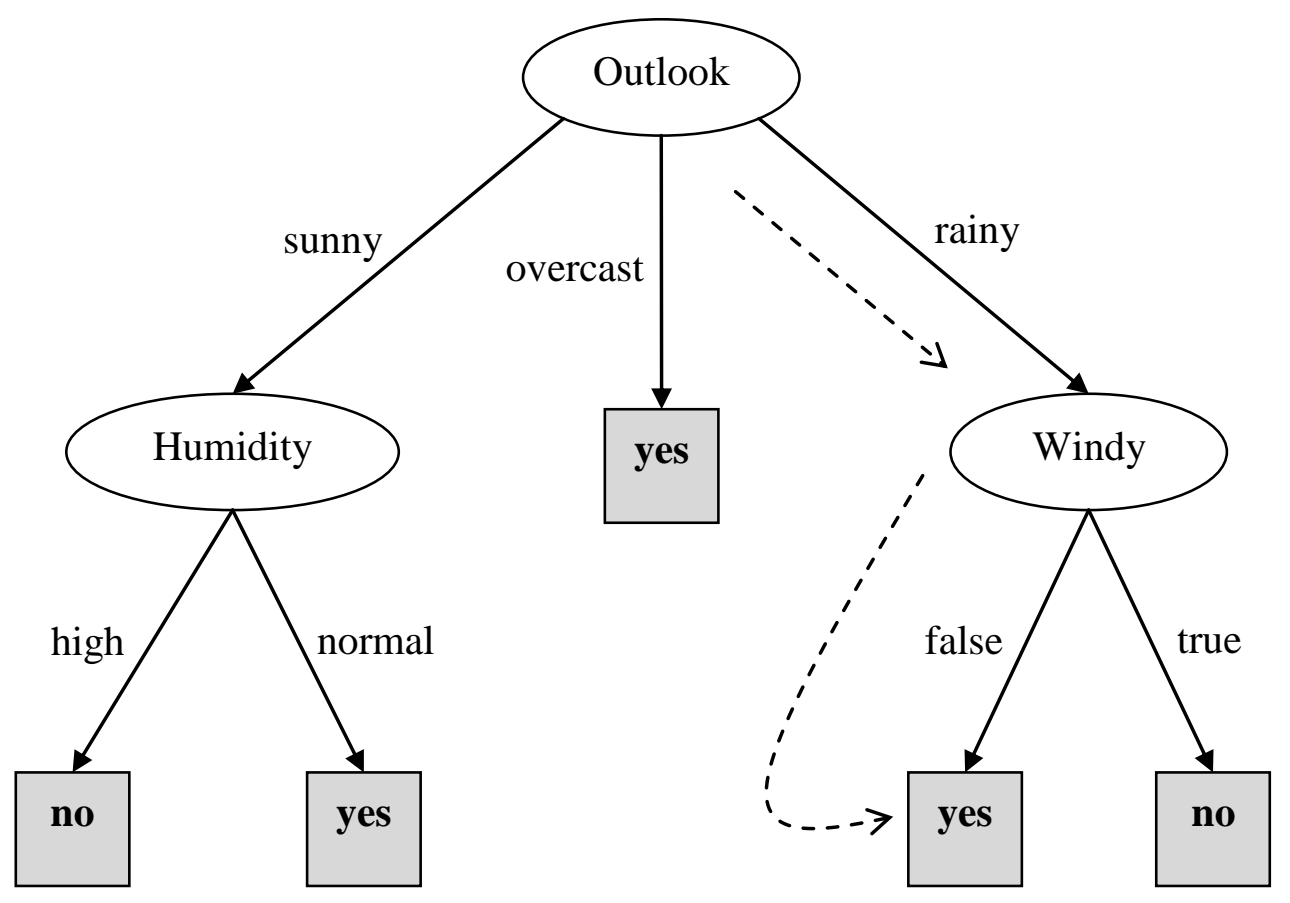

Figure 19. A Decision Tree for the Weather Classification Problem

Given a sample new unknown instance of the weather problem in Table 9, by walking the resulting tree in the figure above, the target class label can be determined via dotted arrows. The record would be classified as yes.

\begin{tabular}{|l|l|l|l|}
\hline Outlook & Humidity & Windy & Play \\
\hline rainy & normal & false & $? ? ?$ \\
\hline
\end{tabular}

Table 9. A New Unknown Instance of the Weather Problem 


\section{The instance-based classifier:}

The decision tree presented above is an example of eager learners because it is designed to immediately learn and build a model from available training records. Instance-based, however, presents an opposite approach. It delays the learning process until it needs to classify a new unknown instance or a test sample record [3, 4]; therefore, it is so called a lazy learner.

The idea behind instance-based classifier is that, as the name implies, it classifies a new unknown instance based on available training instances. Thus, knowledge of an instance-based classifier comes directly from the set of training records themselves. The classifier does not have to generalize the training set into some kind of representation or abstraction. The process of building an instance-based model can be thought to be transparent. The model building process is employed via a mechanism that saves or memorizes the training instances along with an associated distance-metric function to compute distances between each training instance and a new test instance. In the simplest form, this new test instance would be classified in the same class of the closest training instance. The number of closest training instances can be $1,2,3, \ldots, \mathrm{k}$; therefore, the most commonly-used algorithm in designing an instance-based classifier is so called the k-nearest neighbor classification algorithm [3].

The k-nearest neighbor algorithm can be expressed verbally as follows: Let D be a set of training instances, and compute distance between the test instance and each of the training instances. Then select $\boldsymbol{k}$ closest training instances to the test instance to create a list of the nearest-neighbors. The test instance is then classified based on the majority 
class label found in the list. Figure 20 shows the k-nearest neighbors of a test instance, where $\mathrm{k}=1,2,3$.
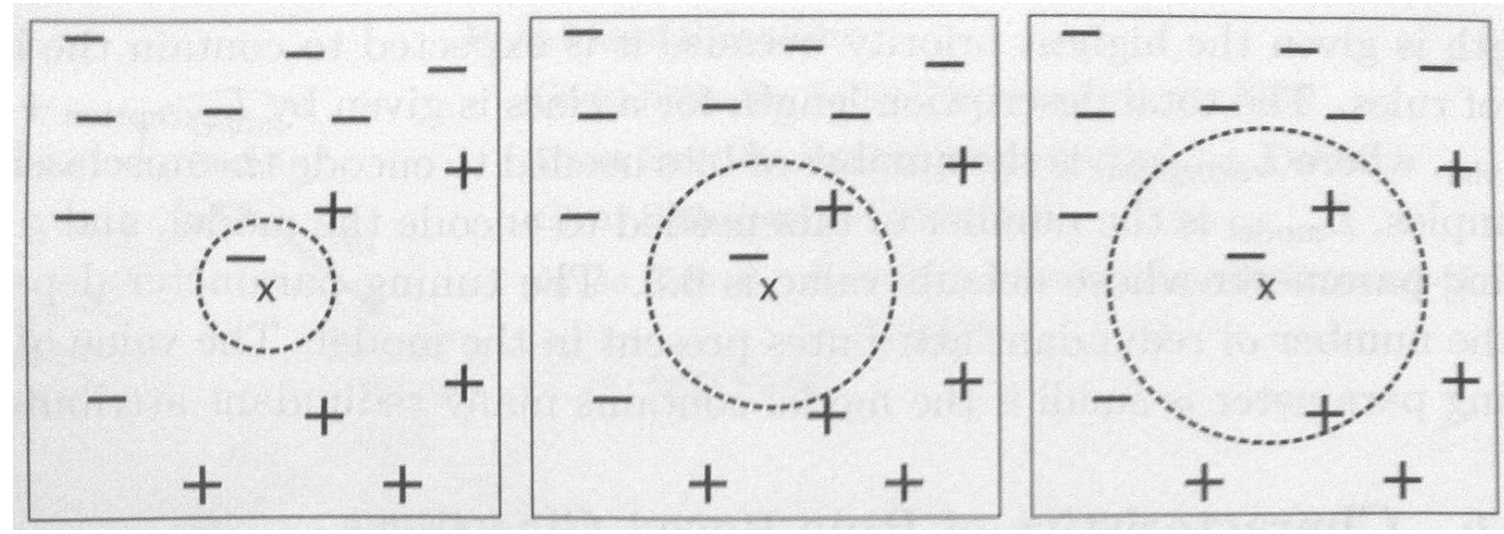

Figure 20. The 1-, 2-, and 3-Nearest Neighbors of an Instance [3]

The only question left is how to compute the distance between any given two instances. The most common method is to use the Euclidean distance formula: Let $\boldsymbol{d}$ be the distance between two points $\boldsymbol{x}$ and $\boldsymbol{y}$ in $\boldsymbol{k}$ dimensional space. The formula is defined in Figure 21. In relation to the k-nearest neighbors algorithm, $\boldsymbol{d}$ is the distance between two instances, $\boldsymbol{x}$ and $\boldsymbol{y}$, where $\boldsymbol{x}_{\boldsymbol{k}}$ and $\boldsymbol{y}_{\boldsymbol{k}}$ are $\mathrm{k}^{\text {th }}$ attributes of $\boldsymbol{x}$ and $\boldsymbol{y}$, respectively.

$$
d(\mathbf{x}, \mathbf{y})=\sqrt{\sum_{k=1}^{n}\left(x_{k}-y_{k}\right)^{2}}
$$

Figure 21. The Euclidean Distance Formula [3] 


\subsection{Concept of Compiler Design}

Just like multi-core OpenMP programming and machine learning, compiler design is another advanced subject that contributes to the inspiration, core design, implementation, and, eventually, completion of this writing project.

A compiler is a translator [5] that translates a program written in a high-level programming language, such as $\mathrm{C}, \mathrm{C}++$, or Java, into a low-level machine language that the target machine understands and executes. At a highest level, a compiler can be illustrated in Figure 22.

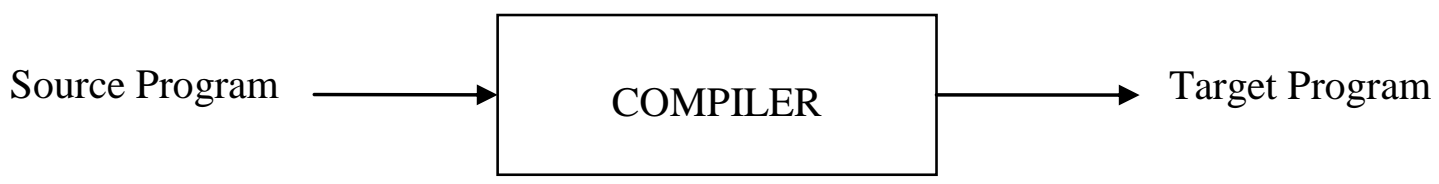

Figure 22. A High-level View of A Compiler

Compilers are everywhere in programming world and play a vital role in software development. One can imagine that behind a successful programming project, either a high-level application or an embedded system, the core contribution of this extremely important special tool, the compiler, should not be taken for granted [5].

Details in depth of an end-to-end compiler design process is not the purpose of this literature review. The following subsections present fundamental but essential concepts of a compiler design framework that practically applies to the design and implementation of this writing project. 


\subsubsection{General Framework of a Typical Compiler}

At a high-level overview, a typical compiler begins its work by scanning a program written in the source language to construct tokens according to the language's lexical specification. The compiler then parses the sequences of tokens to build the corresponding parse tree, also called Abstract Syntax Tree (AST), according to the language's syntactic specification or grammar. The AST is then processed to produce executable target machine code, which is also sometimes called binary code or object code. As a result, a compiler can be practically broken down into three different parts or phases [5]: the front-end, the intermediate tier, and the back-end, as illustrated in Figure 23. Details of each phase are described in each of the next three subsections.

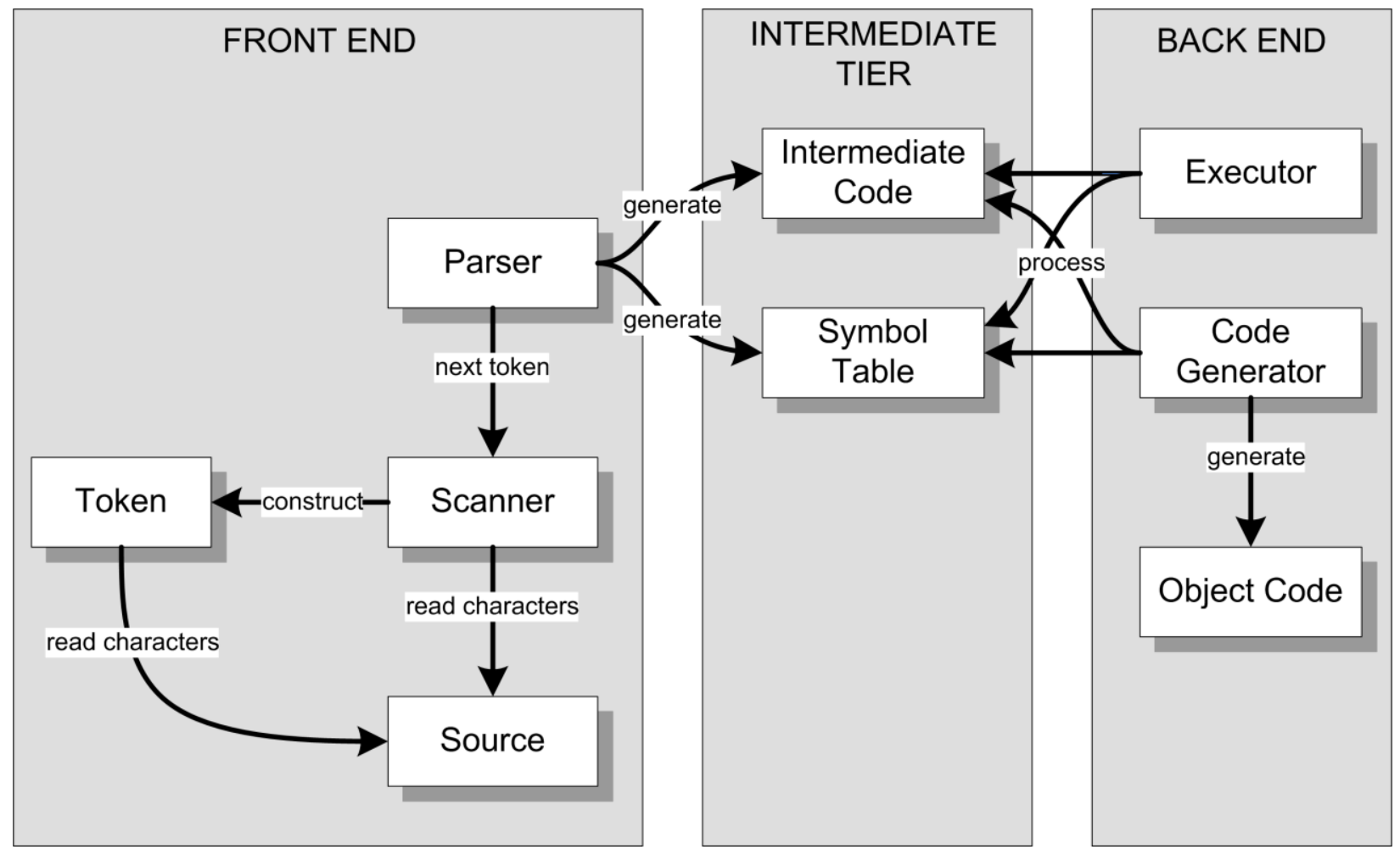

Figure 23. General Framework of a Typical Compiler [5] 


\subsubsection{The Front-End}

The front-end is source language-specific. It must know the programming language grammar, which is a set of rules that specifies the syntax for the language. The source code of a programming language is composed of a variety of language elements called tokens. A token is simply a string of one or more characters that contributes to the language symbol, which can be categorized as an identifier, a number, a character, or any reserved word, and so on. Table 10 gives an example of tokens encountered in the $\mathrm{C}$ language statement:

$$
\text { int } x=5 \text {; }
$$

\begin{tabular}{|l|l|}
\hline Token & Token Type \\
\hline int & reserved word \\
$=$ & identifier \\
5 & assignment operator \\
$;$ & number \\
\hline
\end{tabular}

Table 10. Some C Language Tokens

Two major components of the front-end are scanner and parser. The job of a scanner is to sequentially process the source code one character at a time, according to the language lexical specification, in order to construct tokens for a corresponding parser $[5,35]$. The scanner is also called a lexical analyzer [5] or a tokenizer [38].

According to [5], the scanning algorithm of a scanner can be expressed verbally as follows: The scanner skips any blank characters until it encounters the first character that is non-blank. This current character signals what the next token should be, and it 
becomes the first character of that token. The scanner then constructs or extracts the token by consuming all the characters that belong to that token. The next current character is then determined and the scanning process repeats again. Thus, a result of a scanning process is a sequence of tokens that are ready to be processed by a corresponding parser.

The second important component of the front-end is the parser. The parser understands the grammar of the source language. It receives sequences of tokens from the scanner and makes sure that the tokens are constructed correctly according to the language syntactic specification; hence the parser is also called syntactic analyzer. In that sense, the parser parses the source program according to the grammar and translates the results into intermediate code along with associated symbol tables. Intermediate code and symbol table are the two components representing the next phase in compiling process, the intermediate tier.

The grammar of a programming language can be specified using graphical syntax diagrams or it can be represented using Backus-Naur Form (BNF). Figure 24 shows an example of a tiny grammar of a digit expressed both as a syntax diagram and in BNF. 


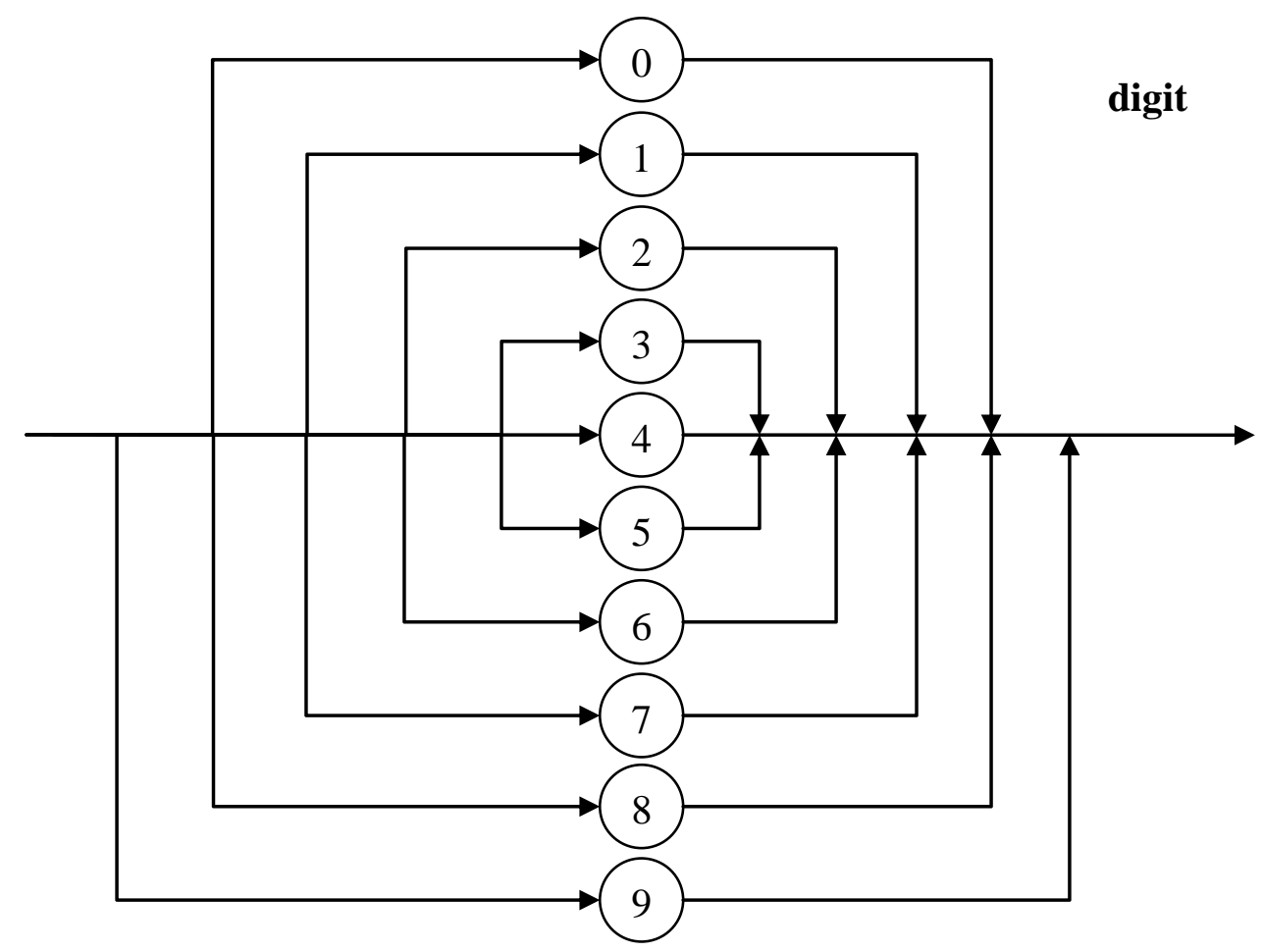

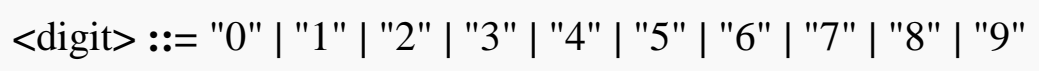

Figure 24. Syntax Diagram and BNF of Digit Grammar

In general, compiler designers do not write a compiler's scanner and parser from scratch $[5,38]$. They use a special tool called a compiler-compiler to write compilers. One of the most popular compiler-compilers is the JavaCC, which is also the tool chosen for this project. It is presented in the next section in greater details.

\subsubsection{The Intermediate Tier}

The symbol table and intermediate code are the two components of this phase, the intermediate tier. Intermediate code is also called intermediate representation, the result 
generated from the front-end phase. Both the intermediate representation and the symbol table are portable and source language-independent [5]. Together they encapsulate all the source program information needed by the back-end for various processing purposes.

The symbol table is a storage structure used to store any useful information found about the source program elements during the parsing process. It is commonly implemented as a stack data structure to allow the compiler to access the information at different scope levels. One example is to use the symbol table to store information about an identifier [5], including, but not limited to, name of the identifier, type of the identifier, location in the source code that it is declared and defined, and so on. Figure 25 is directly extracted from [5] to show structure of the symbol table. Collectively, we can have multiple symbol tables pushed onto a symbol table stack to provide different accessscope levels.

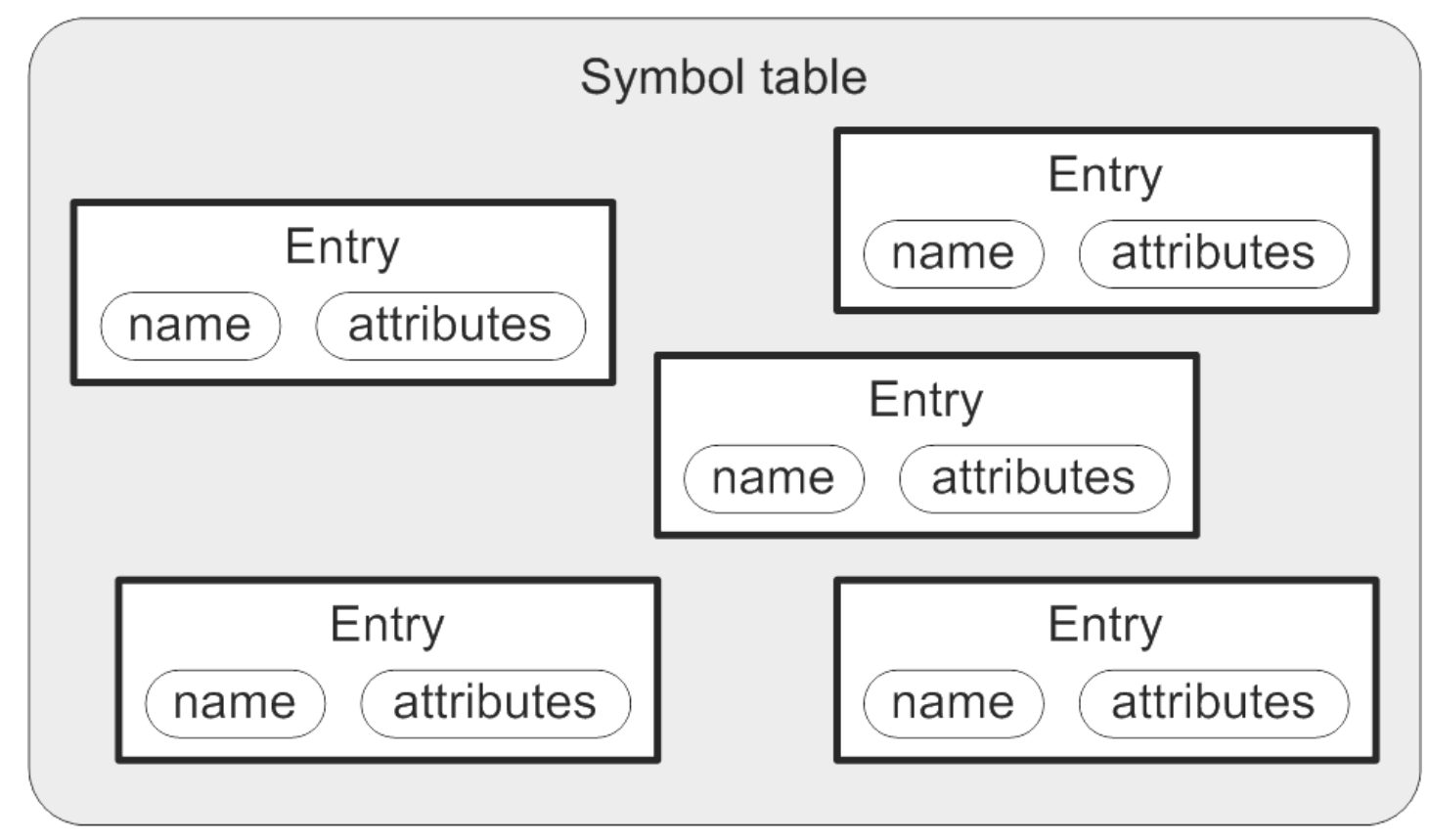

Figure 25. The Symbol Table Structure [5] 
The intermediate code represents a new form of the source program which is portable and source language-independent. This new form is usually a tree structure, commonly known as parse tree or abstract syntax tree (AST) [5, 38]. Back-end components, covered in the next subsection, freely process the AST for many different purposes, including, but not limited to, program execution, program translation, features extraction, and so on. Figure 26 shows an example of a simple assignment expression statement and its corresponding parse tree. By walking this tree, a back-end component is able to perform necessary calculations for the expression.

$a=b+c$

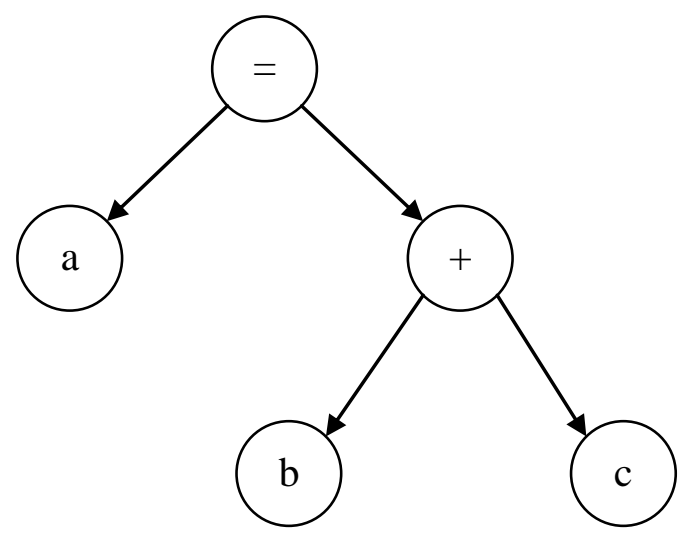

Figure 26. An Expression and Its Corresponding Parse Tree (AST)

\subsubsection{The Back-End}

A major component of the back-end can be either an executor or a code generator for an interpreter or a compiler, respectively [5]. The executor processes the symbol tables and the parse tree to carry out the program execution. The code generator processes the 
symbol tables and the parse tree in order to translate the source program into a target program which can execute or run on a target machine.

Importantly, the back-end does not limit itself as an executor or a code generator. It can be any processing engine to carry out all kinds of specialized computing tasks. The resulting AST can be used for many different purposes. This project uses the parse tree to extract for loops features to perform OpenMP auto-parallelization. 


\section{System Design and Implementation}

This major section describes the design and implementation of the proposed system, which includes various phases. First, the scope and design goals of the system are described. Then, the new approach to design and implement this auto-parallelization system is discussed. Next, development tools and software architecture are explained. Finally, implementation of packages and classes are presented in details.

\subsection{Purpose of the System and Design Goals}

Due to the fact that software programmers have to explicitly produce parallel programs in order to fully take advantage of the potential computing power of the underlying multicore platform [6], this writing project presents a new way to help programmers and/or compiler designers to automatically parallelize serial C programs at the source code level using the OpenMP APIs. Thus, the main purpose of the system is to prove that the chosen approach, presented in section 4.2 , actually works.

Multi-core programming using OpenMP provides a wide range of OpenMP compiler directives to apply to the shared-memory parallel programming model $[1,2]$. Furthermore, loops generally consume all or most of the execution time of a running program. Therefore, the system is reasonably and practically designed and implemented focusing only on for loop constructs while successfully meeting the design goals set forth below.

The system should be portable, scalable, extensible, reliable, and usable [43]. The system is implemented in Java and uses OpenMP as a parallel mechanism. Therefore, it directly and mutually inherits portable and scalable features of Java and OpenMP. The 
system can run on multiple platforms with Java Virtual Machine (JVM) installed. At the

same time, resulted OpenMP programs can be compiled and executed on different multicore machines.

The system is also scalable, extensible, and reliable because it is implemented via machine learning and compiler-based approach. The system can be incrementally trained with new training instances over time via machine learning. Specifically, the chosen instance-based technique allows the system to scale with small or large number of training records. At the same time, the system processes entire $\mathrm{C}$ source files using a $\mathrm{C}$ compiler design technique, which results in extensibility and reliability. It captures all C language elements and builds a corresponding AST which is portable and languageindependent [5] that can be used to extract many other program features, besides for loops, for automatic parallelization purposes. As a result, one can easily extend the system capability to automatically parallelize serial $\mathrm{C}$ programs beyond loop constructs from the already-in-place AST.

Last but not least, the system is also usable simply because it is implemented beyond original goal of research purpose on the way to becoming an actual product. The resulting system provides a user-friendly Graphical User Interface (GUI) and is effectively able to apply auto-parallelism to actual serial C programs.

\subsection{The Machine Learning and Compiler-based Approach}

The goal of this new approach is to automatically apply the OpenMP "for" directive to for loops found in serial $\mathrm{C}$ source files to achieve parallelism. Recall from previous Background section that the OpenMP "for" directive has various clauses to properly control and parallelize for loops. 


\subsubsection{Machine Learning Approach}

The objective of this approach is to predict the OpenMP schedule type static, dynamic, guided, runtime, or auto for the for loop construct (review Table 5). A schedule type cannot be automatically determined from a mathematical formula. Instead, it should be "learned" from those previously-used in OpenMP C source files by a machine learning model. It will apply the learned "knowledge" to a new for loop to determine the best possible schedule type for that loop.

Specifically, an instance-based, also referred to as case-based reasoning, machine learning classification technique, implemented using k-nearest neighbors algorithm, has been selected to build a corresponding classifier for this OpenMP schedule type classification problem. The k-nearest neighbors algorithm is an obvious choice because it is well-known to be used with the instance-based technique. From the general end-to-end data mining process presented in section 3, an in-depth process of implementing this project-specific instance-based classifier is specialized as illustrated in Figure 27.

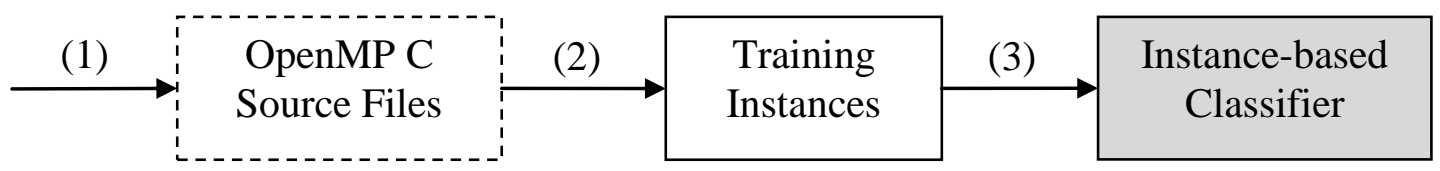

Train the Instance-based Classifier

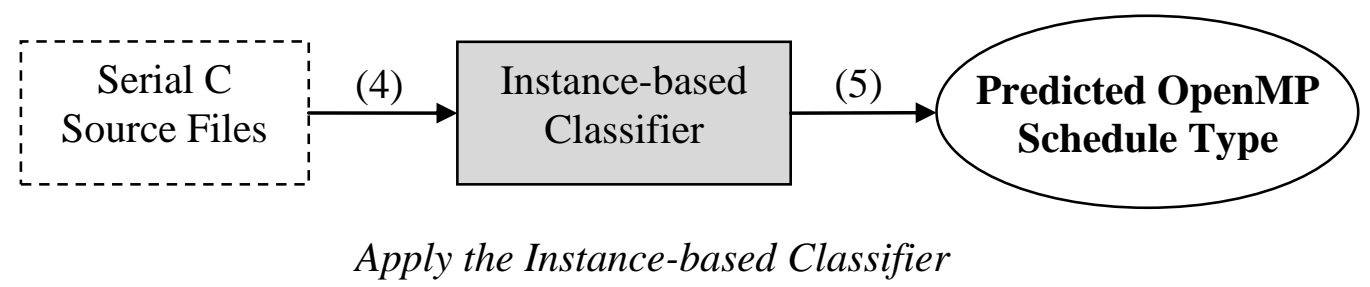

Figure 27. End-to-End Process of the Machine Learning Approach 


\section{(1) Data collection:}

Pre-parallelized C source files using OpenMP are systematically collected in order to ensure having good training samples. They are directly collected from real examples provided in the textbooks or on Web site of a company, such as Intel. They are also methodically constructed from knowledge learned from $[1,2,32]$. These OpenMP files are collectively composed into four files, namely omp_training 1.c, omp_training2.c, omp_training3.c, and omp_training4.c. These files can be found in Appendix B. The benefit of implementing instance-based classifier is that it can start with a small set of training records $[3,4,28]$. These four training files contribute a total of nine training instances, for loops with various features that are pre-parallelized using OpenMP with appropriate schedule types. These training records are different from each other to represent a wide set of training features. This significantly helps the classifier make better and higher quality decisions.

\section{(2) Features extraction:}

This training preprocessing step is to extract representative features of each for loop in order to properly build a relevant set of training instances. Below is the list of loop features that are interested to the application:

- Nested level of the loop

- How many nesting levels the loop has

- Number of iterations of the loop

- Number of operations carried out in the loop

- How many children the loop has 
- How many threads are sharing the work in the loop

- Number of arrays being used in the loop

- Total number of variables being used in the loop

- What is the state of each variable state, i.e., read-only or modified

Figure 28 is an example output of features that are systematically extracted from a for loop. By using compiler design technique, which results in a resourceful AST, features of a for loop can be easily and efficiently extracted with the visitor design pattern. The design pattern is described in later section. Each time the system encounters an operation-typed node in a for loop, it increments the operation count for that loop. Number of iterations is determined at compile time via variable declarations and definitions. Other features are similarly determined from the tree structure. A different set of feature results in a different data record. The system does not process a method call inside the loop due to the $\mathrm{C}$ language library complexity. Method call processing and dynamic calculation of number of iterations are dedicated to future system enhancement.

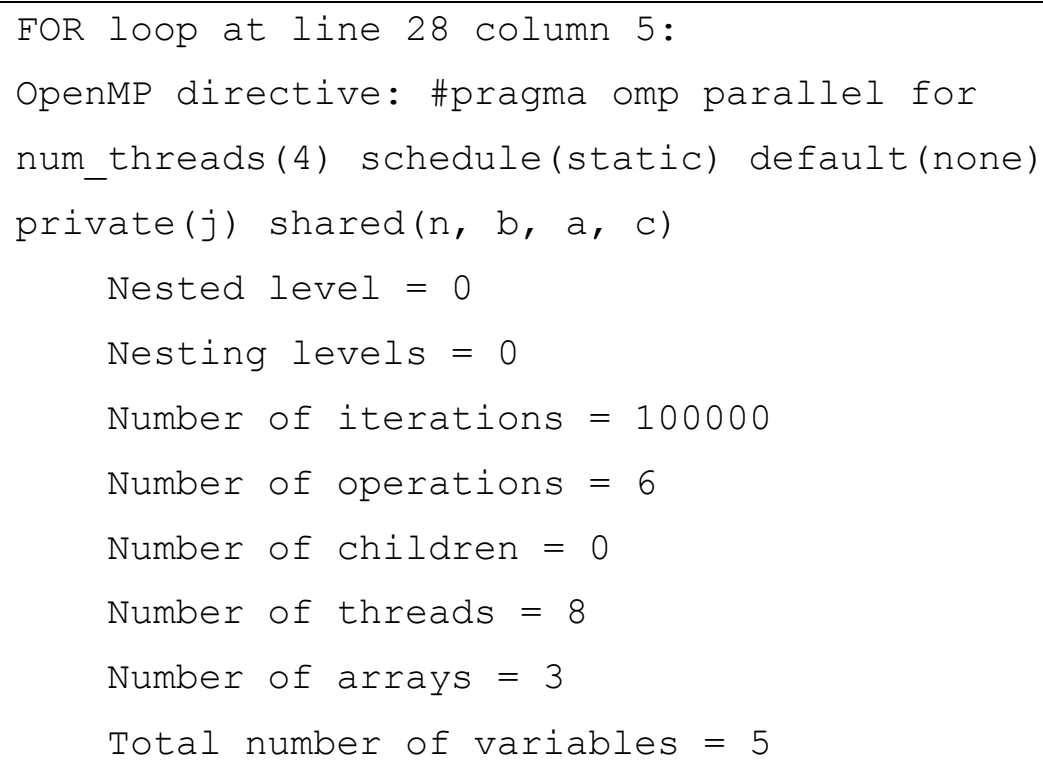




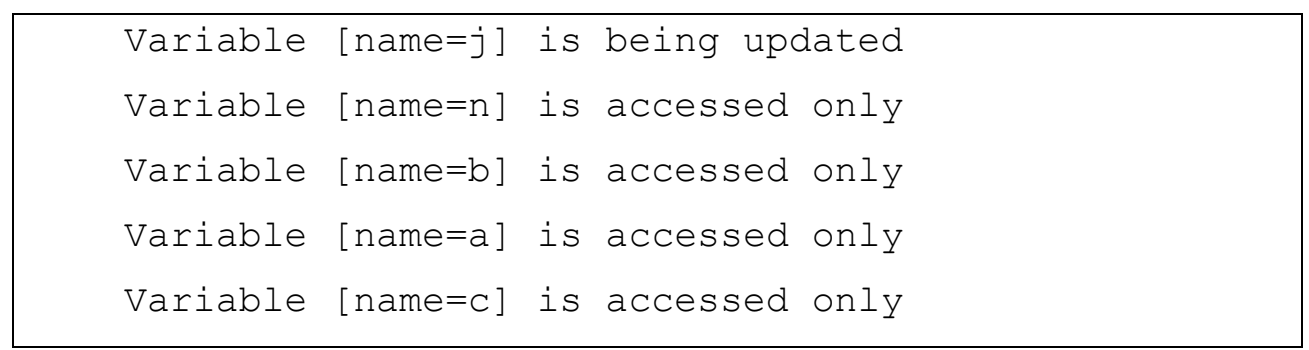

Figure 28. Sample Features Extracted from a for Loop

The results of this training preprocessing step are meaningful training instances that are ready to train the machine learning model, the instance-based classifier. Table 11 shows the results of these training instances, where loop complexity is computed based on the number of iterations, the number of operations being conducted inside the loop, and the imbalanced workload condition of the loop. The imbalanced workload condition of an inner loop is when its number of iterations depends on the iteration control parameter of an enclosing outer loop. Figure 29 is an example of an imbalanced workload condition found in a for loop.

\begin{tabular}{|l|r|c|r|c|}
\hline Threads & Iterations & $\begin{array}{c}\text { Nesting- } \\
\text { levels }\end{array}$ & Complexity & $\begin{array}{c}\text { Schedule } \\
\text { Type }\end{array}$ \\
\hline 4 & 100000 & 0 & 500000 & static \\
4 & 100000 & 0 & 600000 & static \\
4 & 600 & 1 & 4320000 & static \\
4 & 1200 & 1 & 23040000 & static \\
4 & 5 & 2 & 86400000 & dynamic \\
4 & 5 & 3 & 414720000000 & guided \\
4 & 100 & 1 & 240000 & static \\
4 & 100 & 0 & 800 & static \\
dynamic \\
\hline
\end{tabular}

Table 11. The Nine OpenMP Schedule Type Training Instances 


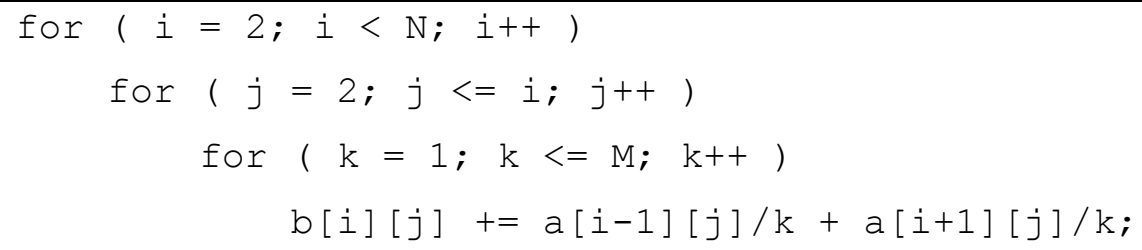

Figure 29. An Example of Loop Imbalanced Workload Condition

\section{(3) Model training:}

This model training step is very straightforward. The scope of this project is not to design a new machine learning algorithm. It makes use of practical machine learning tools introduced from [4], one of which is called Weka. Weka is presented in section 4.3, Development Environment and Tools, with more details. This tool provides a rich set of Java classes and packages to implement various machine learning techniques.

Specifically, the system implements its instance-based classifier using the provided class called weka.classifiers.lazy.IBk. The available schedule type training instances from the previous step are used train and build the classifier, which is then used to predict an OpenMP schedule type of a new unknown record. To be portable, the resulting training instances are also saved in ARFF format, as presented in Appendix A.

Each training instance in the training set extracted in phase (2) is fed to the model for model training purpose. Figure 30 shows part of the training process. For more details, look at Appendix G: The Project Source Code.

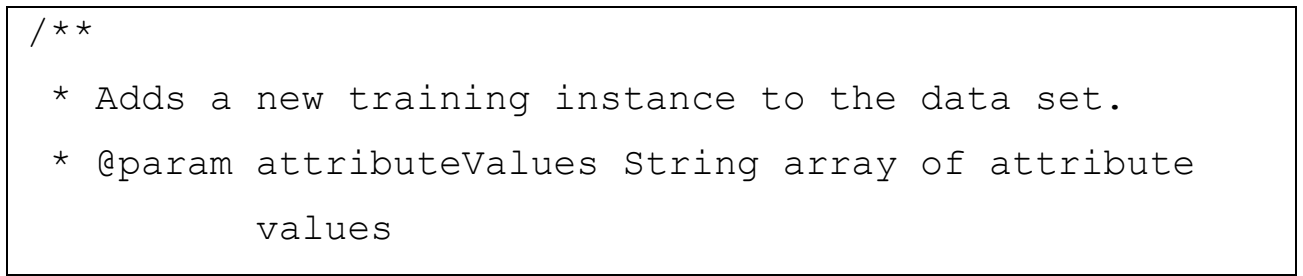




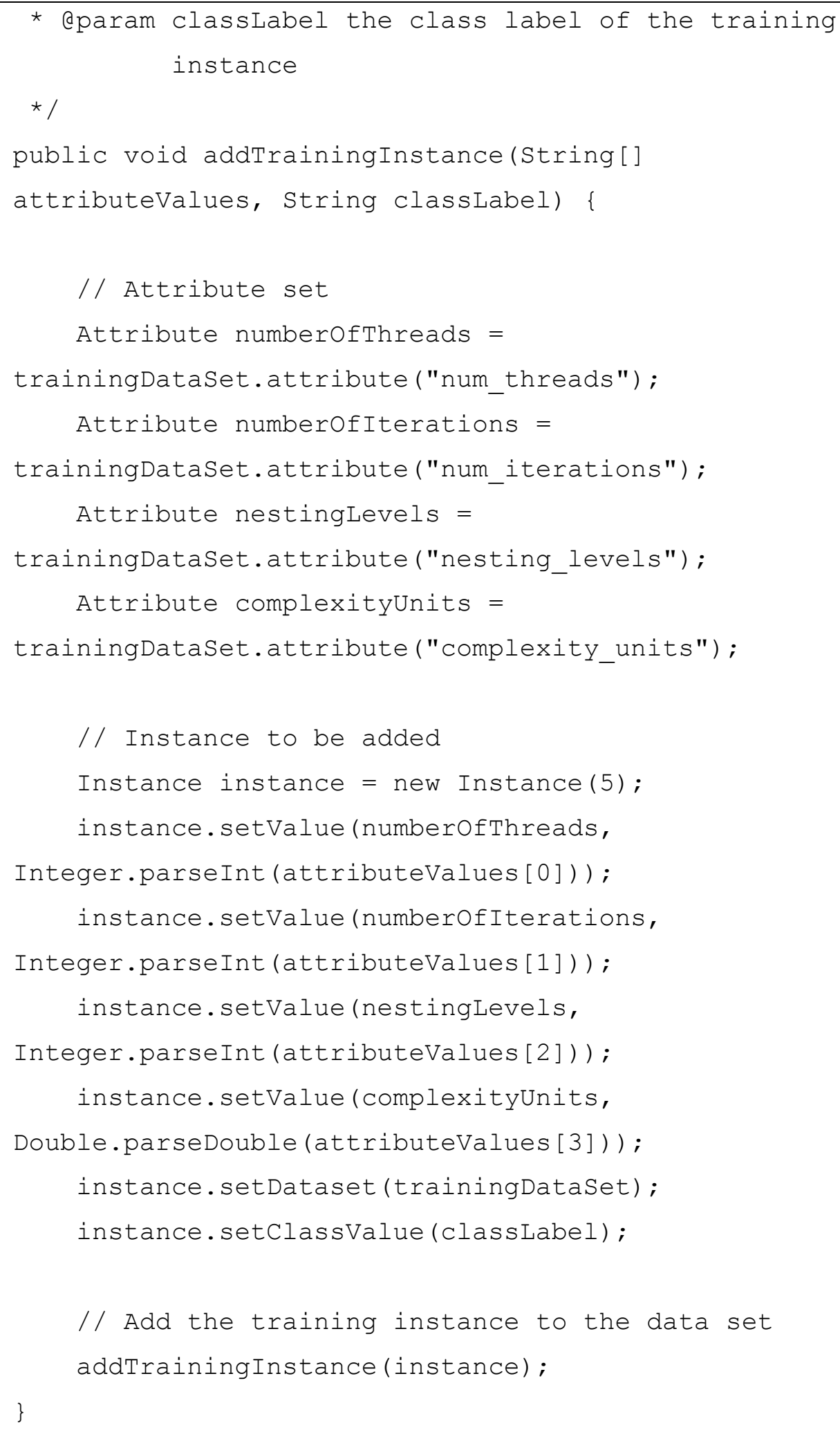

Figure 30. Partial Code for Model Training 


\section{(4) Model applying:}

Similar to model training in phase (3), model applying is very straightforward.

For each new unknown record, loop features are extracted as in phase (2) except for the missing class label, which is the schedule type that the model needs to predict. Figure 31 shows code that implements part of the model applying process. For more details, look at Appendix G: The Project Source Code.

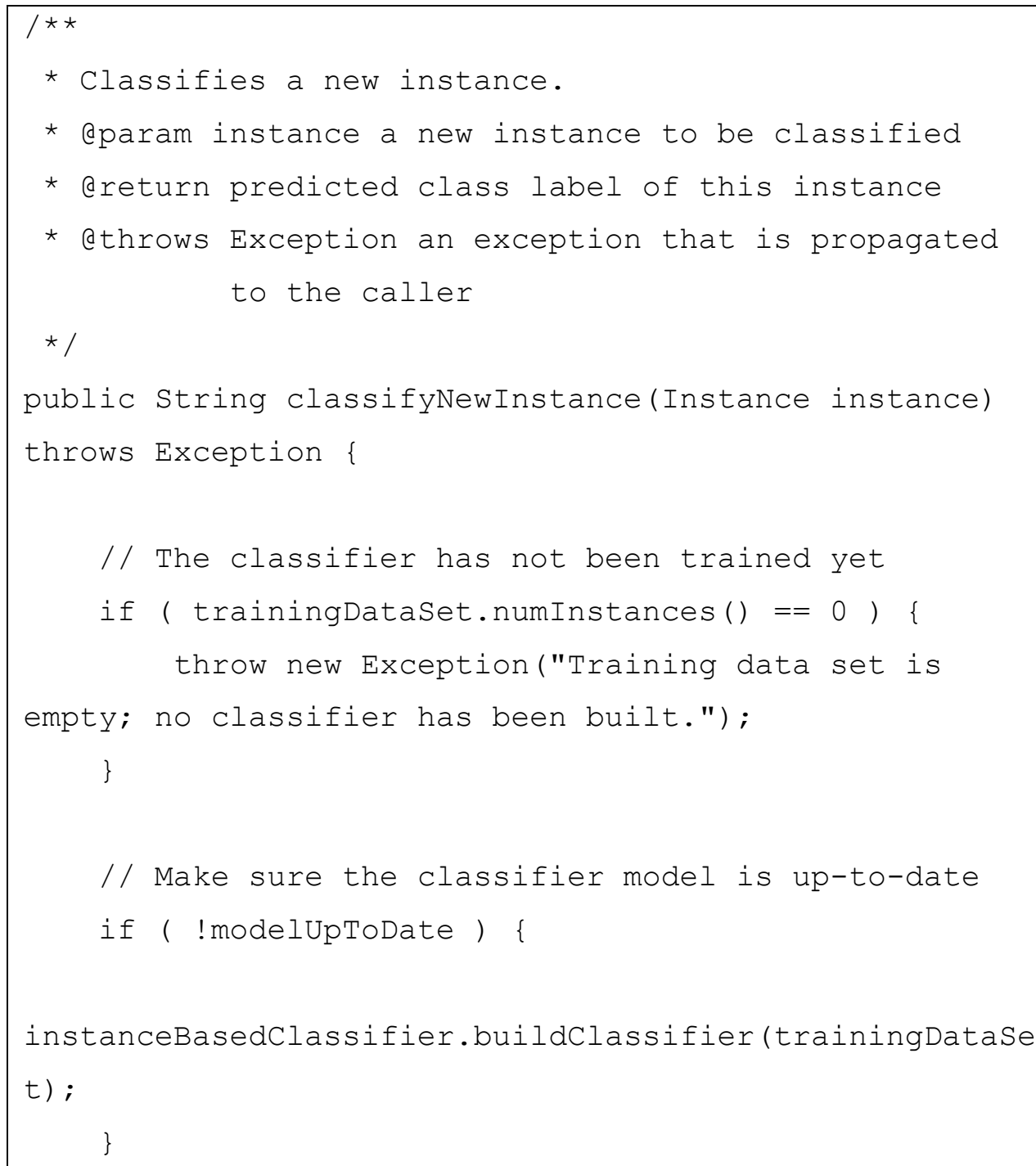




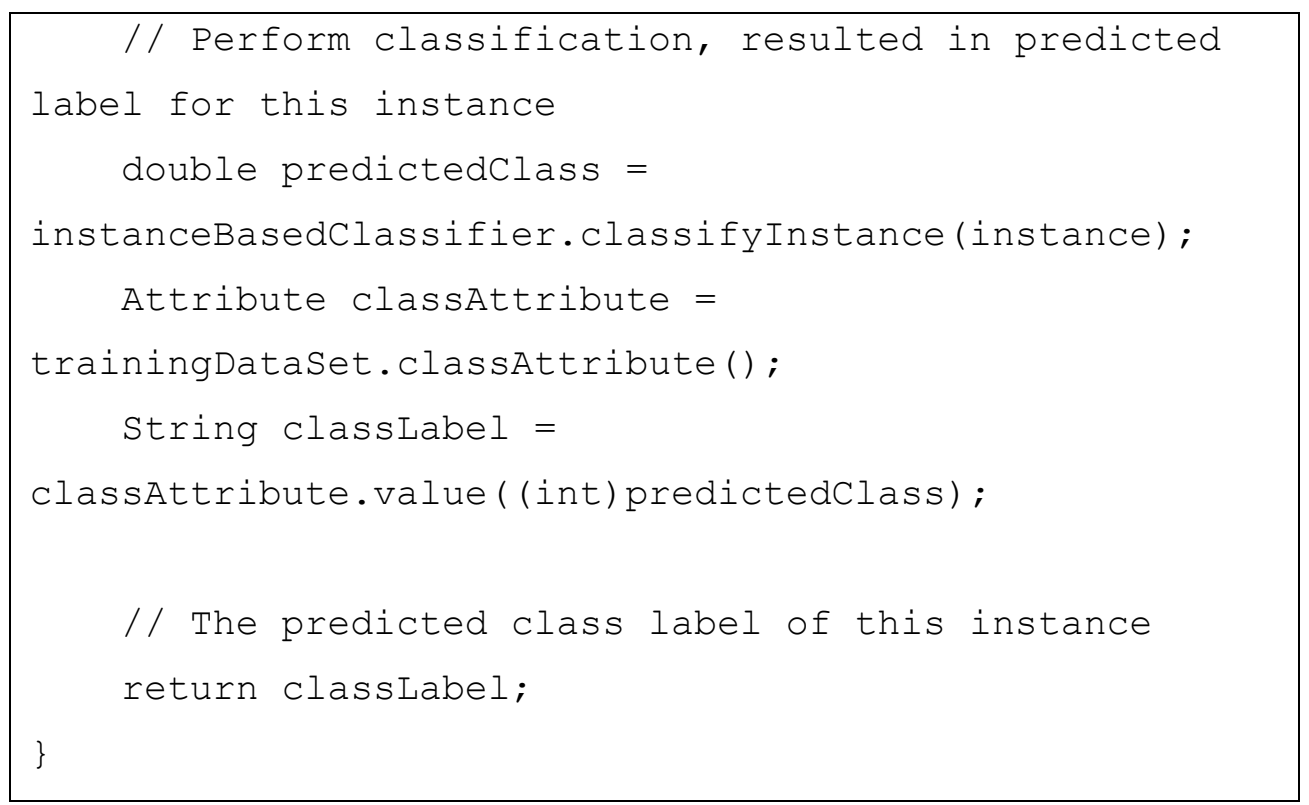

Figure 31. Partial Code for Model Applying

\section{(5) Result interpretation:}

Recall from the Background section that the result obtained from applying a machine learning model can be used for various technological or scientific decisions. The result sometimes can be used directly. In some other cases, it must be processed or interpreted in order to become useful information to an intended application.

In this project, the result, which is a predicted schedule type, obtained from applying the instance-based classifier in phase (4) can be used directly by our autoparallel system to automatically generate corresponding OpenMP directive with the schedule type embedded for the target for loop construct. Importantly, the system is able to learn from the programs it parallelizes because the auto-generated result also becomes a new training record to train back the classifier. As a result, the system gets smarter the more programs it parallelizes.

Below is a partial code showing part of "for" directive construction process. For more details, look at Appendix G: The Project Source Code. 


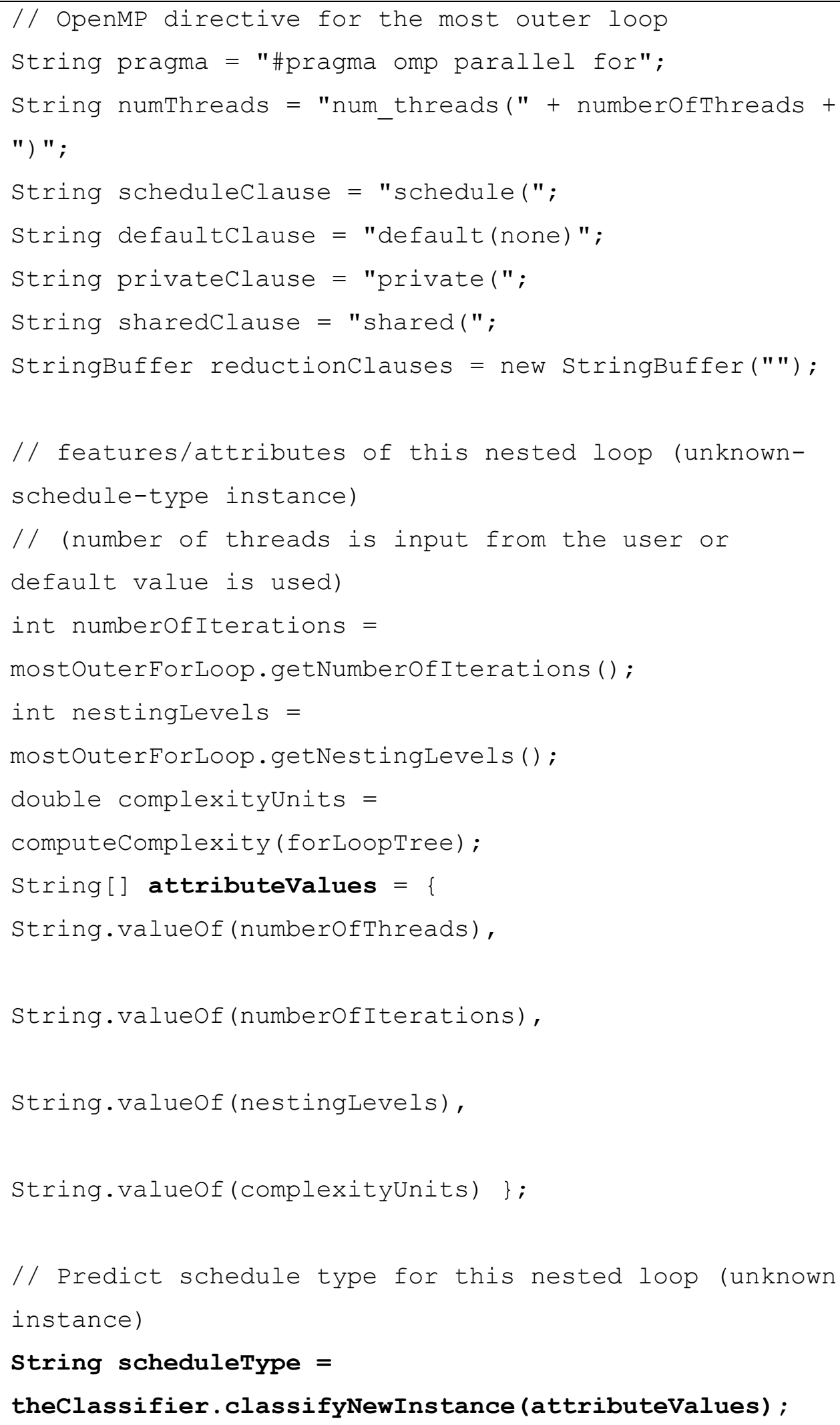




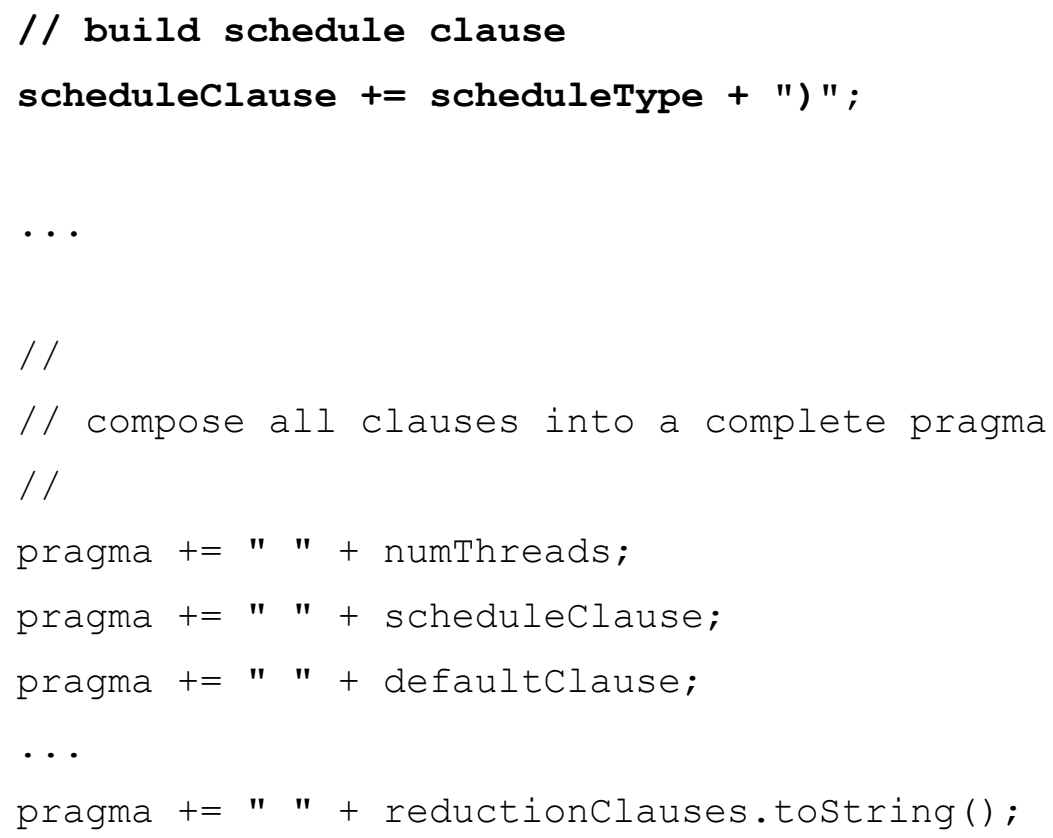

Figure 32. Partial Code to Construct the "for" Directive

\subsubsection{Compiler-based Design Approach}

This compiler-based design approach is naturally embedded into this project's end-to-end design and implementation. Recall in the general Background section that there are three phases in a compiler design framework; therefore, below are the three corresponding phases of compiler design technique applied to the project.

\section{(1) Front-end:}

The project front-end is designed using the compiler-compiler tool called JavaCC. This tool helps to generate a scanner and a parser in the Java language for a given language grammar that is the best fit into the design and implementation of this project. This leaves the project the only, but very important, task to design the grammar for the C language. With information provided from $[39,44]$, the project $\mathrm{C}$ grammar for JavaCC 
has been successfully implemented. Appendices $\mathrm{E}$ and $\mathrm{F}$ contain the $\mathrm{C}$ grammar written in $\mathrm{BNF}$ and the $\mathrm{C}$ grammar written for JavaCC, respectively.

Besides the obvious goal of the grammar to parse $\mathrm{C}$ source programs, the project makes use of this compiler design technique is to analyze and extract for loops features for OpenMP auto-parallelization. Therefore, the JavaCC C grammar for this project goes beyond the basics It is designed and customized to enable the system to extract all information about for loops and their associated variables from the parse tree to properly construct corresponding OpenMP "for" directive. Figure 33 shows a partial example of the grammar to allow an extraction of for loop related information. Appendix F contains the full set of grammar structures.

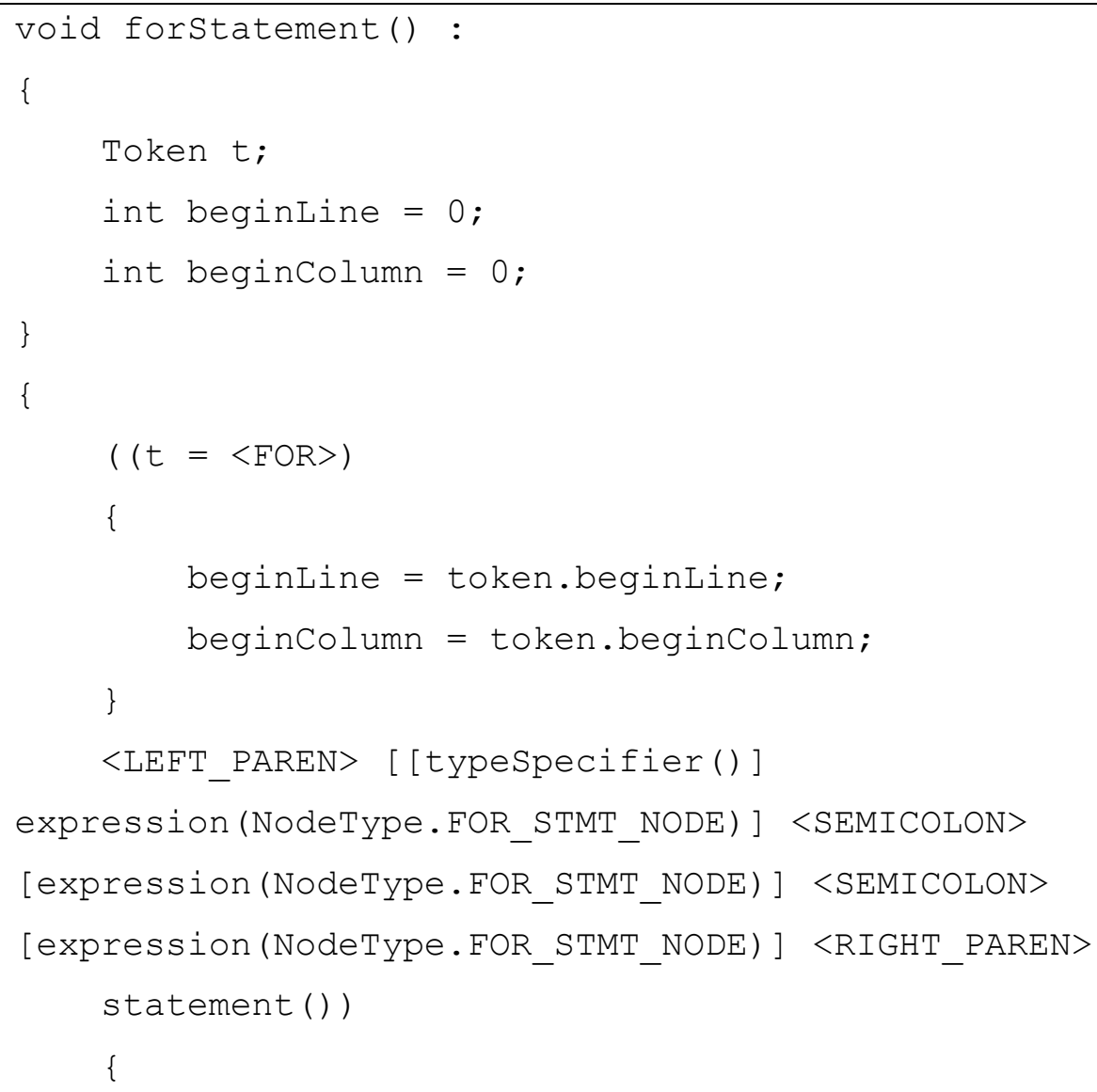




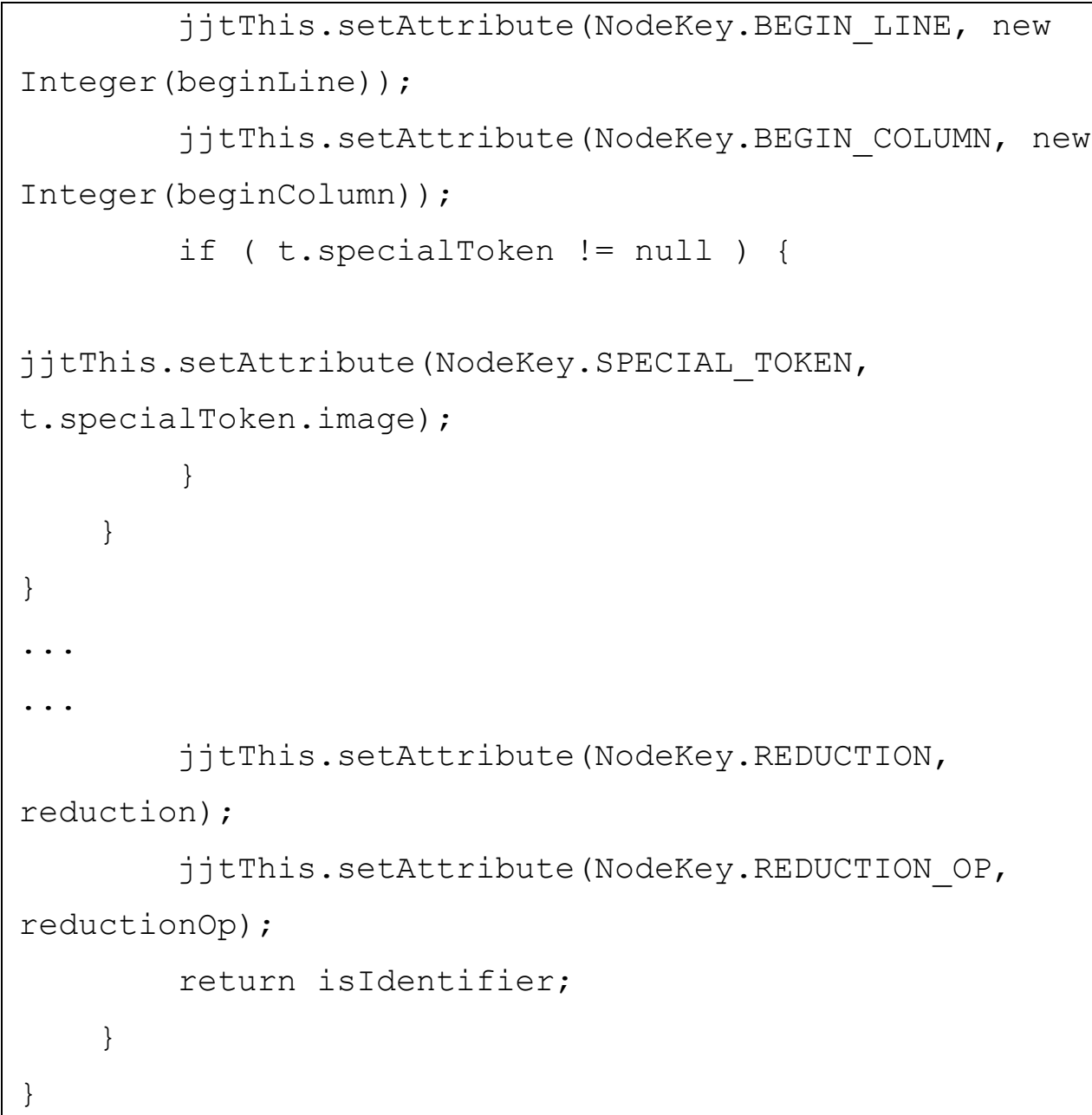

Figure 33. Partial Project C Grammar for JavaCC

\section{(2) Intermediate tier:}

The symbol table and the symbol table stack in this project are designed to store information about declarations and definitions of functions and variables and more during the parsing process in phase (1). Figure 34 illustrates part of the design of symbol table of the project. Symbol tables are used to:

- Retrieve the numeric value of a variable.

- Filter out and retrieve the set of variables that belong to a particular for loop. 
- Separate or distinguish an identifier encountered in a for loop, whether it is a function name or a variable.

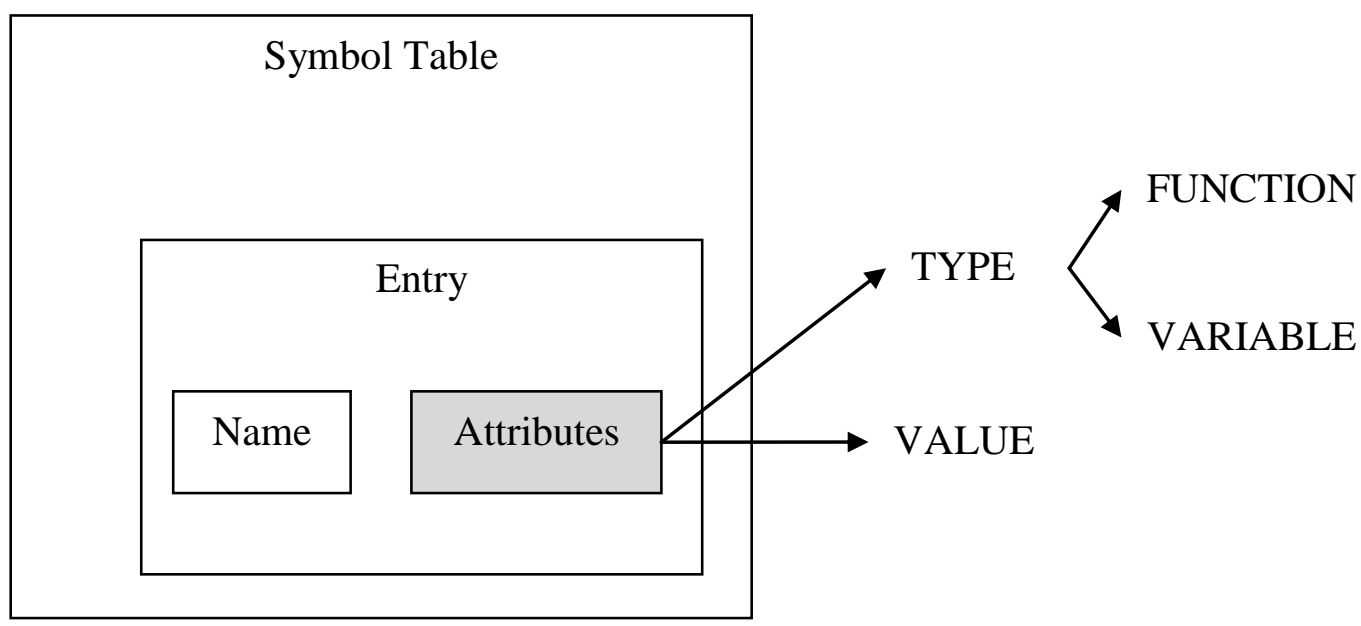

Figure 34. The Symbol Table

JavaCC has a utility called JJTree. It allows the front-end to generate a parse tree for the source program according to the project-specified grammar constructs. Importantly, the resulting parse tree has a built-in feature of the visitor design pattern, which allows the back-end to easily and efficiently process the intermediate information for OpenMP code generation purposes.

\section{(3) Back-end:}

The major back-end component of the project design is the extractor, called ForLoopExtractorVisitor. Its main task is similar to an executor or generator in a sense that it walks the resulting parse tree to obtain the source program information. However, as the name implies, it does not perform any execution or translation, but it rather extract 
a variety information about for loops and associated variables. The information is used for various logic decisions about "for" directive clauses, as indicated below.

The extractor extracts the information from the parse tree and provides the results, which is another tree structure of the extracted for loops, to the main component of the program, called the OpenMPAutoParallelizer. This auto-parallelizer simply performs a breadth-first tree traversal to carry out its major tasks as follows:

- $\quad$ Train the OpenMP schedule type instance-based classifier via extracted for loops features.

- Derive an OpenMP schedule type for the "for" directive via the machine learning method.

- Derive other clauses for the "for" directive, such as the "private" clause, the "shared" clause, the "reduction" clause, and so on.

- Perform loop dependence analysis so that those loops that are not parallelizable are practically rejected. A common form of a loop-carried dependence is illustrated in the figure below, where a computation of an element in the array "a" at a particular iteration $i$ depends on the result of previous $i-1$ iteration. This kind of dependency prevents the loop iterations to be executed in parallel, and it should be rejected by the system.

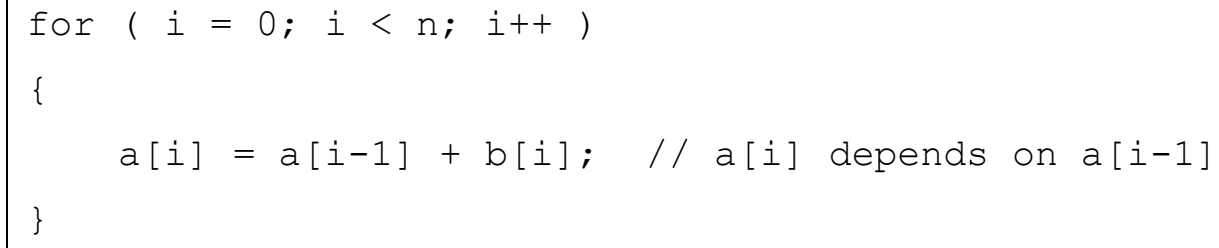

Figure 35. An Example of Loop-carried Dependence 
Figure 36 is an example showing a partial AST generated from the serial C source file called serial_matrix_product_1.c. The tree is composed of various nodes representing structure of the source program. We are interested only in the "forStatement" node. The Figure also shows the features extracted from that node.
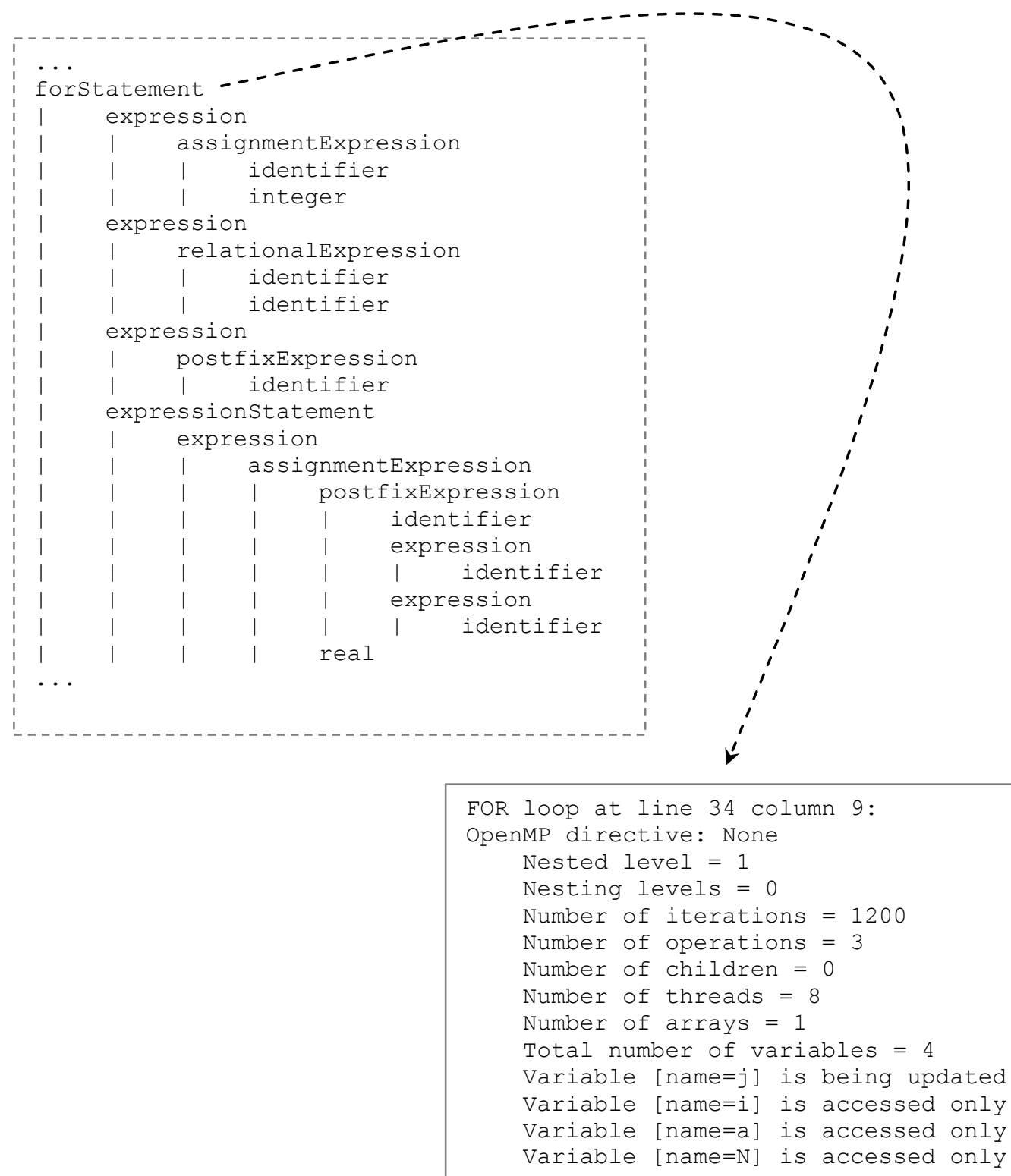

Figure 36. An Example of A Partial AST Generated from serial_matrix_product_1.c 


\subsection{Development Environment and Tools}

The project has been developed and tested under both Windows and Linux platforms. Java development environment is the JDK version 1.7.0. Eclipse Java EE IDE has been chosen as the main designing, coding, and debugging tools with various plug-ins installed to serve different design purposes. UML modeling tools include ObjectAid and UMLet. Two major tools that contribute to the heart of the project are the JavaCC $[5,38.39]$ and the Weka [41].

The JavaCC utility is a compiler-compiler tool that allows compiler designers to design and develop compilers without implement scanners and parsers from scratch. This tool automatically generates corresponding scanner and parser for a given grammar of a programming language. This tool also includes other utilities, such as JJTree and JJDoc. JJTree helps to generate a parse tree, and JJDoc can be used to generate the grammar in BNF documentation.

The Weka library provides a rich set of APIs to help software programmers to develop efficient machine learning applications using a wide range of choices in terms of machine learning techniques and algorithms. The programmers are also able to implement various machine learning techniques for their own designs from the available Weka packages and classes. For instance, this project implements the instance-based classifier from the Weka class called weka.classifiers.lazy.IBk. The classifier can be customized further with the following options as specified in the Weka online documentation [41]: 
- Weight the neighbors by the inverse of their distance.

- Weight the neighbors by 1 - their distance.

- Specify number of nearest neighbors (k value) for the classification.

- Specify a nearest search algorithm to use.

The two core methods of the package used to build the classifier and to classify a new unknown instance are buildClassifier() and classifyInstance(), respectively. For details of all available configurations and methods of the classification classes, see the Weka Java documentation online [41].

\subsection{Software Architecture and Design Patterns}

Software architecture [43] of the system comes directly from the indicated purpose of the system and design goals. That is, the system is decomposed into two main components, machine learning (ML) and compiler design (CD). Under CD, the subsystems are decomposed according to the compiler design framework. The front-end, the intermediate tier, and the back-end components. The software architecture of this project is shown in Figure 37. 


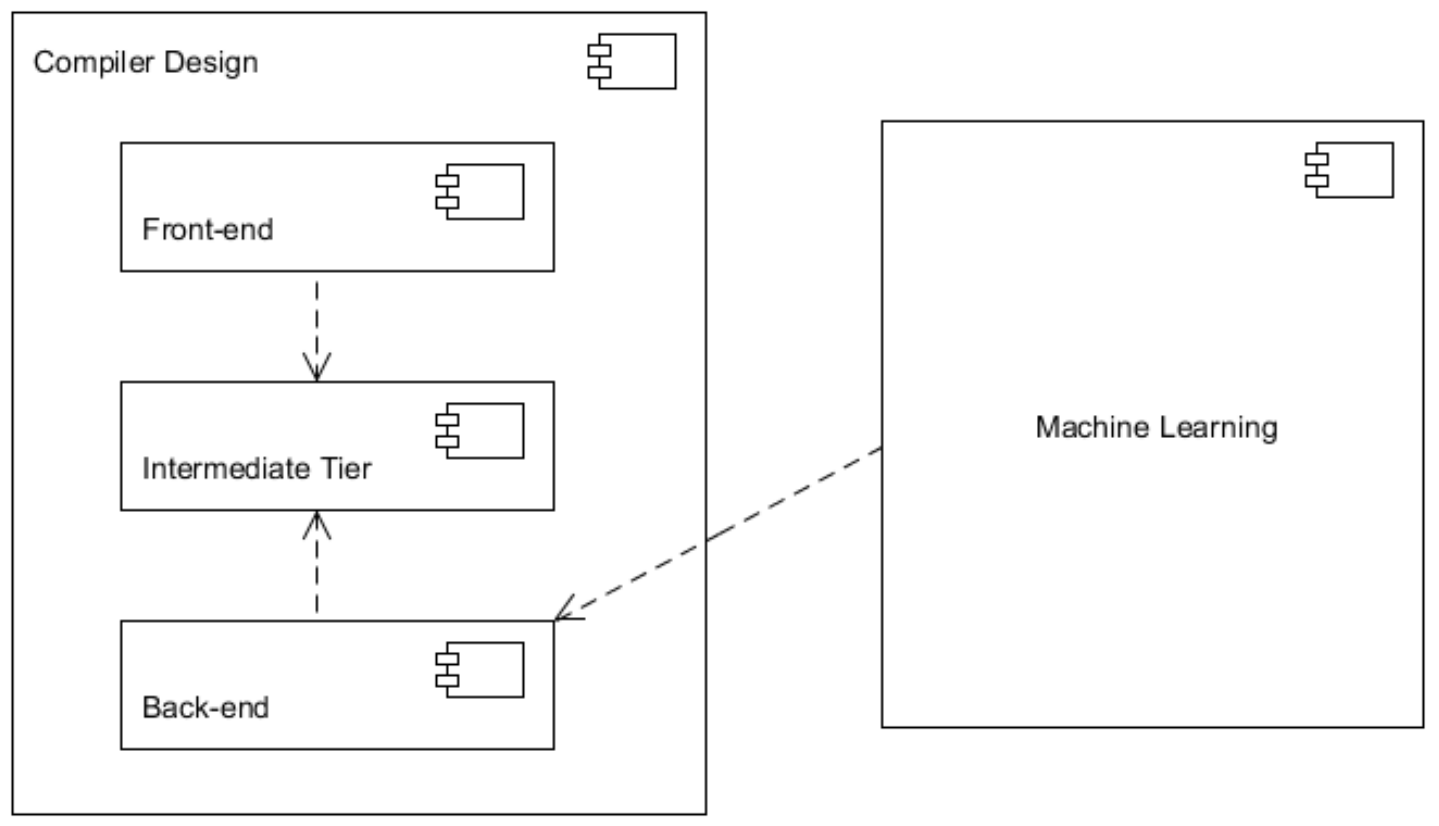

Figure 37. The Project System Software Architecture

Selected design patterns include two main design patterns, which are the abstract factory and the visitor design patterns. The abstract factory provides a mechanism to create and manage symbol tables and symbol table entries, as shown in Figure 38. The visitor design pattern provides an efficient way to traverse the abstract syntax tree in order to extract for loops features for automatic OpenMP parallelization, as shown in Figures 39. 


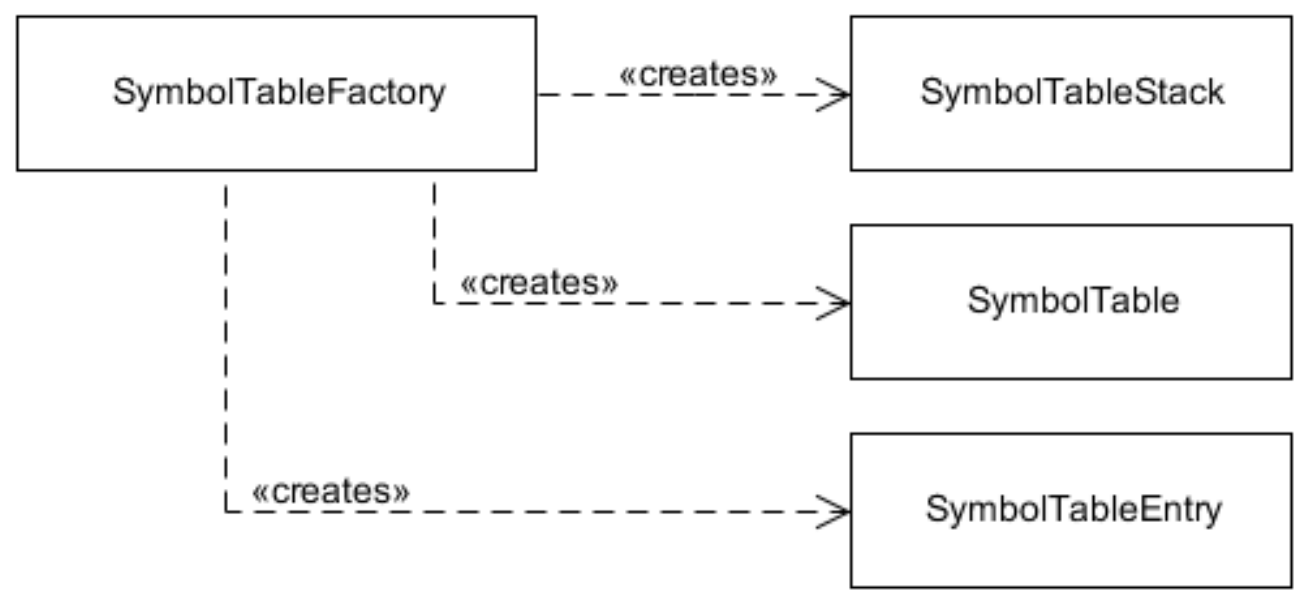

Figure 38. The Abstract Factory Design Pattern

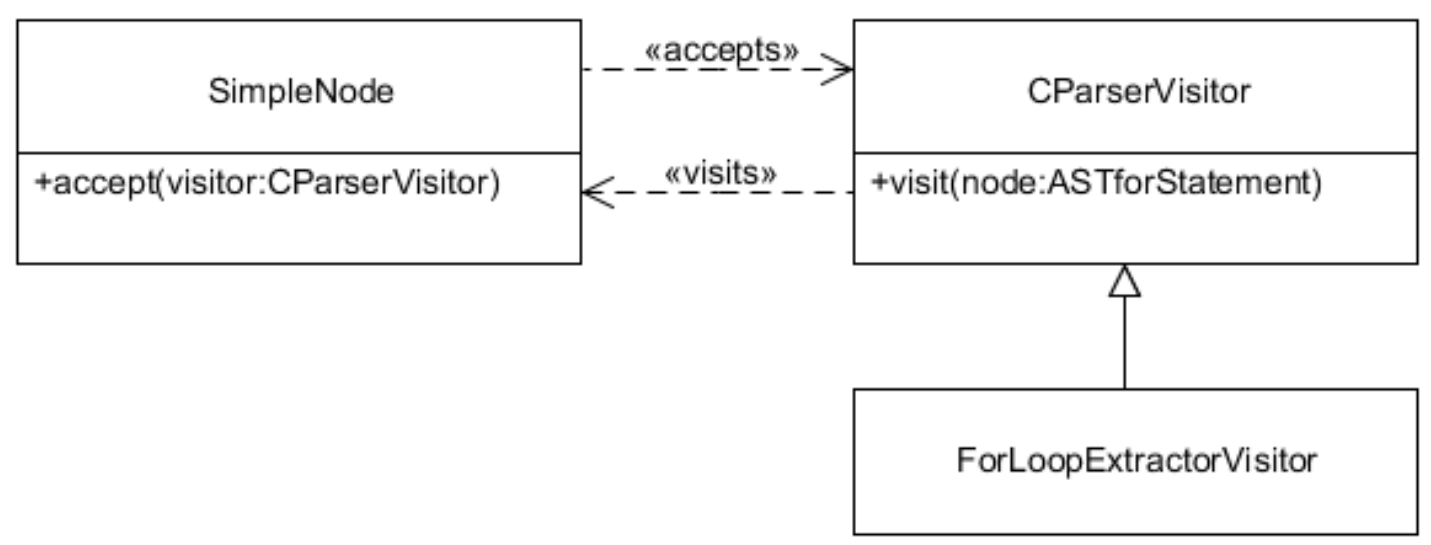

Figure 39. The Visitor Design Pattern

\subsection{Implementation: Packages and Classes}

The project implementation phase is practically and incrementally implemented one feature at a time, exercising the concept "build on something that works" [5] and Scrum 
project backlog [36]. The list in Figure 40 shows the backlog items to implement the system.

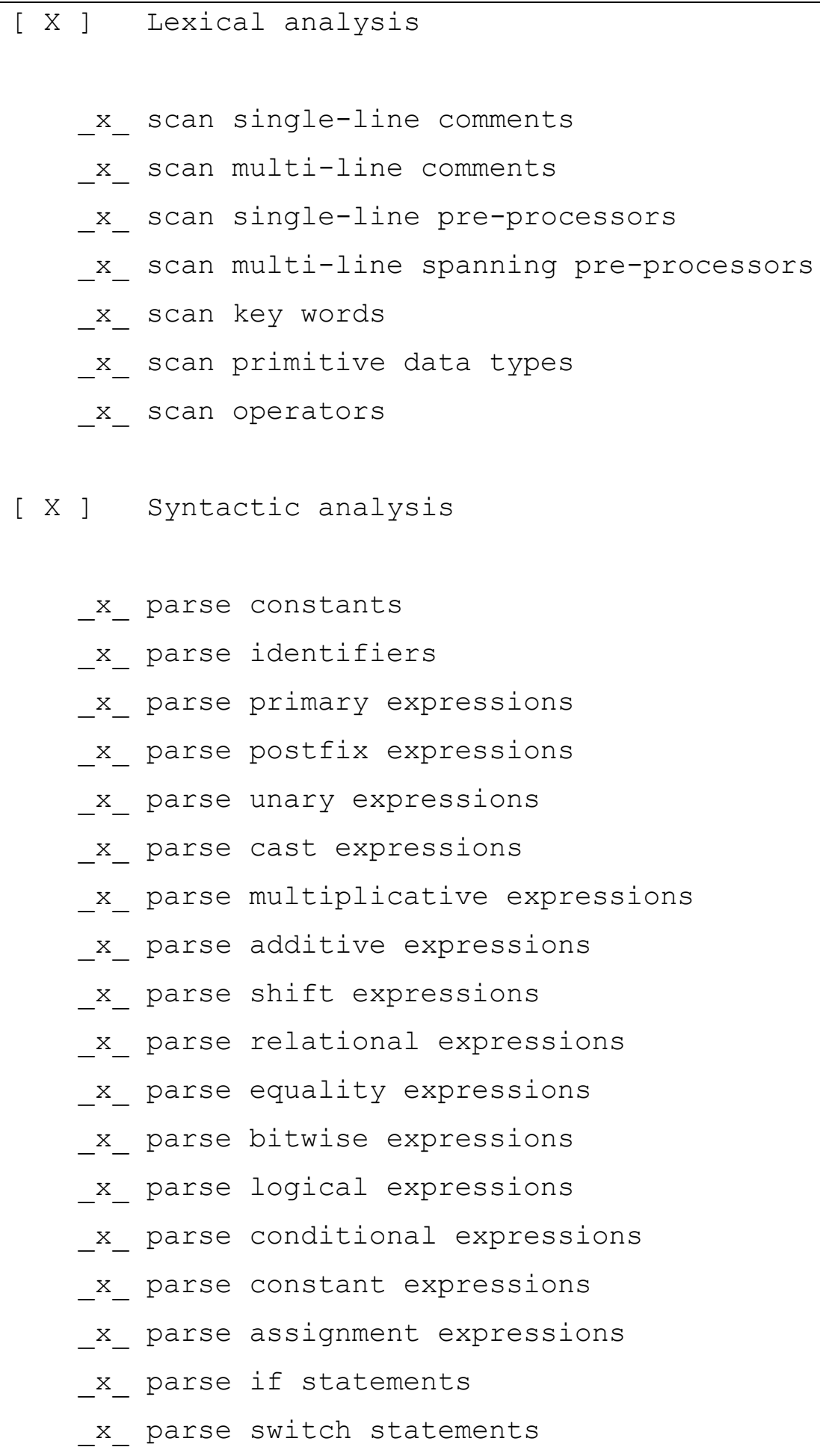




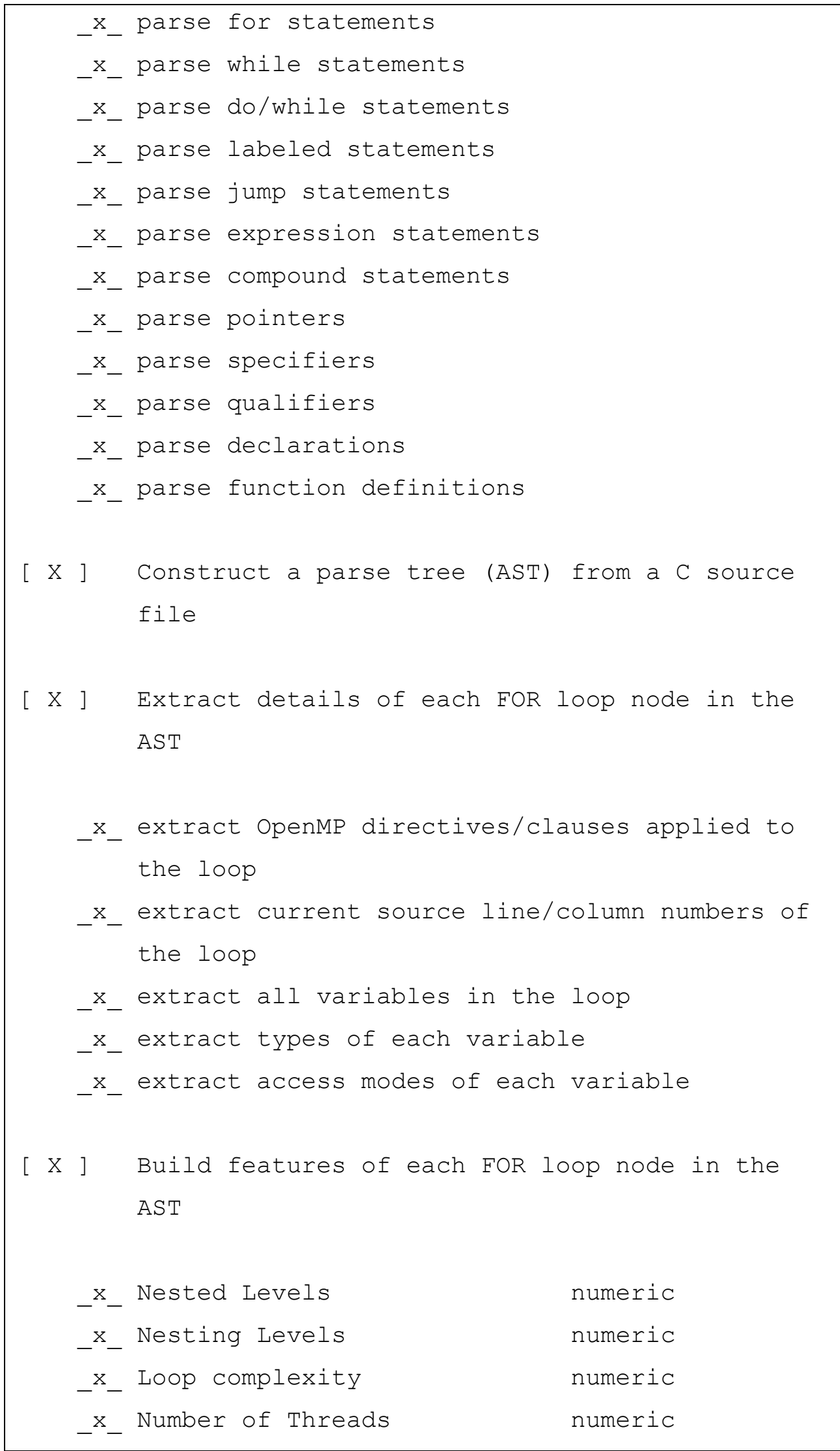




\begin{tabular}{|c|c|c|}
\hline & ${ }_{-}{ }^{x}$ & Number of Iterations \\
\hline & ${ }_{-} x_{-}$ & Number of Operations \\
\hline & $-{ }^{x}$ & Number of Children \\
\hline & $-x_{-}$ & Number of Arrays \\
\hline & $-{ }^{x}-$ & Number of Variables \\
\hline & $-{ }^{x}-$ & Data Dependency \\
\hline$[x$ & & $\begin{array}{l}\text { Able to be trained to classify simple sample } \\
\text { records using the instance-based classifier }\end{array}$ \\
\hline$[x$ & & $\begin{array}{l}\text { Able to be trained to classify a FOR loop node } \\
\text { into an openMP schedule type based on its } \\
\text { extracted features }\end{array}$ \\
\hline$[x$ & & $\begin{array}{l}\text { Build OpenMP num_threads clause for a FOR loop } \\
\text { node based on its extracted information }\end{array}$ \\
\hline$[x$ & & $\begin{array}{l}\text { Build OpenMP schedule clause for a FOR loop } \\
\text { node based on its extracted information }\end{array}$ \\
\hline$[\mathrm{X}$ & & $\begin{array}{l}\text { Build OpenMP default clause for a FOR loop } \\
\text { node based on its extracted information }\end{array}$ \\
\hline$[x$ & & $\begin{array}{l}\text { Build OpenMP private clause for a FOR loop } \\
\text { node based on its extracted information }\end{array}$ \\
\hline$[\mathrm{X}$ & & $\begin{array}{l}\text { Build OpenMP shared clause for a FOR loop node } \\
\text { based on its extracted information }\end{array}$ \\
\hline$[x$ & & $\begin{array}{l}\text { Build OpenMP reduction clause for a FOR loop } \\
\text { node based on its extracted information }\end{array}$ \\
\hline$[x$ & & $\begin{array}{l}\text { Output complete OpenMP \#pragma... for loop } \\
\text { construct for each encounter FOR loop node }\end{array}$ \\
\hline
\end{tabular}




\begin{tabular}{|c|c|}
\hline & from the AST \\
\hline$\left[\begin{array}{ll}\mathrm{X} & ]\end{array}\right.$ & $\begin{array}{l}\text { Output a corresponding OpenMP C source file } \\
\text { resulted from an input sequential C source } \\
\text { file }\end{array}$ \\
\hline$\left[\begin{array}{ll}\mathrm{X} & ]\end{array}\right.$ & $\begin{array}{l}\text { Able to reject not-parallelizable loop based } \\
\text { on loop-carried dependence analysis }\end{array}$ \\
\hline$\left[\begin{array}{ll}\mathrm{X} & ]\end{array}\right.$ & $\begin{array}{l}\text { Able to detect openMP files that are being } \\
\text { asked to be parallelized, and vice versa }\end{array}$ \\
\hline$\left[\begin{array}{ll}\mathrm{X} & ]\end{array}\right.$ & Implement program GUI version \\
\hline$\left[\begin{array}{ll}\mathrm{X} & ]\end{array}\right.$ & $\begin{array}{l}\text { Build test cases to validate the system and } \\
\text { the chosen auto-parallelism approach }\end{array}$ \\
\hline
\end{tabular}

Figure 40. The Simple Scrum Backlog of the Project

For the chosen software architecture, the corresponding packages and major classes are designed and implemented and shown in the UML package and class diagrams in Figures 41-44. For readability, the diagrams show only names of the packages and classes. Refer to Appendix G: The Project Source Code for more details. 


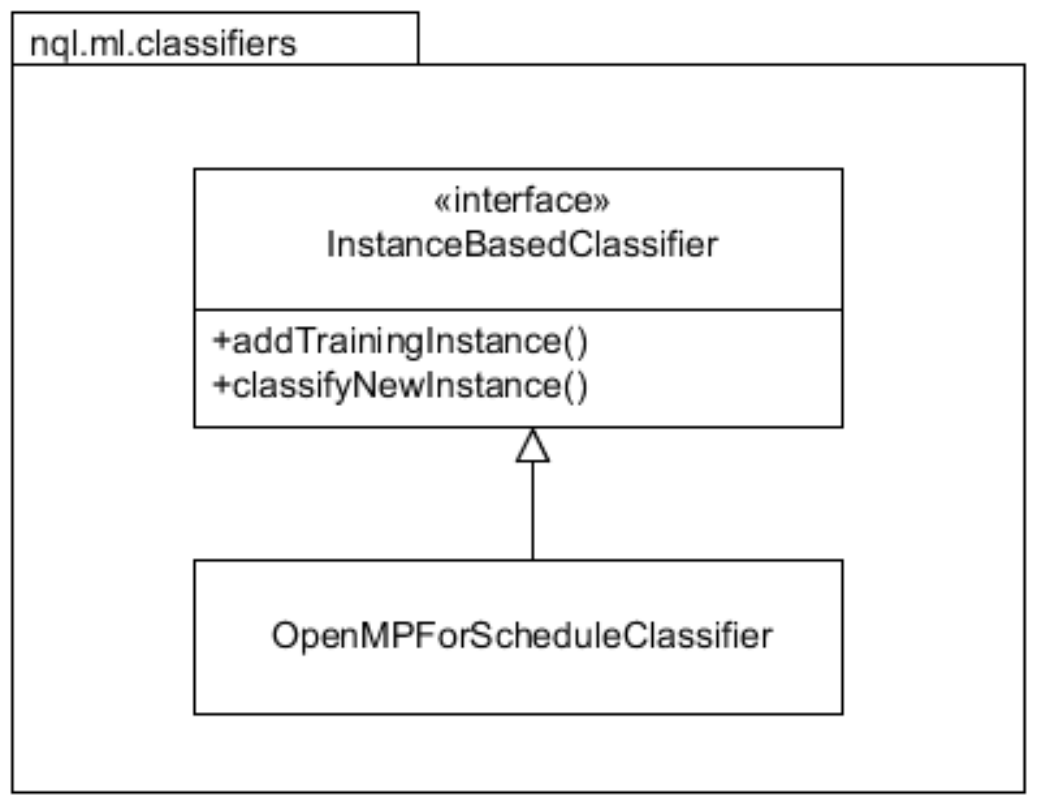

Figure 41. Classes Under nql.ml.classifiers Package

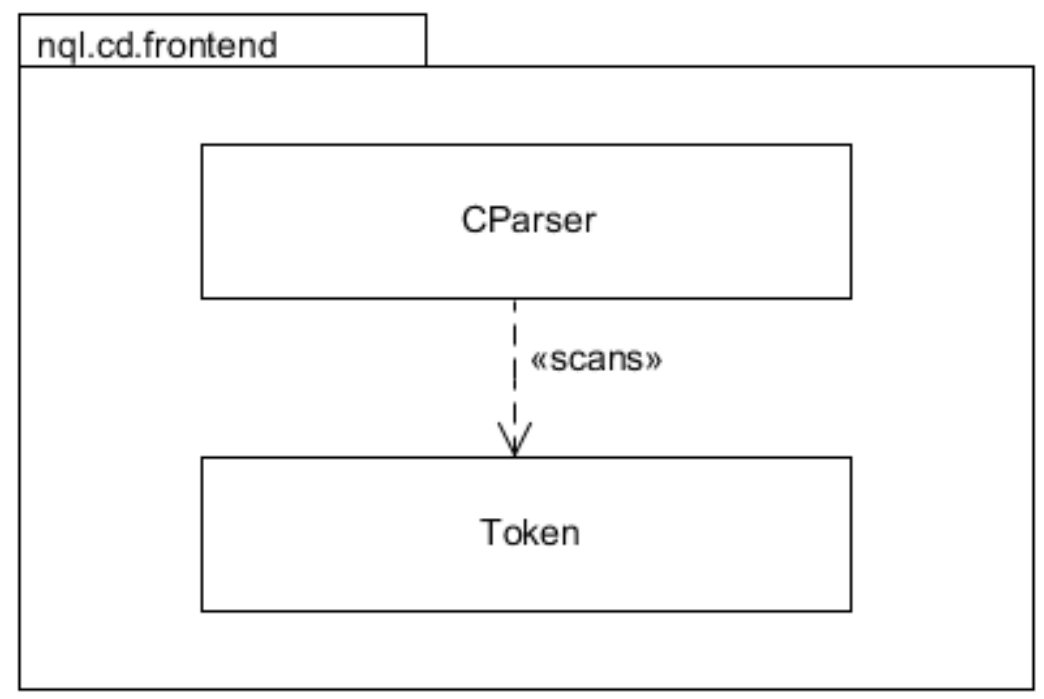

Figure 42. Classes Under nql.cd.frontend Package 


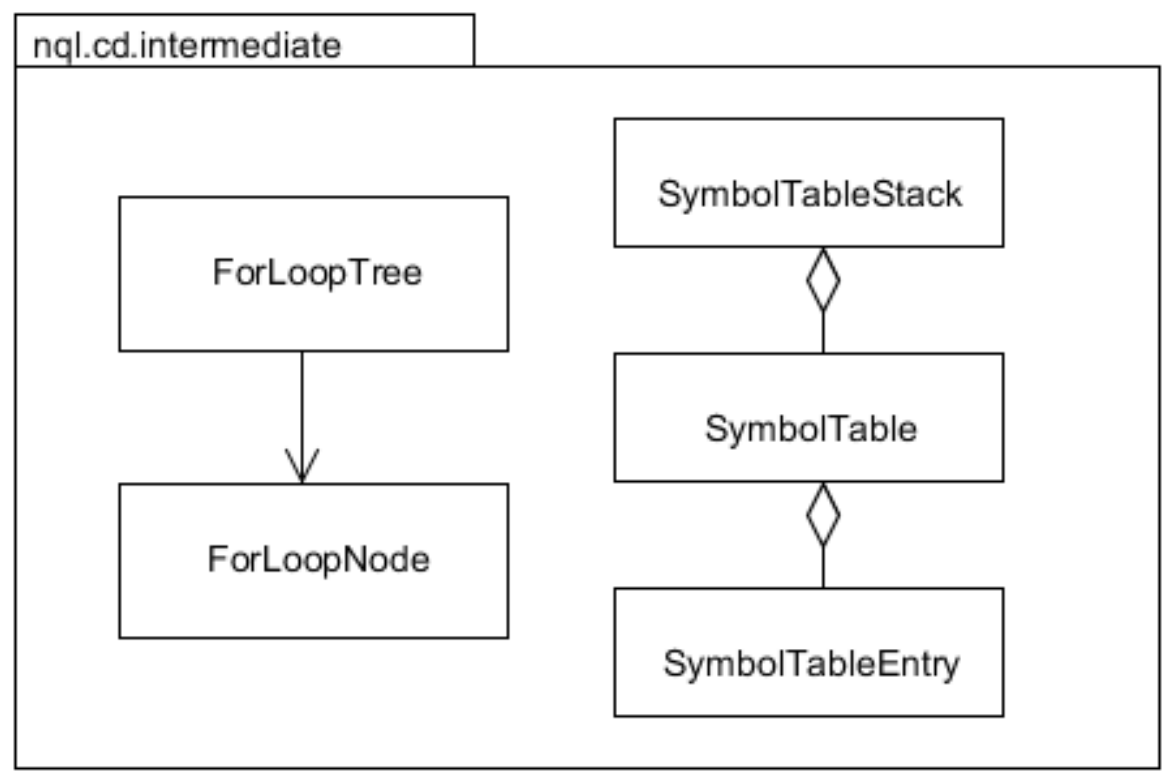

Figure 43. Classes Under nql.cd.intermediate Package

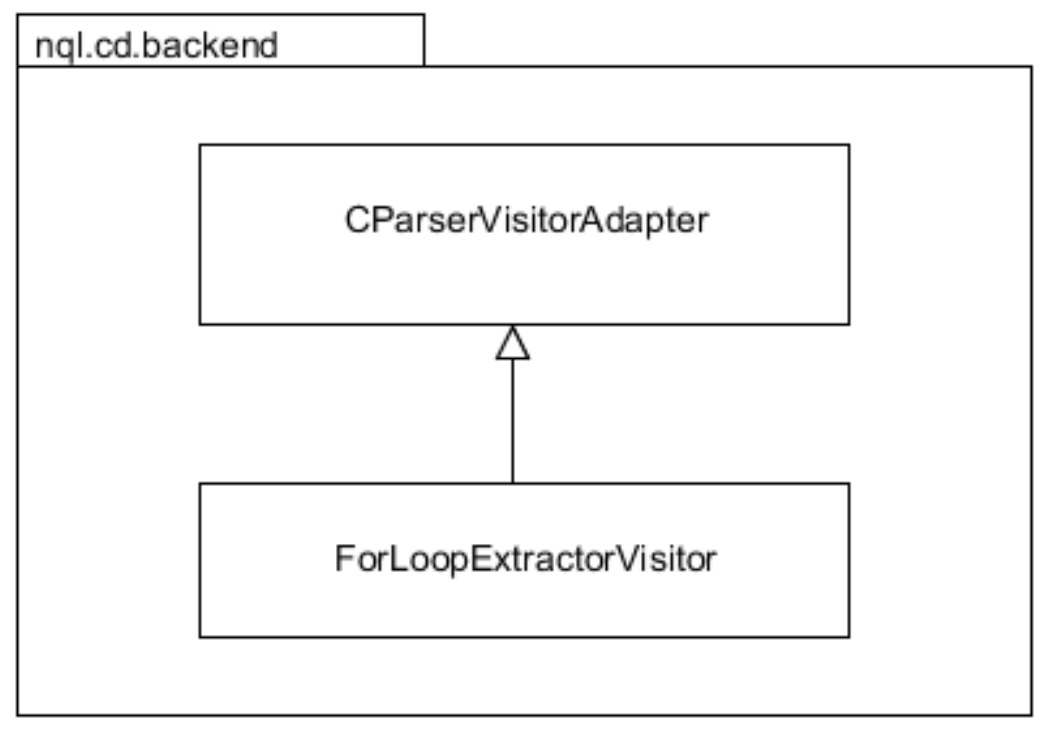

Figure 44. Classes Under nql.cd.backend Package 
The system program can be run in two different ways: command line version or graphical user interface (GUI) version. Figures 45 and 46 show how to run the system with the command line and a snapshot of the GUI version, respectively.

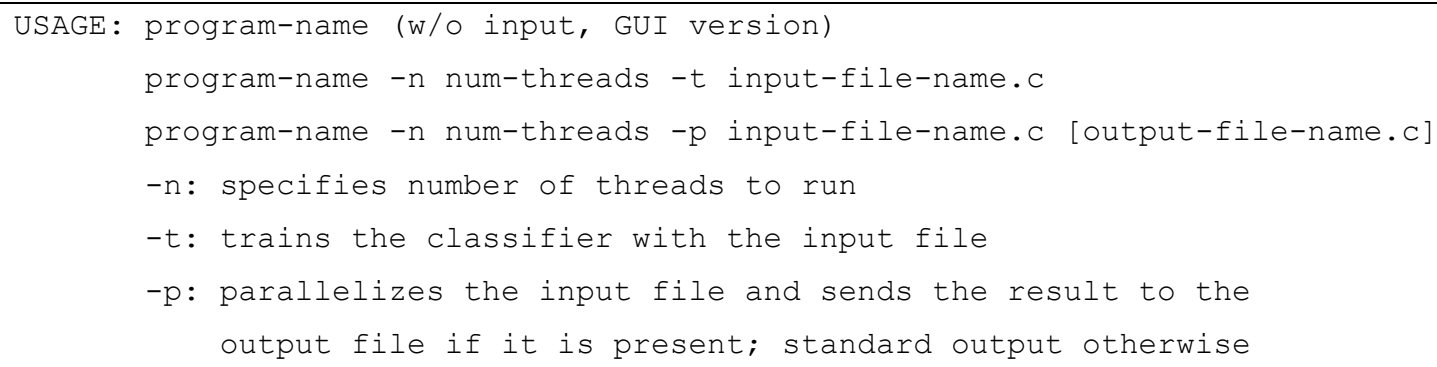

Figure 45. Program Usage for Command Line Version

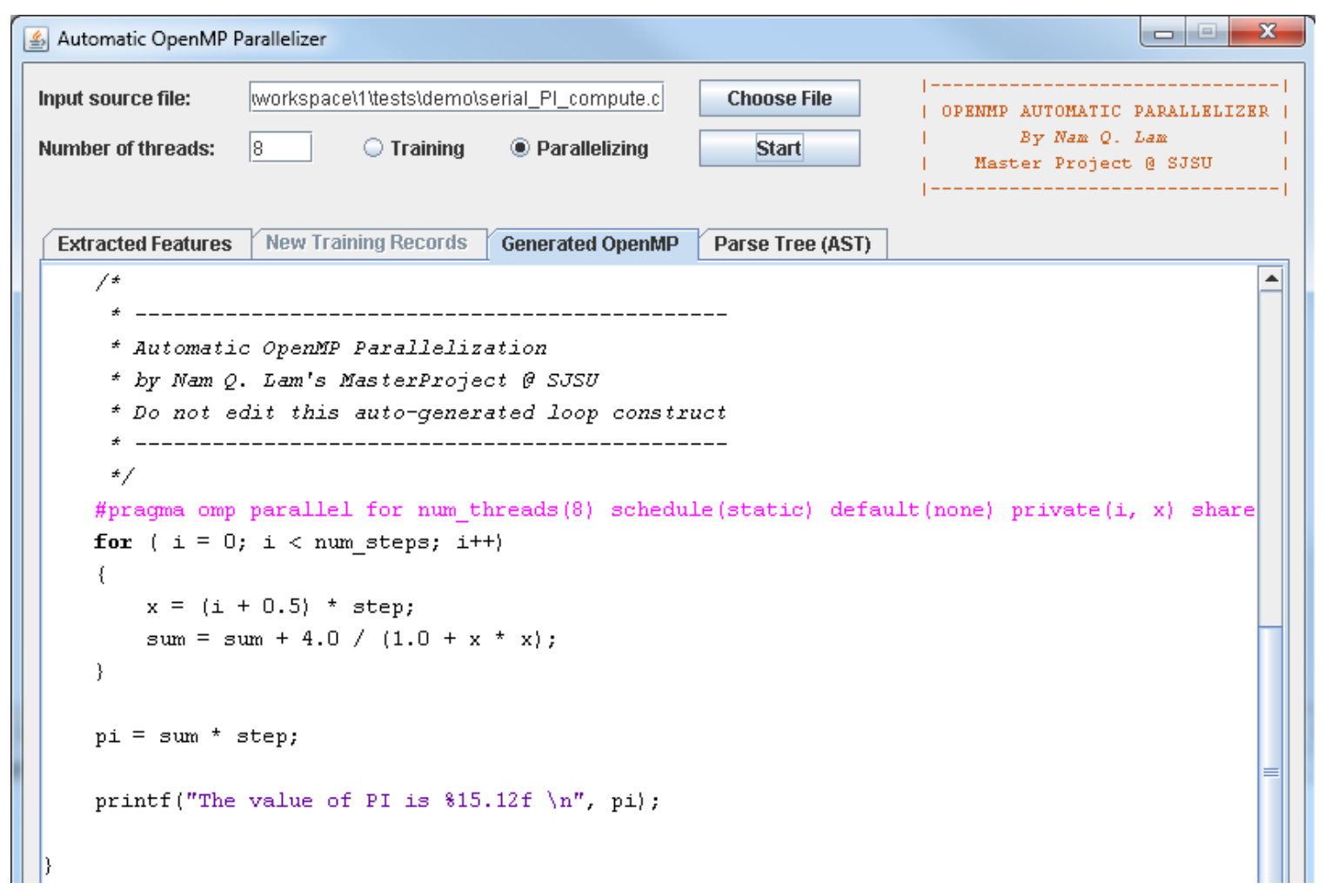

Figure 46. The GUI Version of the Program 


\section{Experimental Results}

OpenMP and auto-parallel performance are measured using the Linux operating system command called "time." This performance measuring method is simple, but practical, as recommended by [2]. The command shows three different time measurements called "real," "user," and "system" for an execution of a program. Among the three, "real" time, also called wall clock time [6], is the most important measurement because it encapsulates the entire time from start to finish of an execution call. This time measurement is used to analyze this project test results.

The objective of this section is to present the experimental results in order to prove the system works and to show the benefits of the machine learning and compilerbased approach chosen for this project. Below are snapshots of various test cases conducted on real samples of test files. Refer to Appendix C for the actual serial C program test files. Some cases show significant speed-up via OpenMP parallelization; while some others show not much of differences. This is understandable and acceptable [2] due to the simplicity of some the test programs and also due to the limitation of the system under test, which is a personal computer equipped with an Intel Core 2 Duo for a total of only four cores. An important concept here is that these resulting OpenMP programs are automatically parallelized.

Figures 47 and 48 contain charts comparing the real (wall clock) execution times between the programs to effectively visualize the test results. Refer to Appendix D for resulting auto-parallelized OpenMP files. Due to significant differences between the real time values of the test cases, two different charts are given for two different groups of test cases. 


\section{Test Case 1}

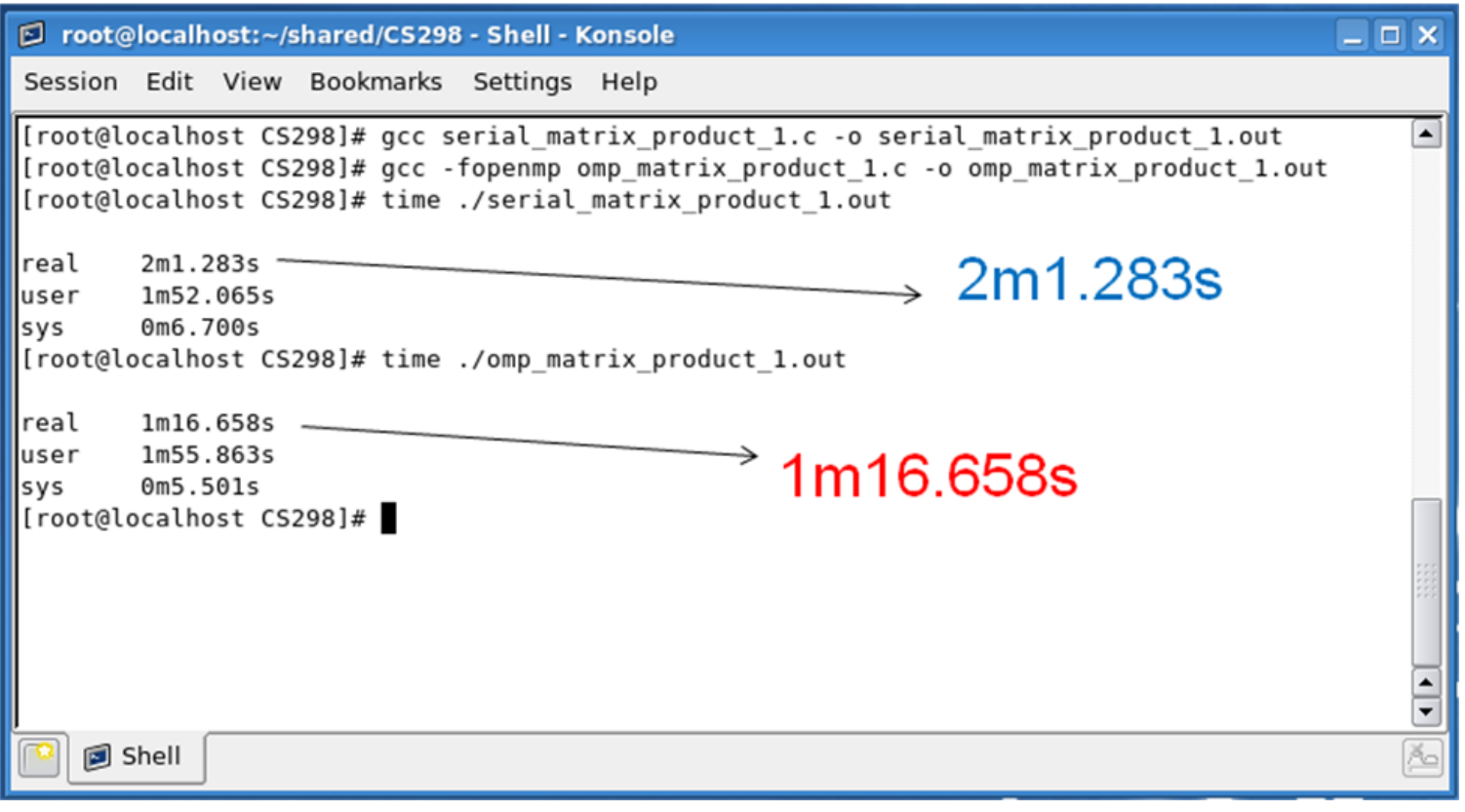

\section{Test Case 2}

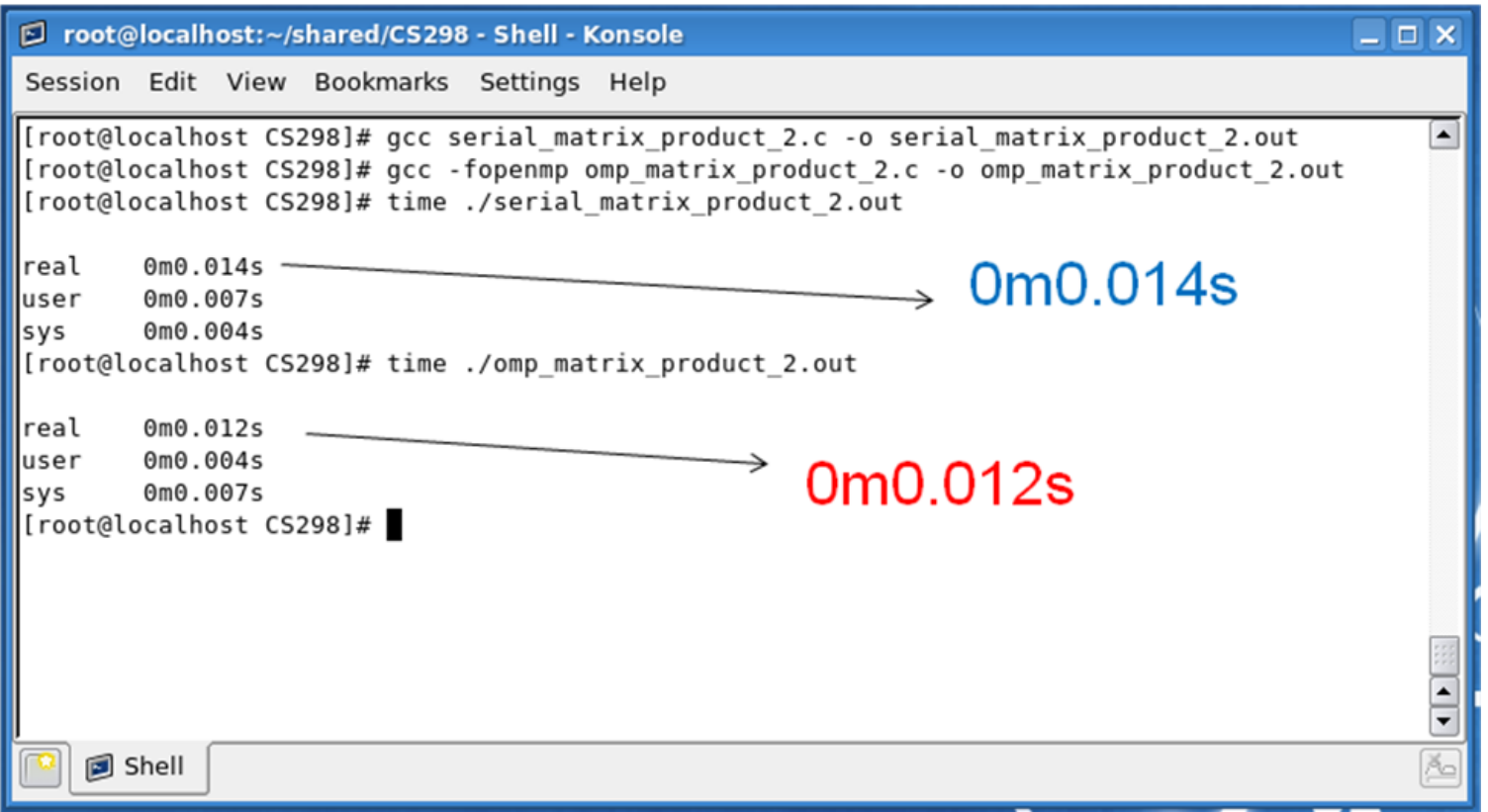




\section{Test Case 3}

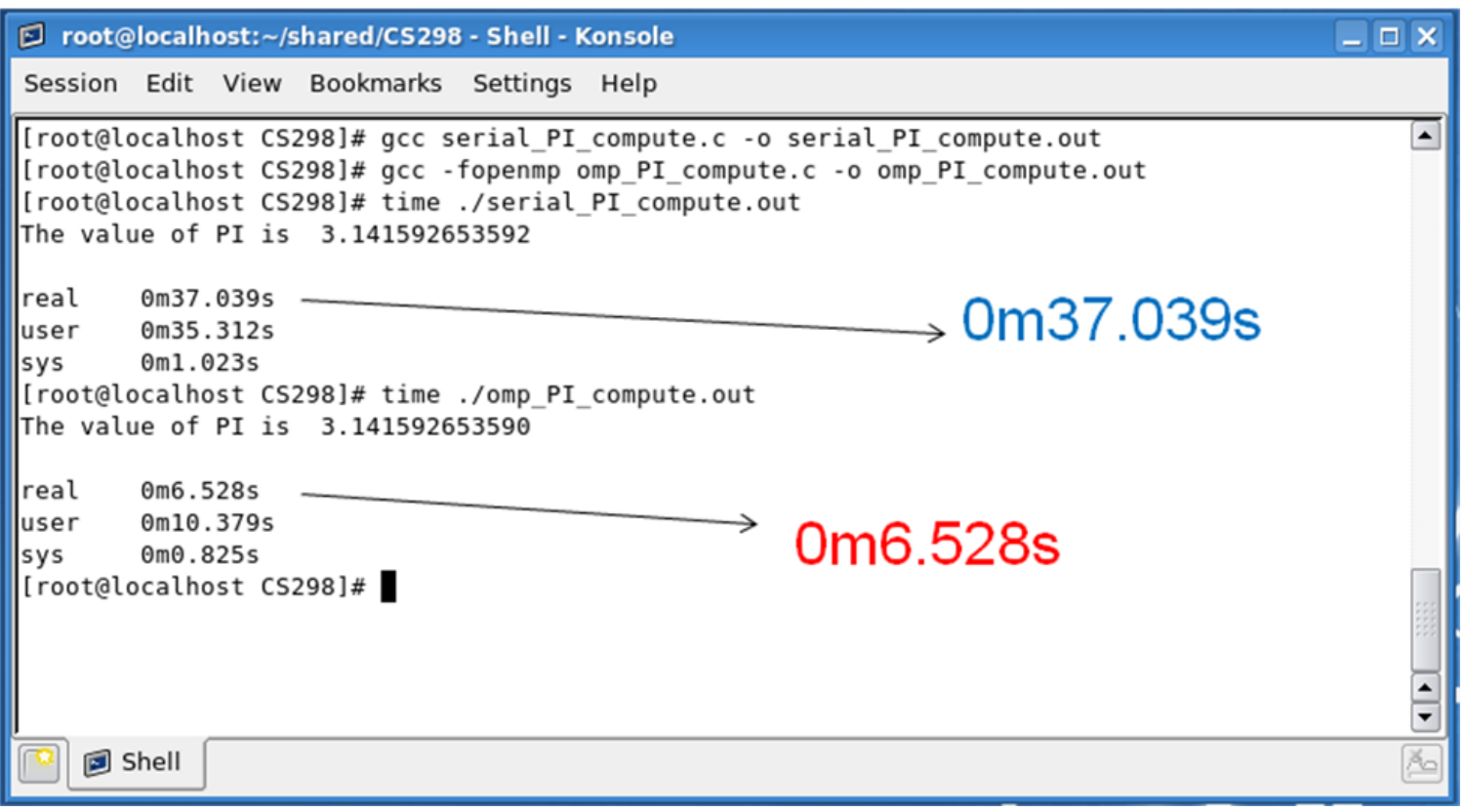

\section{Test Case 4}

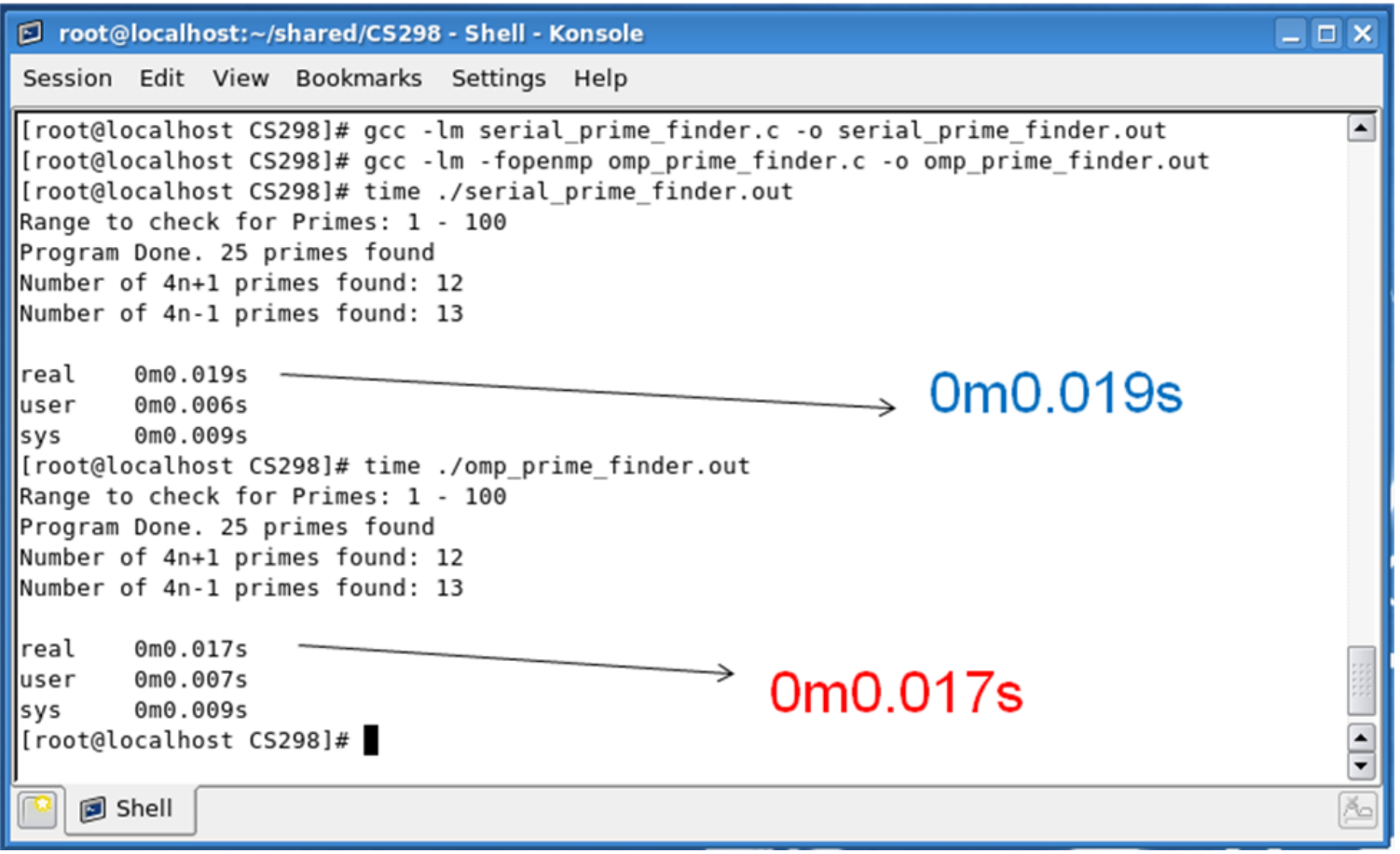




\section{Test Case 5}

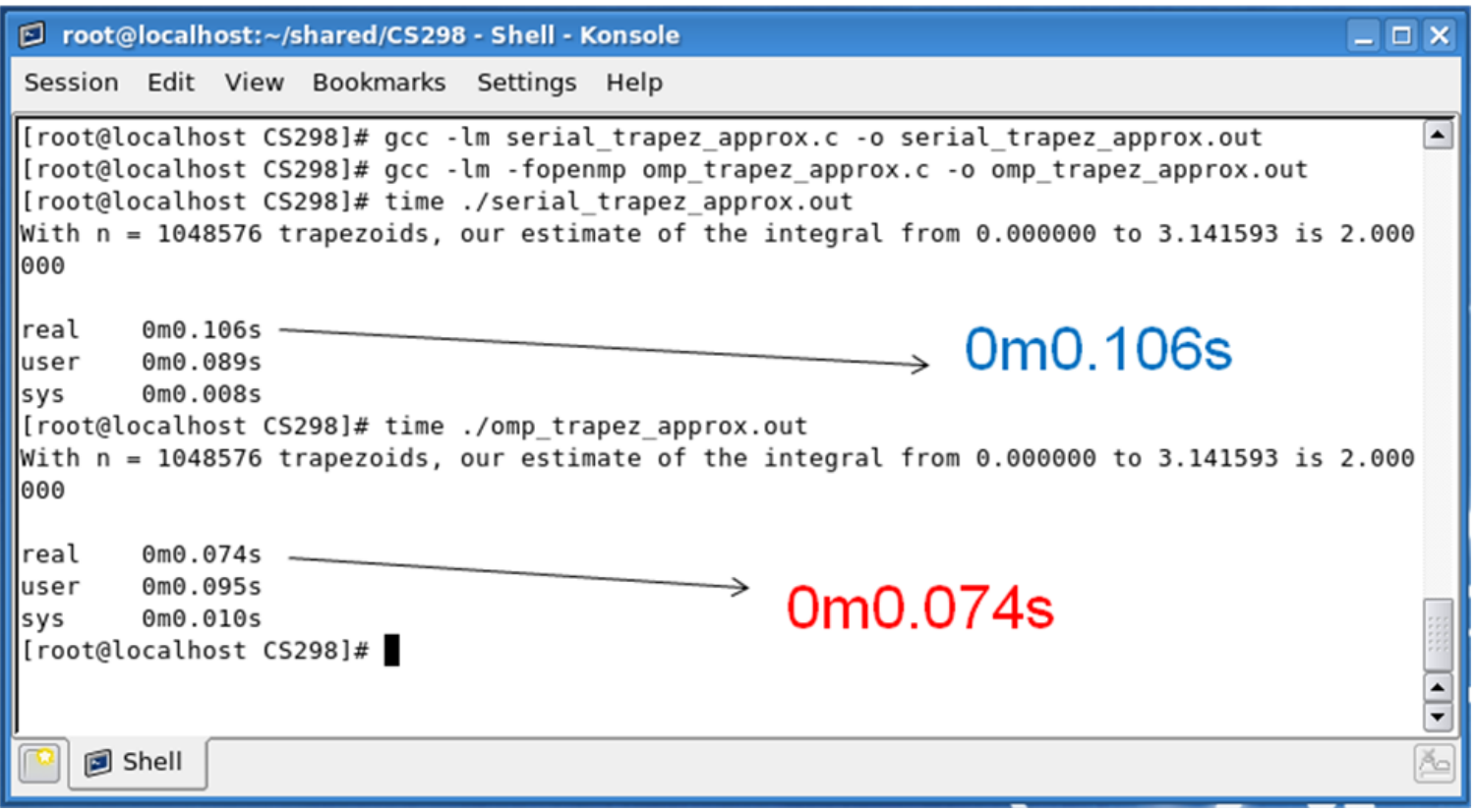

\section{Test Case 6}

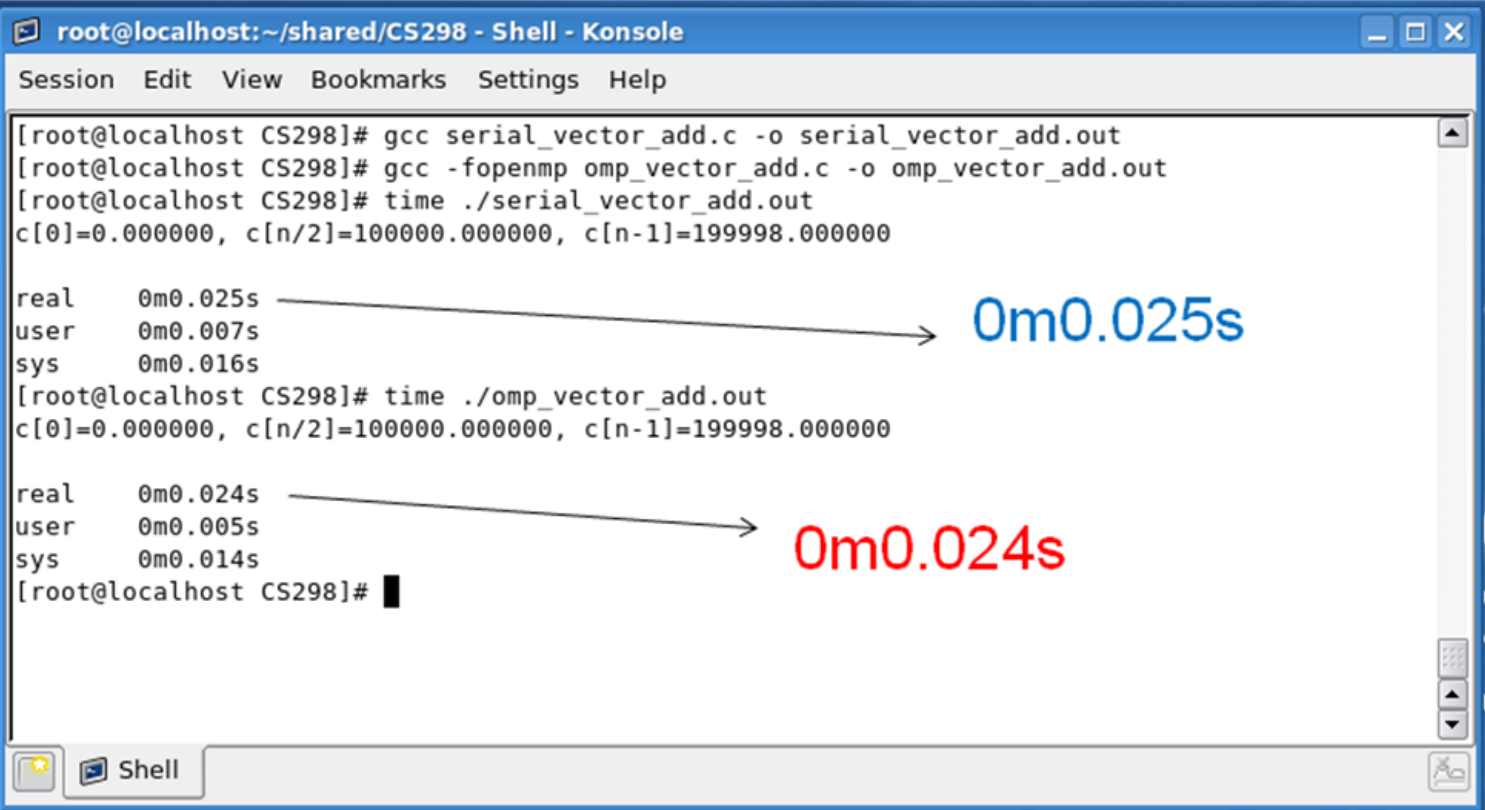




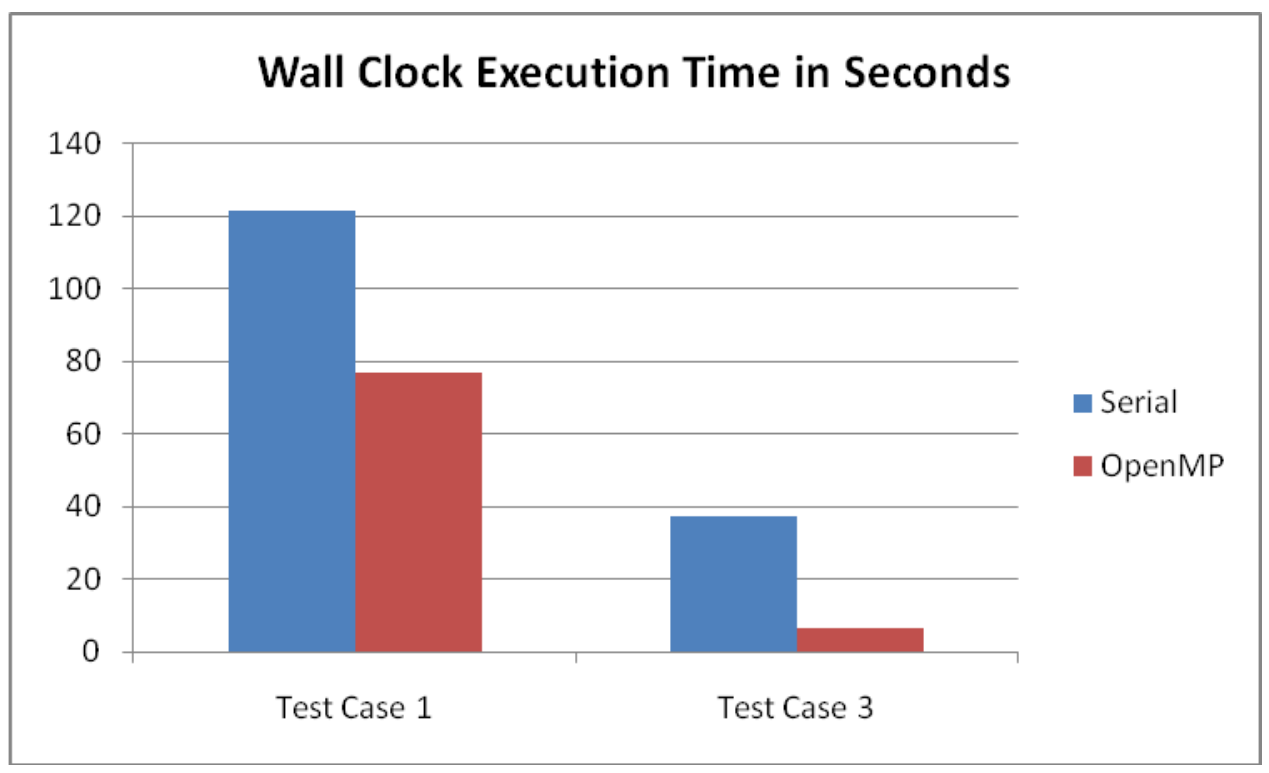

Figure 47. Real Time Chart of Test Cases 1 \& 3

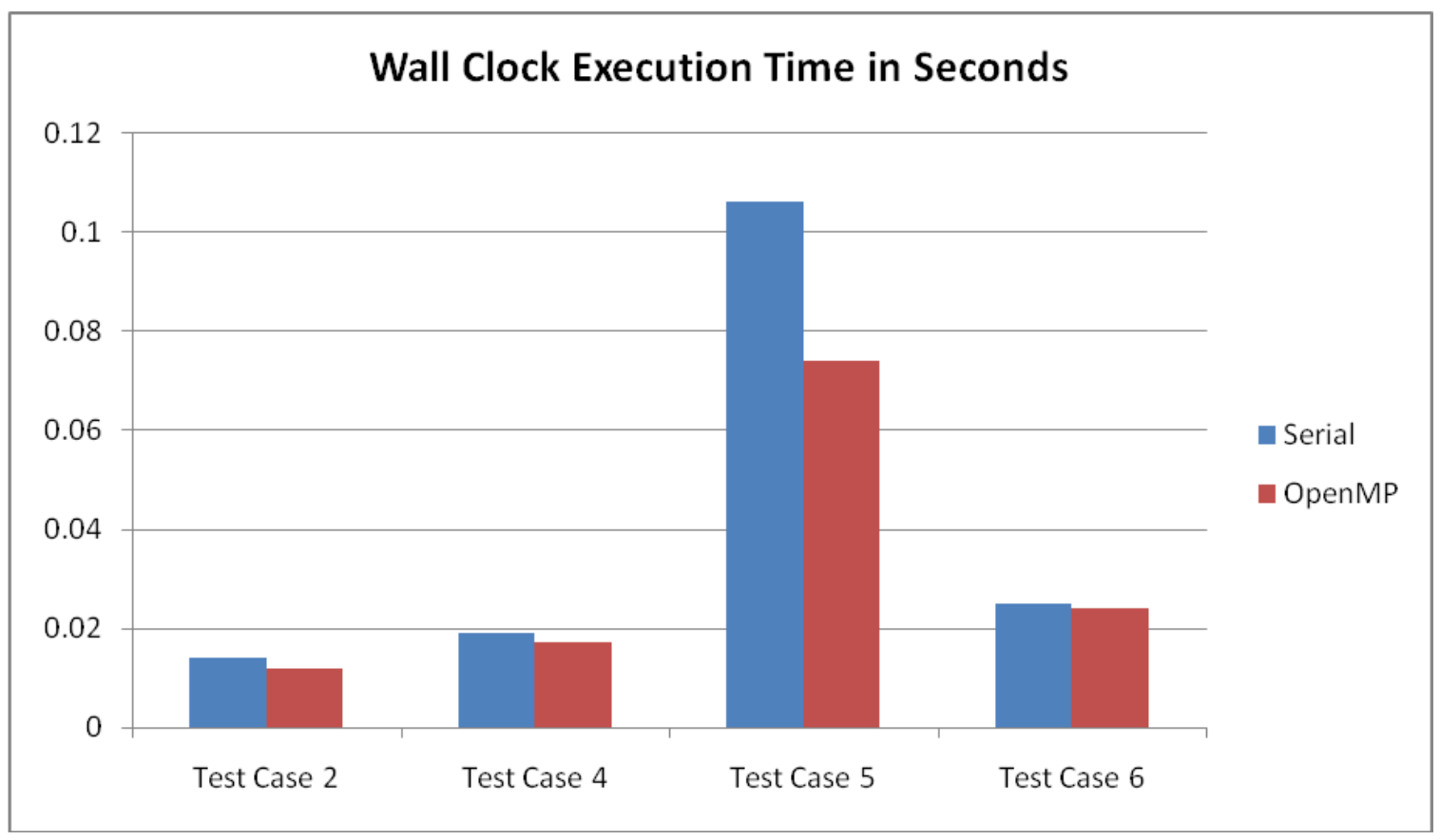

Figure 48. Real Time Chart of Test Cases 2, 4, 5, \& 6 
Based on the results of the test cases and the resulting chart above, we could see that the system has effectively parallelized the serial C programs into OpenMP C programs. Of course, performance of the OpenMP files inherits from performance of OpenMP itself. The project has achieved its goal, that is, the entire parallelizing process has been done automatically. This is the main objective of this writing project. 


\section{Conclusion and Future Work}

In conclusion, this report successfully describes, presents, and proves an advance and practical approach, machine learning and compiler-based design, to implement a novel system to automatically parallelize serial $\mathrm{C}$ programs using OpenMP parallel programming model.

This is my original work. It effectively provides a foundation for automatic parallel programming on multi-core architectures at source code level. The resulting system significantly helps software programmers in many ways. The programmers can use the system as a tool to first automatically parallelize a legacy serial C program, and then from the results, the programmers have the opportunity to fine-tune the program to achieve maximum parallel performance. Furthermore, compiler designers gain different benefits from this tool as well; they can integrate it into a modern compiler to achieve automatic parallelism.

Due to achievements in design goals of the project, specially in scalability and extensibility, in the future, the system can be easily extended to fully achieve parallelism beyond for loops by implementing other OpenMP work-sharing constructs via the already-in-place compiler-based design framework and the machine learning model. 


\section{References}

[1] S. Akhter and J. Roberts. Multi-core Programming: Increasing Performance through Software Multi-threading. Intel Press, 2006.

[2] B. Chapman, G. Jost, and R. Pas. Using OpenMP: Portable Shared Memory Parallel Programming. The MIT Press, 2008.

[3] P. Tan, M. Steinbach, and V. Kumar. Introduction to Data Mining. Addison Wesley, 2005.

[4] I. Witten and E. Frank. Data Mining: Practical Machine Learning Tools and Techniques. Morgan Kaufmann, 2005.

[5] R. Mak. Writing Compilers and Interpreters: A Software Engineering Approach. Wiley, 2009.

[6] R. Chun. CS 159: Parallel Processing. San Jose State University, http://www.cs.sjsu.edu/ rchun/, 2010.

[7] J. Abbate. "Getting Small: A Short History of the Personal Computer." Proceedings of the IEEE, Vol. 87, No. 9, pp. 1695-1698, Sep 1999.

[8] A. Apon, R. Buyya, H. Jin, and J. Mache. "Cluster Computing in the Classroom: Topics, Guidelines, and Experiences." First IEEE/ACM International Symposium on Cluster Computing and the Grid, pp. 476-483, May 2001.

[9] P. Werstein, H. Situ, and Z. Huang. "Load Balancing in a Cluster Computer." Seventh International Conference on Parallel and Distributed Computing, Applications and Technologies (PDCAT'06), pp. 569-577, Jan 2006.

[10] C. Yang and K. Li. "The Anatomy of a Course in Cluster and Grid Computing." 2005 International Conference on Information Technology: Research and Education, pp. 403-407, Jun 2005.

[11] J. Mache and A. Apon. "Teaching Grid Computing: Topics, Exercises, and Experiences.” Proceedings of the IEEE, Vol. 50, No. 1, pp. 3-9, Feb 2007.

[12] Shuai Zhang, Shufen Zhang, X. Chen, and X. Huo. "The Comparison Between Cloud Computing and Grid Computing." 2010 International Conference on Computer Application and System Modeling (ICCASM 2010), pp. V11-72 - V1175 , Oct 2010. 
[13] R. Allan. The History of the Personal Computer: The People and the Technology. Allan Publishing, 2001.

[14] Shufen Zhang, Shuai Zhang, X. Chen, and S. Wu. "Analysis and Research of Cloud Computing System Instance." Second International Conference on Future Networks, pp. 88-92, Jan 2010.

[15] J. Peng, X. Zhang, Z. Lei, B. Zhang, W. Zhang, and Q. Li. "Comparison of Several Cloud Computing Platforms." Second International Symposium on Information Science and Engineering, pp. 23-27, Dec 2009.

[16] C. Gong, J. Liu, Q. Zhang, H. Chen, and Z. Gong. "The Characteristics of Cloud Computing." $39^{\text {th }}$ International Conference on Parallel Processing Workshops, pp. 275-279, Sep 2010.

[17] A. Velte, T. Velte, and R. Elsenpeter. Cloud Computing: A Practical Approach. McGraw-Hill, 2010.

[18] F. Travostino, J. Mambretti, and G. Edwards. Grid Networks: Enabling Grids with Advanced Communication Technology. John Wiley \& Sons, 2006.

[19] B. Brey. Programming the 80286, 80386, 80486, and Pentium Based Personal Computer. Prentice Hall, 1996.

[20] X. Chen and S. Long. "Adaptive Multi-versioning for OpenMP Parallelization via Machine Learning." $15^{\text {th }}$ International Conference on Parallel and Distributed Systems, pp. 907-912, Dec 2009.

[21] S. Mitra and T. Acharya. Data Mining: Multimedia, Soft Computing, and Bioinformatics. Wiley, 2003.

[22] P. Gasper, C. Herbst, J. McGough, C. Rickeet, and G. Stubbendieck. "Automatic Parallelization of Sequential C Code." South Dakota School of Mines and Technology, http://www.mcs.sdsmt.edu/mcgough/research/docs/gasper.pdf.

[23] D. Alpaydin. Introduction to Machine Learning. MIT Press, 2010.

[24] J. Han, M. Kamber, and J. Pei. Data Mining: Concepts and Techniques. Morgan Kaufmann, 2011.

[25] M. Quinn. Parallel Programming in C with MPI and OpenMP. McGraw-Hill, 2004.

[26] Z. Wang and M. O'Boyle. "Mapping Parallelism to Multi-cores: A Machine Learning Based Approach." $14^{\text {th }}$ ACM SIGPLAN Symposium on Principles and 
Practice of Parallel Programming, pp. 75-84, 2009.

[27] Z. Yan and L. Liu. "The Algorithm of Parallel Recognition Based on Data Dependence." 2009 International Conference on Information Technology and Computer Science, pp. 636-639, Jul 2009.

[28] M. Hornick, E. Marcade, and S. Venkayala. Java Data Mining: Strategy, Standard, and Practice. MK Publishers, 2007.

[29] C. Walinsky and D. Banerjee. "A Functional Programming Language Compiler for Massively Parallel Computers." 1990 ACM Conference on LISP and Functional Programming, pp. 131-138, 1990.

[30] T. Gross, A. Zobel, and M. Zolg. "Parallel Compilation for a Parallel Machine." ACM SIGPLAN 1989 Conference on Programming Language Design and Implementation, pp. 91-100, 1989.

[31] M. Areanaz, J. Tourino, and R. Doallo. "A GSA-based Compiler Infrastructure to Extract Parallelism from Complex Loops." $17^{\text {th }}$ Annual International Conference on Supercomputing, pp. 193-204, 2003.

[32] "Intel Guide for Developing Multithreaded Applications," http://software.intel.com/en-us/articles/intel-guide-for-developing-multithreadedapplications/.

[33] C. Wang, Y. Wu, E. Borin, S. Hu, W. Liu, D. Sager, T. Ngai, and J. Fang. "Dynamic Parallelization of Single-Threaded Binary Programs using Speculative Slicing." $23^{\text {rd }}$ International Conference on Supercomputing, pp. 158-168, 2009.

[34] "Introduction to Parallel Computing," https://computing.llnl.gov/tutorials/parallel_comp/.

[35] A. Aho, M. Lam, R. Sethi, and J. Ullman.. Compilers: Principles, Techniques, \& Tools. Pearson/Addison Wesley, 2007.

[36] M. Cohn. Succeeding with Agile: Software Development Using Scrum. Addison Wesley, 2010.

[37] P. Pacheco. Parallel Programming with MPI. MK Publishers, 1997.

[38] T. Copeland. Generating Parsers with JavaCC. Centennial Books, 2007.

[39] "JavaCC is a parser/scanner generator for Java," http://java.net/projects/javacc/.

[40] “ANTLR Parser Generator,” http://www.antlr.org/. 
[41] "Weka 3: Data Mining Software in Java," http://www.cs.waikato.ac.nz/ml/weka/.

[42] “The OpenMP API Specification for Parallel Programming," http://openmp.org/wp/.

[43] B. Bruegge and A. Dutoit. Object-Oriented Software Engineering Using UML, Patterns, and Java. Prentice Hall, 2010.

[44] "C Language Reference," http://msdn.microsoft.com/enus/library/fw5abdx6(v=VS.80).aspx. 


\section{Appendix}

\subsection{Appendix A: Training Instances in ARFF Format}

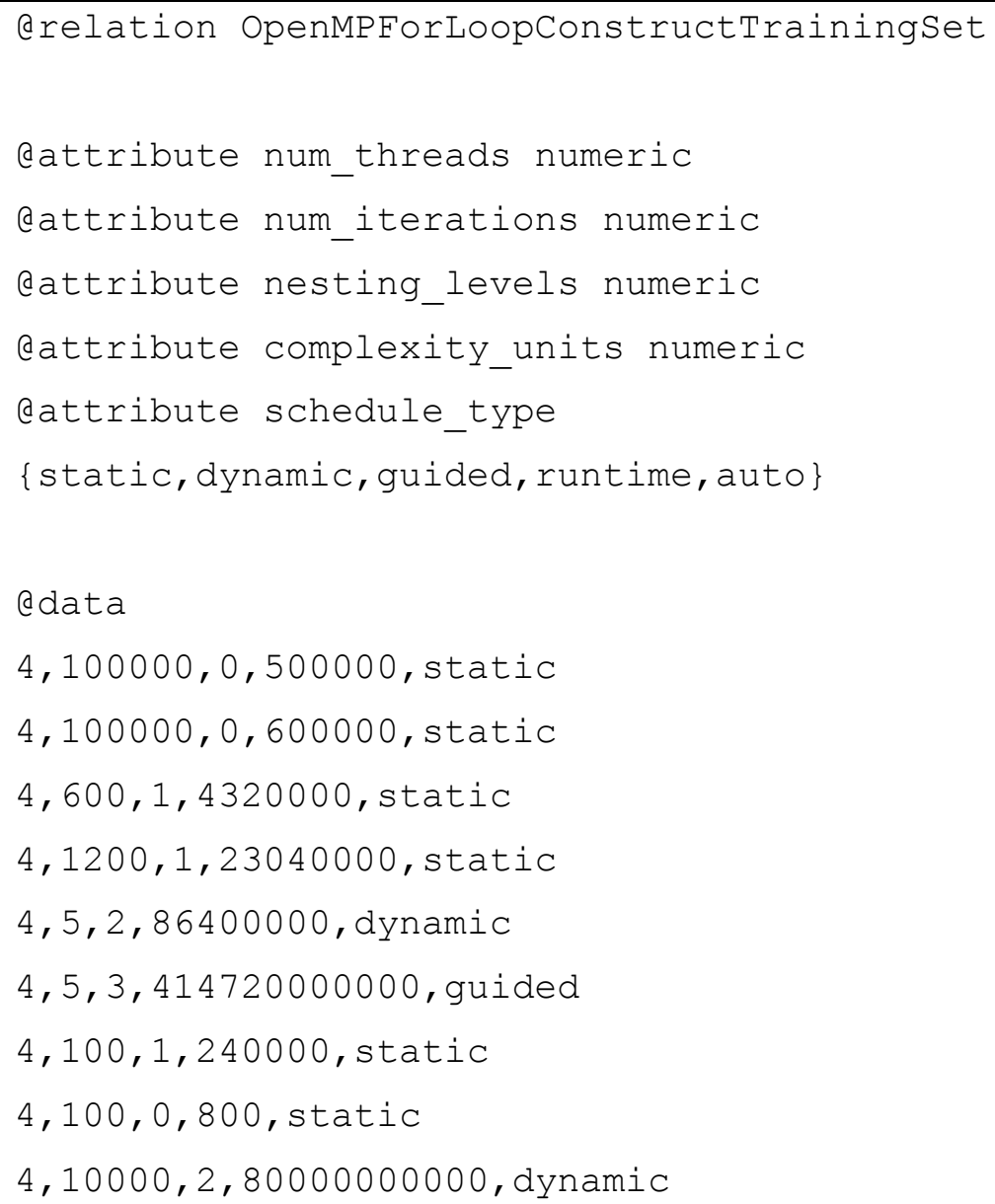

\subsection{Appendix B: OpenMP Training Source Files}

\section{omp_training1.c}

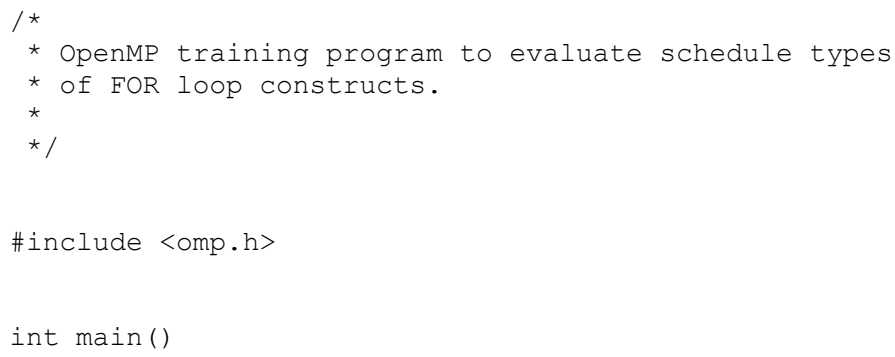




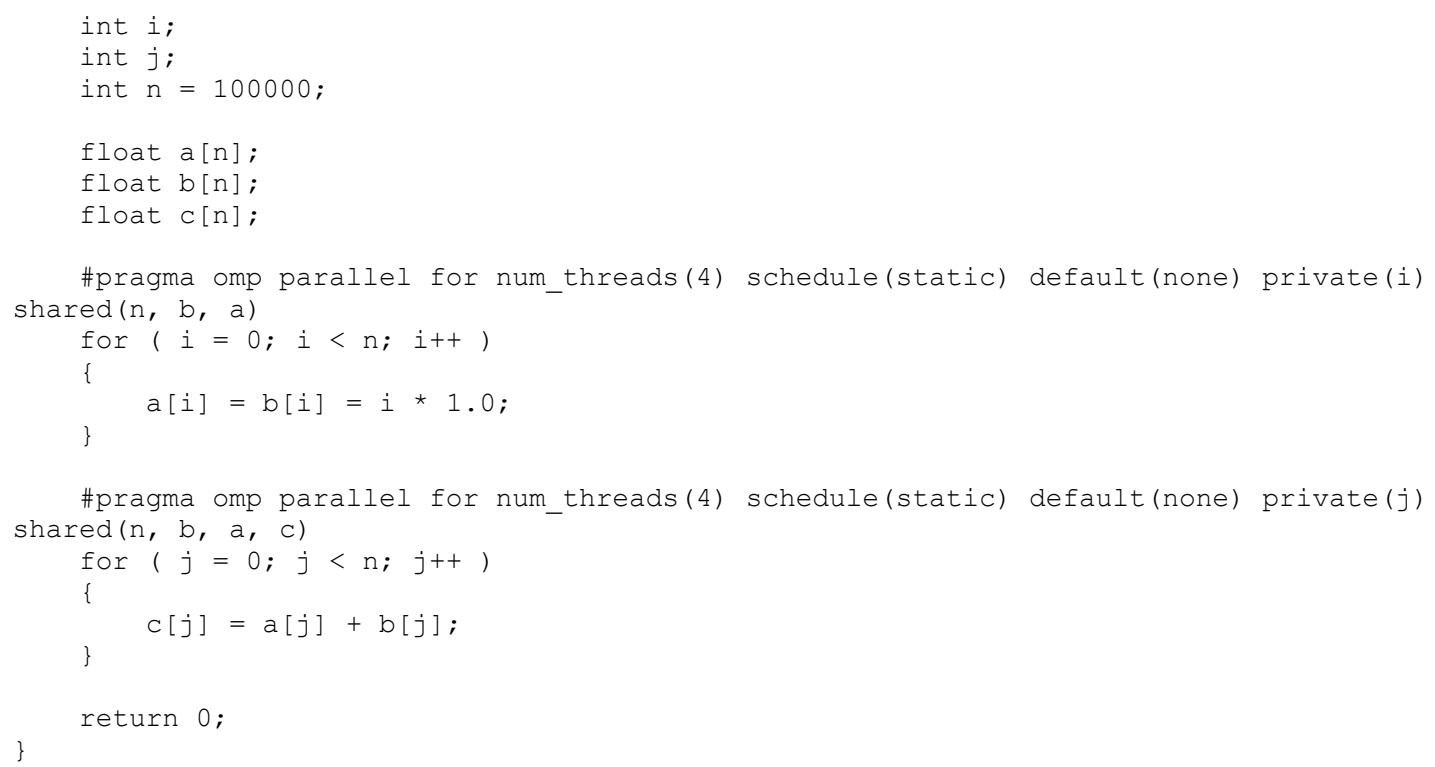

\section{omp_training2.c}

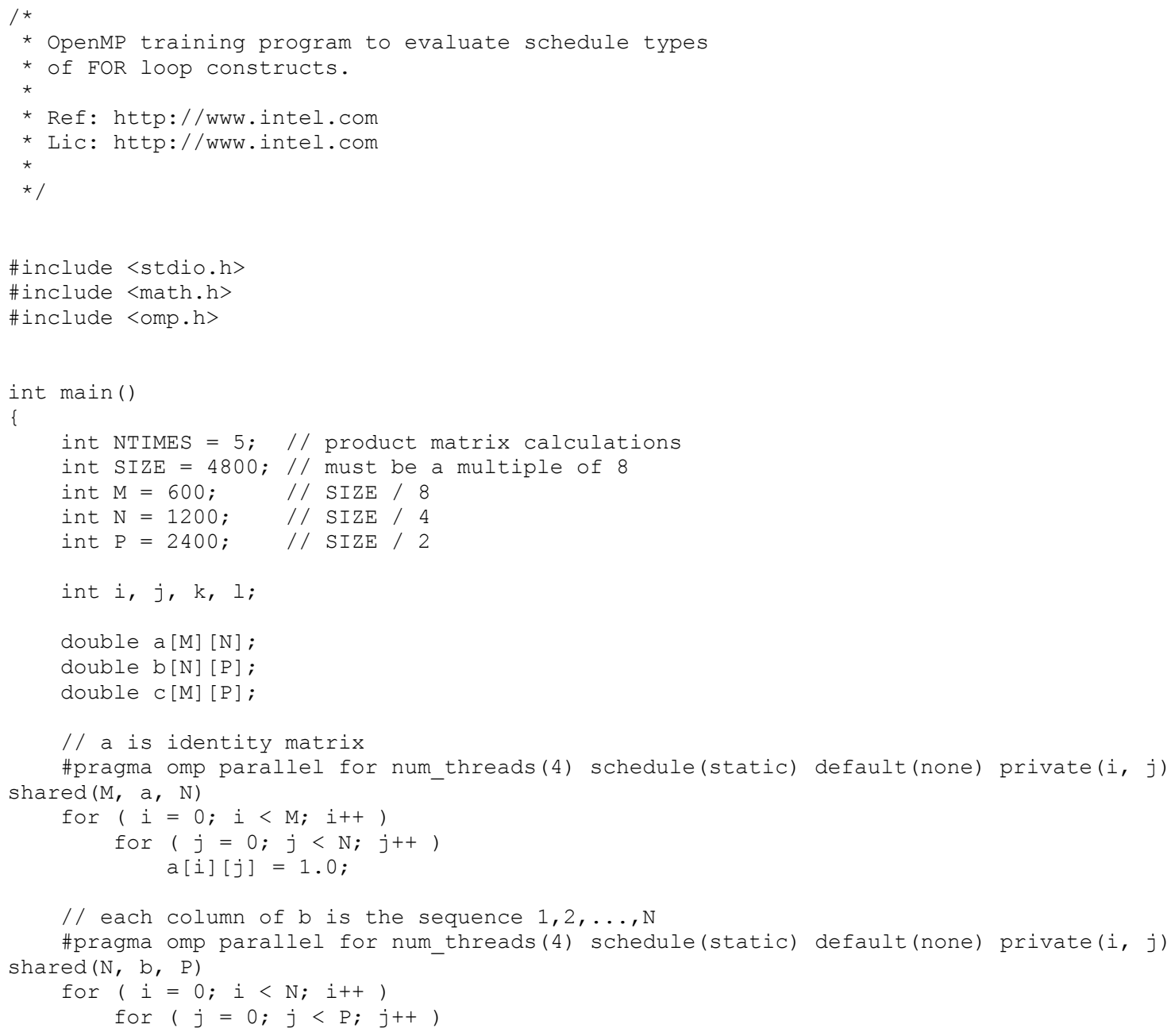


$b[i][j]=i+1 . ;$

// initialize product matrix

\#pragma omp parallel for num_threads(4) schedule(dynamic) default(none) private(l, i, j) shared(NTIMES, M, P, C)

for $(1=0 ; 1<\operatorname{NTIMES} ; 1++)$

for $(i=0 ; i<M ; i++)$

for $(j=0 ; j<P ; j++)$

$c[i][j]=0.0 ;$

// this for loop performs intensive calculations

\#pragma omp parallel for num threads(4) schedule(guided) default(none) private(l, i,

$k$, j) shared (NTIMES, M, N, b, a, P, c)

for $(1=0 ; 1<\operatorname{NTIMES} ; 1++)$

\{

// product calculation

for $(i=0$; $i<M$; $i++)$

\{

for $(\mathrm{k}=0 ; \mathrm{k}<\mathrm{N} ; \mathrm{k}++)$

\{

// each element of the product is just the sum $1+2+\ldots+n$

for $(j=0 ; j<P ; j++)$

\{

\}

$c[i][j]+=a[i][k] * b[k][j] ;$

return 0 ;

\}

\section{omp_training3.c}

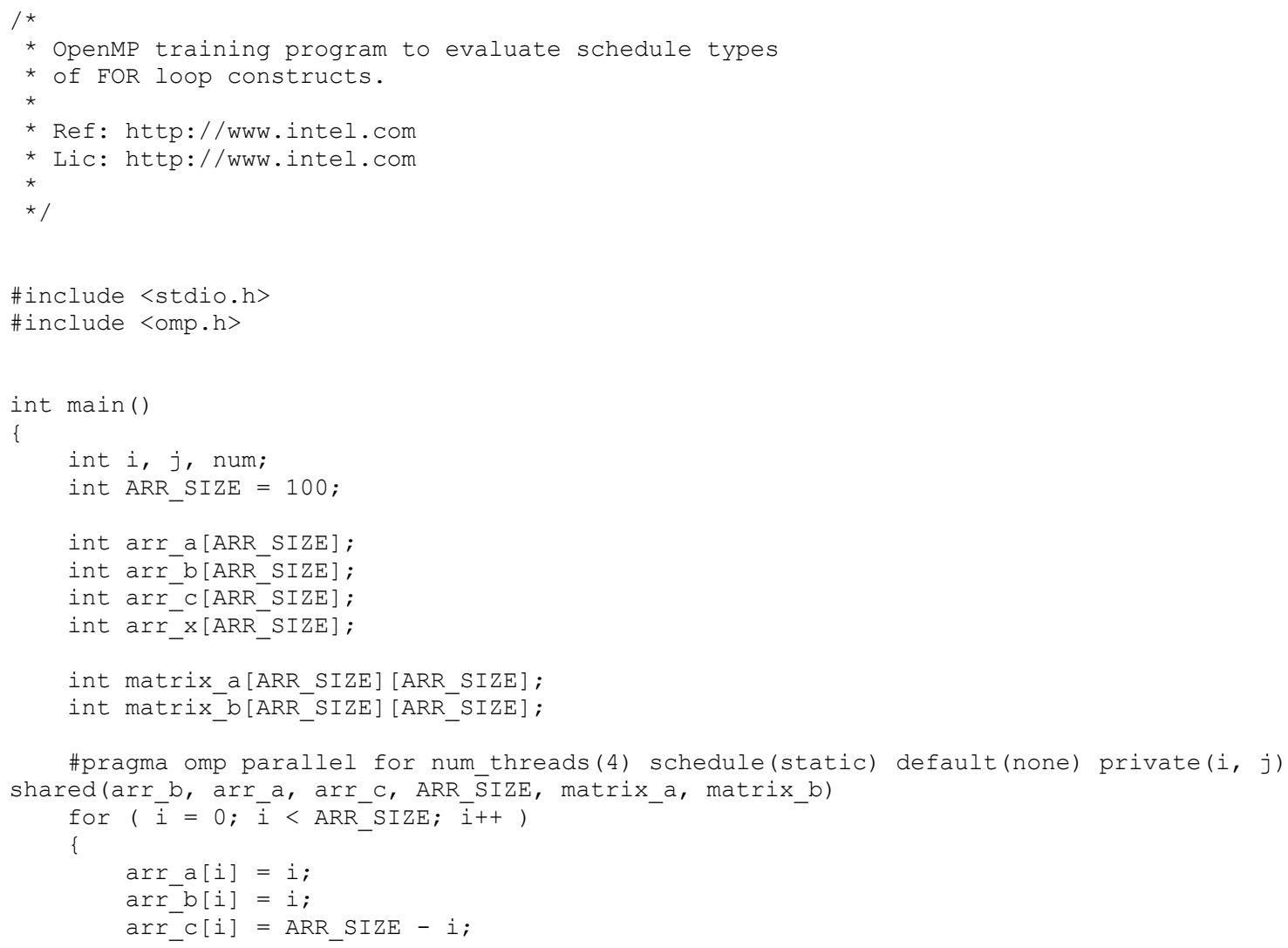




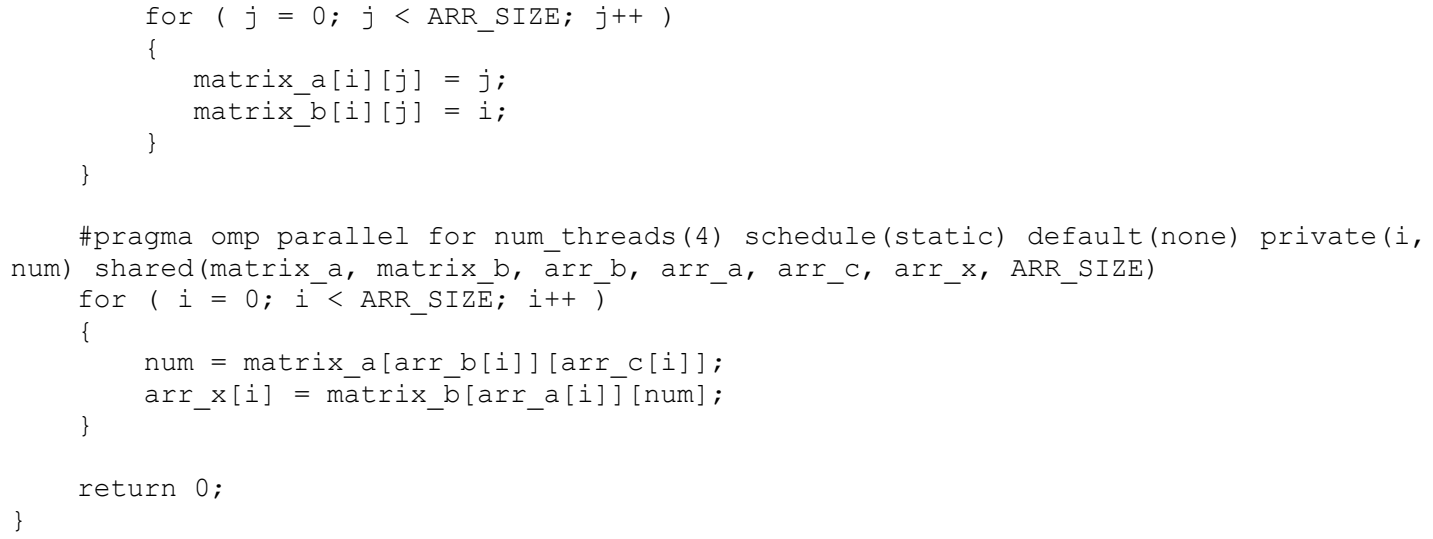

\section{omp_training4.c}

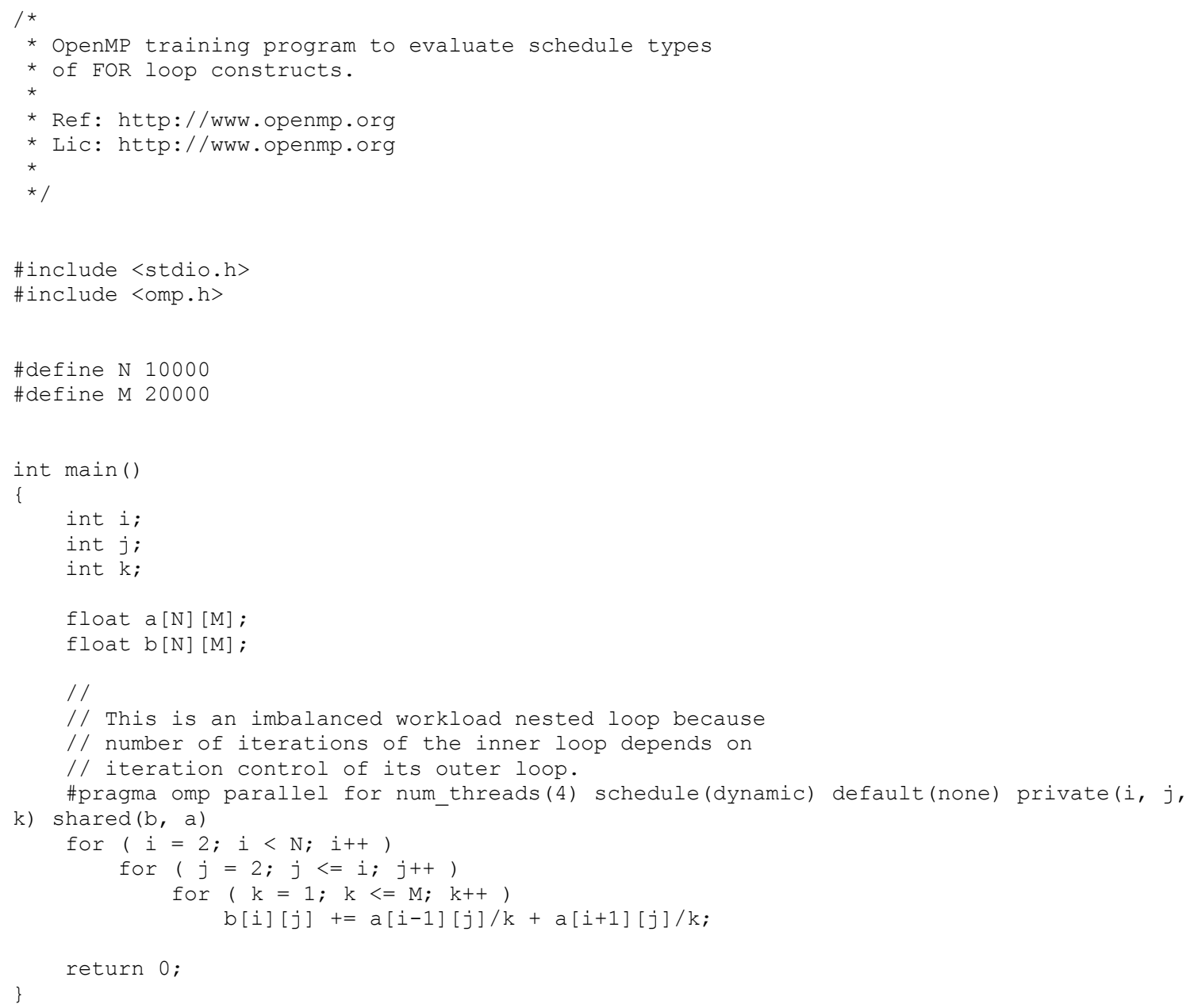

\subsection{Appendix C: Serial Program Test Files}




\section{serial_matrix_product_1.c}

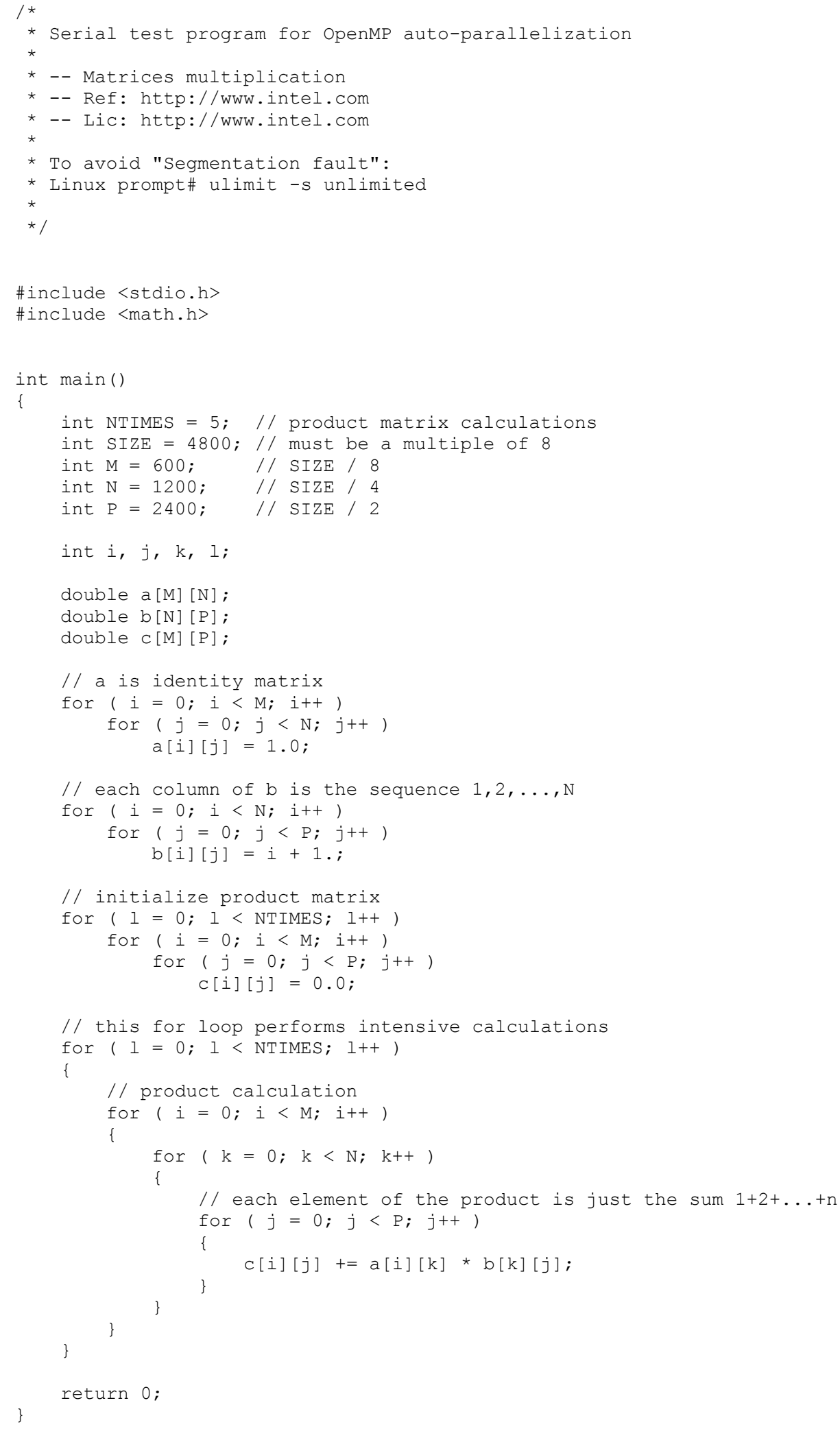




\section{serial_matrix_product_2.c}

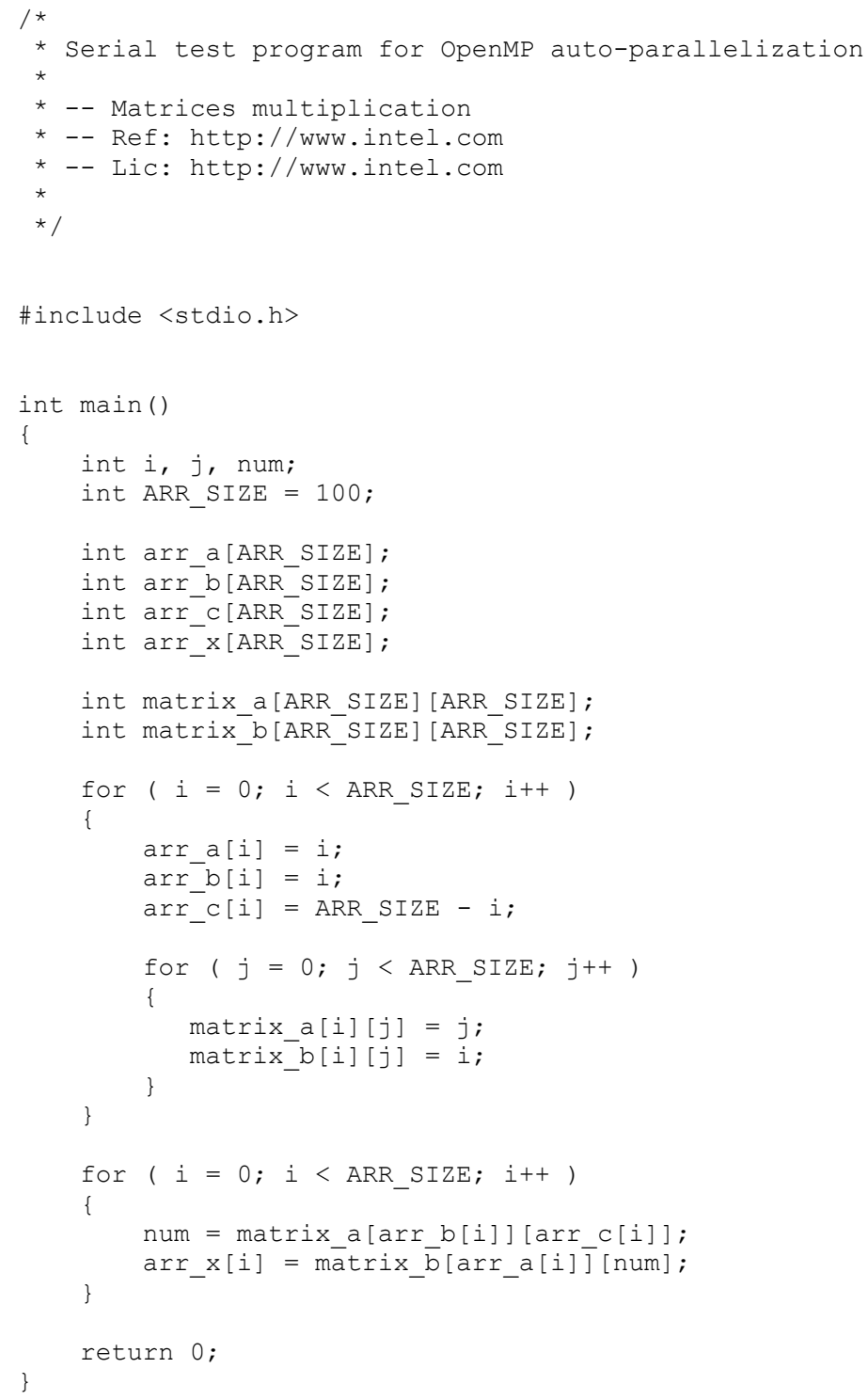

\section{serial_PI_compute.c}

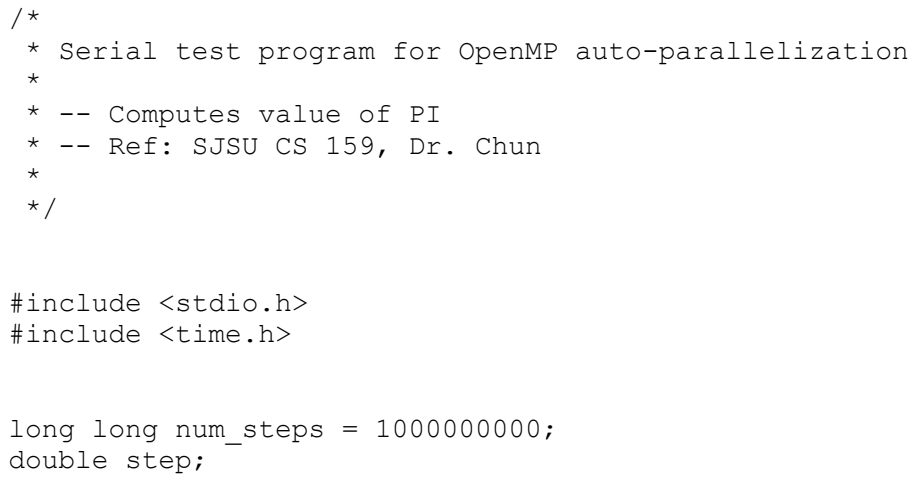




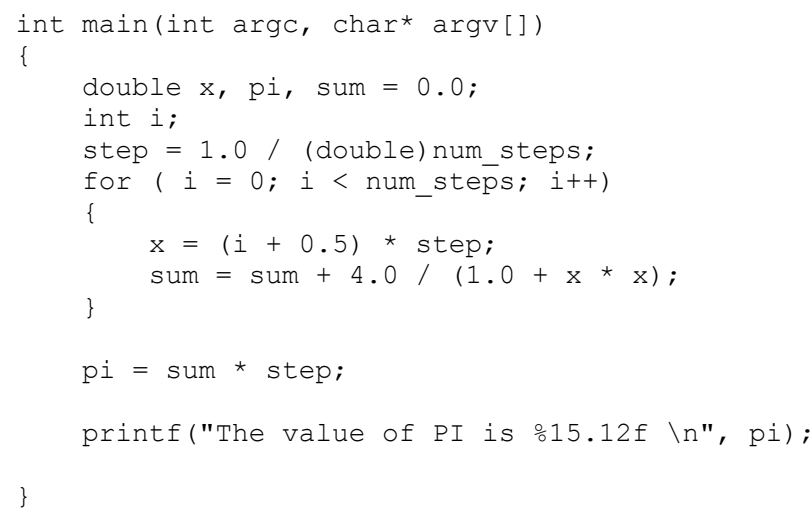

\section{serial_prime_finder.c}

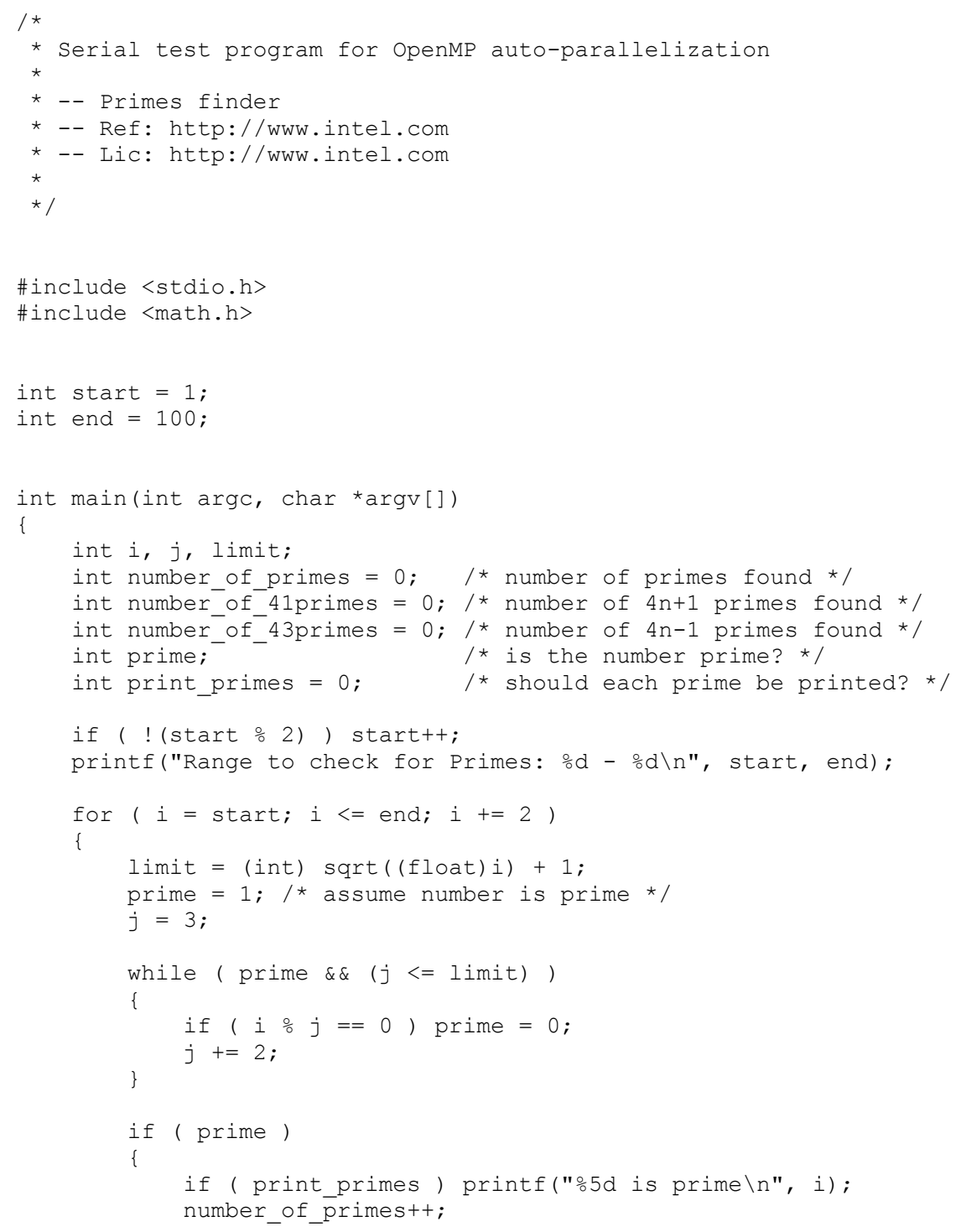




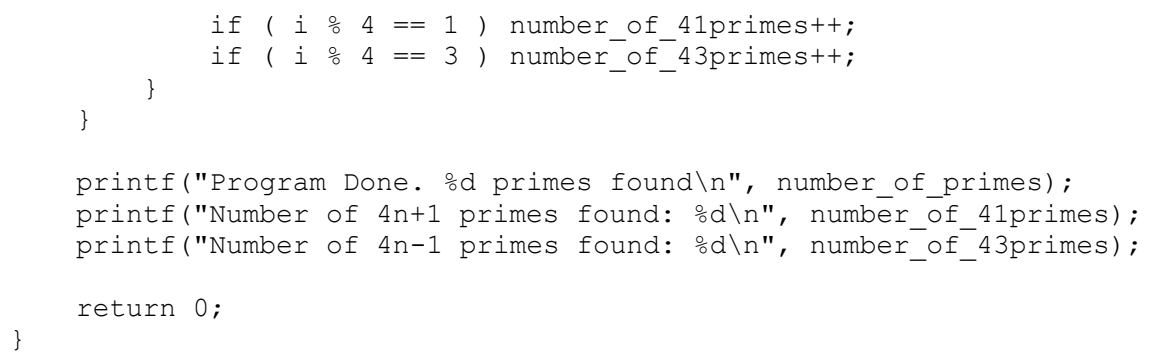

\section{serial_trapez_approx.c}

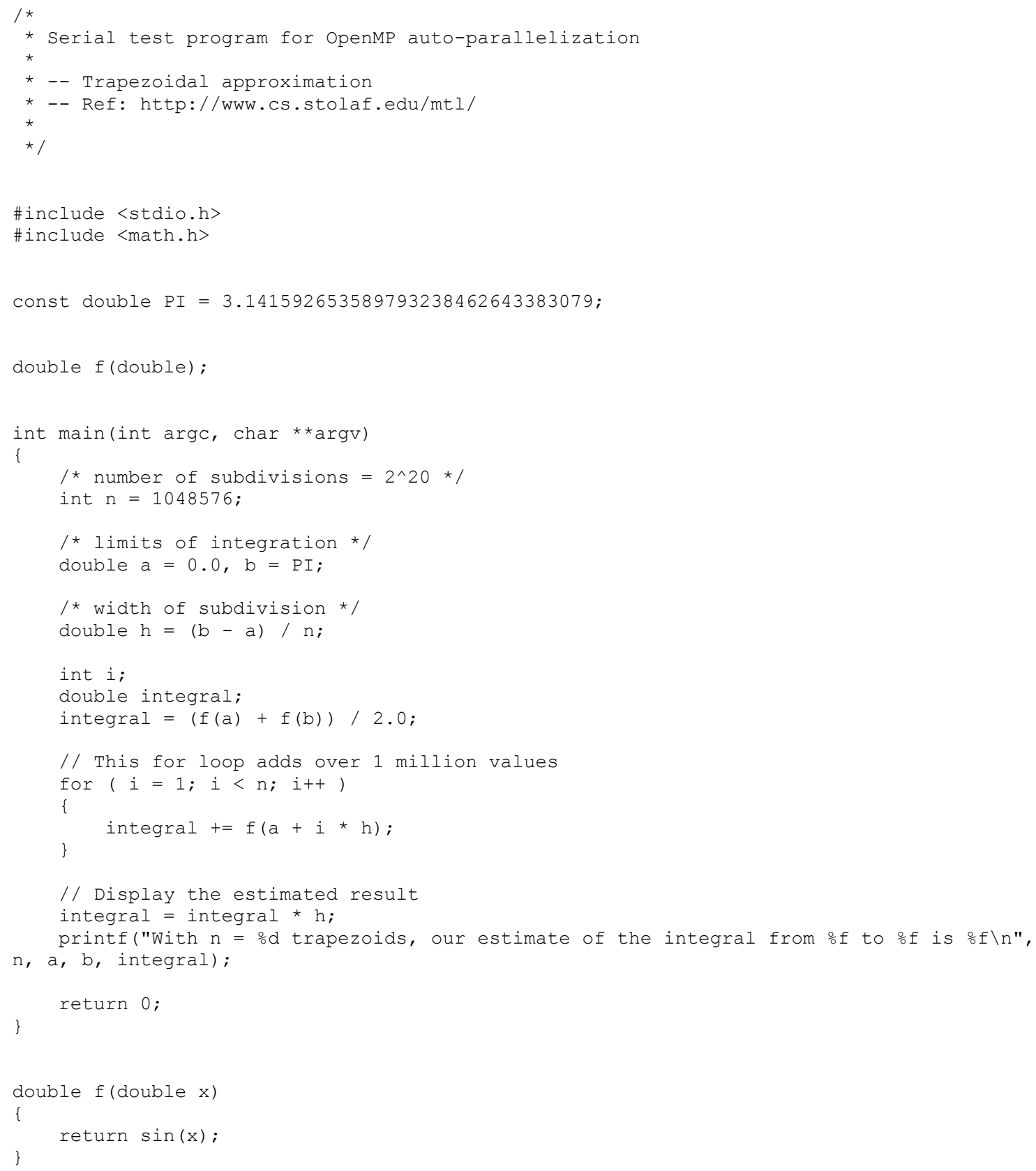




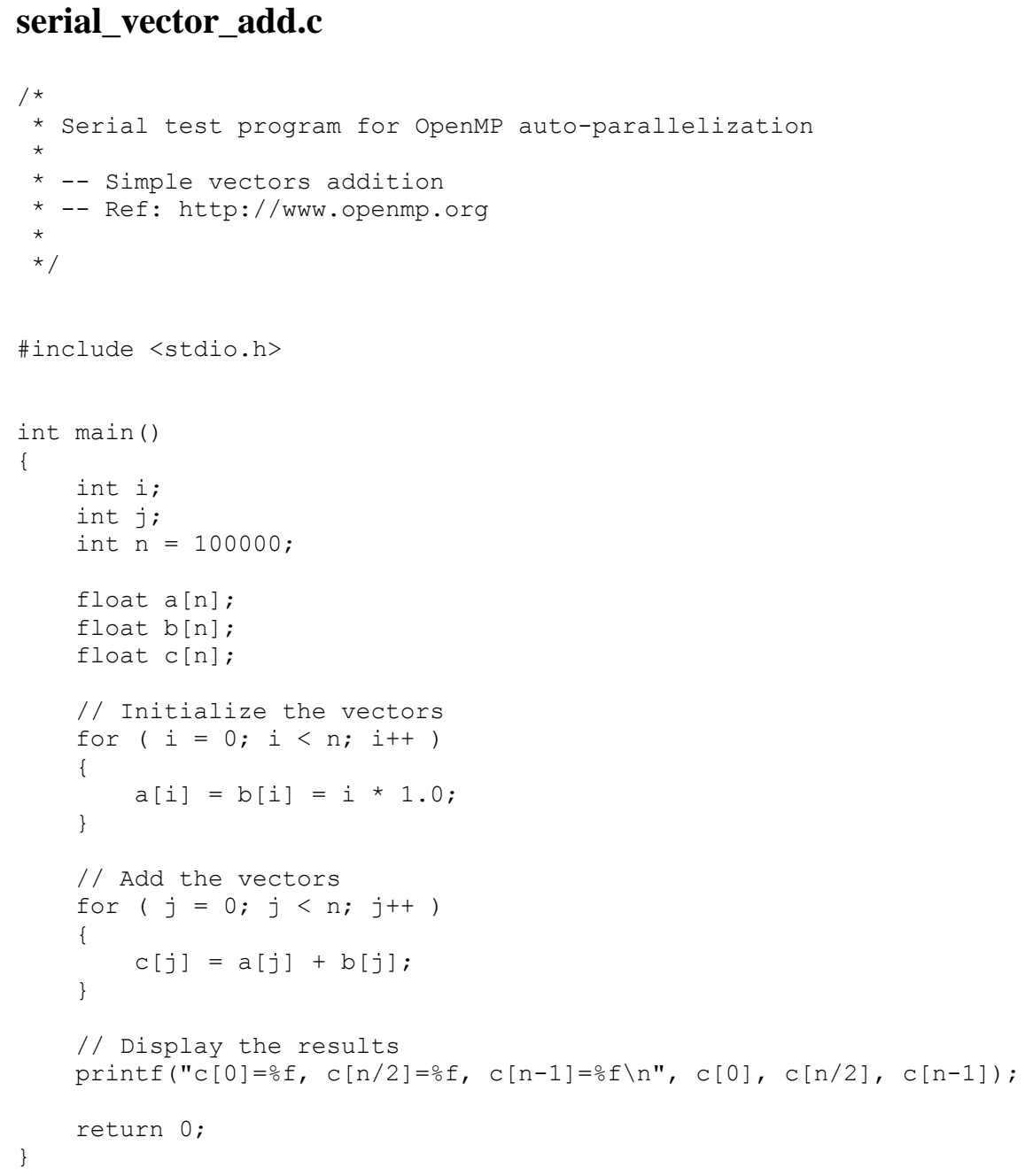

\subsection{Appendix D: Auto-Parallel Result Files}

\section{omp_matrix_product_1.c}

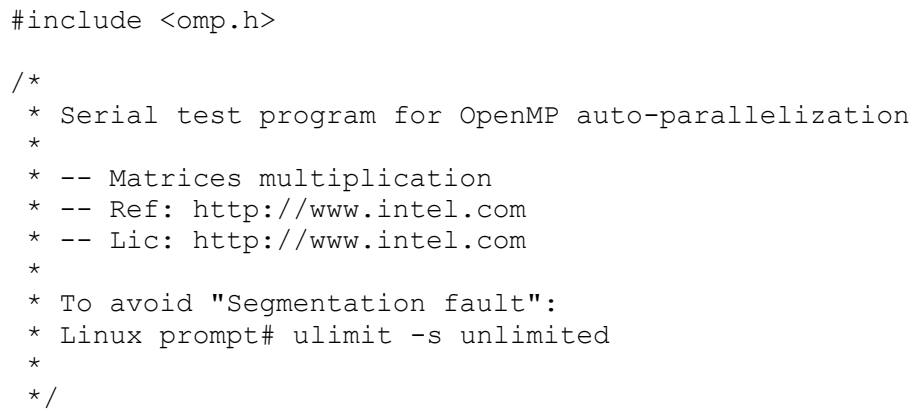


\#include <stdio.h>

\#include <math.h>

int $\operatorname{main}()$

\{

int NTIMES $=5 ; / /$ product matrix calculations

int SIZE $=4800 ; / /$ must be a multiple of 8

int $\mathrm{M}=600 ; \quad / / \mathrm{SIZE} / 8$

int $\mathrm{N}=1200 ; \quad / / \mathrm{SIZE} / 4$

int $\mathrm{P}=2400 ; \quad / / \mathrm{SIZE} / 2$

int $i, j, k, l$;

double a [M] [N];

double b[N] [P];

double C $[\mathrm{M}][\mathrm{P}]$;

// a is identity matrix

/*

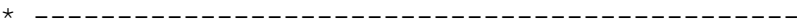

* Automatic openMP Parallelization

* by Nam Q. Lam's MasterProject e SJSU

* Do not edit this auto-generated loop construct

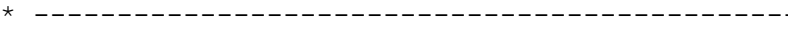

*

\#pragma omp parallel for num_threads(4) schedule(static) default(none) private(i, j) shared $(\mathrm{M}, \mathrm{a}, \mathrm{N})$

for $(i=0 ; i<M$; $i++)$

for $(j=0 ; j<N ; j++)$

$a[i][j]=1.0 ;$

$/ /$ each column of $\mathrm{b}$ is the sequence $1,2, \ldots, \mathrm{N}$

/*

* Automatic OpenmP Parallelization

* by Nam Q. Lam's MasterProject a SJSU

* Do not edit this auto-generated loop construct

* ----

* 1

\#pragma omp parallel for num threads(4) schedule(static) default(none) private(i, $j$ ) shared $(\mathrm{N}, \mathrm{b}, \mathrm{P})$

for $(i=0 ; i<N$; $i++)$

for $(j=0 ; j<P ; j++)$

$b[i][j]=i+1 . ;$

// initialize product matrix

/*

(1)

* Automatic OpenMP Parallelization

* by Nam Q. Lam's MasterProject e SJSU

* Do not edit this auto-generated loop construct

* - -

* /

\#pragma omp parallel for num_threads(4) schedule(dynamic) default(none) private(l, i, j) shared (NTIMES, M, P, C)

for $(1=0 ; 1<\operatorname{NTIMES} ; 1++)$

for $(i=0 ; i<M ; i++)$

for $(j=0 ; j<P ; j++)$

$c[i][j]=0.0 ;$

// this for loop performs intensive calculations

/*

* Automatic OpenMP Parallelization

* by Nam Q. Lam's MasterProject a SJSU

* Do not edit this auto-generated loop construct

*/

\#pragma omp parallel for num_threads(4) schedule(guided) default(none) private(l, $i$, $k$, j) shared (NTIMES, M, N, b, a, ${ }^{-}, c$ ) 


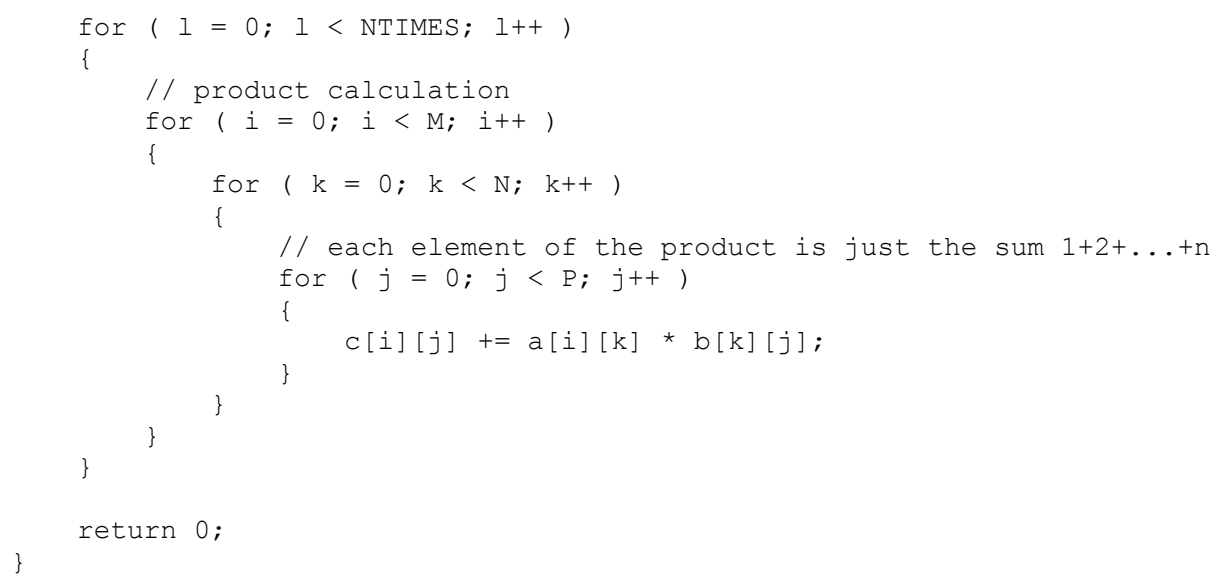

\section{omp_matrix_product_2.c}

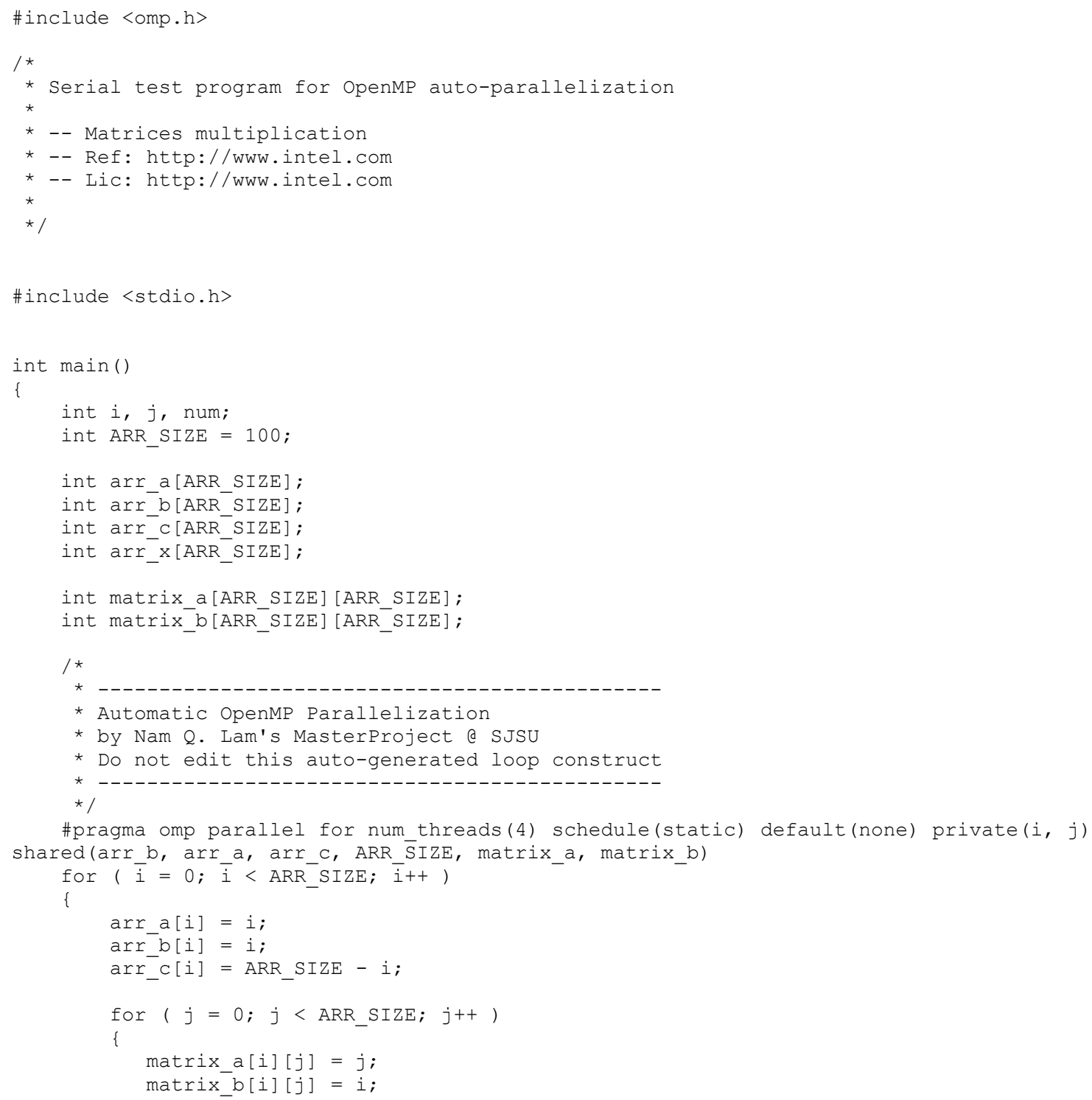




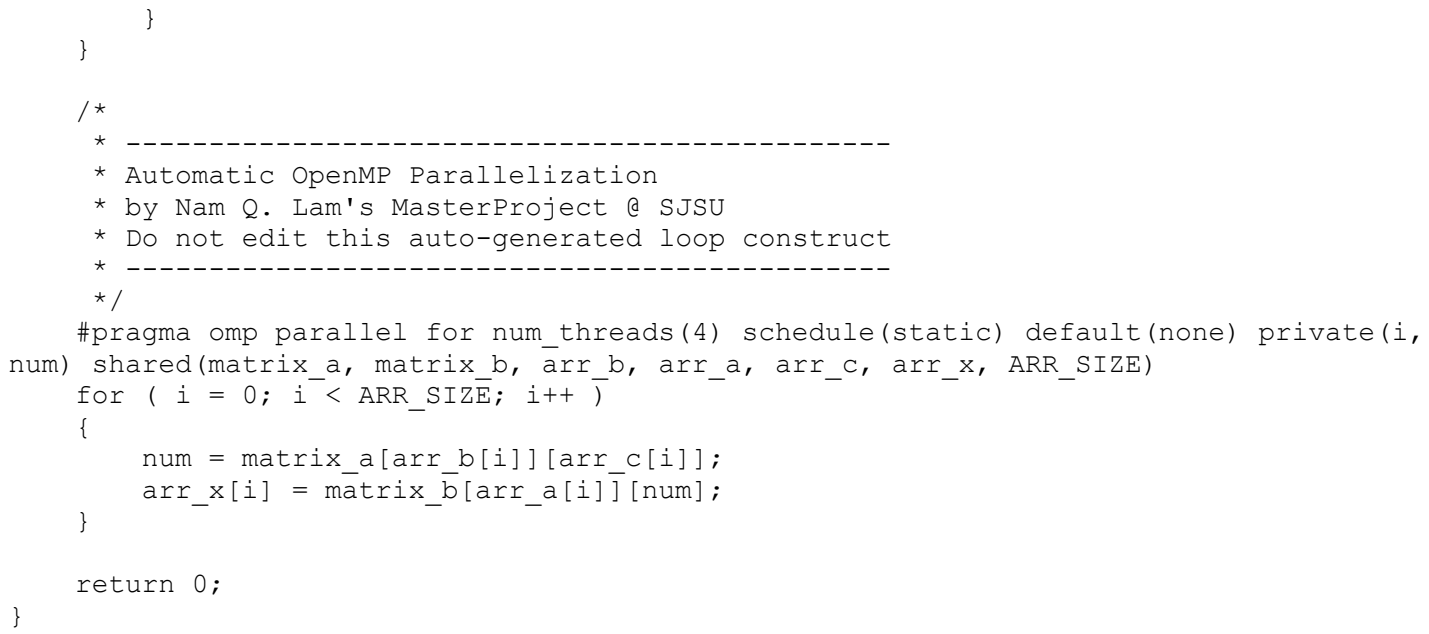

\section{omp_PI_compute.c}

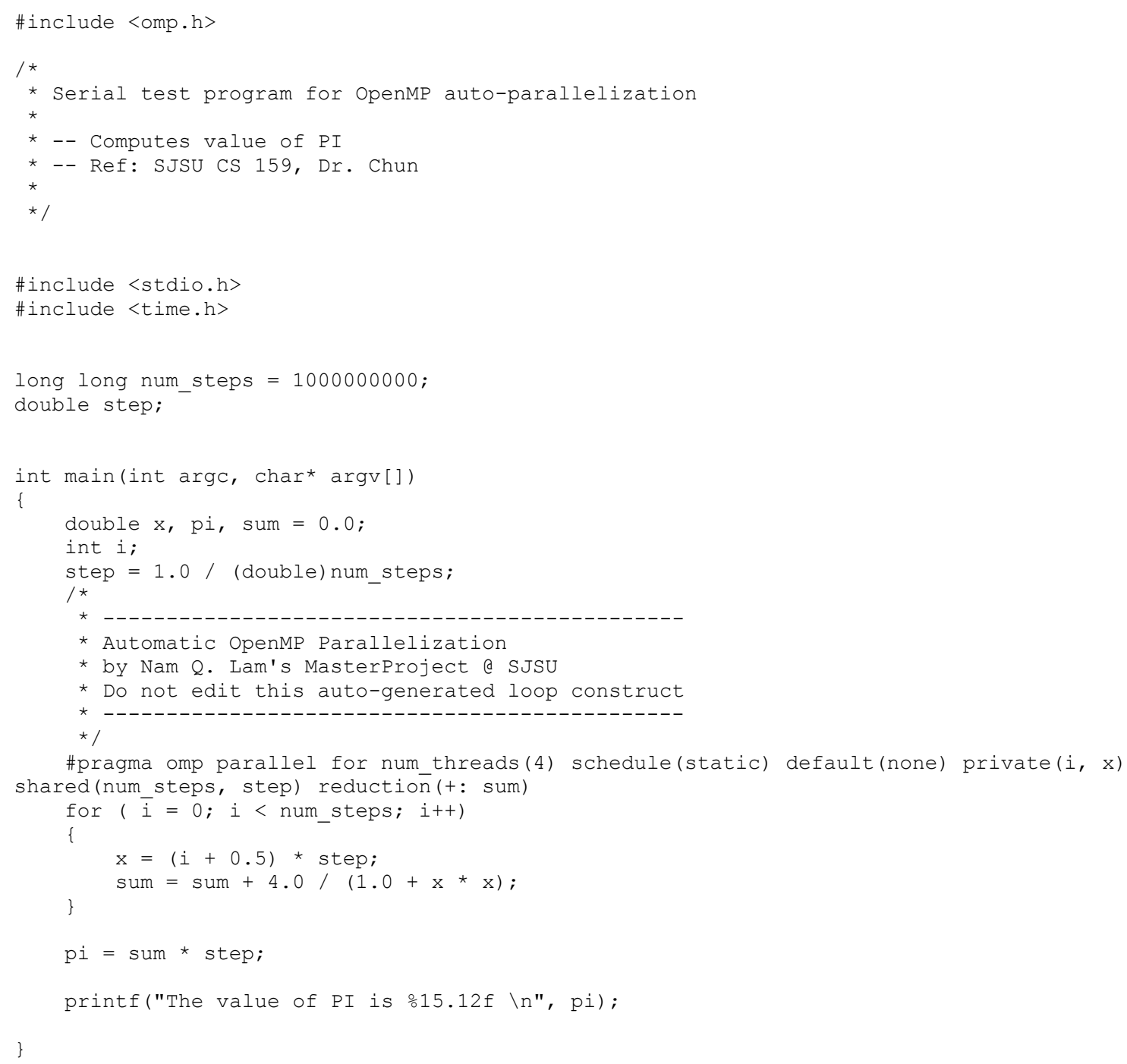




\section{omp_prime_finder.c}

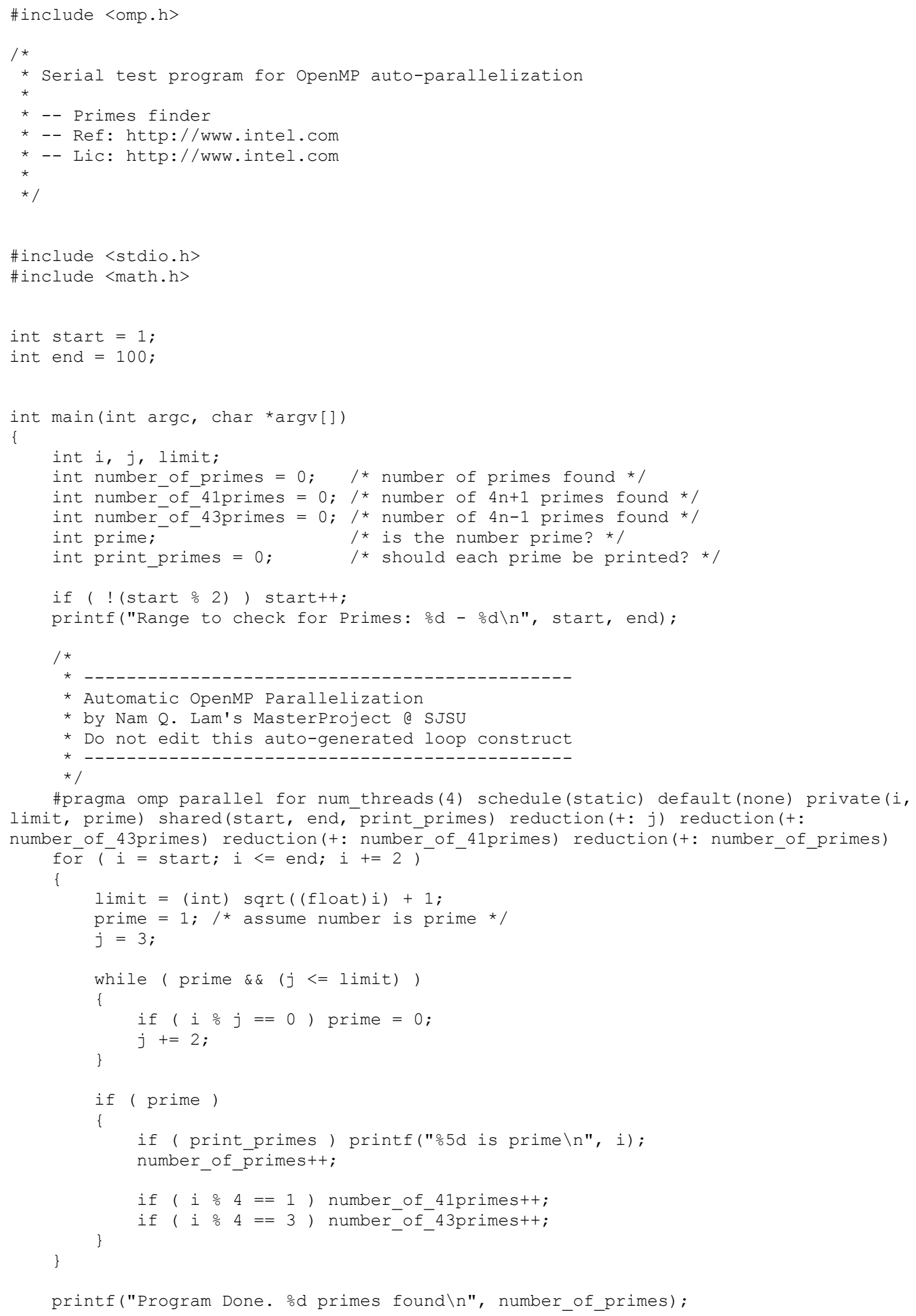


printf("Number of $4 n+1$ primes found: $\% d \backslash n "$, number of 41 primes);

printf("Number of $4 n-1$ primes found: $\% d \backslash n "$, number_of_43primes);

return 0 ;

\}

\section{omp_trapez_approx.c}

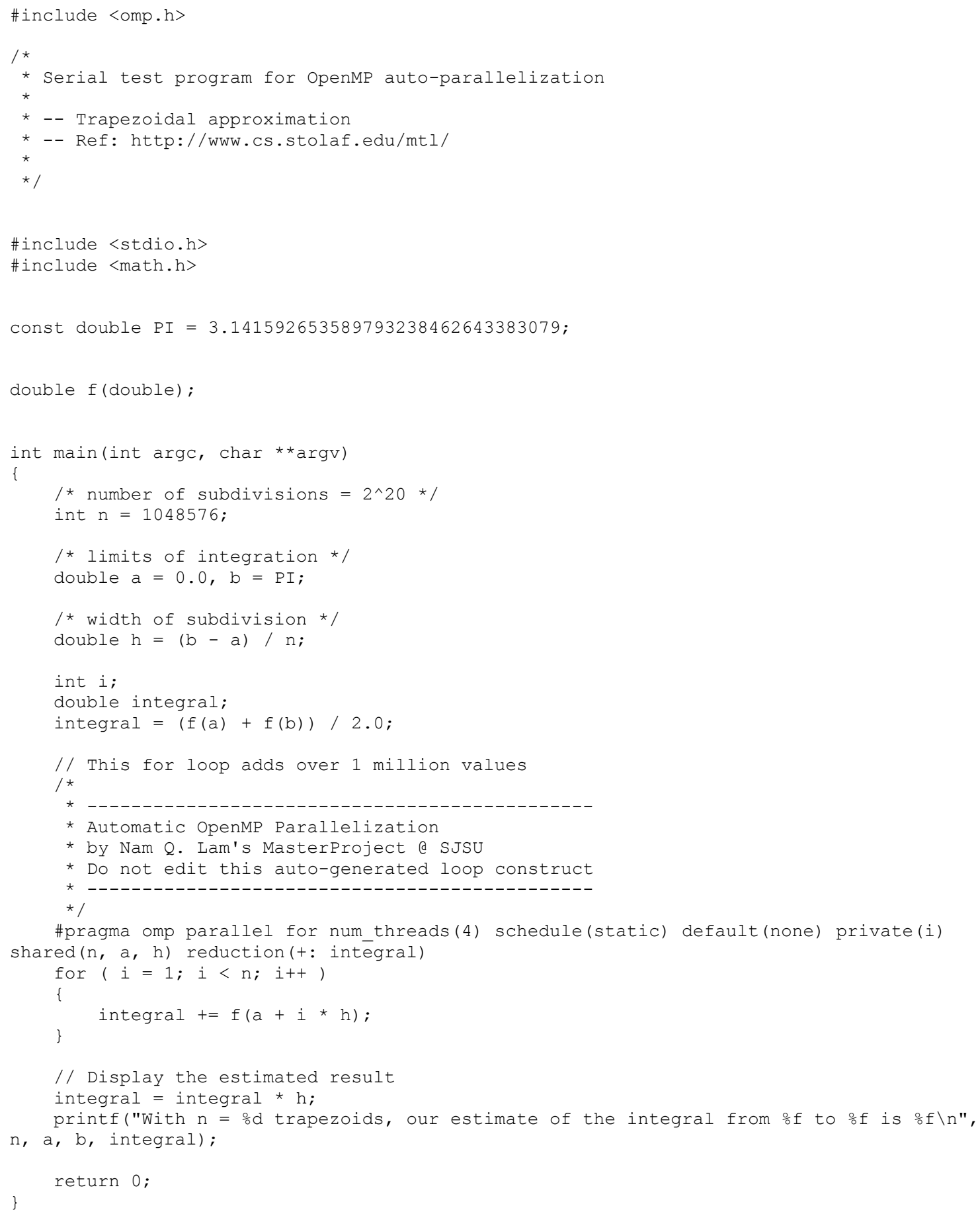




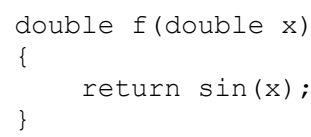

\section{omp_vector_add.c}

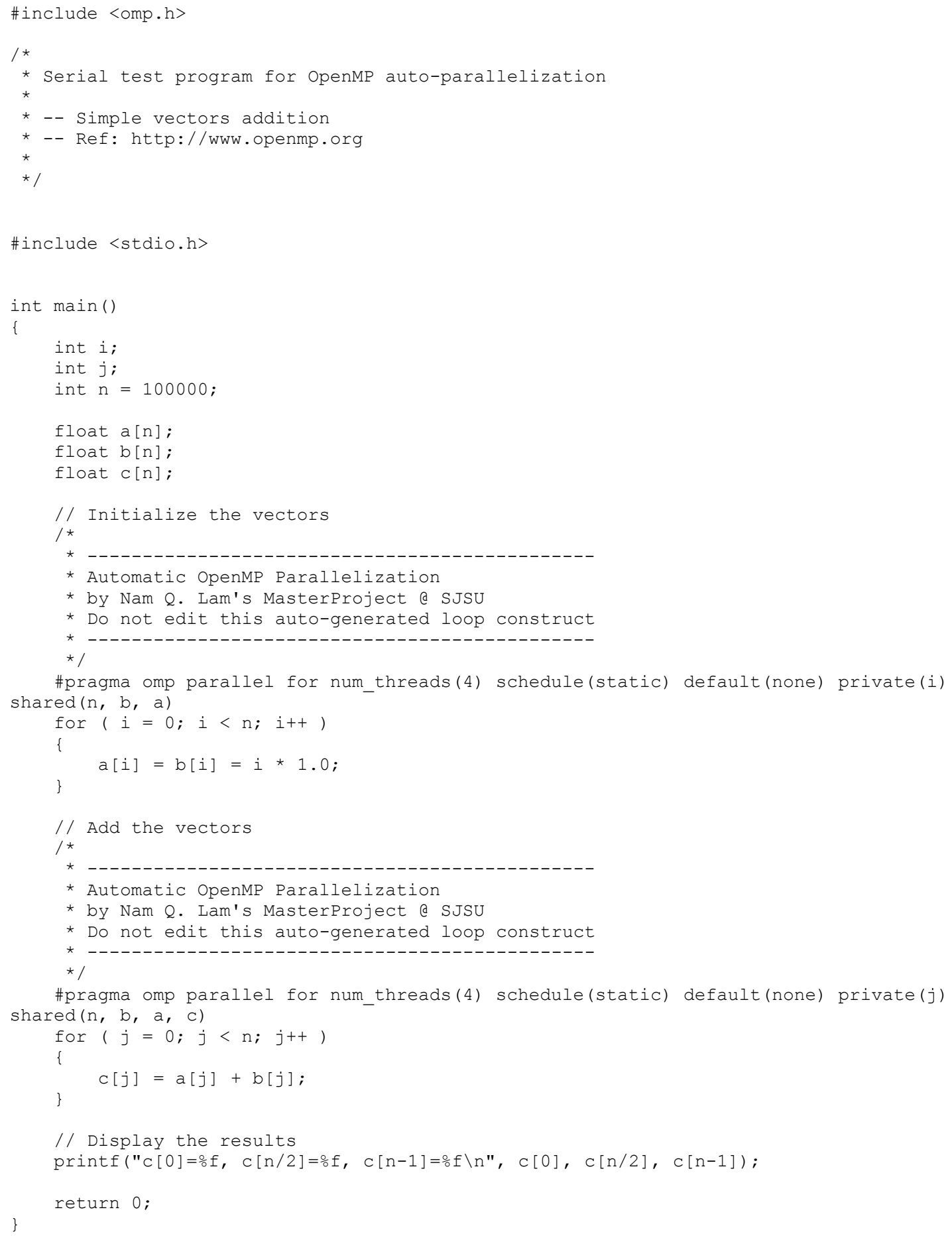




\subsection{Appendix E: C Grammar in BNF Format}

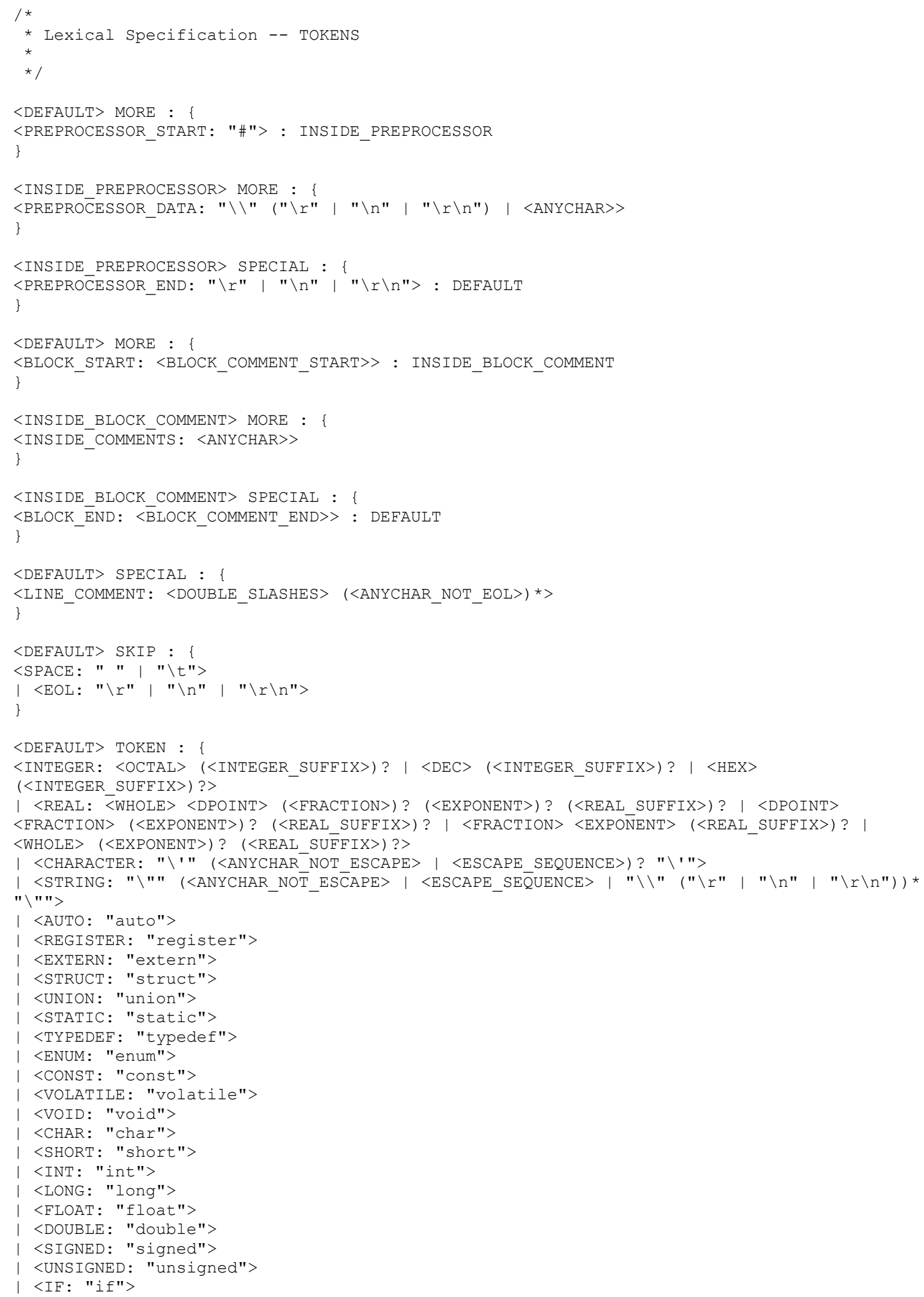




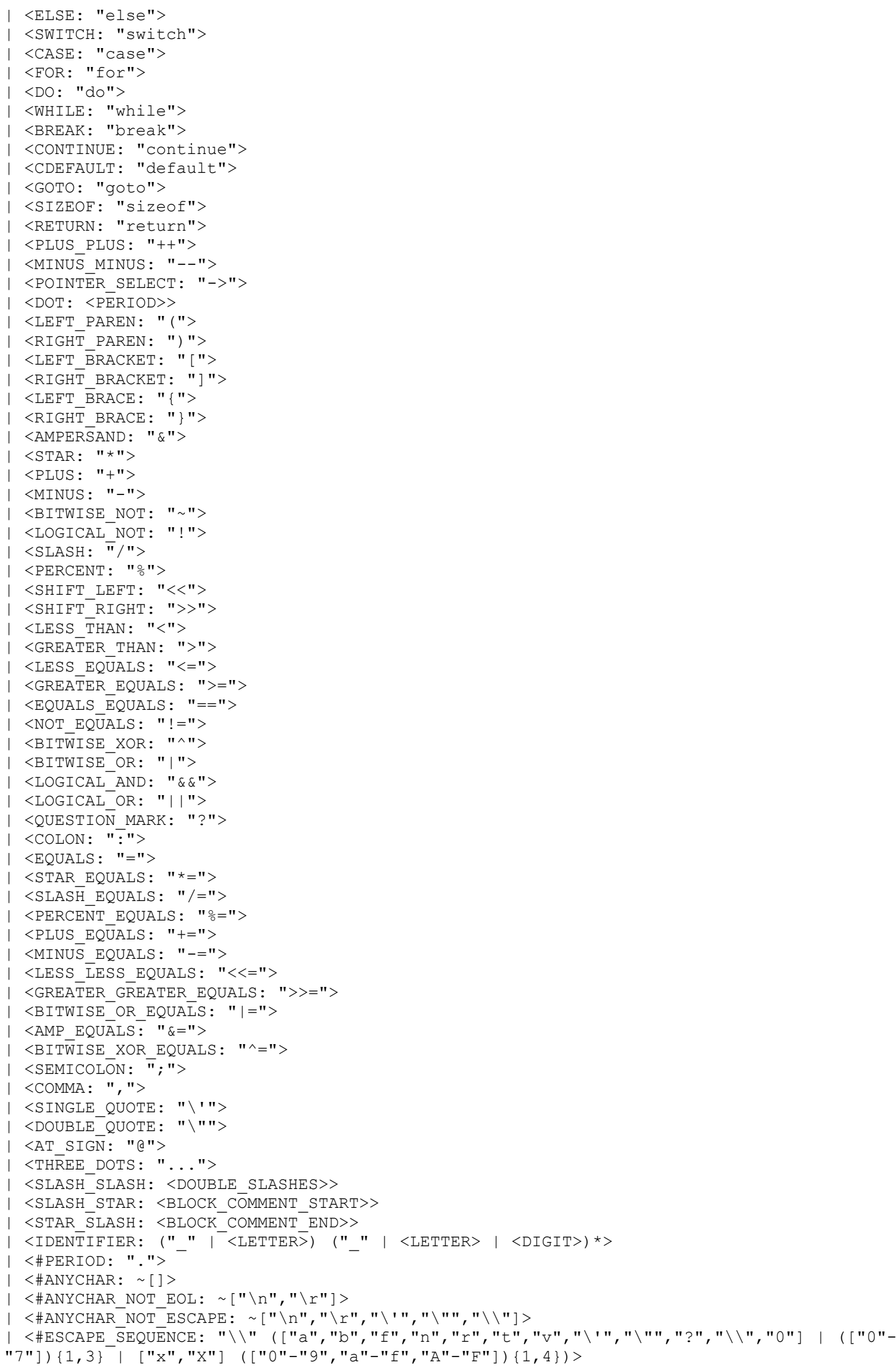




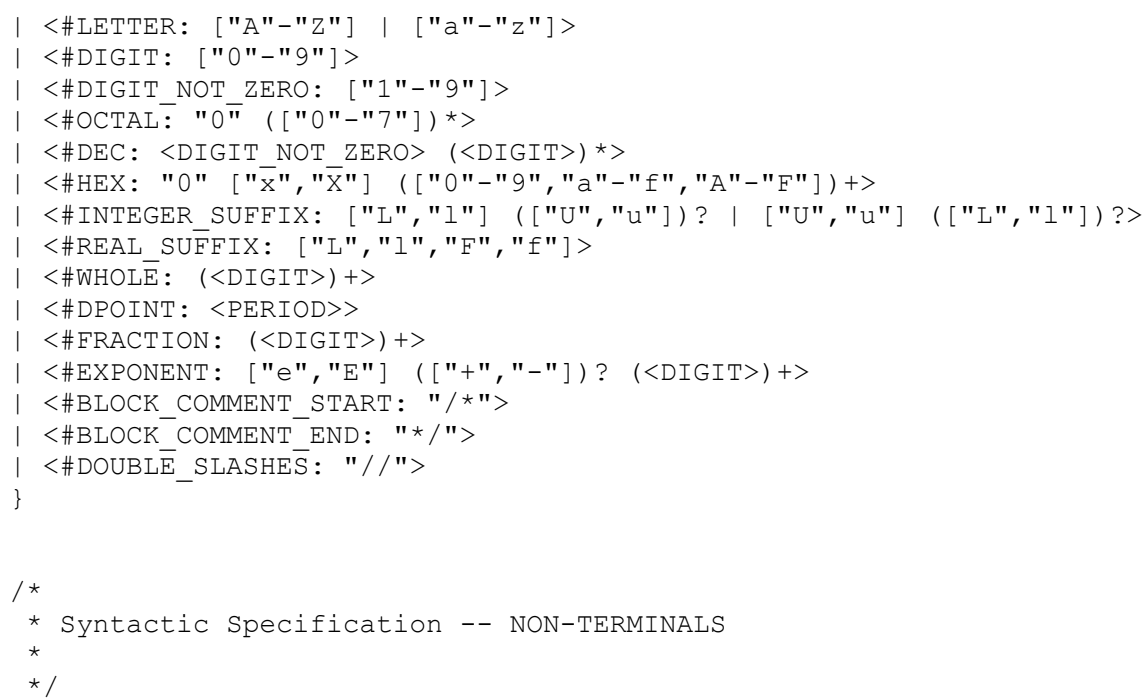




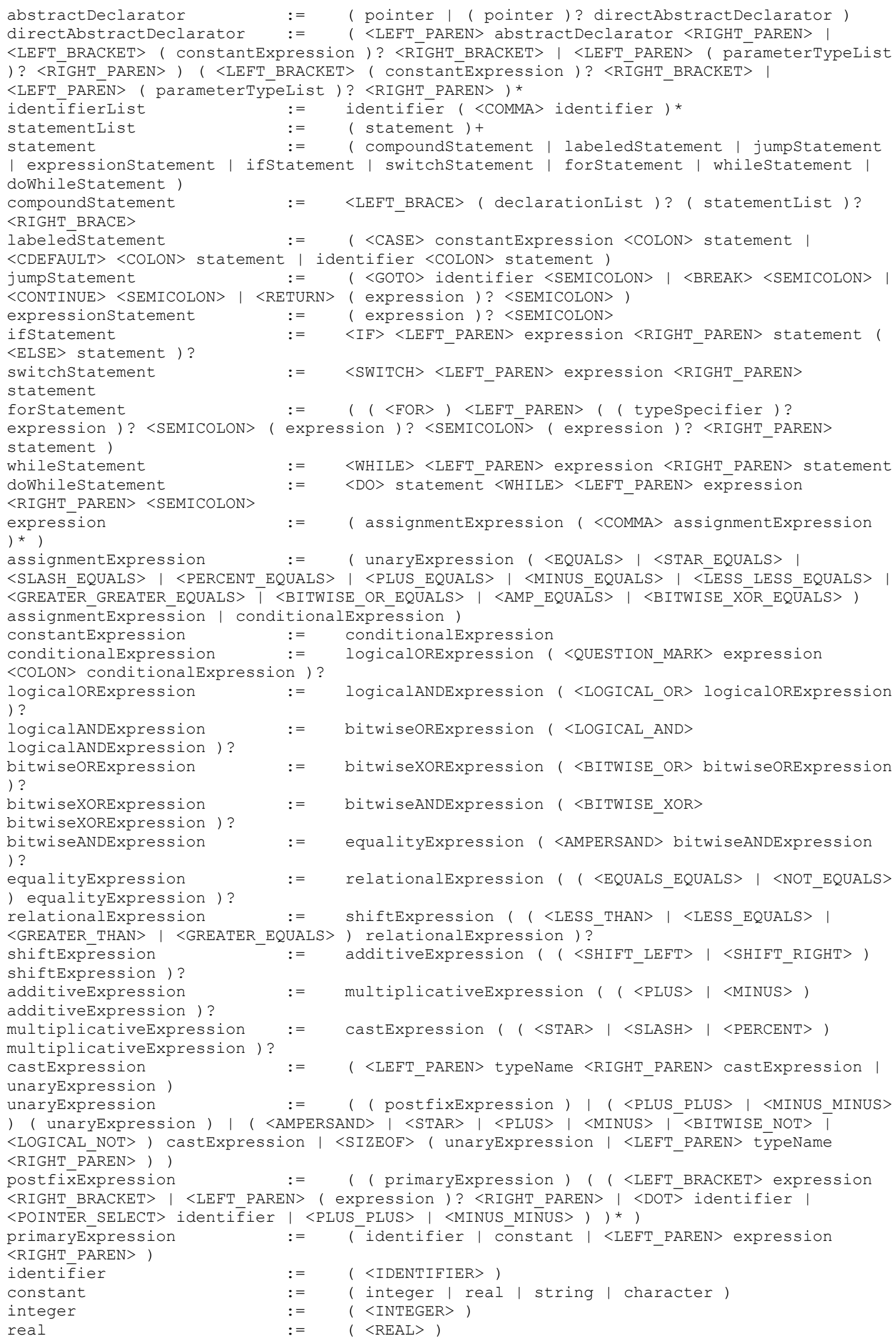



string
character
$:=\quad(<$ STRING $>)$
$:=\quad(<$ CHARACTER $>)$

\subsection{Appendix F: C Grammar for JavaCC}

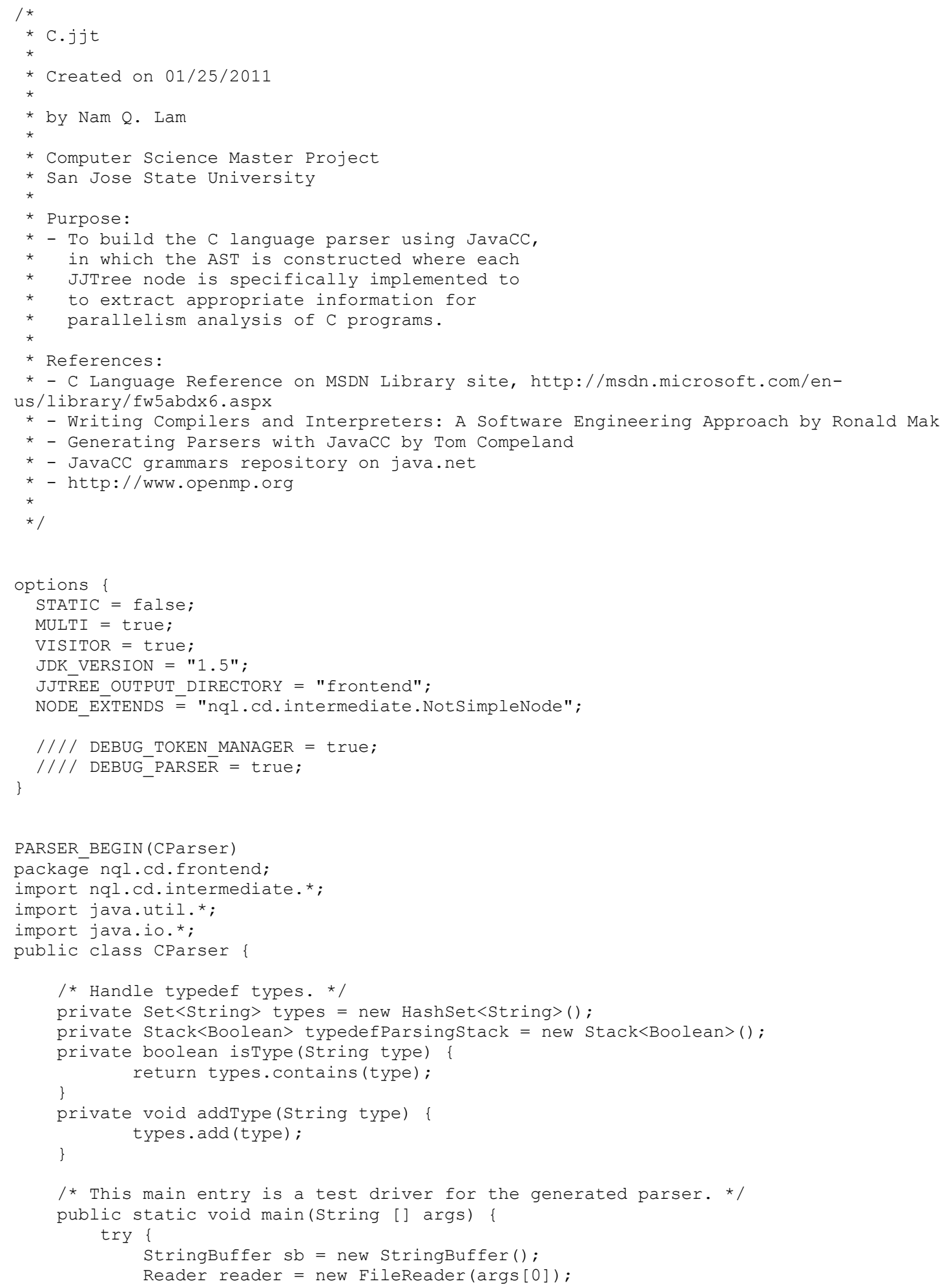




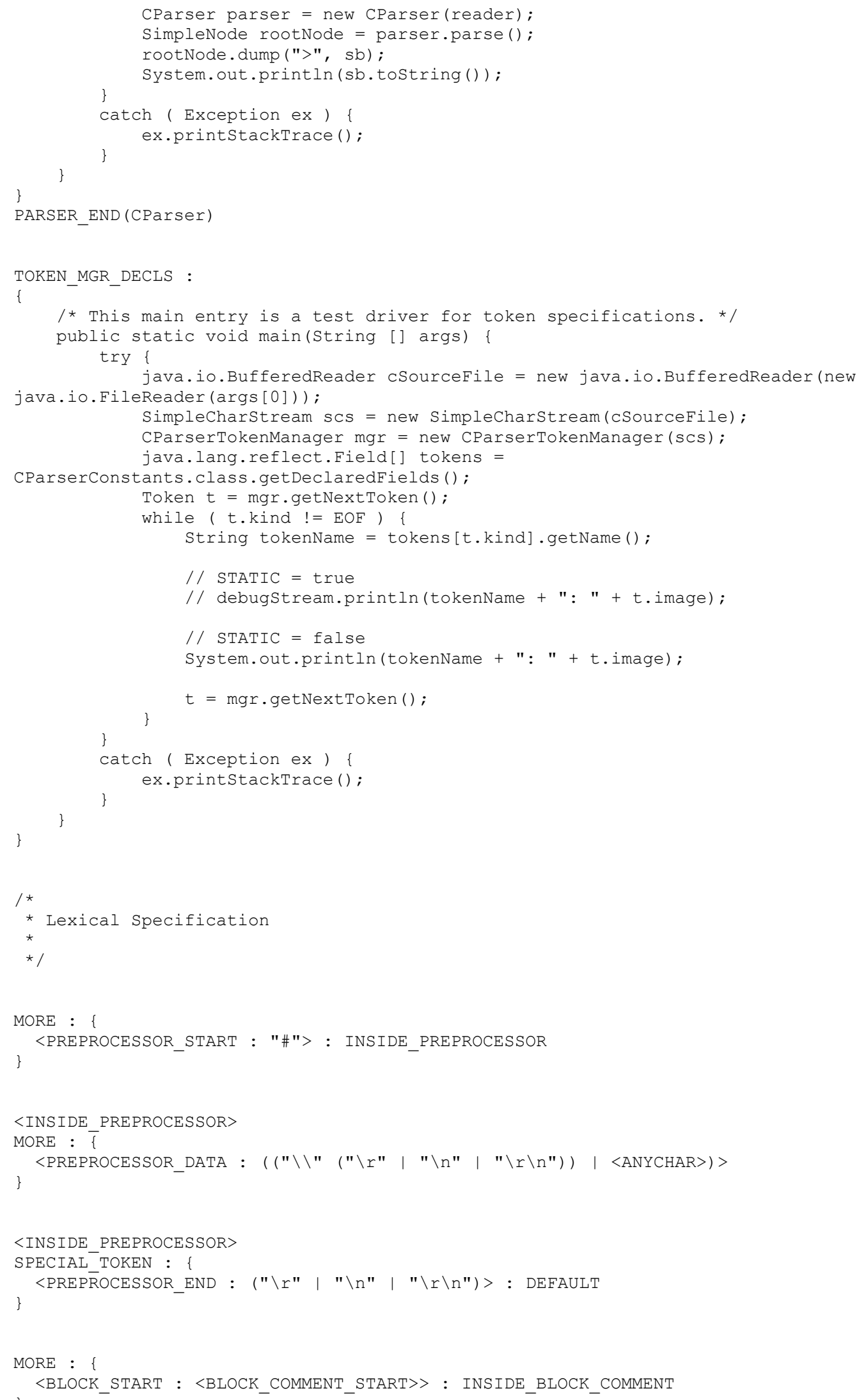




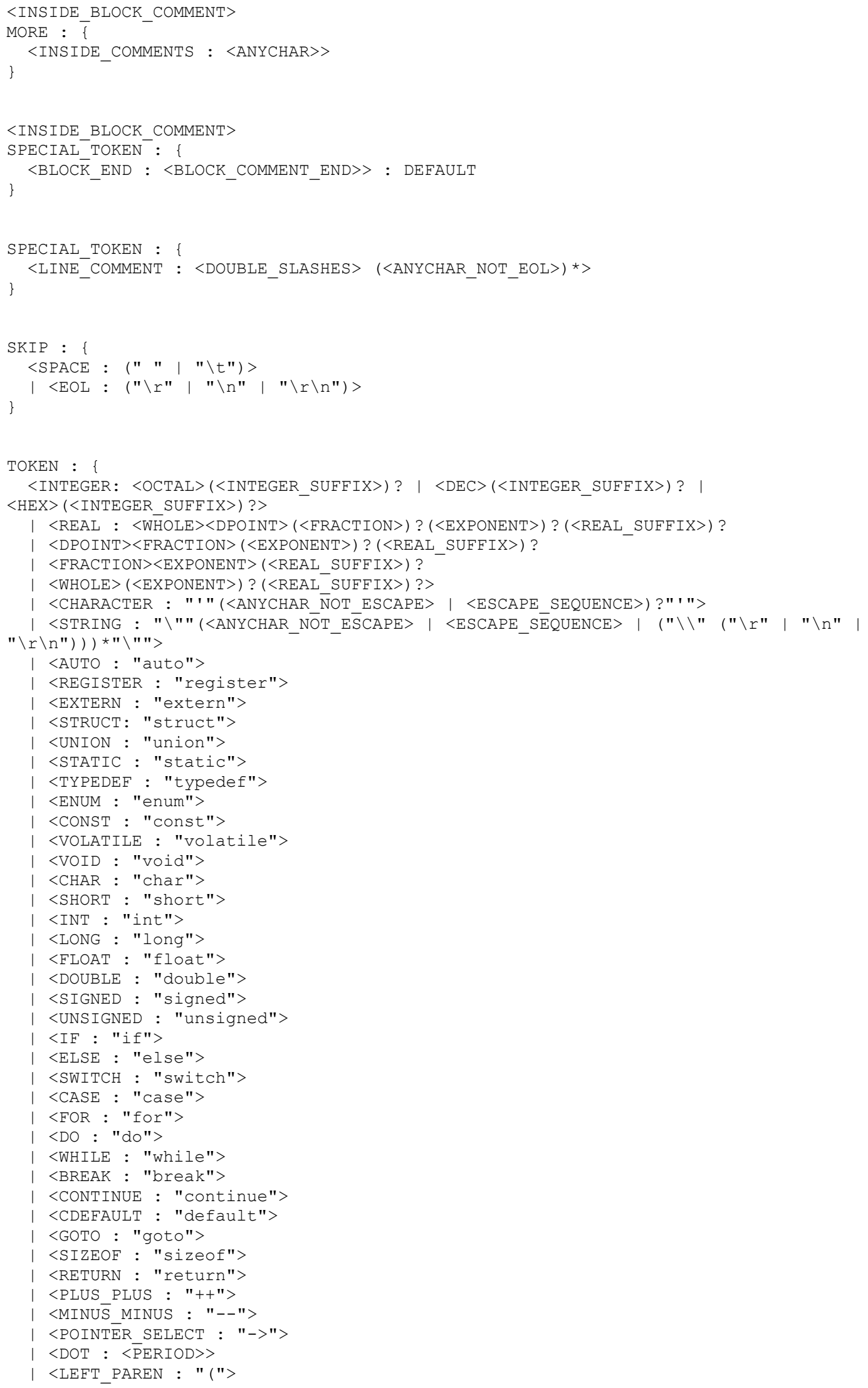




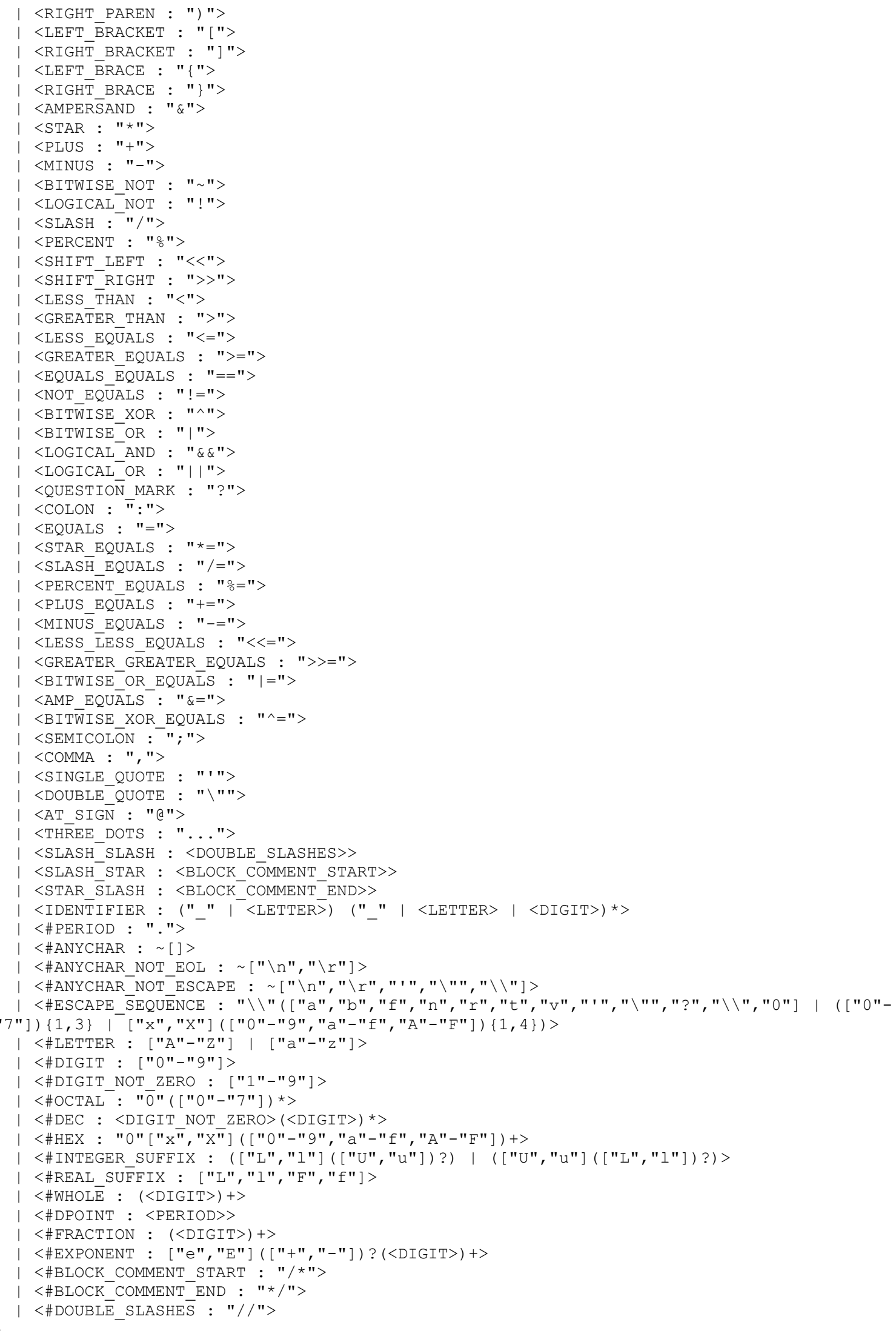




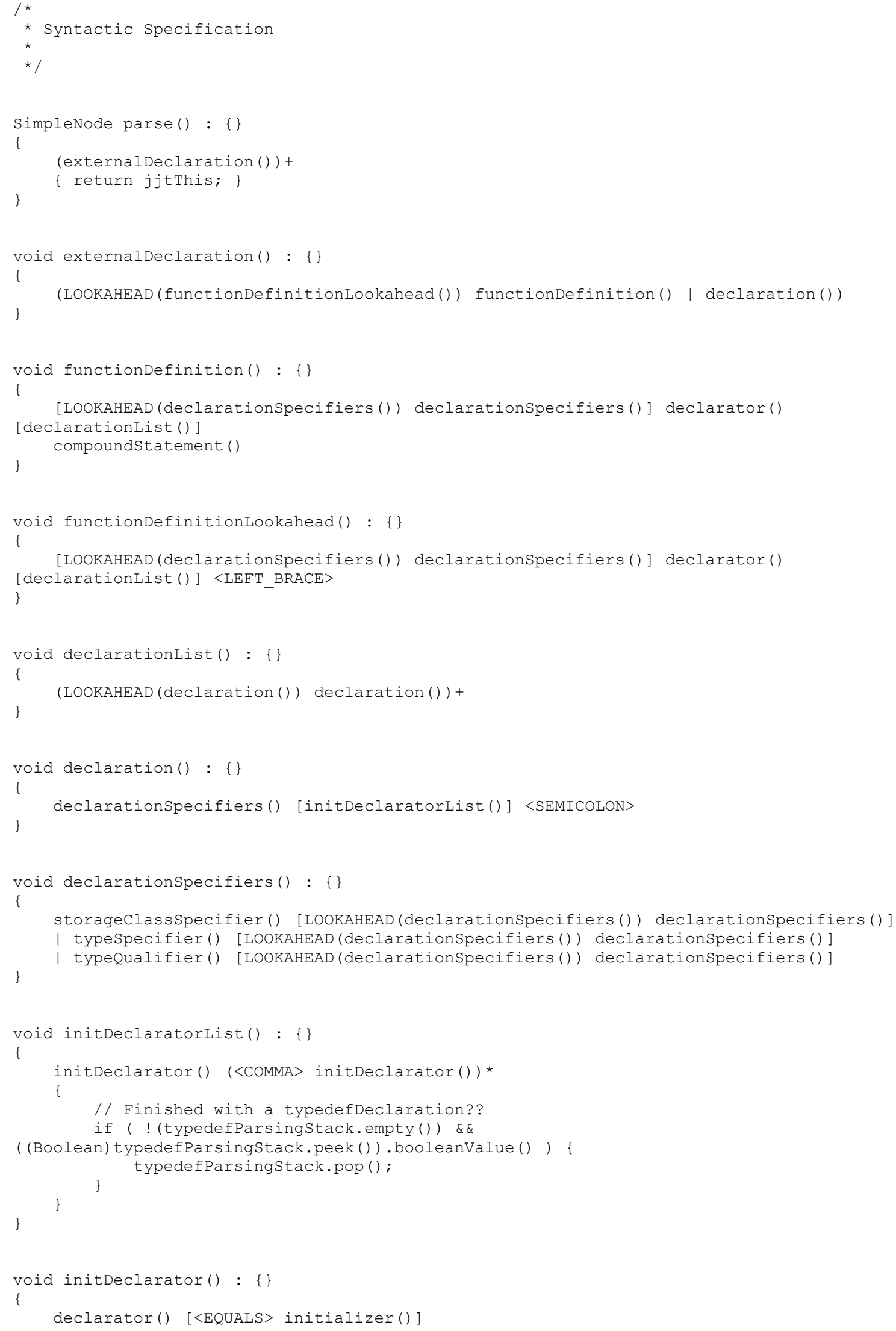




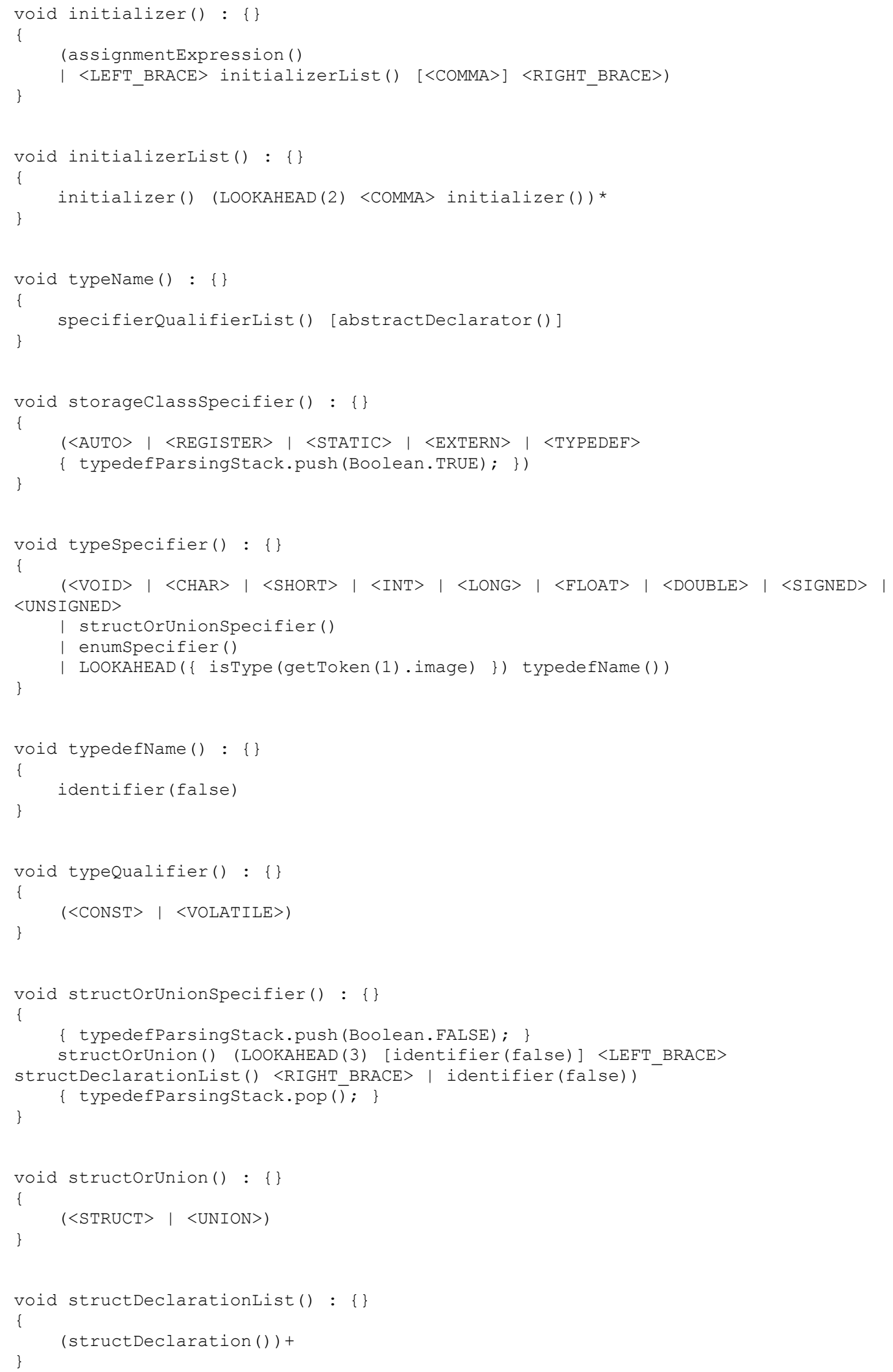




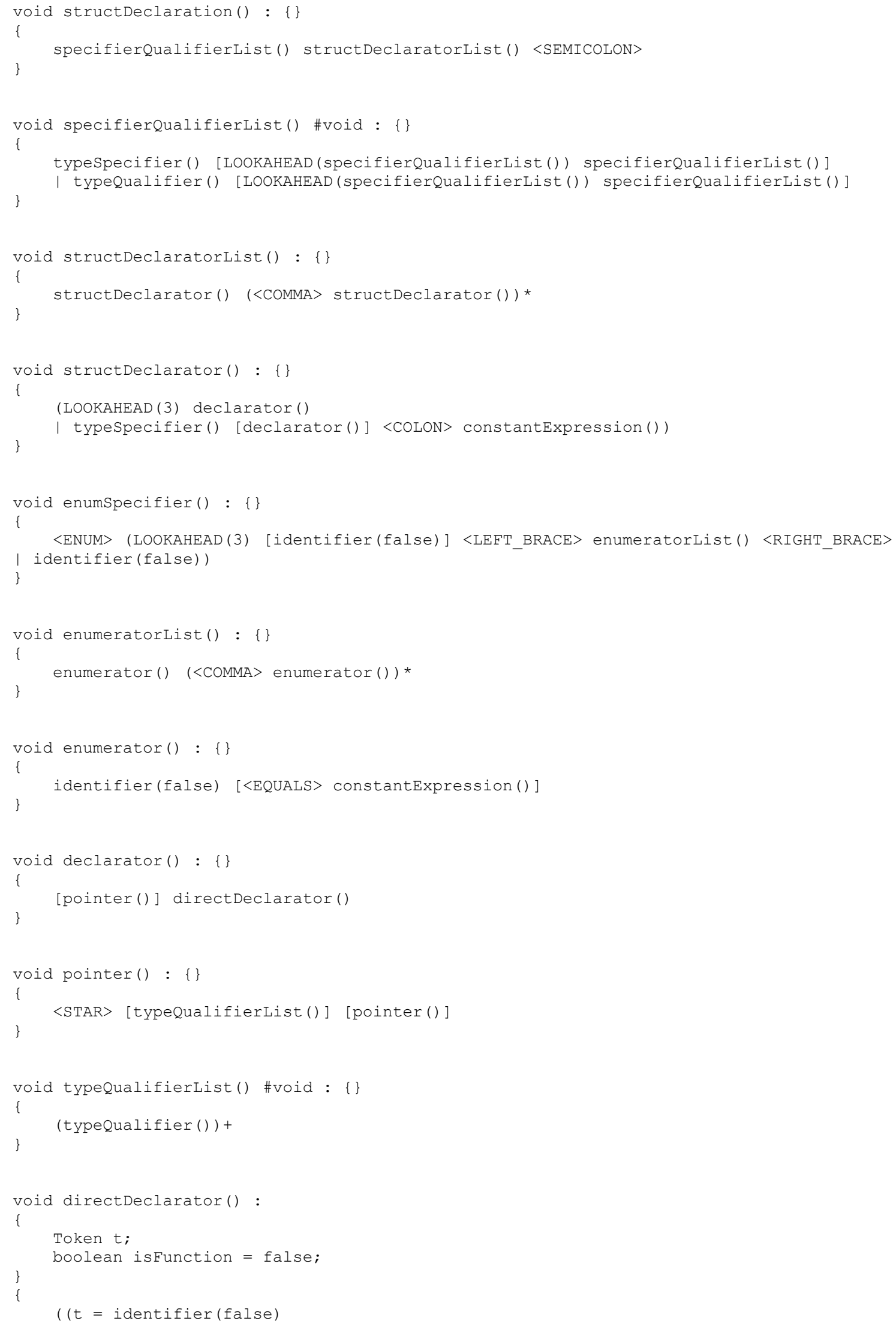




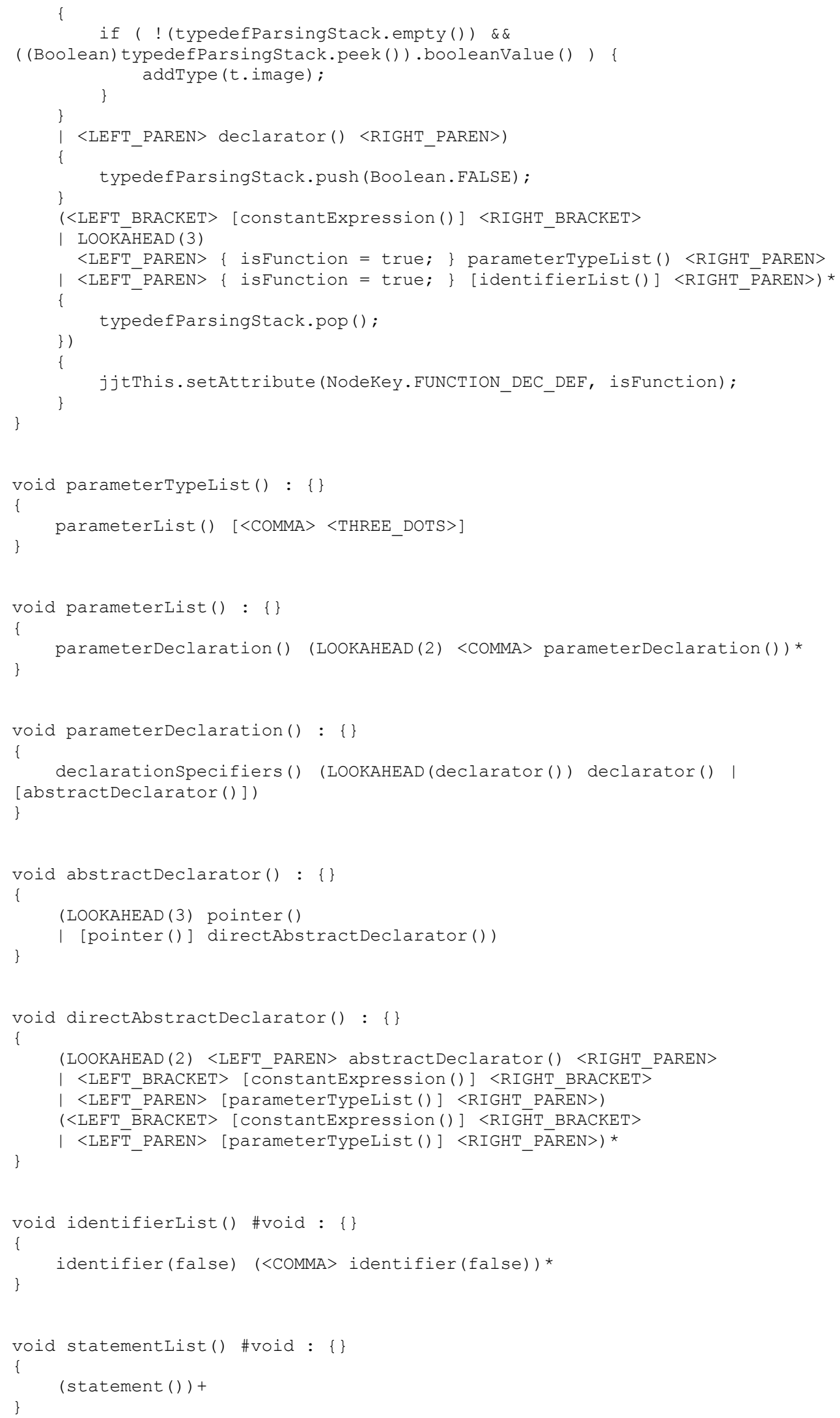




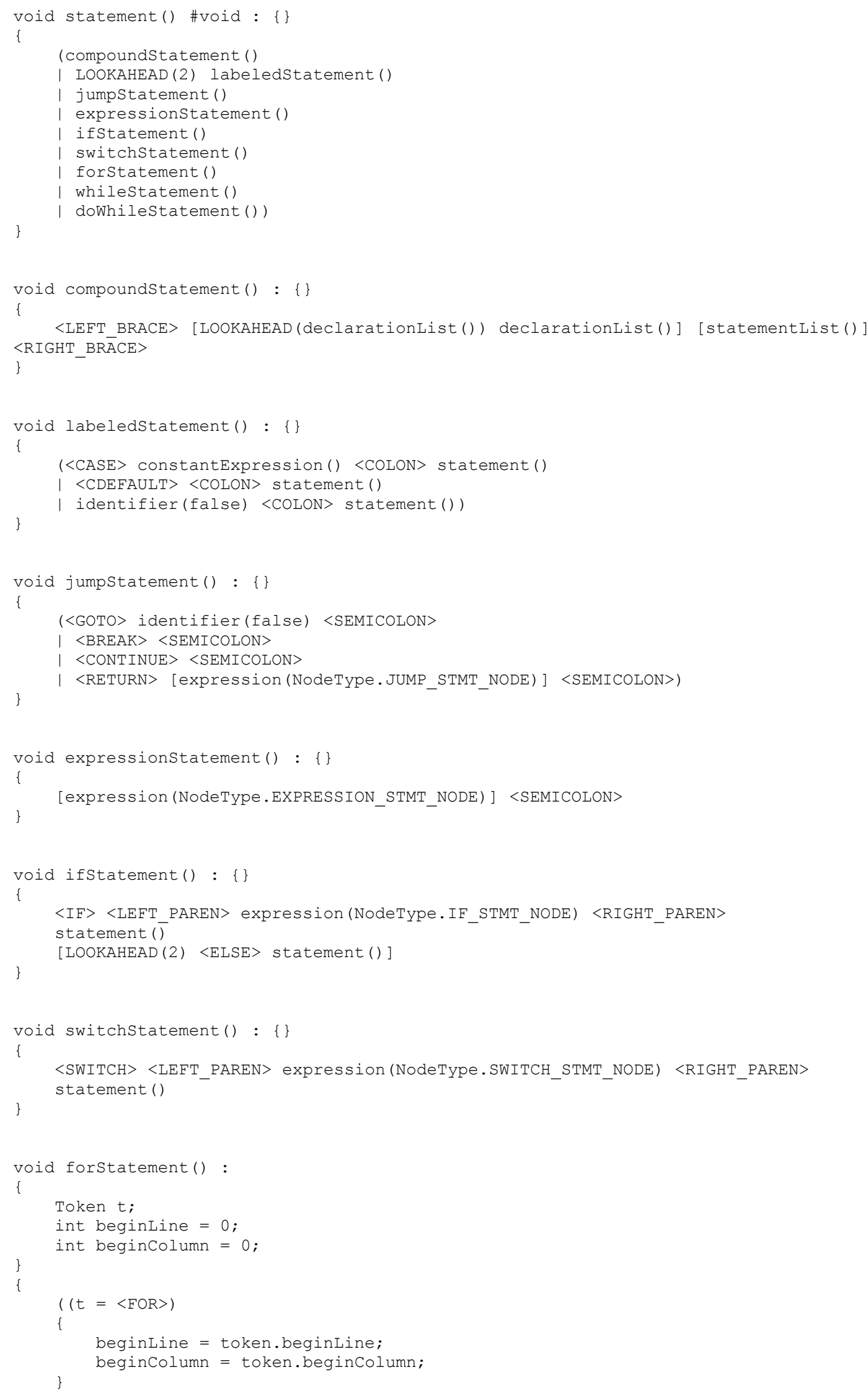




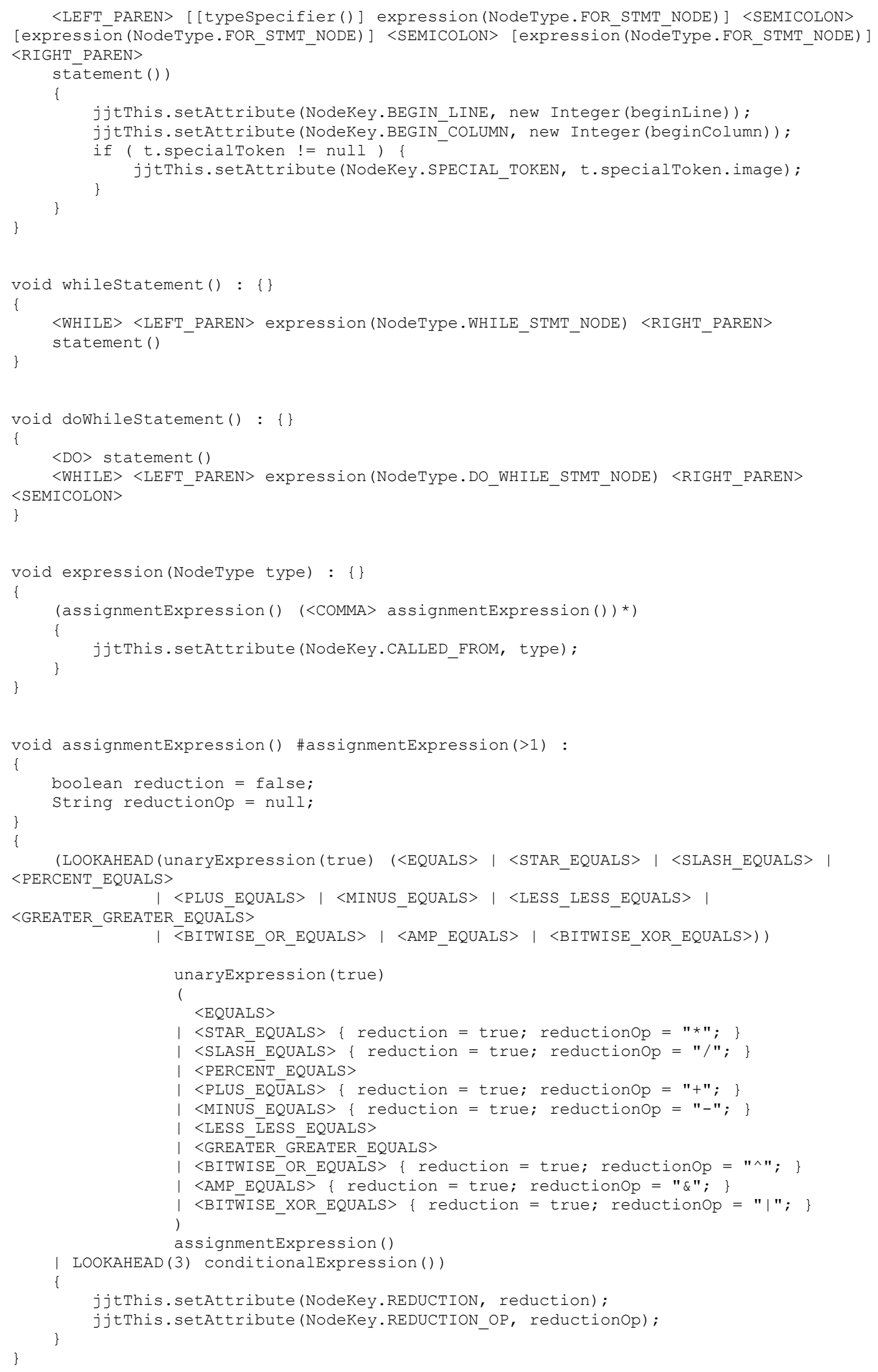




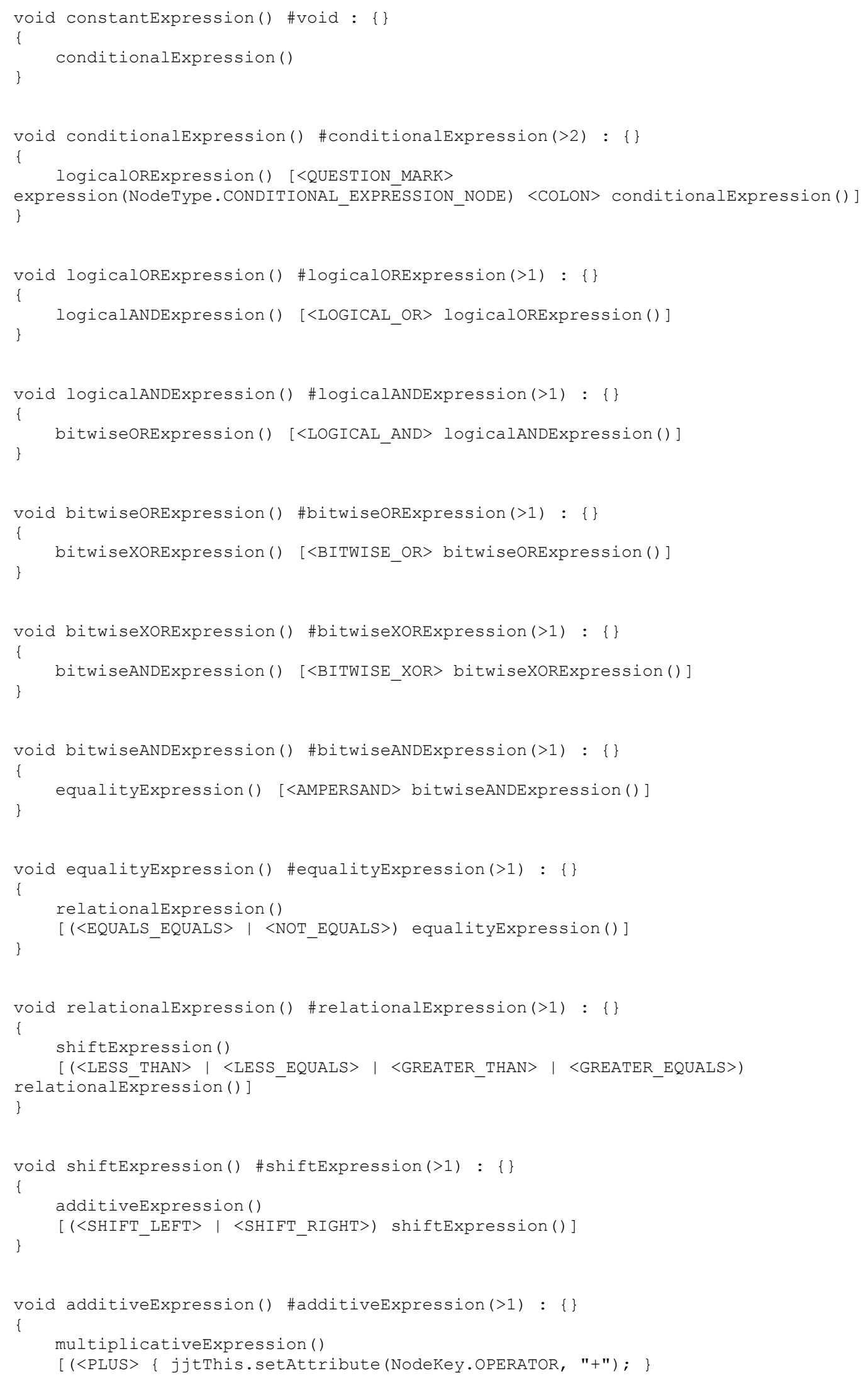




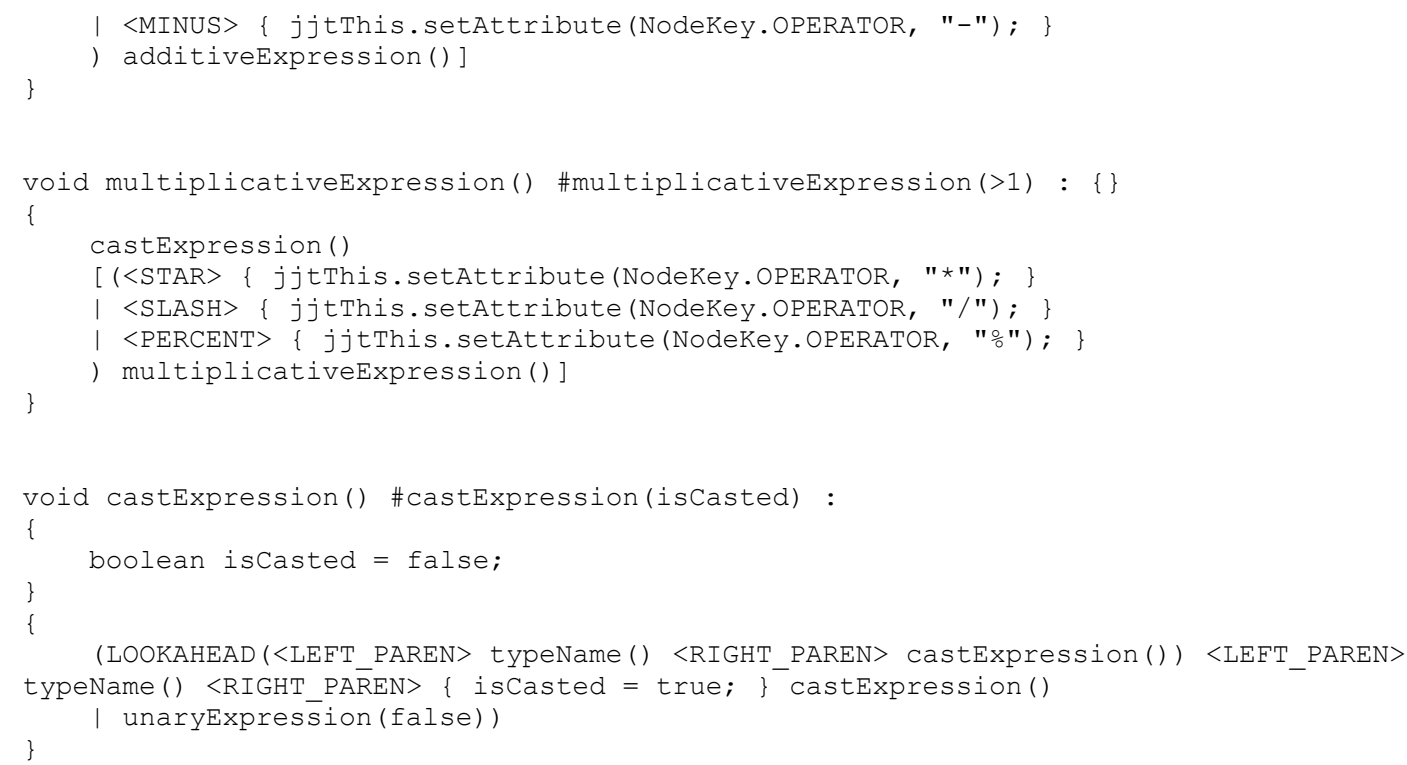




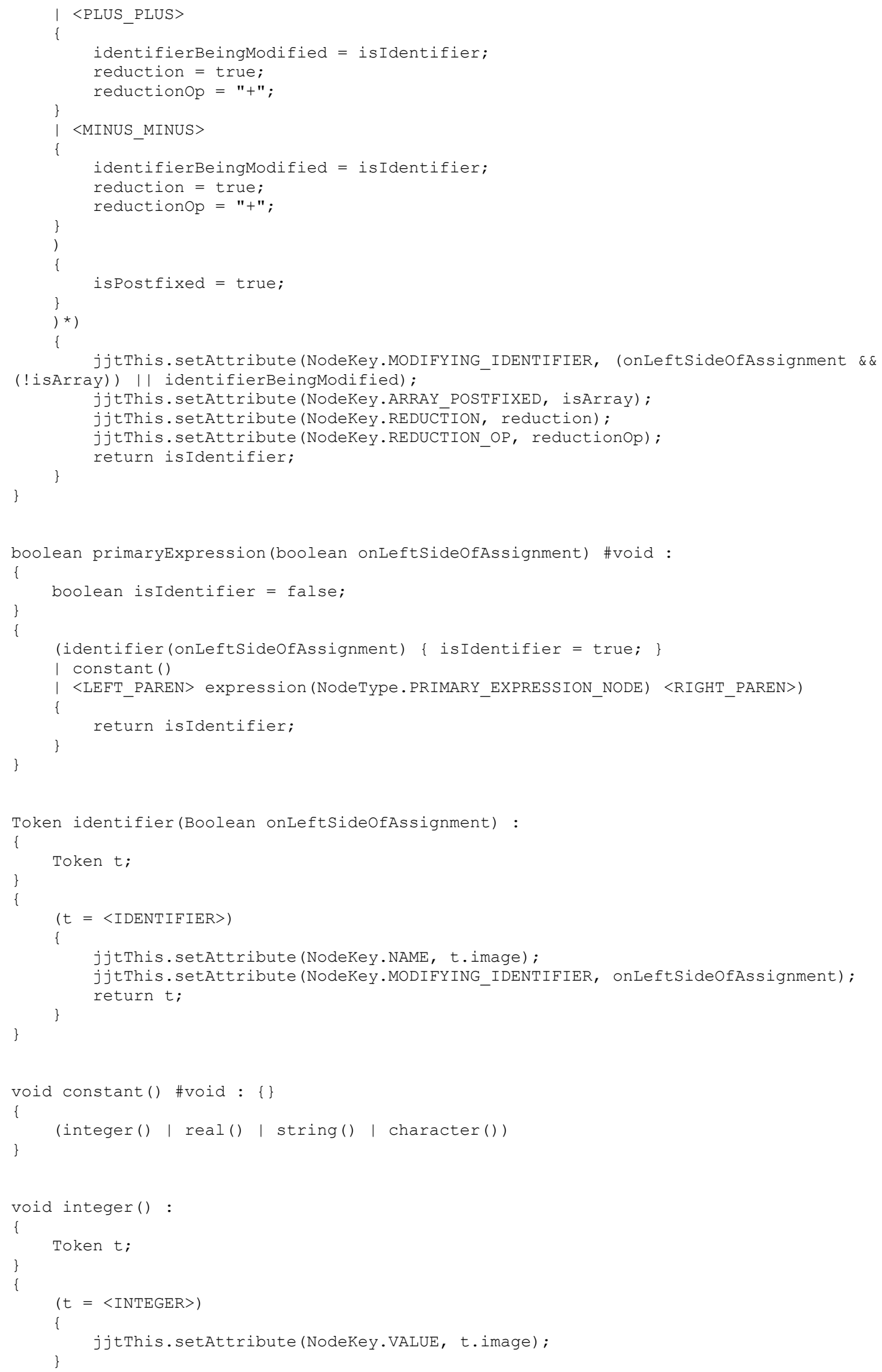




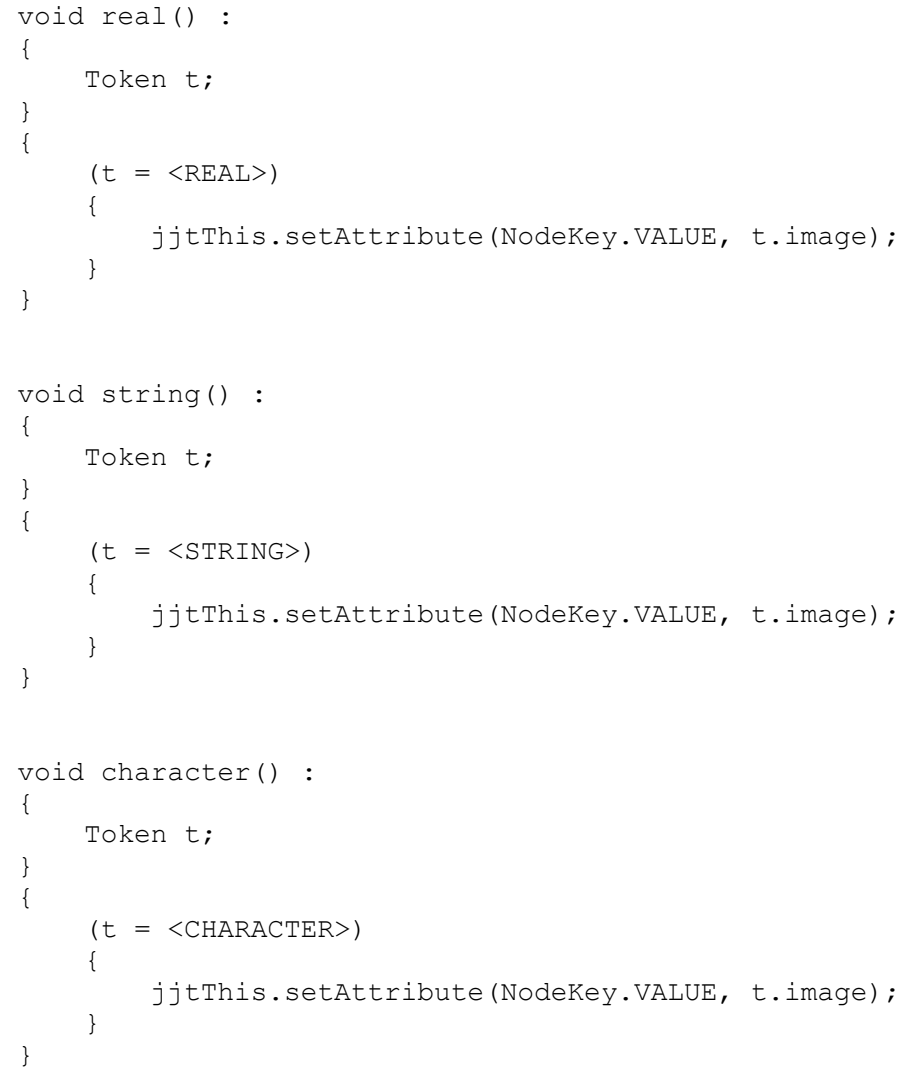

\subsection{Appendix G: The Project Source Code}

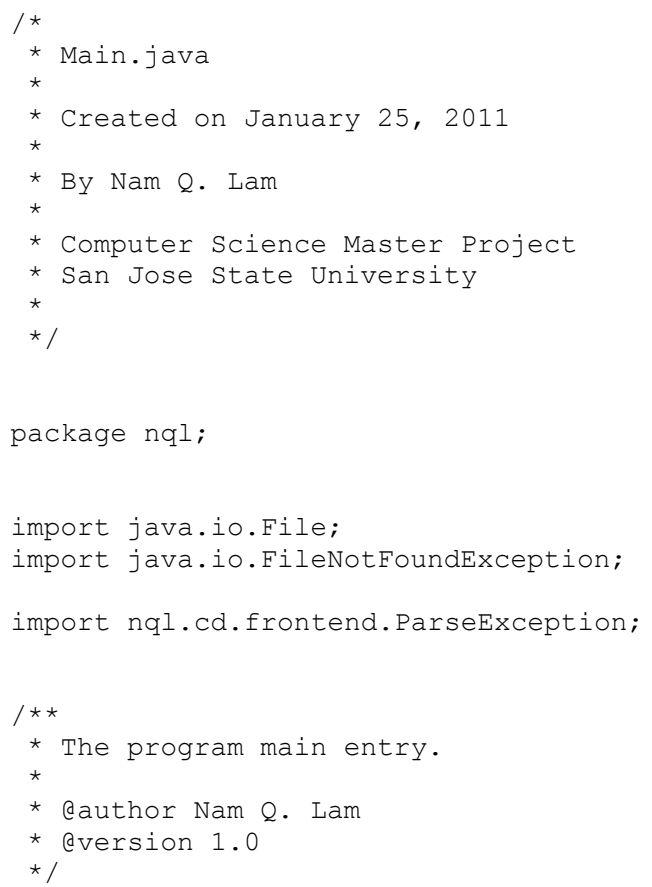




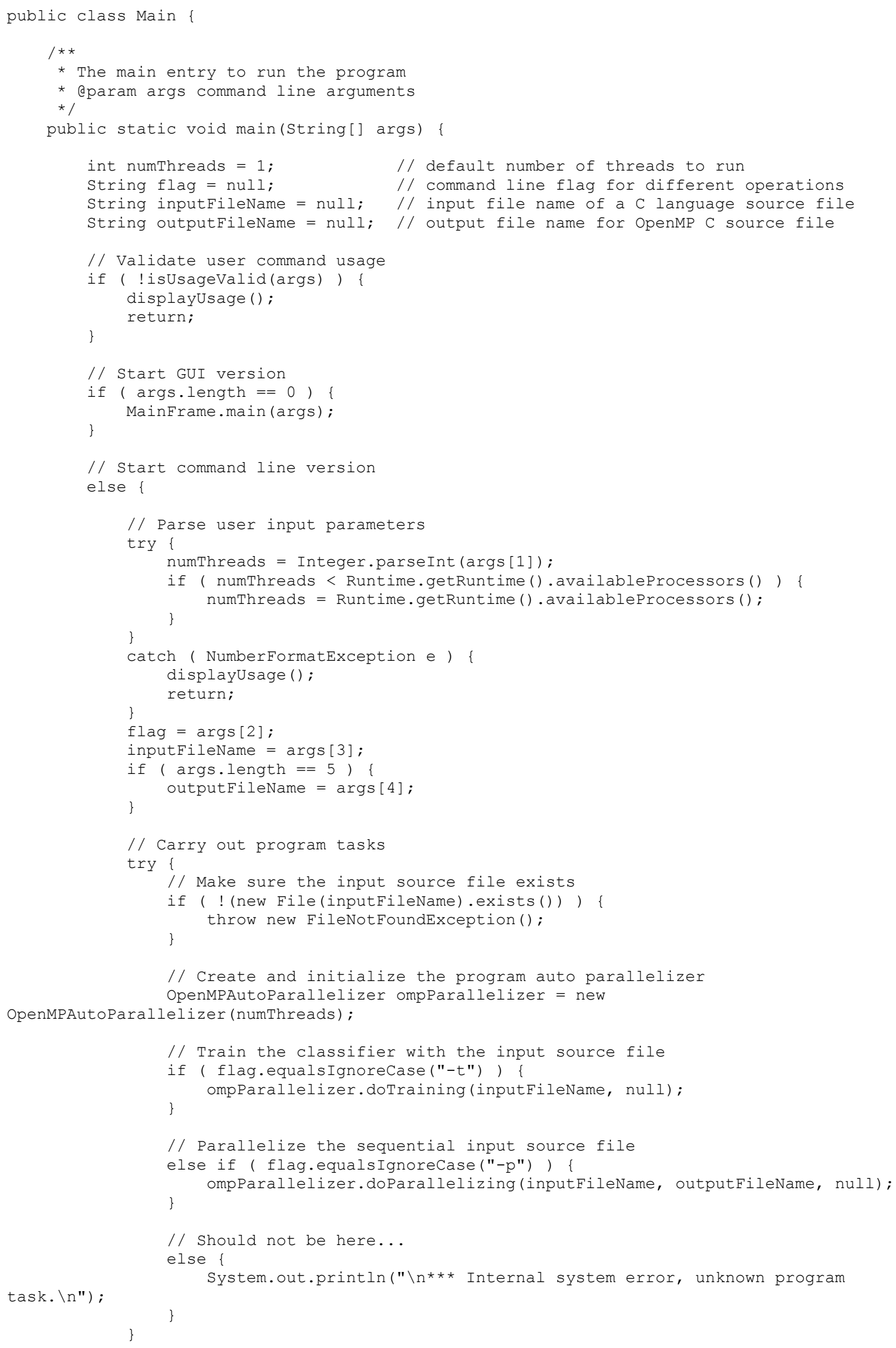




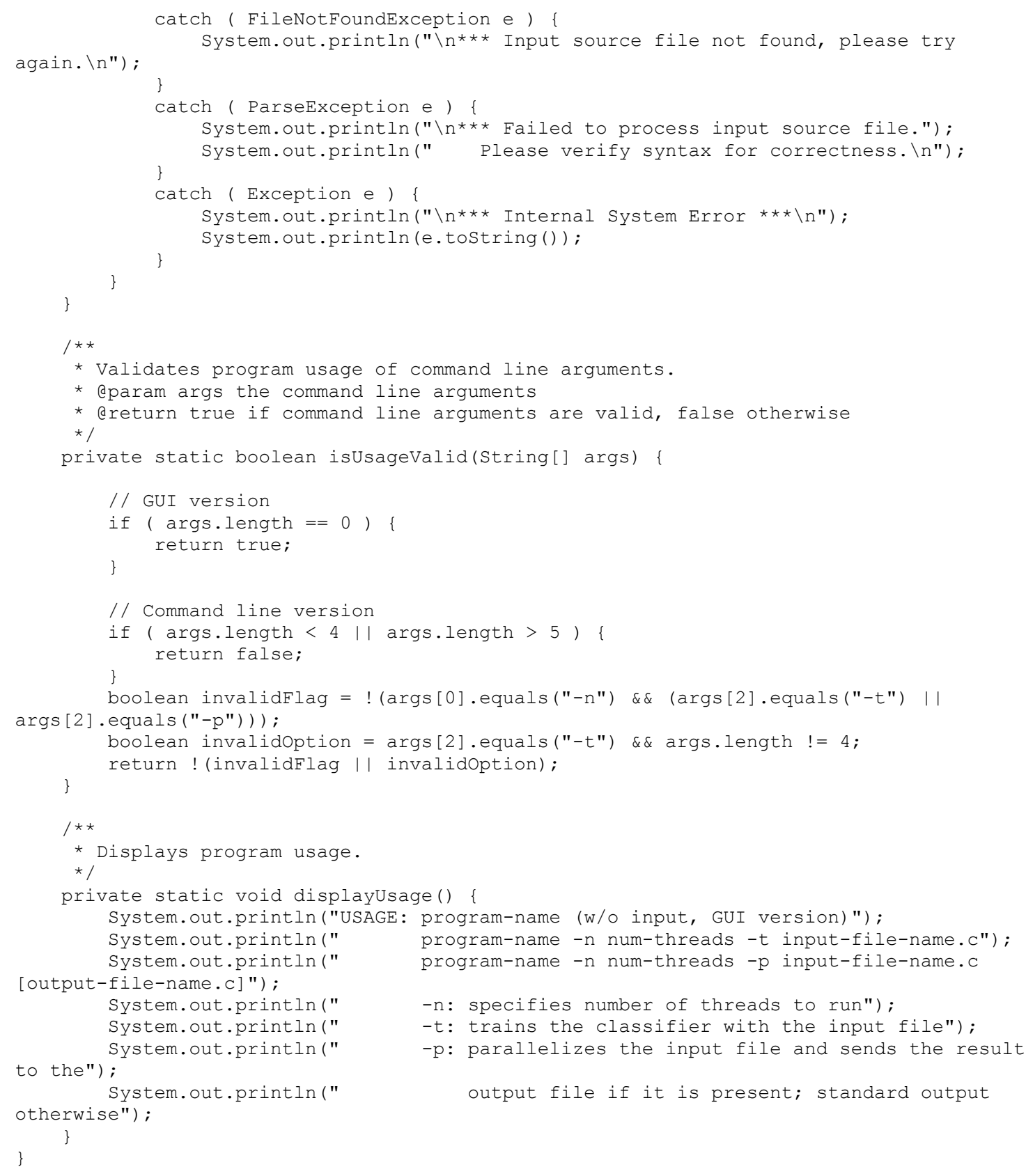




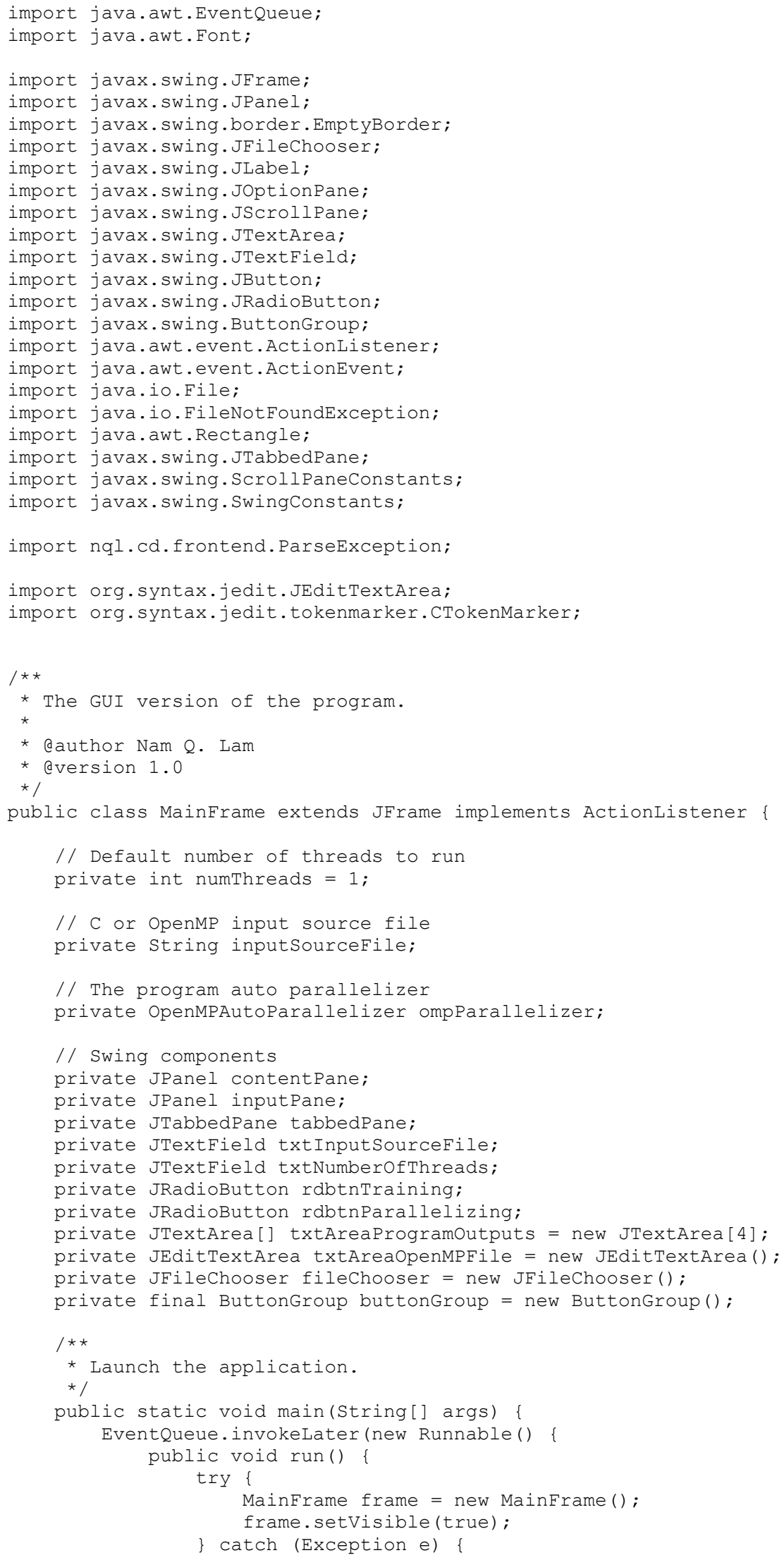




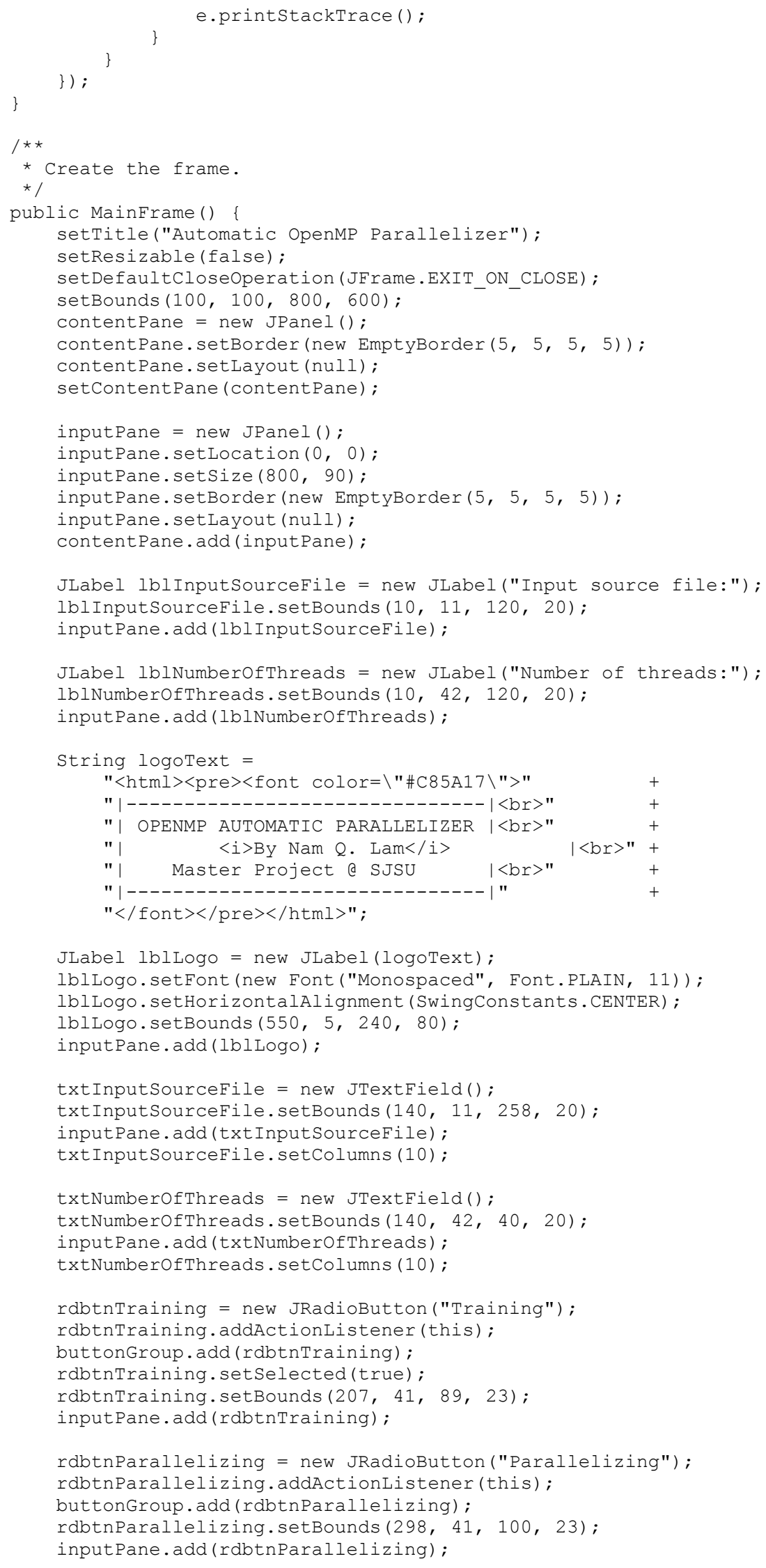


JButton btnChooseInputSourceFile = new JButton("Choose File");

btnChooseInputSourceFile.addActionListener (this);

btnChooseInputSourceFile.setBounds (419, 10, 100, 23);

inputPane. add (btnchooseInputSourceFile);

JButton btnstart = new JButton ("Start");

btnstart.addActionListener(this);

btnStart. setBounds (419, 41, 100, 23);

inputPane.add (btnstart);

tabbedPane = new JTabbedPane (JTabbedPane.TOP);

tabbedPane. setBounds $(10,100,775,465)$;

contentPane. add (tabbedPane);

txtAreaProgramoutputs $[0]=$ new JTextArea();

txtAreaProgramoutputs [1] = new JTextArea();

txtAreaProgramoutputs [2] = new JTextArea();

txtAreaProgramoutputs [3] = new JTextArea();

txtAreaProgramoutputs [0] .setEditable (false);

txtAreaProgramoutputs [1]. setEditable (false);

txtAreaProgramoutputs [2] . setEditable (false);

txtAreaProgramoutputs [3] . setEditable (false);

txtAreaProgramoutputs [0] . setLineWrap (false);

txtAreaProgramoutputs [1]. setLineWrap (false);

txtAreaProgramoutputs [2]. setLineWrap (false);

txtAreaProgramoutputs [3]. setLineWrap (false);

txtAreaProgramoutputs [0]. setFont (new Font ("Monospaced", Font.PLAIN, 12));

txtAreaProgramoutputs [1]. setFont (new Font ("Monospaced", Font.PLAIN, 12)) ;

txtAreaProgramoutputs [2]. setFont (new Font("Monospaced", Font.PLAIN, 12));

txtAreaProgramoutputs [3]. setFont (new Font ("Monospaced", Font.PLAIN, 12));

JScrollPane pScroll0 = new JScrollPane(txtAreaProgramoutputs[0], ScrollpaneConstants.VERTICAL SCROLLBAR AS NEEDED,

ScrollPaneConstants.HORIZONTĀL_SCROLLBĀR_ $\bar{A} S$ NEEDED);

JScrollPane pScroll1 = new JScrō̄lPāne(txtAreaProgramoutputs[1],

ScrollPaneConstants.VERTICAL_SCROLLBAR_AS_NEEDED,

ScrollPaneConstants.HORIZONTĀL SCROLLBĀR $\bar{A} S$ NEEDED) ;

JScrollPane pScroll2 = new JScrollipane(txtAreaProgramoutputs[2],

ScrollPaneConstants.VERTICAL_SCROLLBAR_AS_NEEDED,

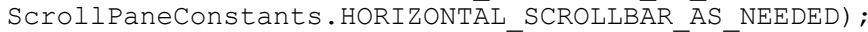

JScrollPane pScroll3 = new JScrōllPäne (txtAreaProgramoutputs [3],

ScrollPaneConstants.VERTICAL SCROLLBAR AS NEEDED,

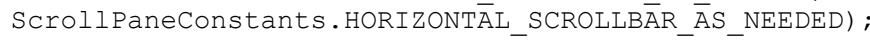

// Enable C syntax highlight for the auto-generated OpenMP file;

// otherwise, pscroll2 can be used instead in the tabbedPane.add() below txtAreaOpenMPFile.setEditable (false);

txtAreaOpenMPFile. setCaretBlinkEnabled (false);

txtAreaOpenMPFile.setTokenMarker(new CTokenMarker());

txtAreaOpenMPFile.setText ("");

tabbedPane.add("Extracted Features", pScroll0);

tabbedPane.add("New Training Records", pScroll1);

tabbedPane.add("Generated OpenMP", txtAreaOpenMPFile);

tabbedPane.add("Parse Tree (AST)", pScroll3);

tabbedPane.setEnabledAt (2, false);

\}

public void actionPerformed(ActionEvent event) \{

String cmd = event.getActionCommand();

// Disable appropriate tabs if Training option is selected

if ( cmd.equals("Training") ) \{

tabbedPane. setEnabledAt (1, true);

tabbedPane.setEnabledAt (2, false);

\} 


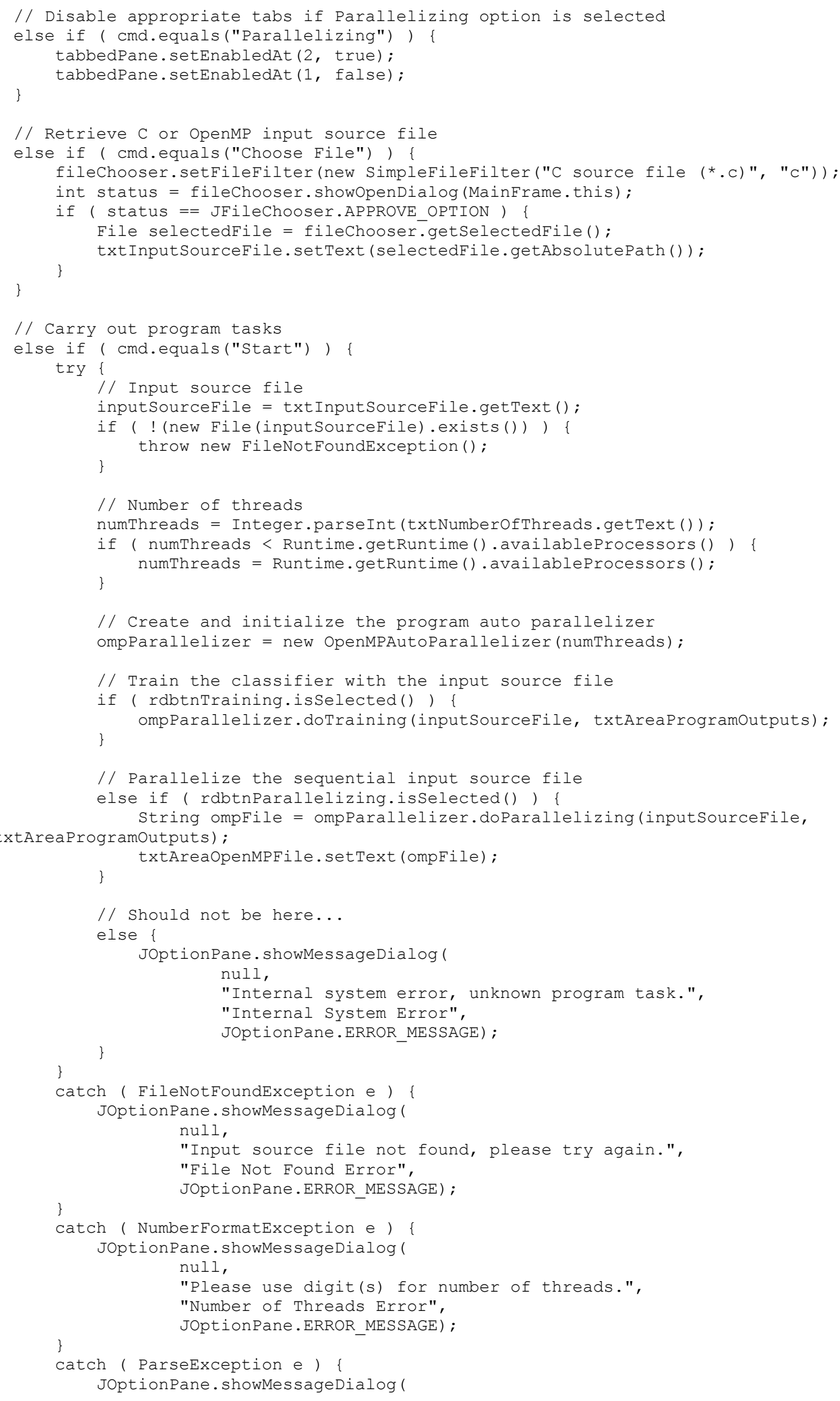


null,

"Failed to process input source file. InPlease verify syntax for

correctness.",

"Syntax Error",

JOptionPane.ERROR_MESSAGE);

\}

catch ( Exception e ) \{

JoptionPane. showMessageDialog (

null,

e.tostring (),

"Internal Error",

JOptionPane.ERROR MESSAGE);

(

// Should not be here...

else \{

JoptionPane. showMessageDialog(

null,

"Internal system error, unknown action command.",

"Internal system Error",

JOptionPane.ERROR_MESSAGE);

\}

$/ *$

* OpenMPAutoParallelizer.java

* Created on March 11, 2011

* By Nam Q. Lam

* Computer Science Master Project

* San Jose State University

*

package nql;

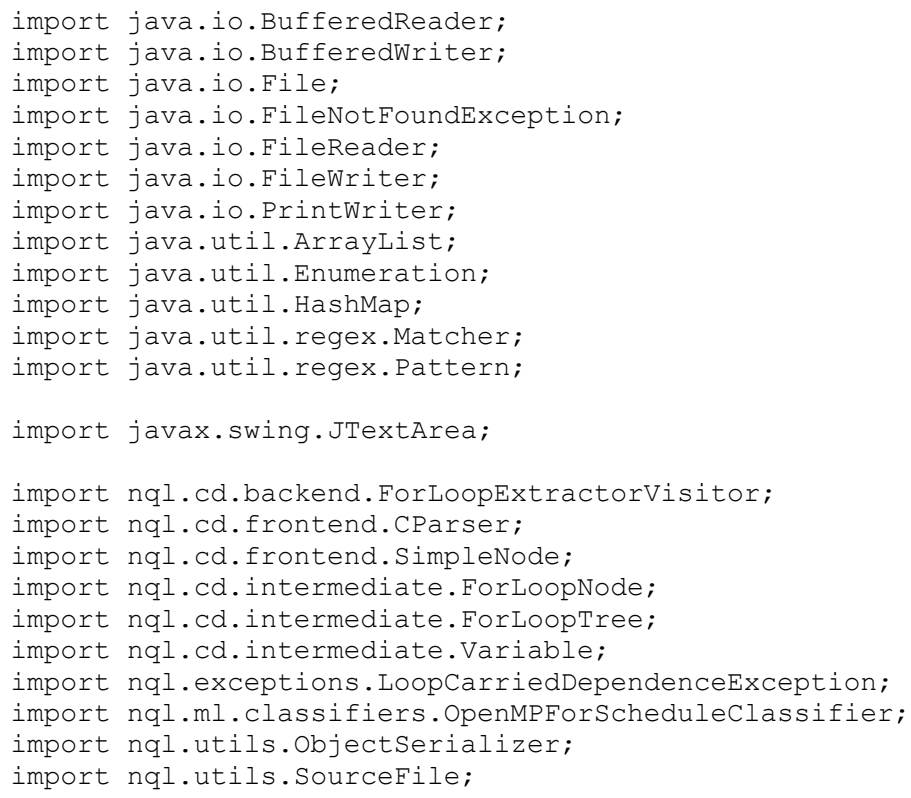




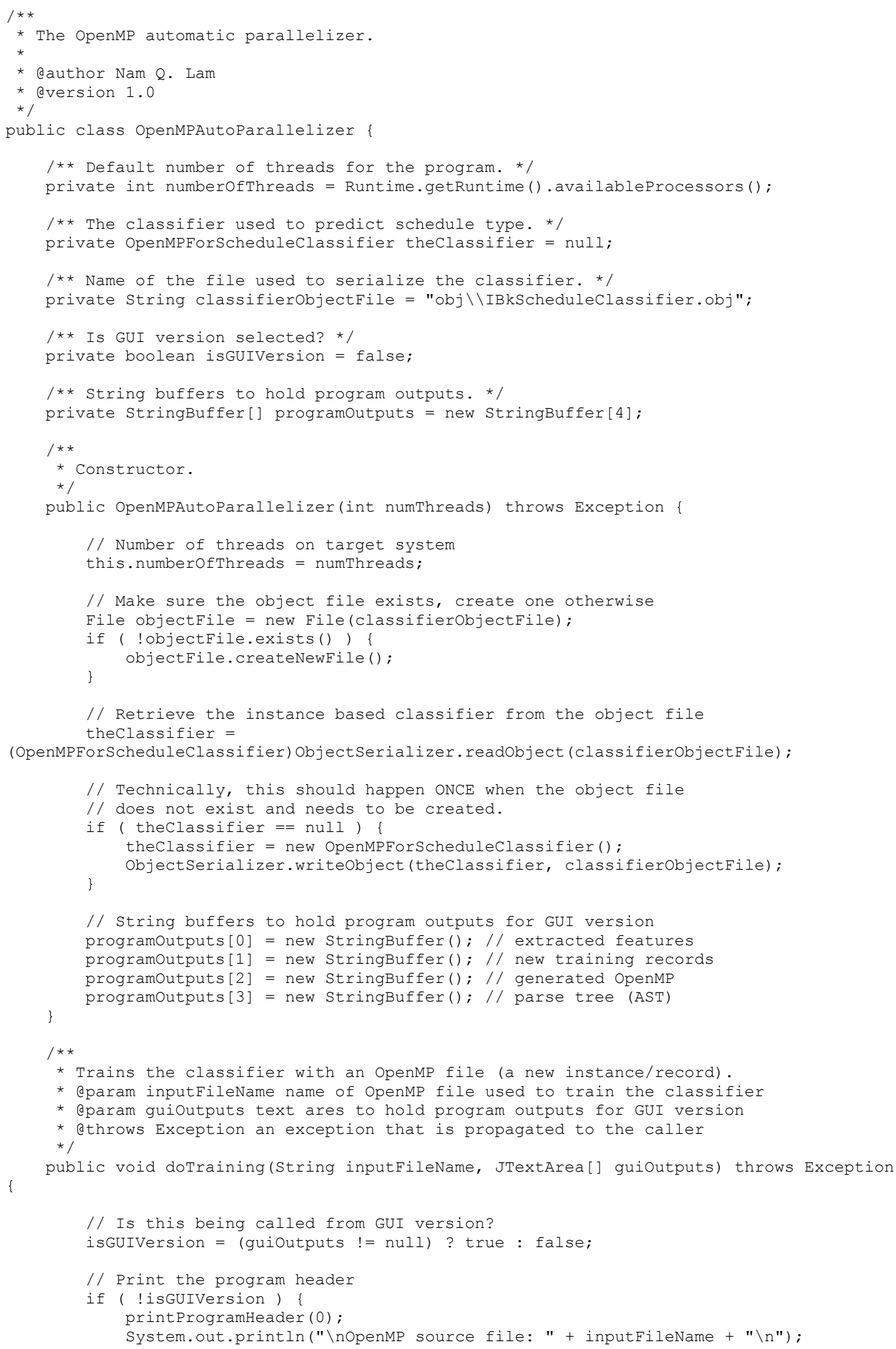


// Extract all FOR loops from the input file ArrayList<ForLoopTree> forLoopTreeList = extractForLoops (inputFileName);

// Display information of each extracted FOR loop printForLoopInfo (forLoopTreeList);

// Use extracted information/features to train the classifier trainTheclassifier (forLoopTreeList);

// If called from GUI version, send outputs to text areas

if ( isGUIVersion ) \{ guioutputs [0]. setText (programoutputs [0] .tostring ()); guioutputs [1]. setText (programoutputs [1] .toString()); guioutputs [3] . setText (programoutputs [3] . toString()); 


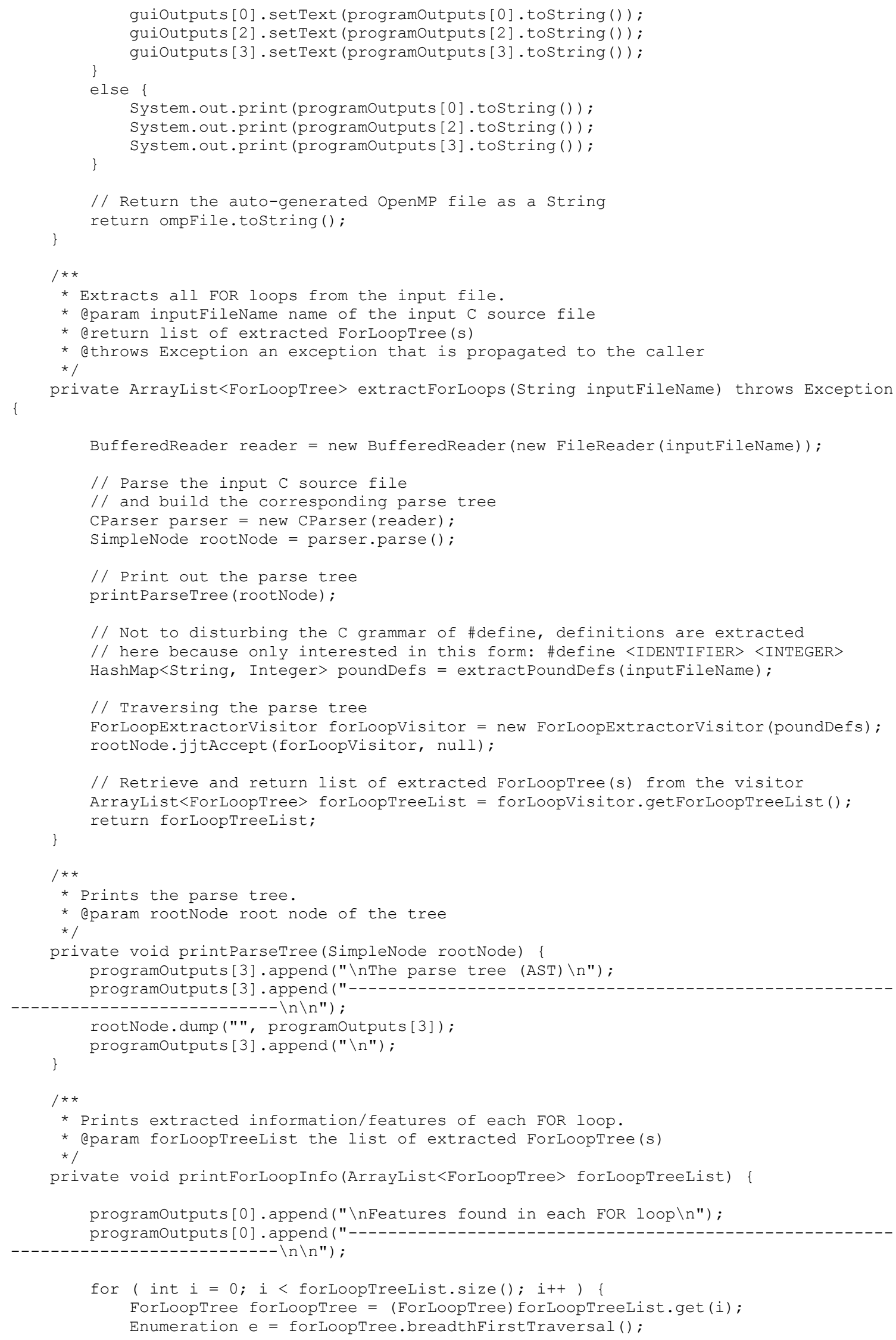




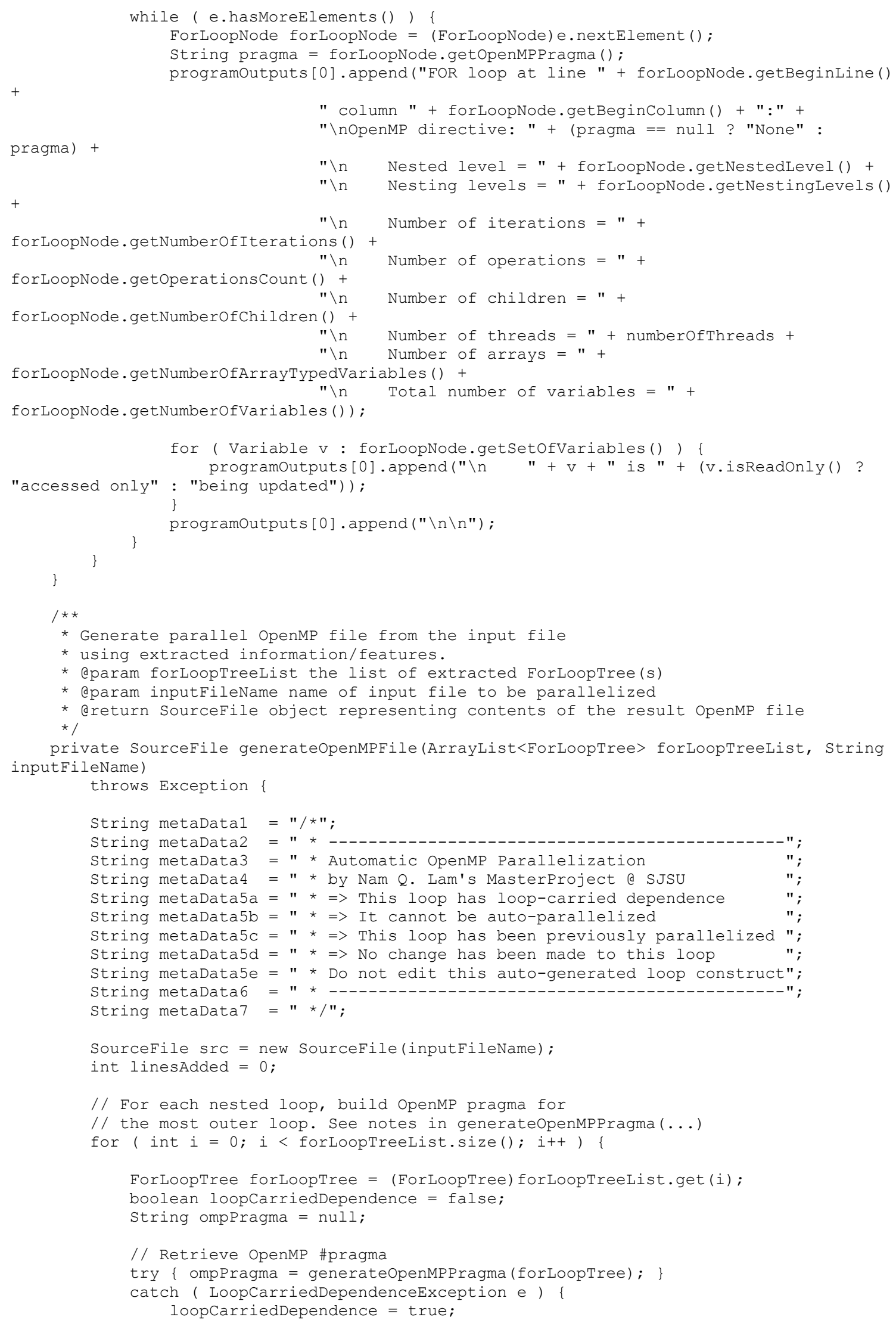


ForLoopNode mostOuterForLoop = (ForLoopNode) forLoopTree.getRootNode (); int row $=$ mostouterForLoop.getBeginLine ();

int $\operatorname{col}=$ mostouterForLoop.getBeginColumn();

// Loop-carried dependence found? If so,

// print appropriate information to indicate so

if ( loopCarriedDependence $==$ true ) \{

src.addLine (linesAdded + row - 1, col - 1, metaDatal);

linesAdded++;

src.addLine (linesAdded + row - 1, col - 1, metaData2);

linesAdded++;

src.addLine (linesAdded + row - 1, col - 1, metaData3);

linesAdded++;

src.addLine (linesAdded + row - 1, col - 1, metaData4);

linesAdded ++;

src.addLine (linesAdded + row - 1, col - 1, metaData5a);

linesAdded++;

src.addLine (linesAdded + row - 1, col - 1, metaData5b):

linesAdded ++;

src.addLine(linesAdded + row - 1, col - 1, metaData6);

linesAdded ++ ;

src.addLine (linesAdded + row - 1, col - 1, metaData7);

linesAdded++;

// Already been previously parallelized using OpenMP,

// print appropriate information to indicate so

else if ( ompPragma $==$ null ) \{

src.addLine(linesAdded + row - 2, col - 1, metaDatal);

linesAdded++;

src.addLine(linesAdded + row - 2, col - 1, metaData2);

linesAdded ++ ;

src.addLine (linesAdded + row - 2, col - 1, metaData3);

linesAdded++;

src.addLine (linesAdded + row - 2, col - 1, metaData4);

linesAdded ++ ;

src.addLine (linesAdded + row - 2, col - 1, metaData5c);

linesAdded++;

src.addLine (linesAdded + row - 2, col - 1, metaData5d);

linesAdded ++ ;

src.addLine (linesAdded + row - 2, col - 1, metaData6);

linesAdded ++ ;

src.addLine (linesAdded + row - 2, col - 1, metaData7);

linesAdded++;

// Just been parallelized via this program auto-parallelizer,

// print resulting information

else \{

src.addLine (linesAdded + row - 1, col - 1, metaDatal);

linesAdded++;

src.addLine(linesAdded + row - 1, col - 1, metaData2);

linesAdded++;

src.addLine (linesAdded + row - 1, col - 1, metaData3);

linesAdded++;

src.addLine (linesAdded + row - 1, col - 1, metaData4);

linesAdded++;

src.addLine (linesAdded + row - 1, col - 1, metaData5e);

linesAdded++;

src.addLine (linesAdded + row - 1, col - 1, metaData6);

linesAdded ++ ;

src.addLine (linesAdded + row - 1, col - 1, metaData7);

linesAdded ++;

src.addLine (linesAdded + row - 1, col - 1, ompPragma);

linesAdded++;

// insert OpenMP header omp.h

insertopenMPHeaderFile (src);

// return result OpenMP parallelized source file 


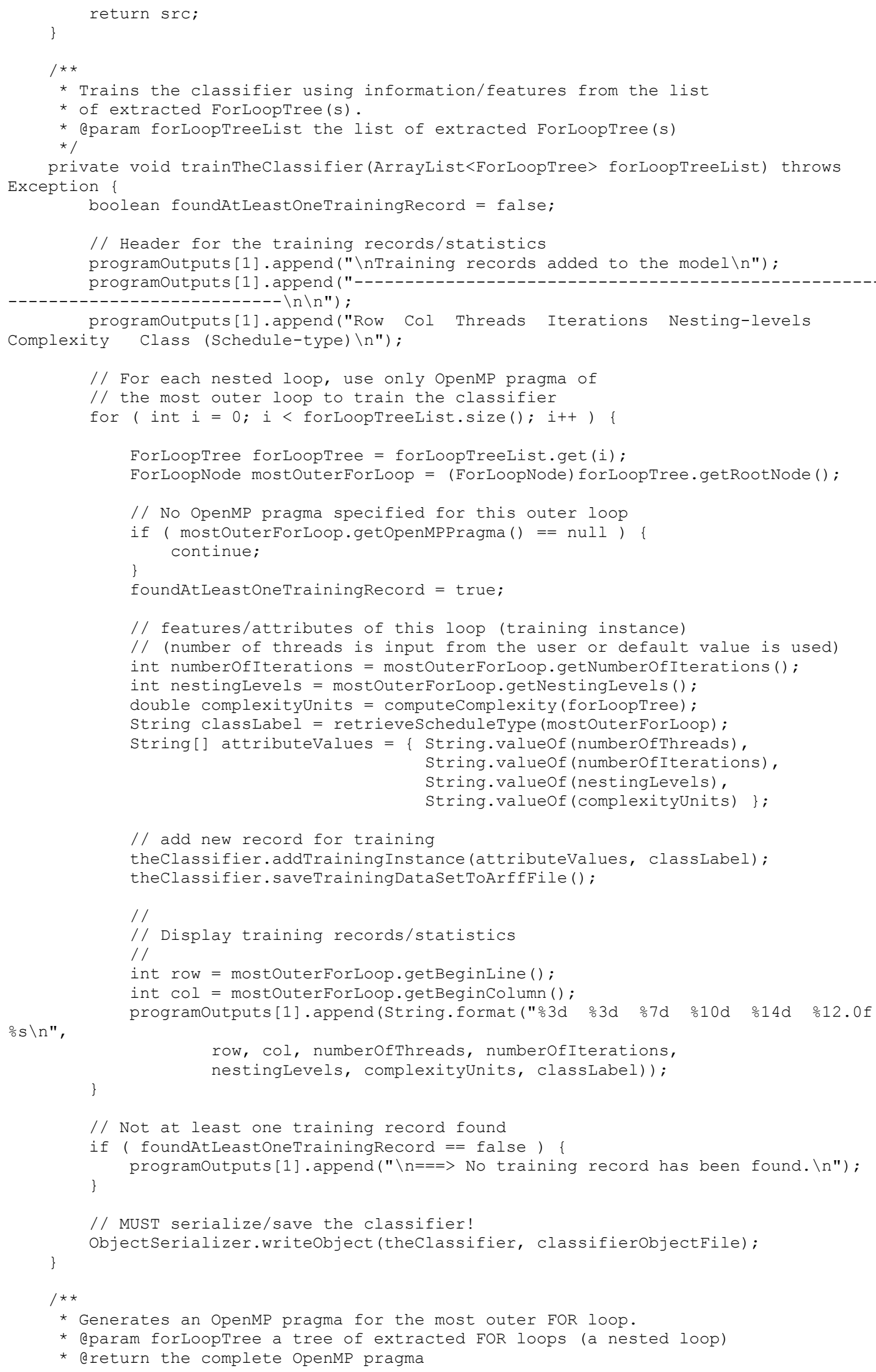




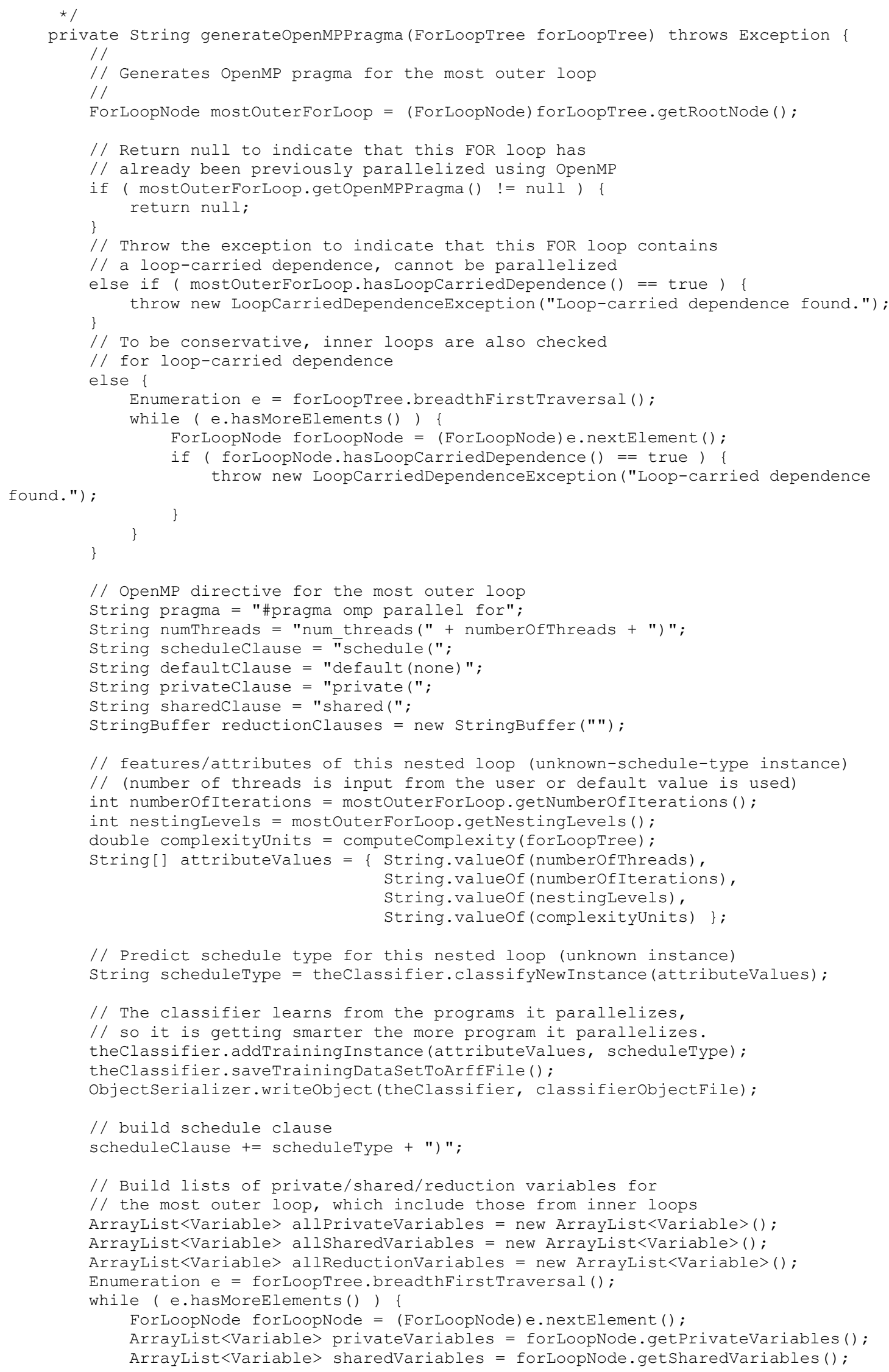




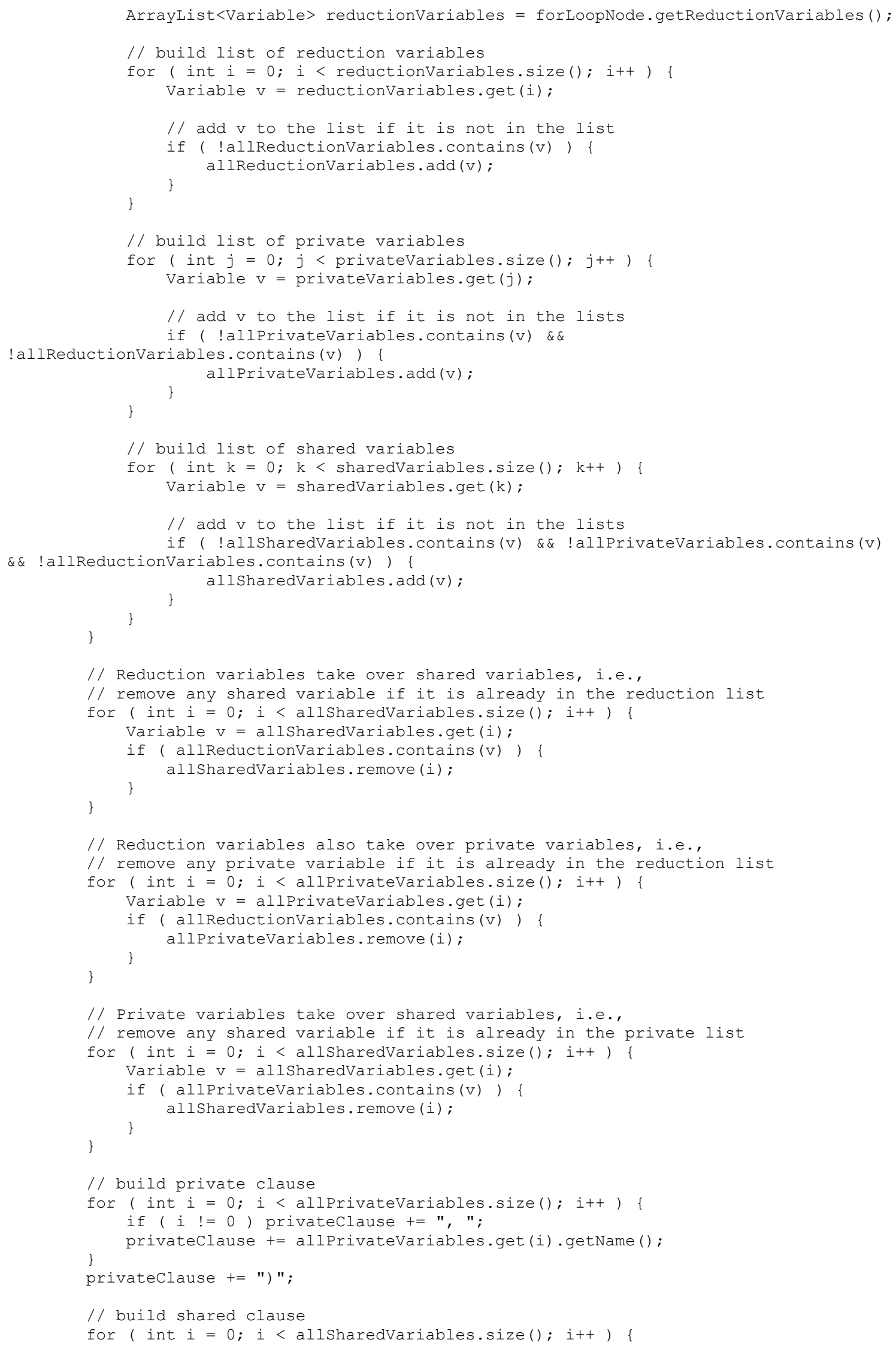




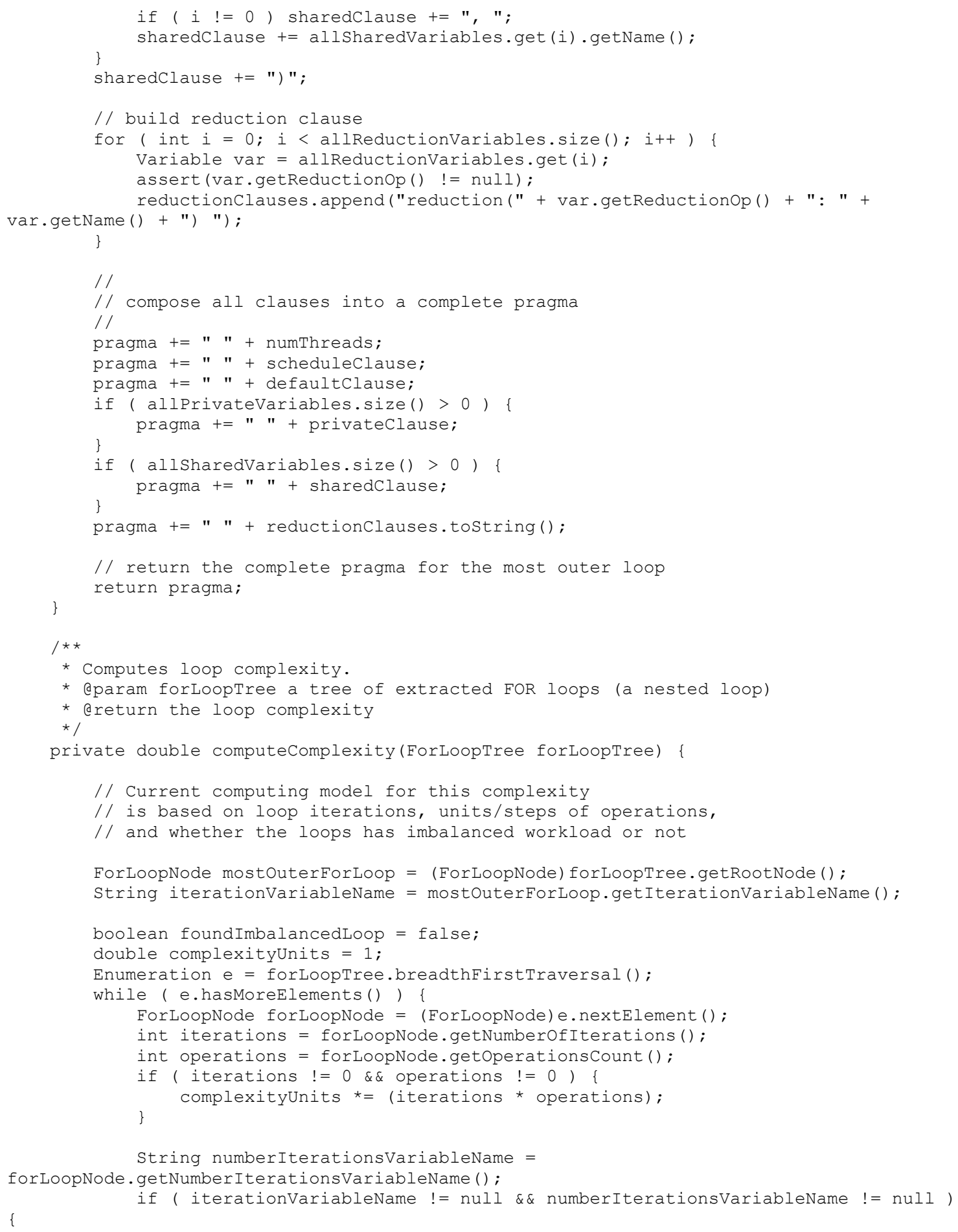




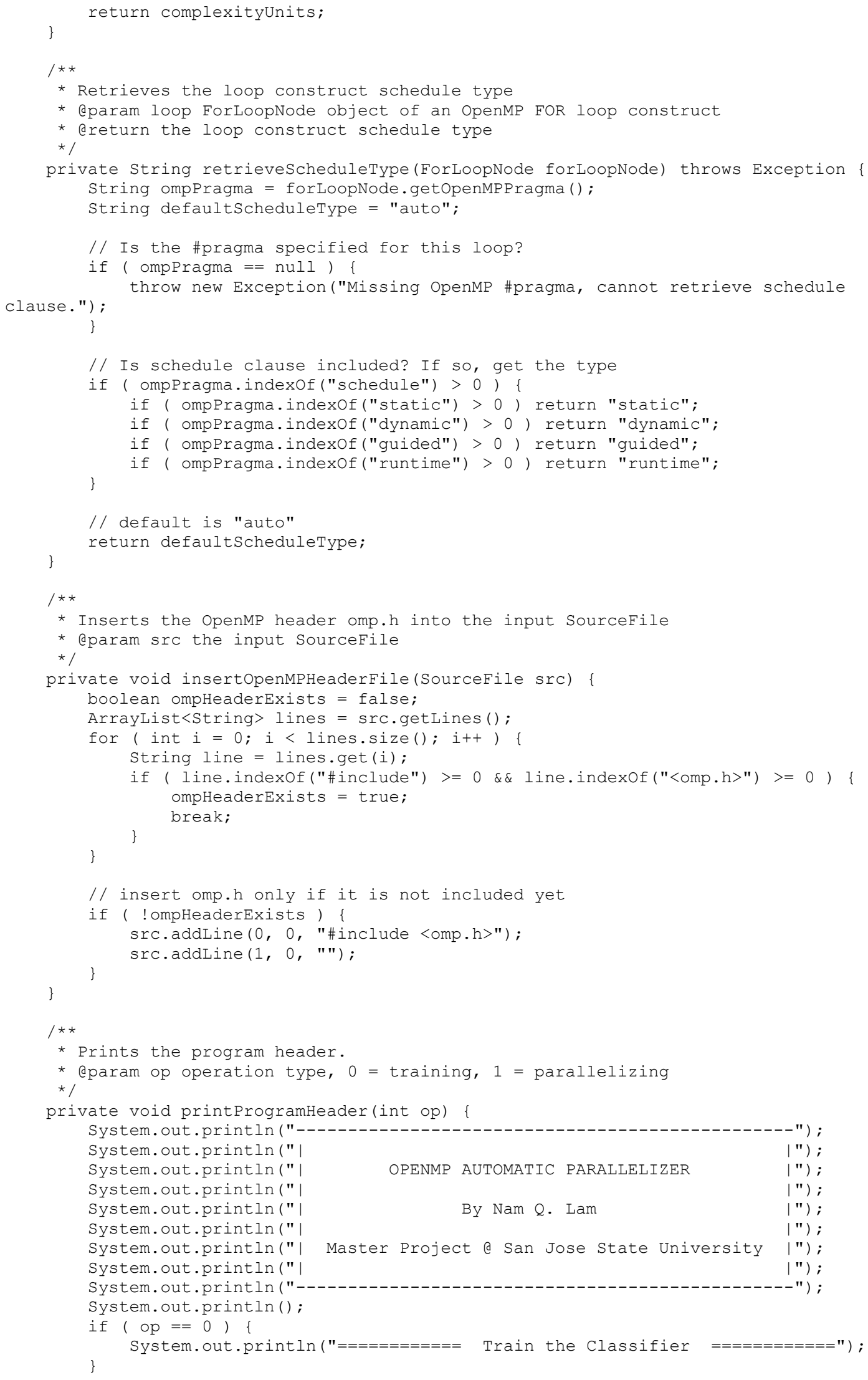




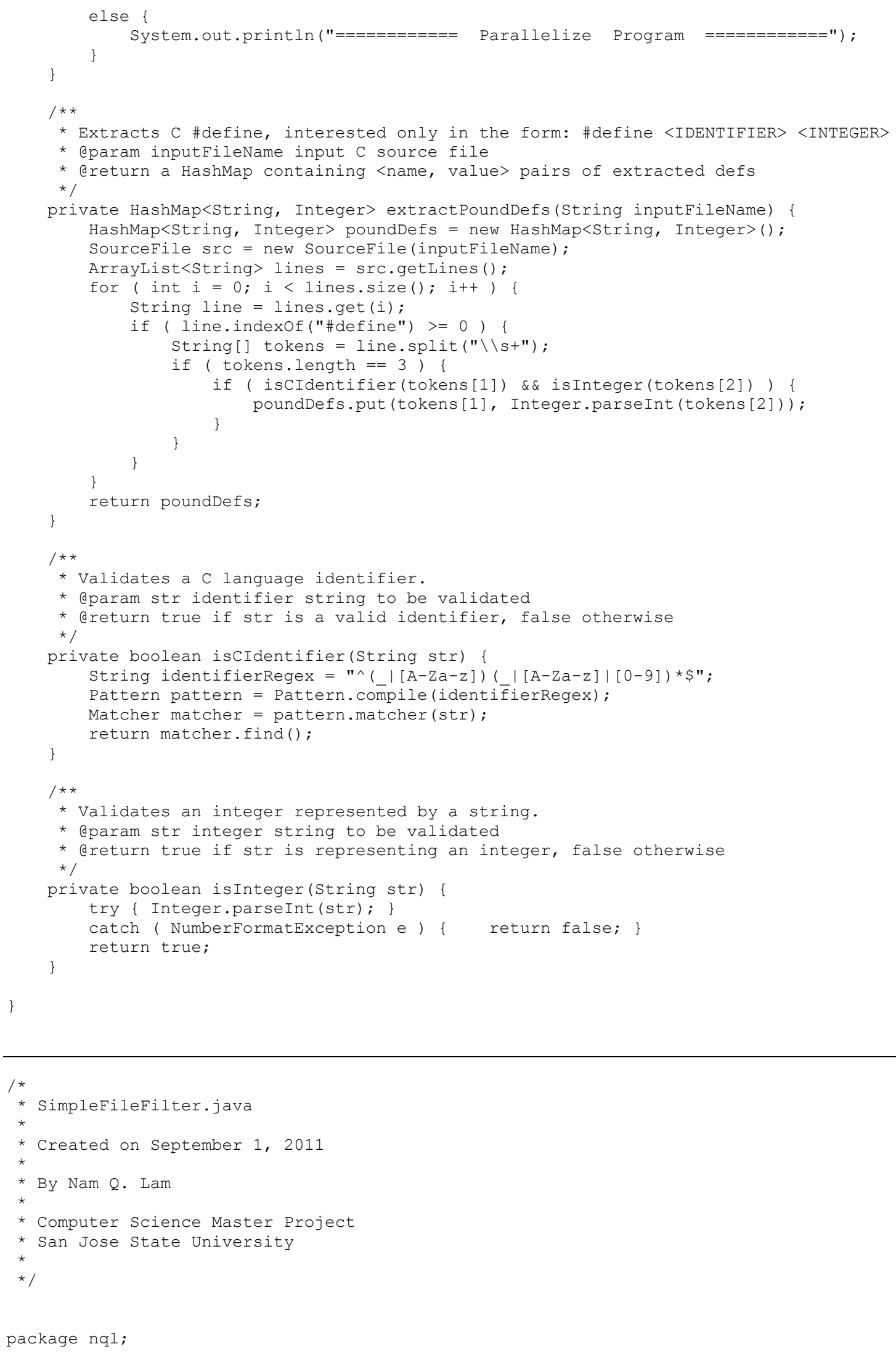




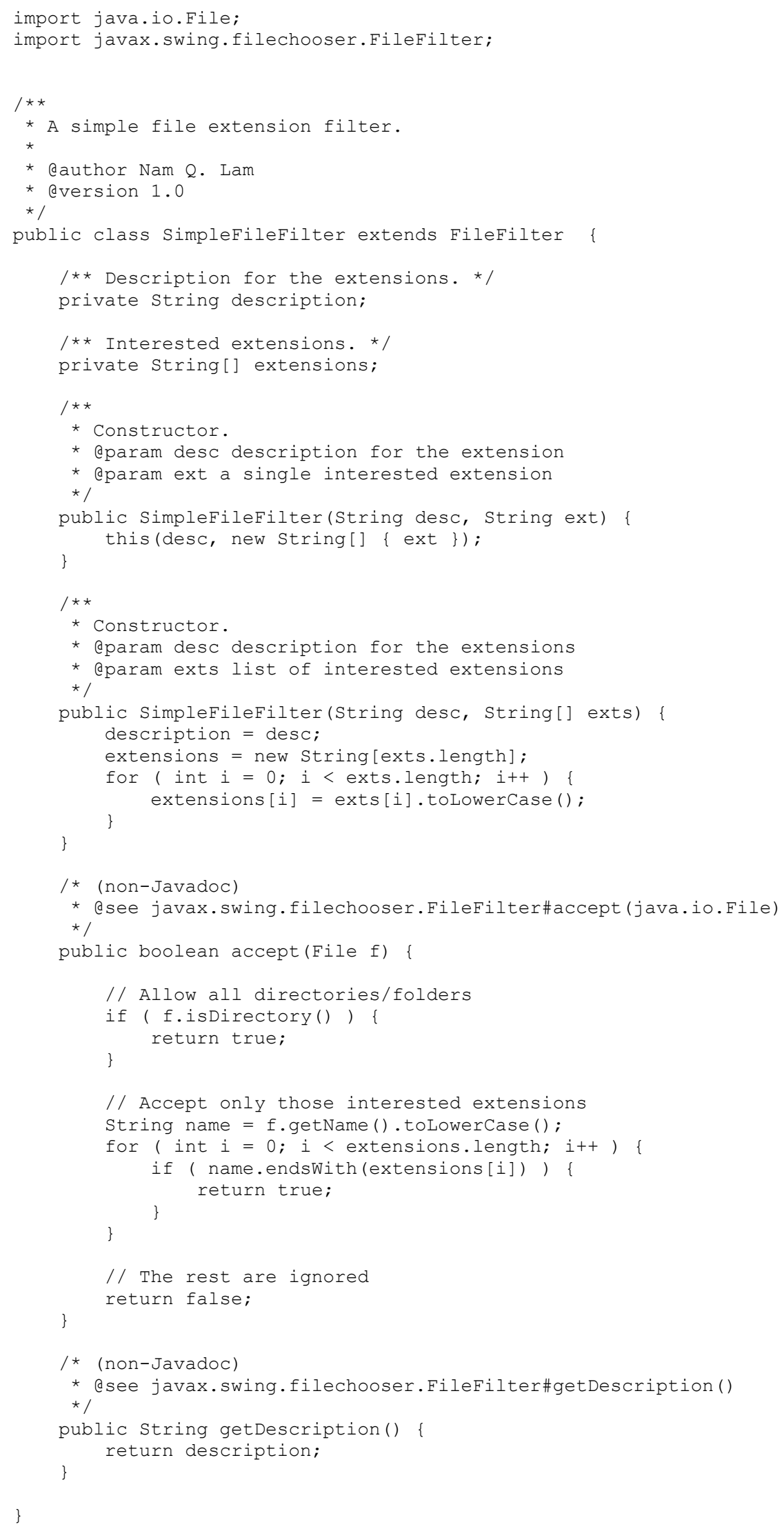




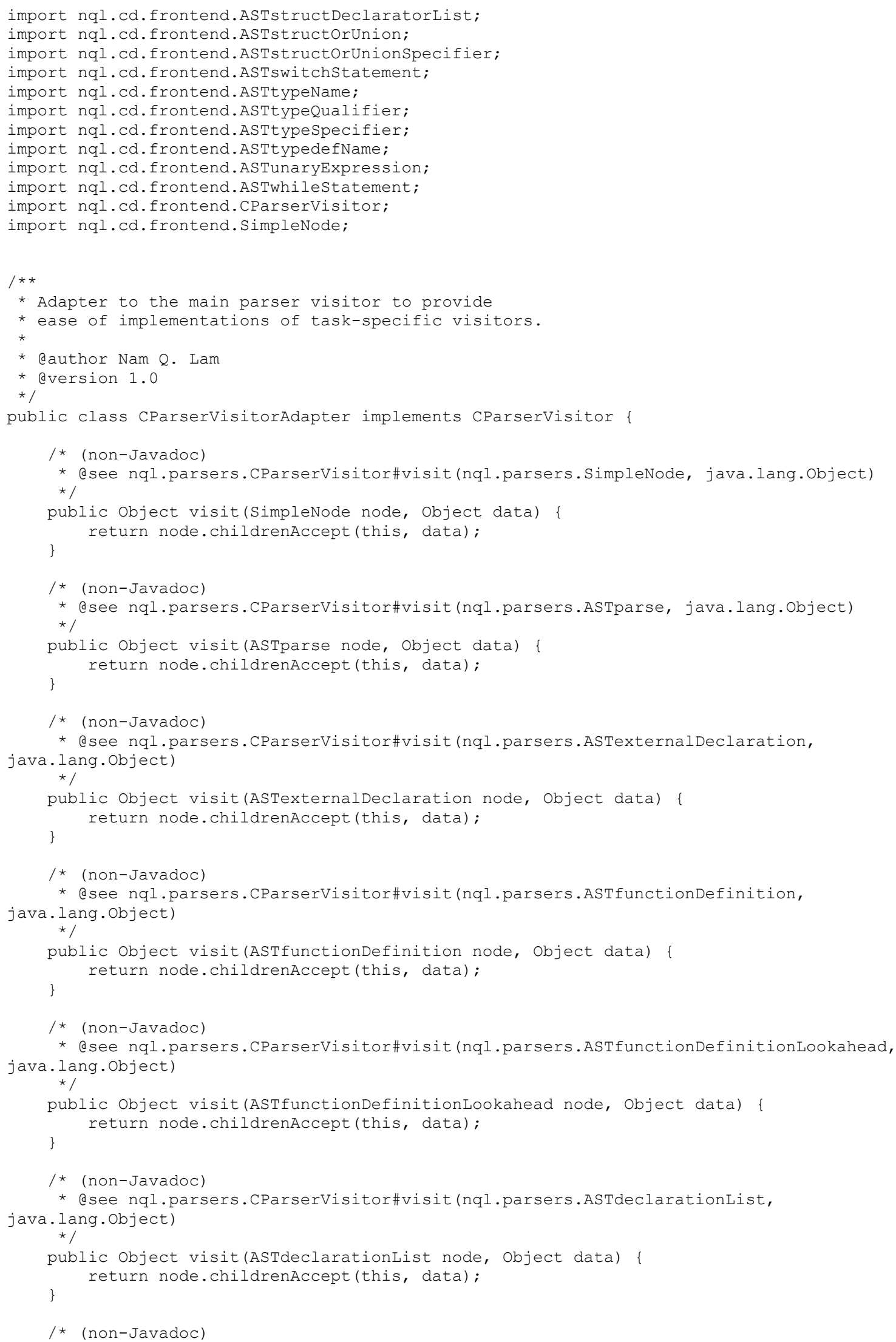




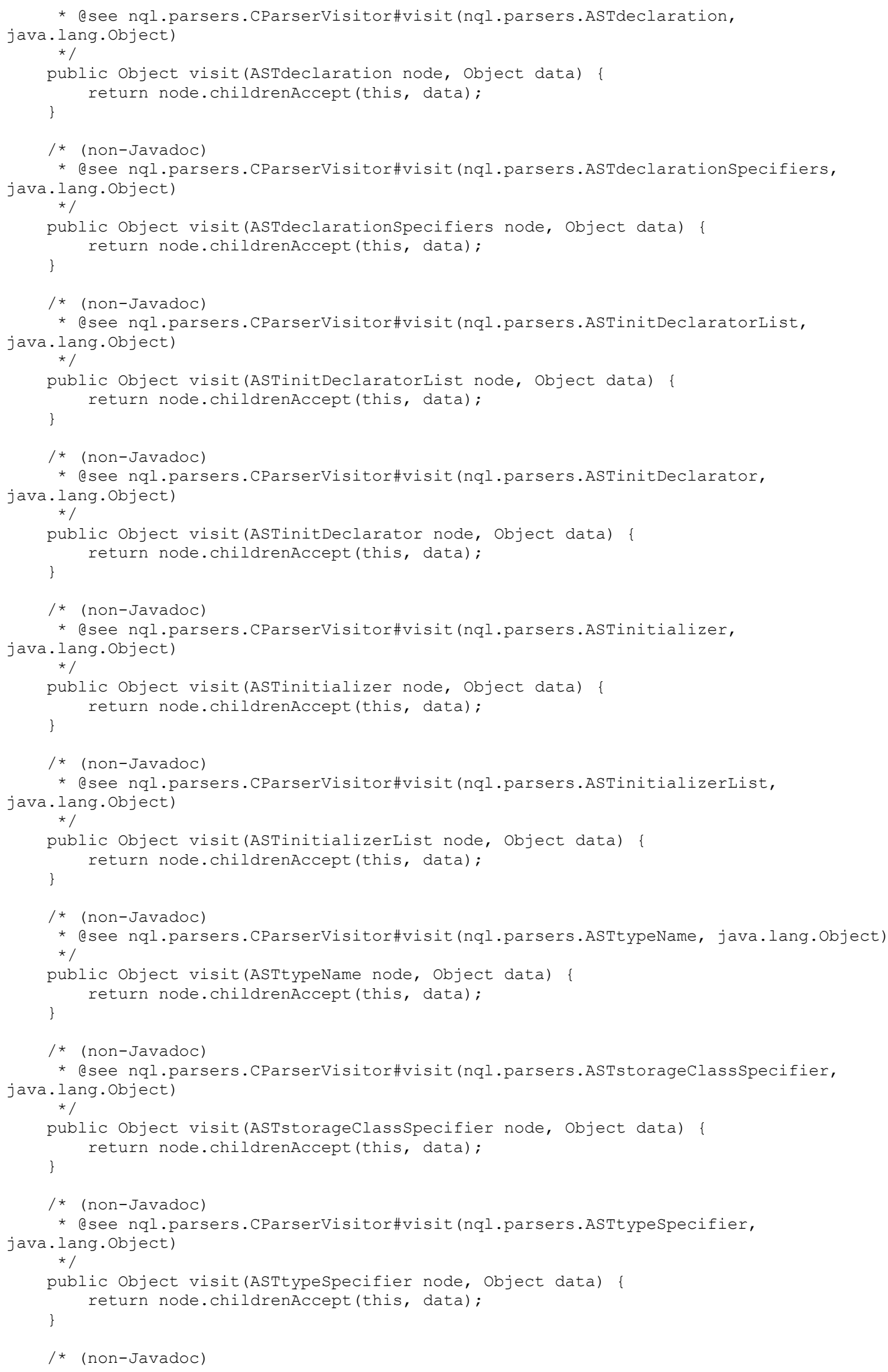




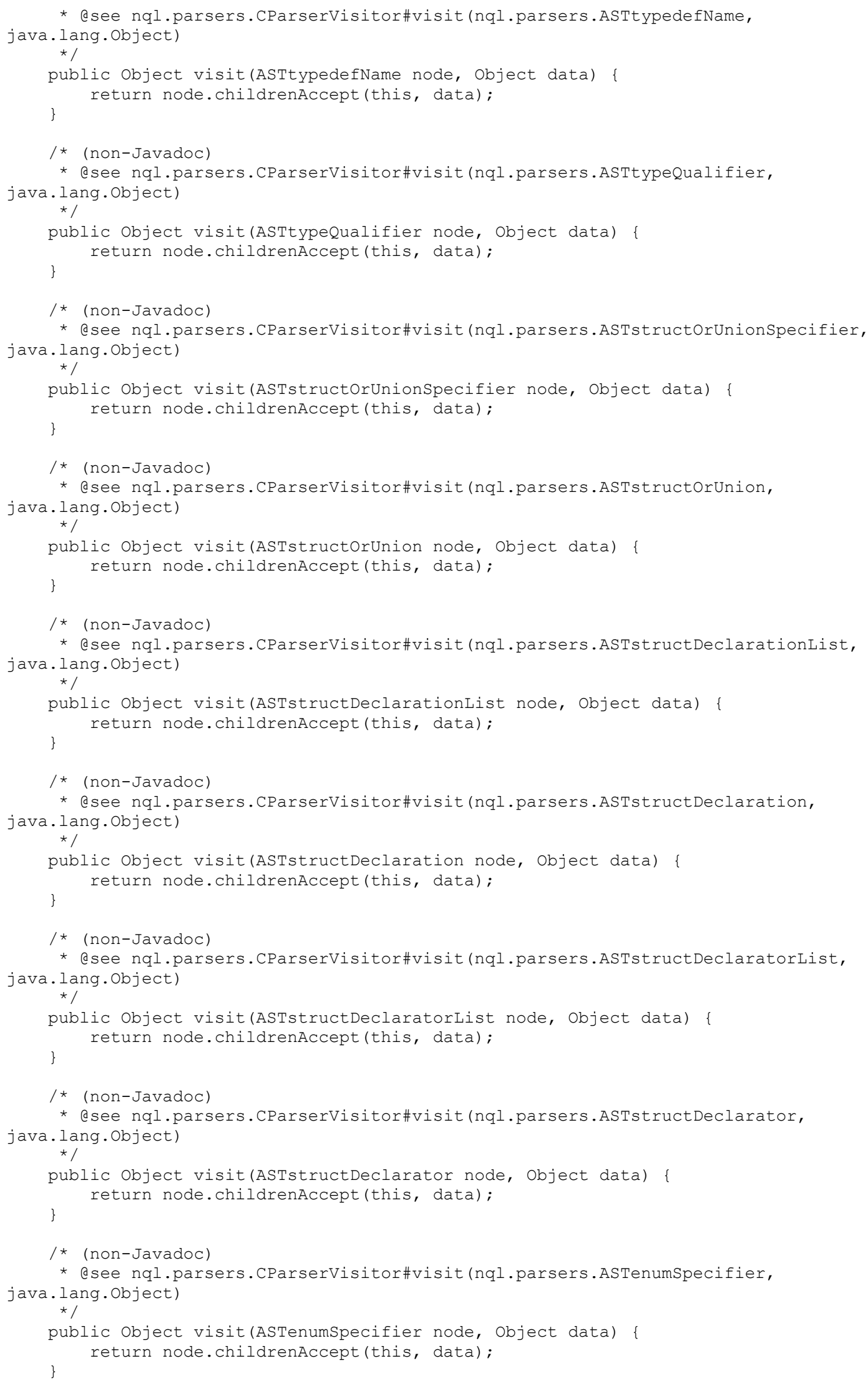




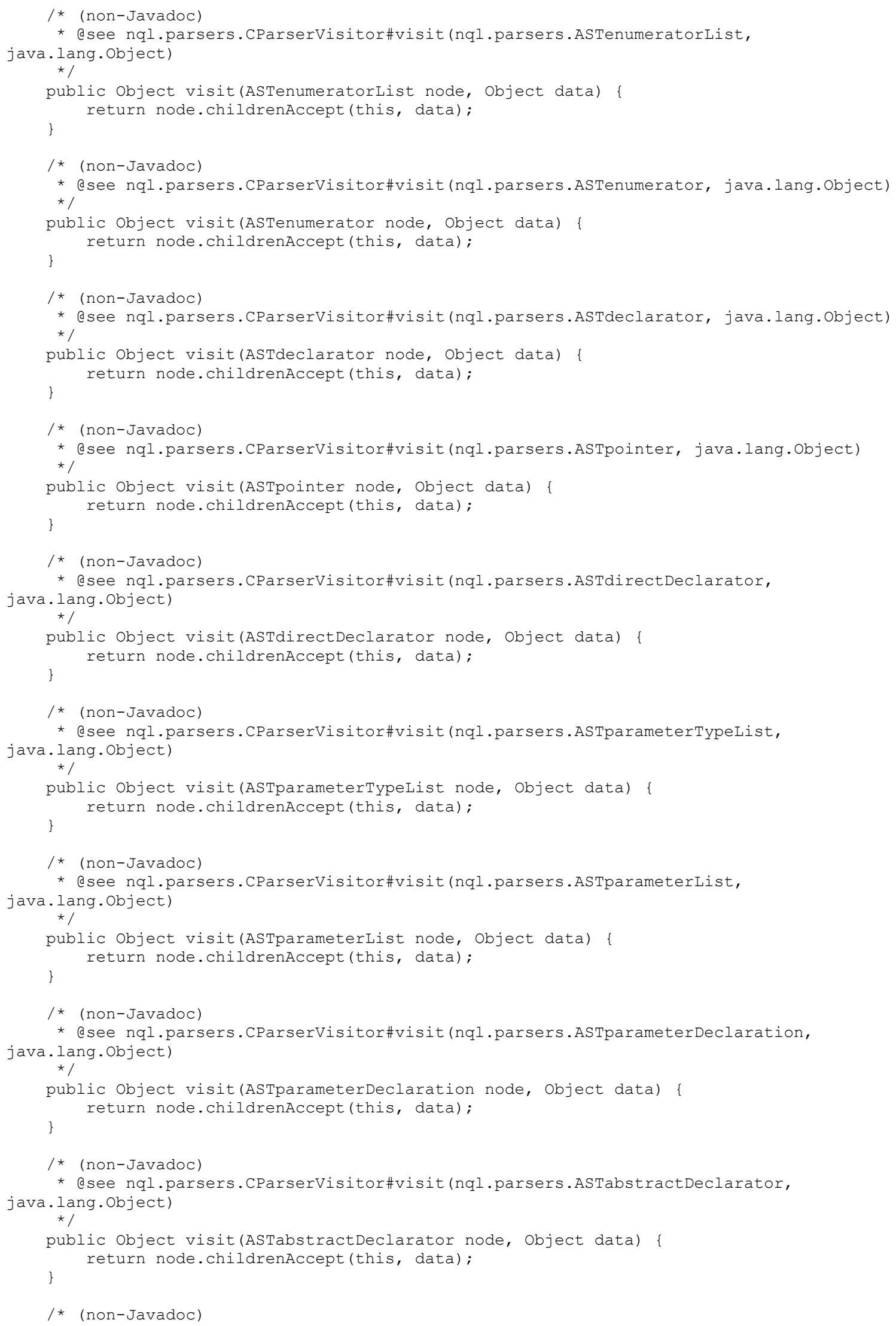




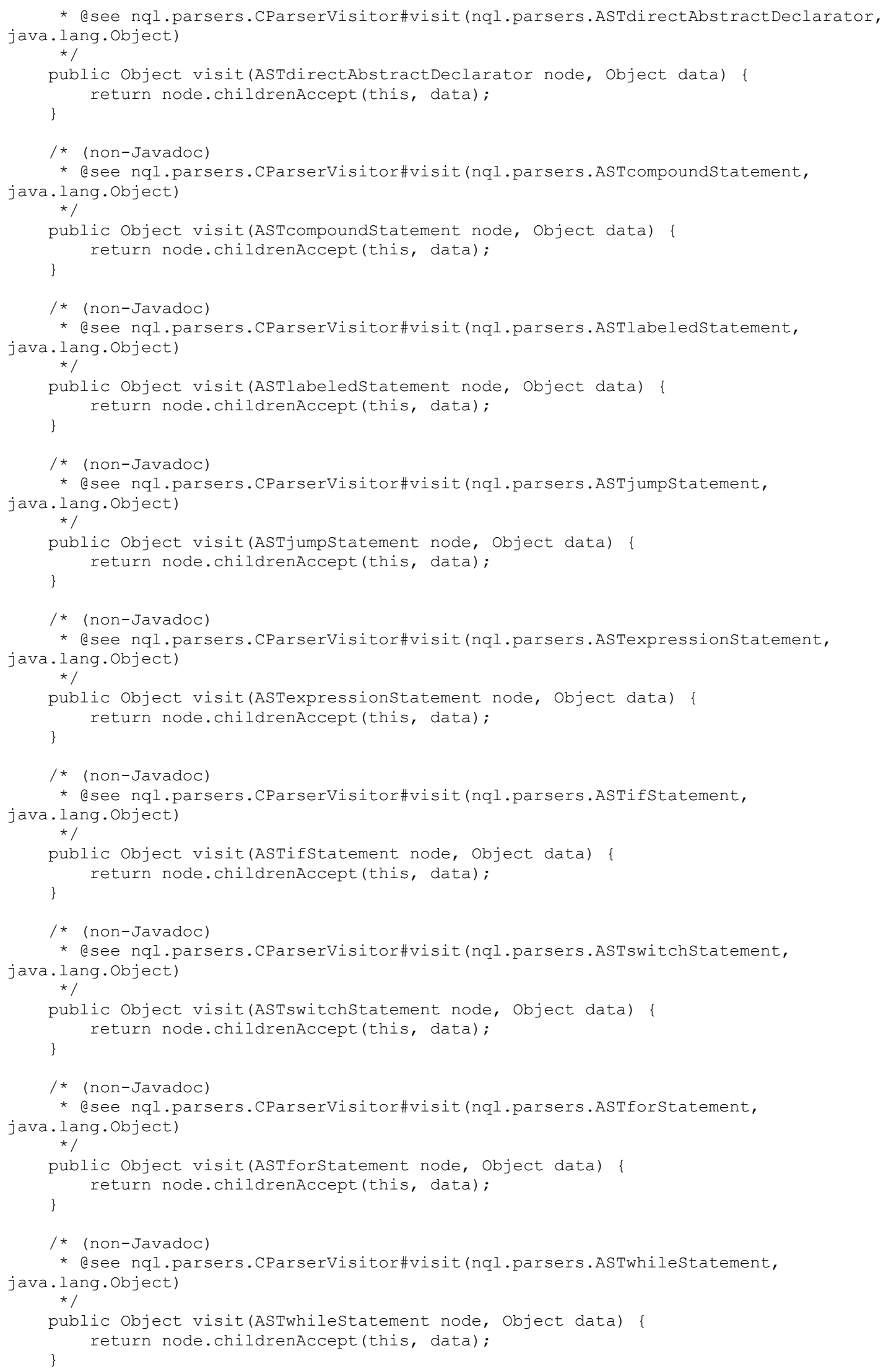




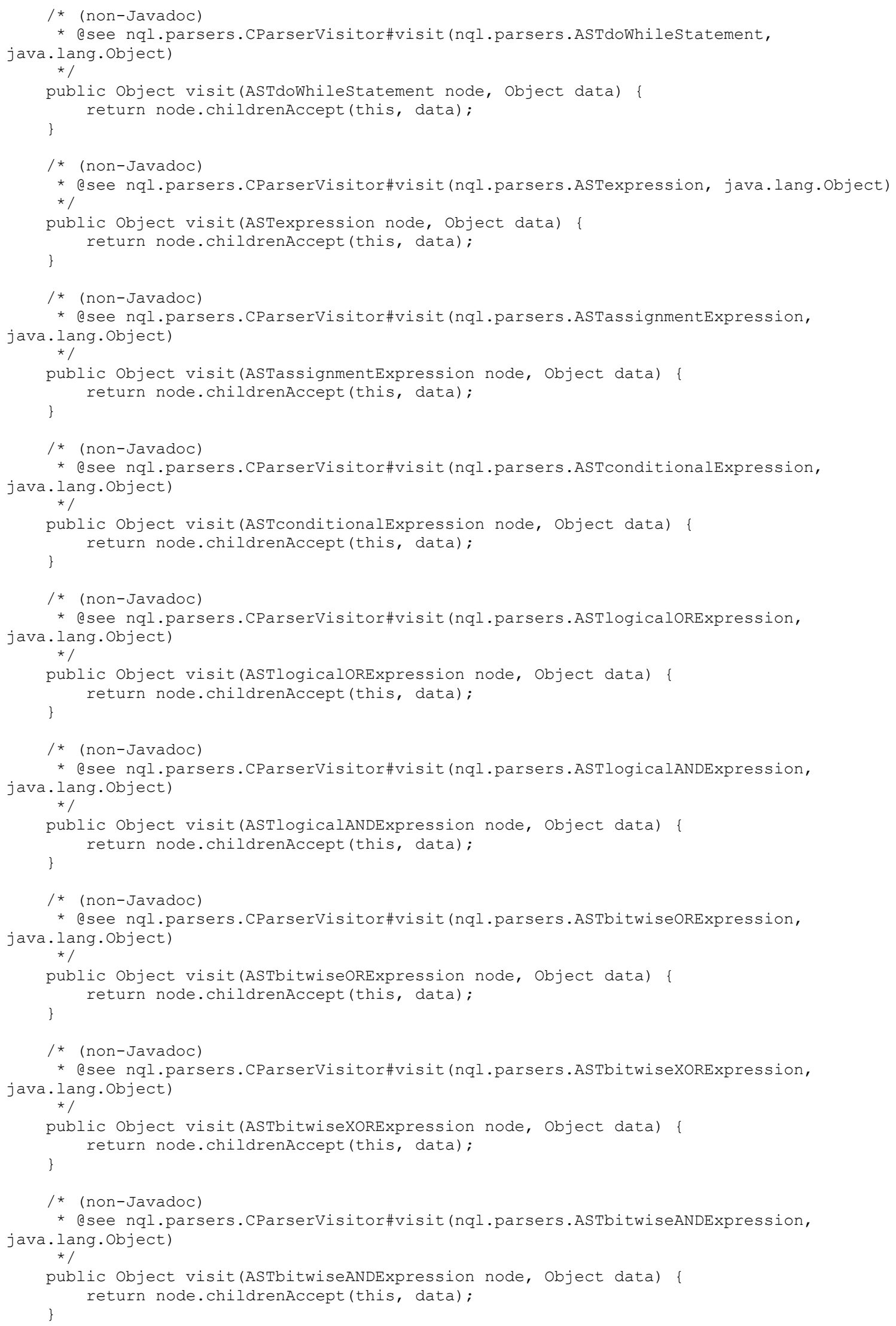




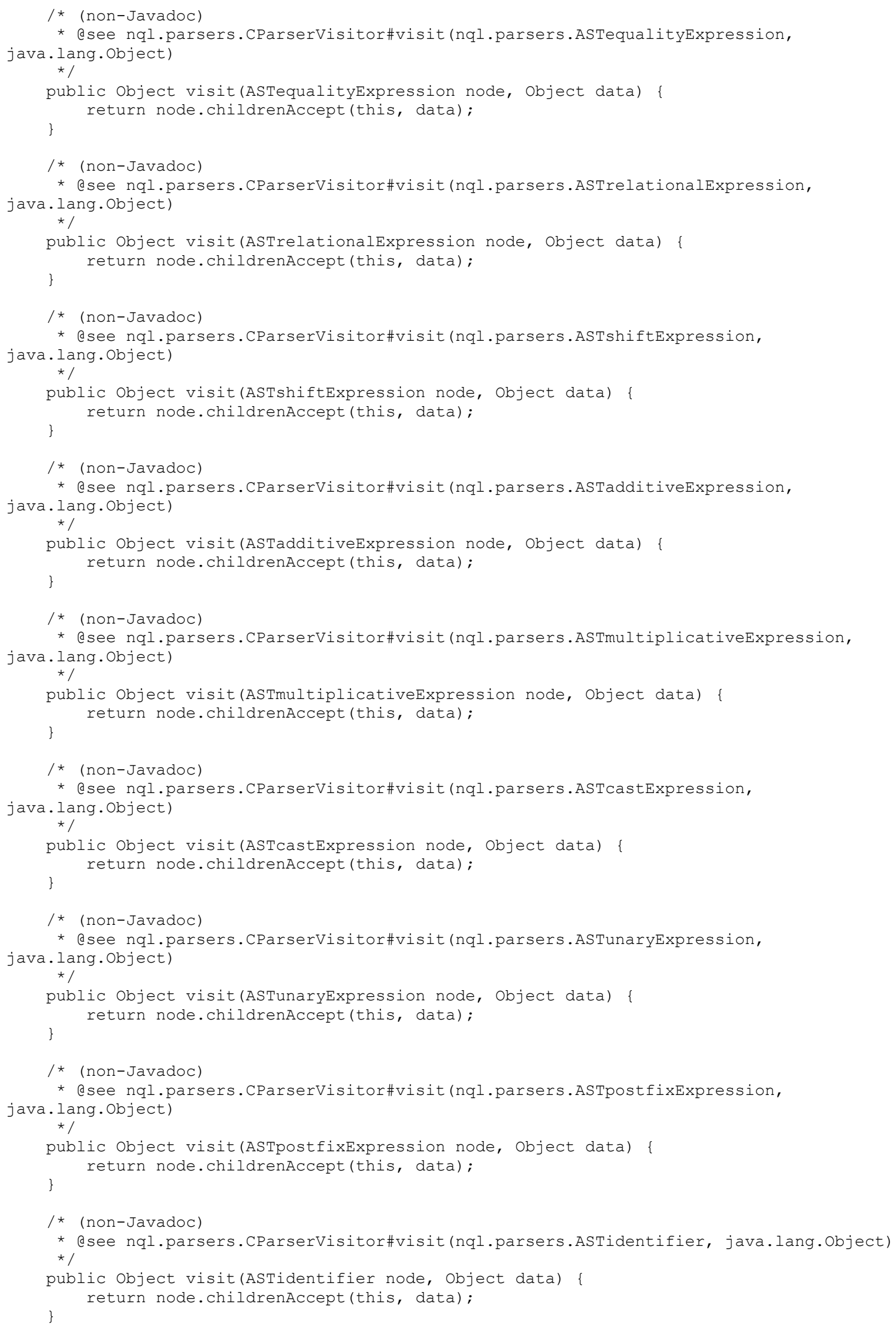




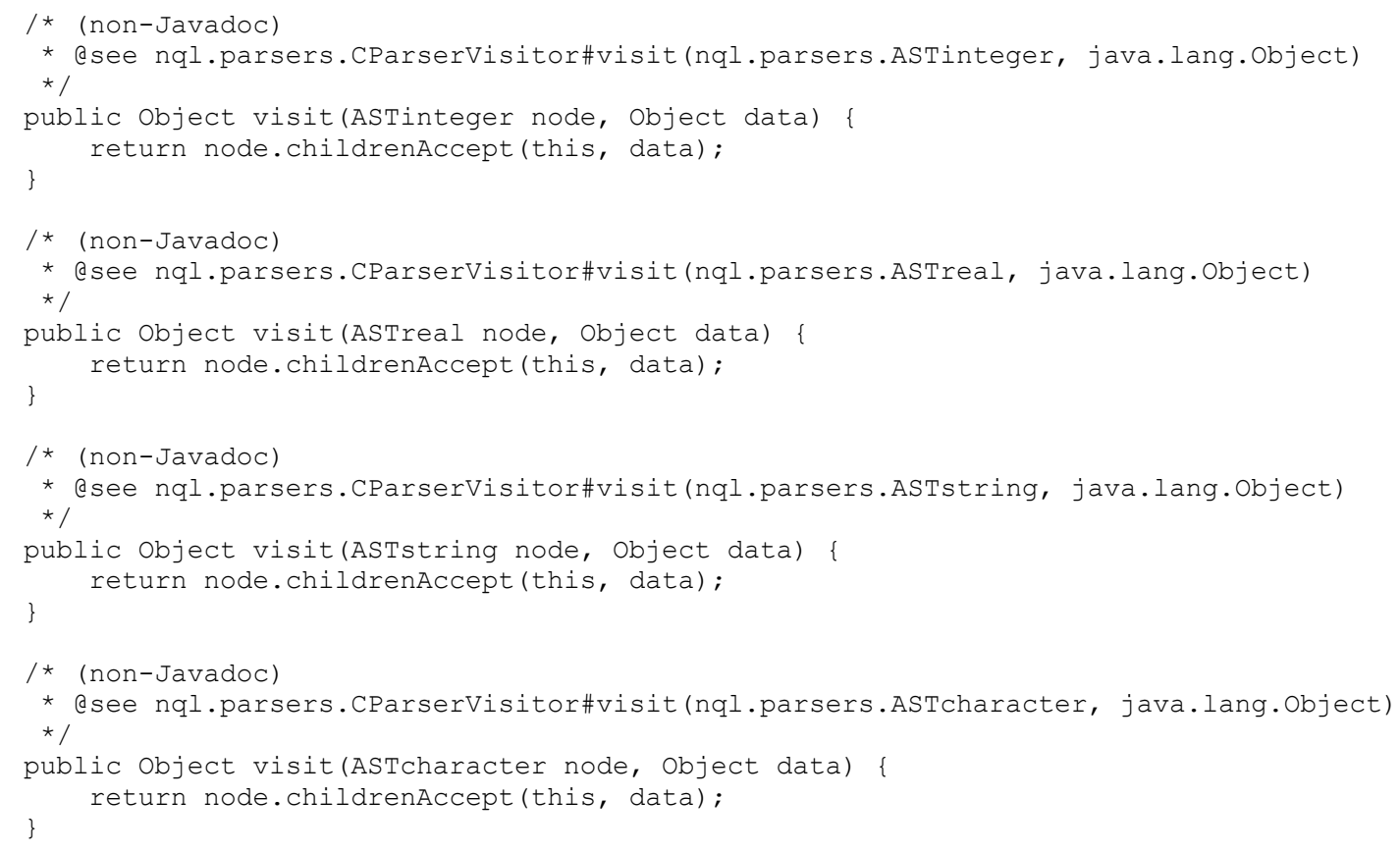


import nql.cd.frontend.ASTpostfixExpression;

import nql.cd.frontend.ASTrelationalExpression;

import nql.cd.frontend.ASTshiftExpression;

import nql.cd.frontend.ASTunaryExpression;

import nql.cd.frontend.CParserTreeConstants;

import nql.cd.frontend.SimpleNode;

import nql.cd.intermediate.ForLoopNode;

import nql.cd.intermediate.ForLoopTree;

import nql.cd.intermediate.NodeKey;

import nql.cd.intermediate.NodeType;

import nql.cd.intermediate.SymbolTableEntry;

import nql.cd.intermediate.SymbolTableFactory;

import nql.cd.intermediate. SymbolTablekey;

import nql.cd.intermediate.SymbolTableStack;

import nql.cd.intermediate.SymbolTableType;

import nql.cd.intermediate.Variable;

import nql.cd.intermediate.VariableType;

import nql.exceptions.AutoParallelizerRestrictionException;

$1 * *$

* Visitor to extract information of all FOR loop nodes in the AST.

* Qauthor Nam Q. Lam

* eversion 1.0

* 1

public class ForLoopExtractorVisitor extends CParserVisitorAdapter \{

/** Nested level of a current FOR loop node. */

private int currentNestedLevel;

/** Nesting level of a current FOR loop node. */

private int currentNestingLevel;

/** Is the traversal currently inside a variable declaration? */

private boolean insideVariableDeclaration;

/** Is the traversal currently inside a function declaration/definition? */

private boolean insideFunctionDecOrDef;

/** Is the traversal currently inside a function definition? */

private boolean insideFunctionDefinition;

/** Is the traversal currently inside a top-level FOR loop node? */

private boolean insideTopLevelForLoopNode;

$1 * *$

* Is the traversal currently inside an assignment expression

* that contributes to OpenMP deduction?

*/

private boolean insideAssignmentExpressionAndReduction;

$1 * *$

* Is the traversal currently inside a postfix expression

* that contributes to openMP deduction?

*

private boolean insidePostfixExpressionAndReduction;

/** Is the traversal currently inside a post increment/decrement expression? */ private boolean insidePostIncrementDecrementExpression;

/** Is the traversal currently inside a pre increment/decrement expression? */ private boolean insidePreIncrementDecrementExpression;

/** Is the traversal currently inside a FOR loop control parameters? */

private boolean expInsideForLoopControlparameters;

/** Bi-operator of an assignment expression inside a FOR loop. */

private String assignmentExpressionop;

/** Operator of a postfix expression inside a FOR loop. */ 
private String postfixExpressionop;

$1 * \star$

* List of array-typed postfixExpression nodes that are

* on the left of an assignment expression inside a FOR loop.

* 1

private ArrayList<SimpleNode> leftArrayPostfixExpressionList;

$/ * \star$

* List of array-typed postfixExpression nodes that are

* on the right of an assignment expression inside a FOR loop. * 1

private ArrayList<SimpleNode> rightArrayPostfixExpressionList;

/** Tree of FOR loops (a nested loop). */

private ForLooptree forLoopTree;

/** List of extracted ForLoopTree(s). */

private ArrayList<ForLoopTree> forLoopTreeList;

/** Symbol table stack to store identifier information. */

private SymbolTablestack symbolTablestack;

/**

* Constructor.

* /

public ForLoopExtractorVisitor(HashMap<String, Integer> poundDefs) \{

currentNestedLevel $=-1$;

currentNestingLevel $=-1$;

insideVariableDeclaration = false;

insideFunctionDecorDef = false;

insideFunctionDefinition = false;

insideTopLevelForLoopNode = false;

insideAssignmentExpressionAndReduction = false;

assignmentExpressionop = null;

insidePostfixExpressionAndReduction = false;

postfixExpressionop = null.

expInsideForLoopControlparameters = false;

leftArrayPostfixExpressionList = new ArrayList $<$ SimpleNode $>()$.

rightArrayPostfixExpressionList = new ArrayList $<$ SimpleNode $>$ ();

forLoopTree = new ForLoopTree();

forLooptreeList $=$ new ArrayList $<$ ForLooptree $>()$;

symbolTablestack = SymbolTableFactory.createSymbolTablestack ();

// Process \#define definitions

if ( !poundDefs.isEmpty () ) \{

Set<string $>$ keyset $=$ poundDefs. $\operatorname{keyset}()$;

Iterator $\langle$ String $>$ it $=$ keyset.iterator () ;

while ( it.hasNext() ) \{

String name = it.next ();

Integer value $=$ (Integer) poundDefs.get (name);

SymbolTableEntry definedEntry = symbolTablestack.enterGlobal (name);

definedEntry.setAttribute (SymbolTableKey.TYPE, SymbolTableType.DEFINE);

definedEntry.setAttribute (SymbolTableKey.VALUE, value);

// Populate pre-defined library function names

symbolTableStack. enterGlobal ("scanf") . setAttribute (SymbolTableKey.TYPE,

Symboltabletype. FUNCTION);

symbolTableStack. enterGlobal ("printf") . setAttribute (SymbolTableKey.TYPE,

SymboltableType. FUNCTION);

symbolTablestack. enterGlobal ("sin") . setAttribute (SymbolTableKey.TYPE,

Symboltabletype. FUNCTION);

symbolTablestack. enterGlobal ("cos") . setAttribute (SymbolTableKey.TYPE,

SymboltableType. FUNCTION);

symbolTablestack. enterGlobal ("tan") . setAttribute (SymbolTableKey. TYPE,

SymbolTableType.FUNCTION);

symbolTablestack. enterGlobal ("sqrt") . setAttribute (SymbolTableKey.TYPE,

SymbolTableType. FUNCTION); 
symbolTableStack.enterGlobal ("pow") . setAttribute (SymbolTableKey.TYPE, SymbolTableType.FUNCTION);

symbolTableStack.enterGlobal ("log") . setAttribute (SymbolTableKey. TYPE, SymboltableType.FUNCTION);

\}

$/ * \star$

* Retrieves the extracted FOR loops as a list of ForLooptree(s).

* @return list of extracted ForLooptree(s)

*

public Arraylist<ForLoopTree> getForLoopTreeList() \{ return forLoopTreeList;

/* (non-Javadoc)

* esee

nql.cd.intermediate.CParserVisitorAdapter\#visit (nql.cd.frontend.ASTfunctionDefinition, java.lang.object)

* /

public Object visit(ASTfunctionDefinition node, Object data) \{

// Enter a function scope

symbolTableStack.push();

// Set the flag

insideFunctionDefinition = true;

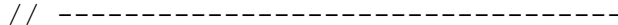

// Traversing down the tree...

// --------------------------------

object obj = super.visit(node, data);

// Clear the flag

insideFunctionDefinition = false;

// Leave the function scope

symbolTableStack.pop ();

return obj;

\}

/* (non-Javadoc)

* esee CParserVisitorAdapter\#visit(nql.parsers.ASTinitDeclarator, java.lang.object) $* /$

public Object visit(ASTinitDeclarator node, Object data) \{

// Retrieve variable and its initial value

String name = null;

Integer value = null;

SymbolTableEntry varEntry = null;

if ( node.jjtGetNumChildren() > 1 ) \{

// Interested in initialization declaration of the form <identifier> =

<integer>,

// which will be used to compute number of iterations of a FOR loop SimpleNode identifierNode =

(SimpleNode) node.jjtGetChild(0).jjtGetChild(0).jjtGetChild(0);

SimpleNode integerNode $=$ (SimpleNode) node.jjtGetChild(1).jjtGetChild(0);

ASTinteger )

if ( identifierNode instanceof ASTidentifier \&\& integerNode instanceof

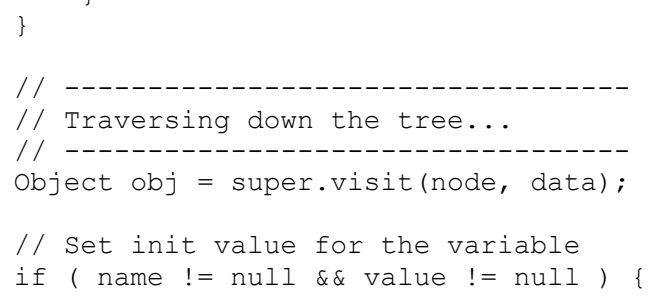




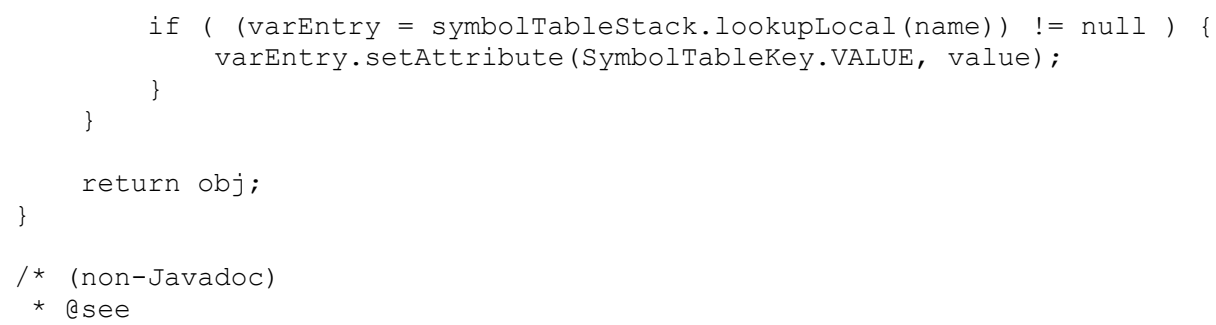




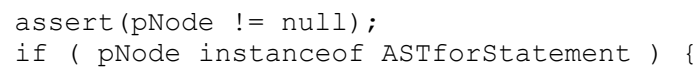




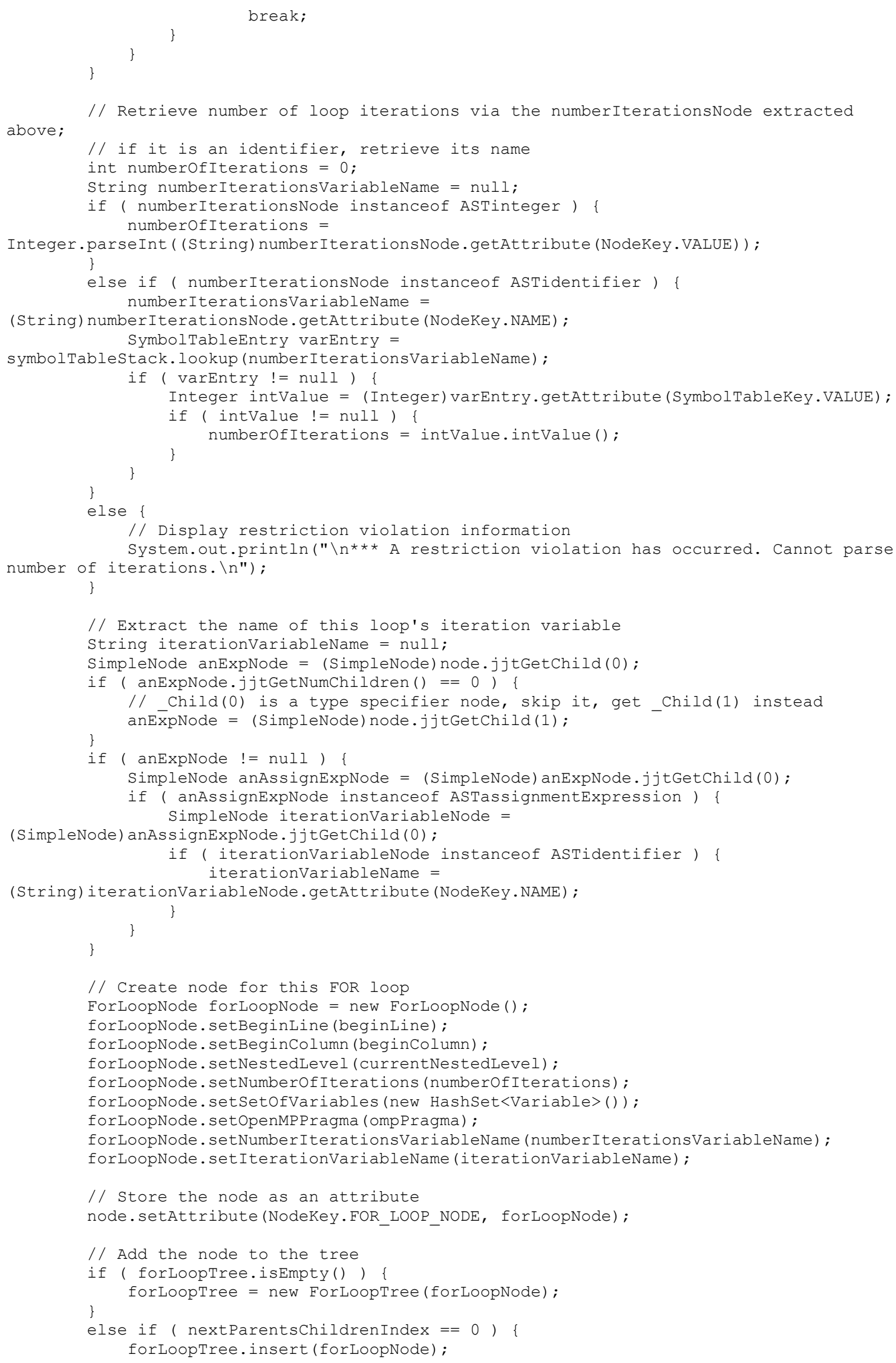




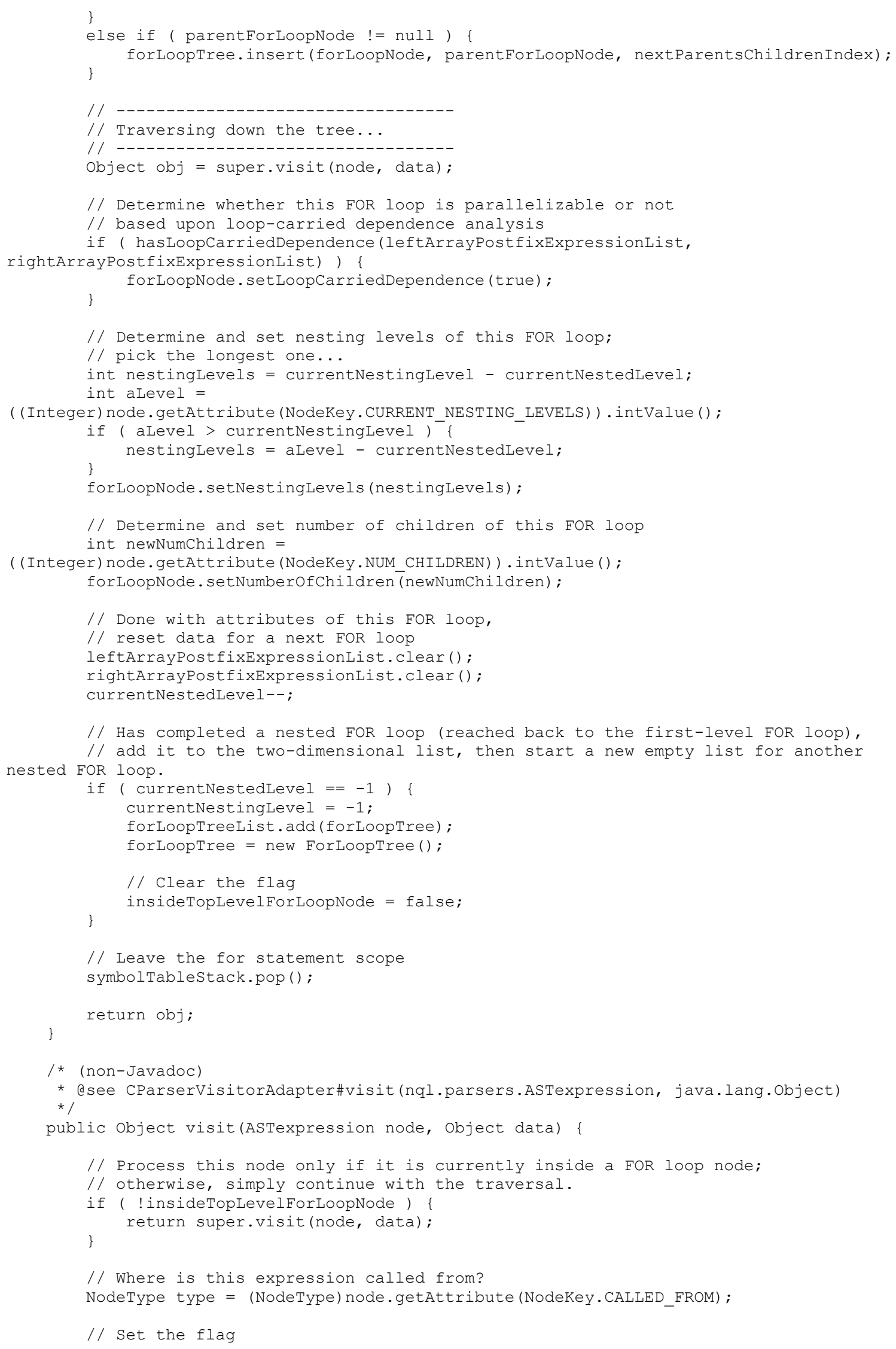




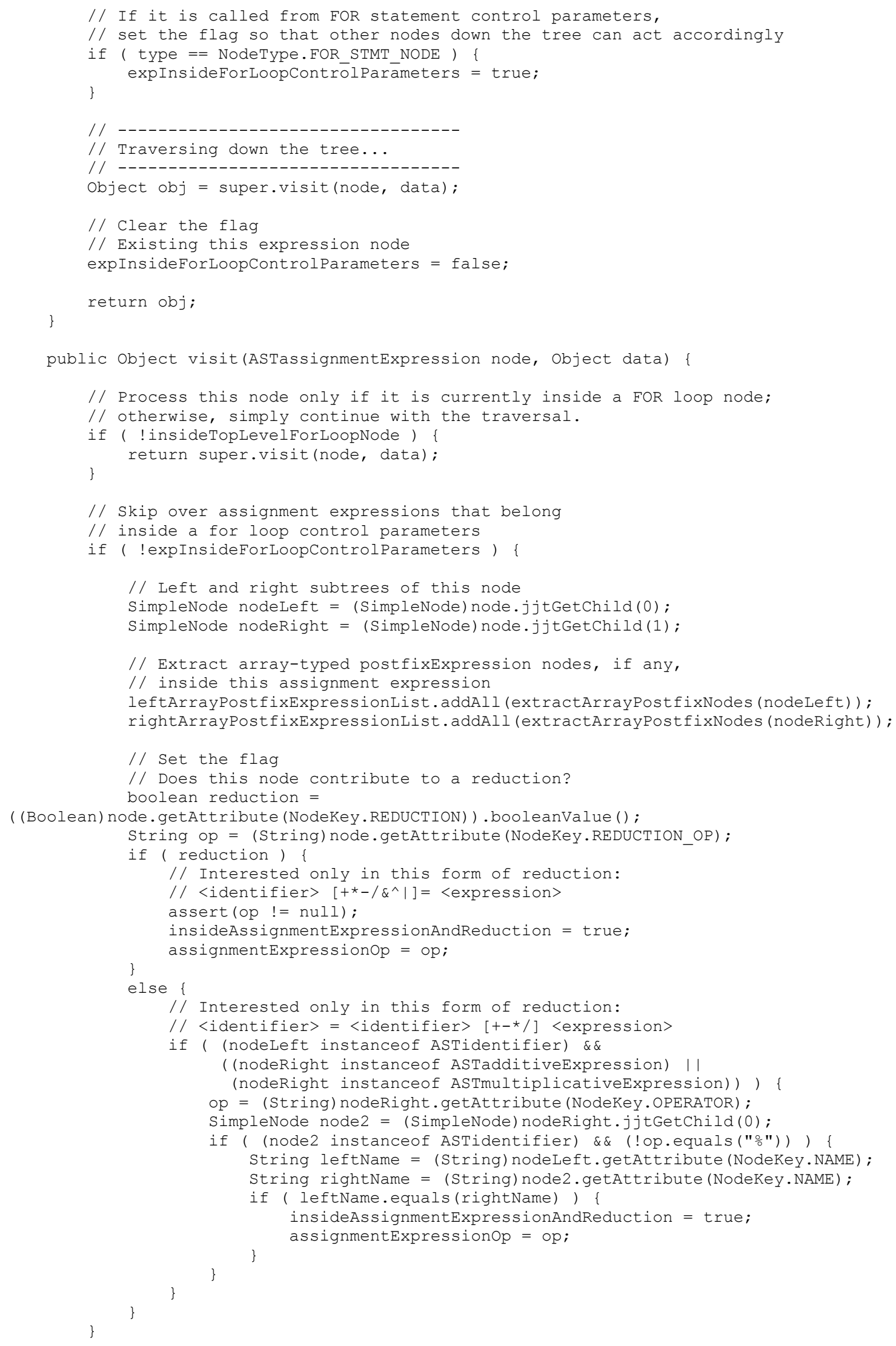




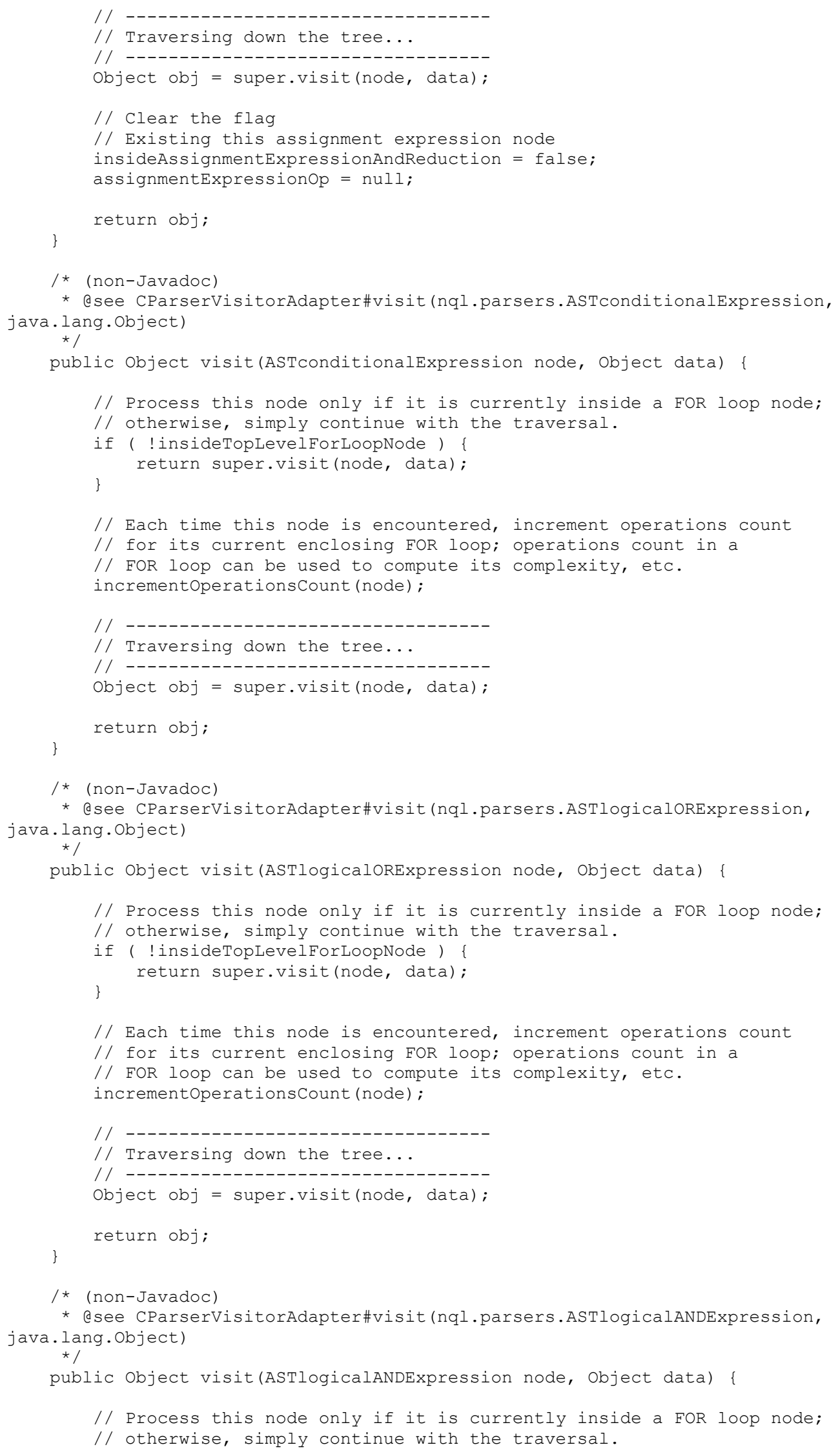




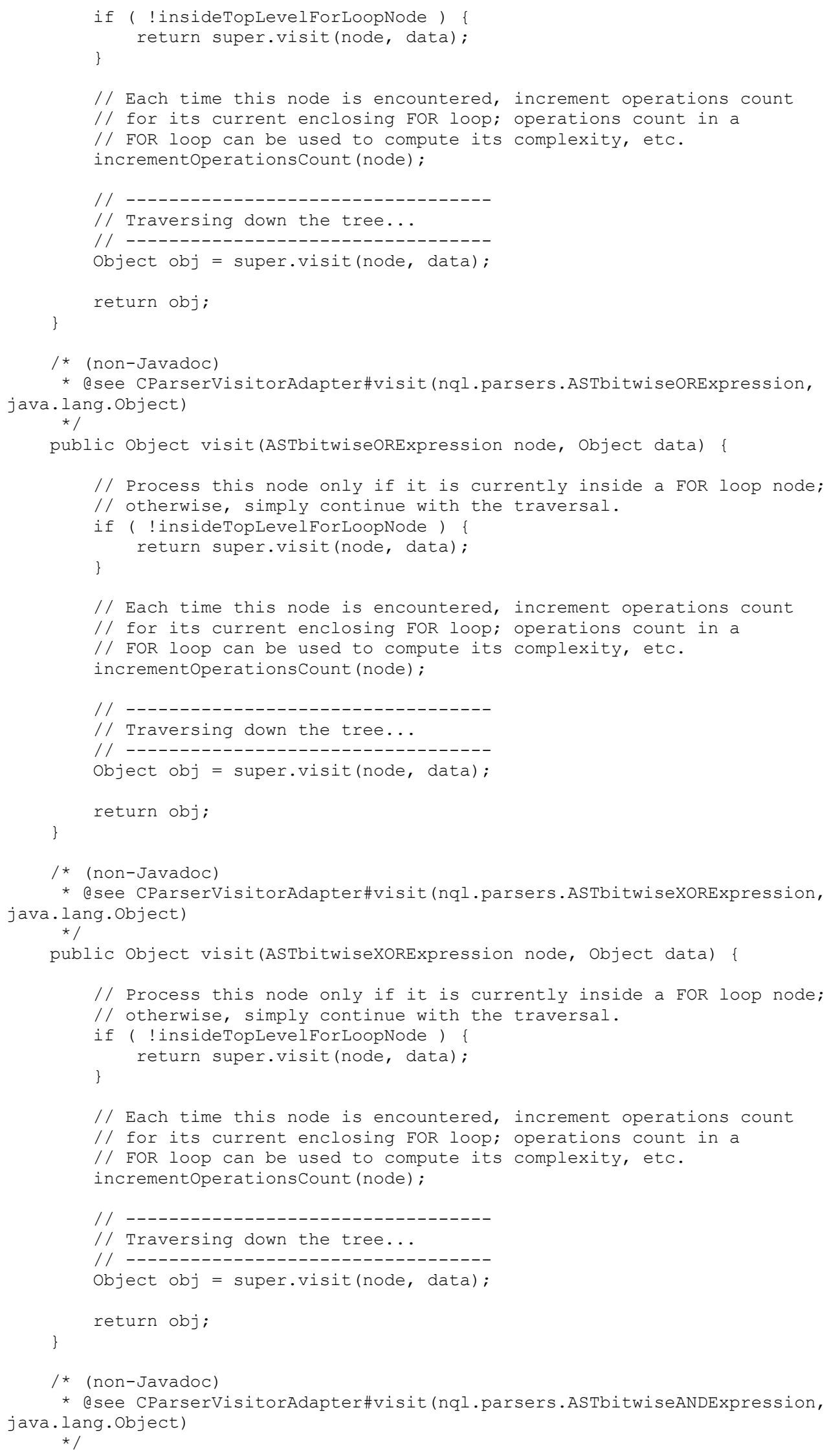




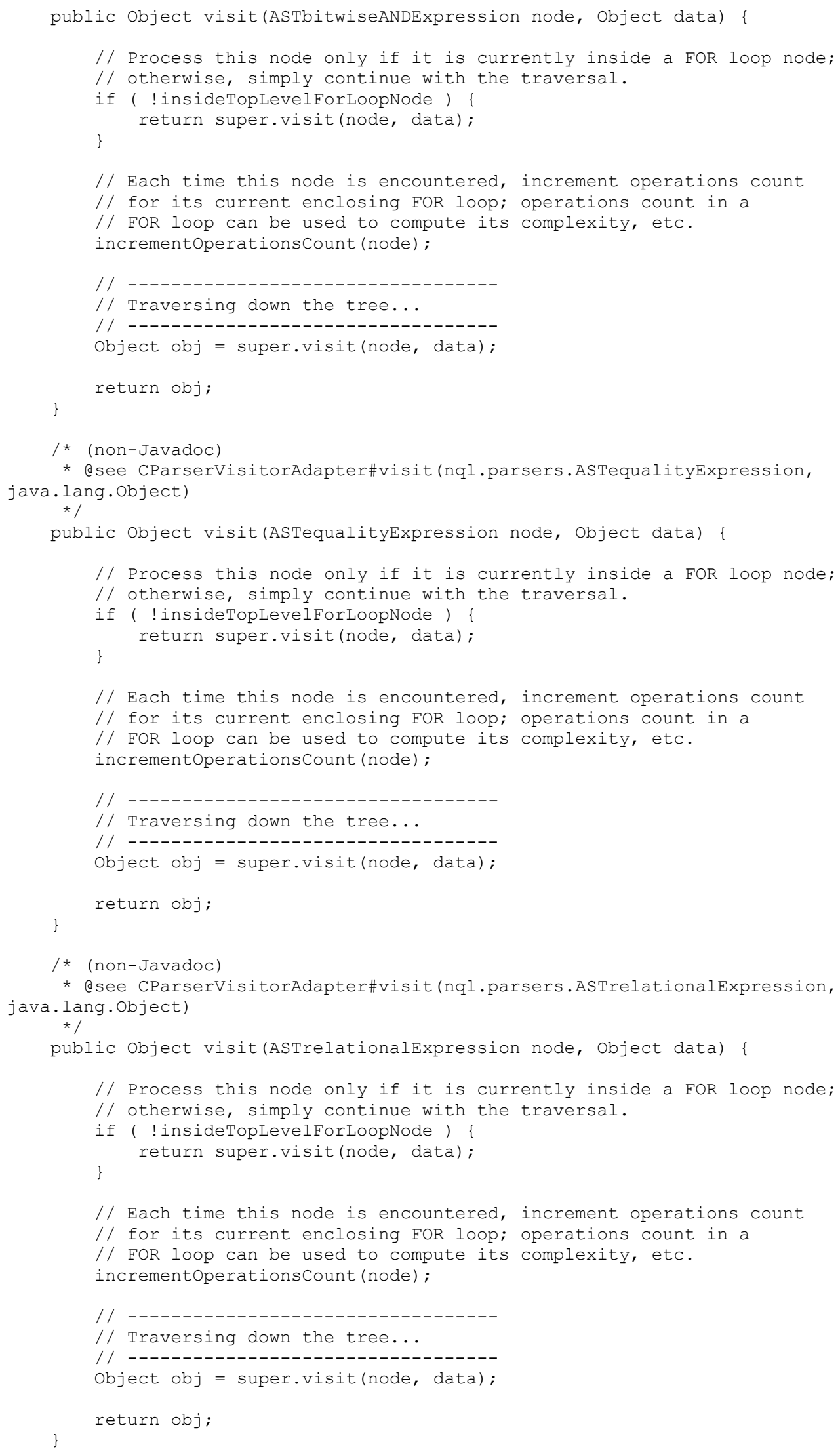




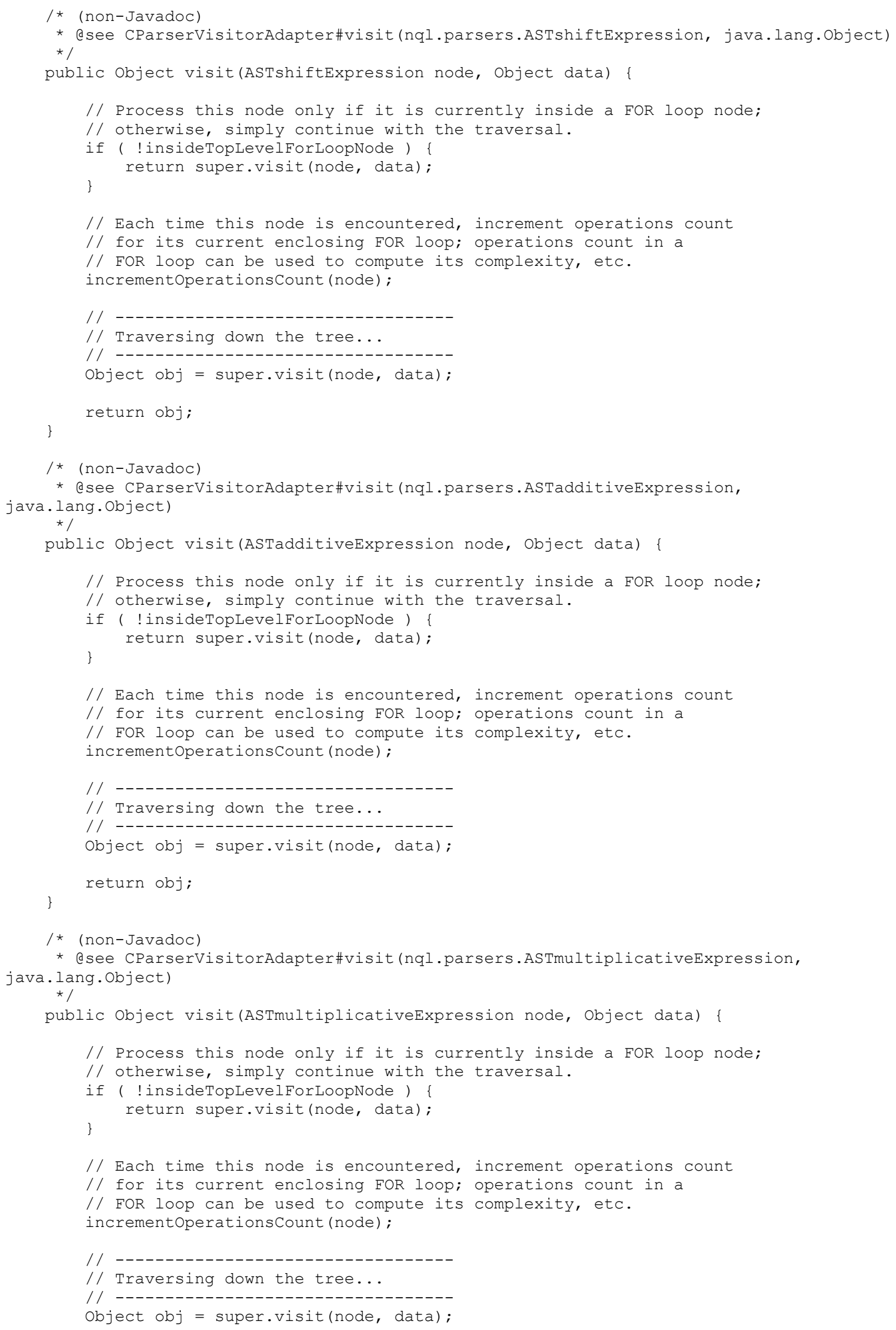




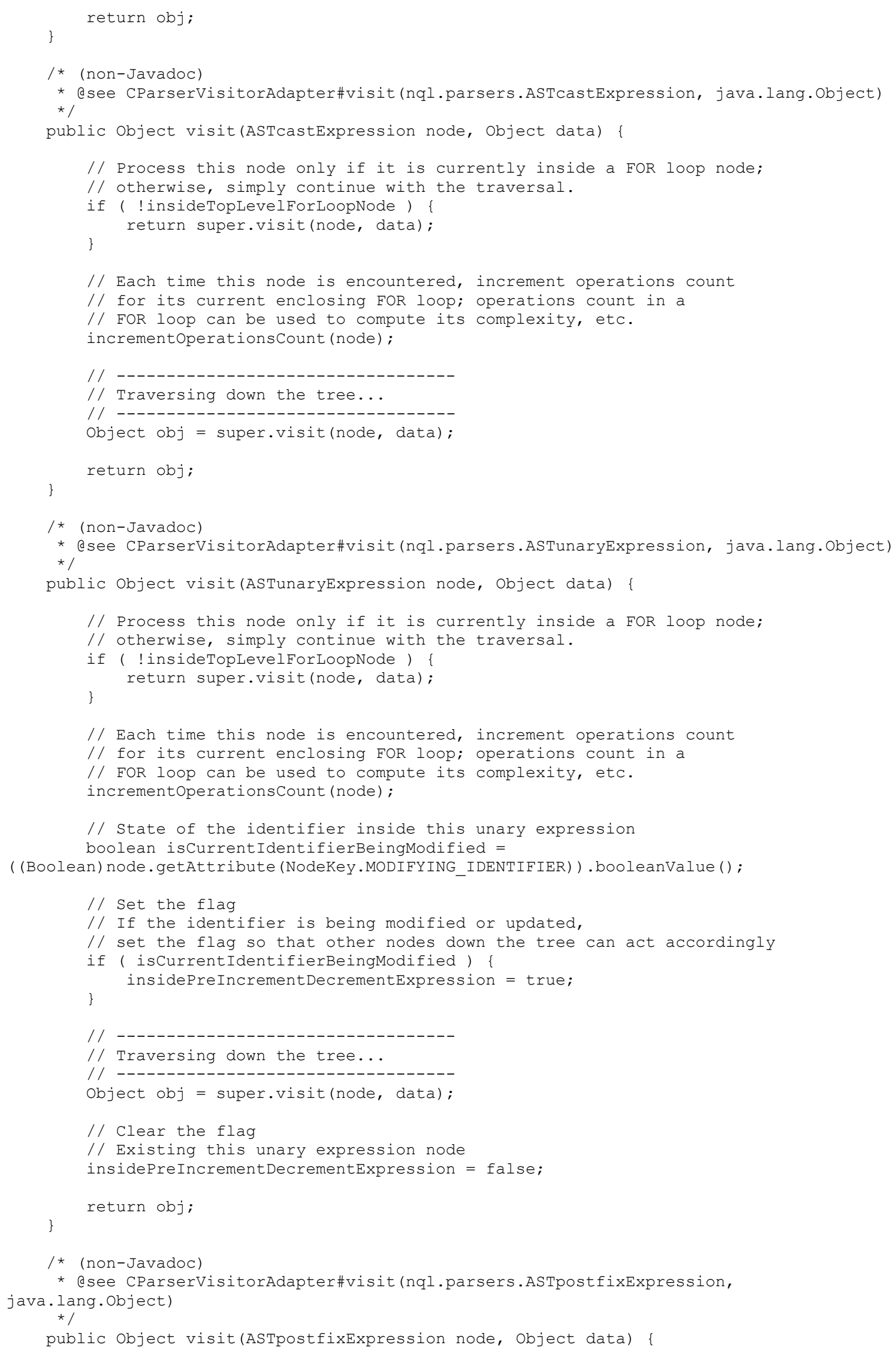


// Process this node only if it is currently inside a FOR loop node;

// otherwise, simply continue with the traversal.

if ( !insideTopLevelForLoopNode ) \{

return super.visit(node, data);

\}

// Each time this node is encountered, increment operations count

// for its current enclosing FOR loop; operations count in a

// FOR loop can be used to compute its complexity, etc.

incrementoperationscount (node);

// State of the identifier inside this postfix expression

boolean isCurrentIdentifierBeingModified =

( (Boolean) node.getAttribute (NodeKey.MODIFYING IDENTIFIER)).booleanValue();

// Set the flags

// If the identifier is being modified or updated,

// set the flag so that other nodes down the tree can act accordingly

if ( iscurrentIdentifierBeingModified ) \{

insidePostIncrementDecrementExpression = true;

// Does this node contribute to a reduction?

if ( expInsideForLoopControlparameters ) \{

boolean reduction $=$

( (Boolean) node.getAttribute (NodeKey.REDUCTION)) .booleanValue (); String op = (String) node.getAttribute (NodeKey.REDUCTION OP) ; if ( reduction ) \{

assert (op != null);

insidePostfixExpressionAndReduction = true;

postfixExpressionop = op;

// Is this node an array postfix? If so, retrieve the array name.

String arrayName = null;

boolean isArrayPostfixed $=$

( (Boolean) node.getAttribute (NodeKey. ARRAY POSTFIXED)) . booleanValue ();

if ( isArraypostfixed ) \{

SimpleNode aNode $=$ (SimpleNode) node.jjtGetChild(0);

if ( aNode instanceof ASTidentifier ) \{

arrayName $=$ (String) aNode.getAttribute (NodeKey.NAME) ;

// ----------------------------------

// Traversing down the tree...

// ---------------------------------

object obj = super.visit (node, data);

// Clear the flags

// Existing this postfix expression

insidePostIncrementDecrementExpression = false;

insidePostfixExpressionAndReduction = false;

postfixexpressionop = null;

// Update the identifier state if this node is an array postfix, i.e.,

// although the identifier (array name) happens to be on the left side

// of an assignment, but array name itself is not updated or modified,

// only its elements, if any.

if ( isArrayPostfixed \&\& (arrayName != null) ) \{

Variable oldVariable = new Variable (arrayName, false);

Variable newVariable = new Variable (arrayName, true);

newVariable.setType (VariableType.ARRAY) ;

SimpleNode pNode = getParentForLoop (node);

assert (pNode != null);

if ( pNode instanceof ASTforstatement ) \{ 
ForLoopNode forLoopNode $=$

(ForLoopNode) pNode.getAttribute (NodeKey.FOR_LOOP_NODE) ; HashSet<Variable> setofVariābles ${ }^{-}$forLoopNode.getSetofVariables (); setofVariables.remove (oldVariable); setofVariables.add (newVariable); \} forLoopNode.setSetofVariables (setofVariables);

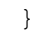




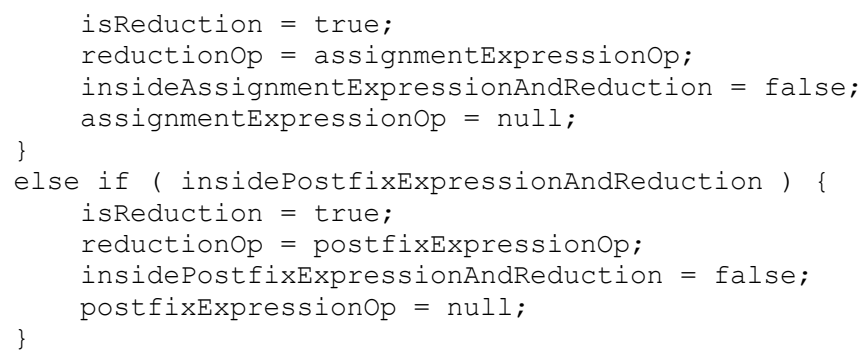




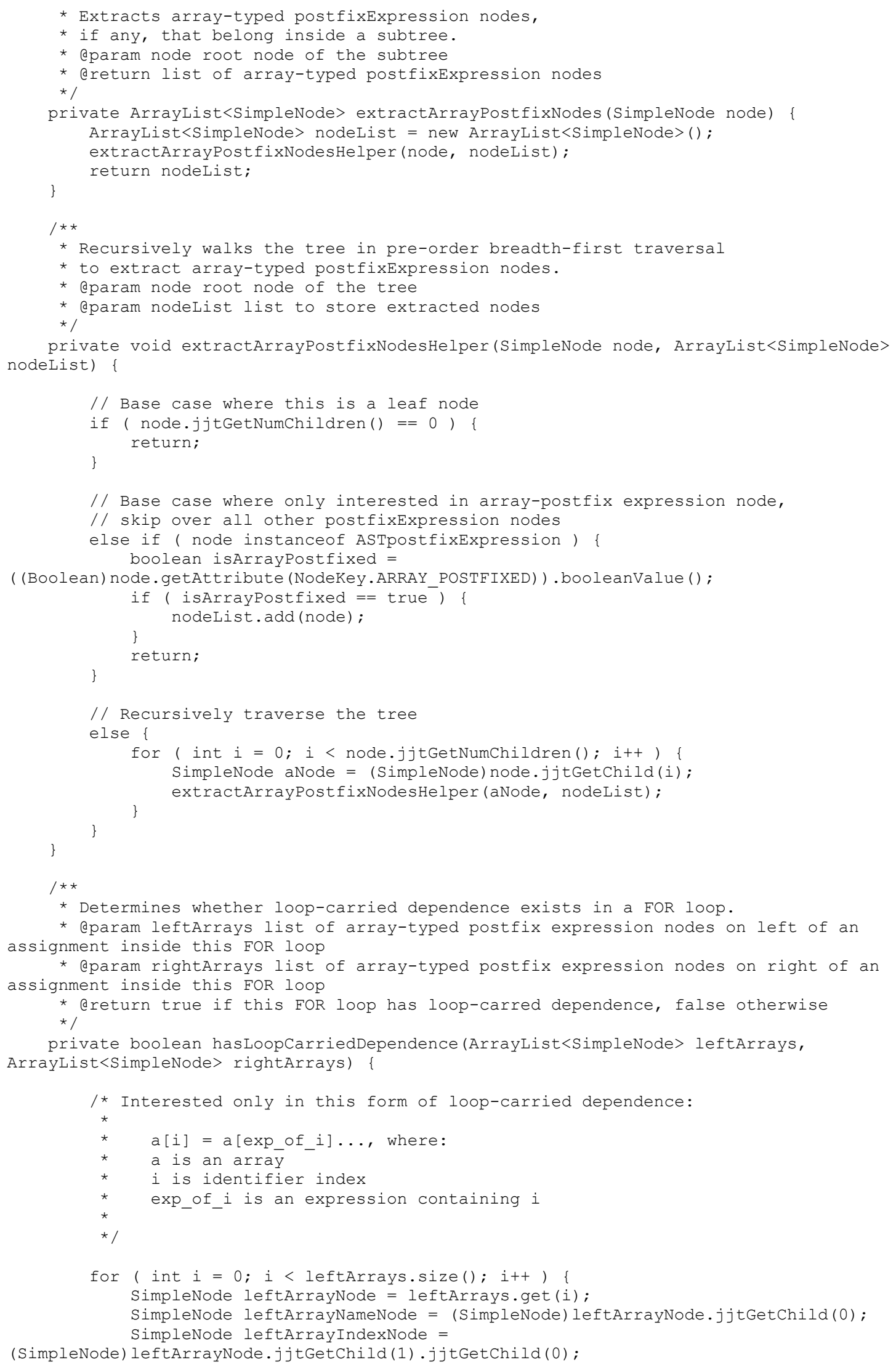


for ( int $j=0 ; j<$ rightArrays.size(); $j++$ ) \{

SimpleNode rightArrayNode = rightArrays.get $(j)$;

SimpleNode rightArrayNameNode =

(SimpleNode) rightArrayNode.jjtGetChild (0);

SimpleNode rightArrayIndexNode =

(SimpleNode) rightArrayNode.jjtGetChild(1) ·jjtGetChild(0);

String leftArrayName =

(String) leftArrayNameNode.getAttribute (NodeKey.NAME) ; String rightArrayName =

(String) rightArrayNameNode.getAttribute (NodeKey.NAME) ;

// Same array name?

if (leftArrayName.equals (rightArrayName) ) \{

// Left array index should be an identifier and the identifier

// should appear inside the rightArrayIndexNode

if ( (leftArrayIndexNode instanceof ASTidentifier) \&\&

(doesExpressionContainIdentifier(rightArrayIndexNode,

leftArrayIndexNode) $==$ true) ) \{

// Different index distance?

if (

! (leftArrayIndexNode.getClass ().equals (rightArrayIndexNode.getClass ())) ) \{

loop

// Same C array but different index distance;

// therefore, a loop-carried dependence exist in this FOR

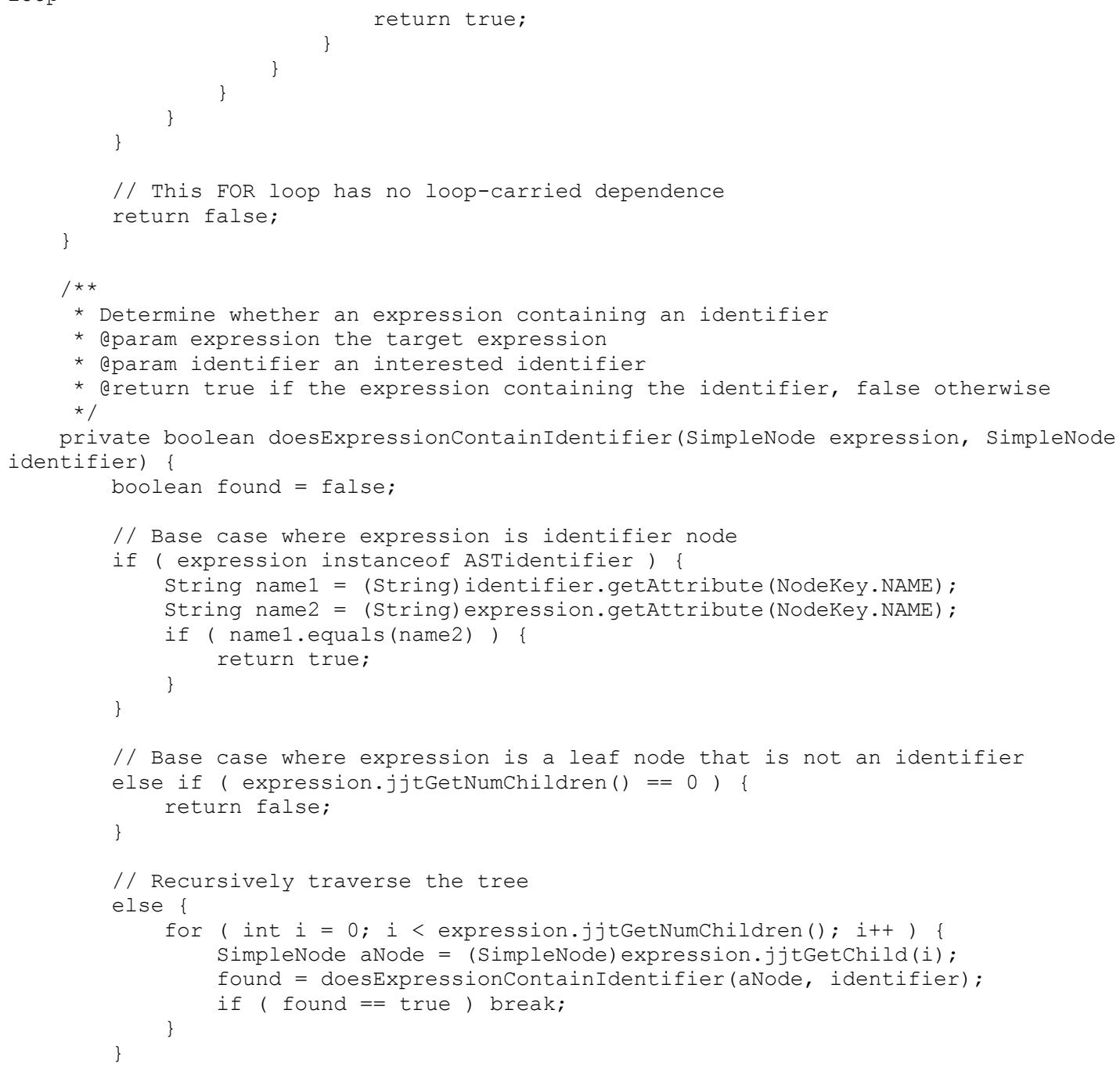




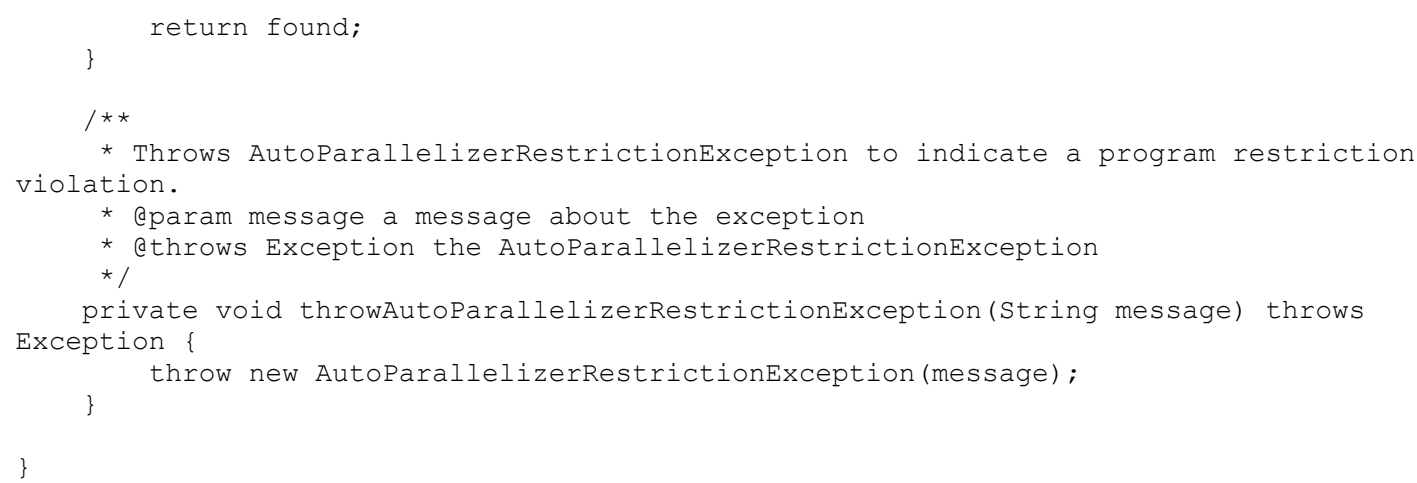




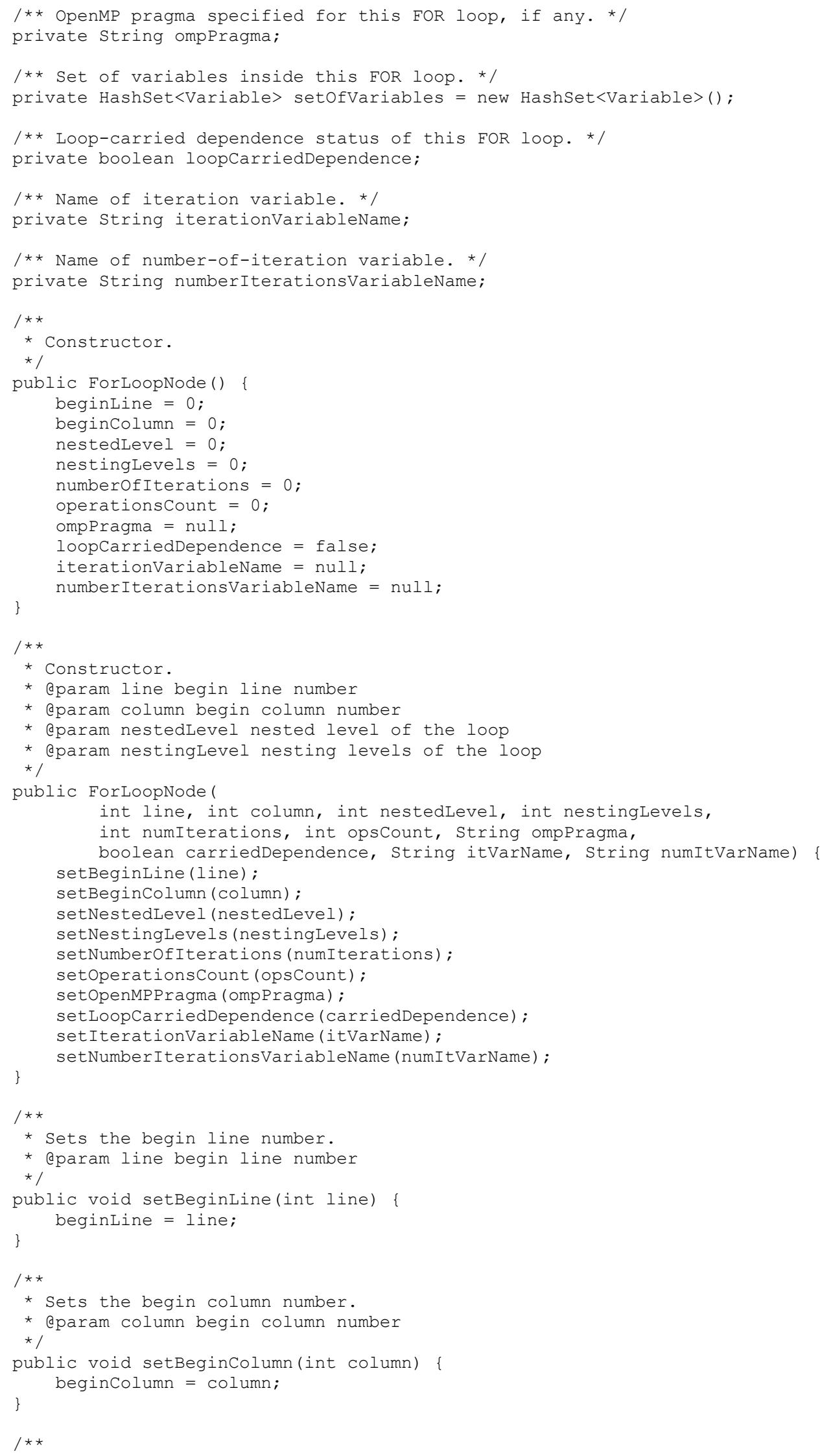




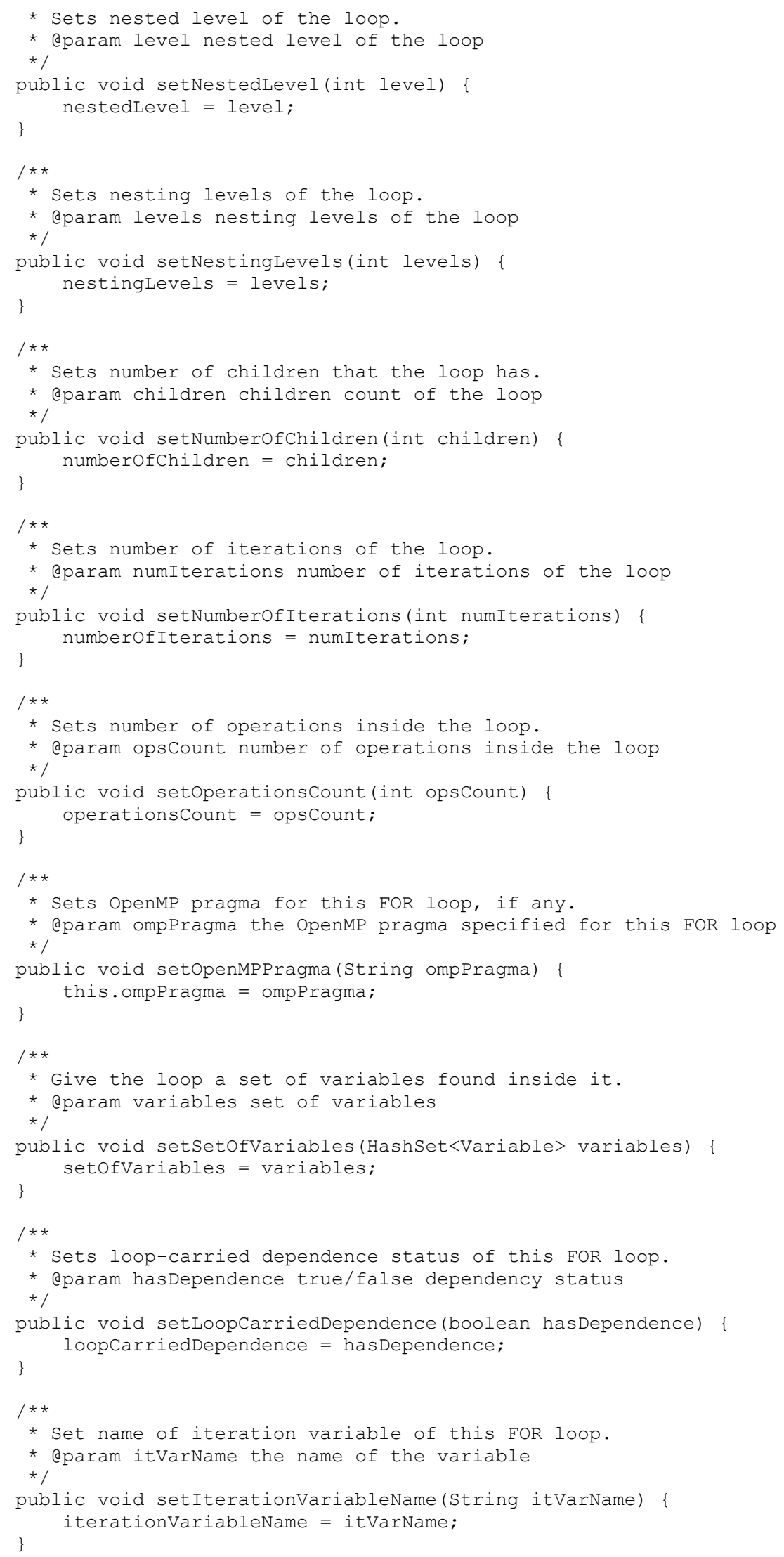




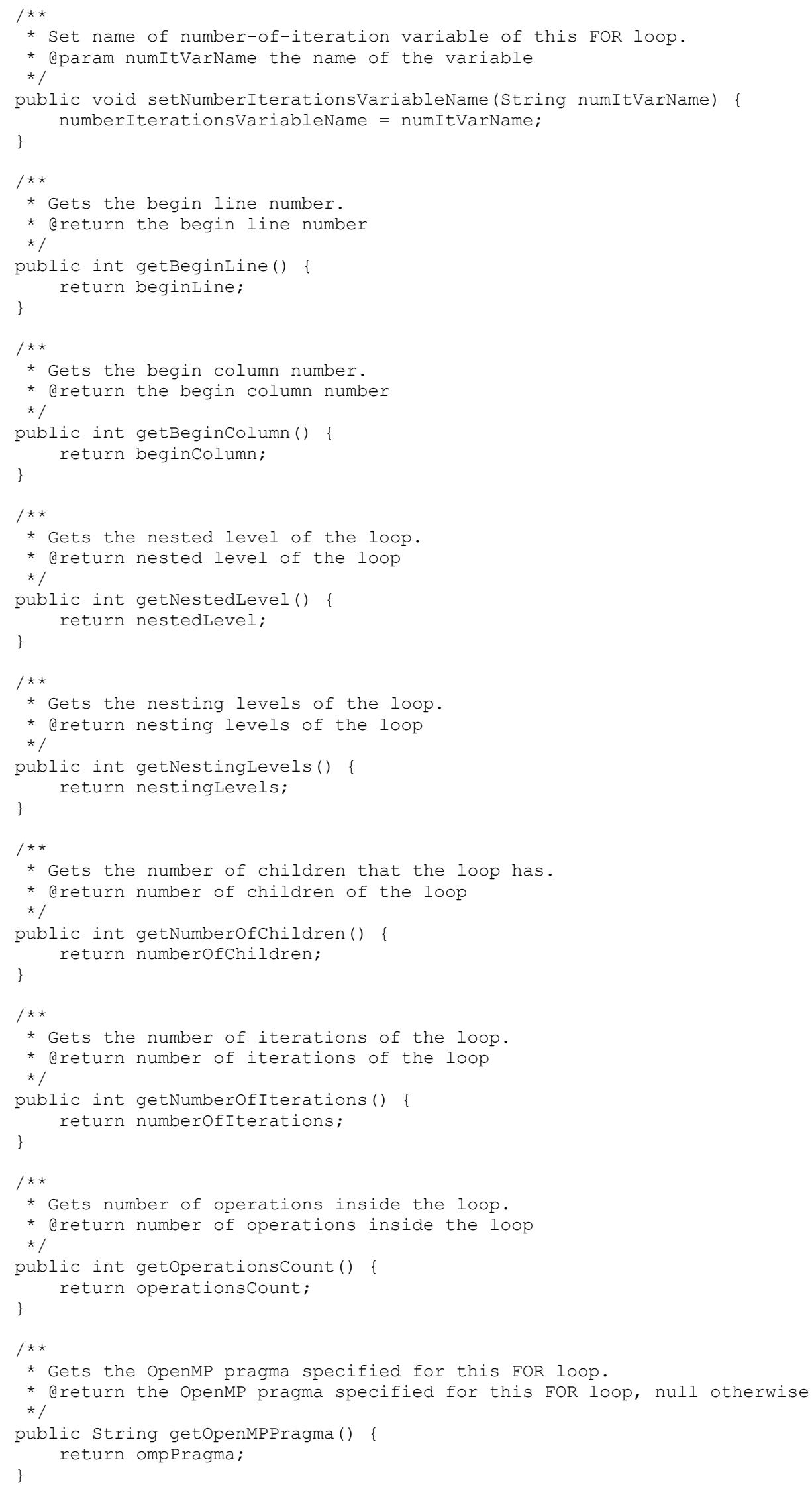




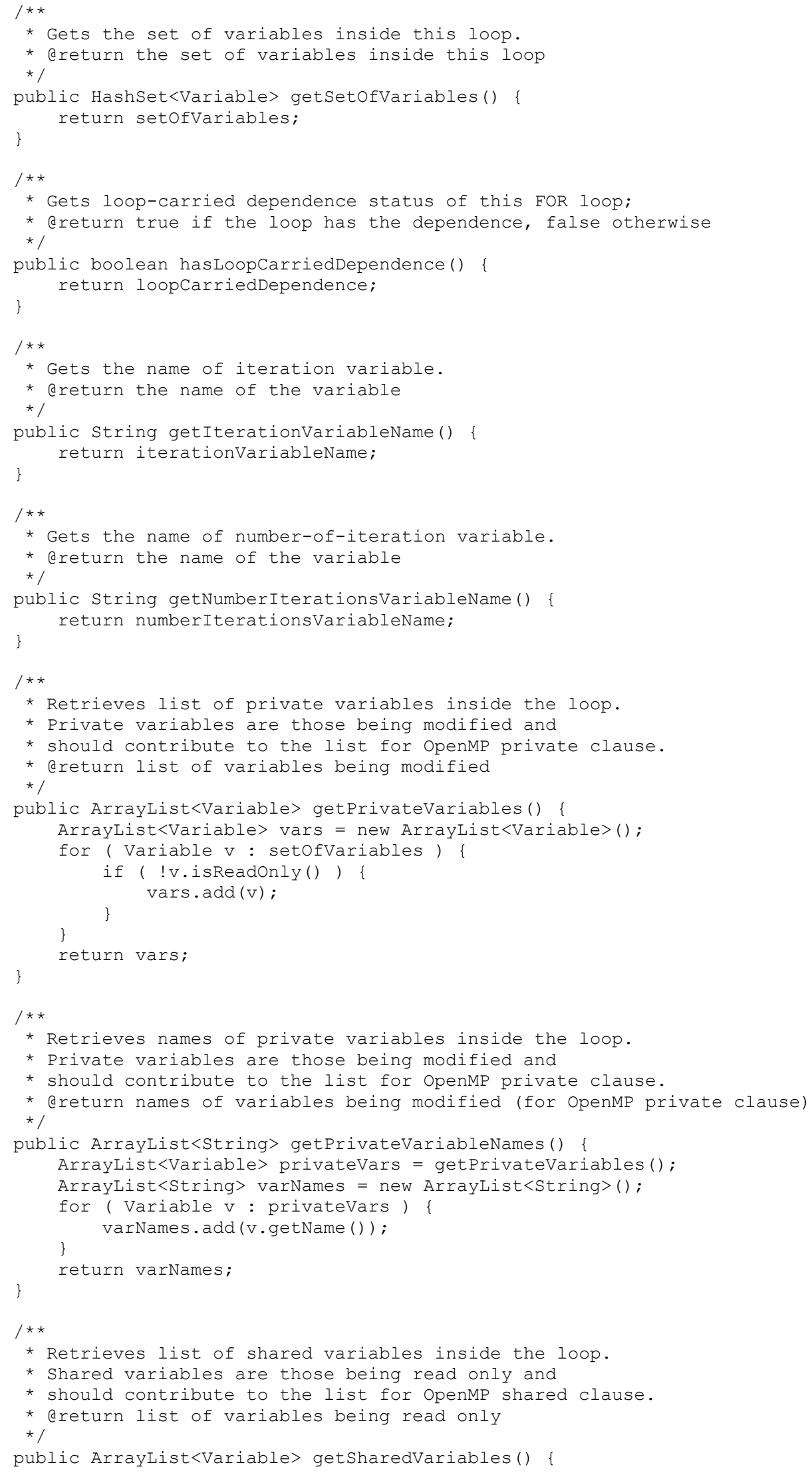




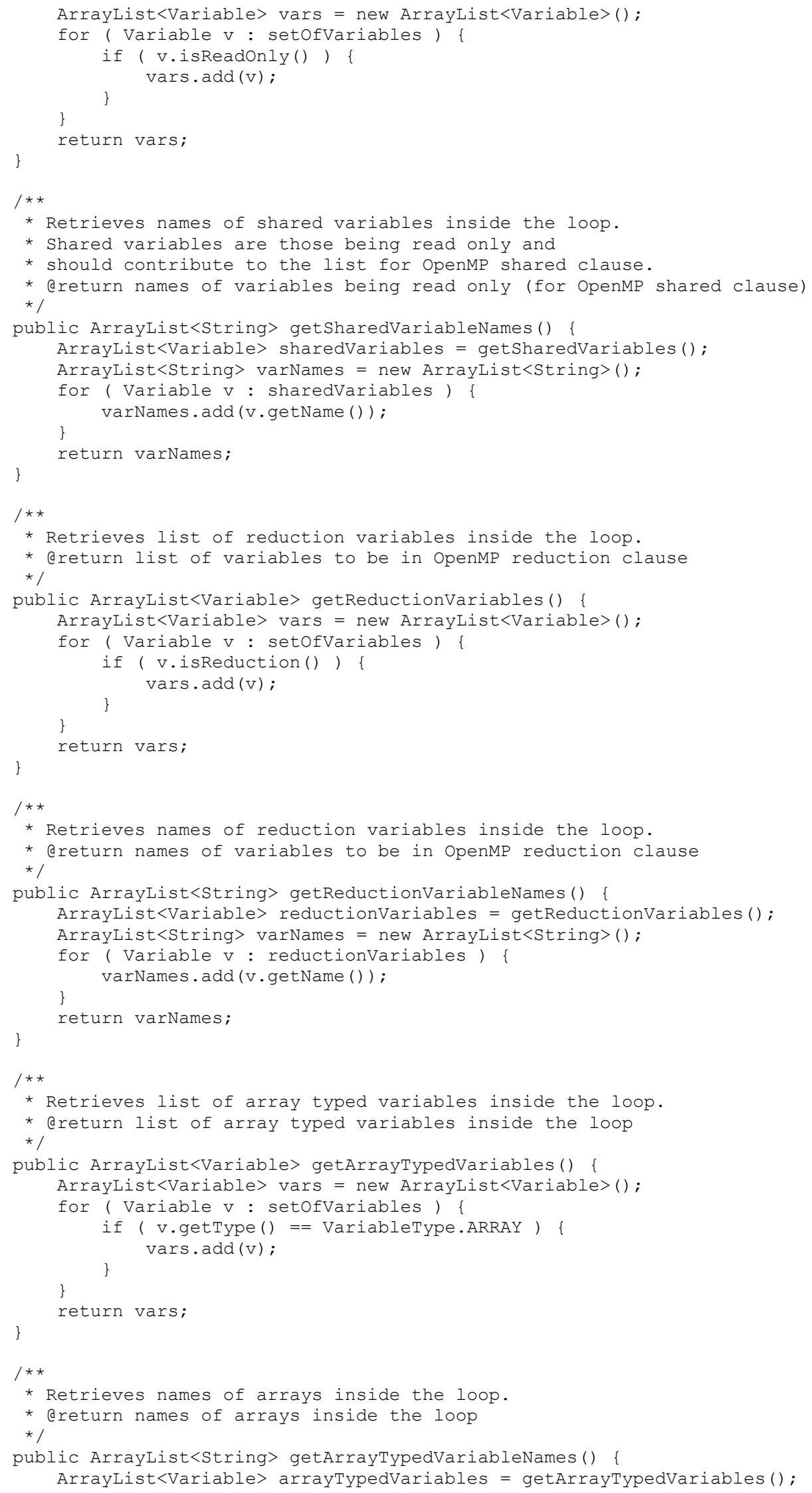




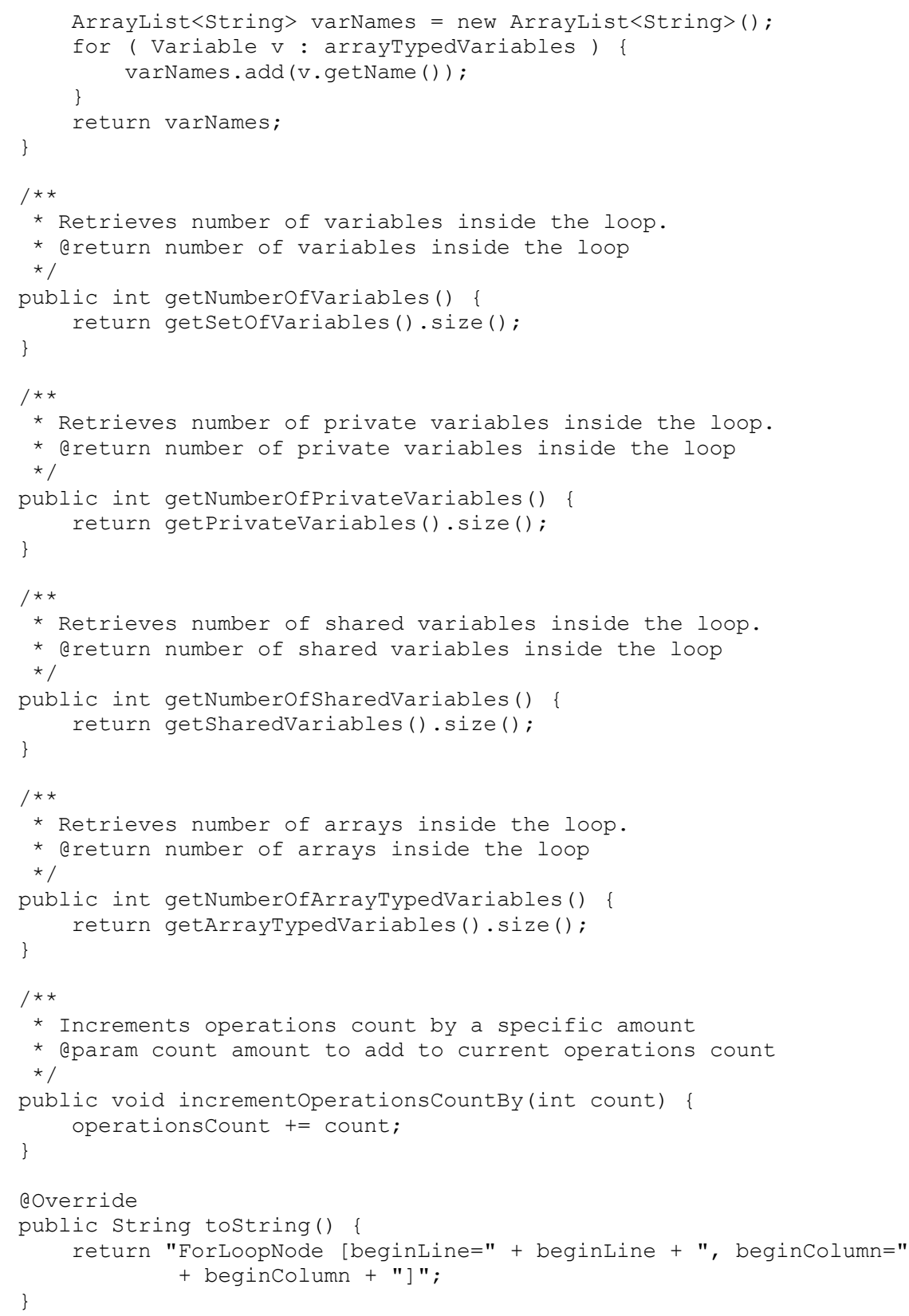




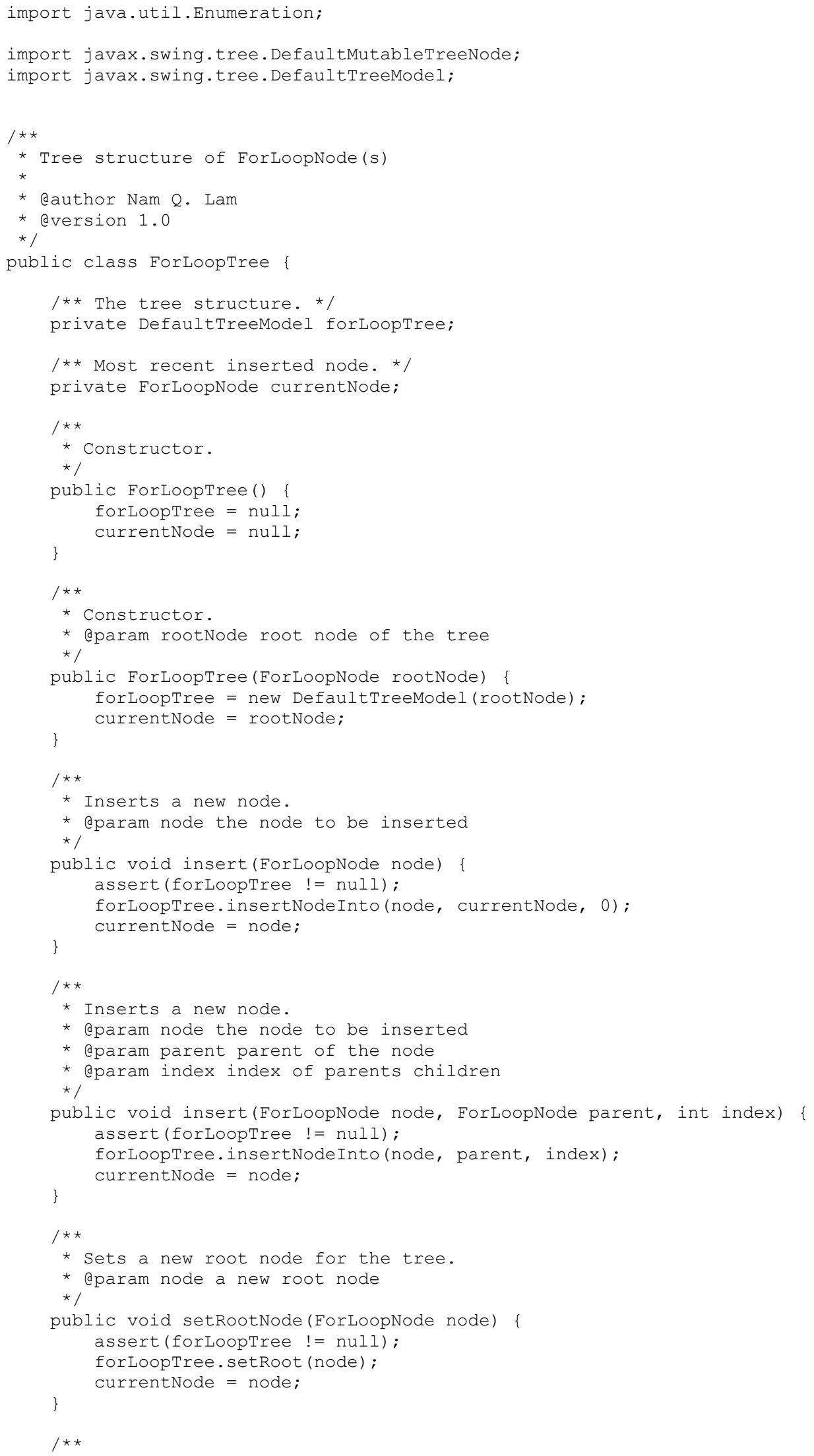




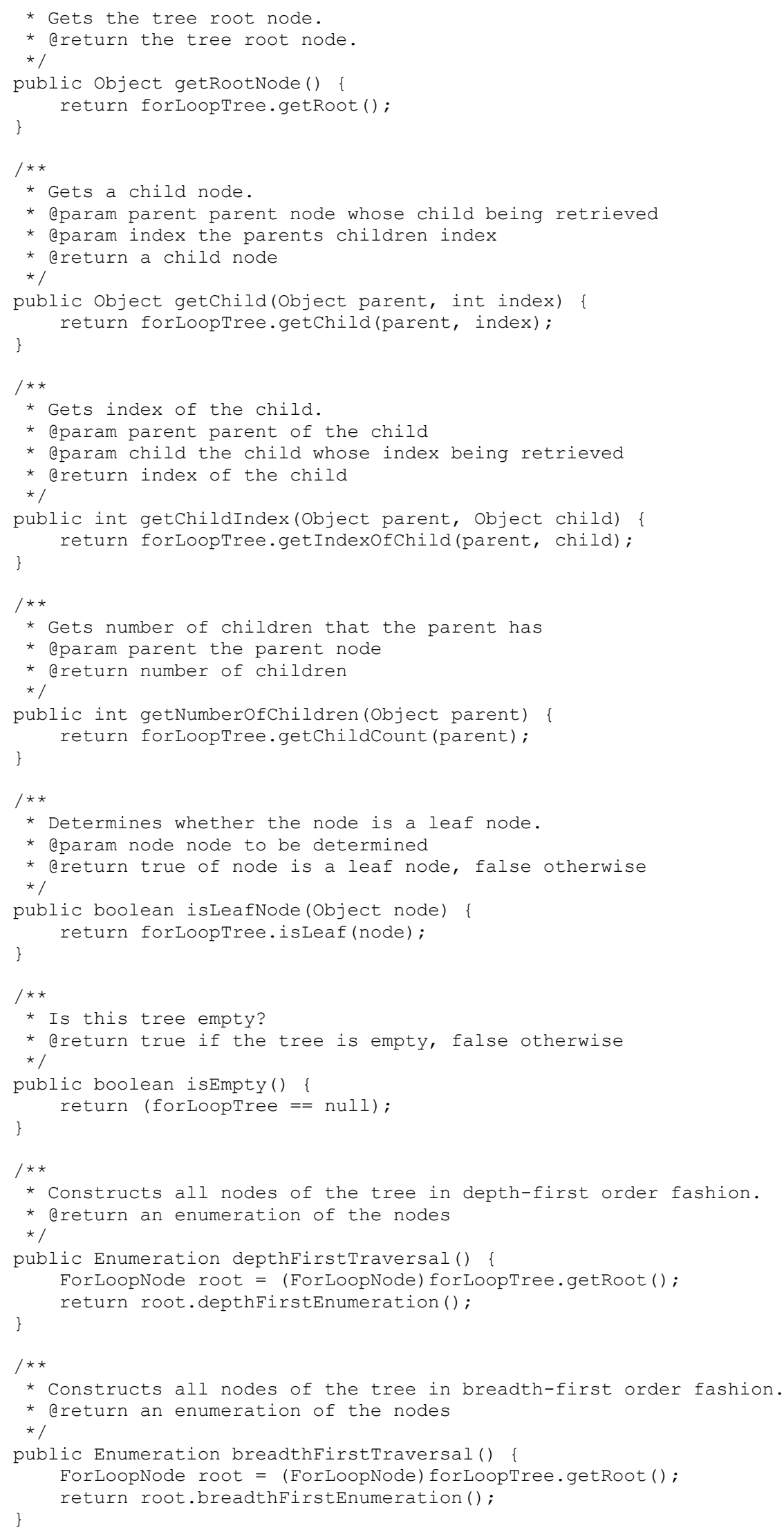




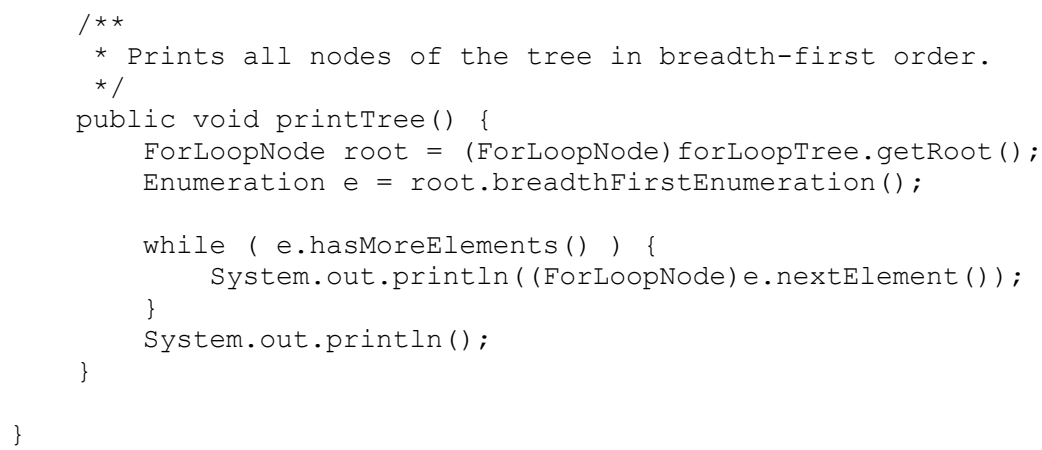




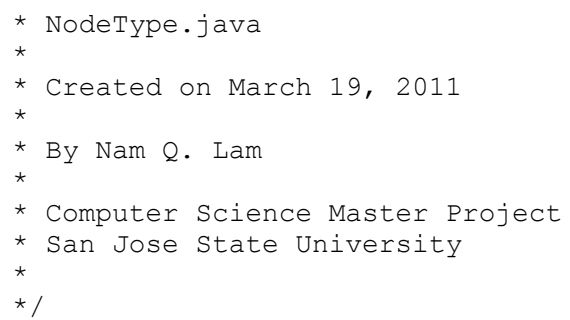




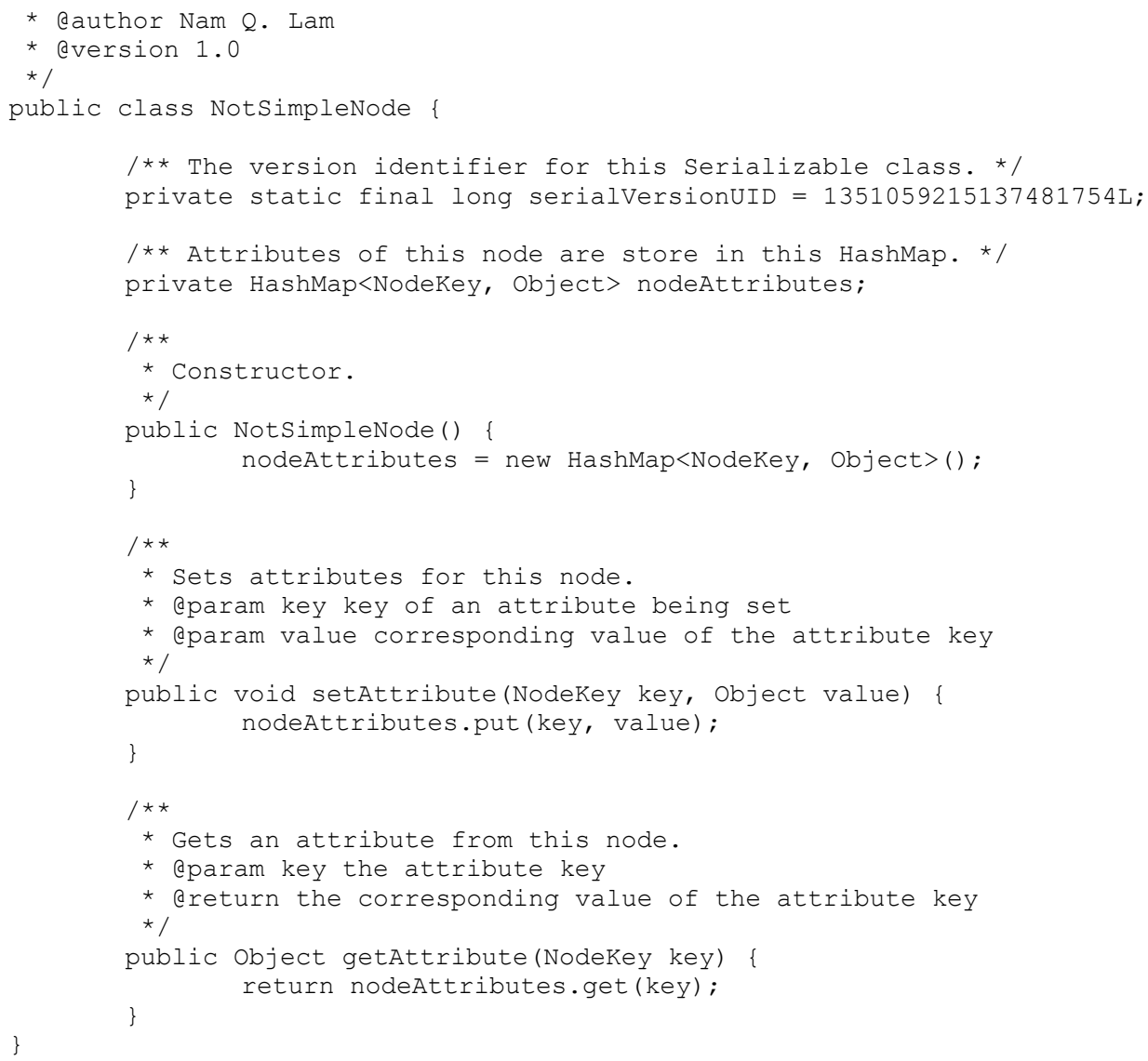

/*

* SymbolTable.java

* Created on September 1, 2011

* By Nam Q. Lam

* Computer Science Master Project

* San Jose State University

*

package nql.cd.intermediate;

import java.util.*;

$1 * \star$

* Symbol table used to store symbol table entries

* at a particular nesting level or scope.

* eauthor Nam Q. Lam

* Qversion 1.0

* /

public class symboltable \{

/** Nesting level of this symbol table. */

private int nestingLevel;

/** Data structure for symbol table entries. */ 


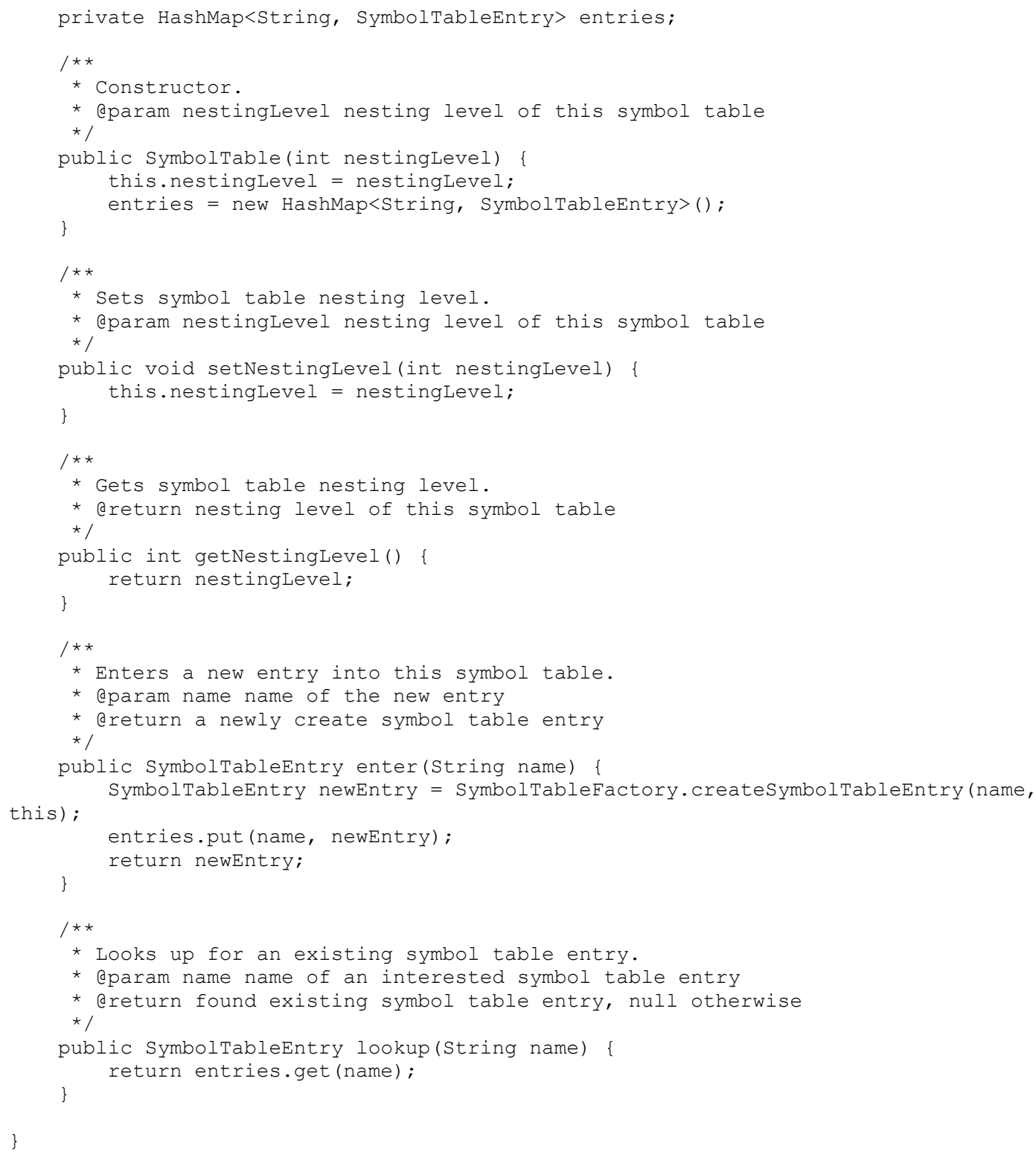




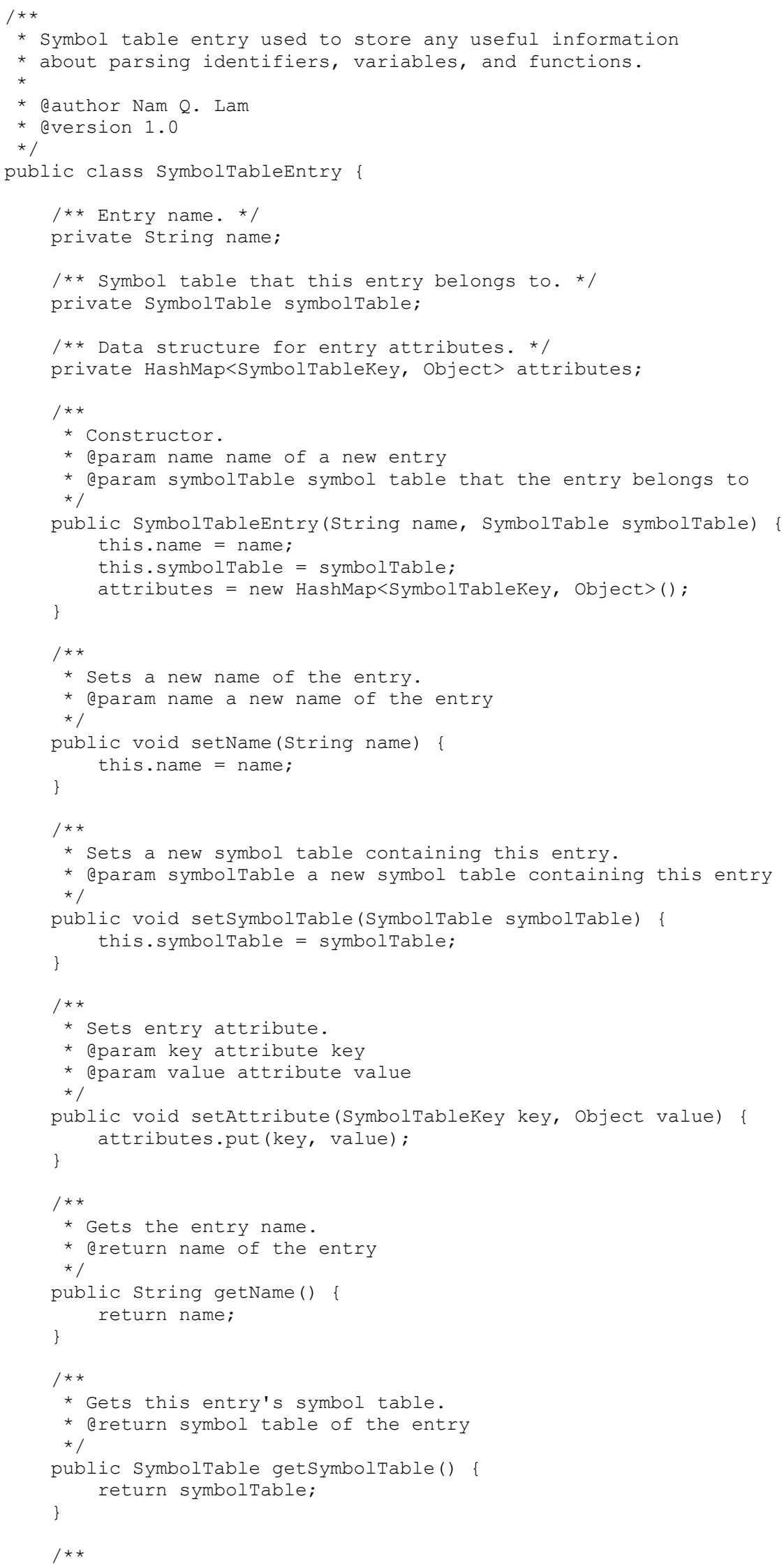




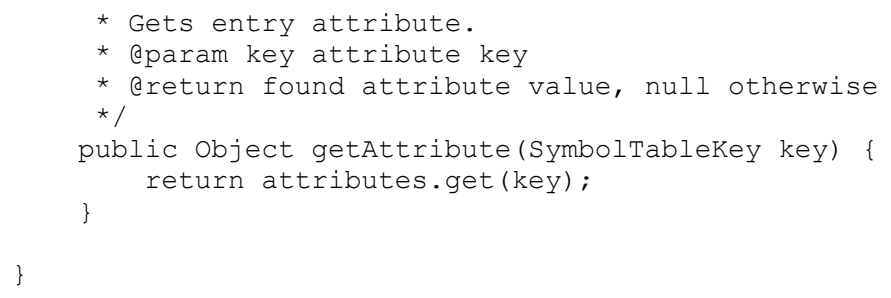


* Created on September 1, 2011

* By Nam Q. Lam

* Computer Science Master Project

* San Jose State University

* /

package nql.cd.intermediate;

$/ * \star$

* Attributes of a symbol table entry.

* Qauthor Nam Q. Lam

* Qversion 1.0

* $/$

public enum symbolTablekey \{

TYPE,

VALUE,

\}

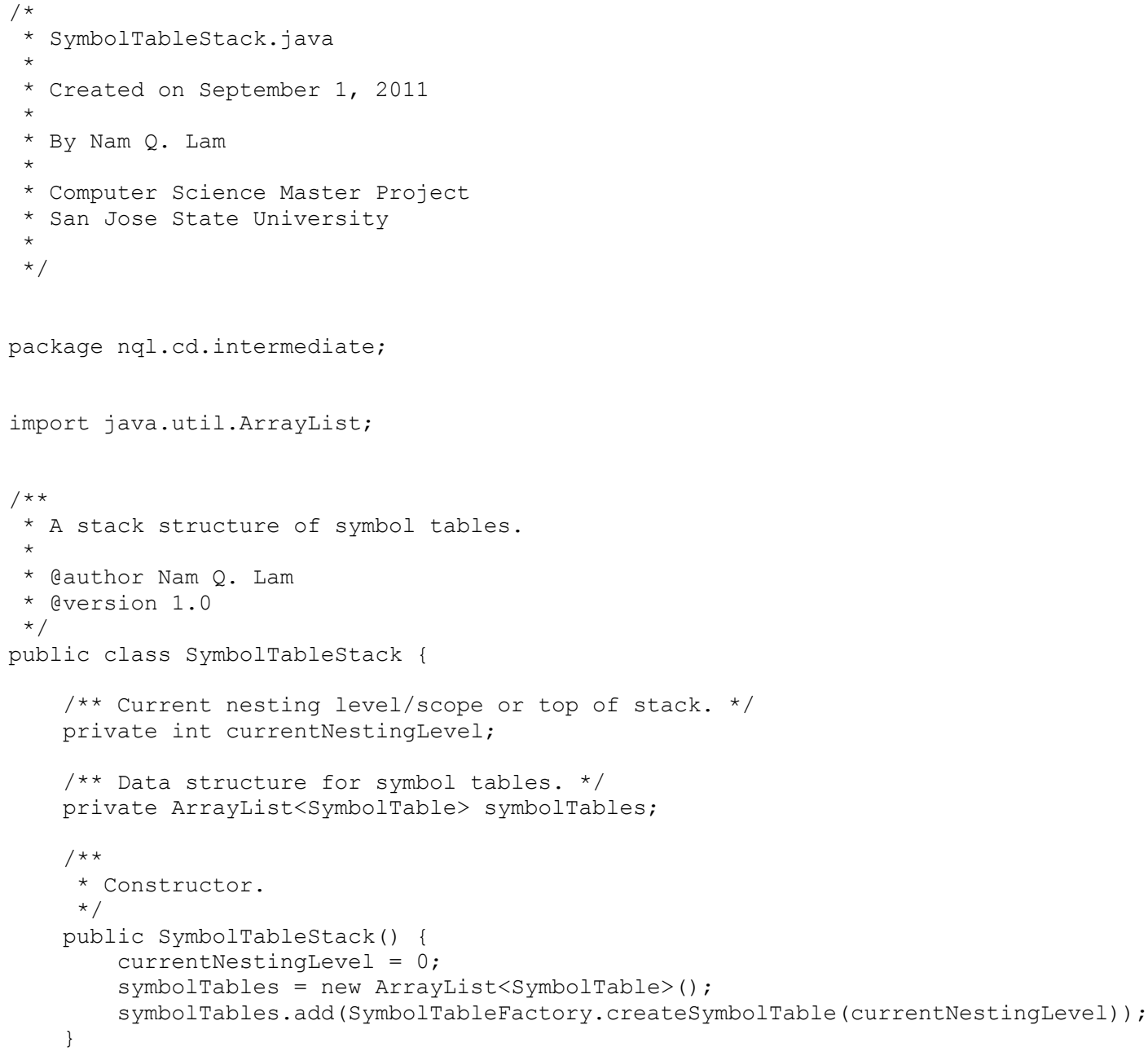




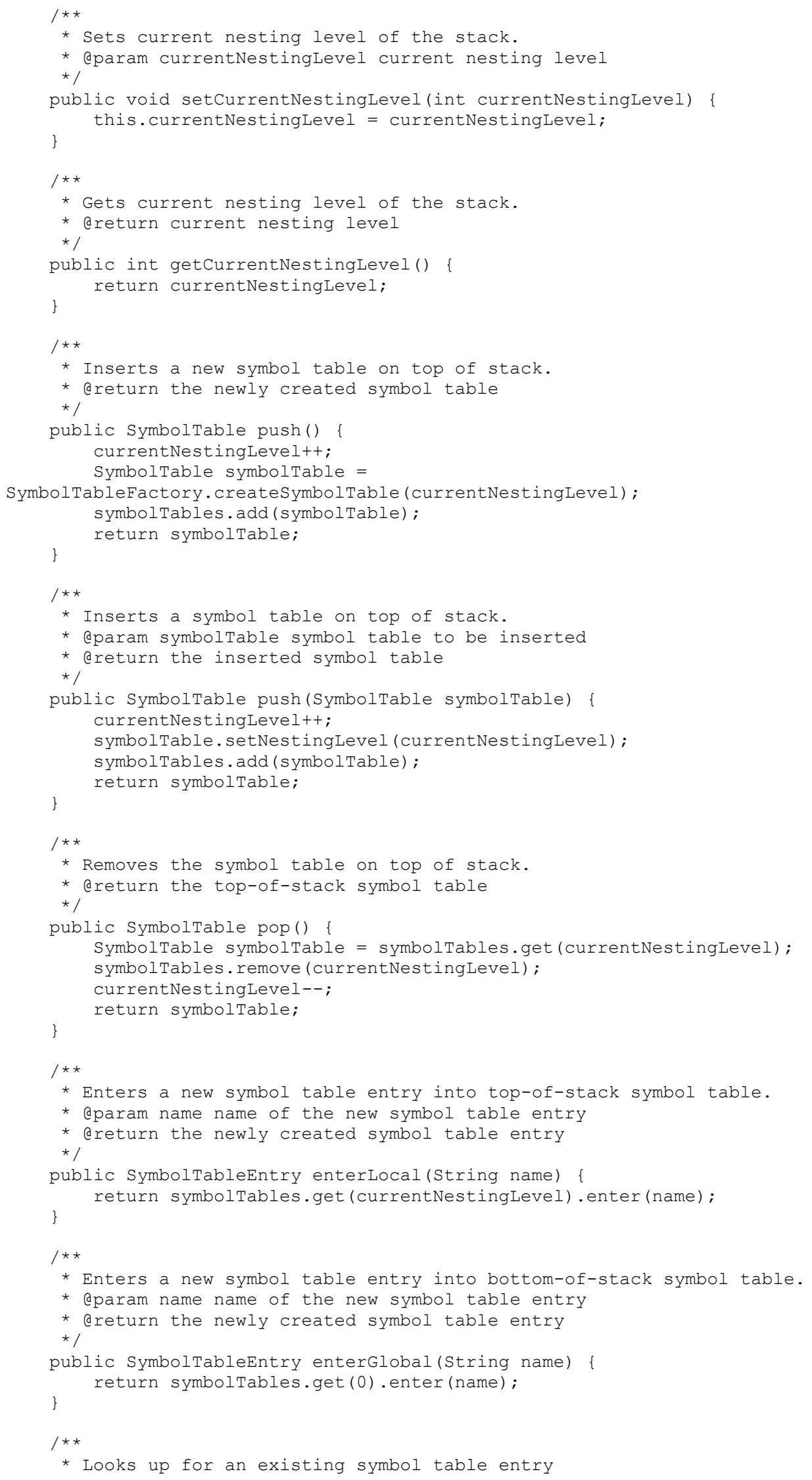




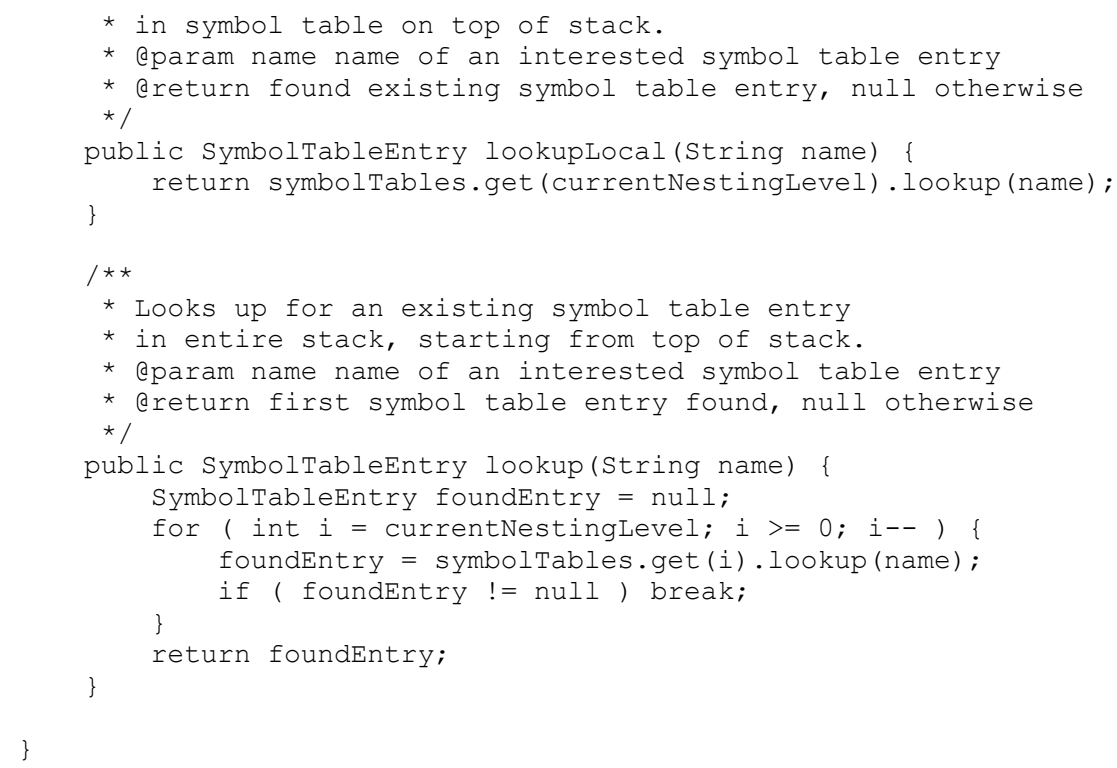




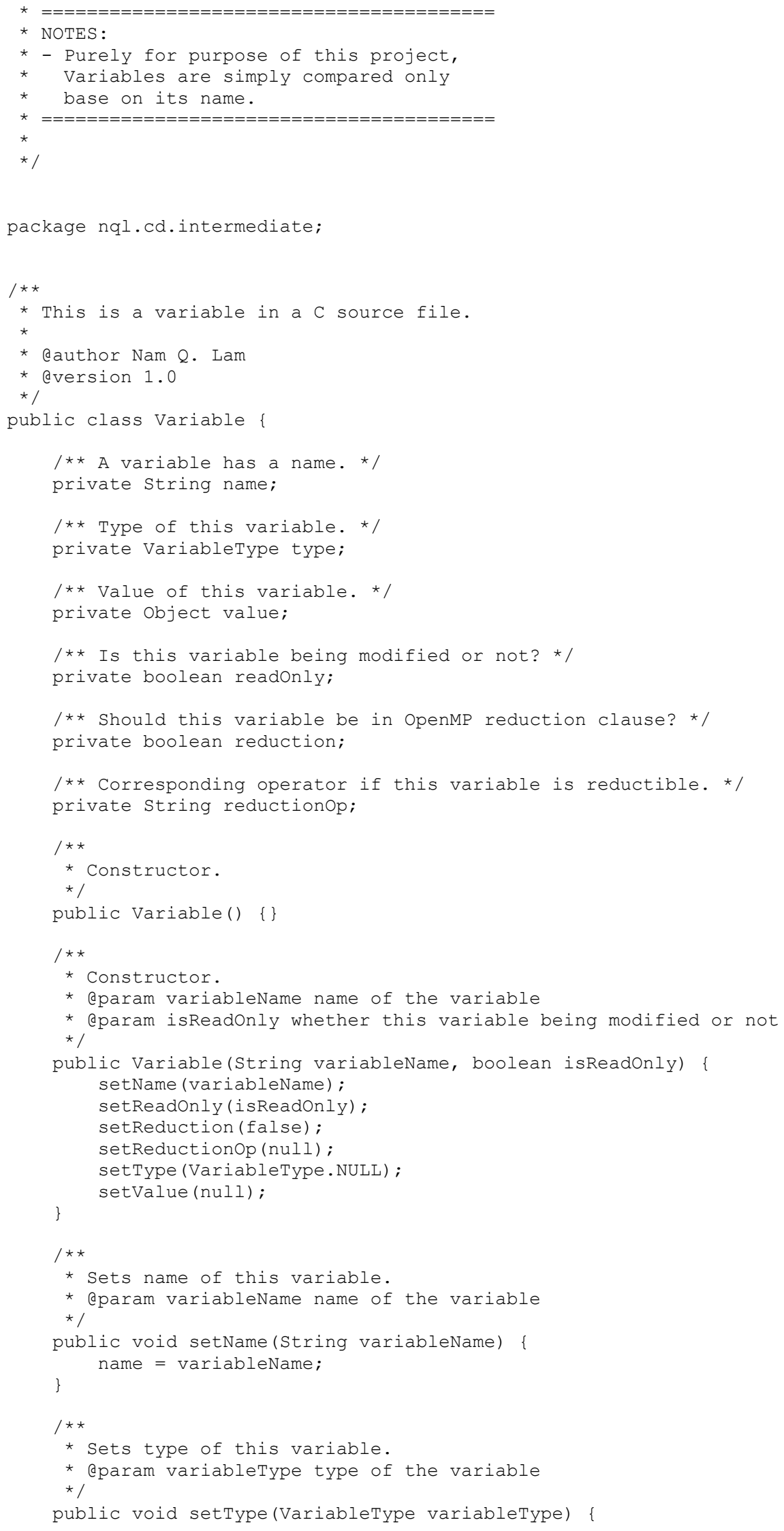




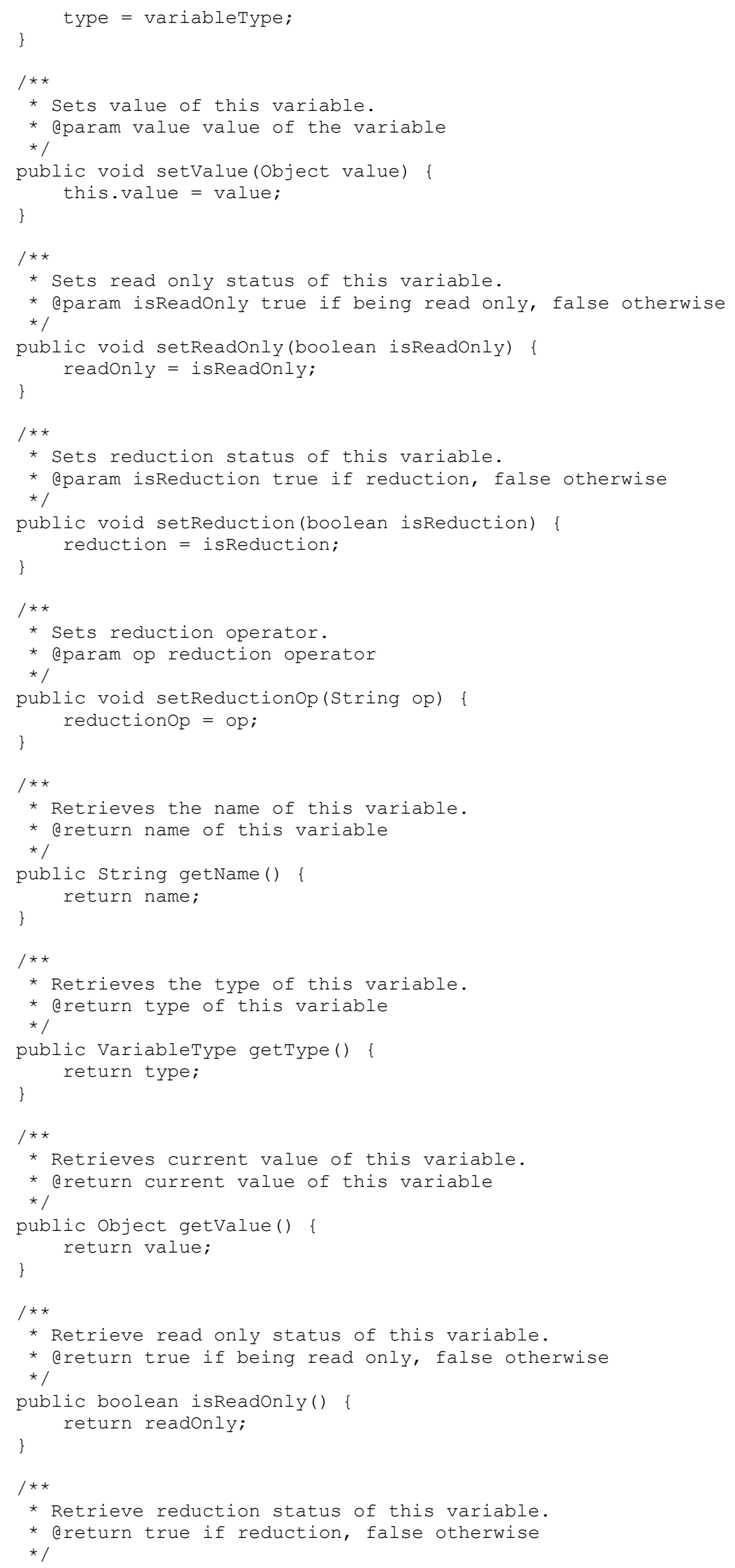




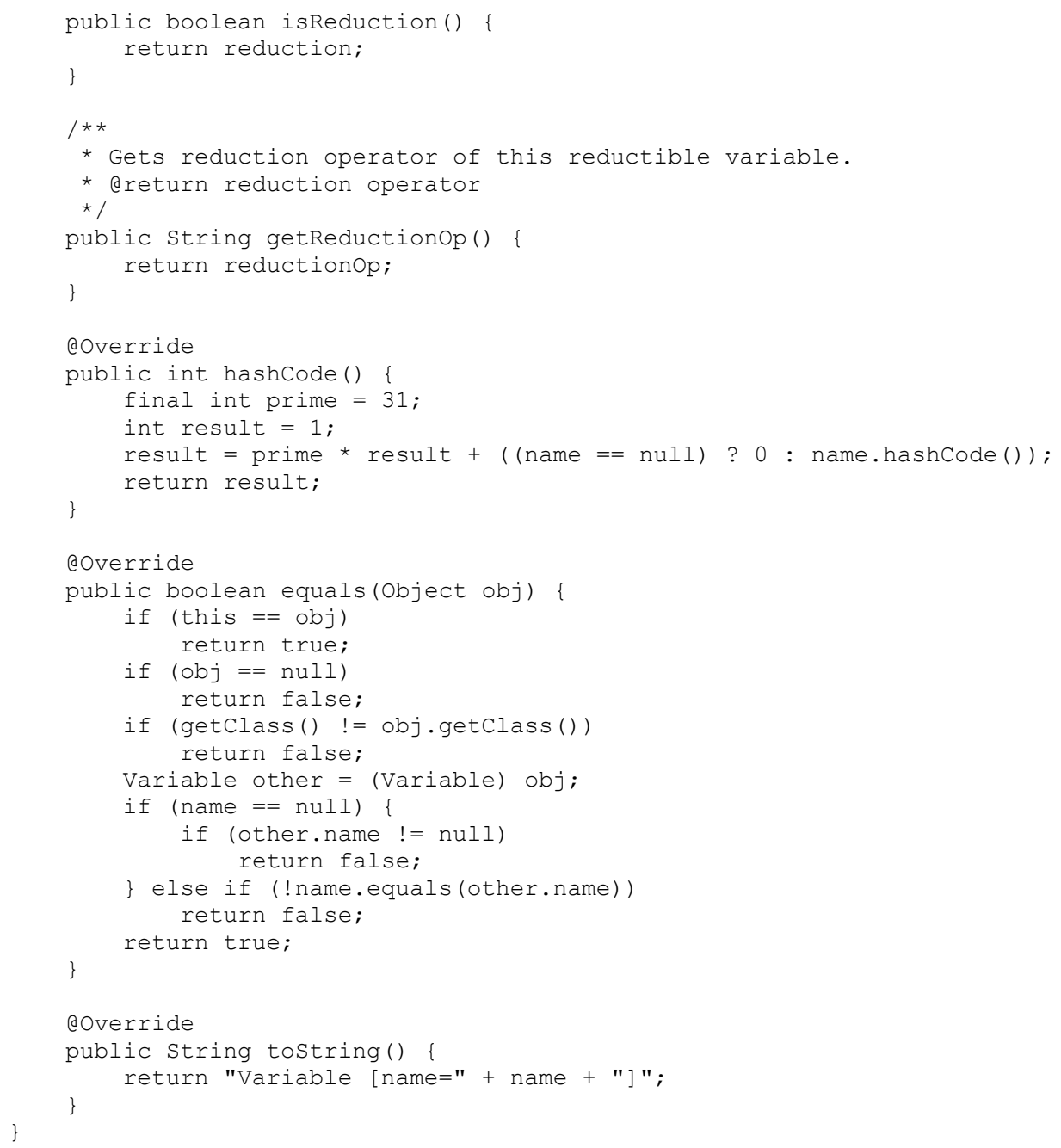

$/ *$

* VariableType.java

* Created on March 19, 2011

* By Nam Q. Lam

* Computer Science Master Project

* San Jose State University

* /

package nql.cd.intermediate;

$1 * \star$

* Type of a Variable (identifier node in the AST).

$\star$

* Qauthor Nam Q. Lam

* Qversion 1.0

* /

public enum VariableType \{

ARRAY, 
INTEGER，

REAL，

STRING，

CHARACTER，

NULL,

\}

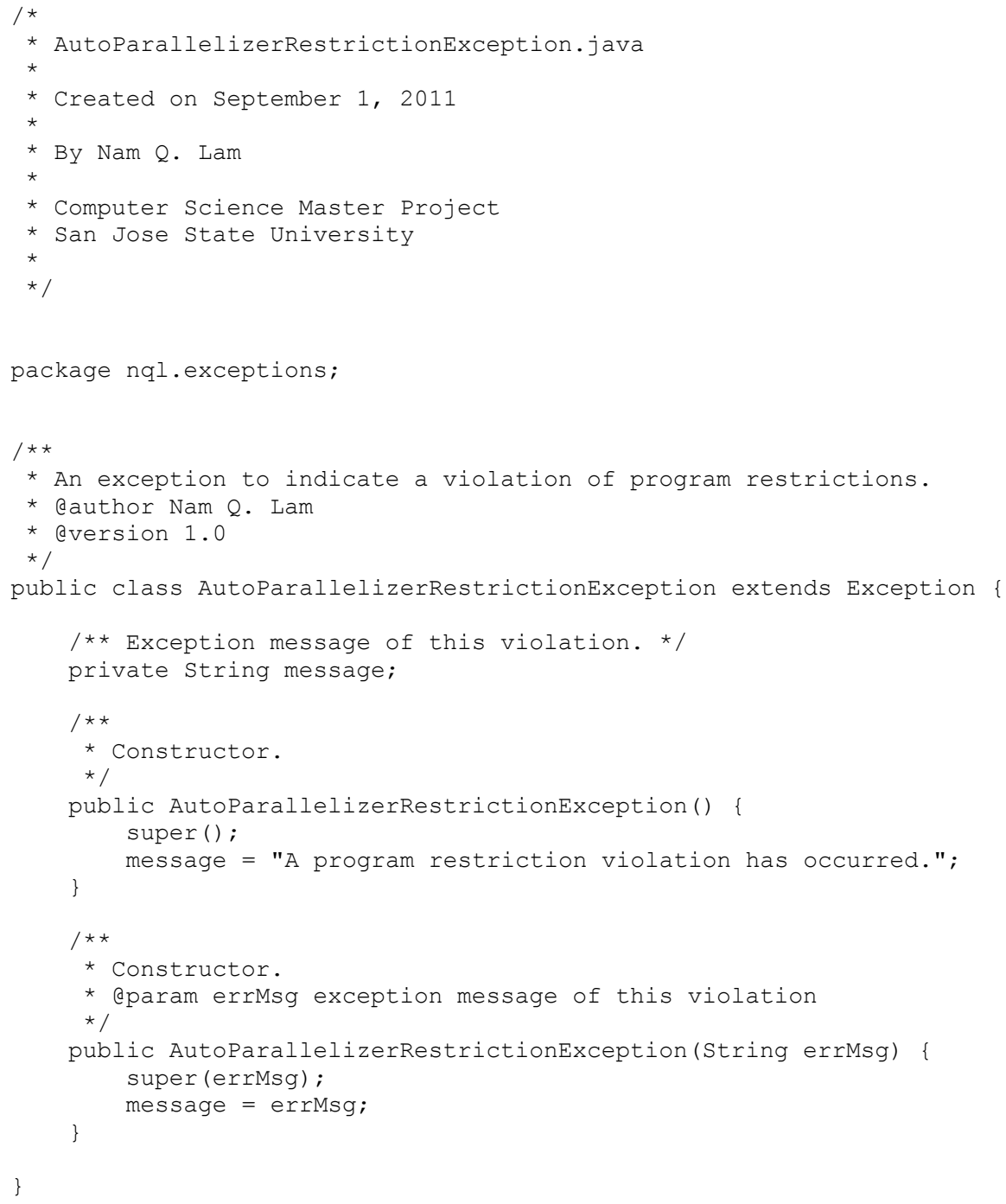

/*

* LoopCarriedDependenceException.java

* Created on September 1, 2011

* By Nam Q. Lam

* Computer Science Master Project

* San Jose State University

* 


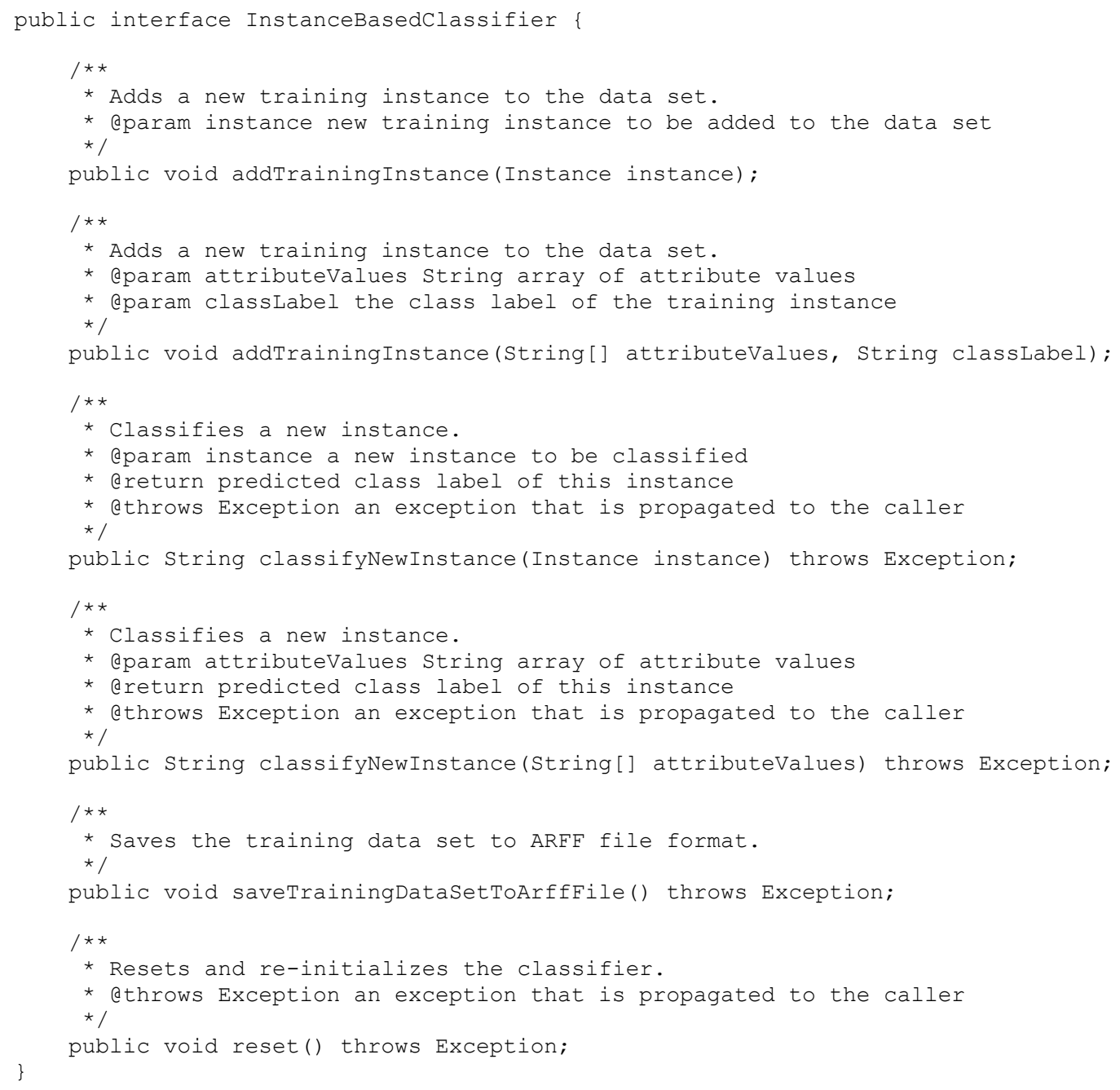

/*

* OpenMPForScheduleclassifier.java

* Created on February 14, 2011

* By Nam Q. Lam

* Computer Science Master Project

* San Jose State University

* /

package nql.ml.classifiers;

import java.io.File;

import java.io.Serializable;

import weka.classifiers.Classifier;

import weka.classifiers.lazy.IBk;

import weka.core.Attribute;

import weka.core.FastVector;

import weka.core.Instance;

import weka.core. Instances;

import weka.core.converters. ArffSaver; 


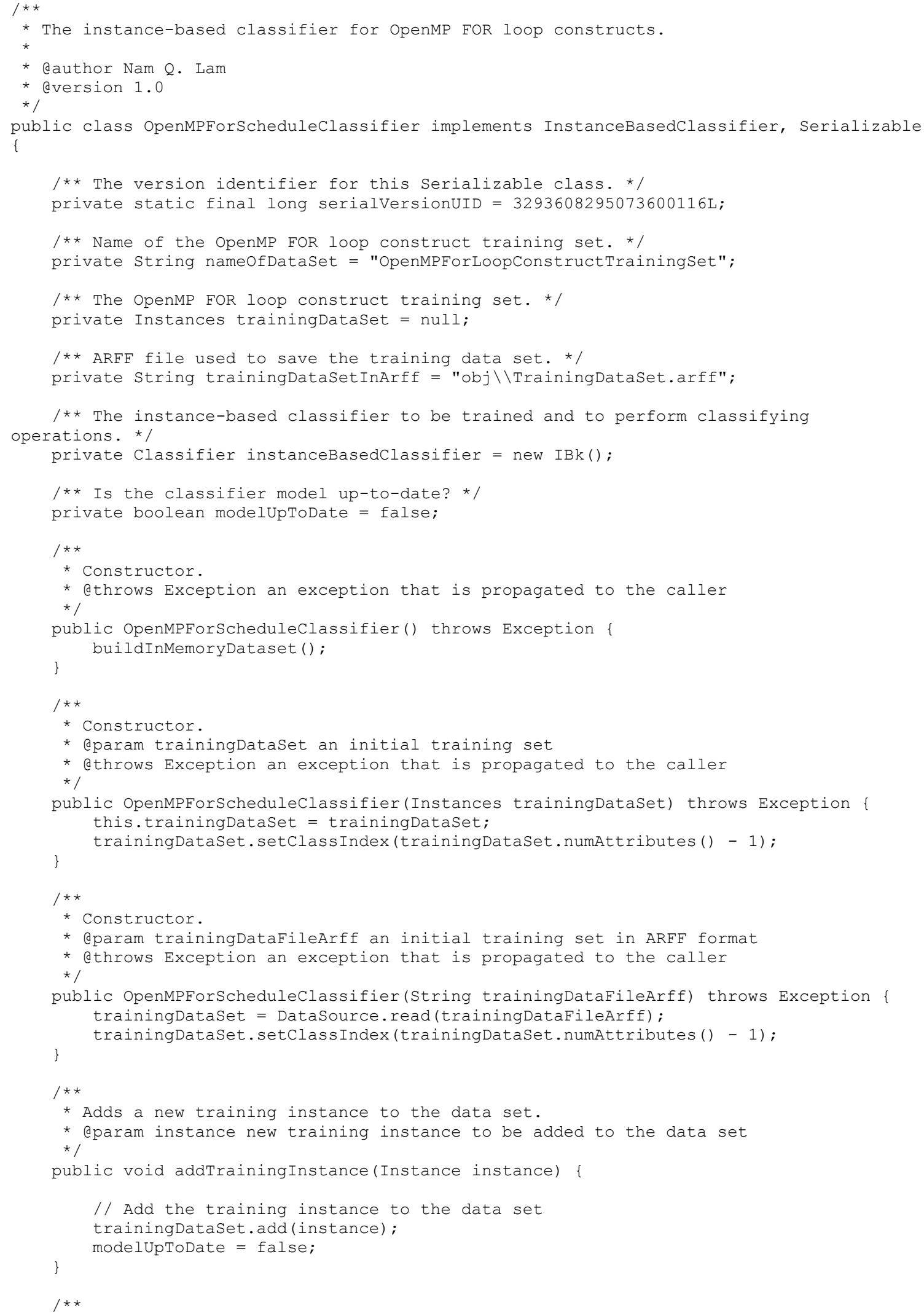




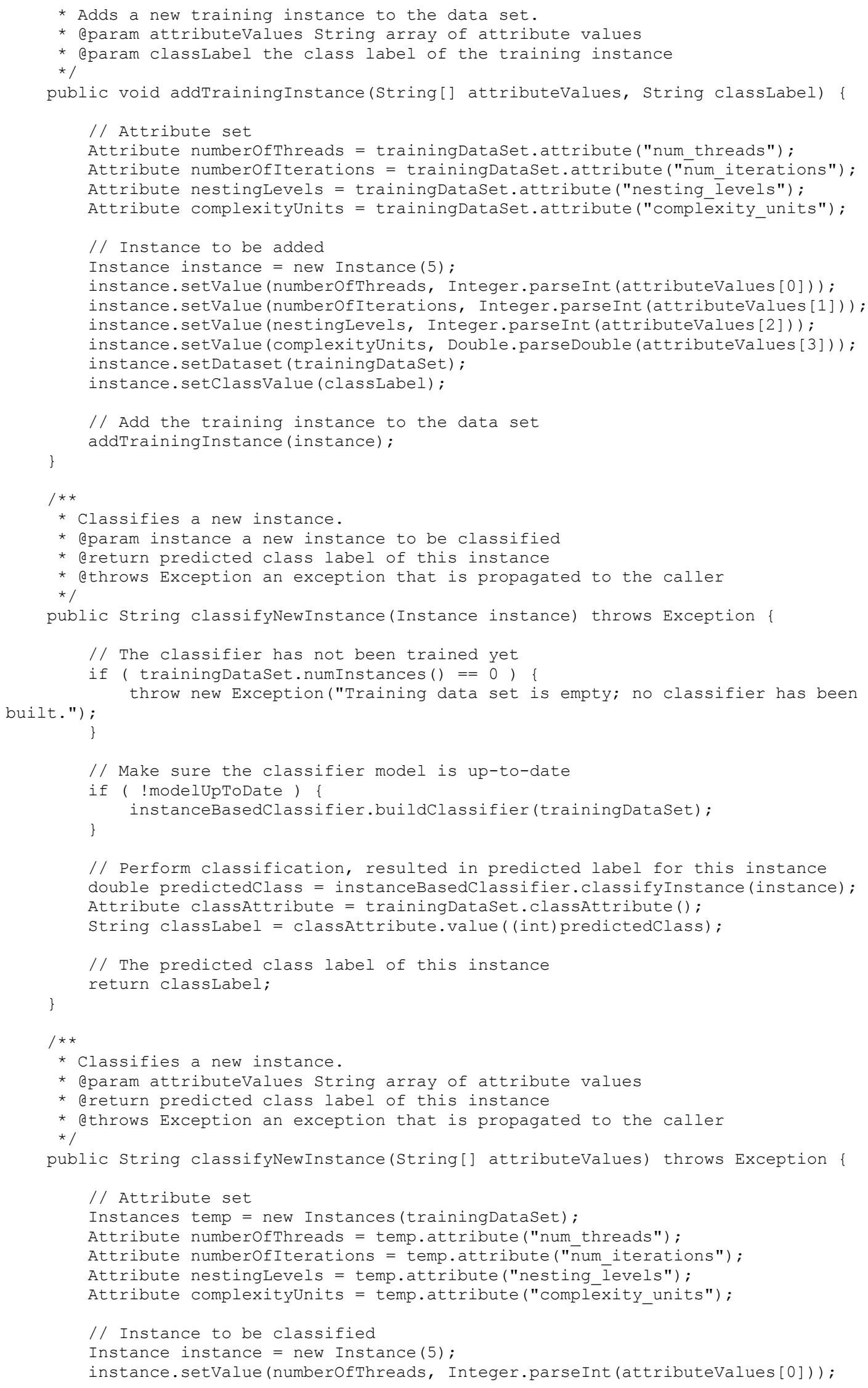




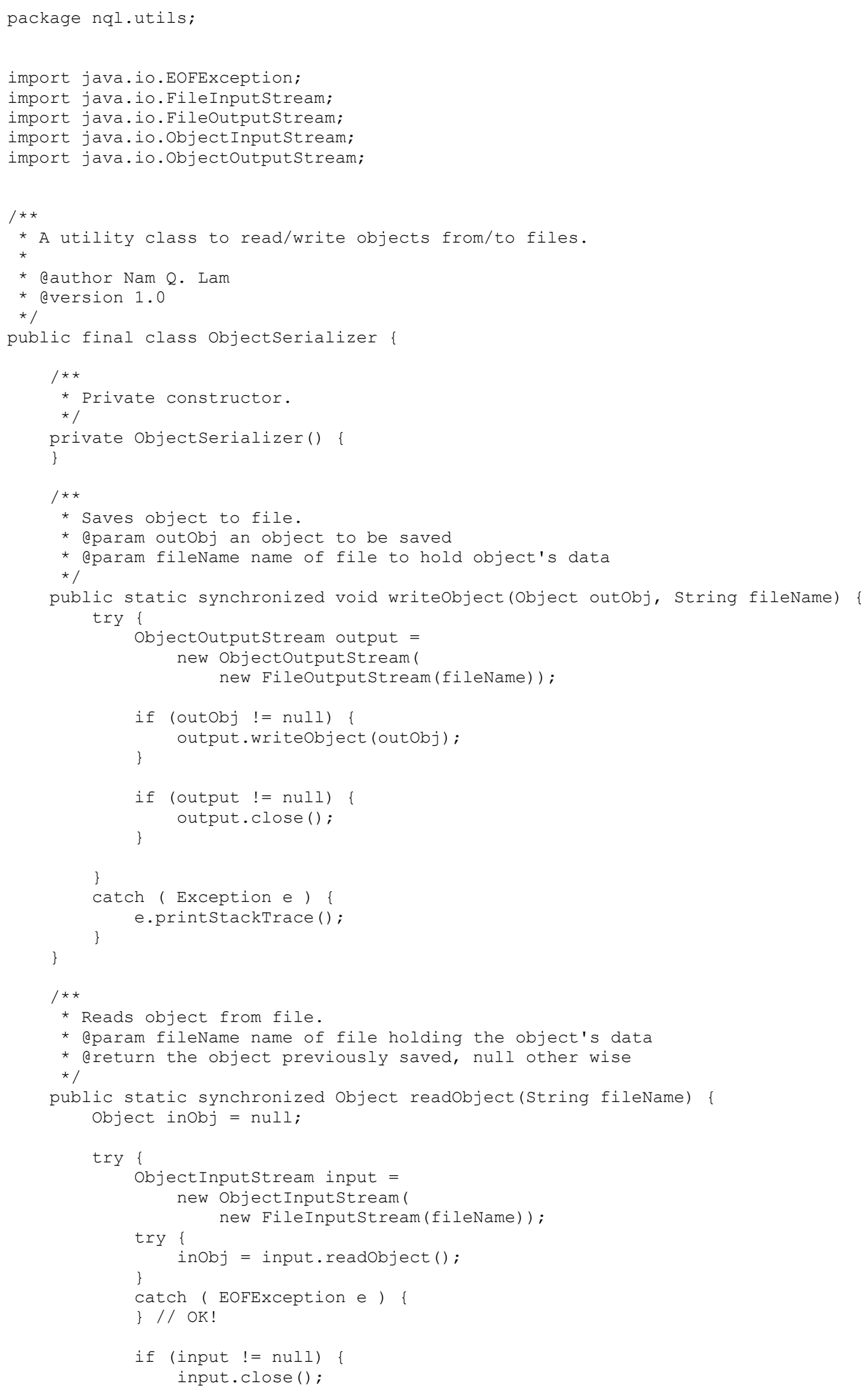




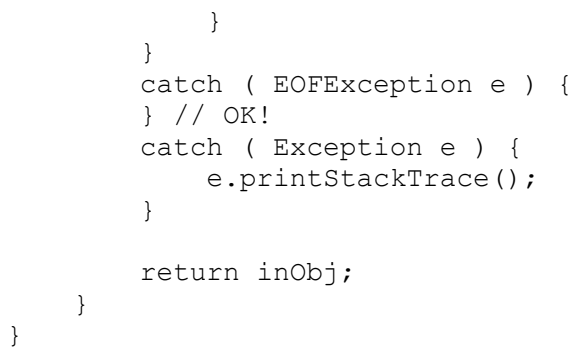

$$
\text { /* }
$$




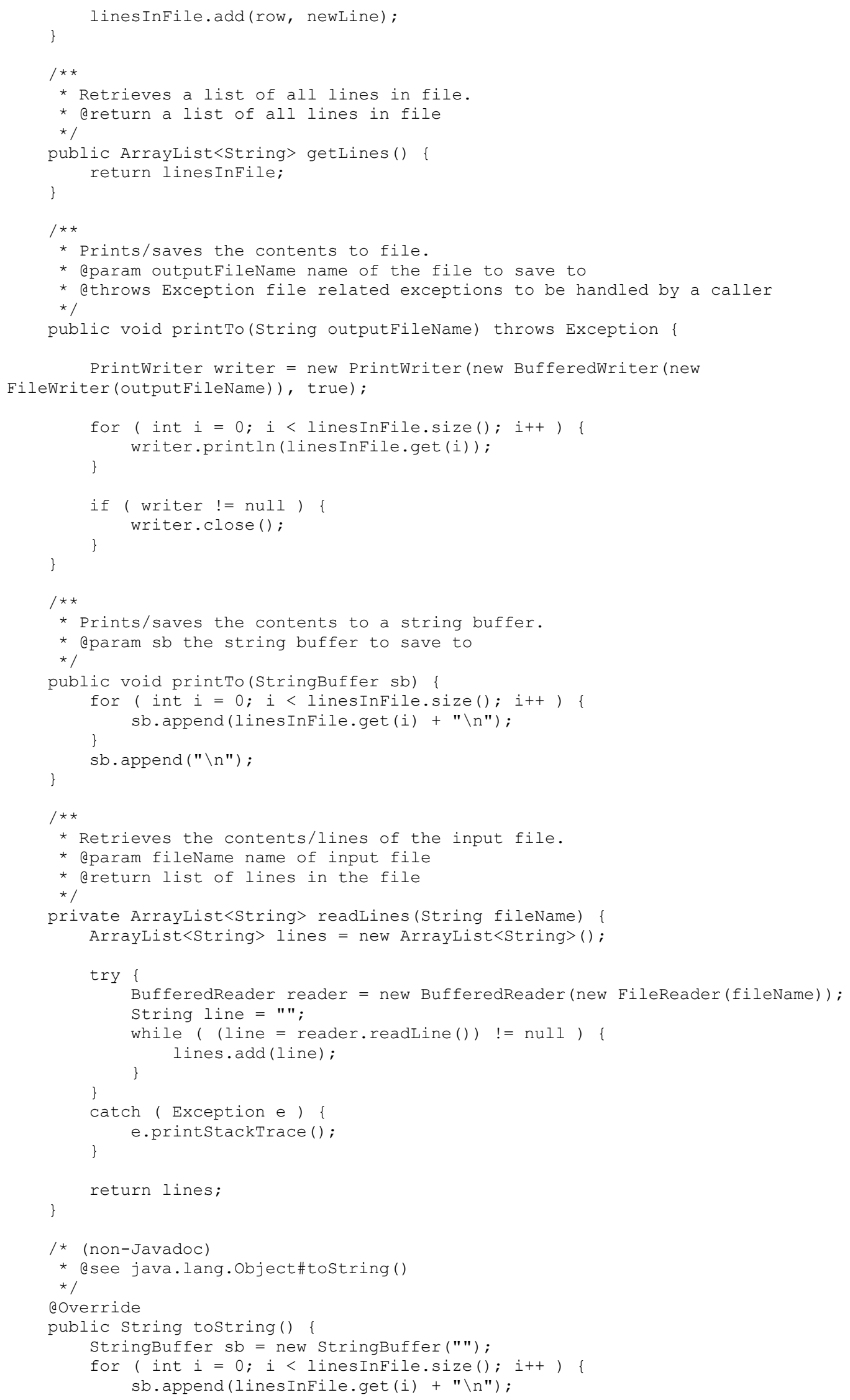


/* Generated By:JJTree\&JavaCC: Do not edit this line. CParser.java */ package nql.cd.frontend;

import nql.cd.intermediate. *;

import java.util.*;

import java.io.*;

public class CParser/*abgen(jjtree)*/implements CParserTreeConstants, CParserConstants

$\{/ *$ abgen (jjtree)*/

protected JJTCParserState jjtree = new JJTCParserState ();

/* Handle typedef types. */

private Set<String $>$ types = new HashSet<String $>()$;

private Stack<Boolean $>$ typedefParsingStack = new Stack<Boolean $>$ ();

private boolean isType (String type) \{

\} return types.contains (type);

private void addType (String type) \{

\} types.add (type);

/* This main entry is a test driver for the generated parser. */ public static void main(String [] args) \{

try \{

StringBuffer sb = new StringBuffer();

Reader reader $=$ new FileReader (args [0]);

CParser parser $=$ new $\mathrm{CParser}($ reader $)$;

SimpleNode rootNode = parser.parse();

rootNode.dump (">", sb);

\}

System.out.println(sb.toString());

catch ( Exception ex ) \{

ex.printStackTrace();

/*

* Syntactic Specification

*

* 1

final public SimpleNode parse() throws ParseException \{ /*abgen (jjtree) parse * /

ASTparse jjtn000 = new ASTparse (JJTPARSE);

boolean jjtc000 = true;

jjtree.openNodeScope (jjtn000);

try \{

label 1:

while (true) \{

externalDeclaration();

if (jj_2_1(1)) \{

;

\} else \{ (

break label_1;

jjtree.closeNodeScope (jjtn000, true);

jjtc000 = false;

\{if (true) return jjtn000;

\} catch (Throwable jjte000) \{

if $(j j t c 000)$ \{

jjtree.clearNodeScope (jjtno00);

jjtc000 = false;

\} else

jjtree.popNode();

if (jjte000 instanceof RuntimeException) \{ 


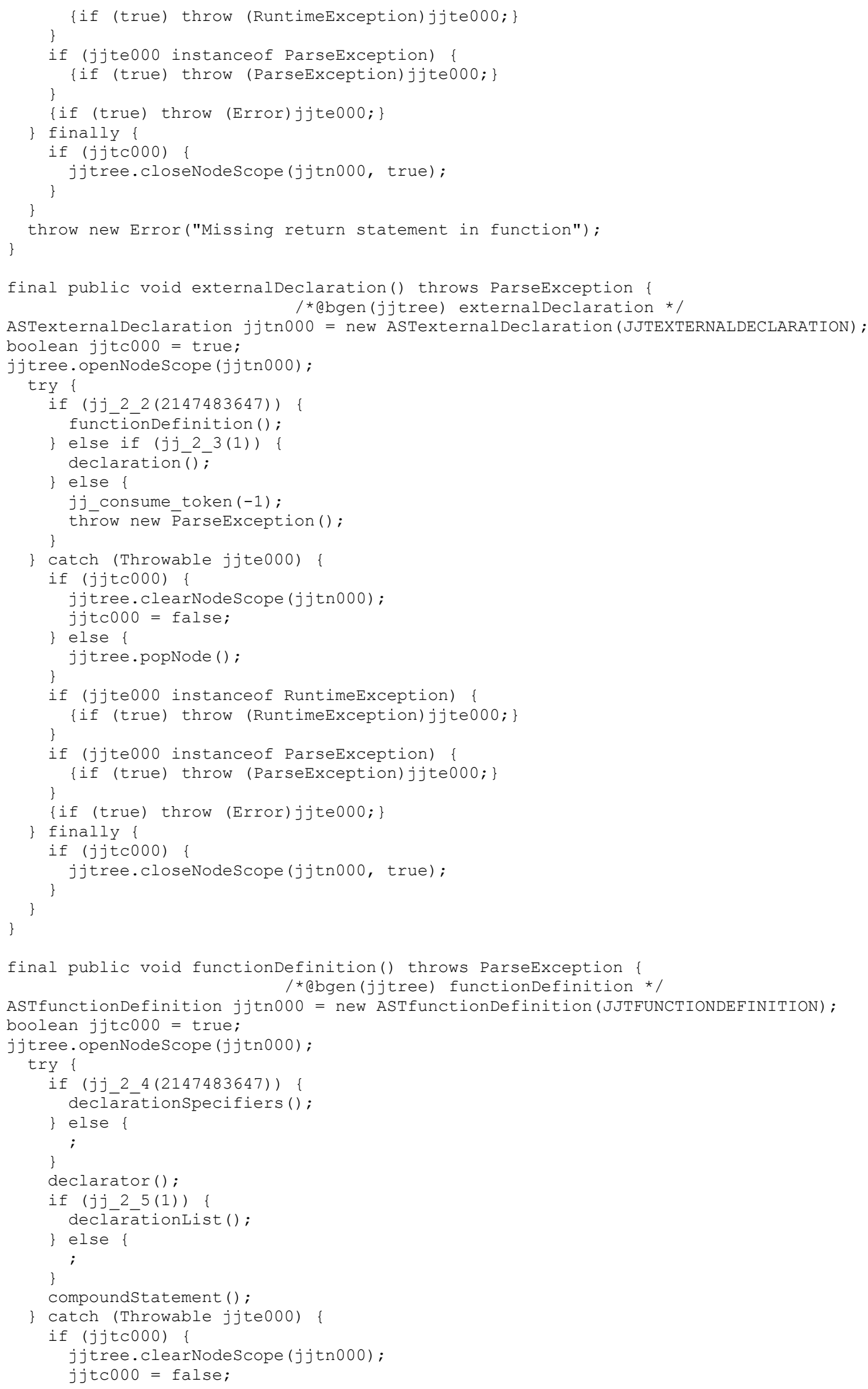




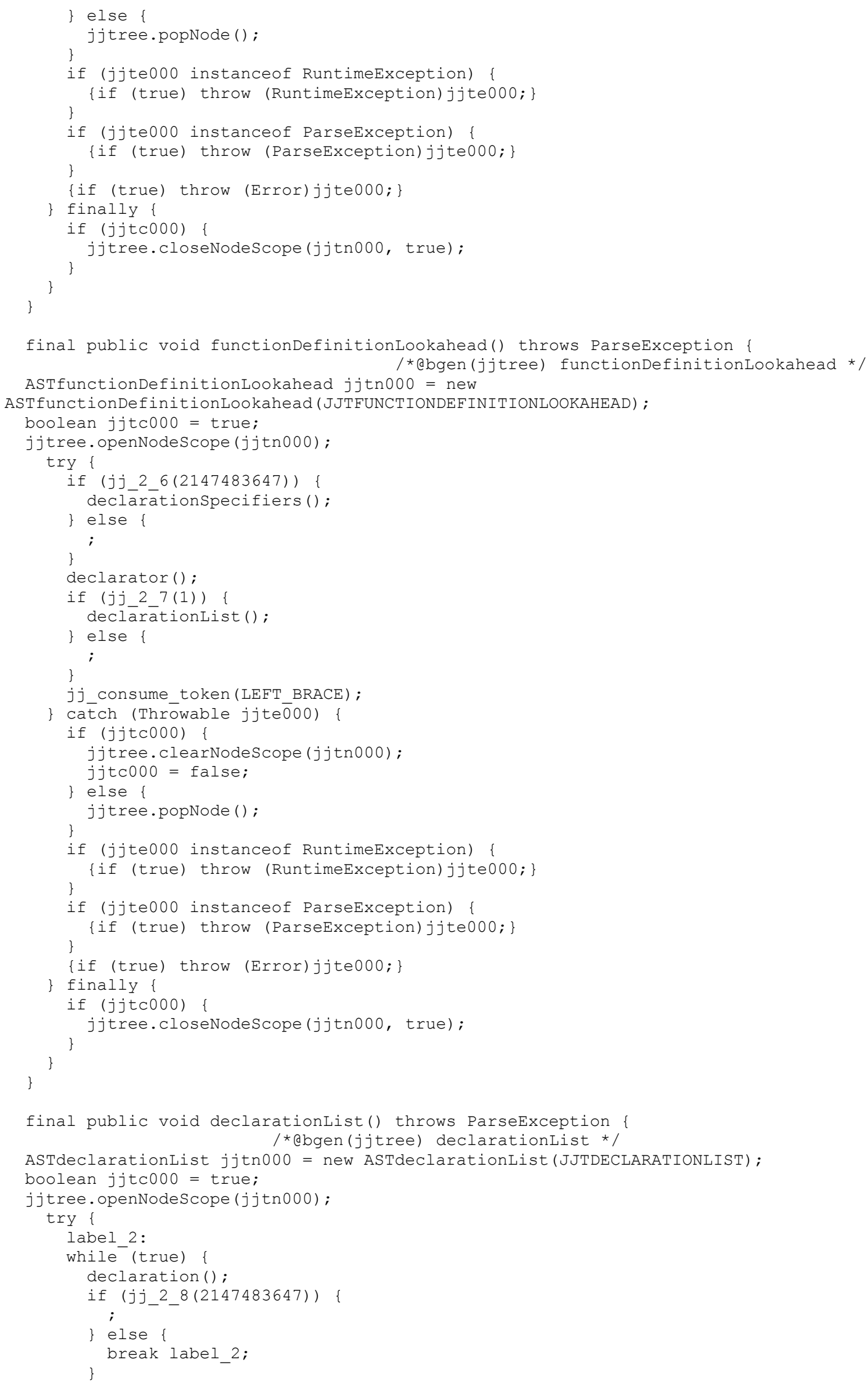




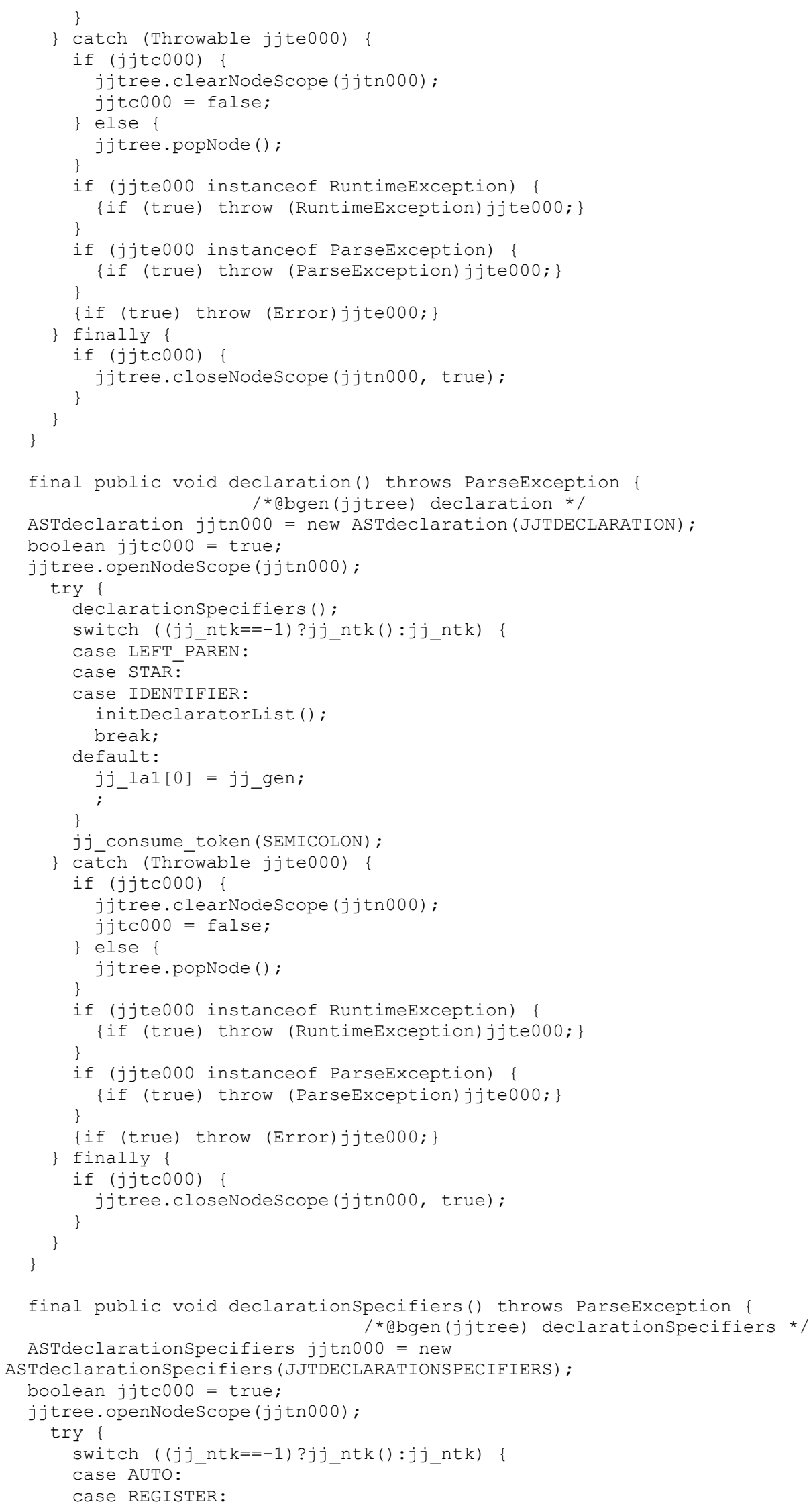




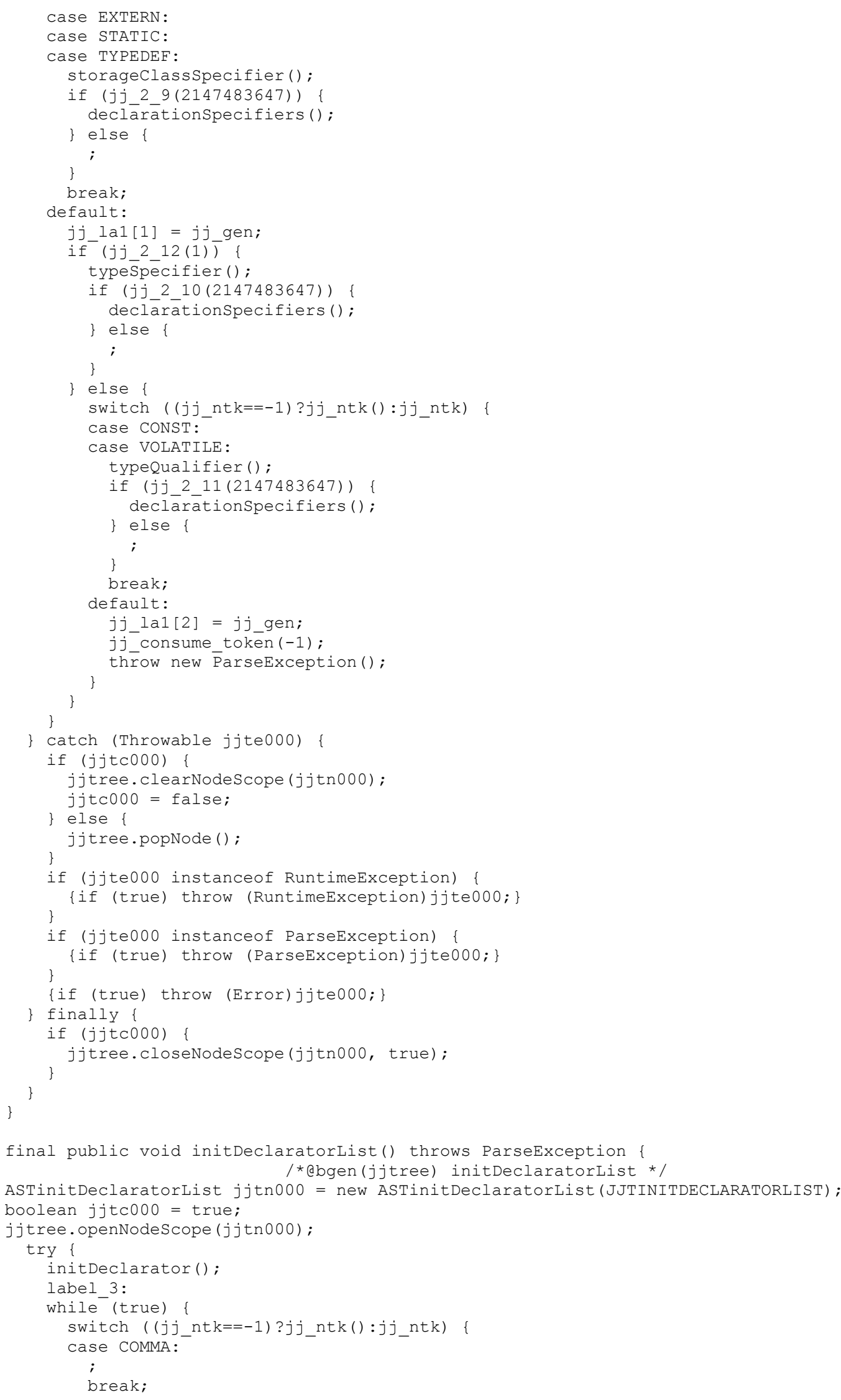




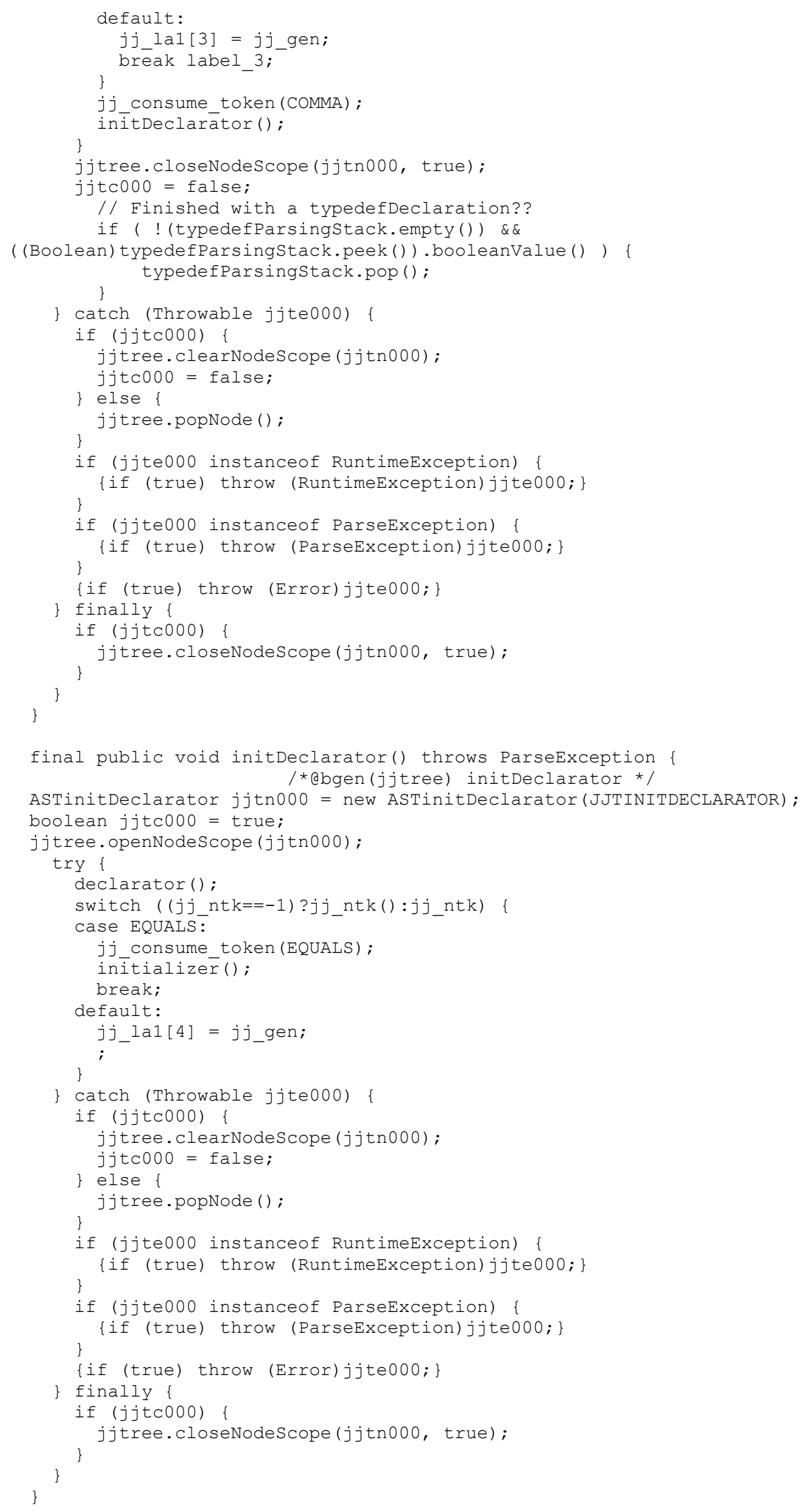




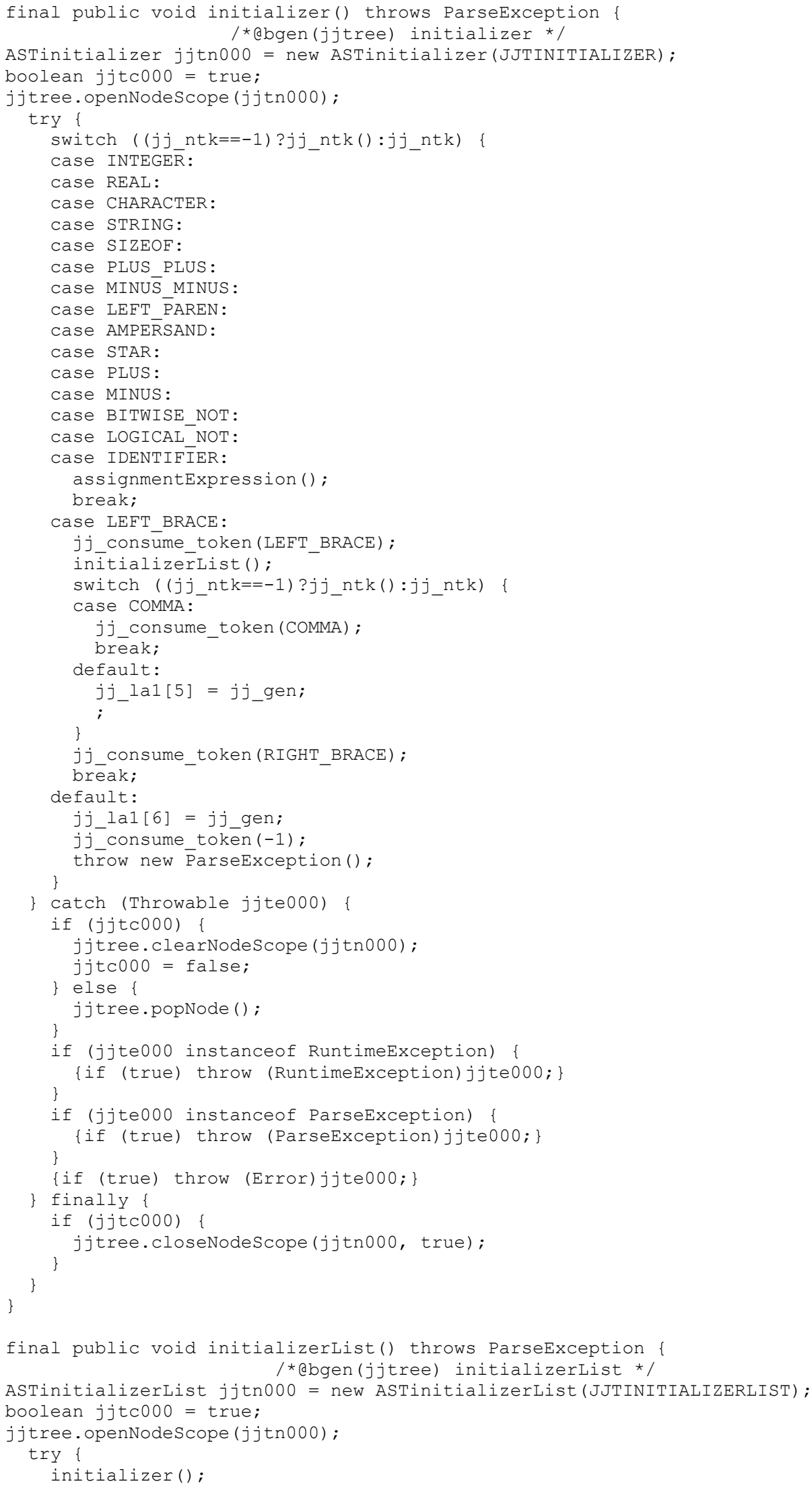




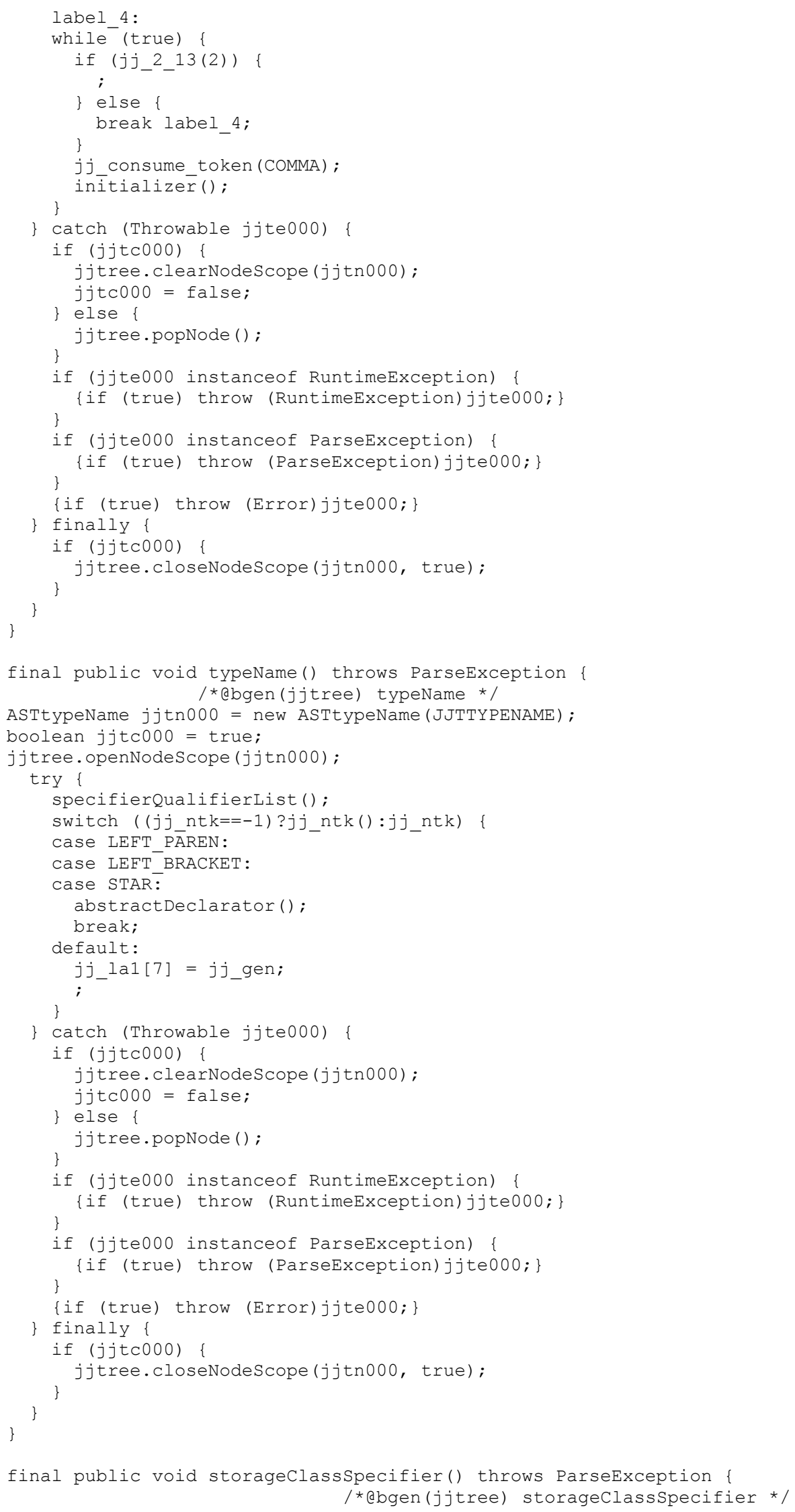


ASTstorageClasspecifier jjtn000 = new

ASTstorageClassSpecifier (JJTSTORAGECLASSSPECIFIER) ;

boolean jjtc000 = true;

jjtree.openNodeScope (jjtn000);

try \{

switch $\left(\left(j j \_n t k==-1\right) ? j j \_n t k(): j j \_n t k\right) \quad\{$

case AUTO:

jj_consume_token (AUTO);

bréak;

case REGISTER:

jj_consume_token (REGISTER);

break;

case STATIC:

jj_consume_token (STATIC);

break;

case EXTERN:

jj_consume_token (EXTERN);

brēak;

case TYPEDEF:

jj_consume_token (TYPEDEF);

jjtrēe.close $\bar{N}$ odeScope (jjtn000, true);

jjtc000 = false;

typedefParsingstack.push (Boolean.TRUE) ;

break;

default:

jj_la1[8] = jj_gen;

jj_consume token $(-1)$;

\}

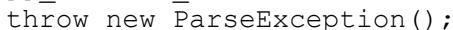

\} finally

if $(j j t c 000)\{$

jjtree.closeNodeScope (jjtn000, true) ; \}

\}

\}

final public void typespecifier() throws ParseException \{ /*abgen(jjtree) typespecifier */

ASTtypeSpecifier jjtn000 = new ASTtypeSpecifier (JJTTYPESPECIFIER); boolean jjtc000 = true;

jjtree.openNodeScope (jjtn000);

try \{

switch ( (jjntk==-1) ?jjntk():jjntk) \{

case VOID:

jj_consume_token (VOID) ;

break;

case CHAR:

jj_consume token (CHAR);

bréak;

case SHORT:

jj_consume_token (SHORT);

break;

case INT:

jj_consume_token (INT);

break;

case LONG:

jj_consume token (LONG);

brēak;

case FLOAT:

jj_consume_token (FLOAT) ;

break;

case DOUBLE:

jj_consume_token (DOUBLE) ;

break;

case SIGNED:

jj_consume_token (SIGNED);

break;

case UNSIGNED:

jj_consume_token (UNSIGNED) ;

break;

case STRUCT: 


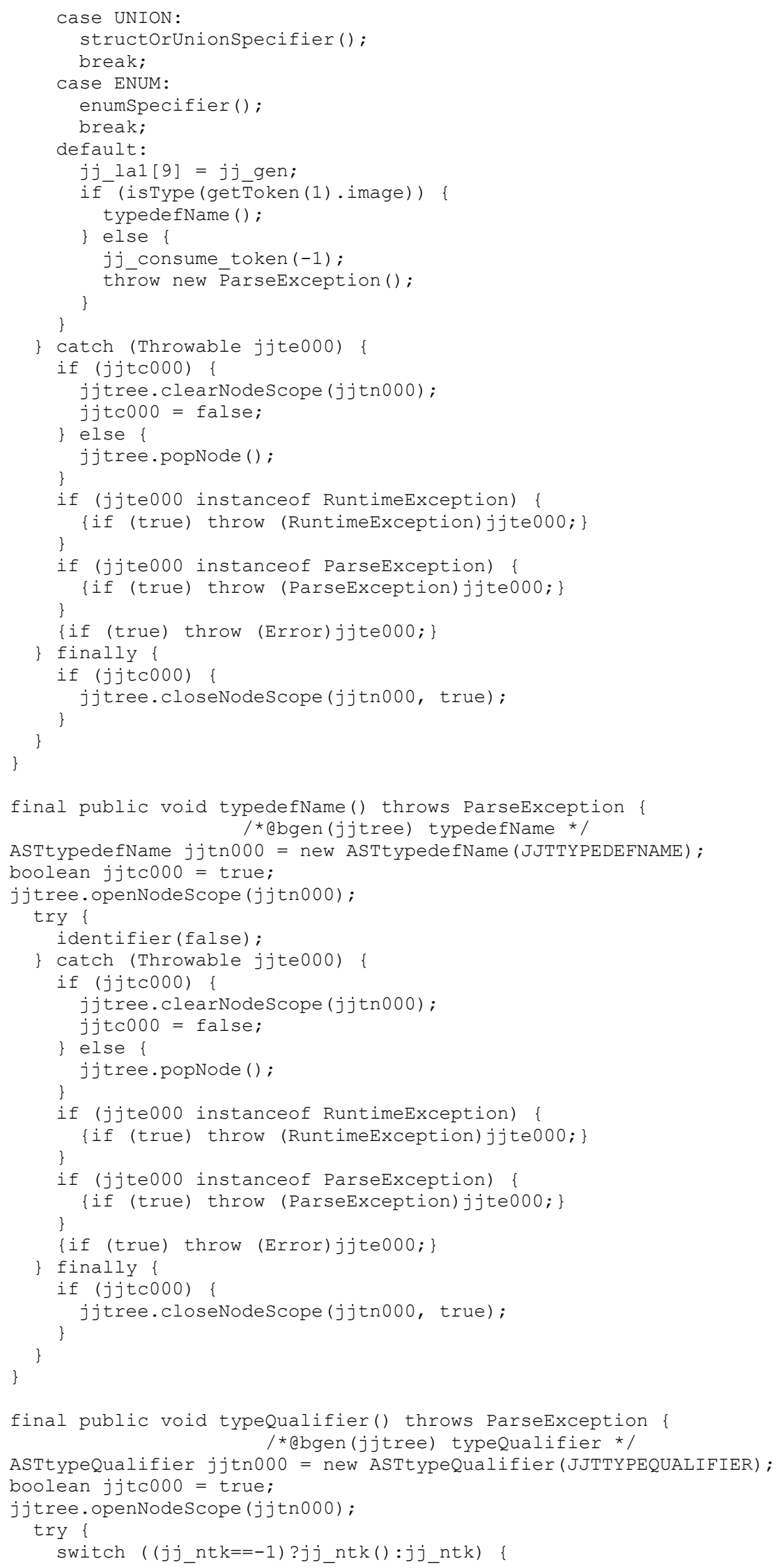




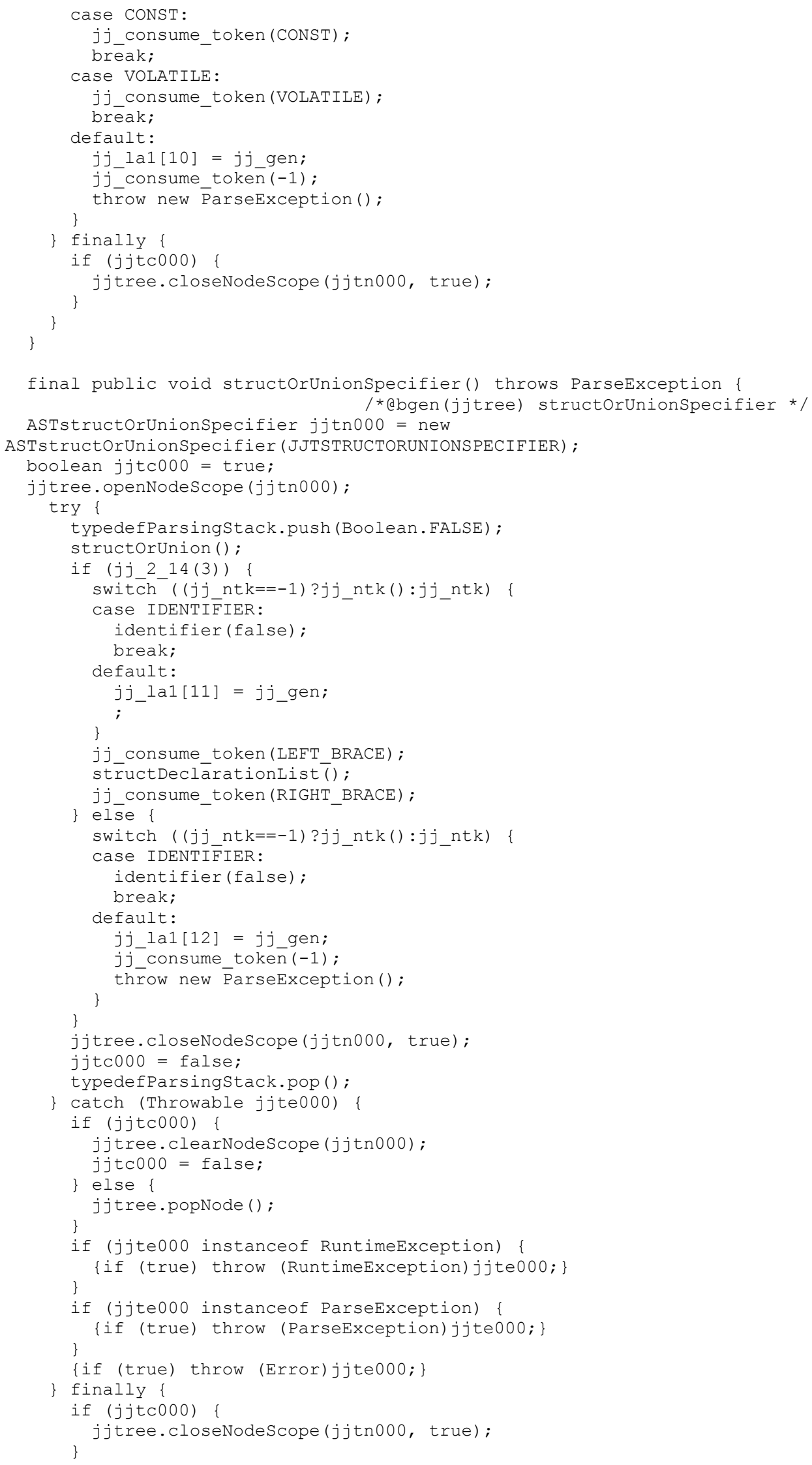


final public void structorUnion() throws ParseException \{
$/ *$ abgen(jjtree) structorUnion */

ASTstructorUnion jjtn000 = new ASTstructorUnion (JJTSTRUCTORUNION);

boolean jjtc000 = true;

jjtree.openNodeScope (jjtn000);

try \{

switch ( (jj_ntk==-1) ?jjntk ():jjntk) \{

case STRUCT:

jj_consume_token (STRUCT);

brēak;

case UNION:

jj_consume_token (UNION) ;

brēak;

default:

jj_la1[13] = jj_gen;

jj_consume_token $(-1)$; \}

throw new $\bar{P}$ arseException();

\} finally \{

if $(j \mathrm{jtc000)}\{$

jjtree.closeNodeScope (jjtn000, true);

\}

final public void structDeclarationList() throws ParseException \{ /*abgen (jjtree) structDeclarationList */

ASTstructDeclarationList jjtn000 = new

ASTstructDeclarationList (JJTSTRUCTDECLARATIONLIST) ;

boolean jjtc000 = true;

jjtree.openNodescope (jjtn000);

try \{

label 5 :

while (true) \{

structDeclaration();

if (jj_2_15(1)) \{

;

\} else \{ \}

break label_5;

\}

\} catch (Throwable jjte000) \{

if $(j j t c 000) \quad\{$

jjtree.clearNodeScope (jjtn000);

jjtc000 = false;

\} else \{

\}

jjtree.popNode ();

if (jjte000 instanceof RuntimeException) \{

\}

\{if (true) throw (RuntimeException)jjte000;

if (jjte000 instanceof ParseException) \{

\{if (true) throw (ParseException)jjte000;

\{if (true) throw (Error)jjte000;

\} finally

if $(j j t c 000) \quad\{$

jjtree.closeNodeScope (jjtn000, true) ;

\}

\}

final public void structDeclaration() throws ParseException \{ /*abgen (jjtree) structDeclaration */

ASTstructDeclaration jjtn000 = new ASTstructDeclaration (JJTSTRUCTDECLARATION); boolean jjtc000 = true;

jjtree.openNodeScope (jjtn000);

try 


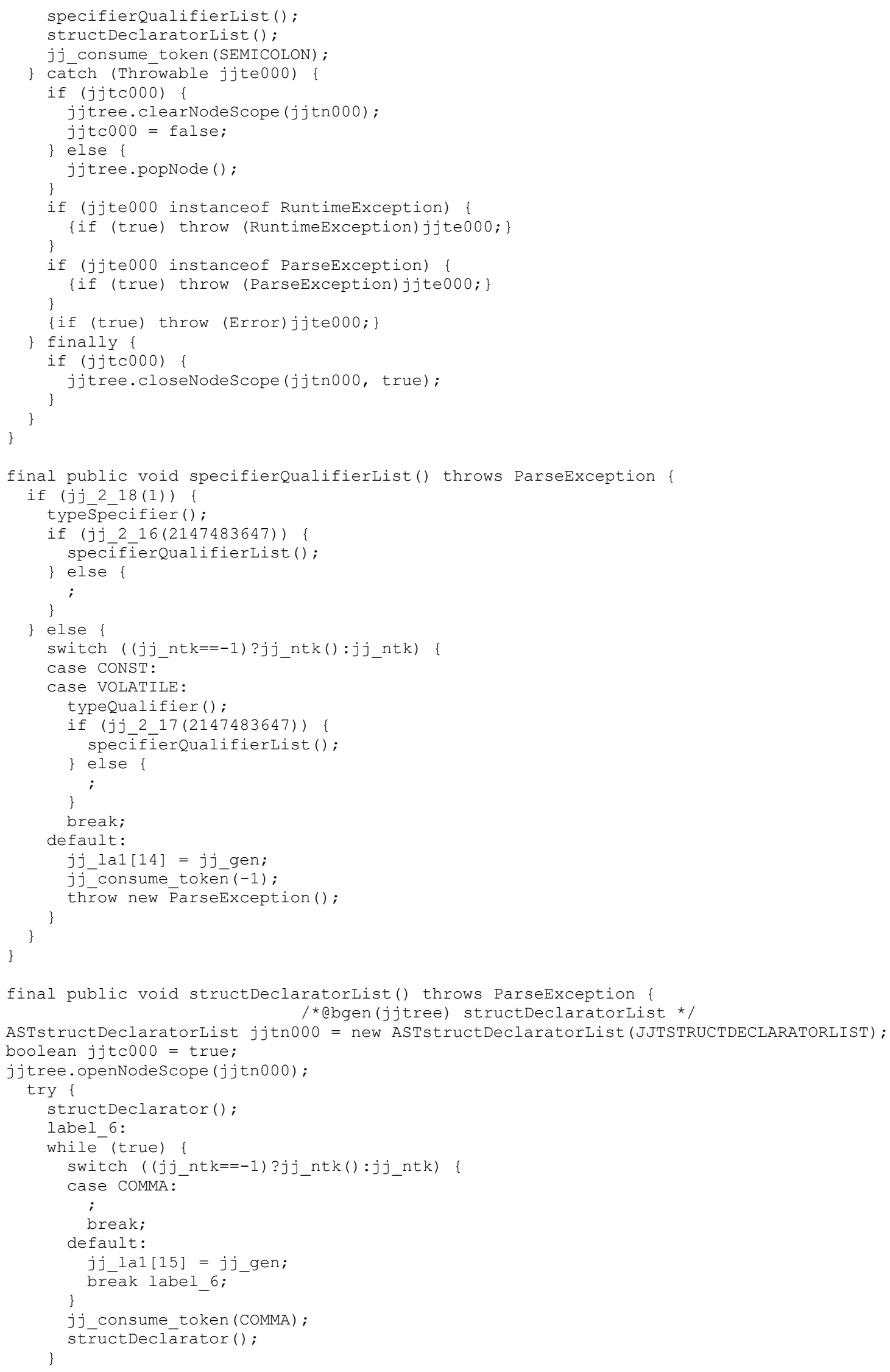




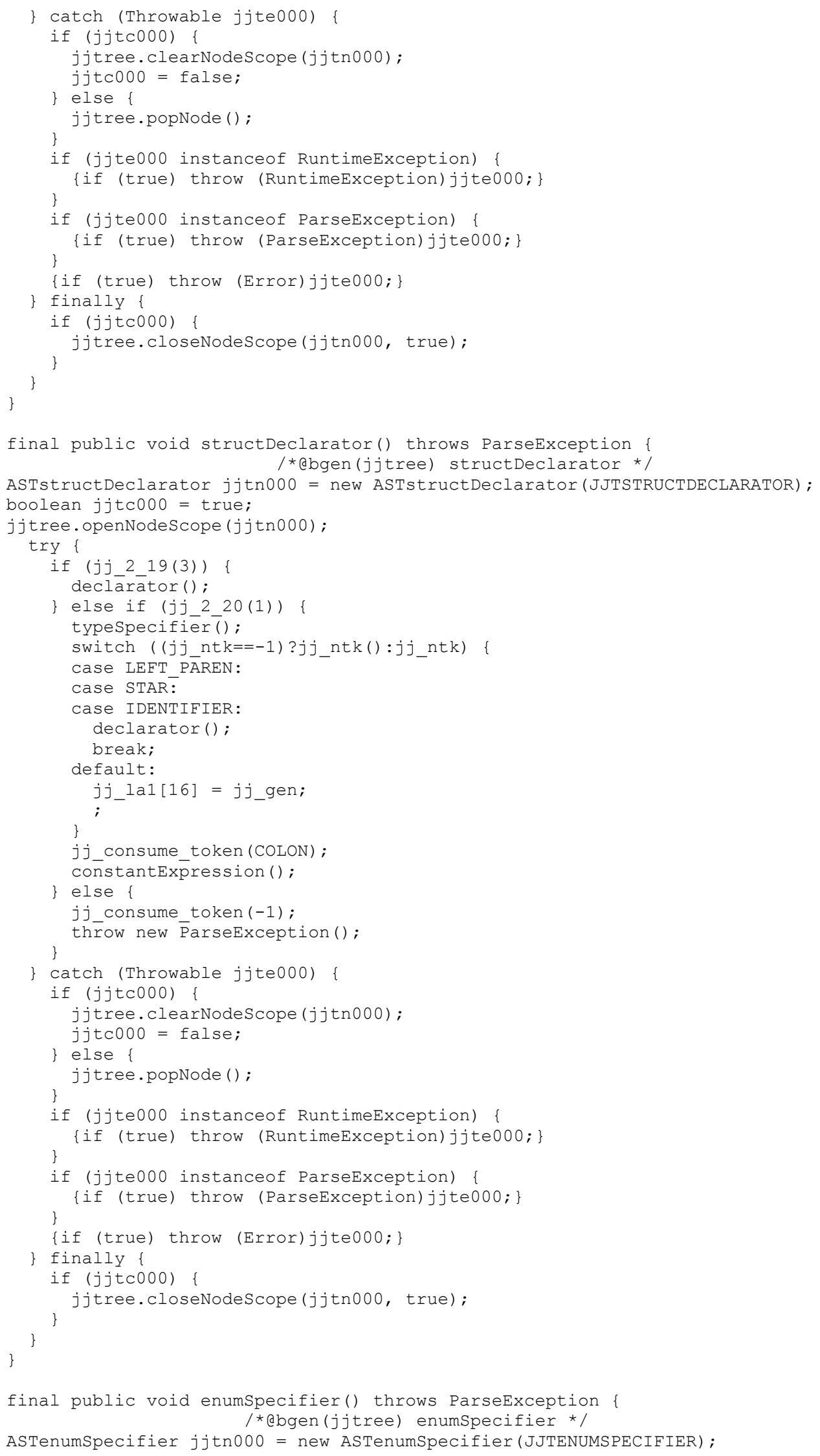




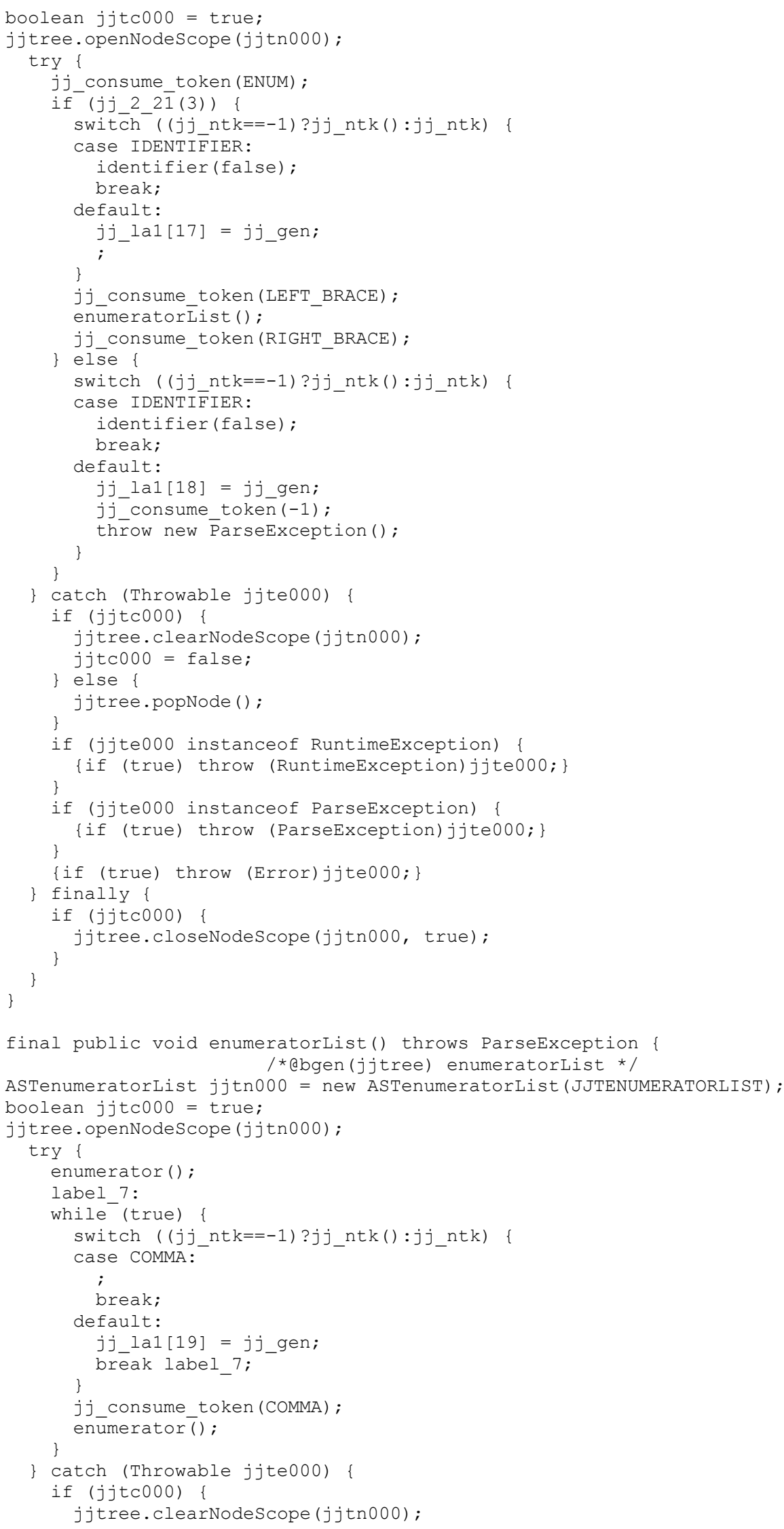




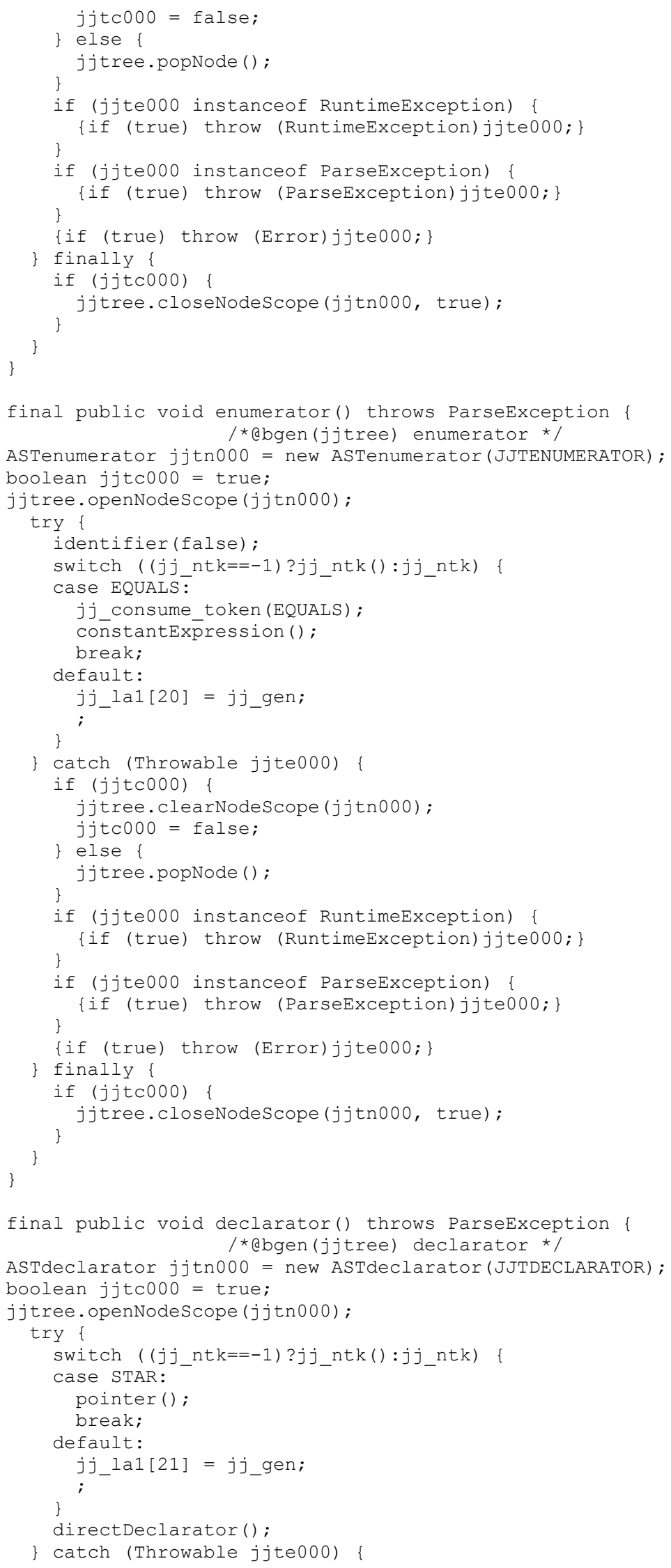




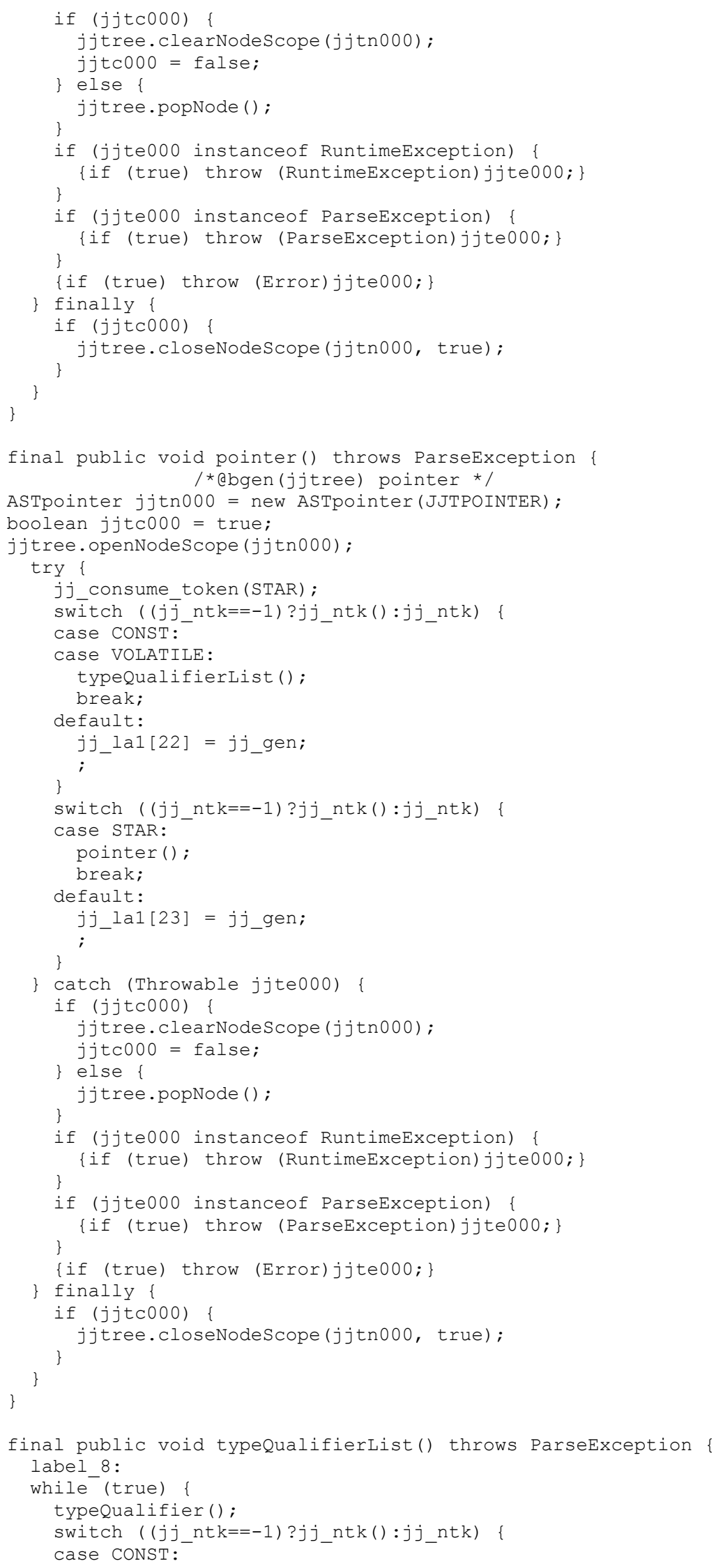




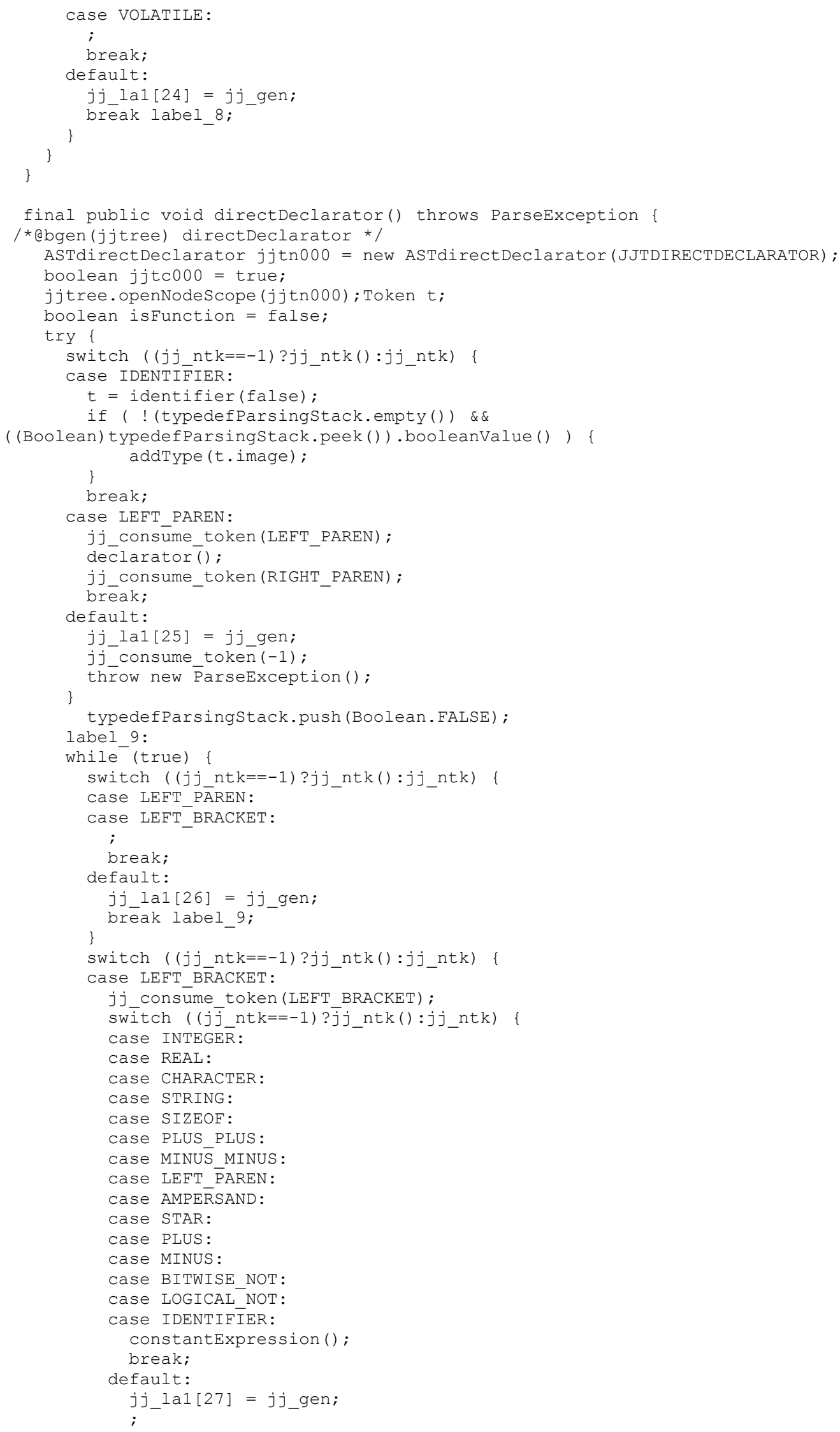




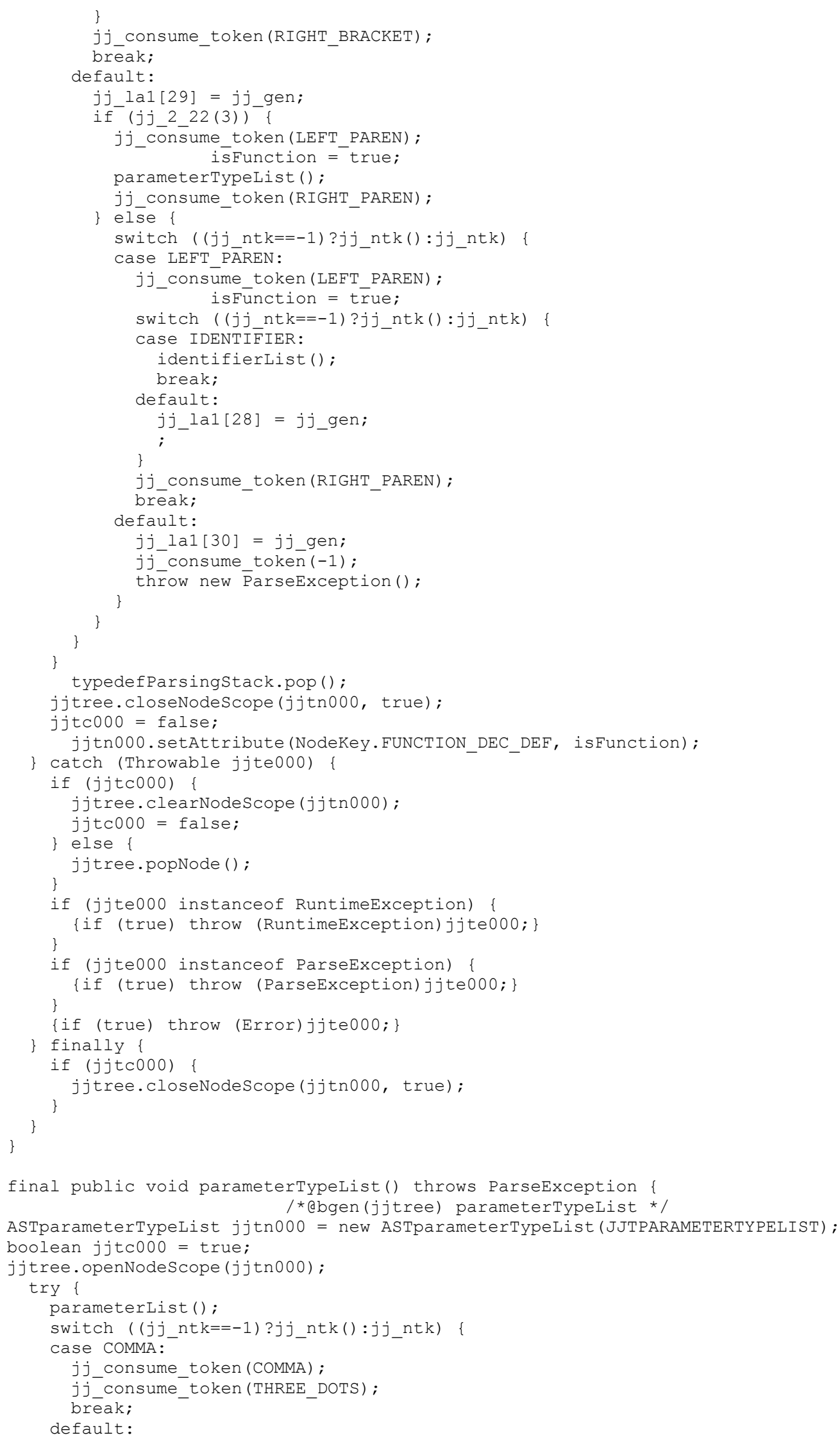




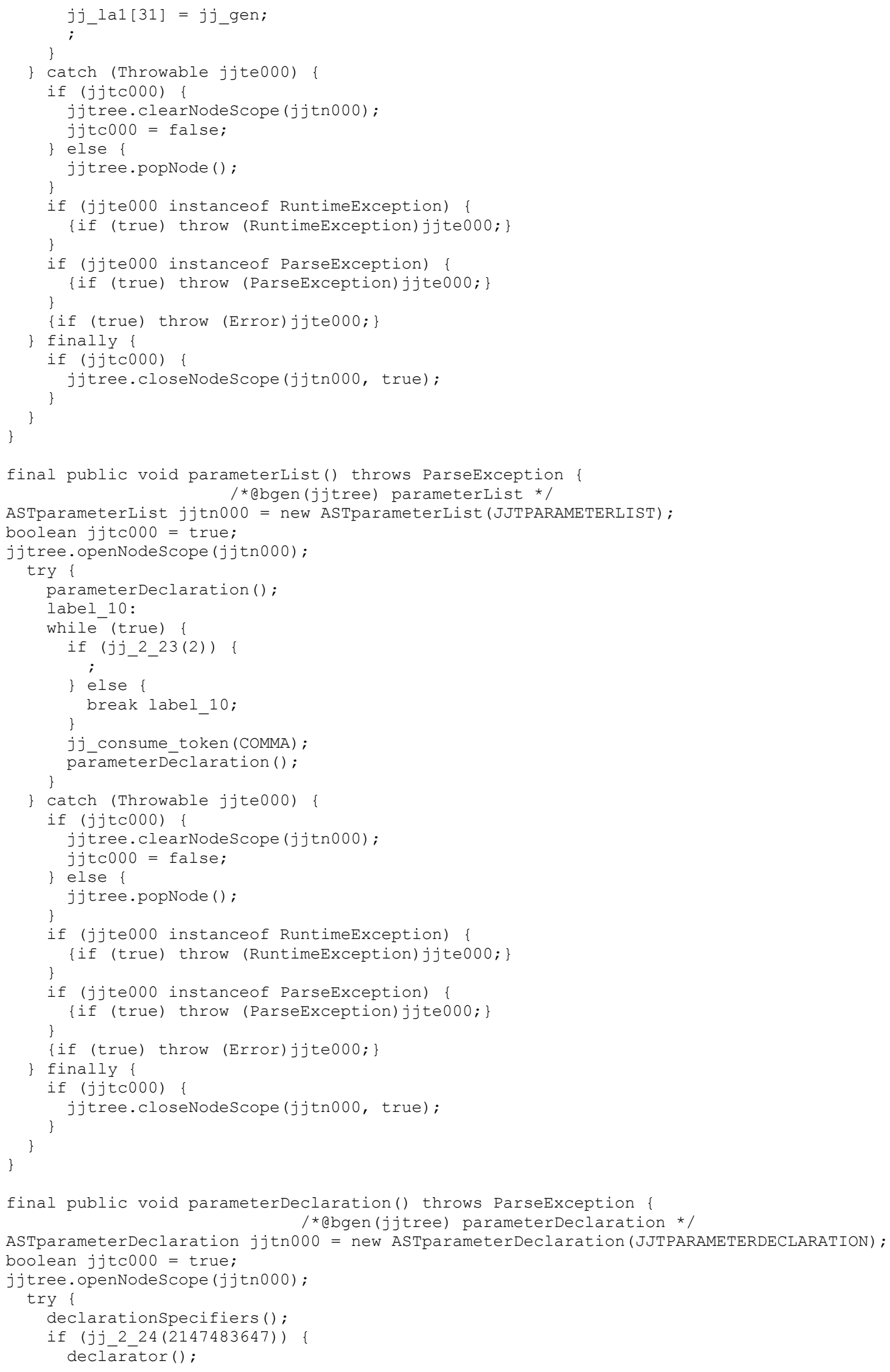




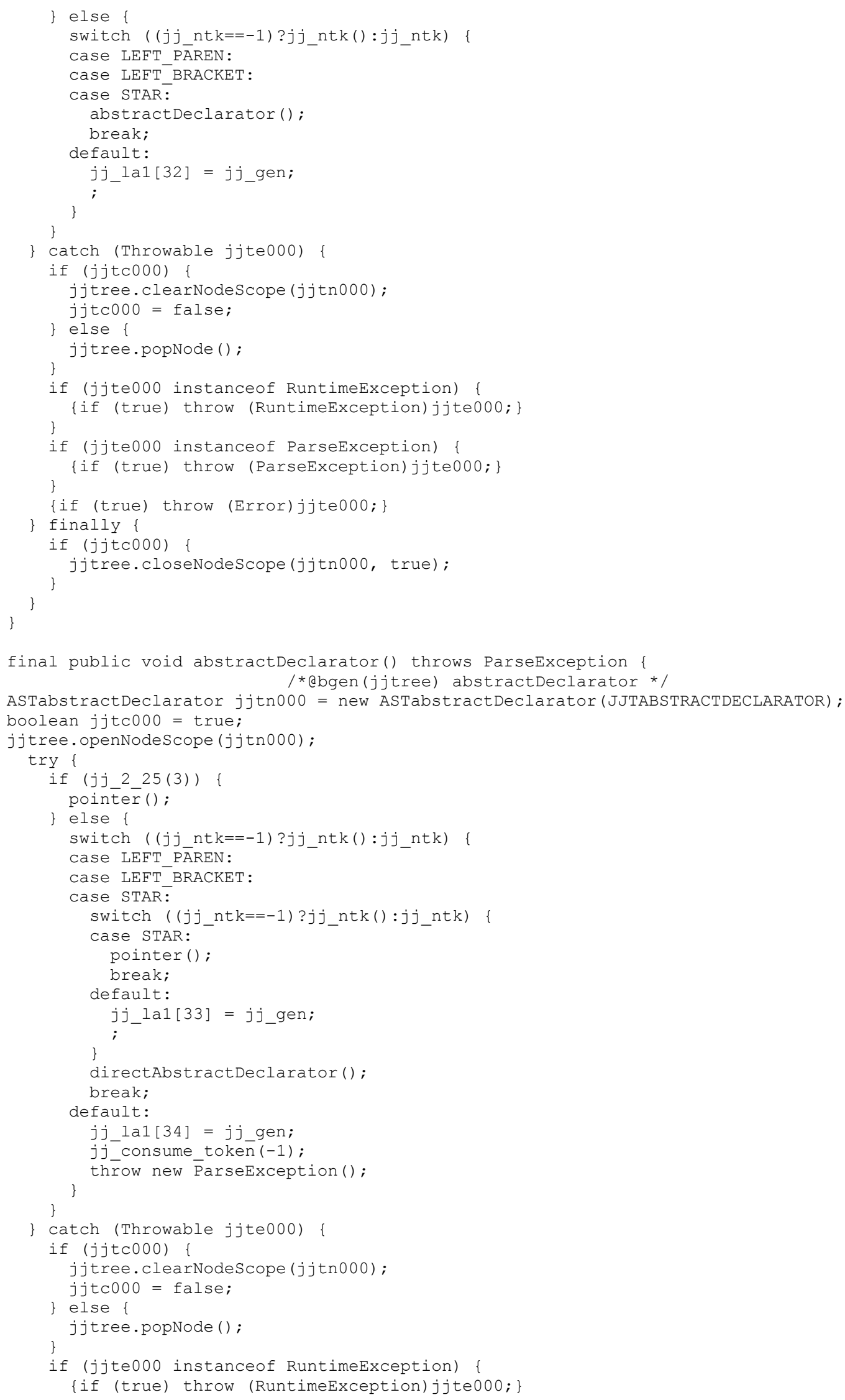




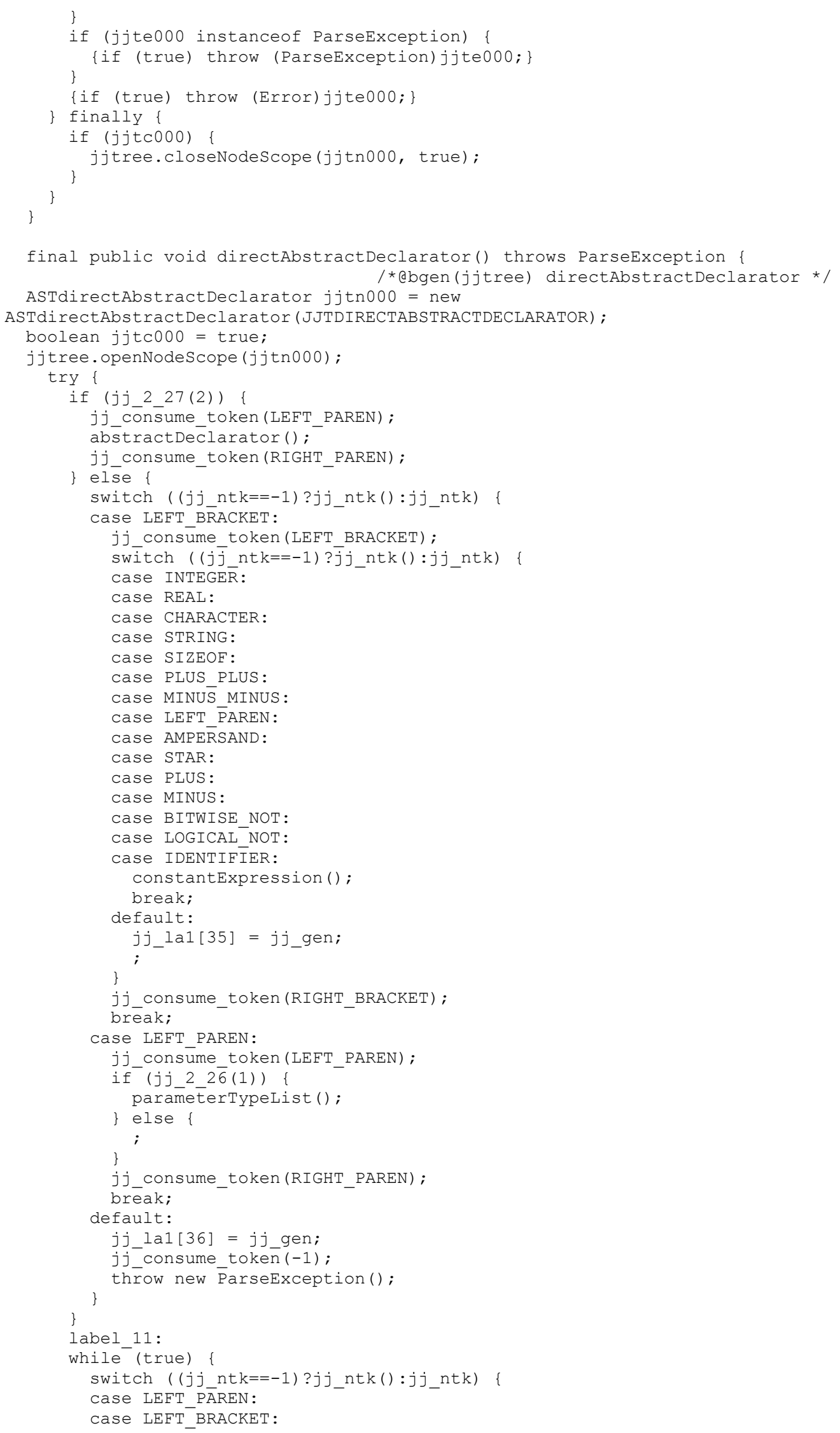




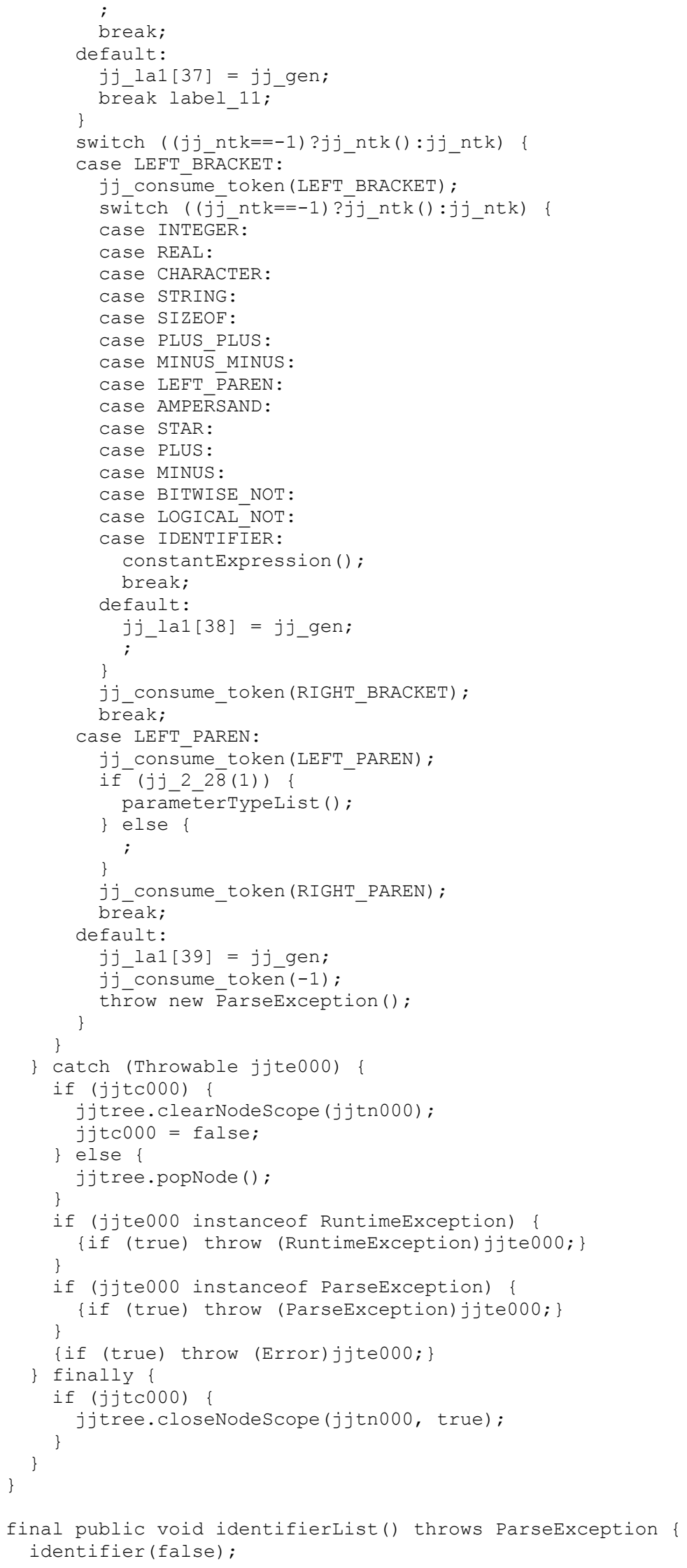




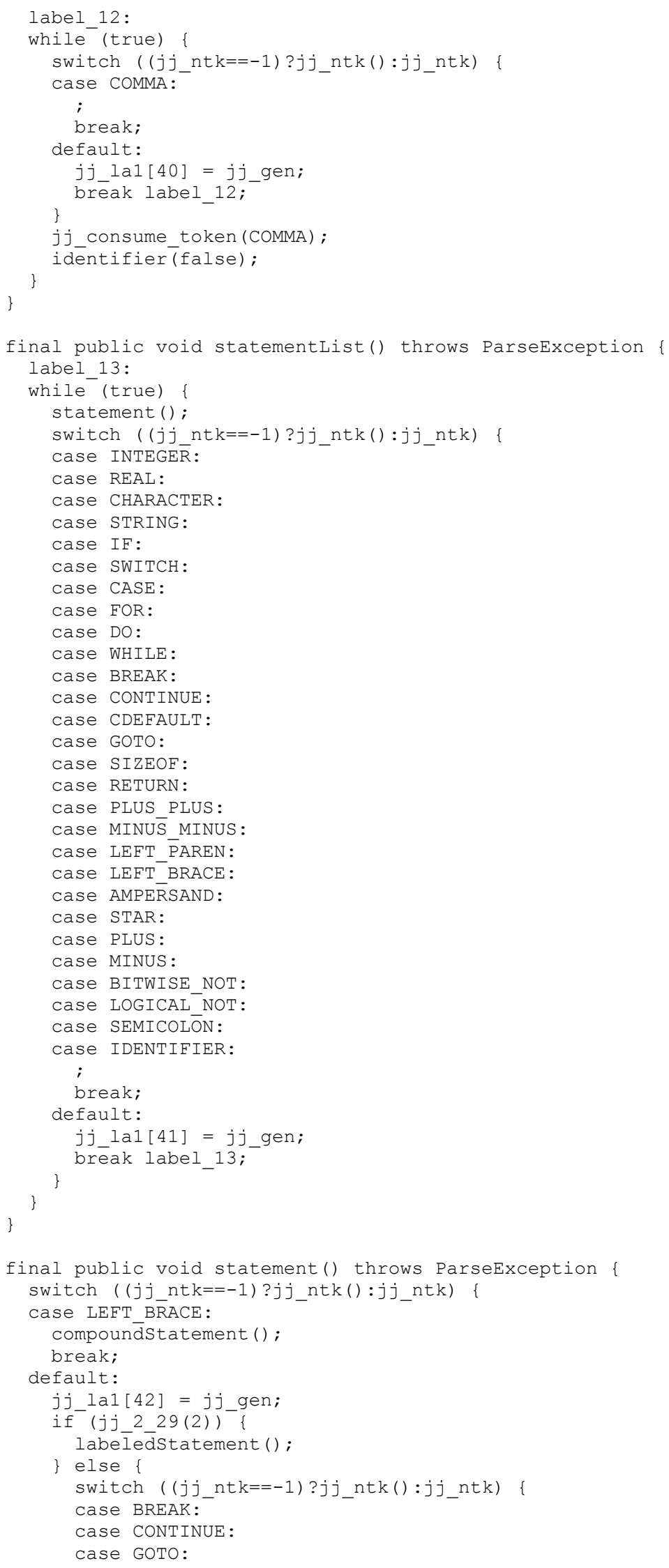




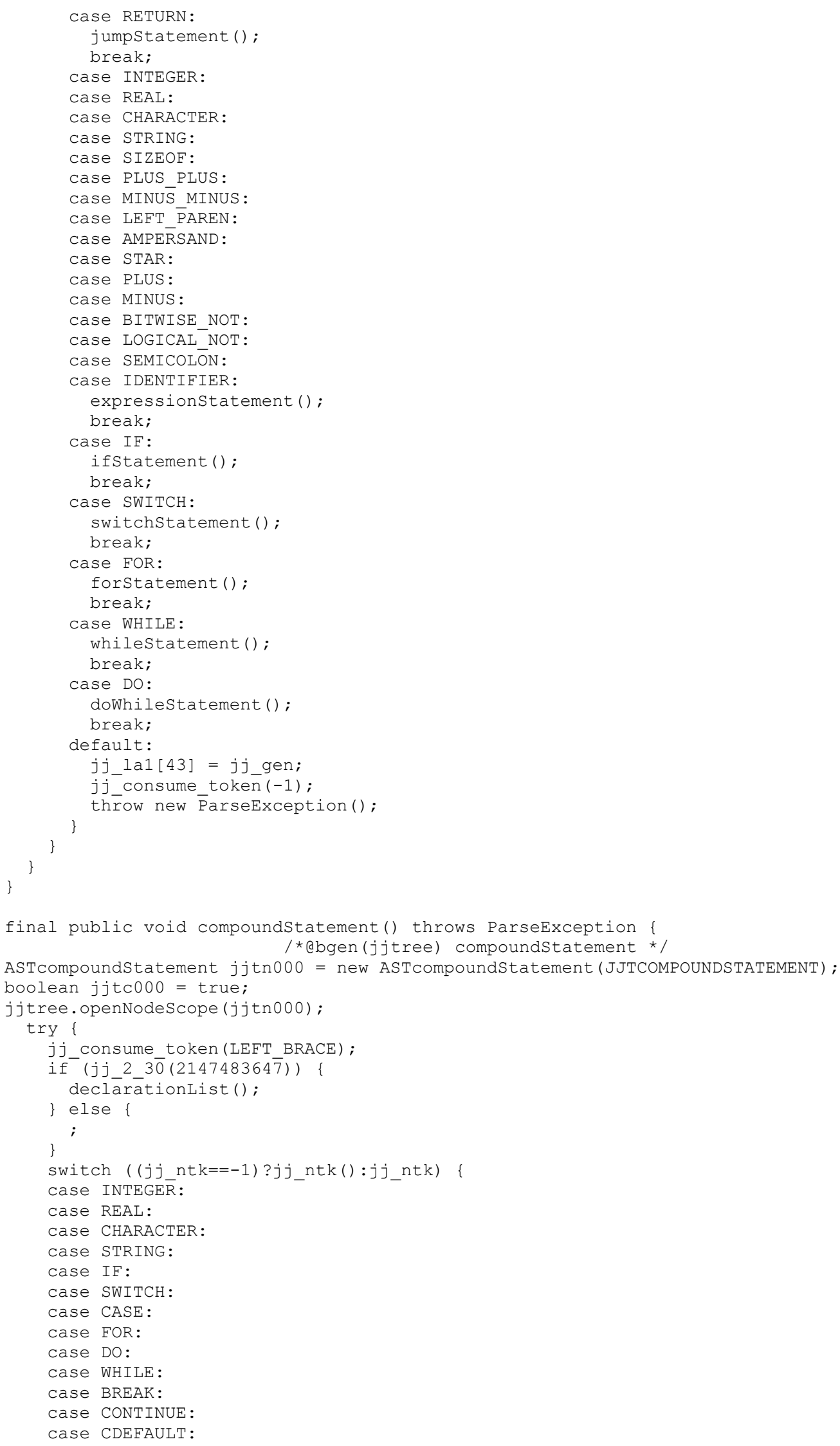




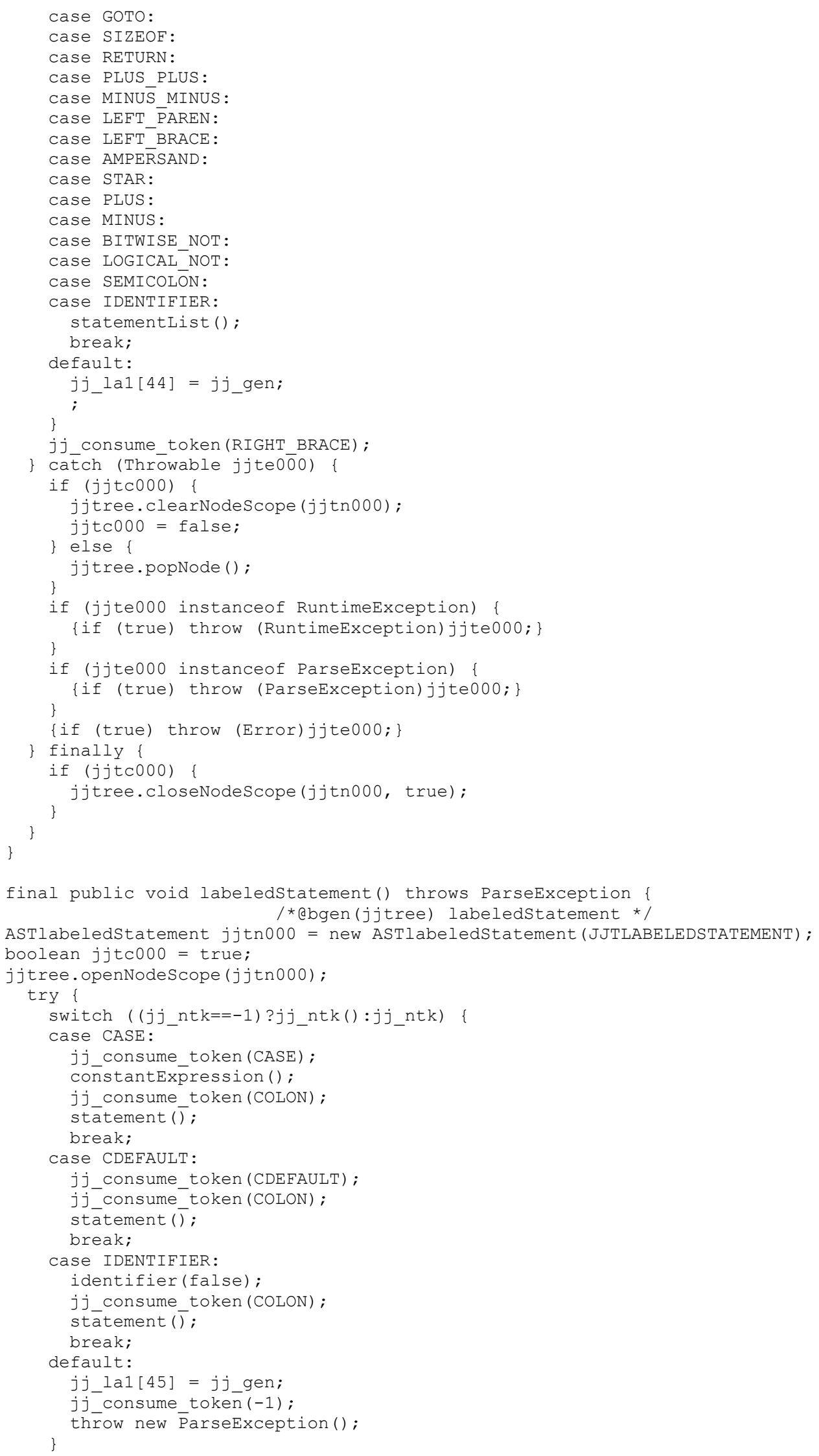




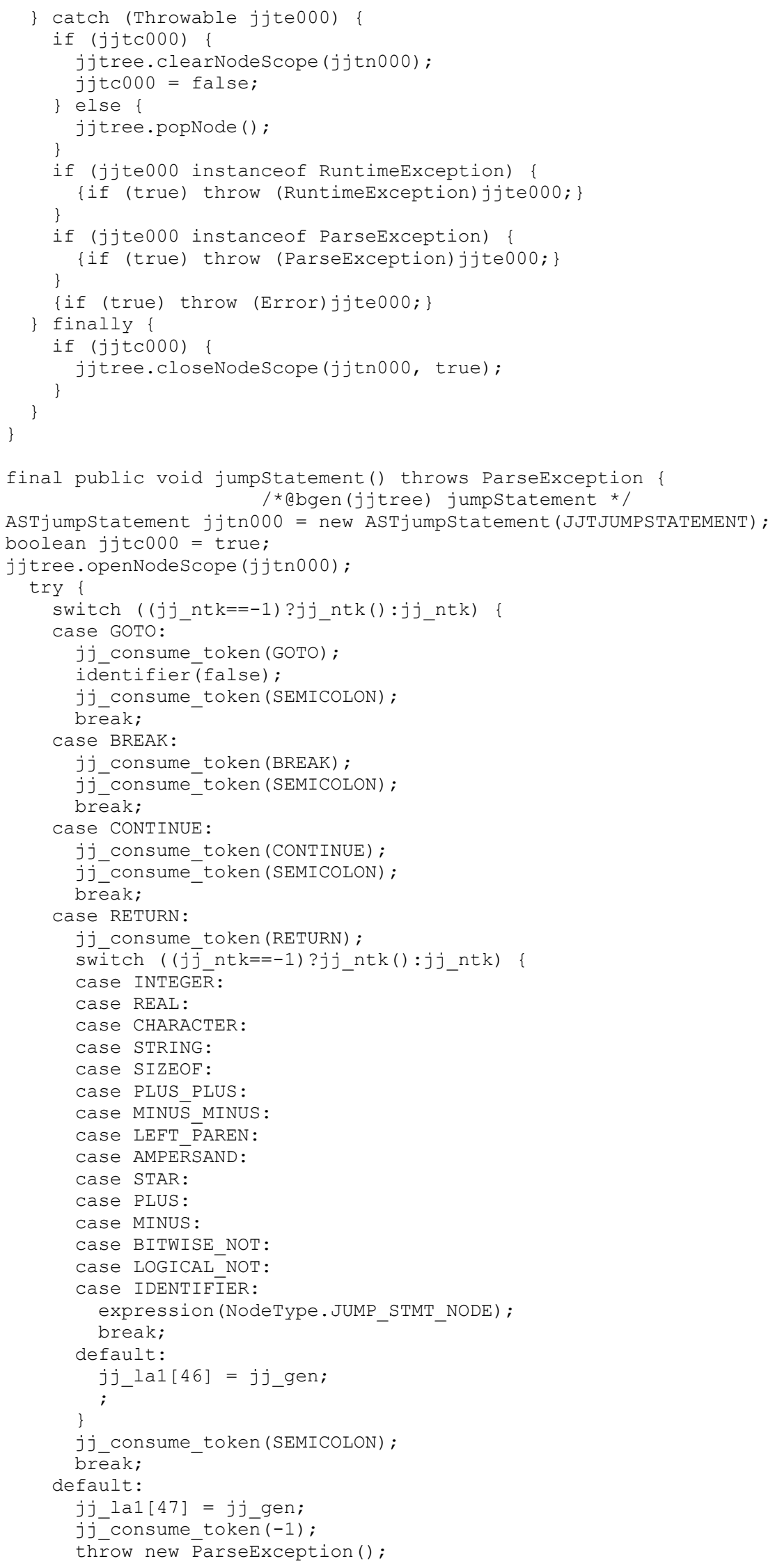




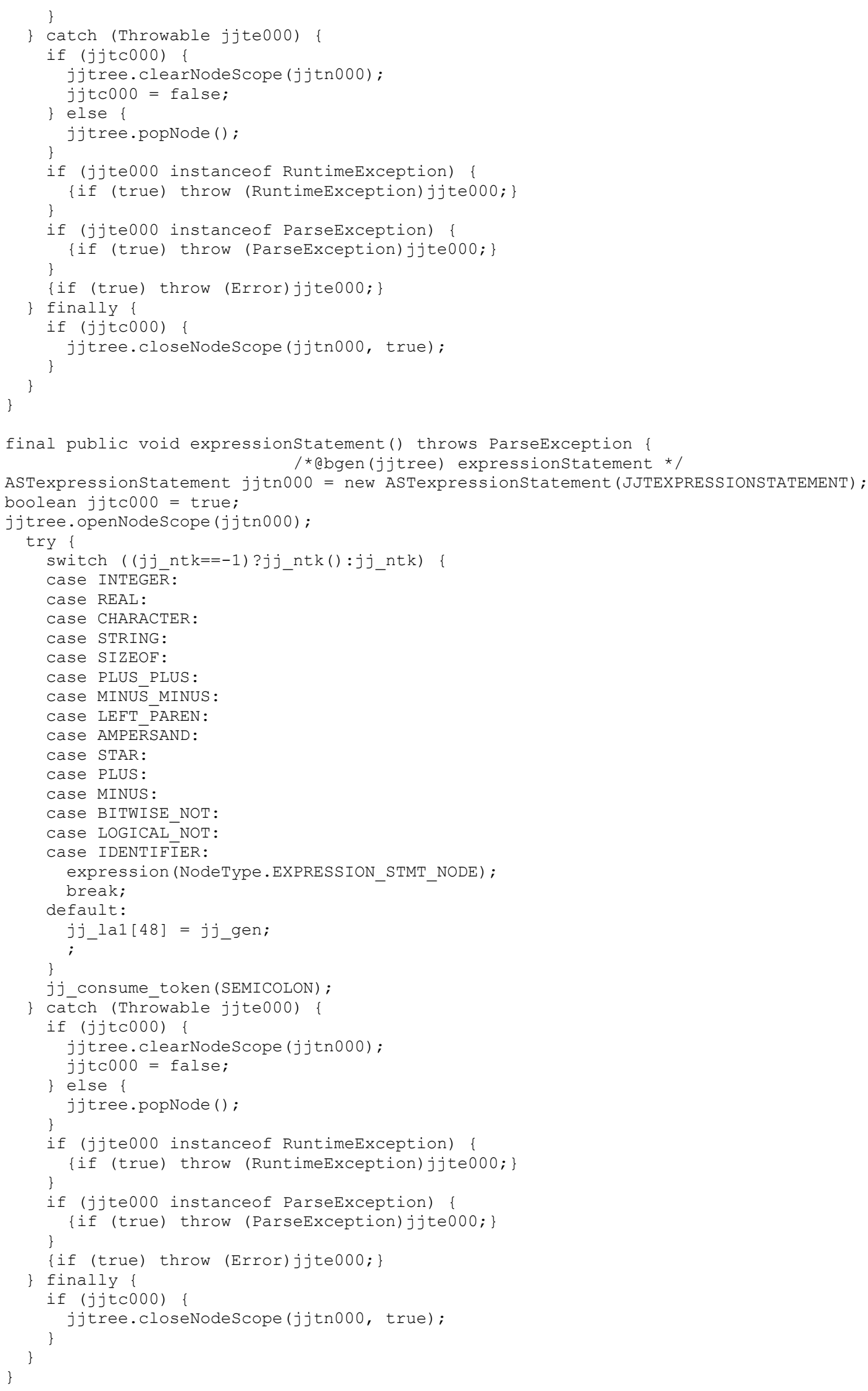




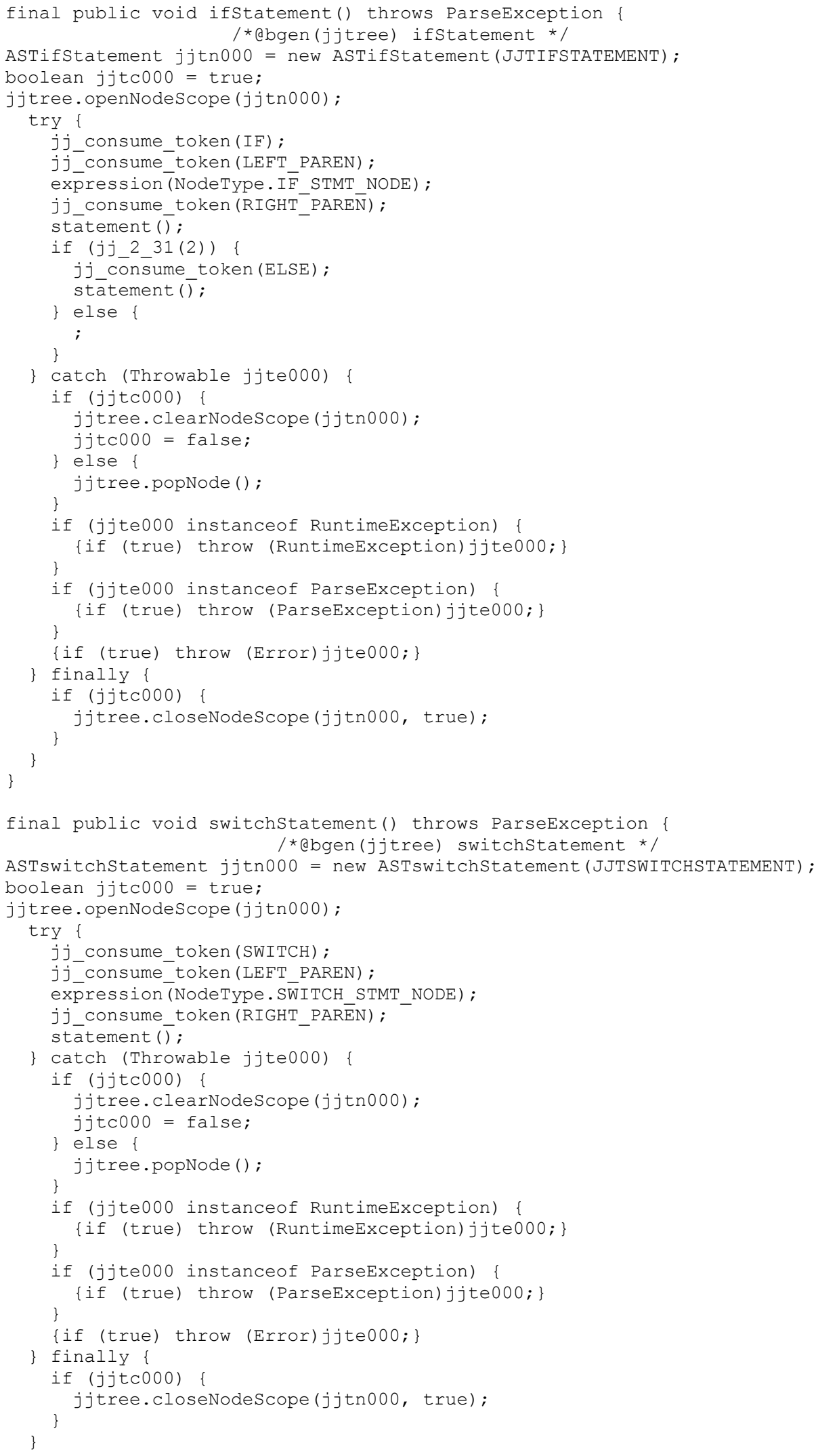




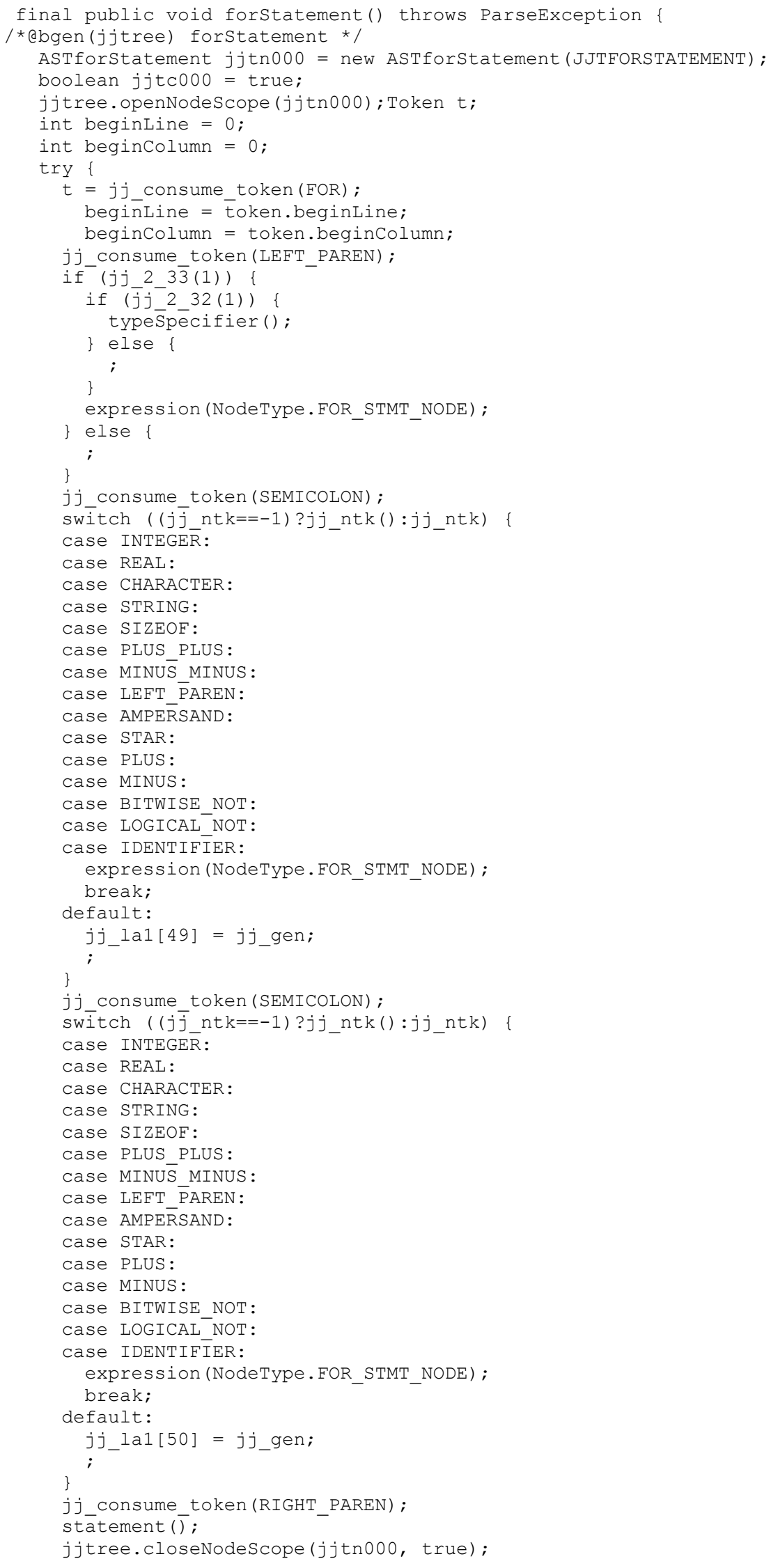




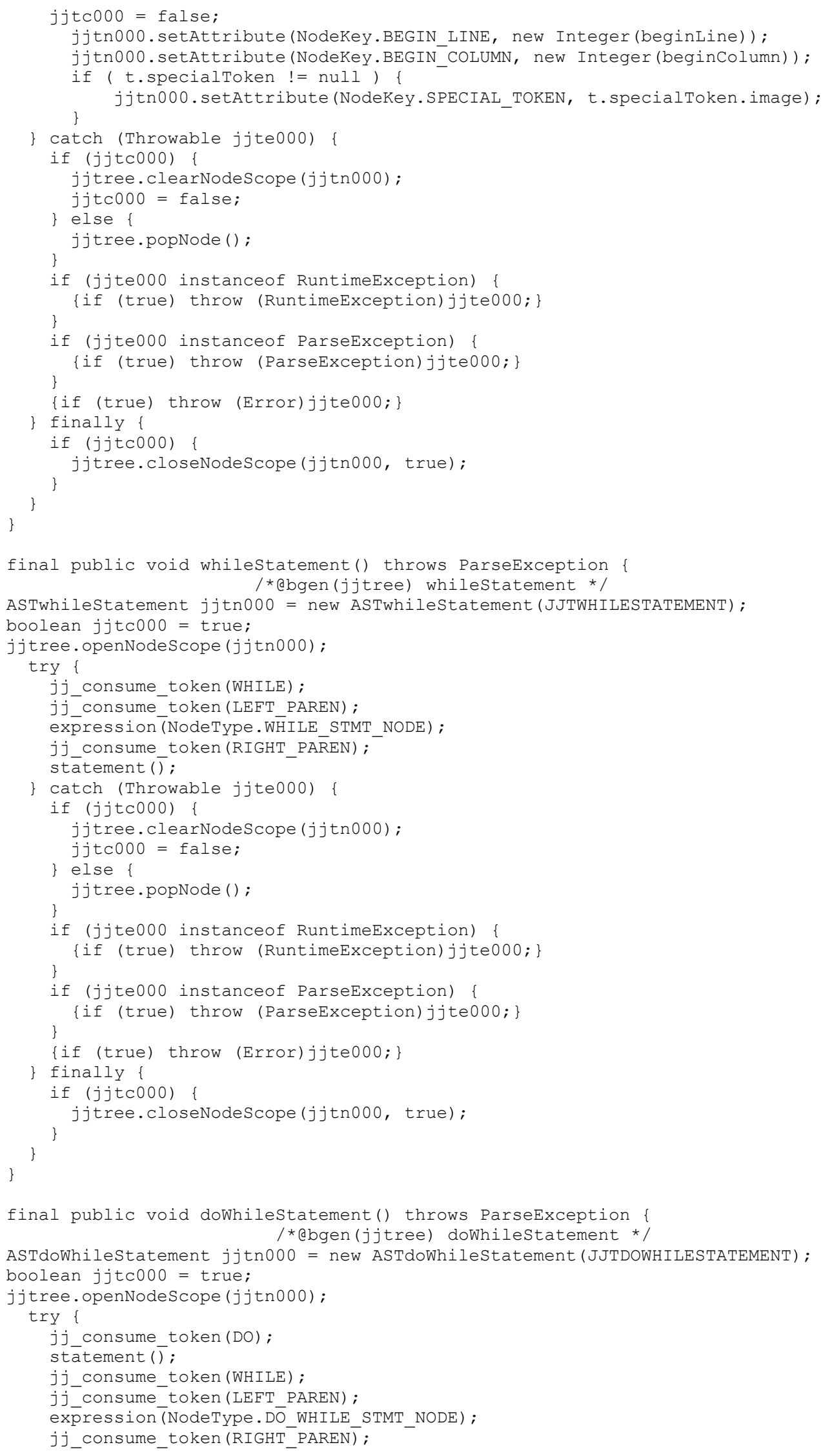




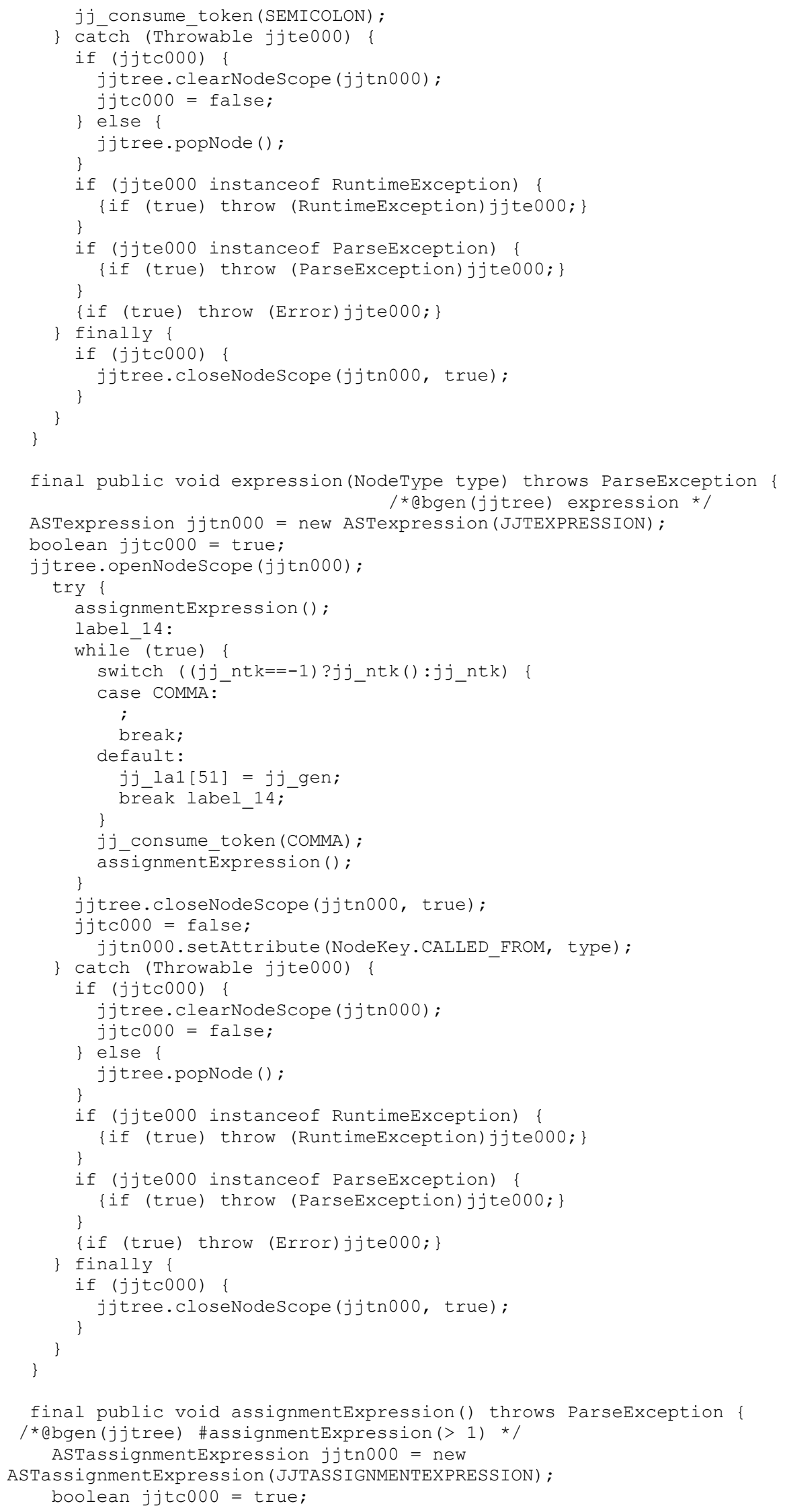




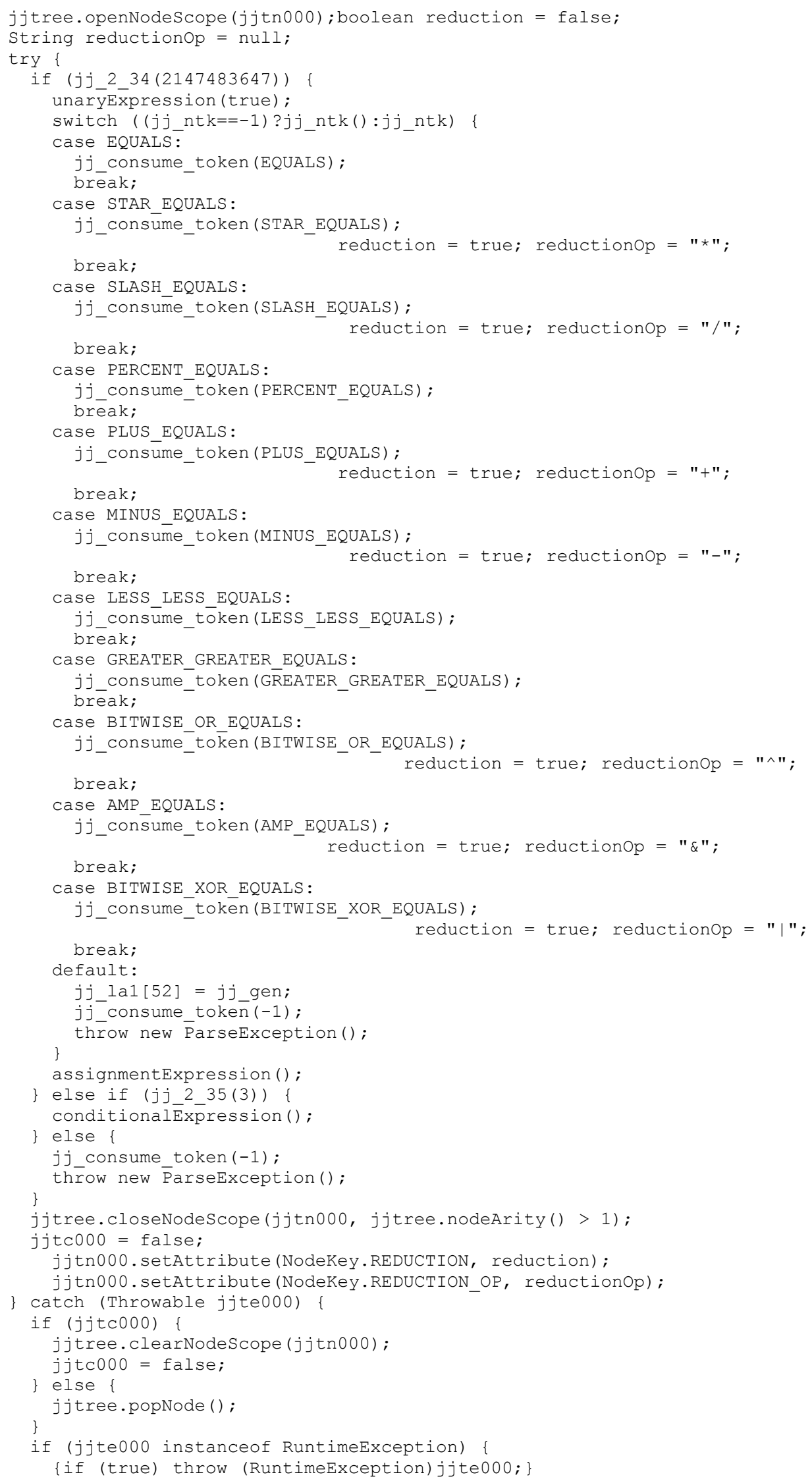




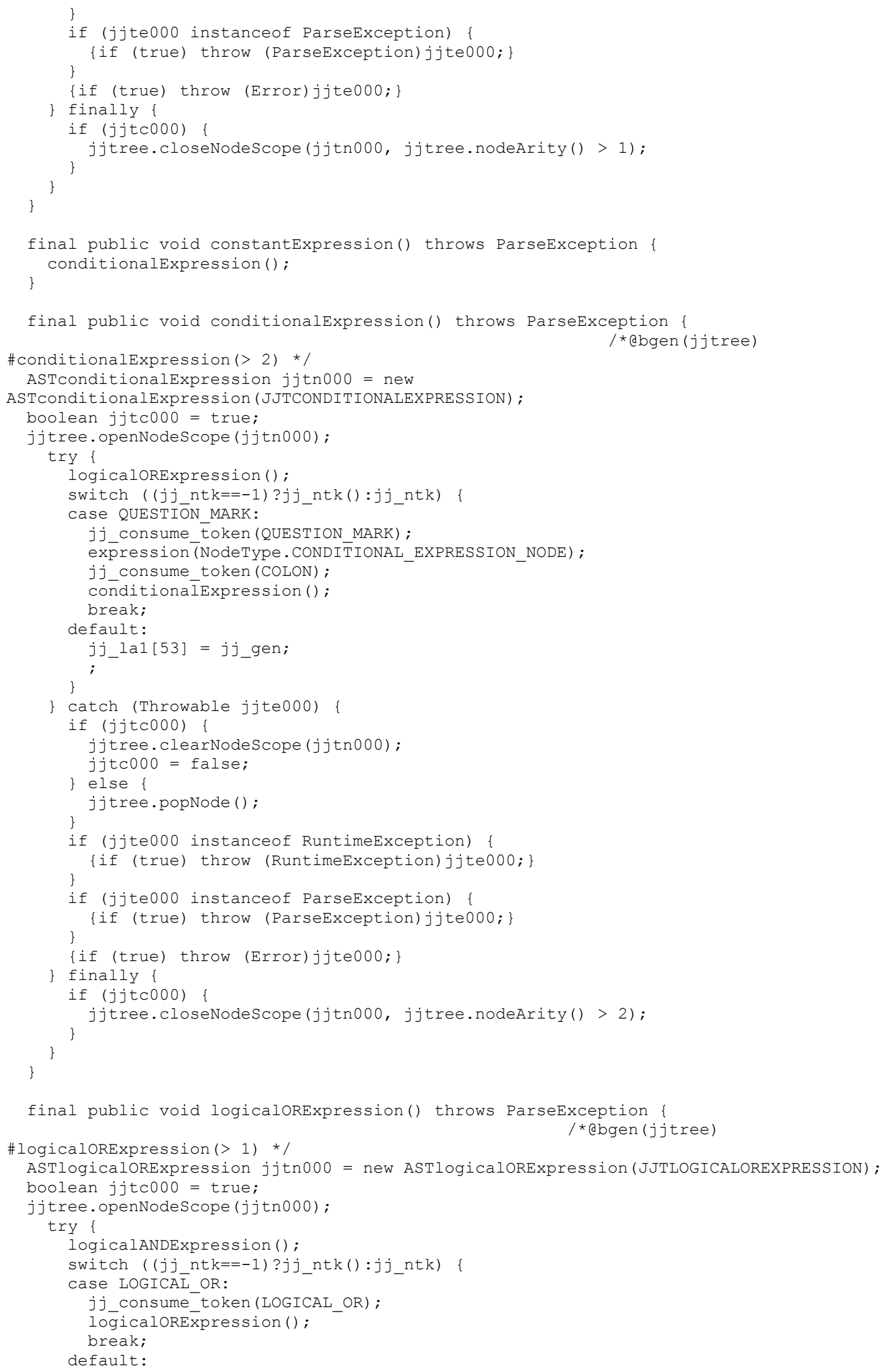




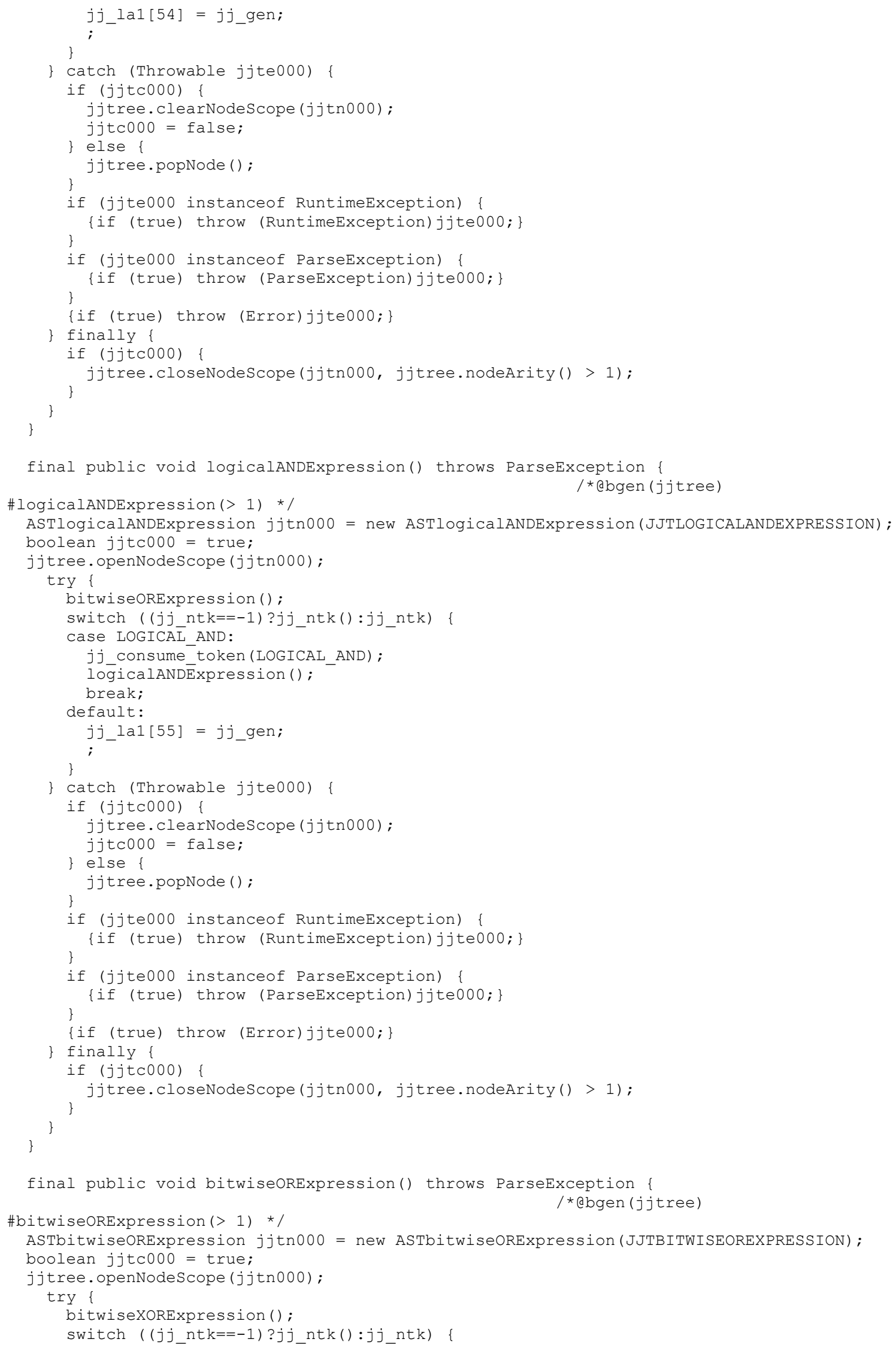




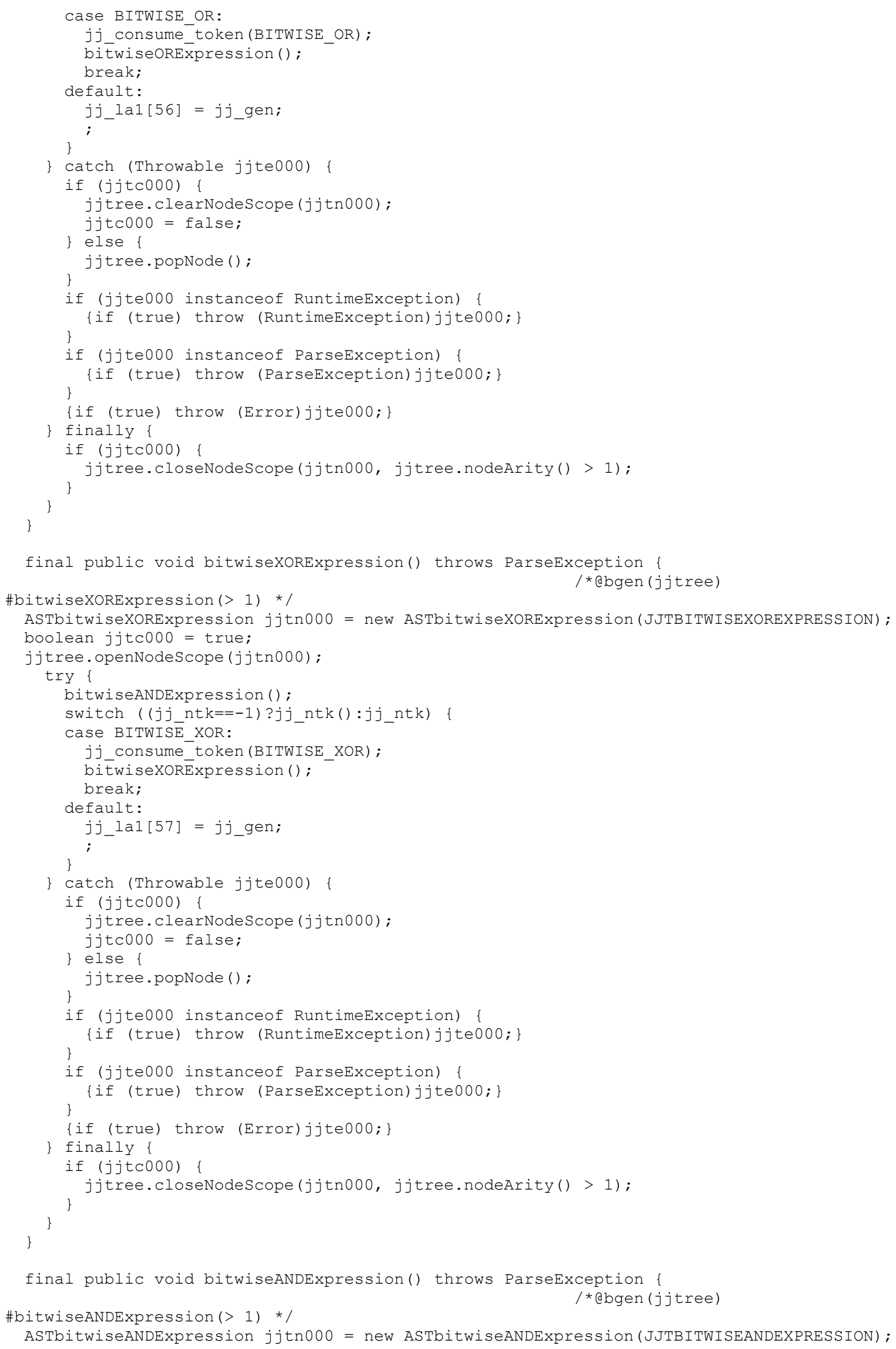




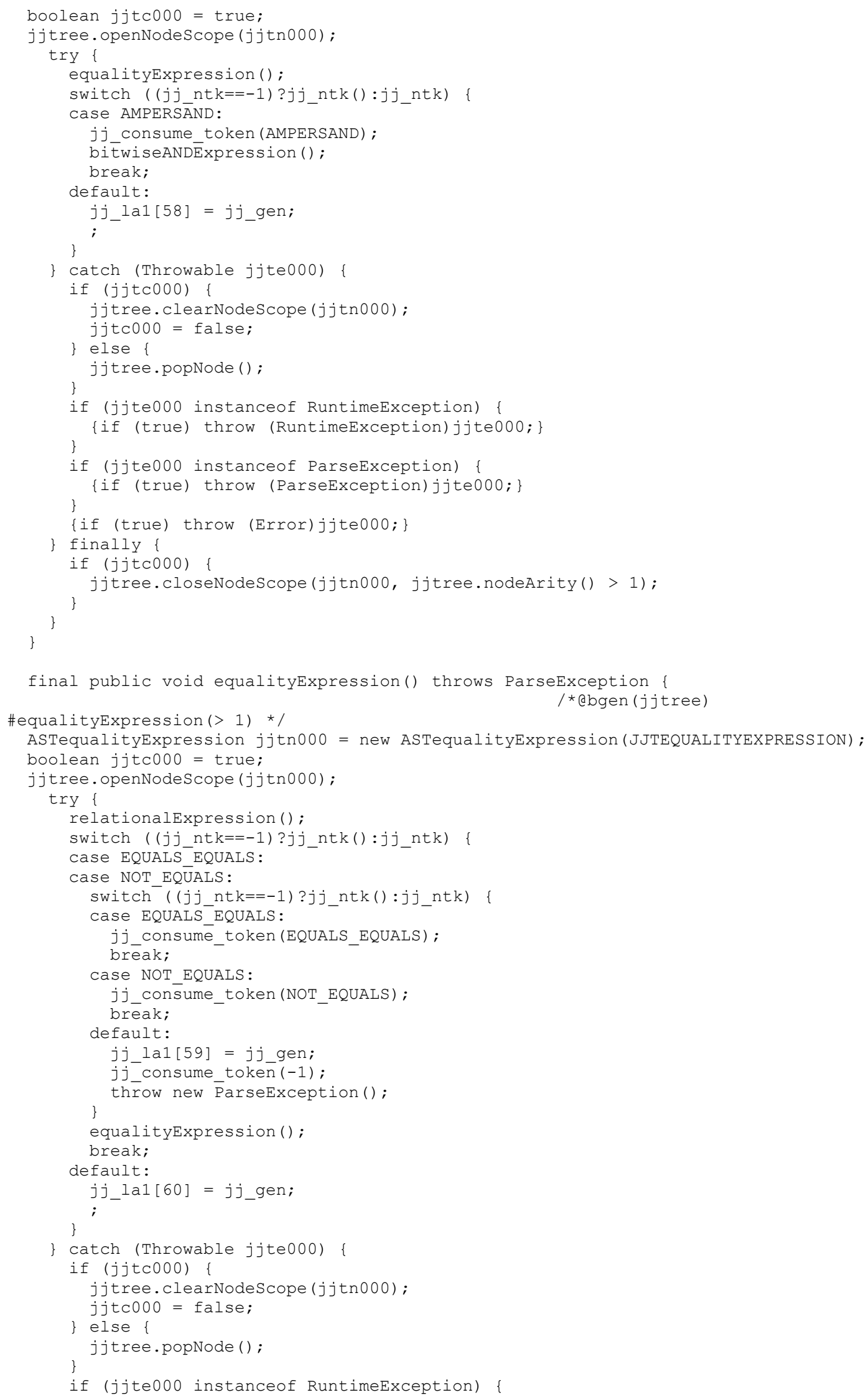




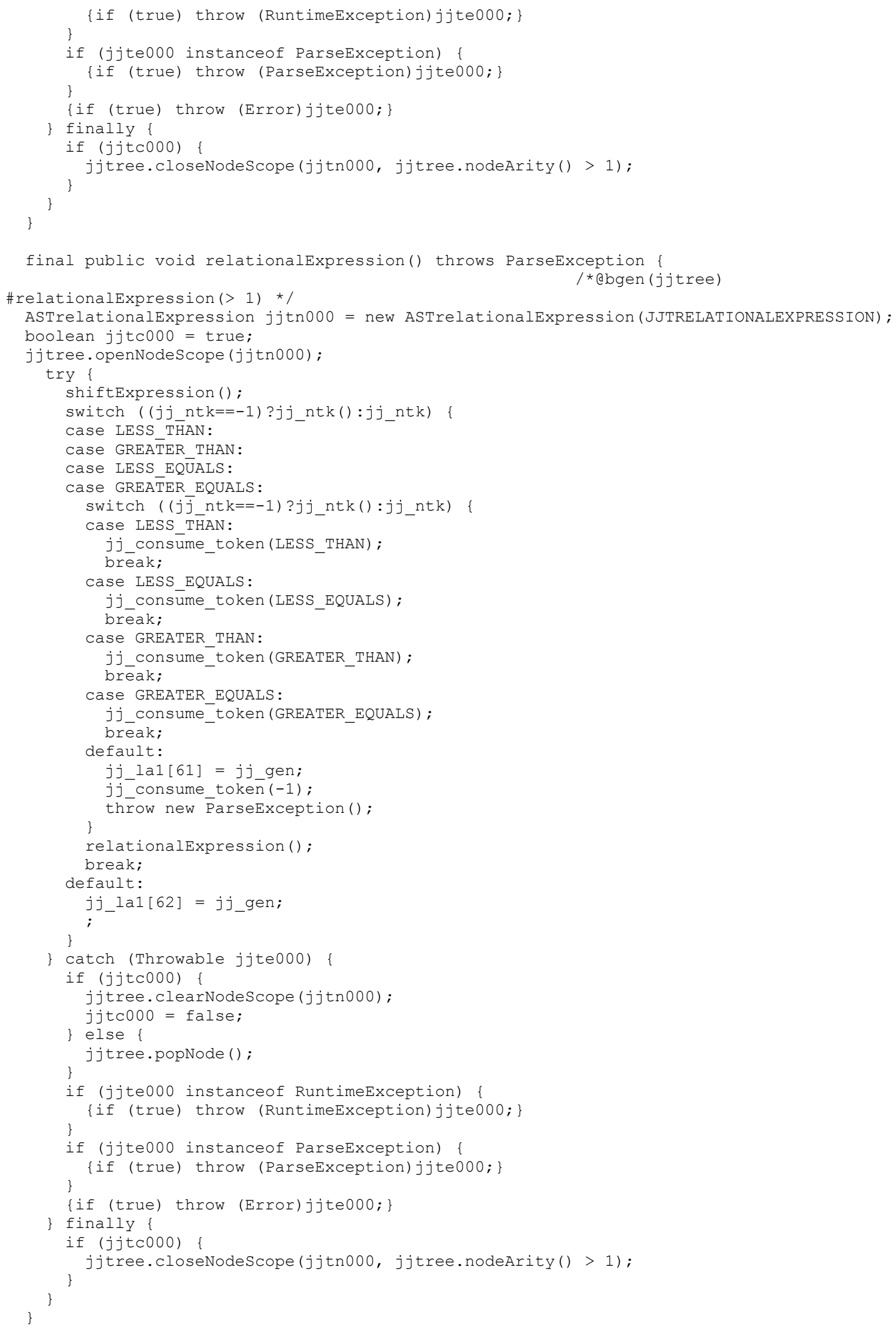




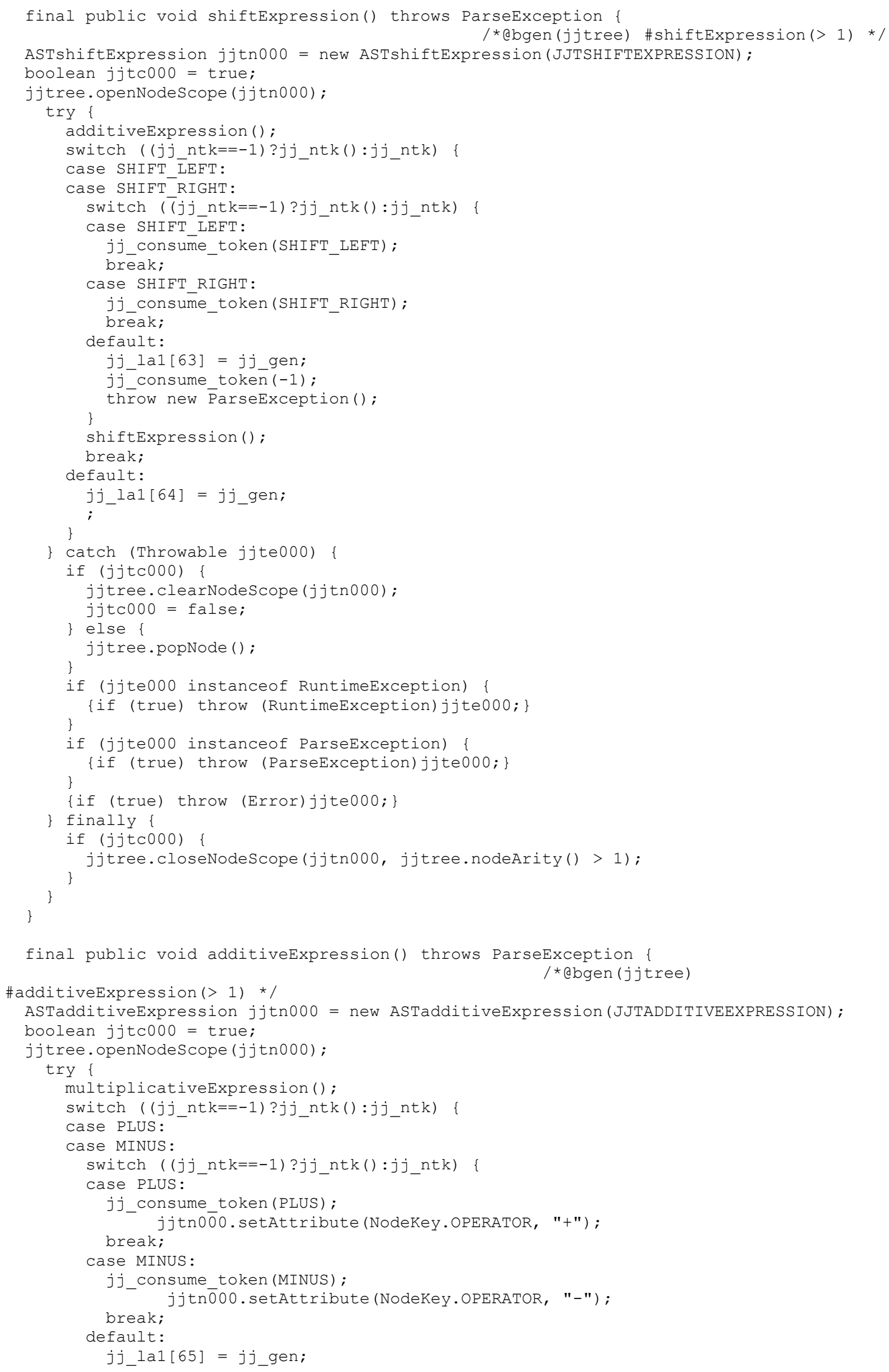




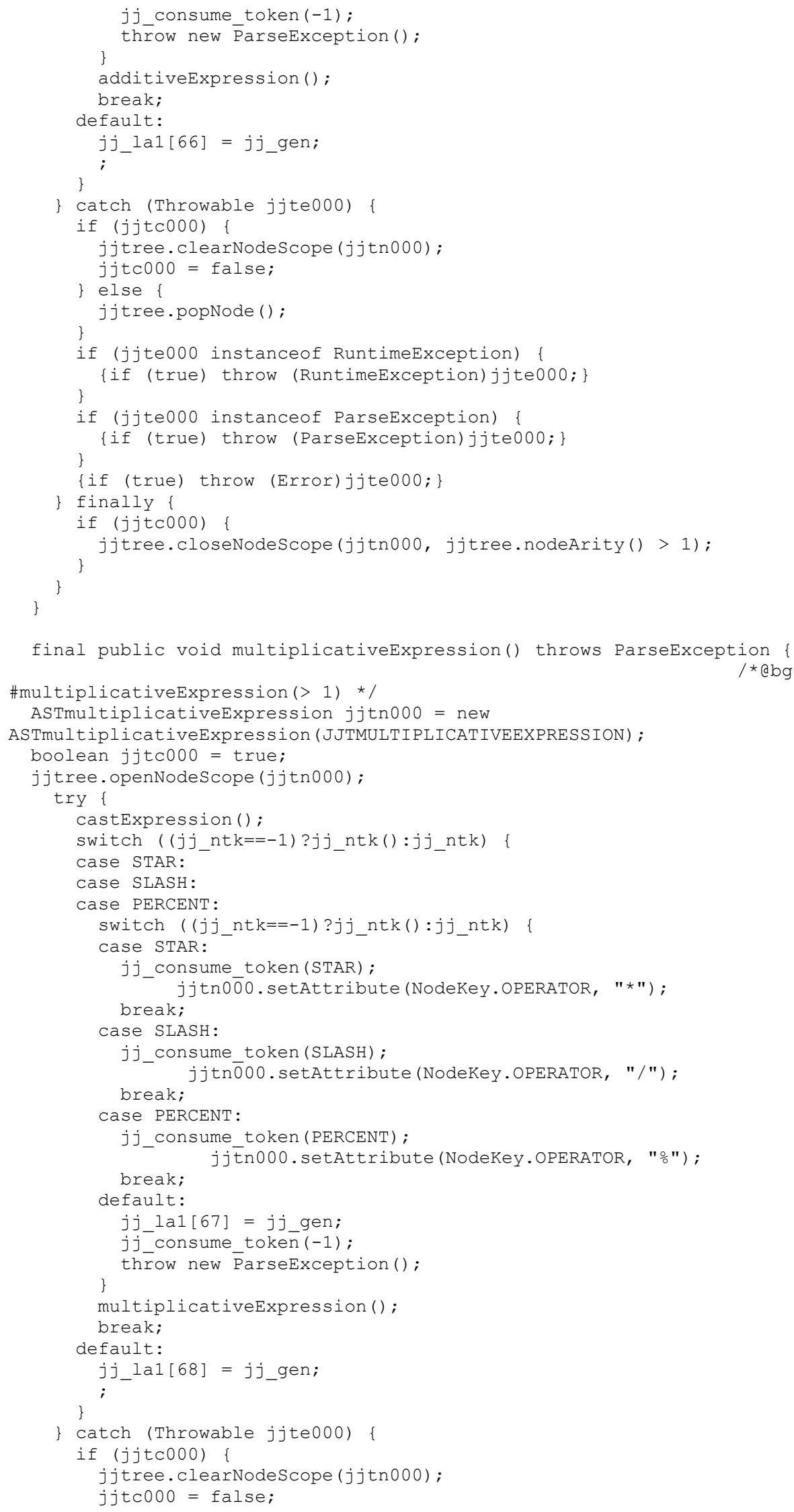




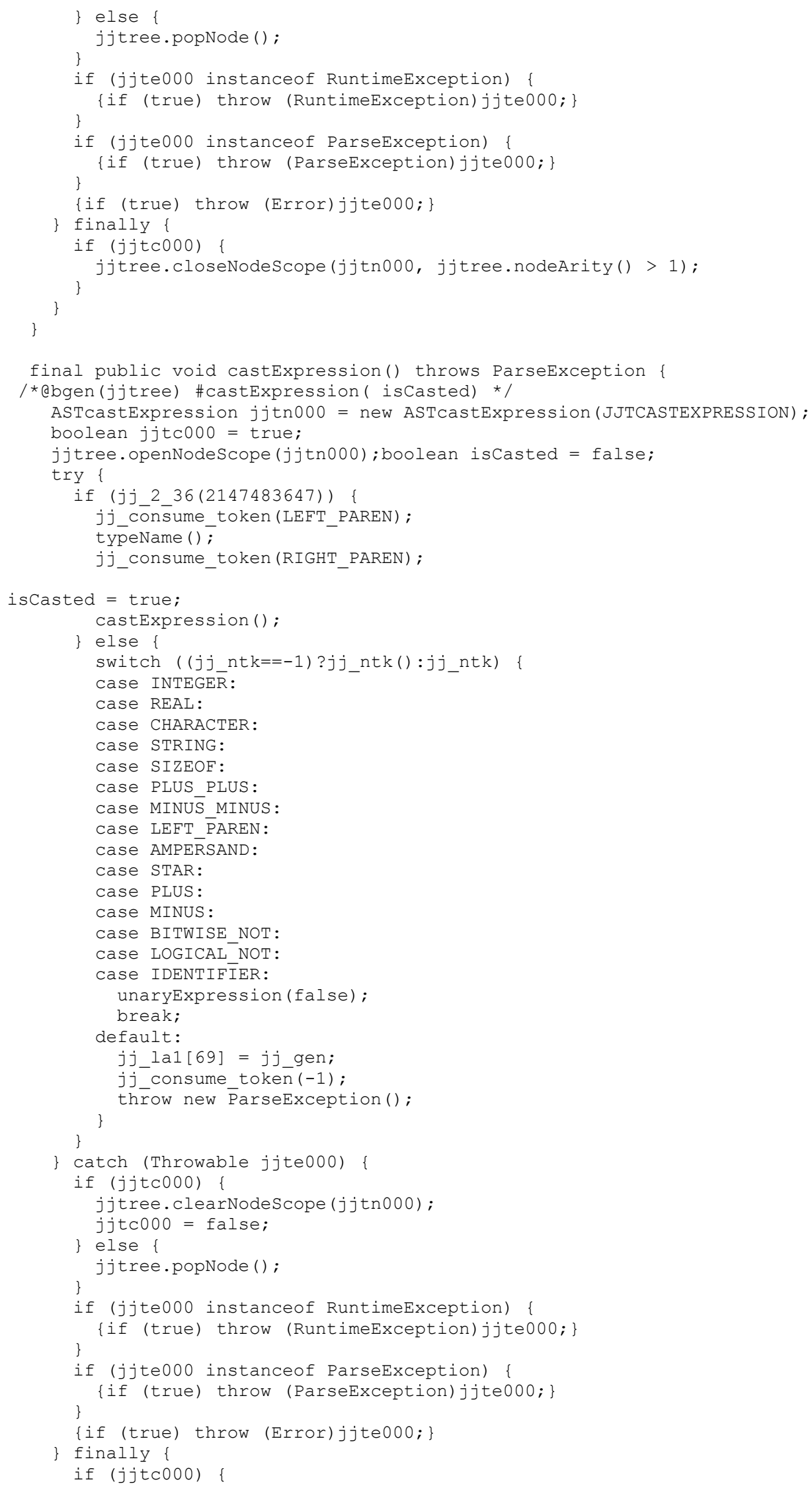




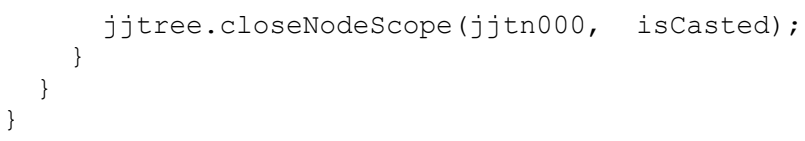

final public boolean unaryExpression(boolean onLeftSideOfAssignment) throws ParseException \{ 


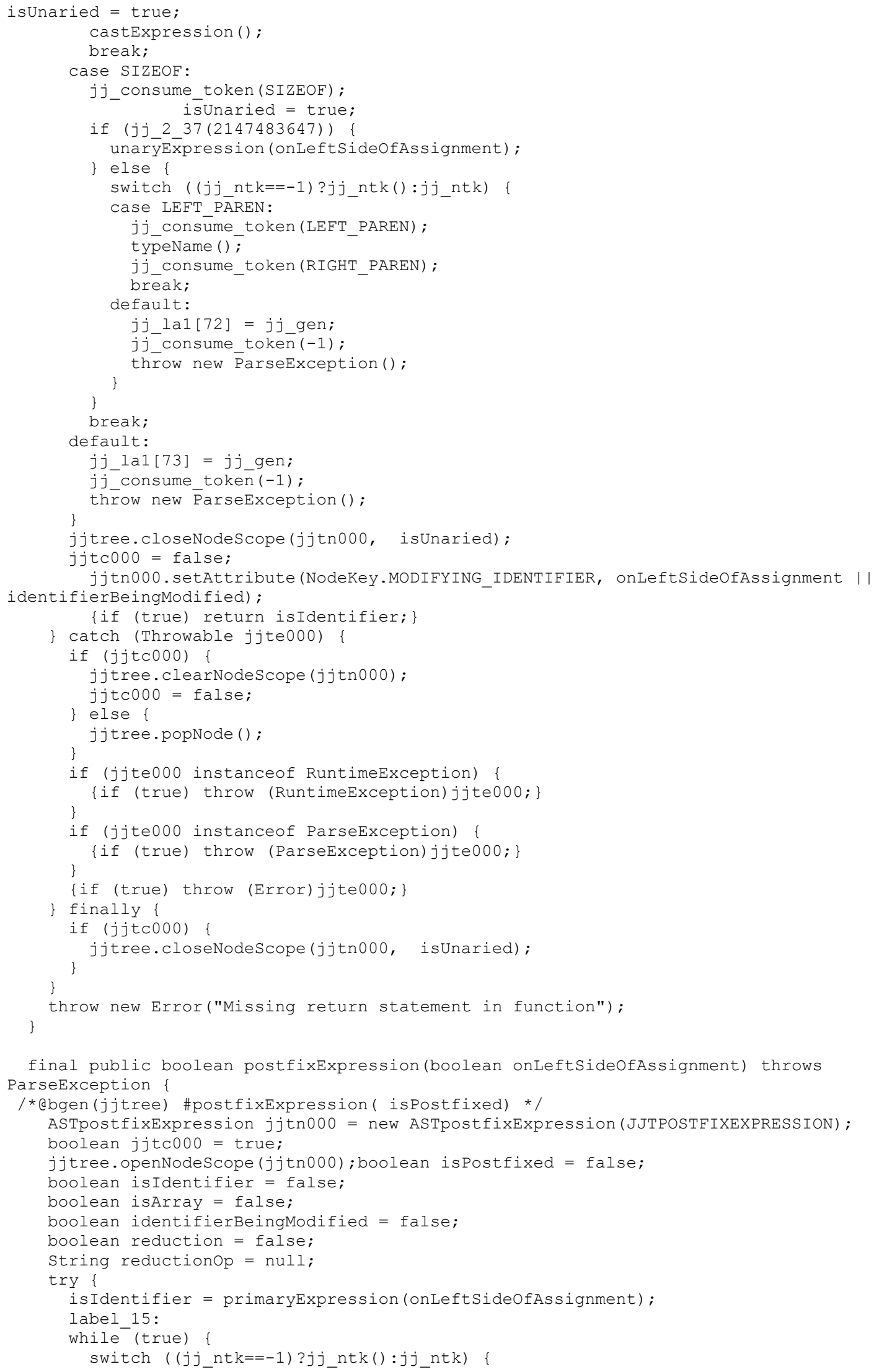




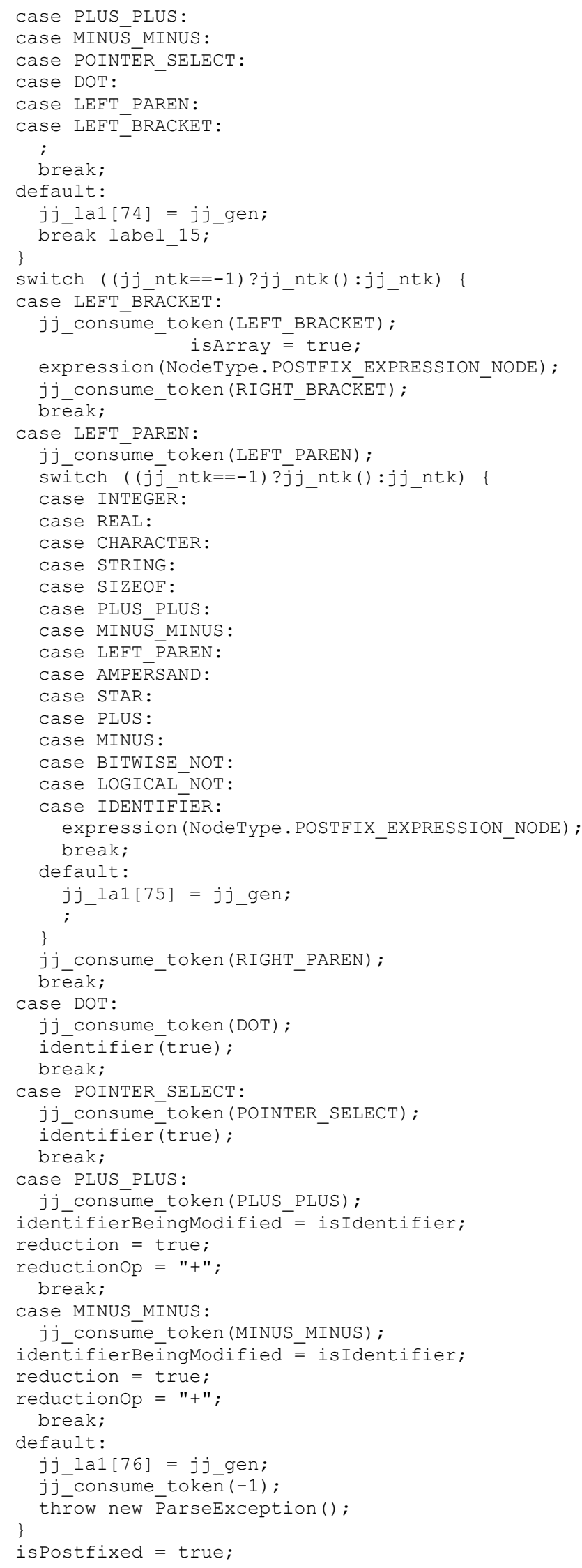




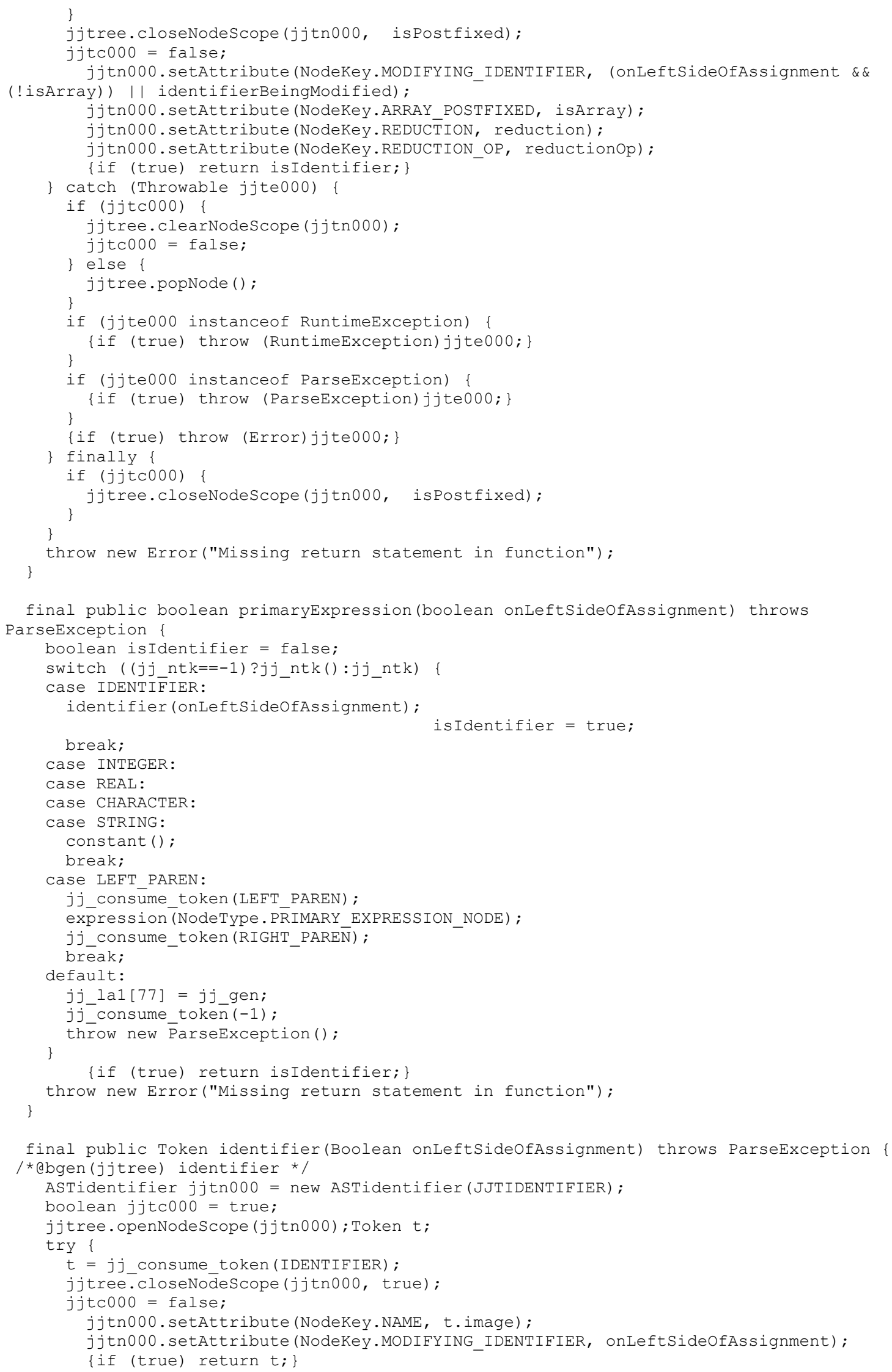




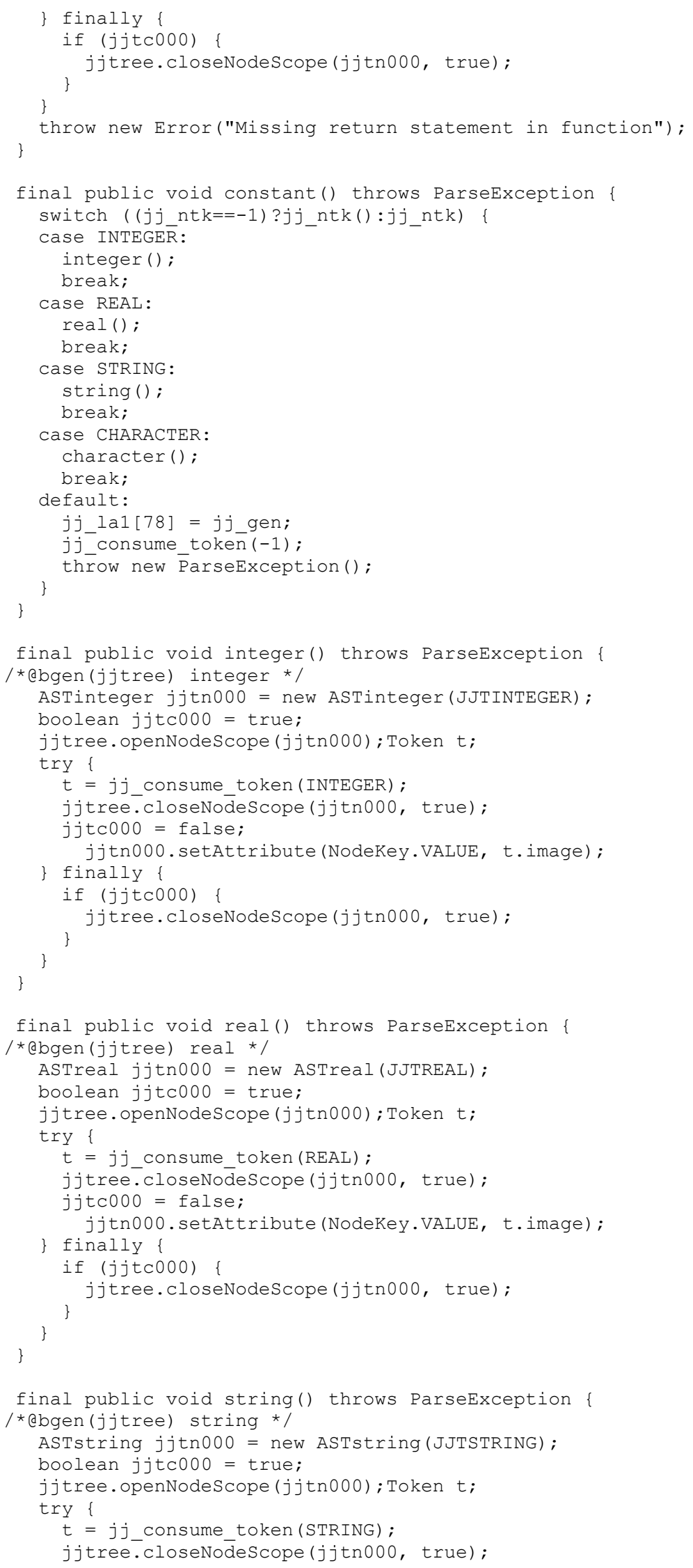




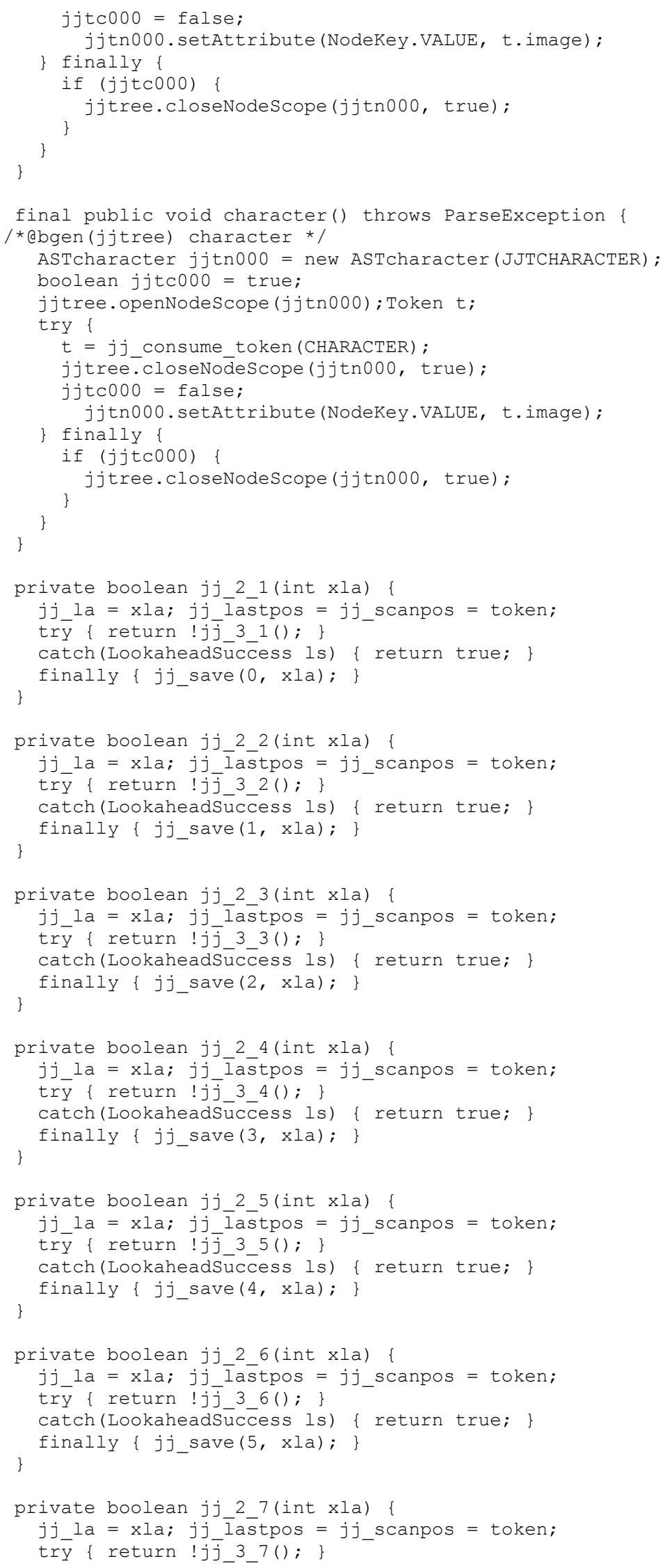




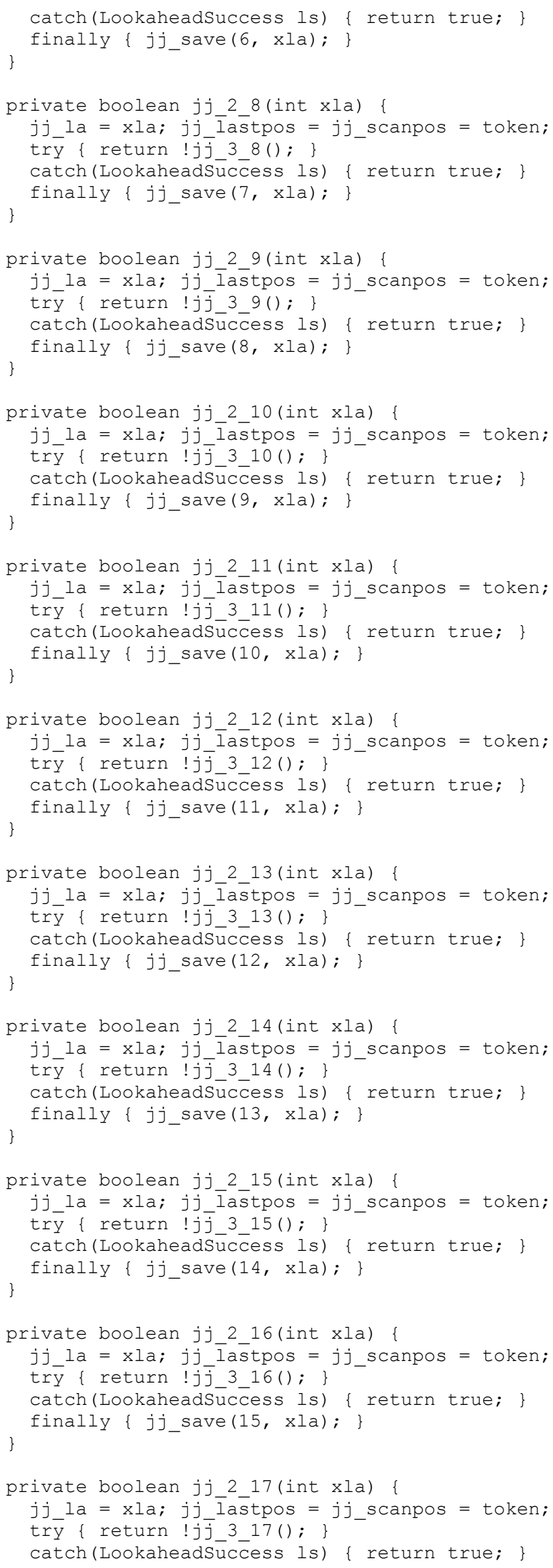




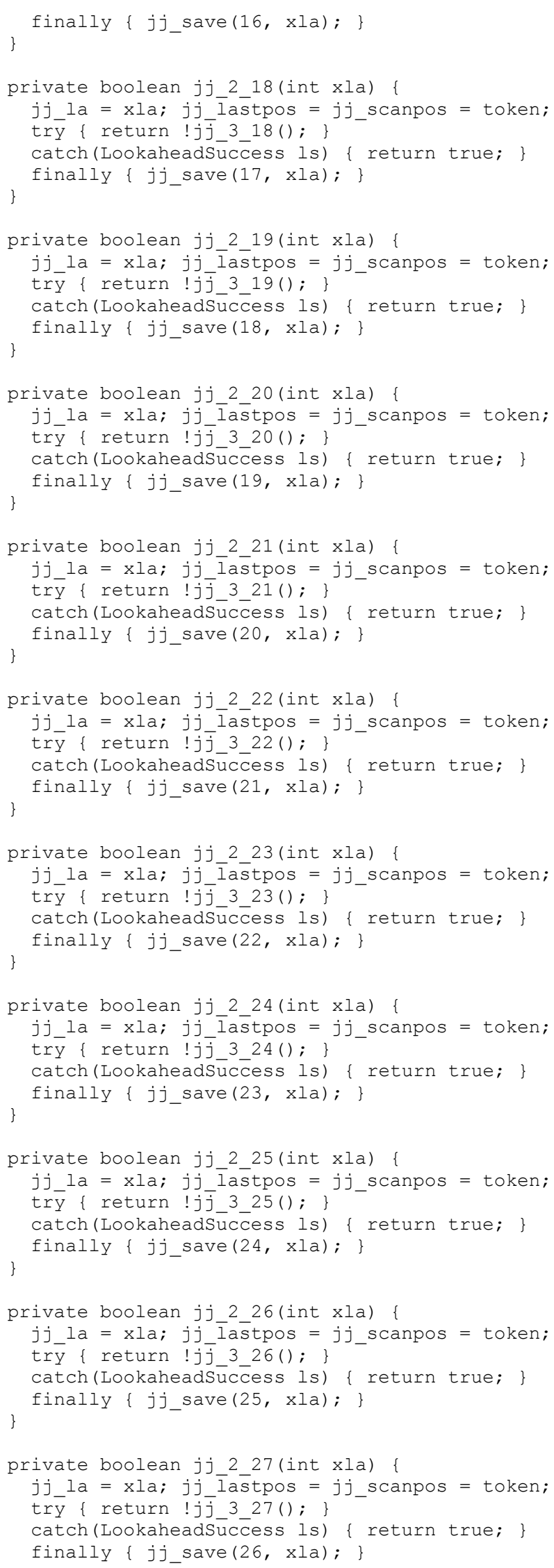




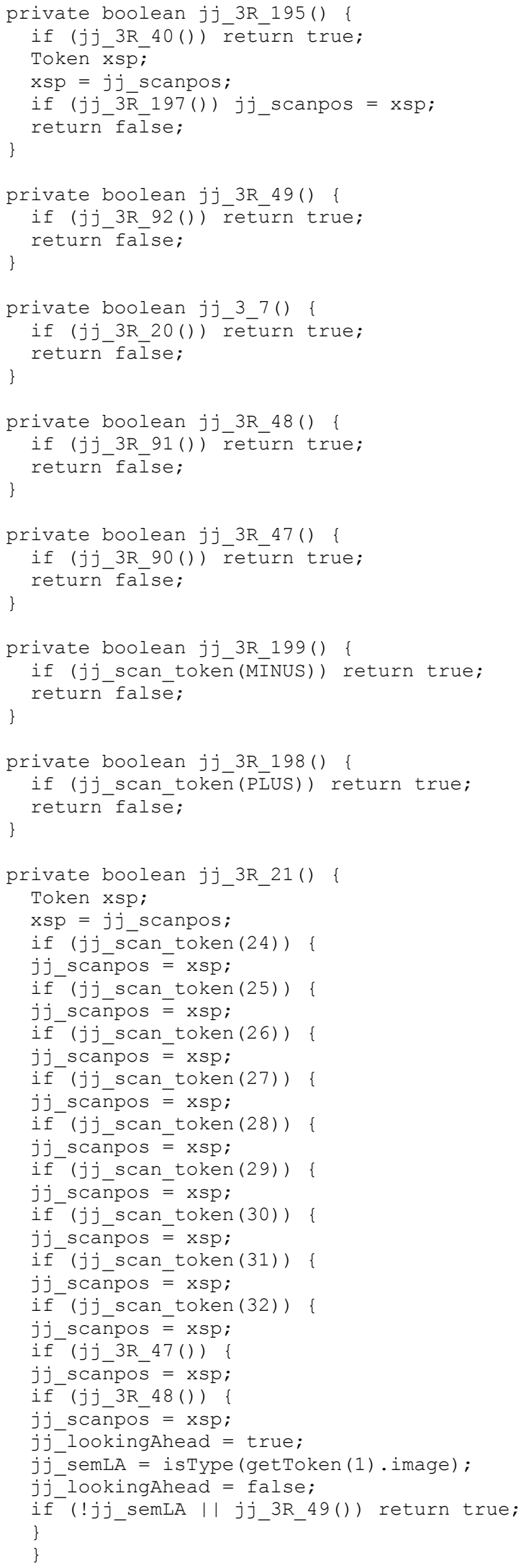




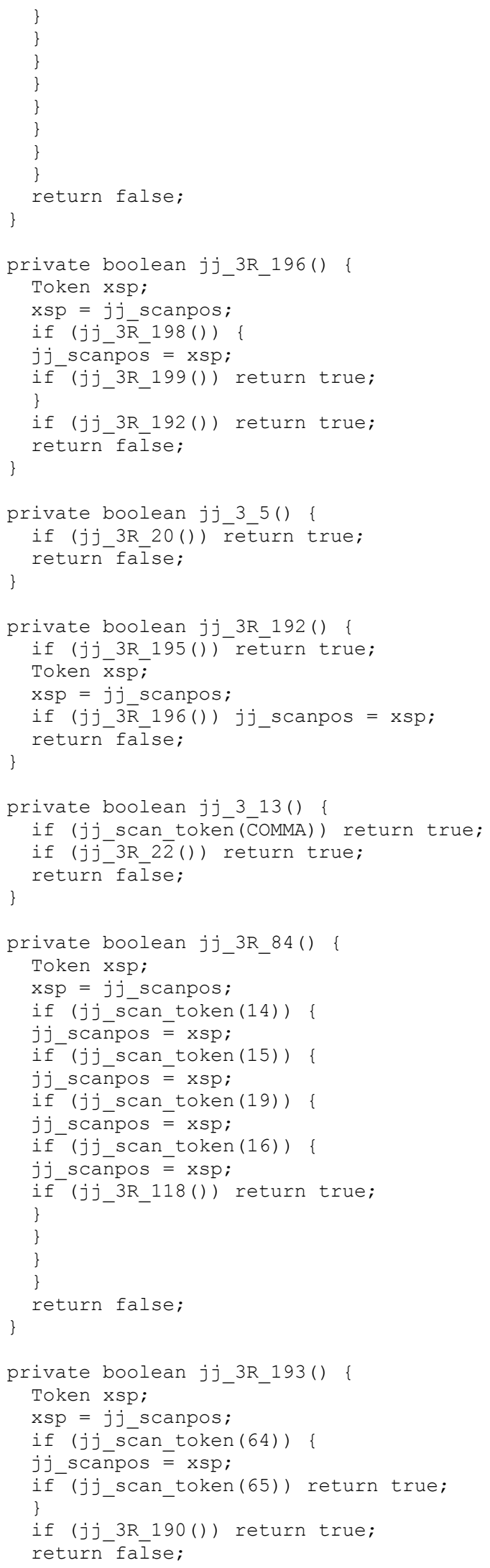




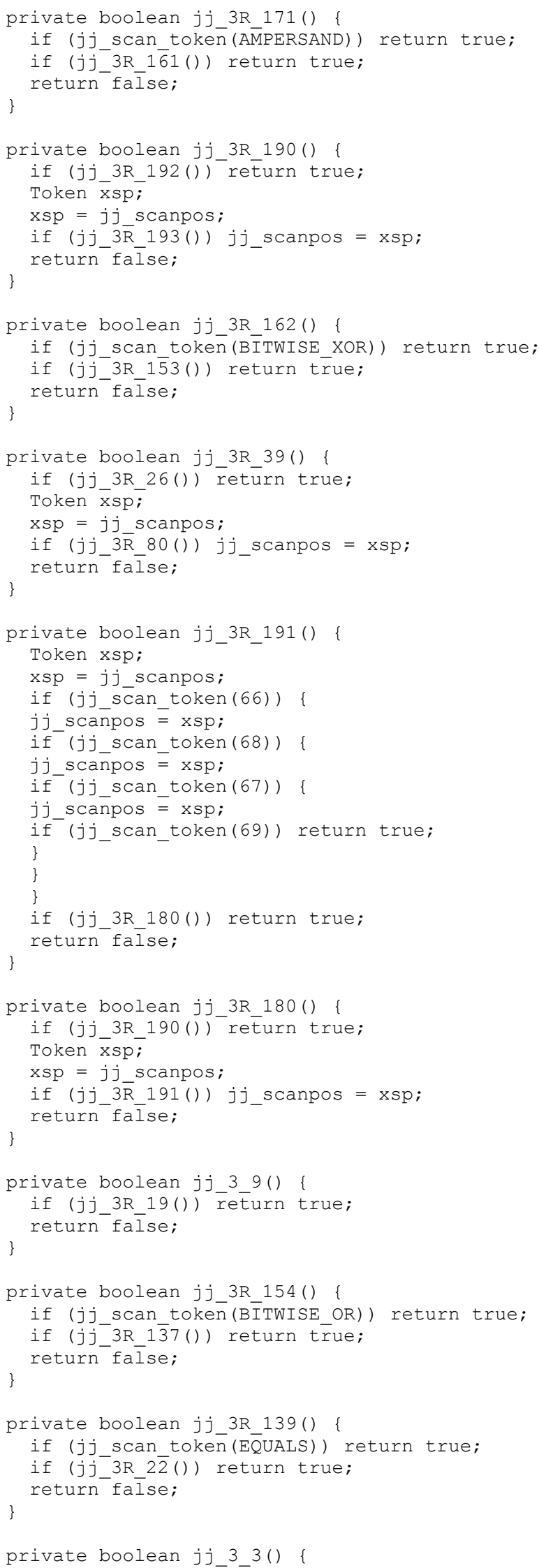




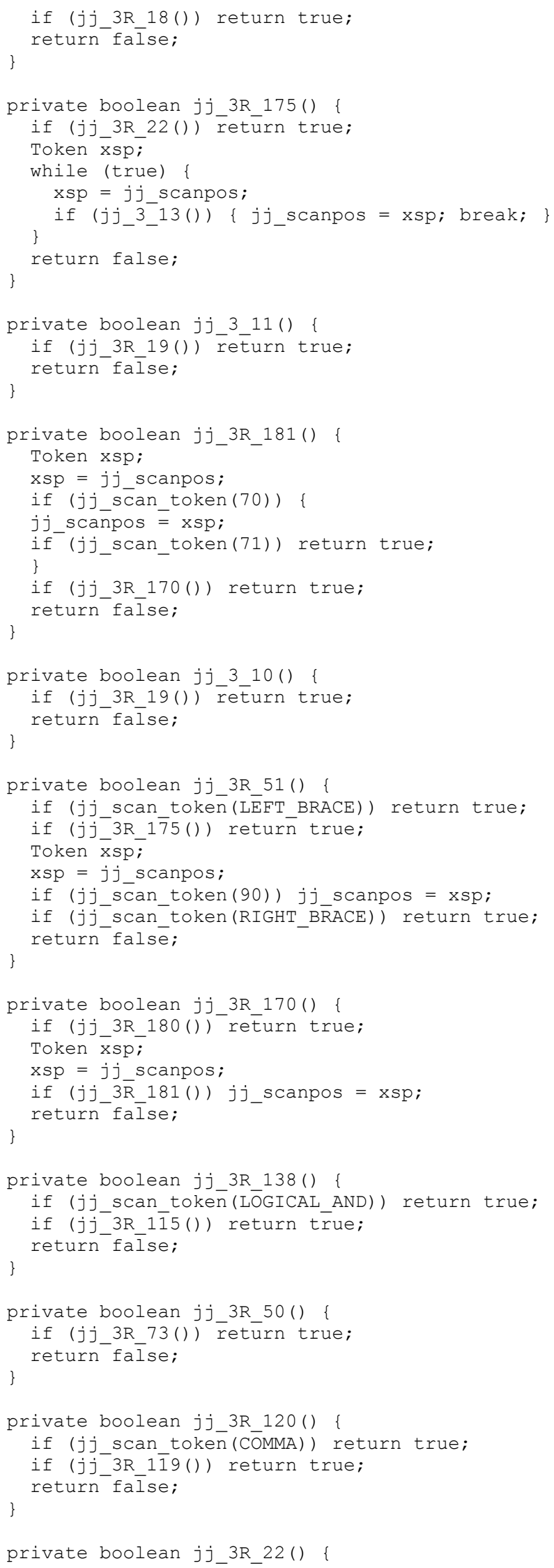




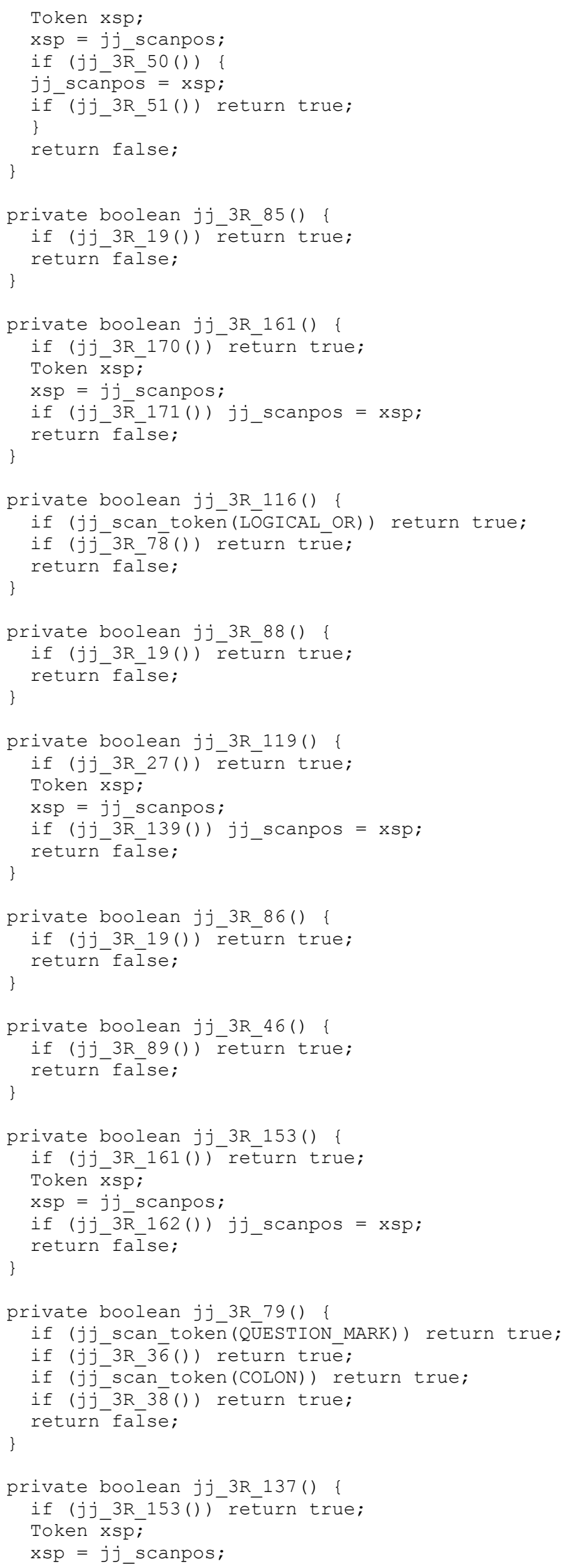




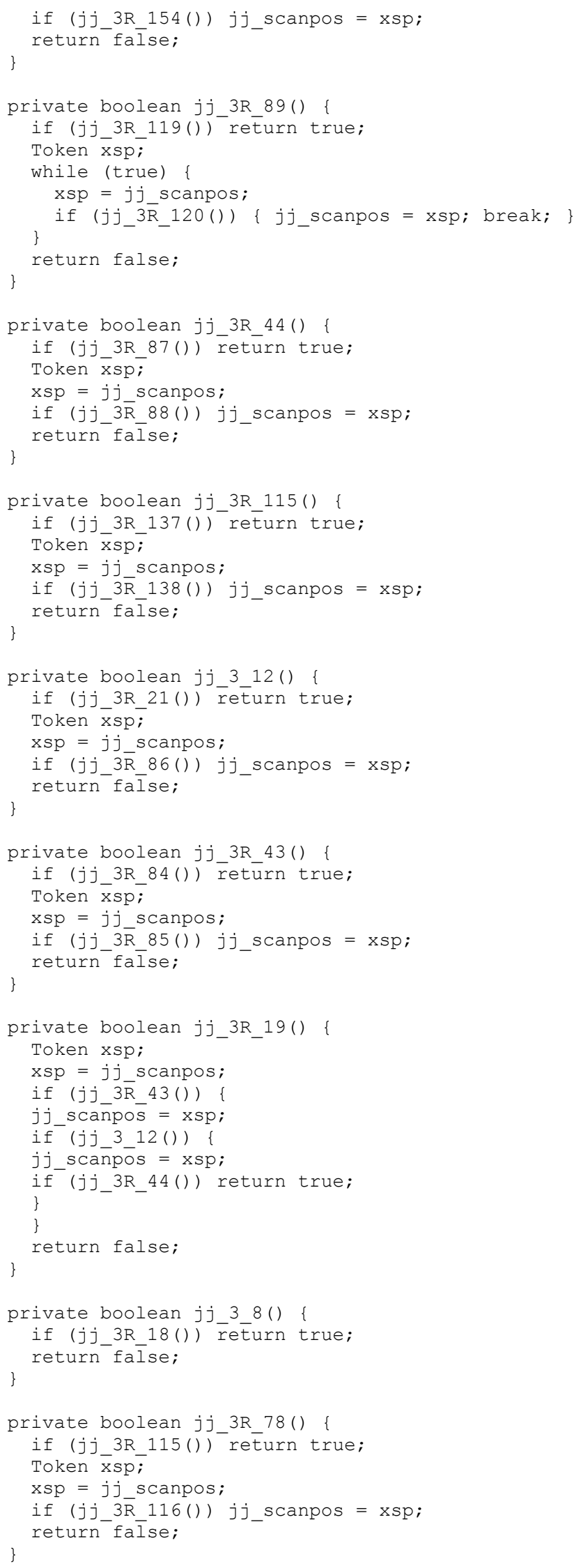




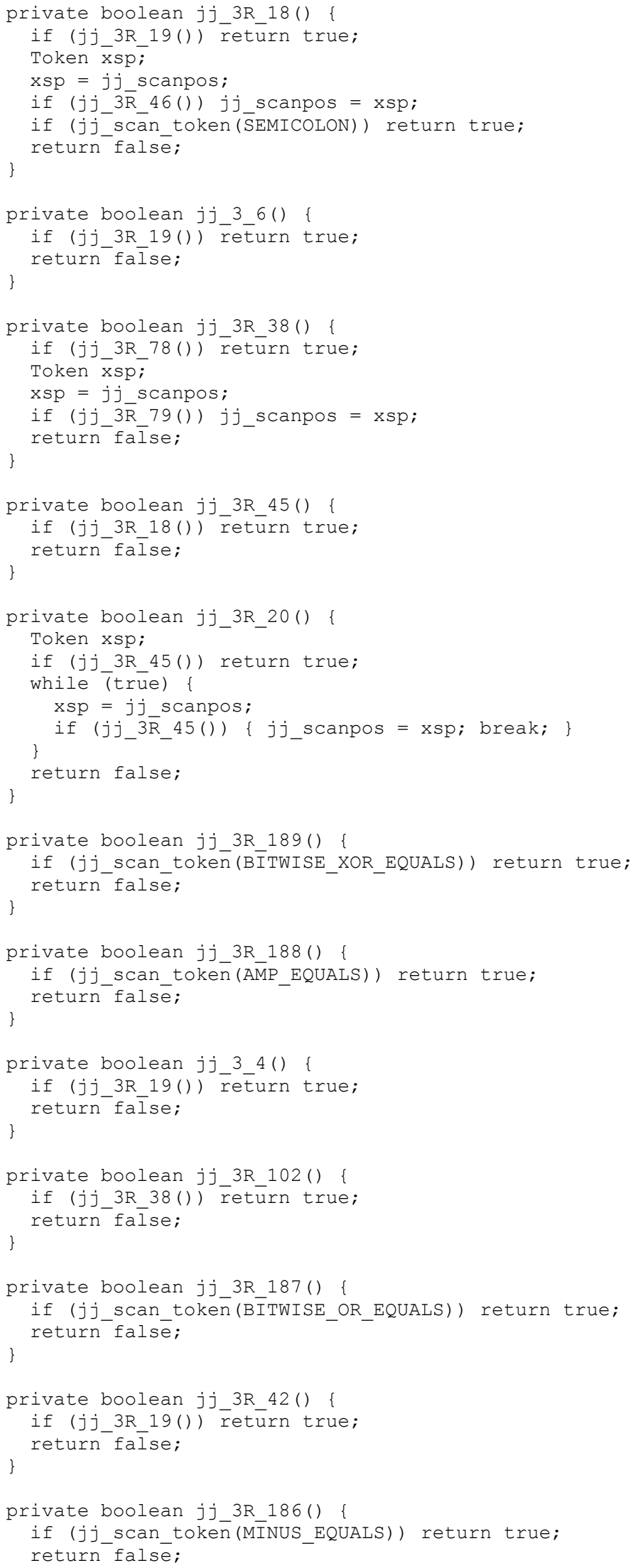




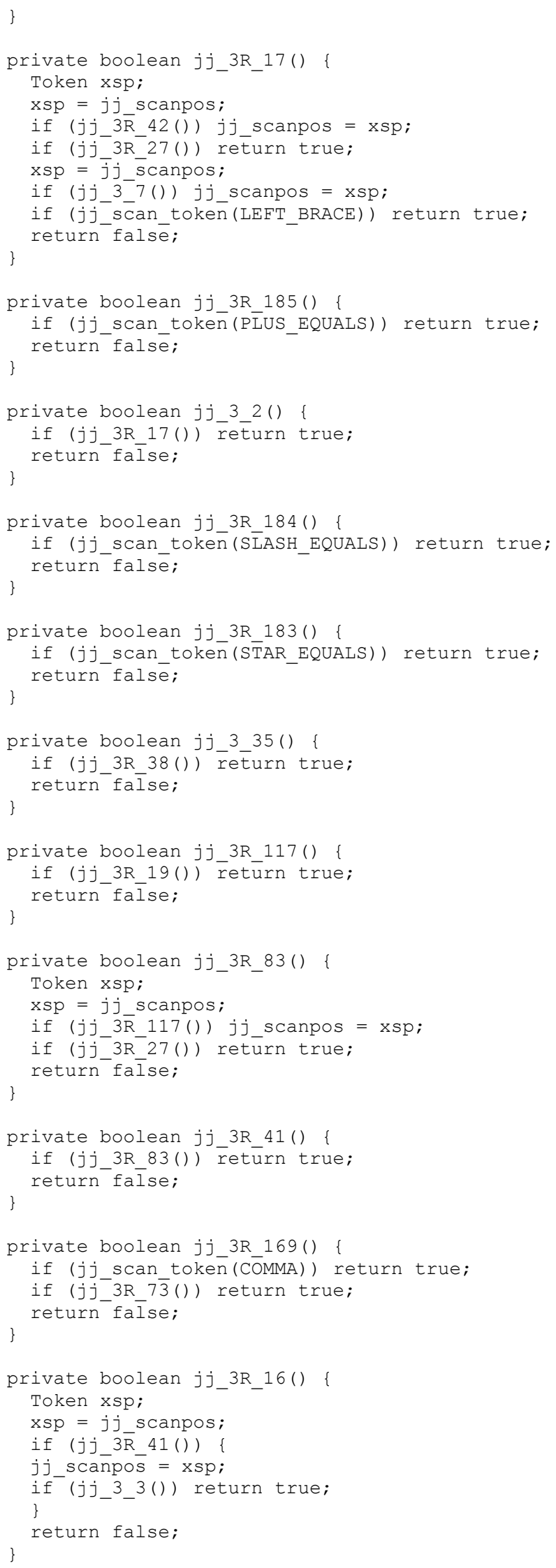




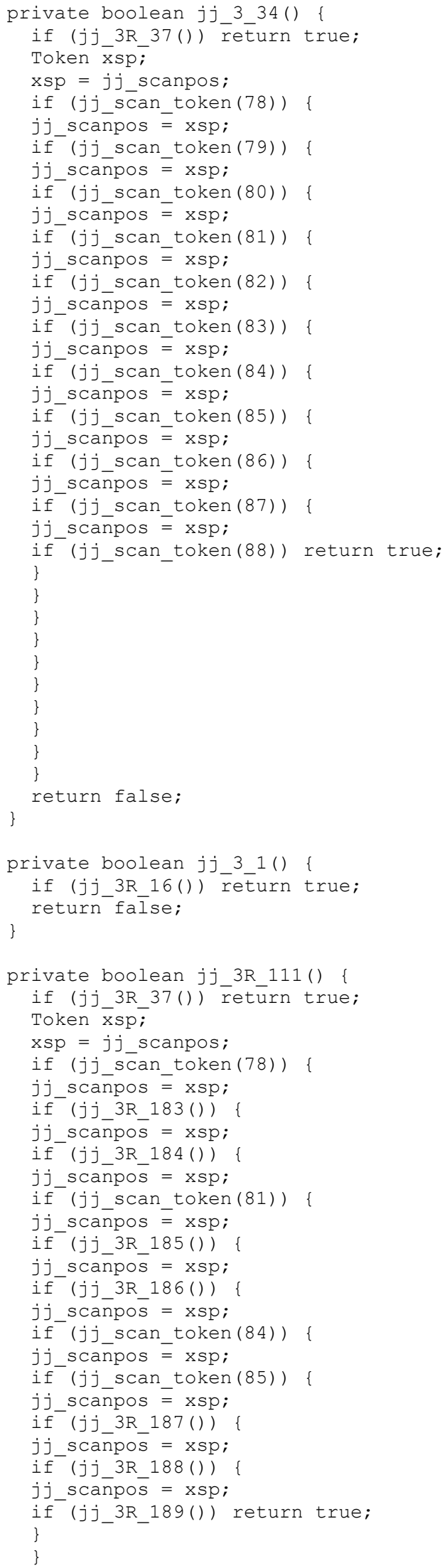




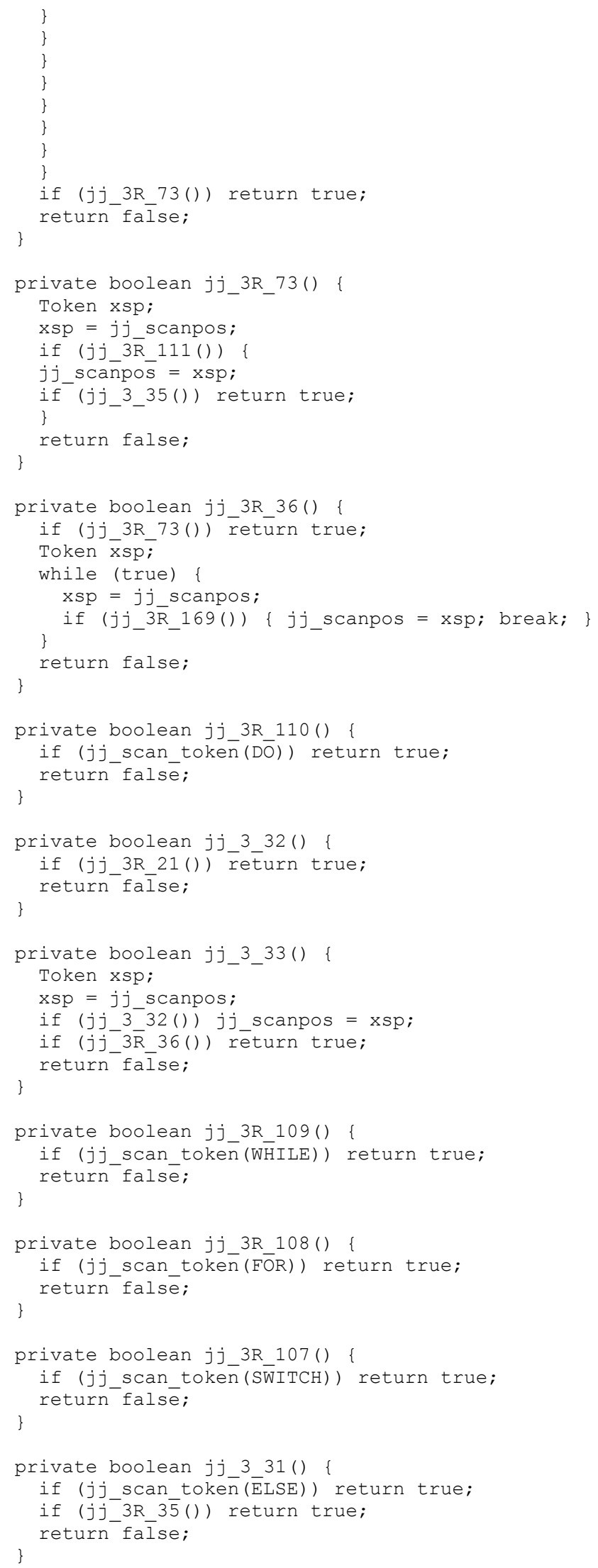




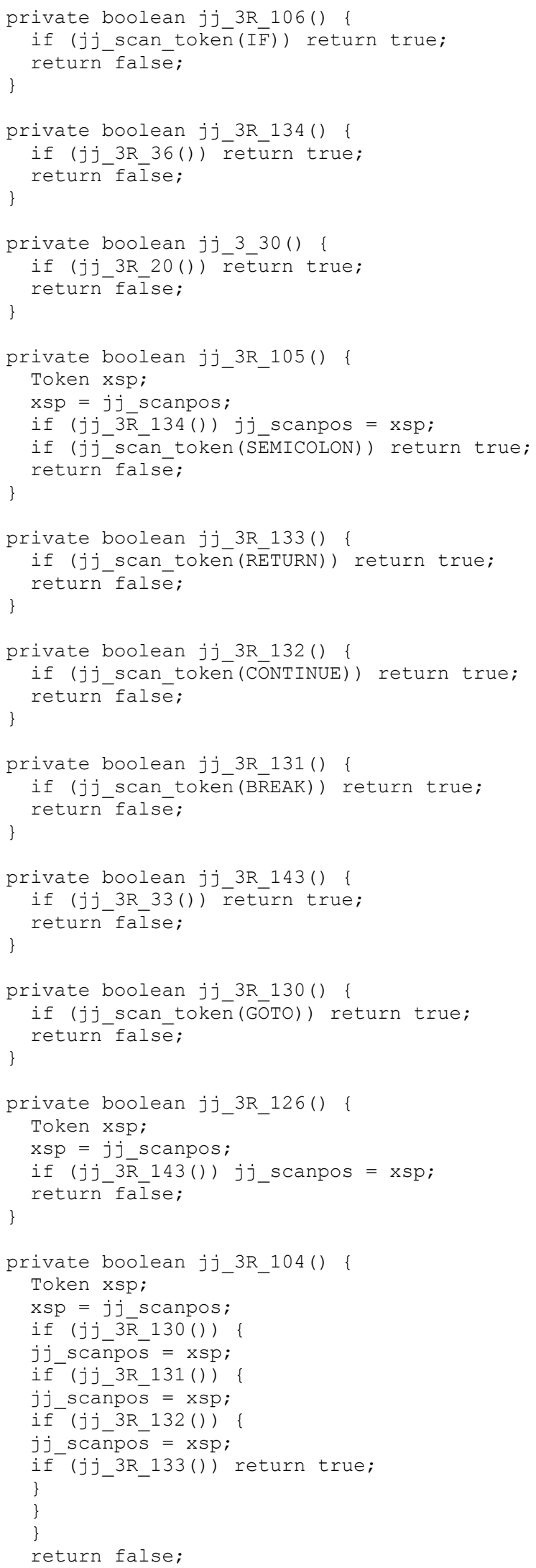




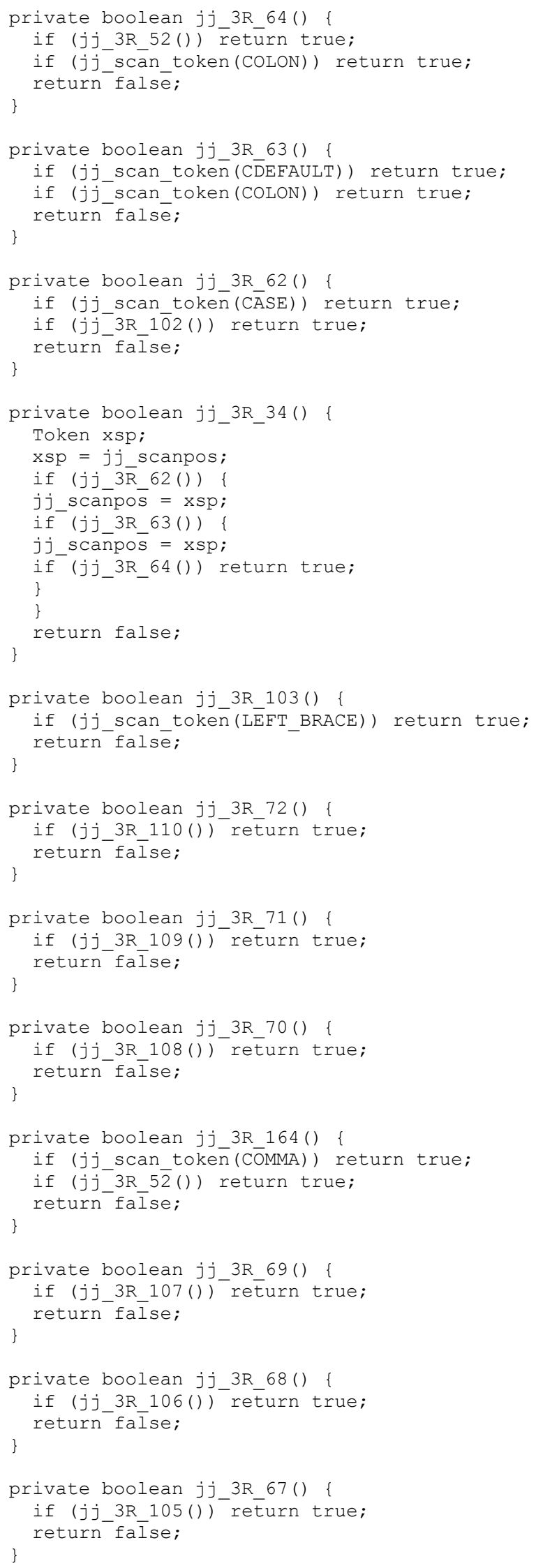




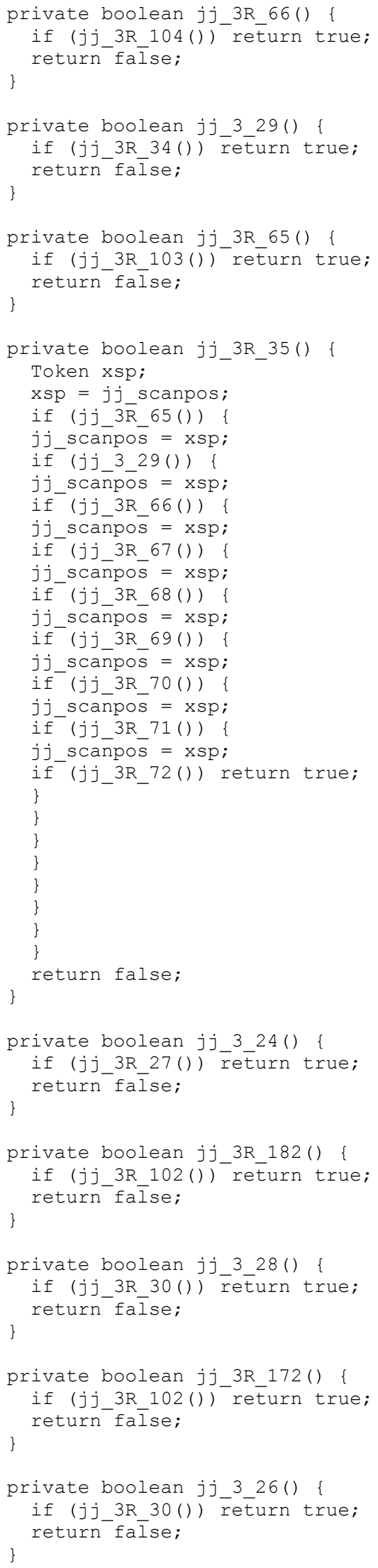




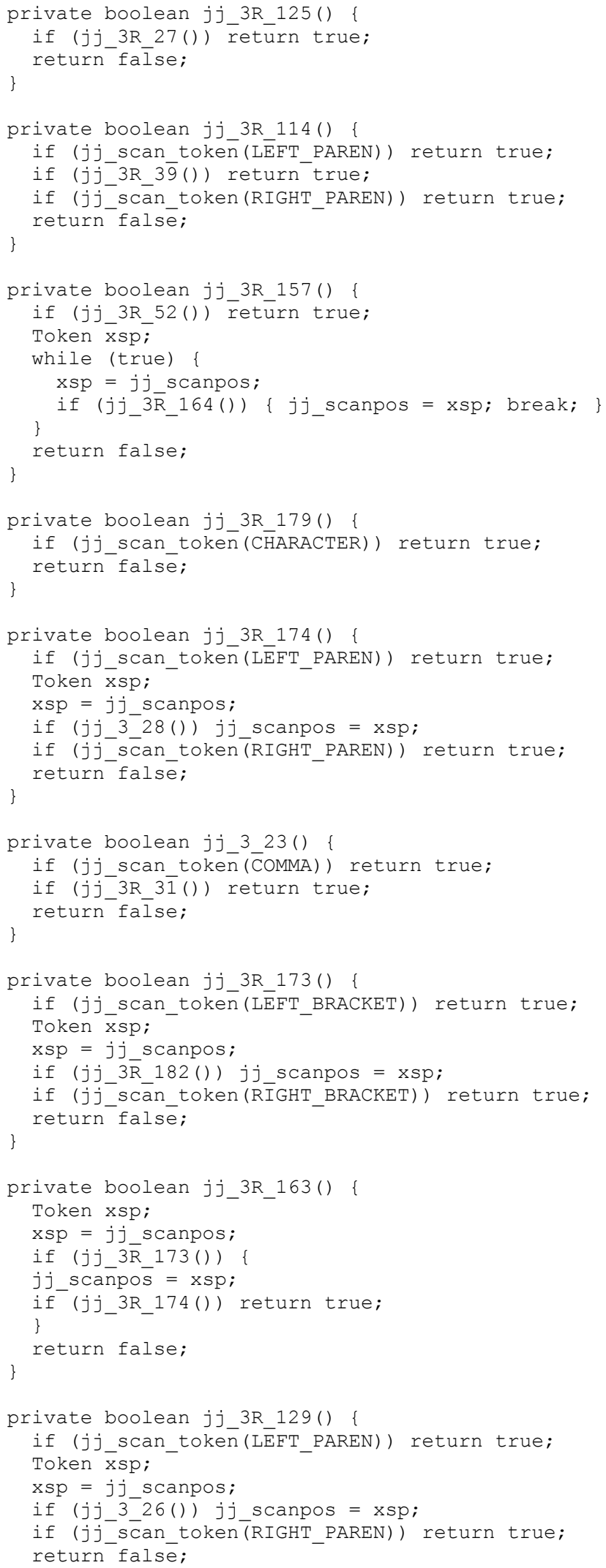




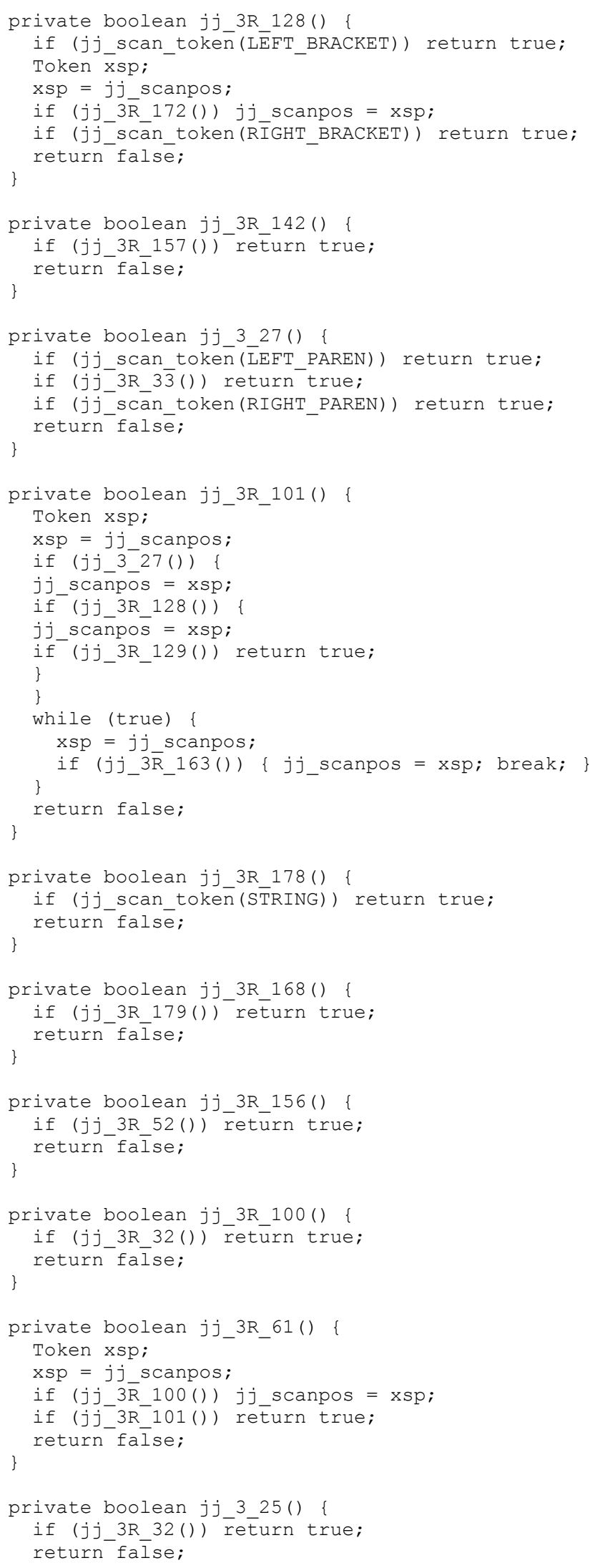




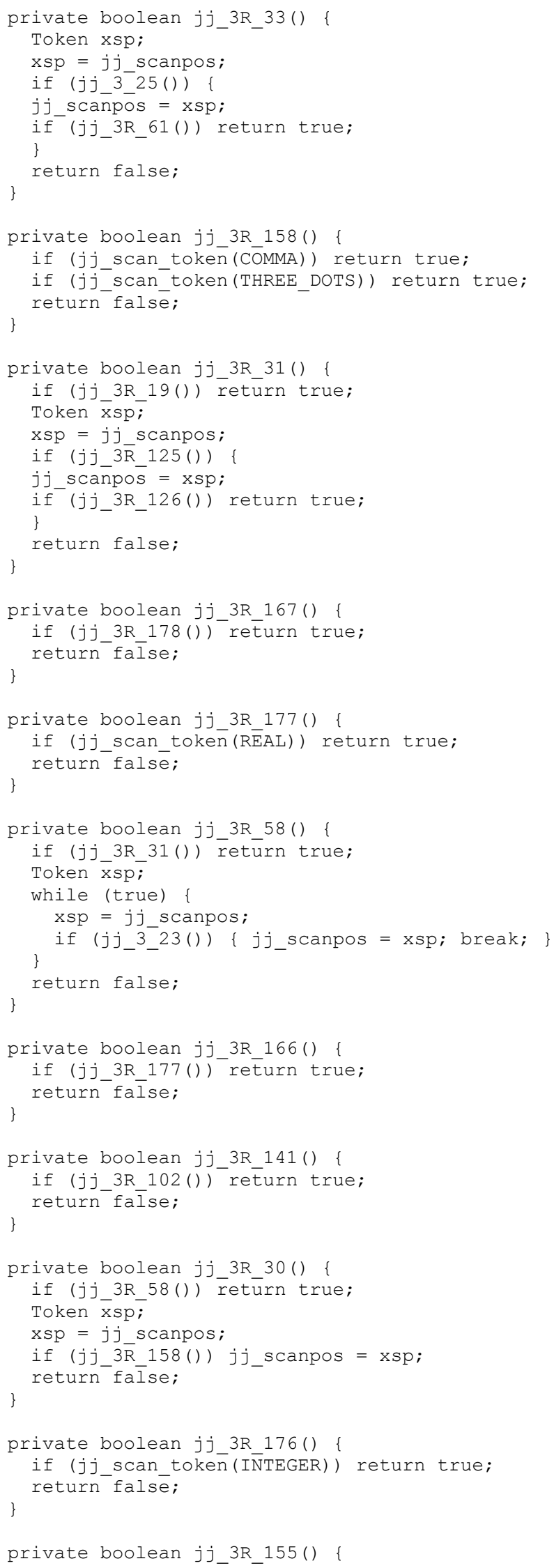




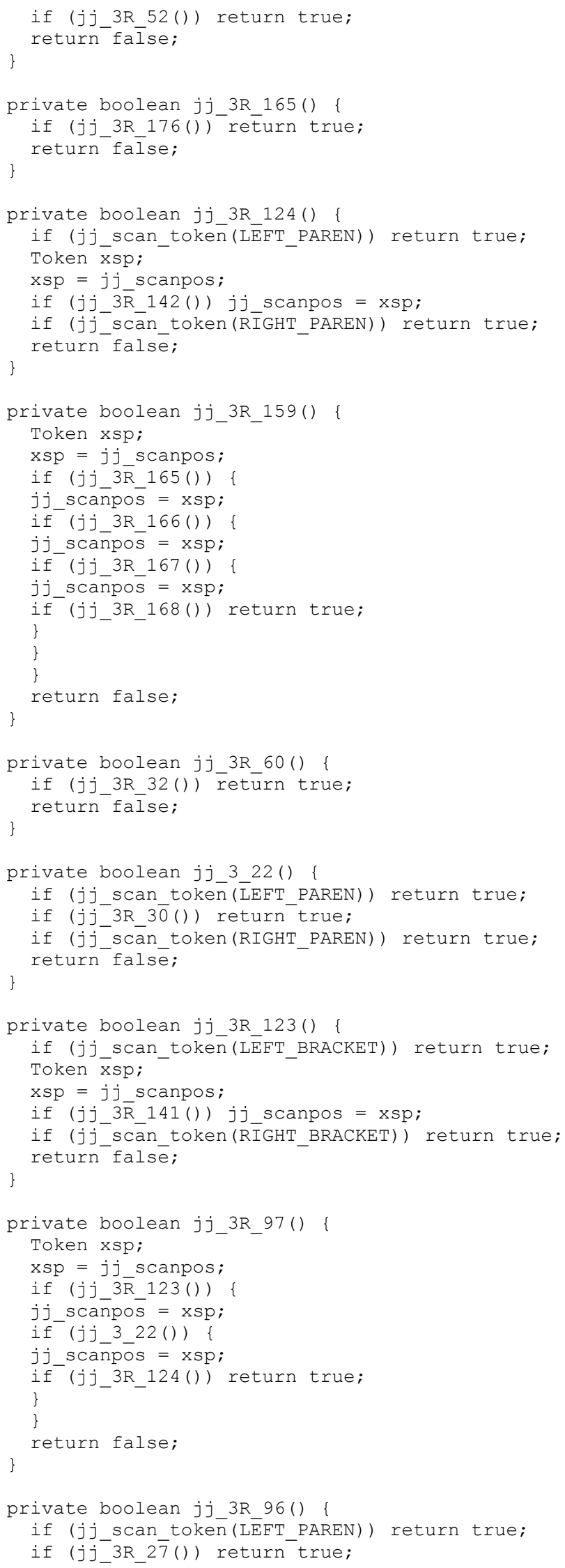




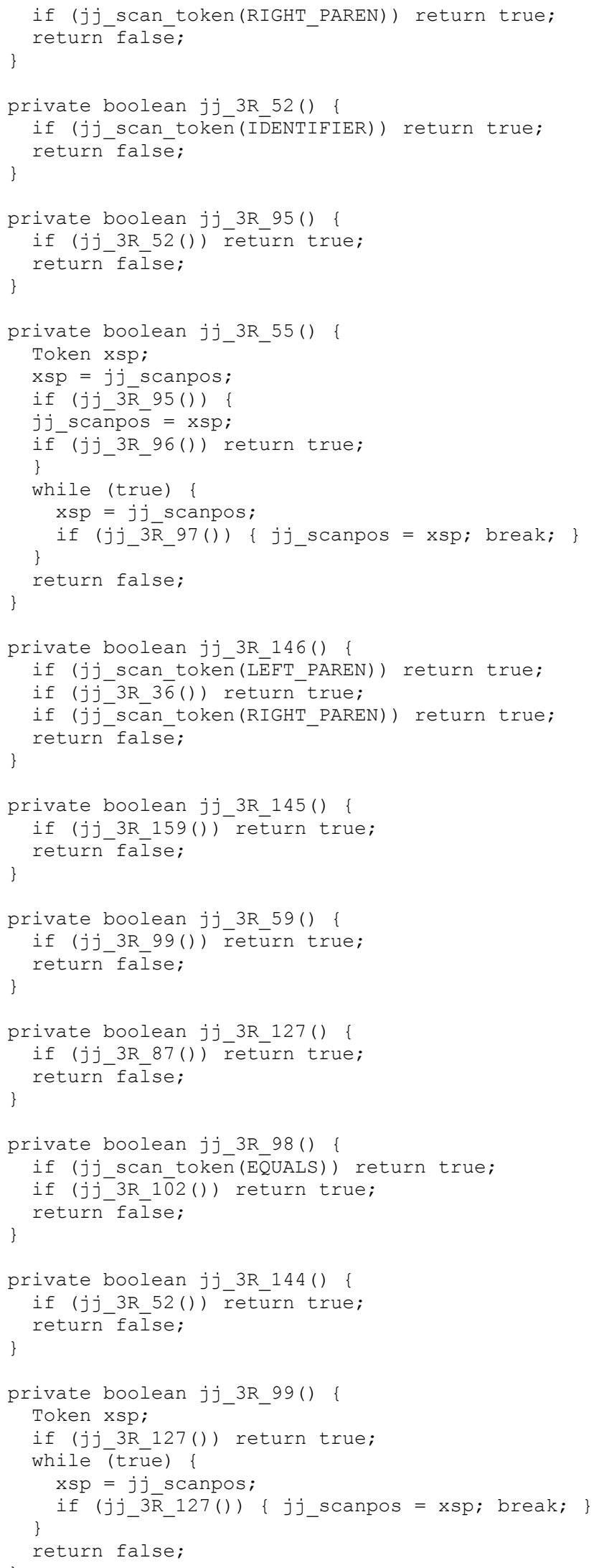




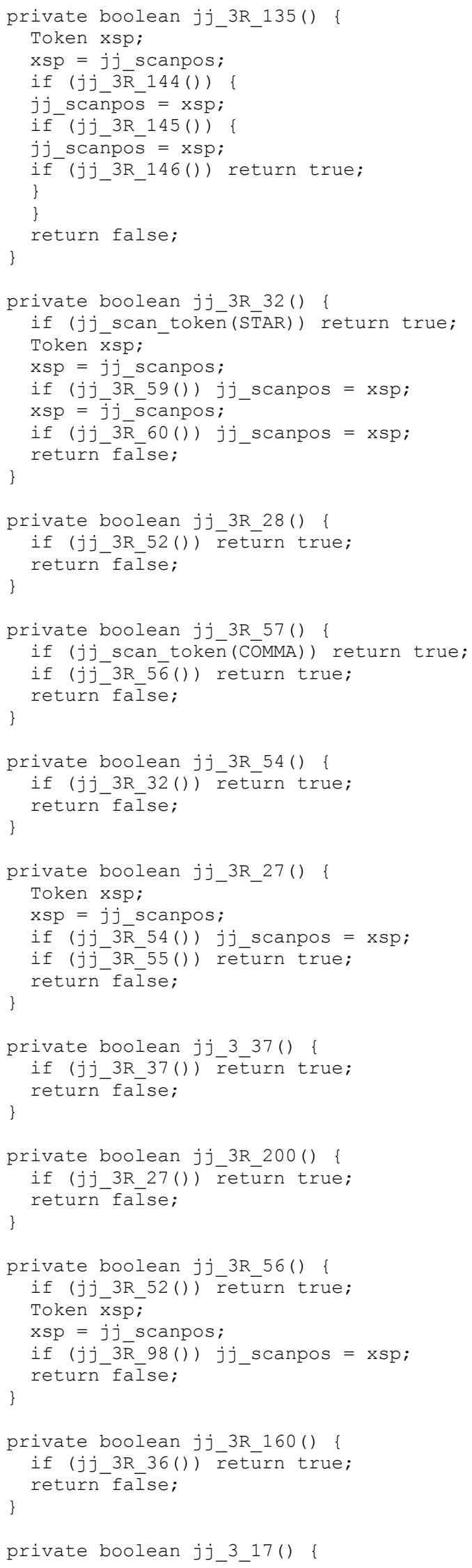




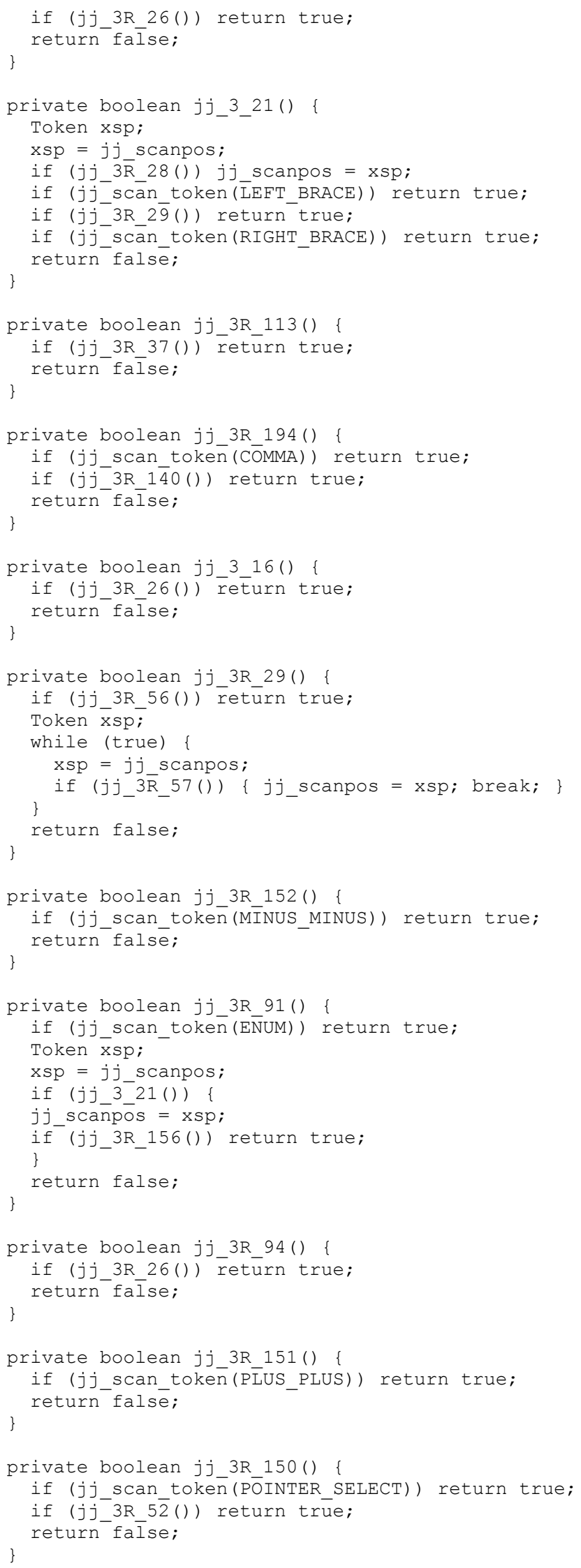




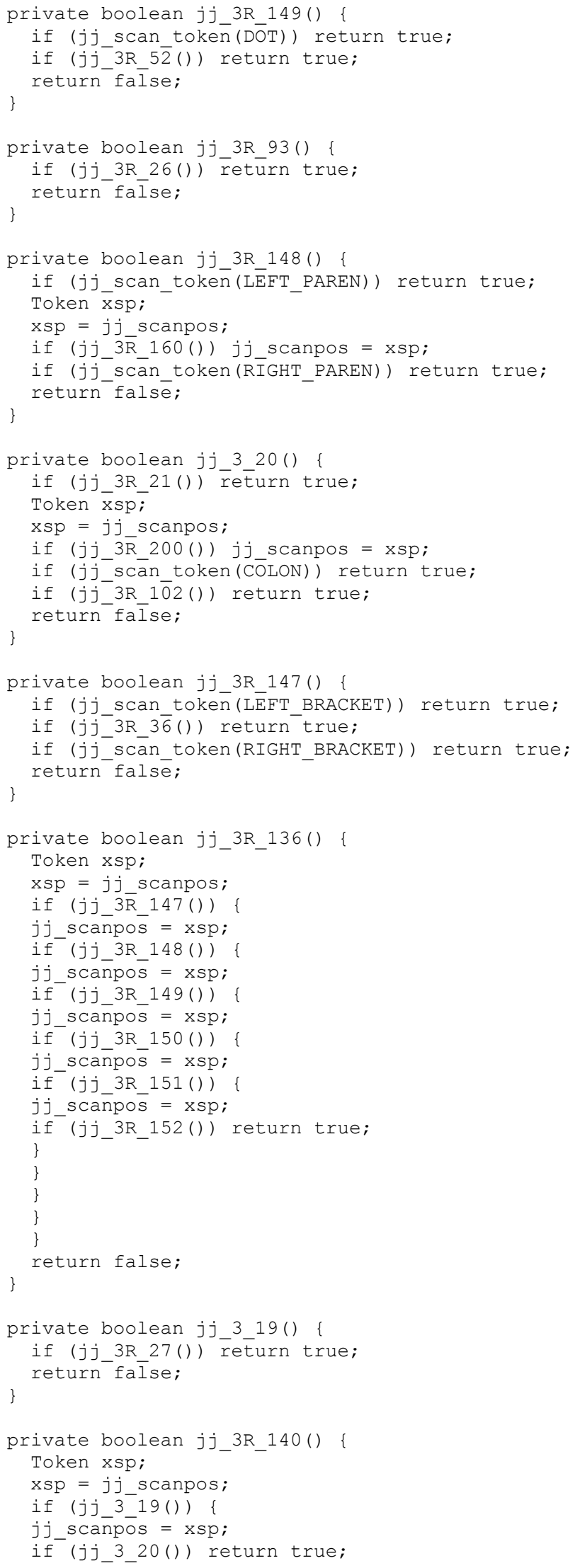




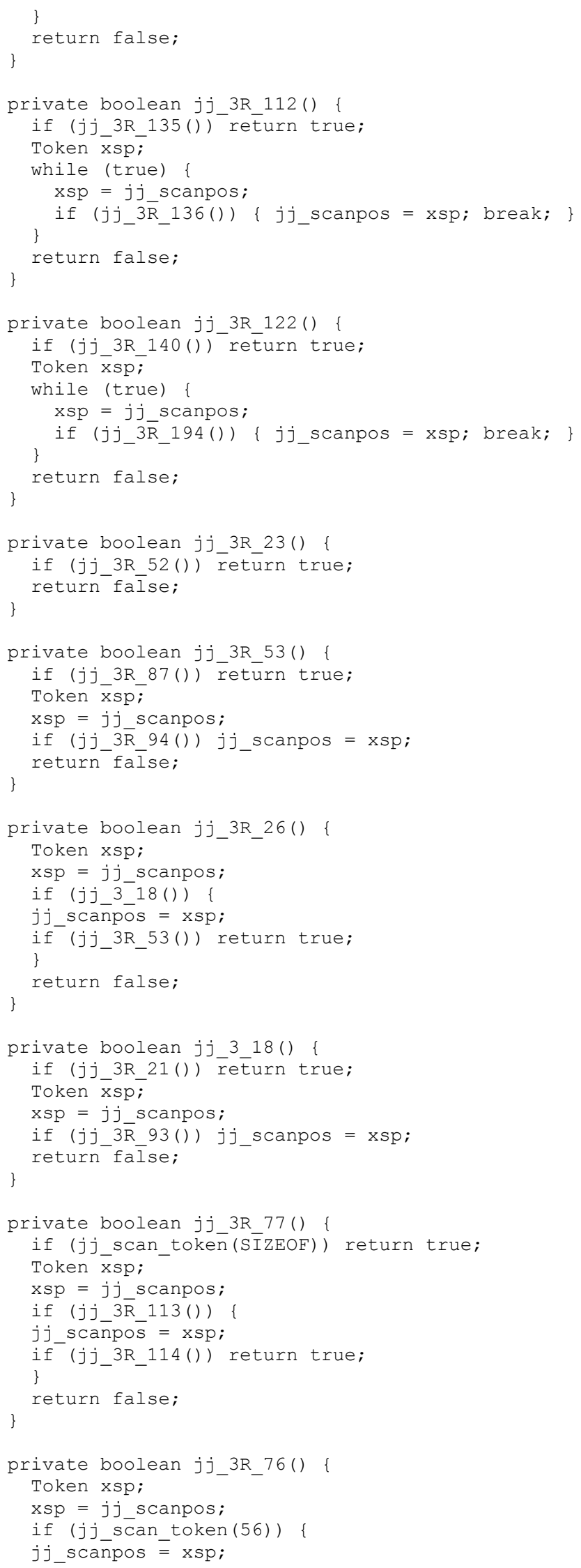




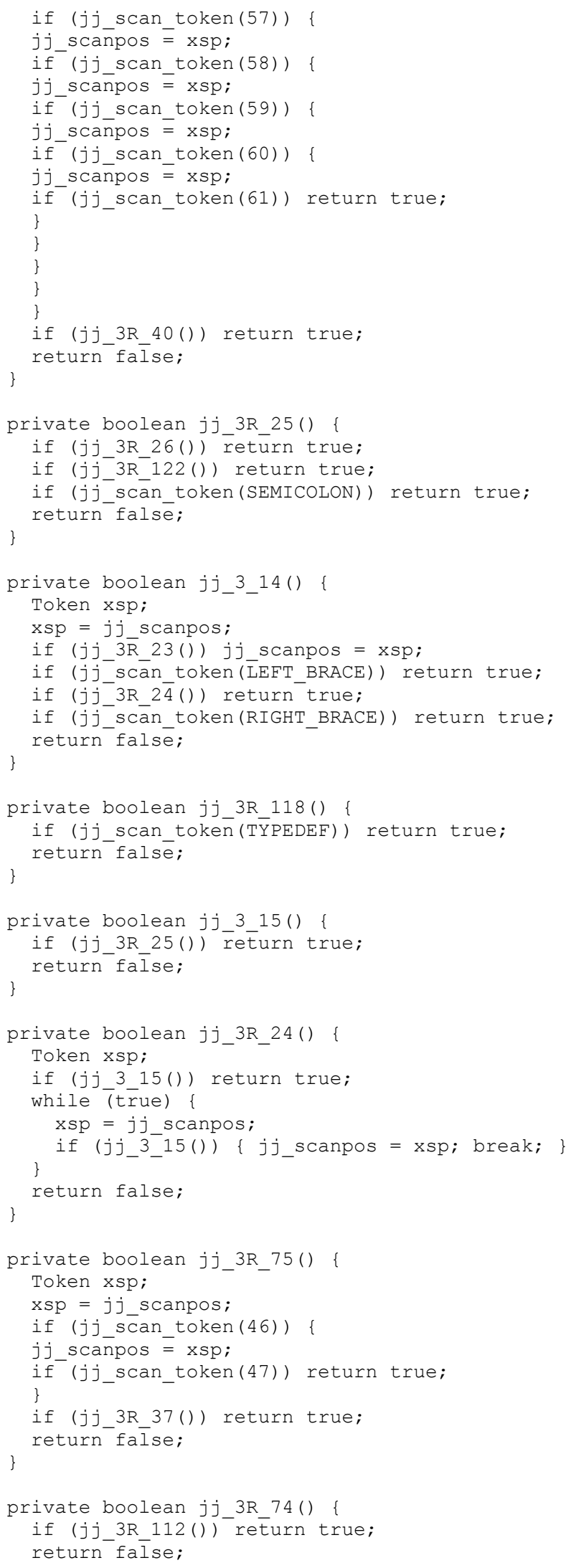




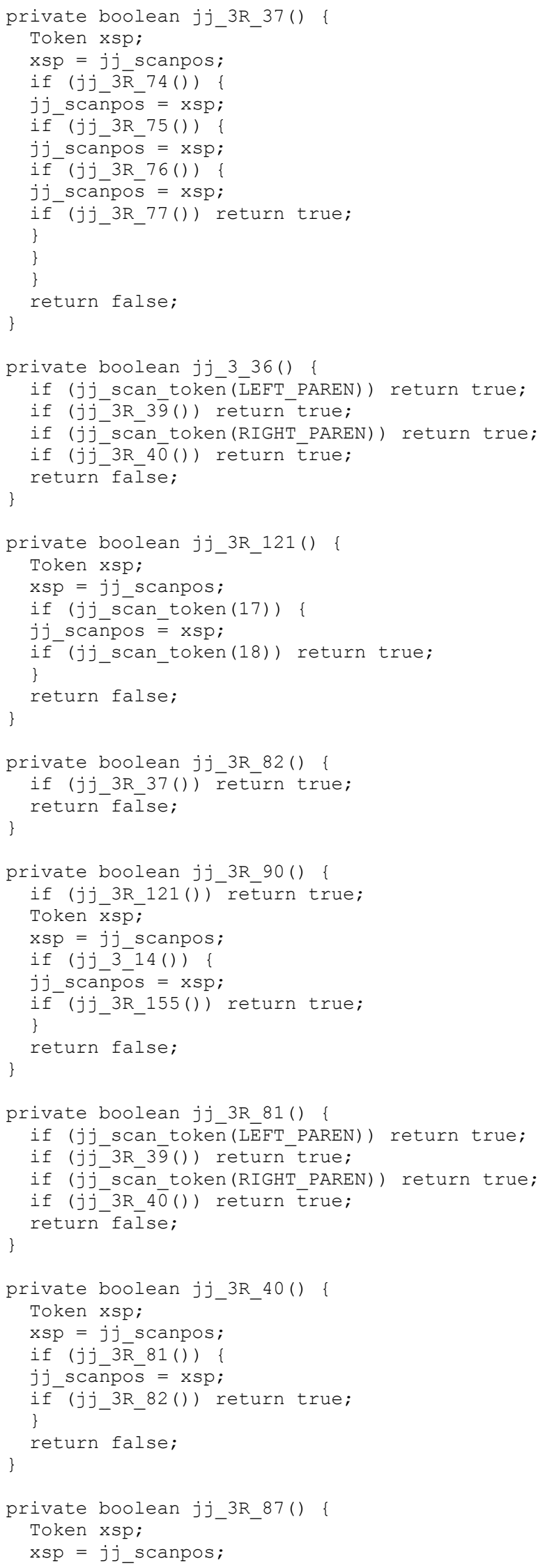




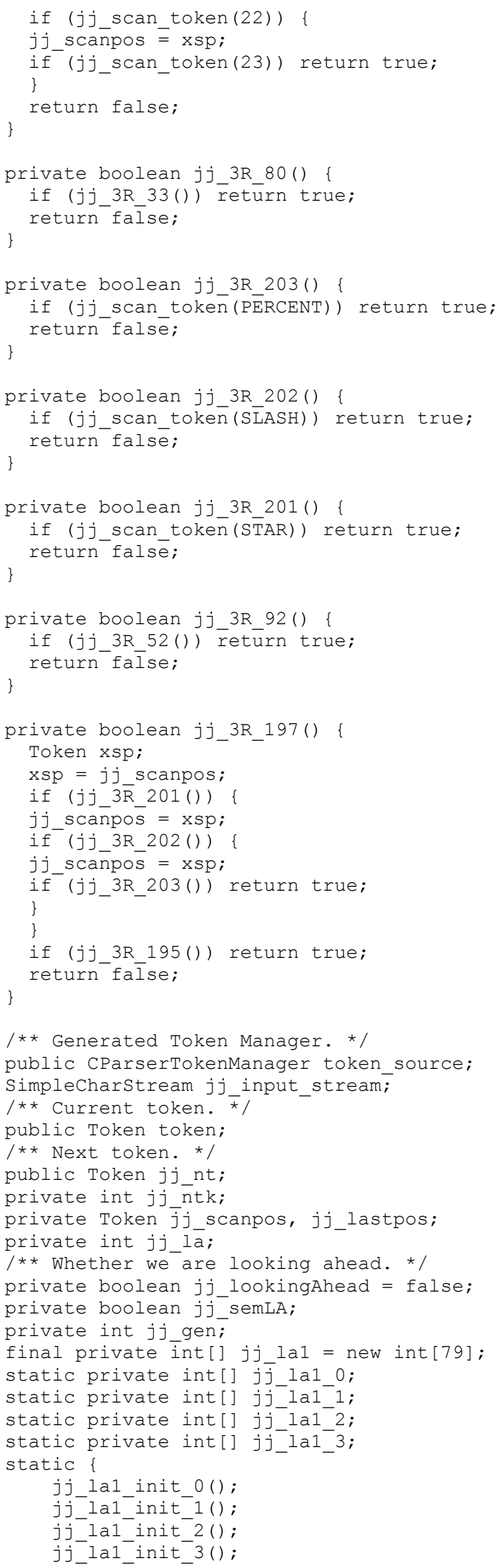


private static void jjlal init_o() \{

jj_lal_o = new int []

$\{0 \times 0,0 \times 1 \overline{9} \mathrm{c} 00 \overline{0}, 0 \times \mathrm{xc} 00000,0 \times 0,0 \times 0,0 \times 0,0 \times 3 \mathrm{c} 00,0 \times 0,0 \times 19 \mathrm{c} 000,0 \times f f 260000,0 \times c 00000,0 \times 0,0 \times 0,0 \times 6000$ $0,0 \times c 00000,0 \times 0,0 \times 0,0 \times 0,0 \times 0,0 \times 0,0 \times 0,0 \times 0,0 \times c 00000,0 \times 0,0 \times c 00000,0 \times 0,0 \times 0,0 \times 3 c 00,0 \times 0,0 \times 0,0 \times 0,0$ $\mathrm{x} 0,0 \times 0,0 \times 0,0 \times 0,0 \times 3 \mathrm{c} 00,0 \times 0,0 \times 0,0 \times 3 \mathrm{c} 00,0 \times 0,0 \times 0,0 \times 3 \mathrm{c} 00,0 \times 0,0 \times 3 \mathrm{c} 00,0 \times 3 \mathrm{c} 00,0 \times 0,0 \times 3 \mathrm{c} 00,0 \times 0,0 \times 3 \mathrm{c}$ $00,0 \times 3 \mathrm{c} 00,0 \times 3 \mathrm{c} 00,0 \times 0,0 \times 0,0 \times 0,0 \times 0,0 \times 0,0 \times 0,0 \times 0,0 \times 0,0 \times 0,0 \times 0,0 \times 0,0 \times 0,0 \times 0,0 \times 0,0 \times 0,0 \times 0,0 \times 0,0 \times 0$, $0 \times 3 \mathrm{c} 00,0 \times 0,0 \times 0,0 \times 0,0 \times 3 \mathrm{c} 00,0 \times 0,0 \times 3 \mathrm{c} 00,0 \times 0,0 \times 3 \mathrm{c} 00,0 \times 3 \mathrm{c} 00$,$\} ;$

\}

private static void jj_lal_init_1() \{

jj_la1_1 = new int []

$\{0 \times 20400 \overline{0} 0,0 \bar{x} 0,0 \times 0,0 \times 0,0 \times 0,0 \times 0,0 \times 3 f 44 \mathrm{~d} 000,0 \times 2140000,0 \times 0,0 \times 1,0 \times 0,0 \times 0,0 \times 0,0 \times 0,0 \times 0,0 \times 0,0 \times 204$ $0000,0 \times 0,0 \times 0,0 \times 0,0 \times 0,0 \times 2000000,0 \times 0,0 \times 2000000,0 \times 0,0 \times 40000,0 \times 140000,0 \times 3 f 04 d 000,0 \times 0,0 \times 100000$ $, 0 \times 40000,0 \times 0,0 \times 2140000,0 \times 2000000,0 \times 2140000,0 \times 3 f 04 d 000,0 \times 140000,0 \times 140000,0 \times 3 f 04 d 000,0 \times 1400$ $00,0 \times 0,0 \times 3 f 44 \mathrm{fffa}, 0 \times 400000,0 \times 3 \mathrm{f} 04 \mathrm{fbea}, 0 \times 3 \mathrm{f} 4 \mathrm{fffa}, 0 \times 410,0 \times 3 \mathrm{f} 04 \mathrm{~d} 000,0 \times 2 \mathrm{~b} 00,0 \times 3 \mathrm{f} 04 \mathrm{~d} 000,0 \times 3 \mathrm{f} 0$ $4 \mathrm{~d} 000,0 \times 3 \mathrm{f} 04 \mathrm{~d} 000,0 \times 0,0 \times 0,0 \times 0,0 \times 0,0 \times 0,0 \times 0,0 \times 0,0 \times 1000000,0 \times 0,0 \times 0,0 \times 0,0 \times 0,0 \times 0,0 \times 0,0 \times c 000000$, $0 \times c 000000,0 x c 2000000,0 x c 2000000,0 \times 3 f 04 d 000,0 x c 000,0 \times 3 f 000000,0 \times 40000,0 \times 3 f 04 d 000,0 \times 17 c 000$, $0 \times 3 \mathrm{f} 04 \mathrm{~d} 000,0 \times 17 \mathrm{c} 000,0 \times 40000,0 \times 0$,$\} ;$

\}

private static void jjlal_init_2() \{

jj_la1_2 = new int []

$\{0 \times 0,0 \times 0,0 \times 0,0 \times 4000000,0 \times 4000,0 \times 4000000,0 \times 0,0 \times 0,0 \times 0,0 \times 0,0 \times 0,0 \times 0,0 \times 0,0 \times 0,0 \times 0,0 \times 4000000,0 \times 0$ $, 0 \times 0,0 \times 0,0 \times 4000000,0 \times 4000,0 \times 0,0 \times 0,0 \times 0,0 \times 0,0 \times 0,0 \times 0,0 \times 0,0 \times 0,0 \times 0,0 \times 0,0 \times 4000000,0 \times 0,0 \times 0,0 \times 0,0$ $\mathrm{x} 0,0 \times 0,0 \times 0,0 \times 0,0 \times 0,0 \times 4000000,0 \times 2000000,0 \times 0,0 \times 2000000,0 \times 2000000,0 \times 0,0 \times 0,0 \times 0,0 \times 0,0 \times 0,0 \times 0,0 \times$ $4000000,0 \times 1 \mathrm{ffc} 000,0 \times 1000,0 \times 800,0 \times 400,0 \times 200,0 \times 100,0 \times 0,0 \times c 0,0 \times c 0,0 \times 3 c, 0 \times 3 c, 0 \times 3,0 \times 3,0 \times 0,0 \times 0$, $0 \times 0,0 \times 0,0 \times 0,0 \times 0,0 \times 0,0 \times 0,0 \times 0,0 \times 0,0 \times 0,0 \times 0,0 \times 0,0 \times 0$,$\} ;$

\}

private static void jj_lal_init_3() \{

jj 1 al 3 = new int []

$\{0 \times 4,0 \times 0,0 \times 0,0 \times 0,0 \times 0,0 \times 0,0 \times 4,0 \times 0,0 \times 0,0 \times 0,0 \times 0,0 \times 4,0 \times 4,0 \times 0,0 \times 0,0 \times 0,0 \times 4,0 \times 4,0 \times 4,0 \times 0,0 \times 0,0 \times 0$, $0 \times 0,0 \times 0,0 \times 0,0 \times 4,0 \times 0,0 \times 4,0 \times 4,0 \times 0,0 \times 0,0 \times 0,0 \times 0,0 \times 0,0 \times 0,0 \times 4,0 \times 0,0 \times 0,0 \times 4,0 \times 0,0 \times 0,0 \times 4,0 \times 0,0 \times 4,0$ $\mathrm{x} 4,0 \times 4,0 \times 4,0 \times 0,0 \times 4,0 \times 4,0 \times 4,0 \times 0,0 \times 0,0 \times 0,0 \times 0,0 \times 0,0 \times 0,0 \times 0,0 \times 0,0 \times 0,0 \times 0,0 \times 0,0 \times 0,0 \times 0,0 \times 0,0 \times 0,0 x$ $0,0 \times 0,0 \times 0,0 \times 4,0 \times 0,0 \times 0,0 \times 0,0 \times 4,0 \times 0,0 \times 4,0 \times 0,0 \times 4,0 \times 0$,$\} ;$

\}

final private JJCalls[] jj 2 rtns = new JJCalls[37];

private boolean jj_rescan = false;

private int jj_gc $=0$;

/** Constructor with InputStream. */

public CParser(java.io.InputStream stream) \{

this(stream, null);

/** Constructor with InputStream and supplied encoding */

public CParser(java.io.InputStream stream, String encoding) \{

try $\{j j$ input_stream = new SimplecharStream(stream, encoding, 1, 1); \}

catch (java.io. UnsupportedEncodingException e) \{ throw new RuntimeException(e); \}

token source $=$ new CParserTokenManager (jj_input_stream);

token ${ }^{-}=$new Token ();

jj ntk = -1;

jj_gen $=0$;

for (int $i=0 ; i<79 ; i++) j j$ lal $[i]=-1$;

for (int $i=0 ; i<j j \_2$ rttns.lēngth; $\left.i++\right)$ jj_2_rtns[i] = new JJCalls () ;

\}

/** Reinitialise. */

public void ReInit(java.io.InputStream stream) \{

ReInit (stream, null);

/** Reinitialise. */

public void ReInit (java.io.InputStream stream, String encoding) \{

try \{jj_input_stream.ReInit(stream, encoding, 1, 1); \}

catch (java.io. UnsupportedEncodingException e) \{ throw new RuntimeException(e); \}

token_source.ReInit(jj_input_stream);

token ${ }^{-}=$new Token () ;

jj_ntk $=-1$;

jjĒree.reset ();

jj_gen $=0$;

for (int $i=0 ; i<79 ; i++)$ jjlal[i] $=-1$;

for (int $\left.i=0 ; i<j j \_2 \_r t n s . l e n g t h ; i++\right) j j \_2$ rtns[i] = new JJCalls();

/** Constructor. */ 


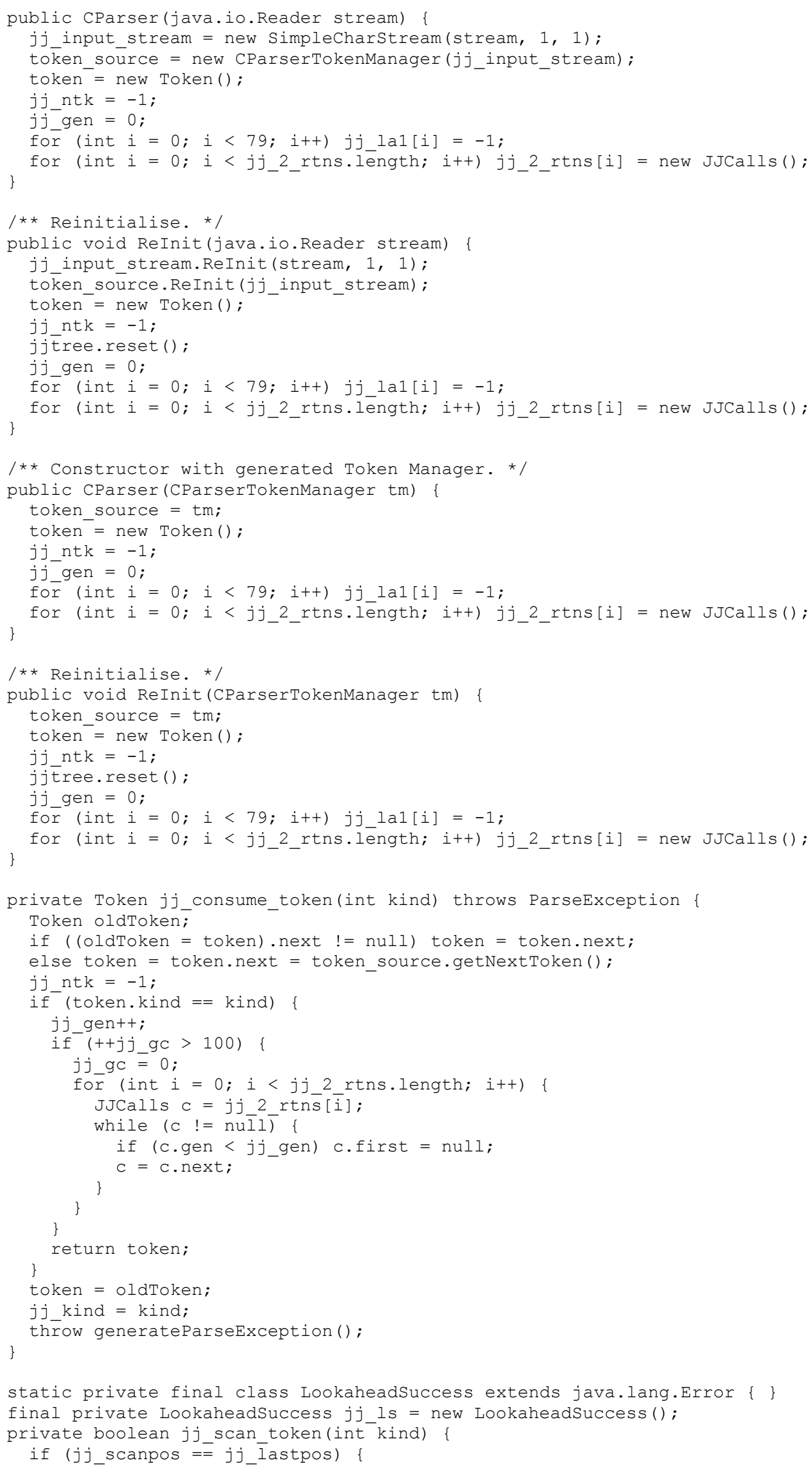




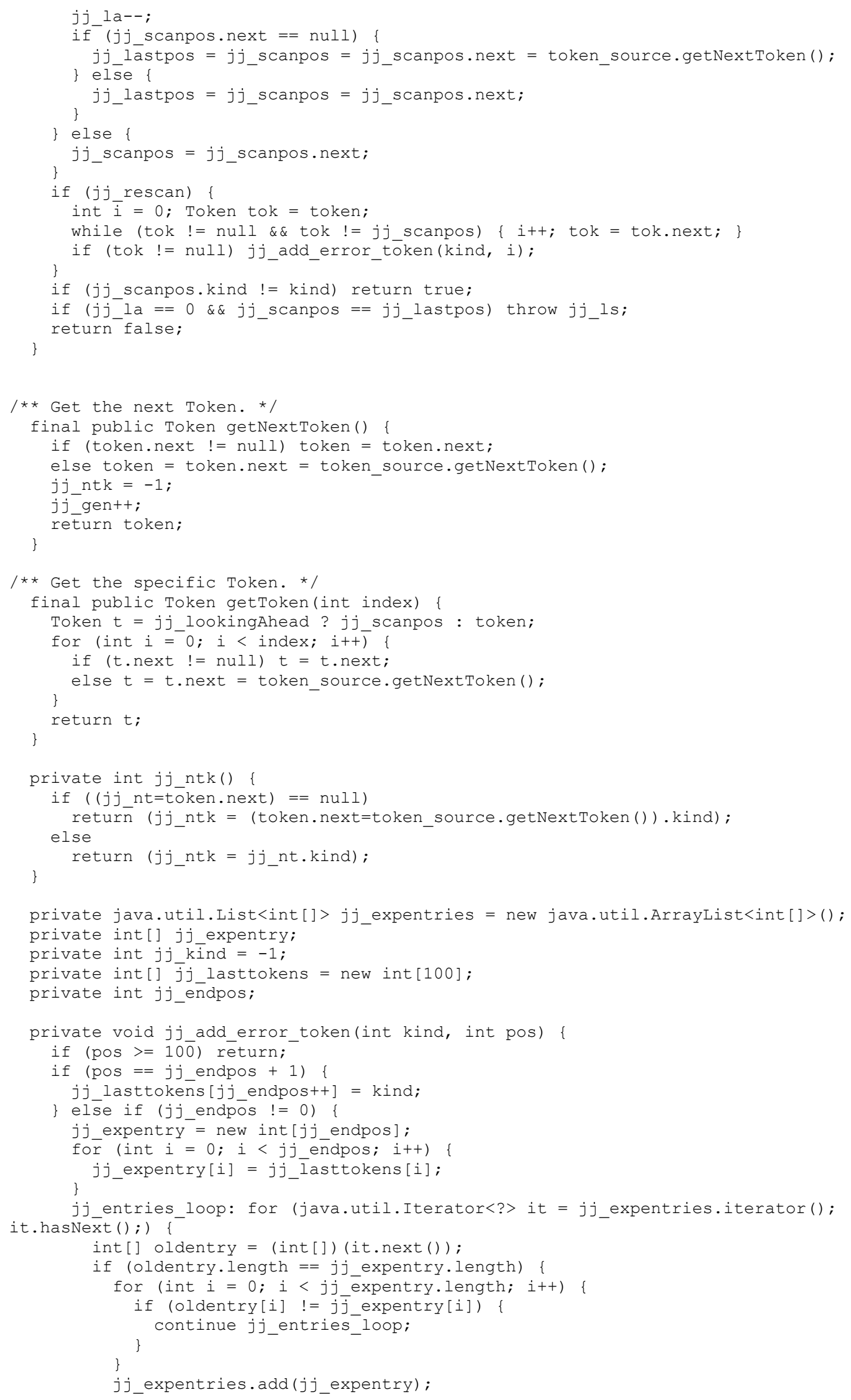




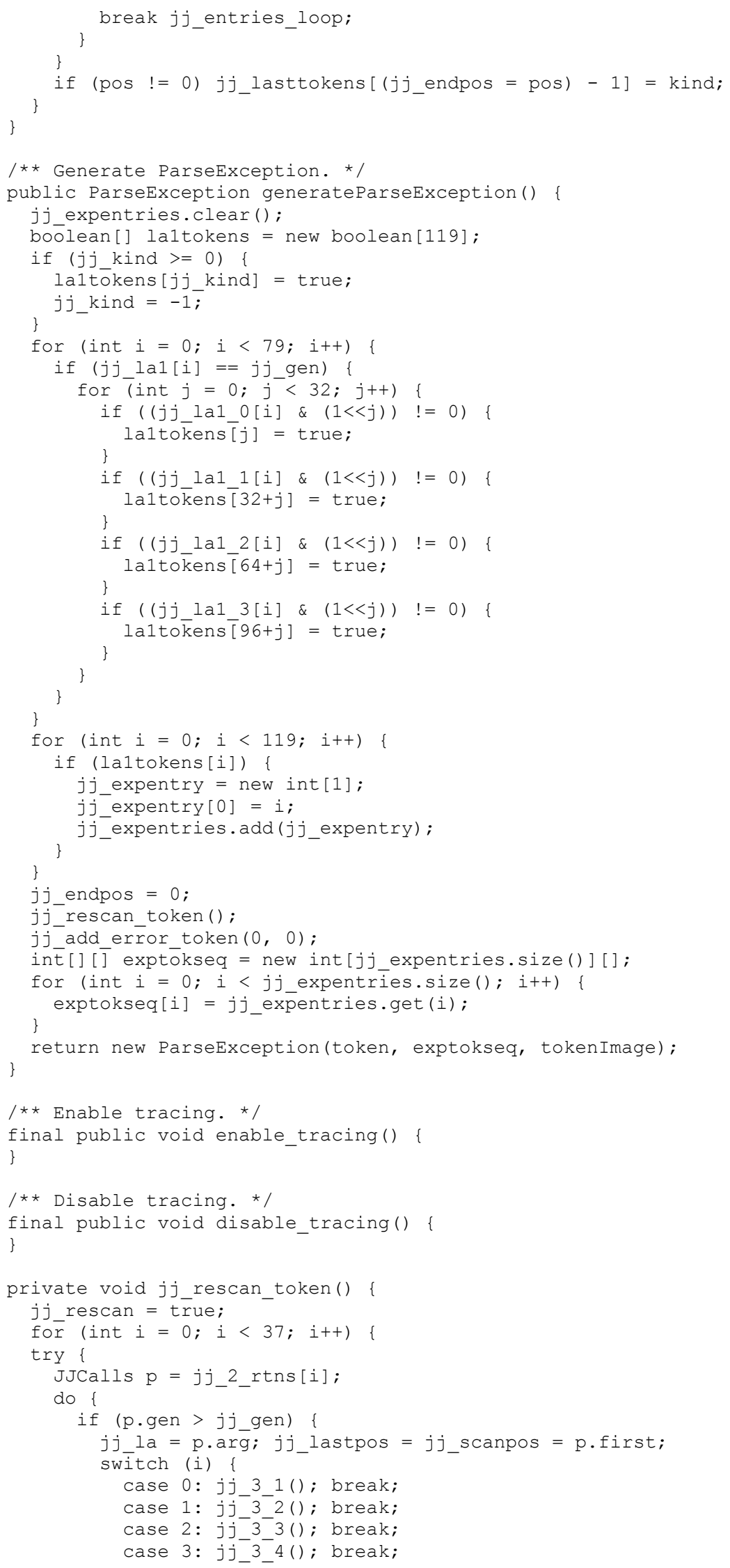




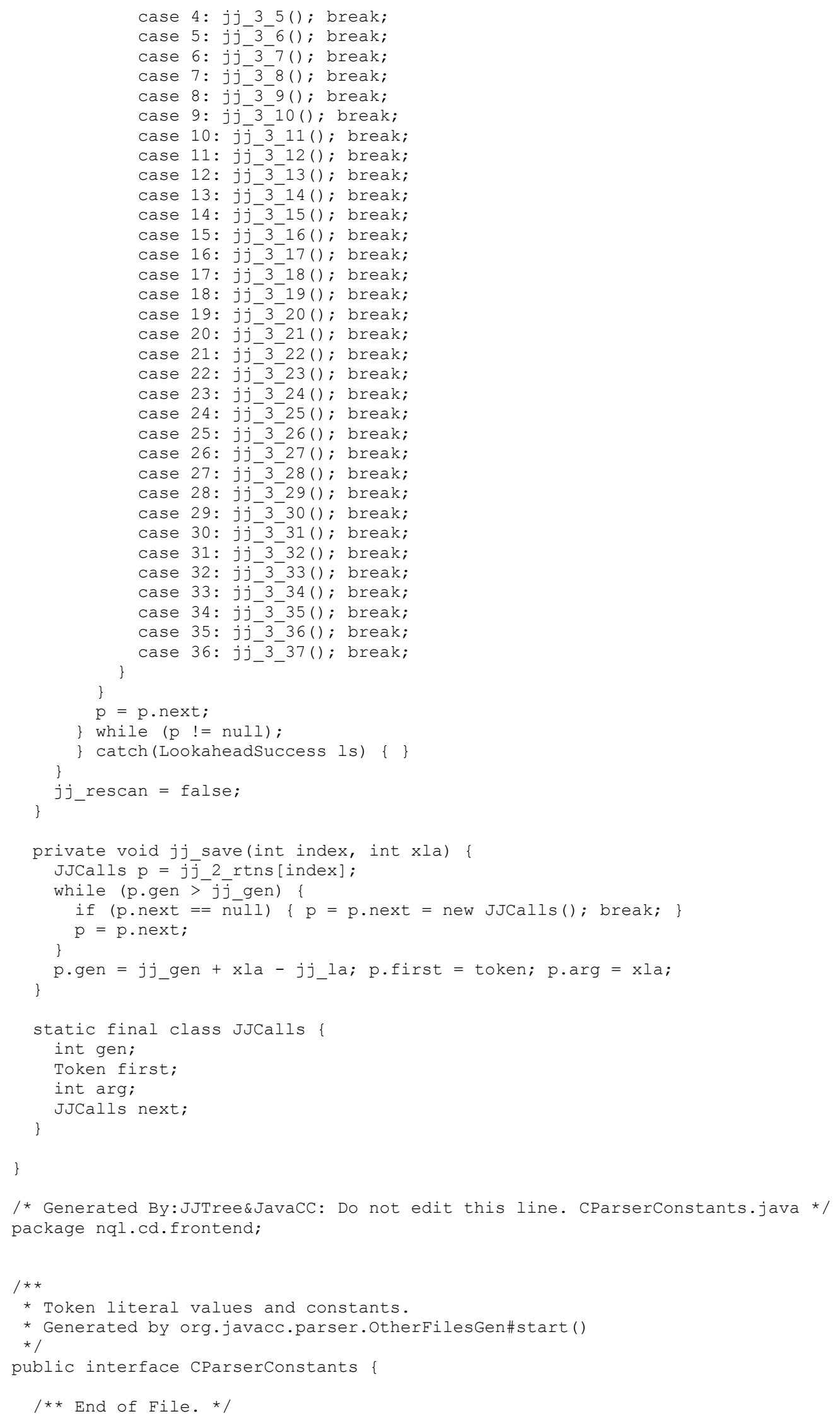




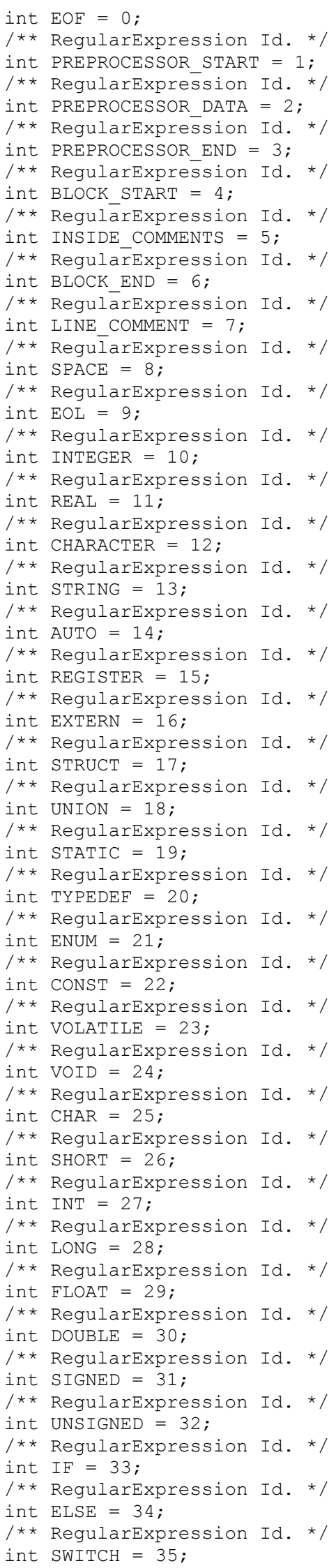




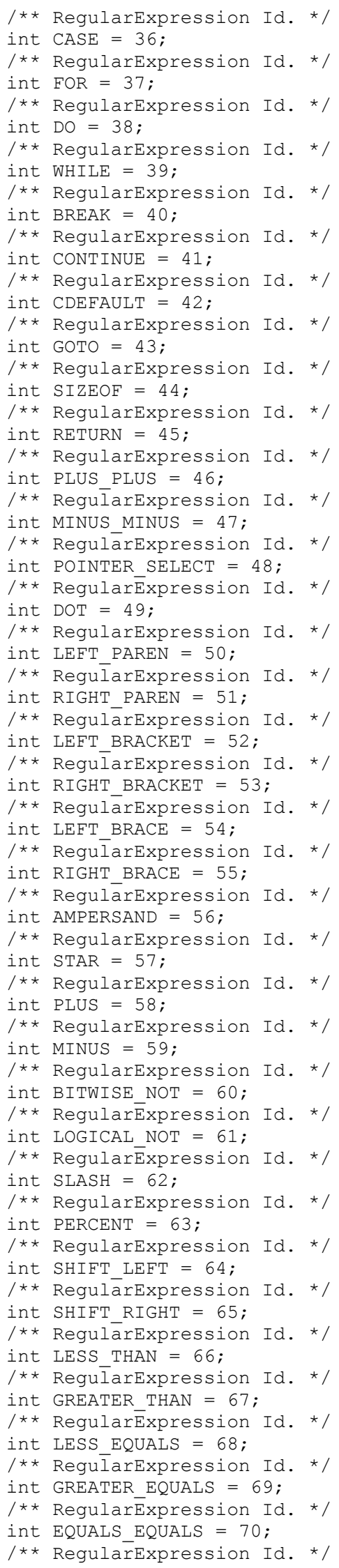




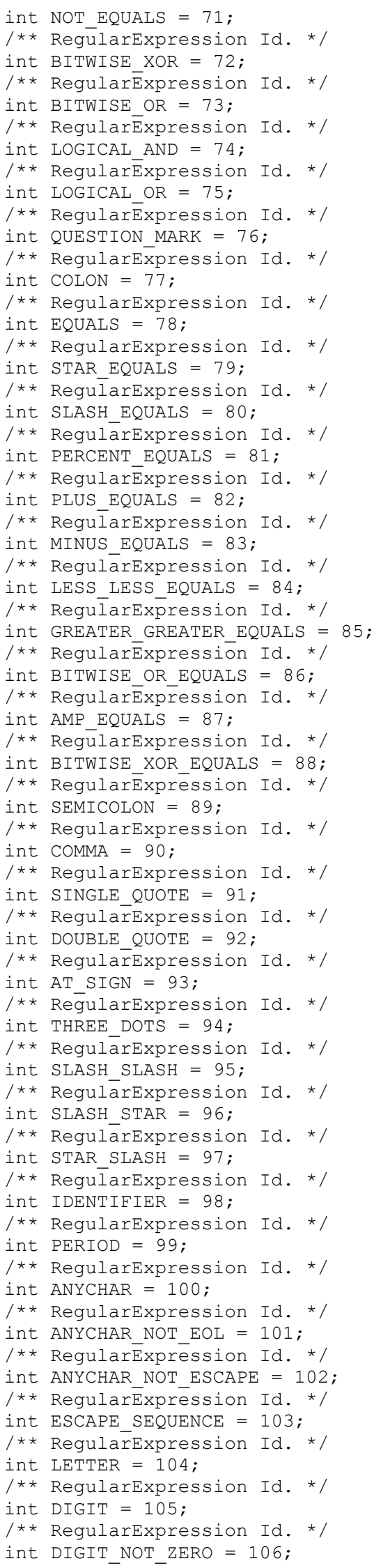




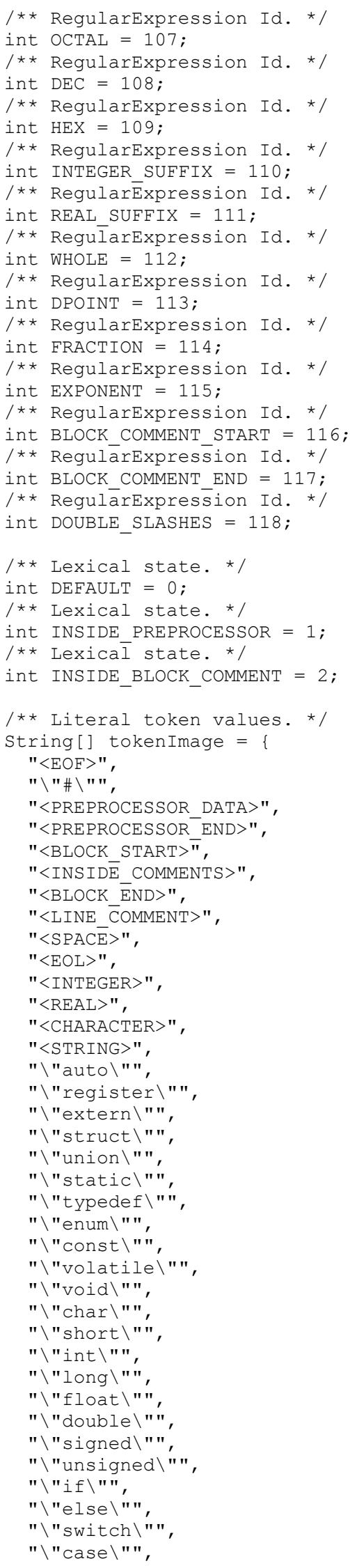




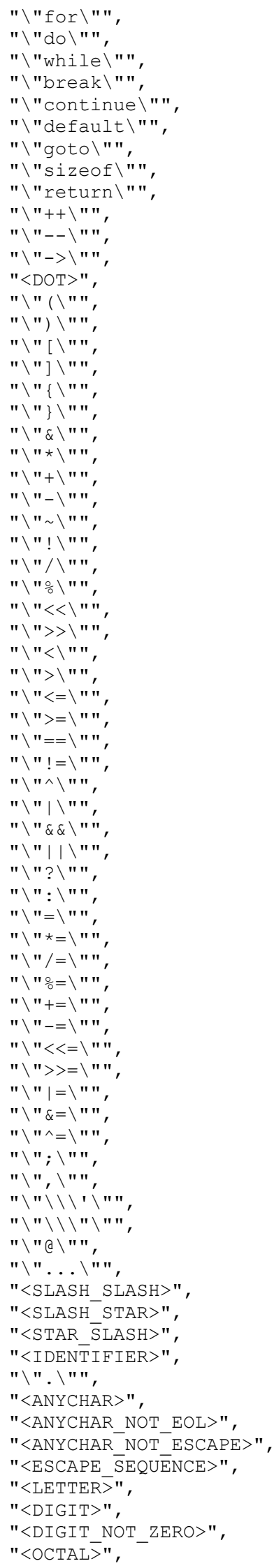




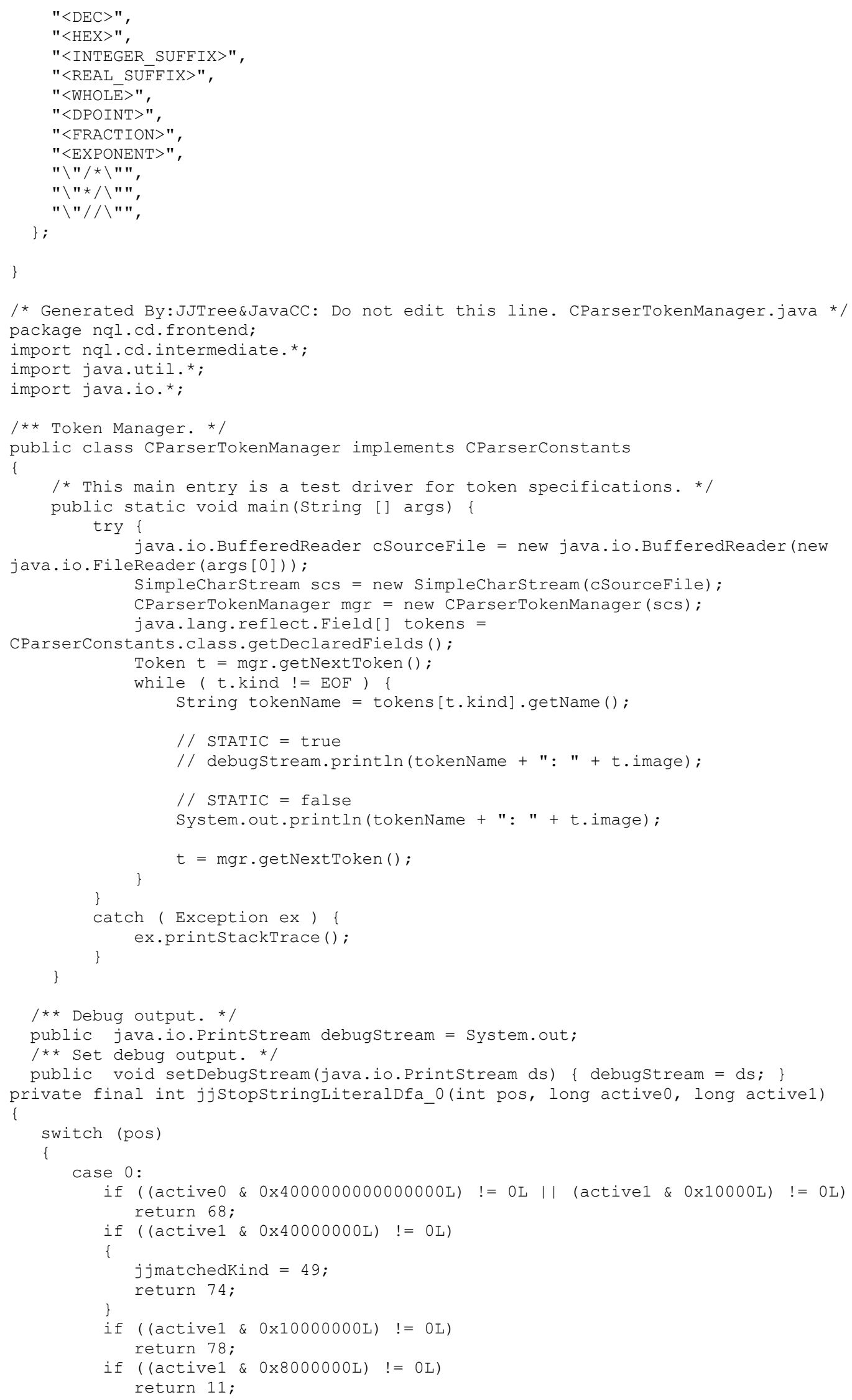




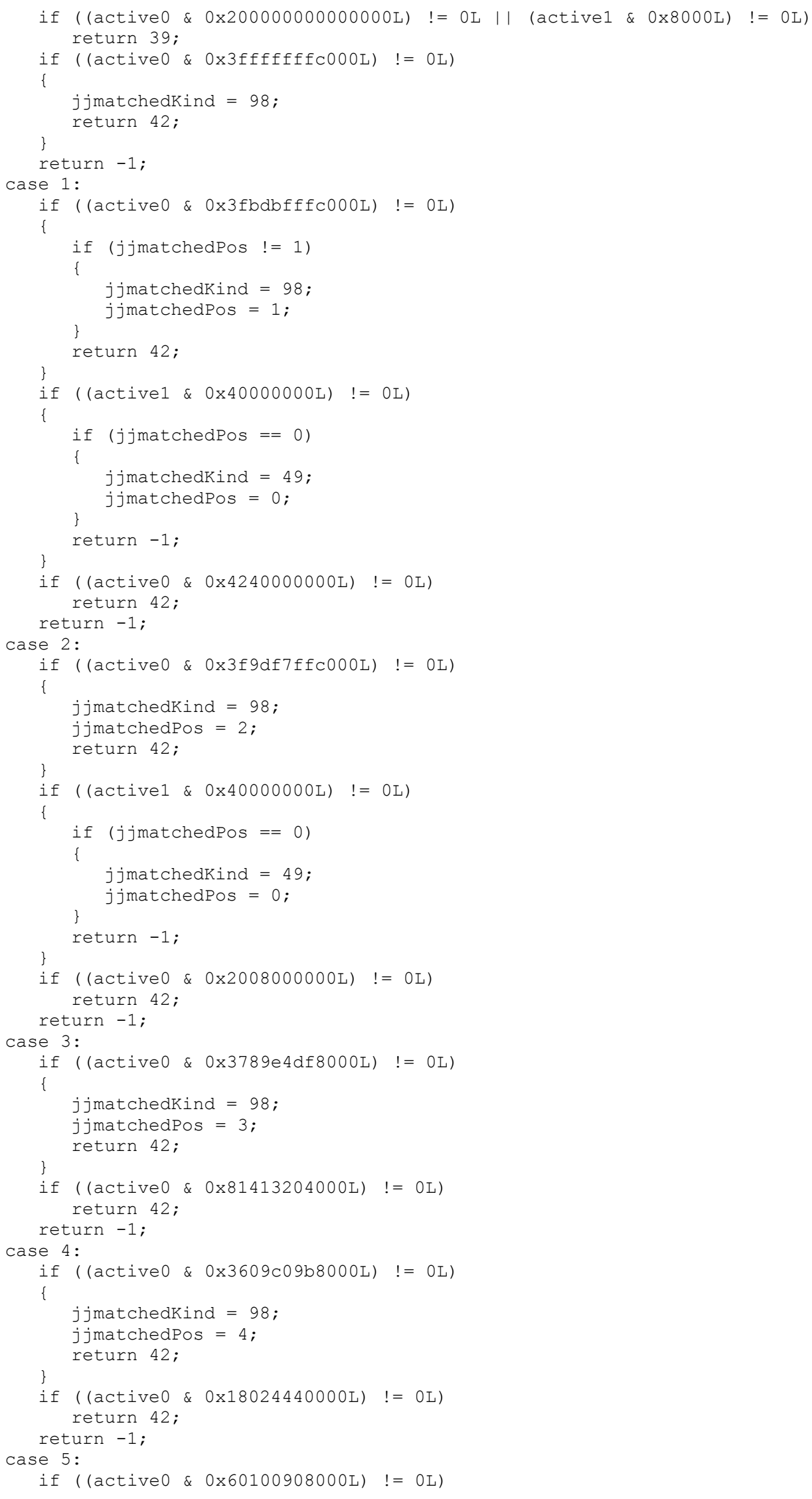




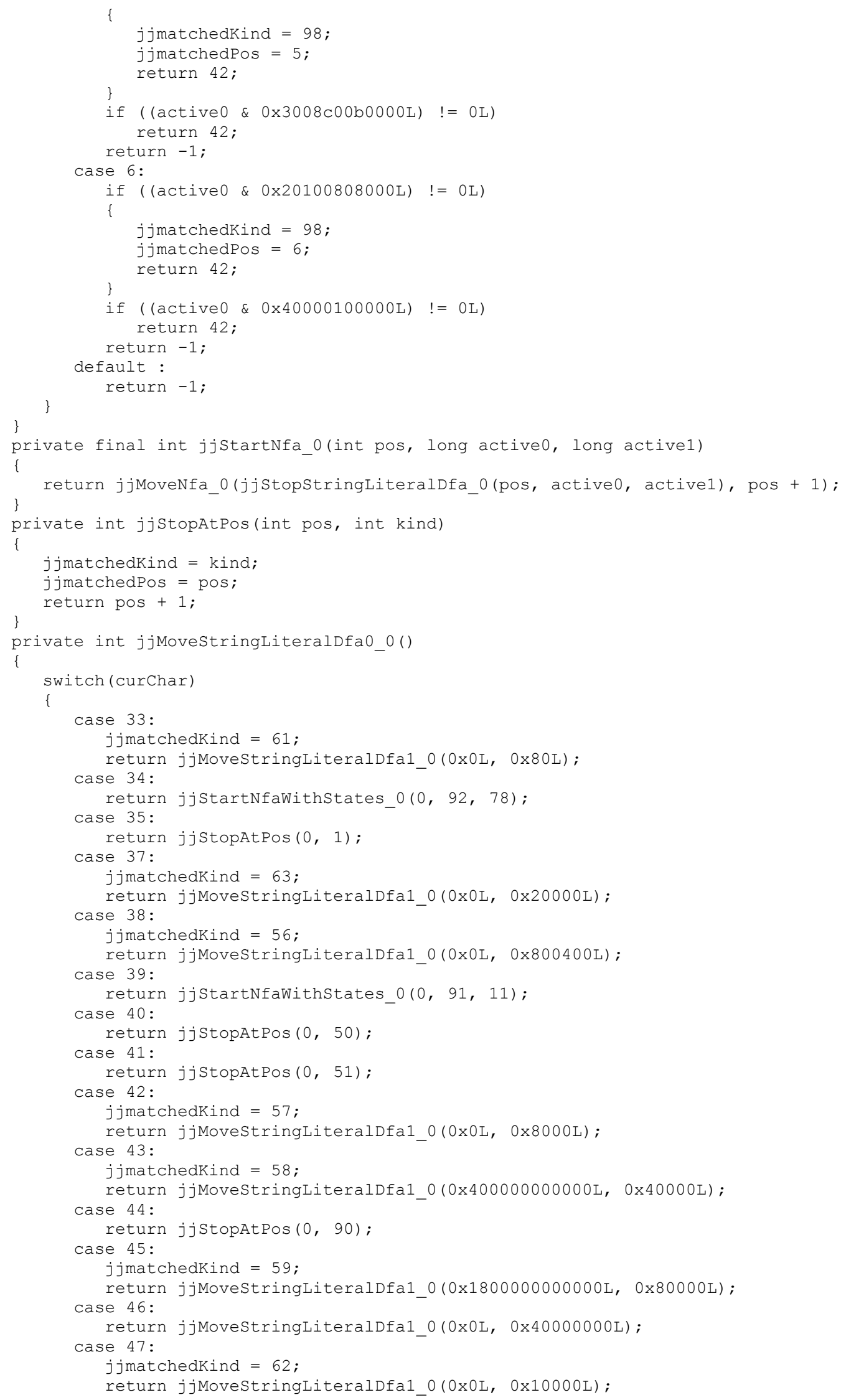




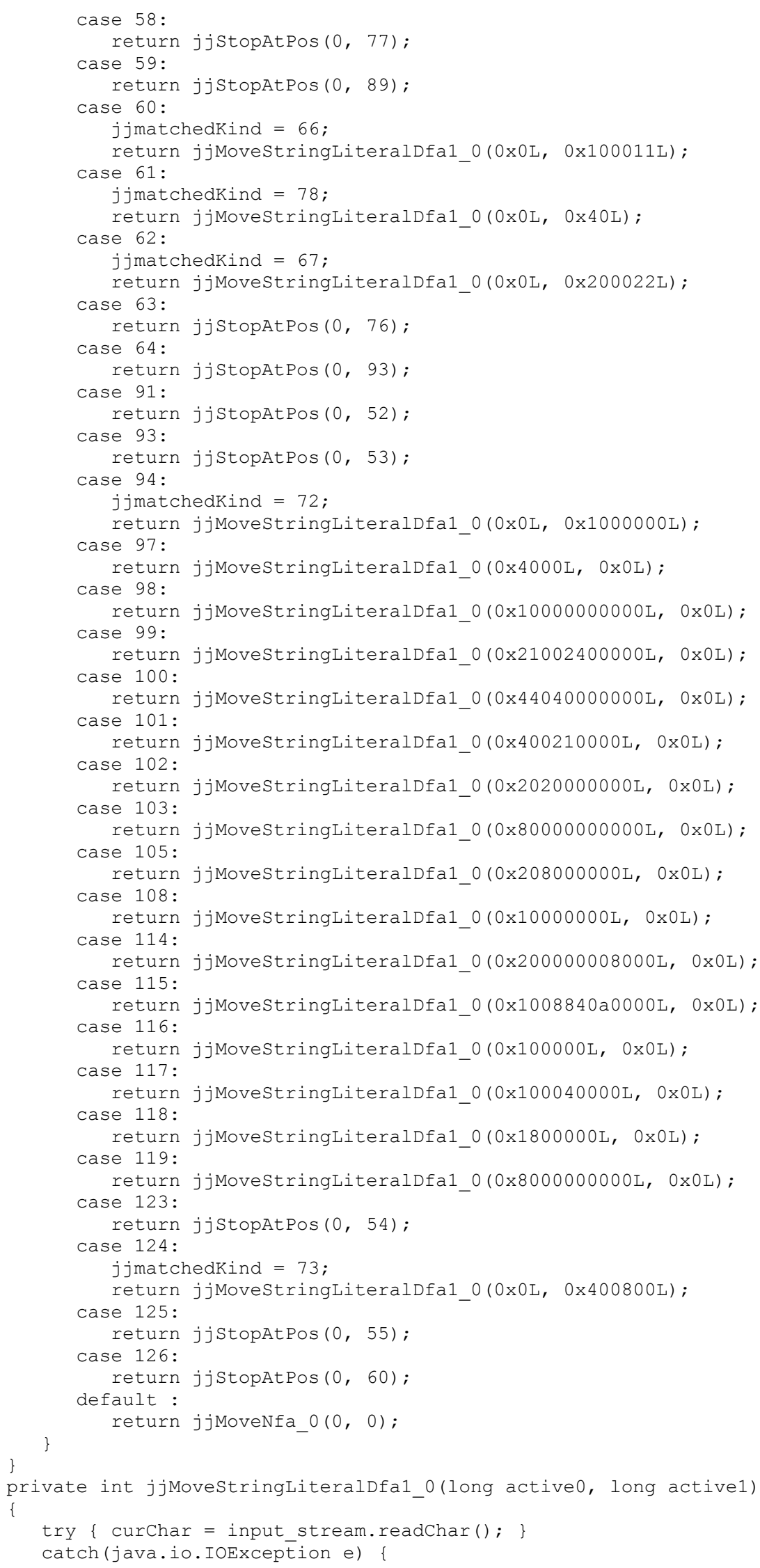




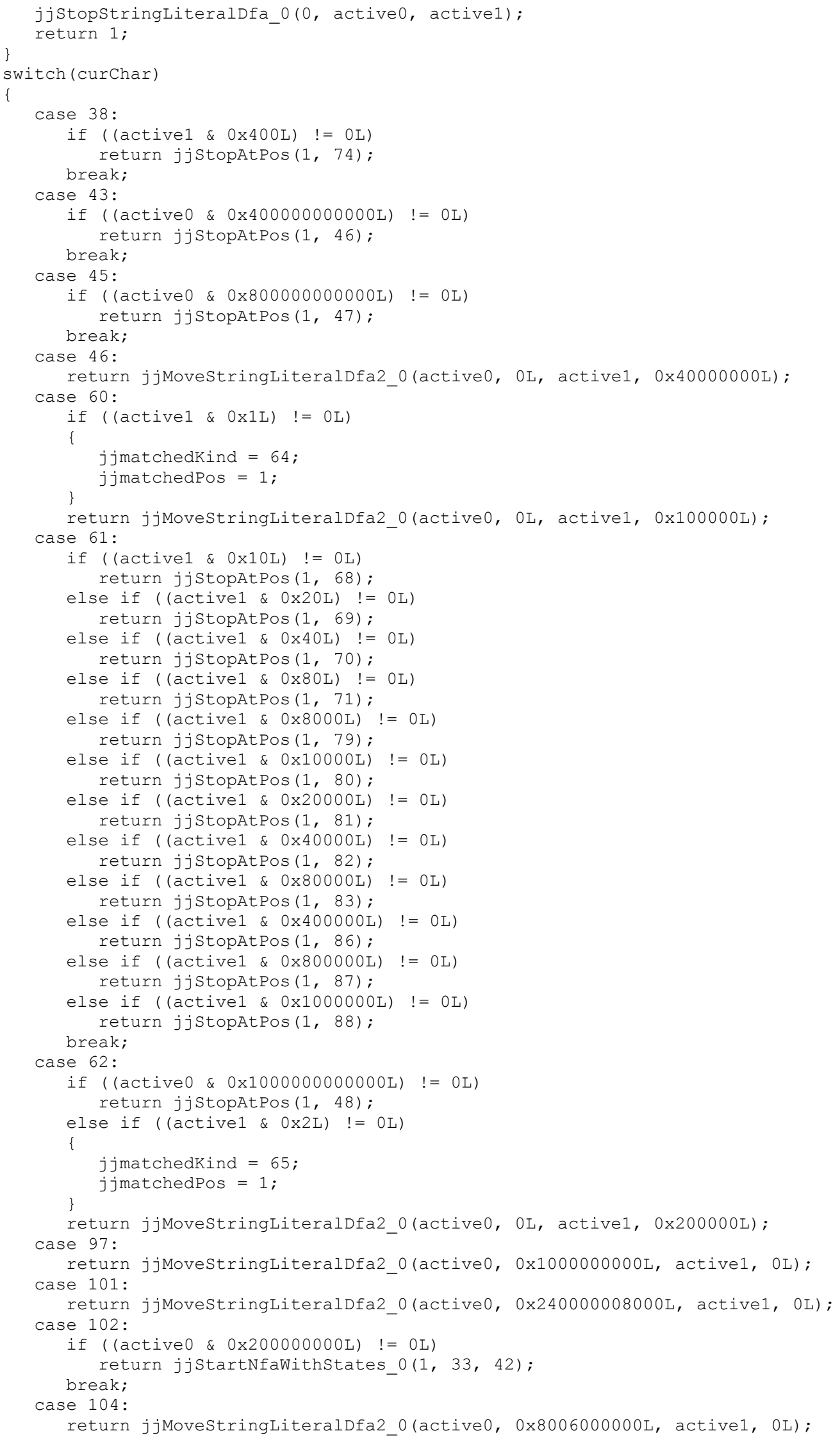




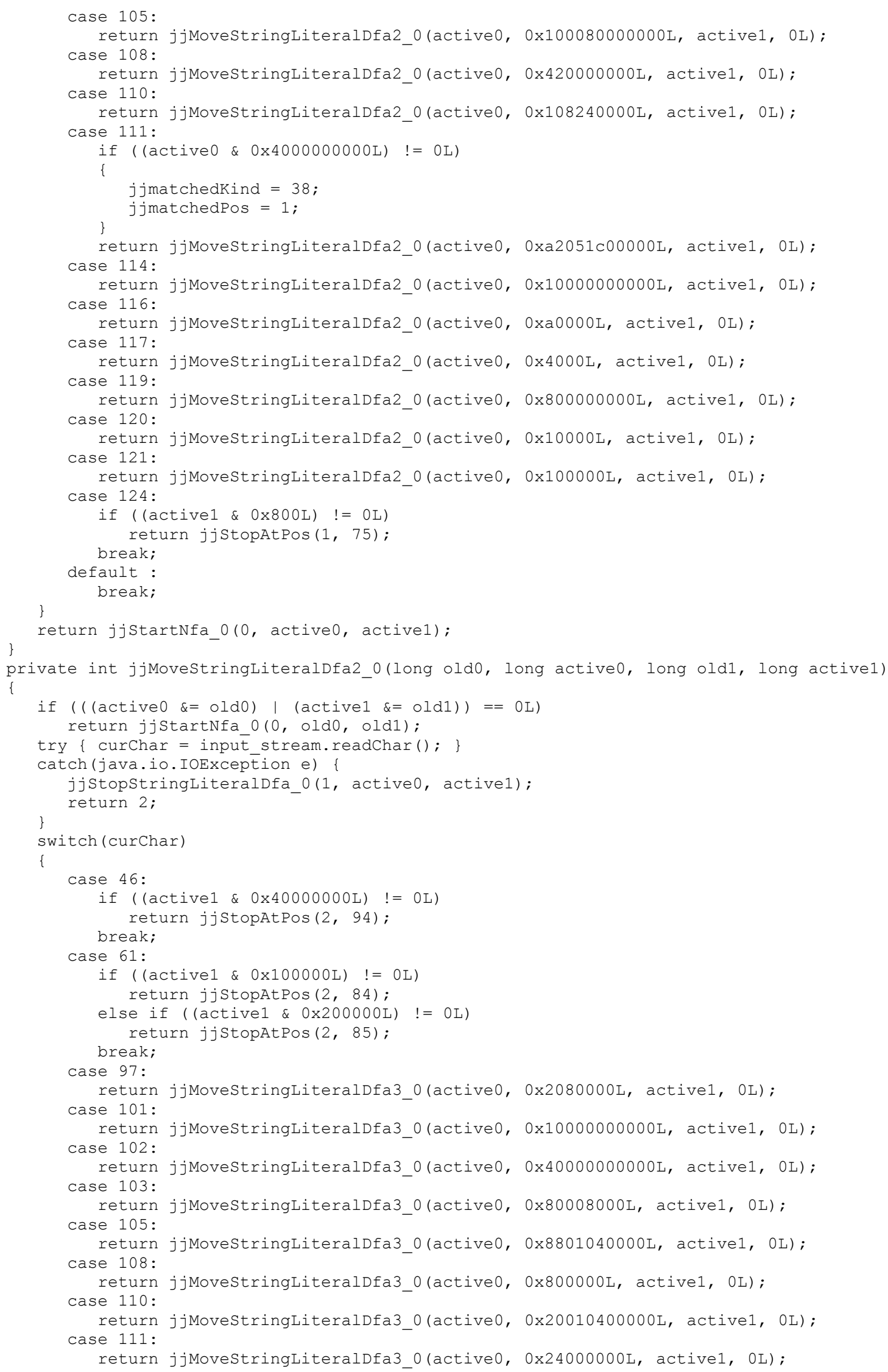




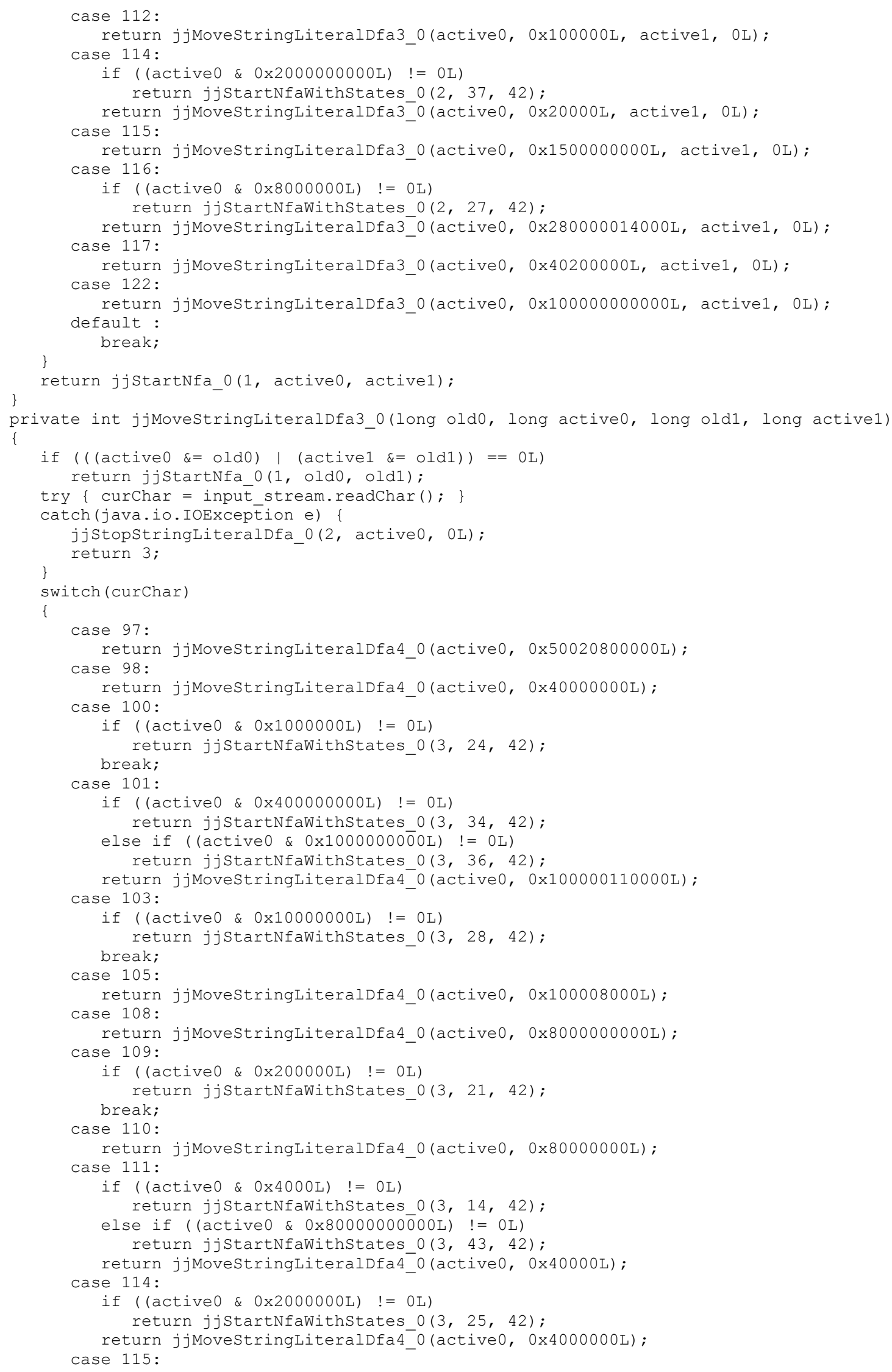




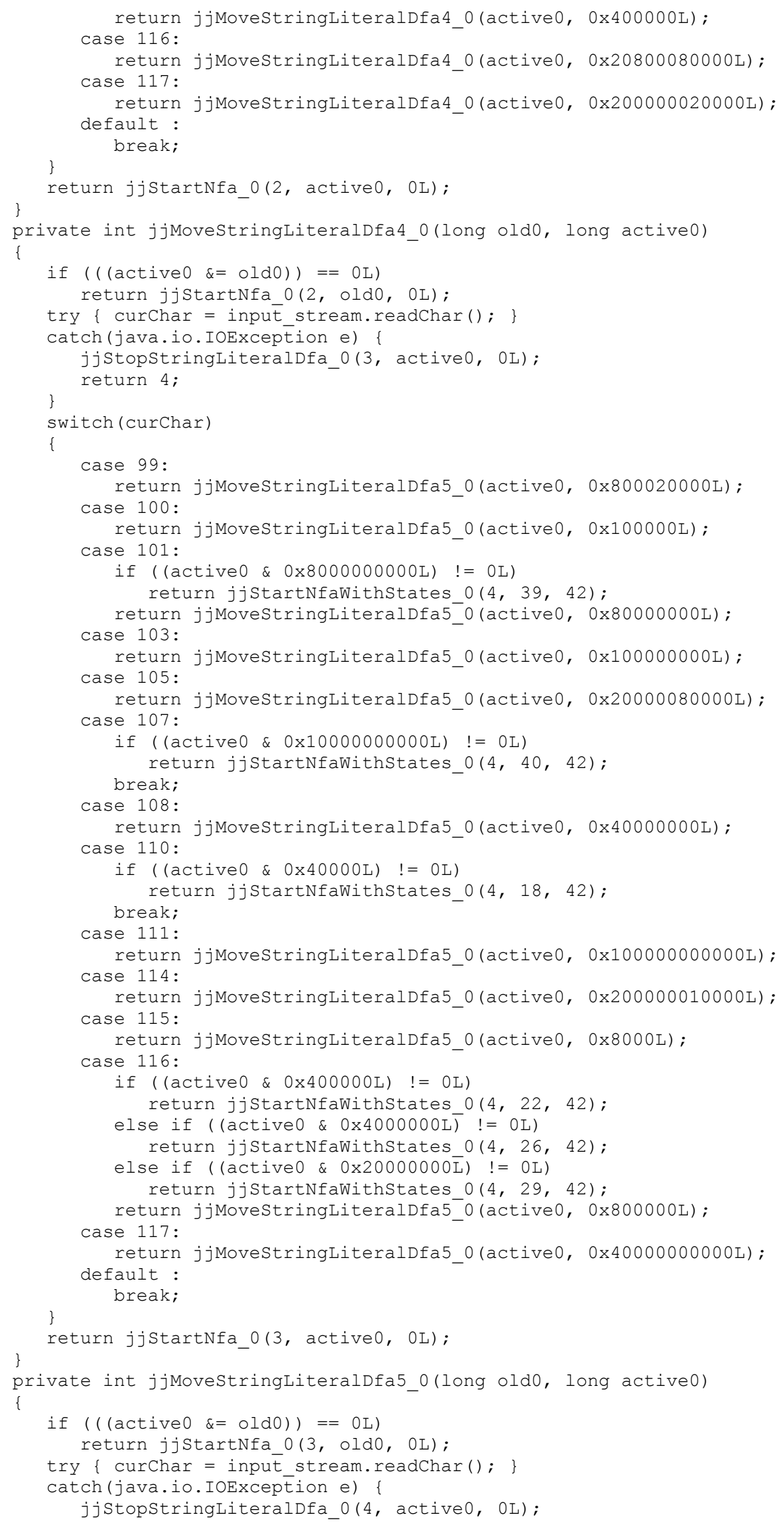




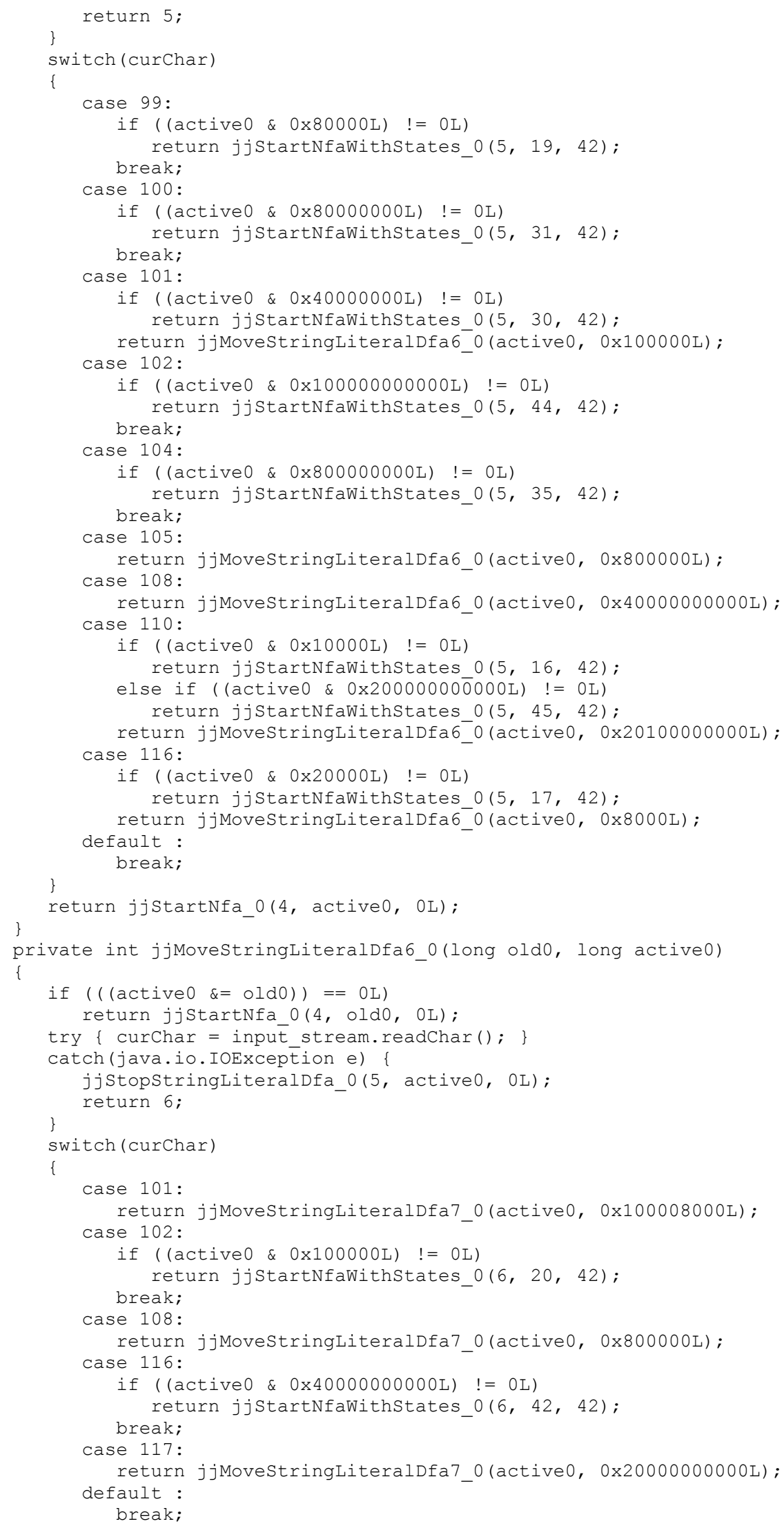




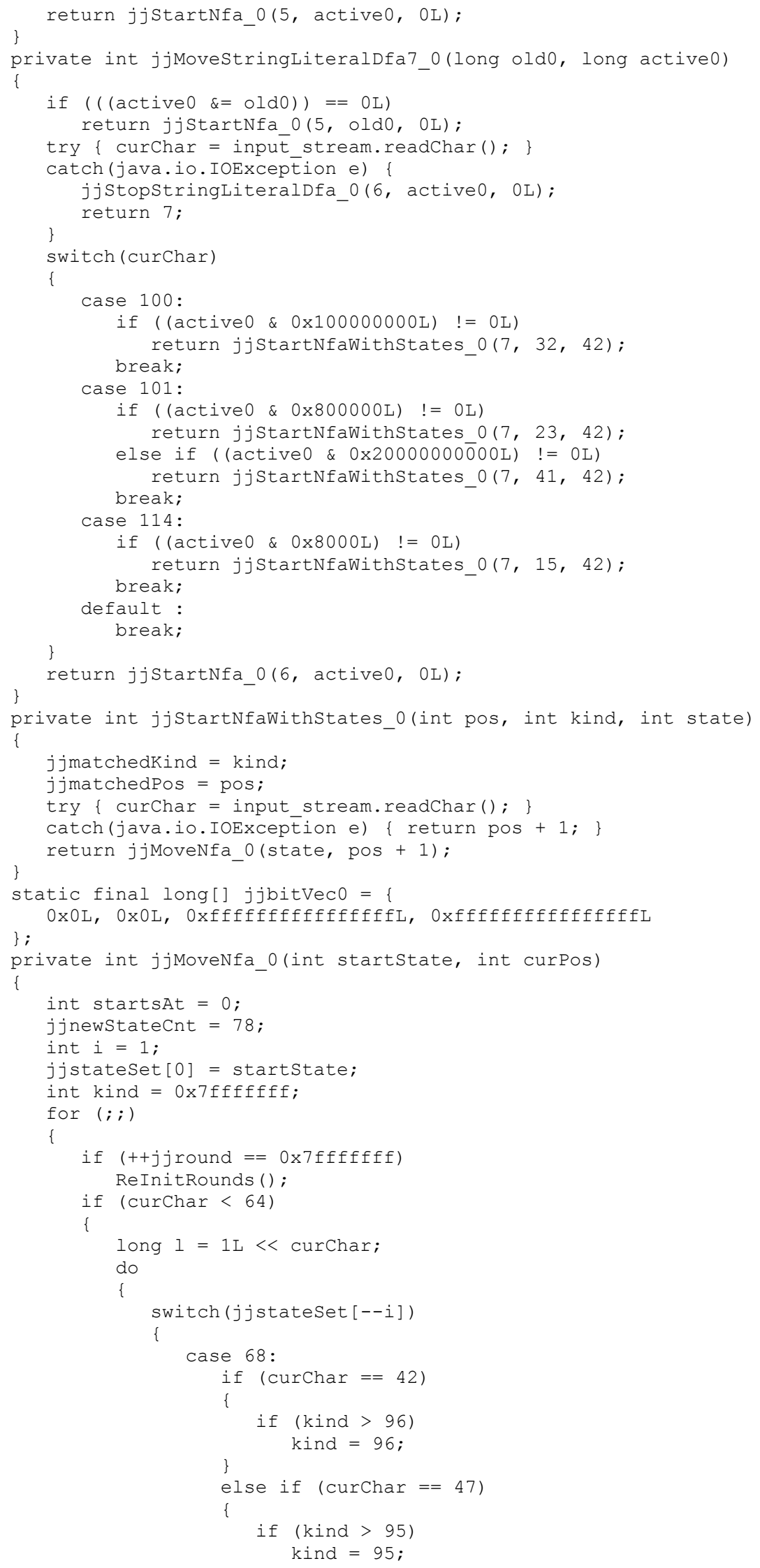




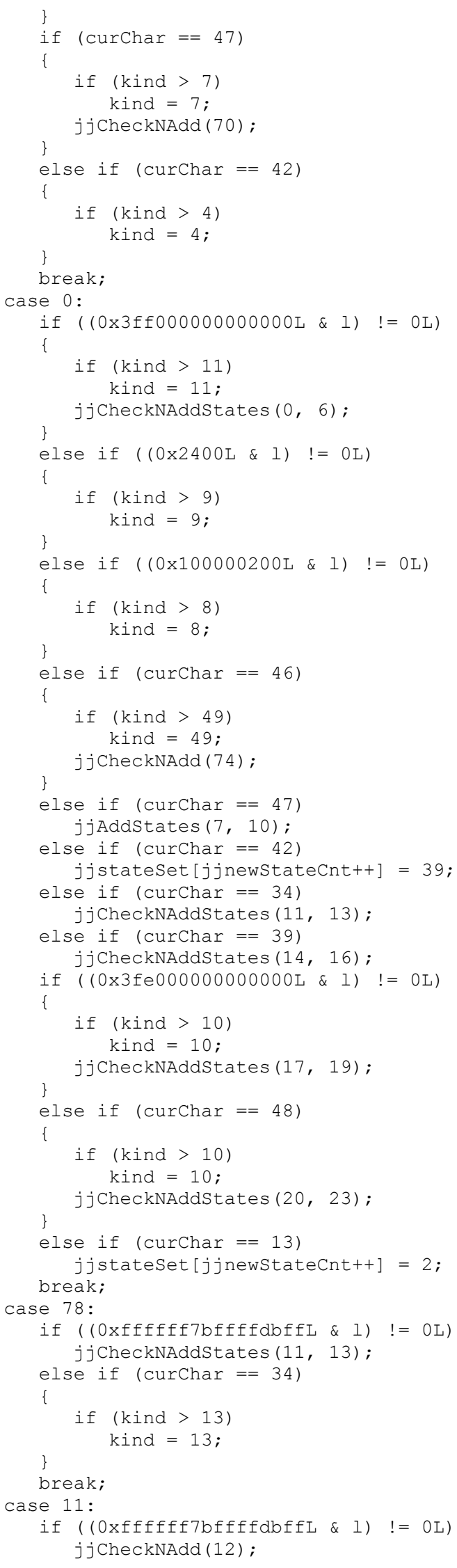




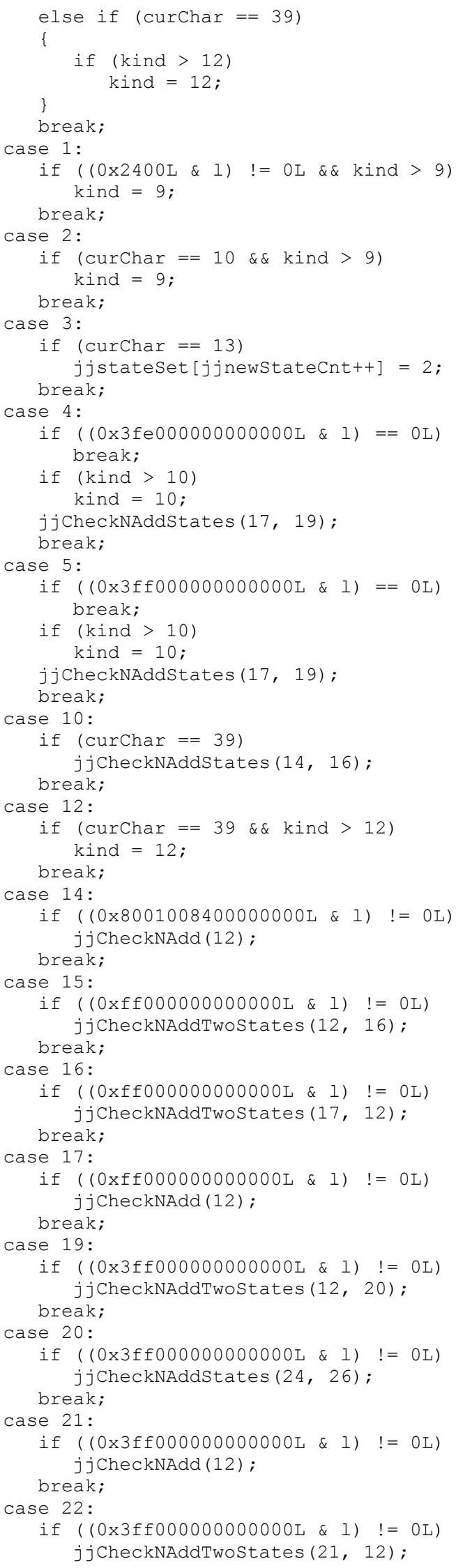




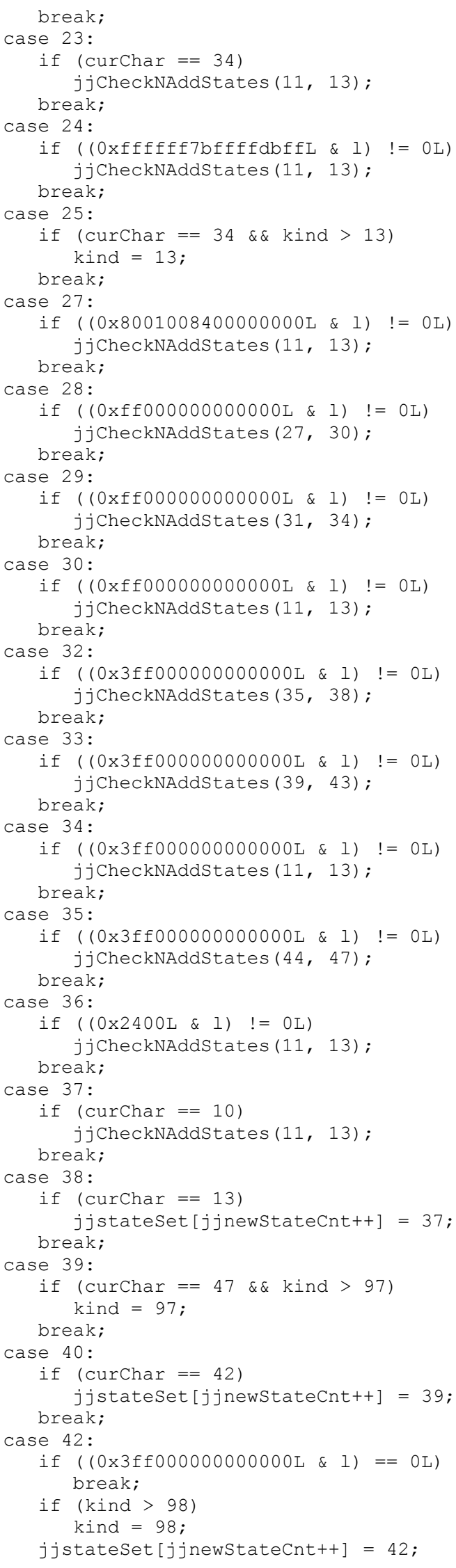




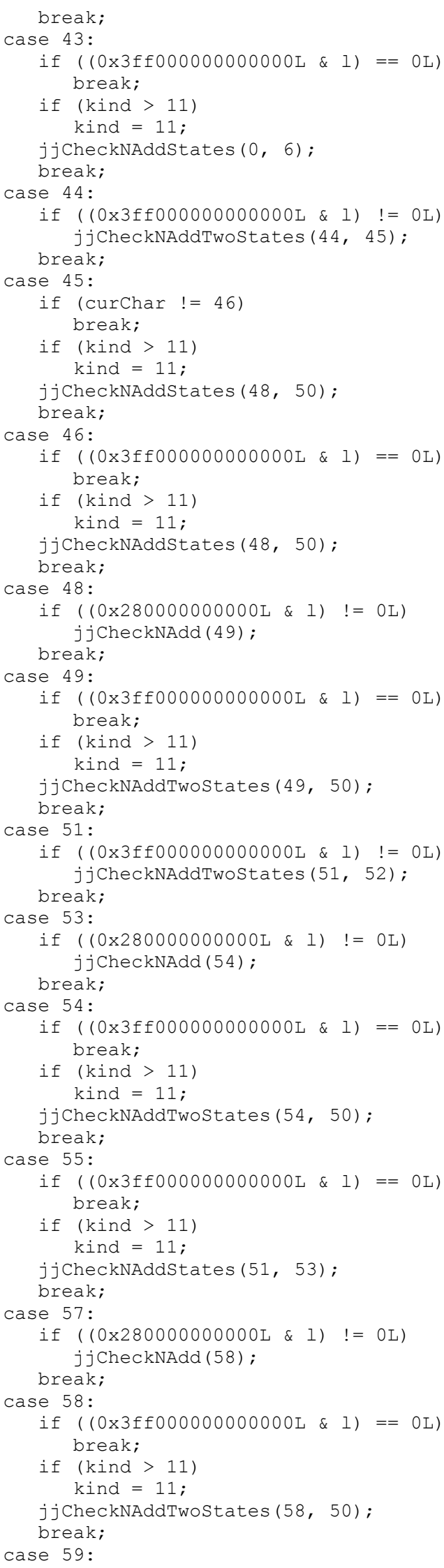




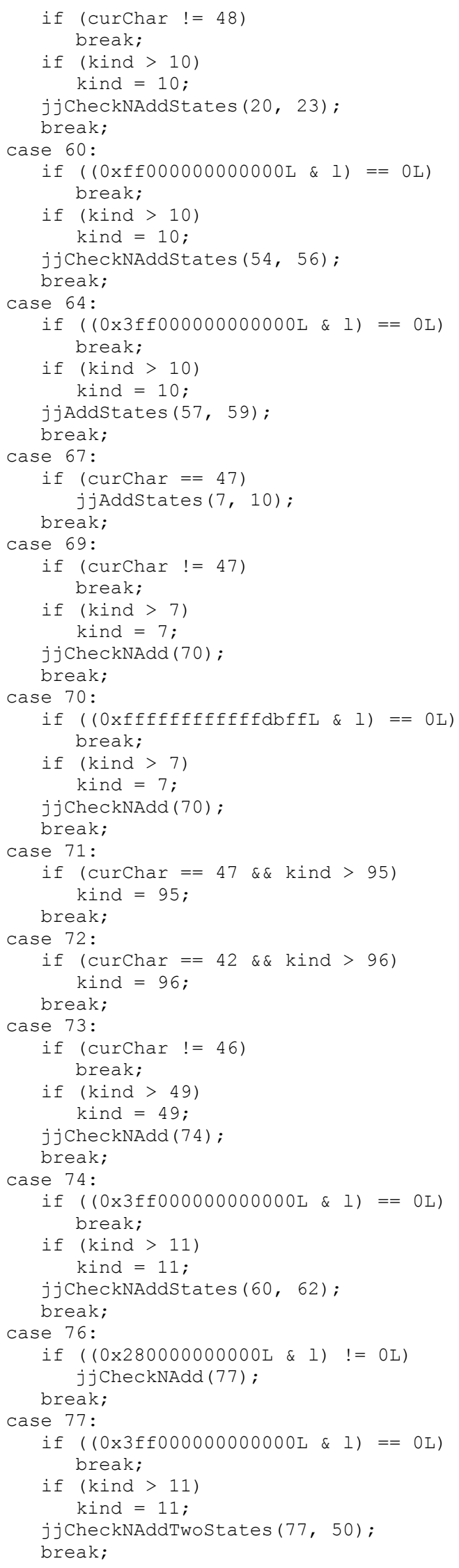




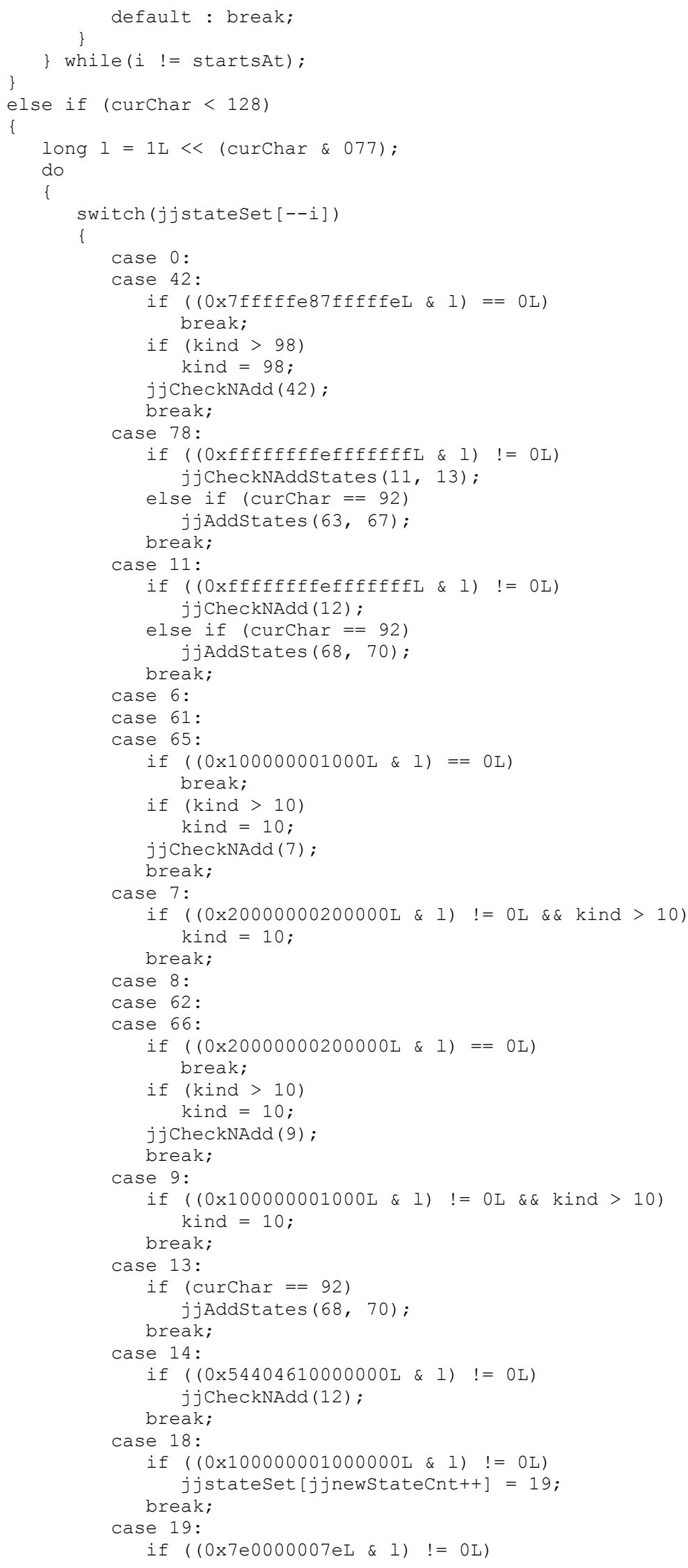




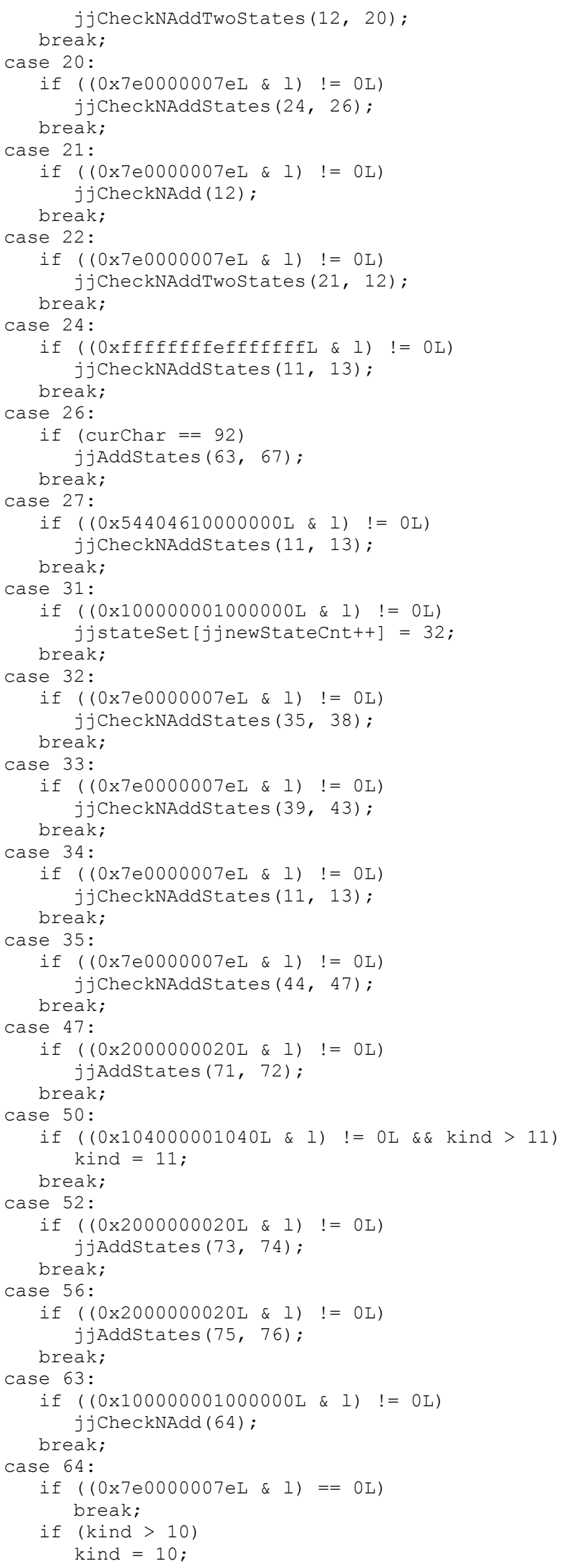




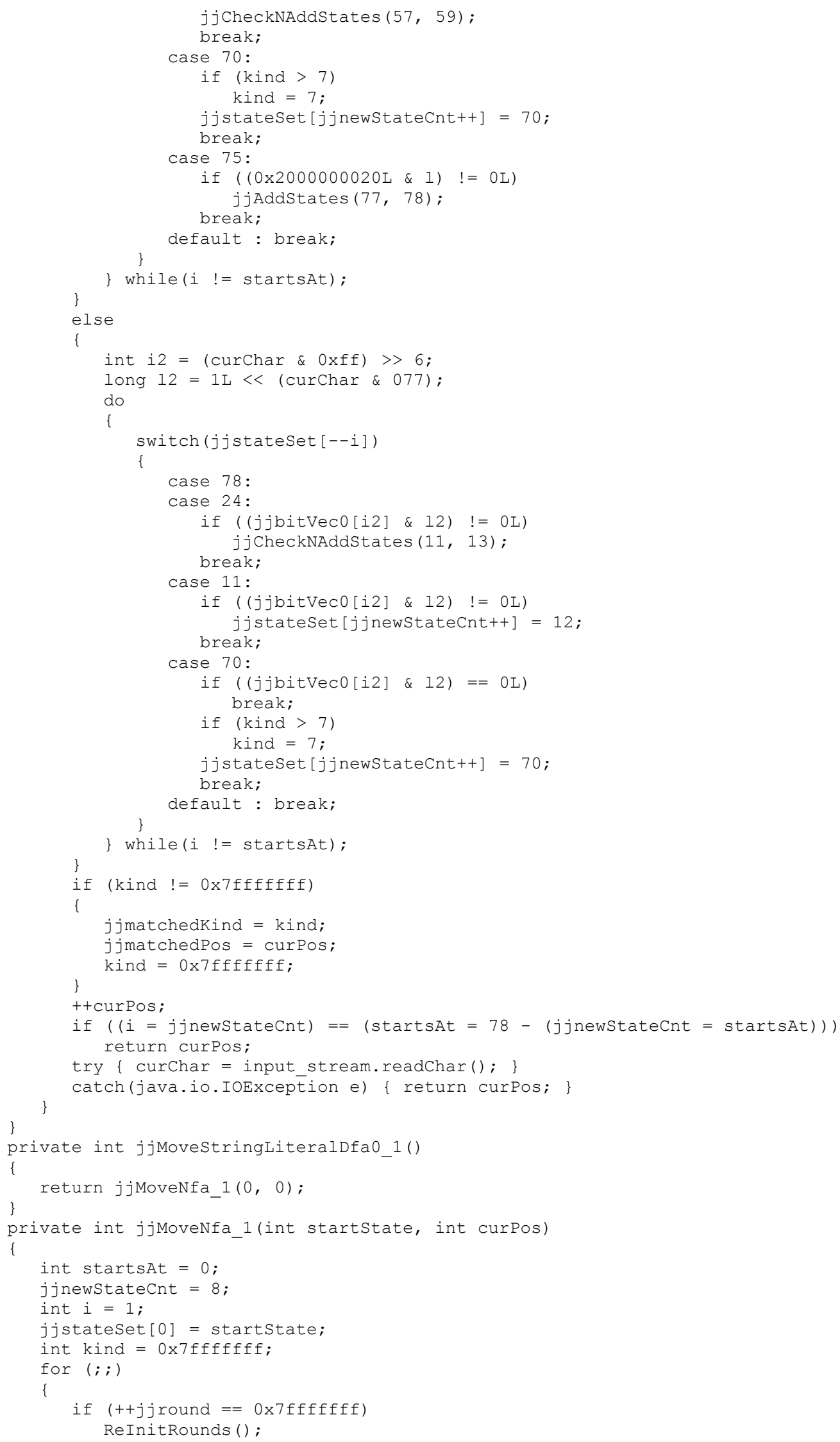




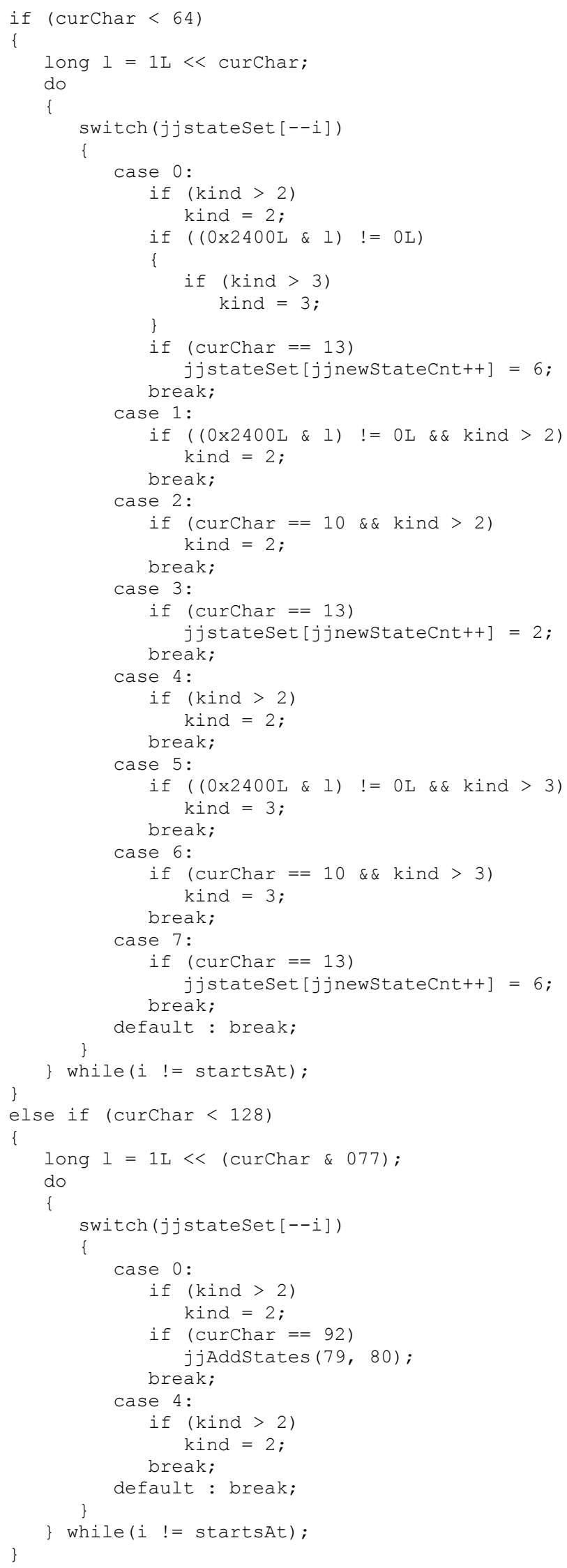




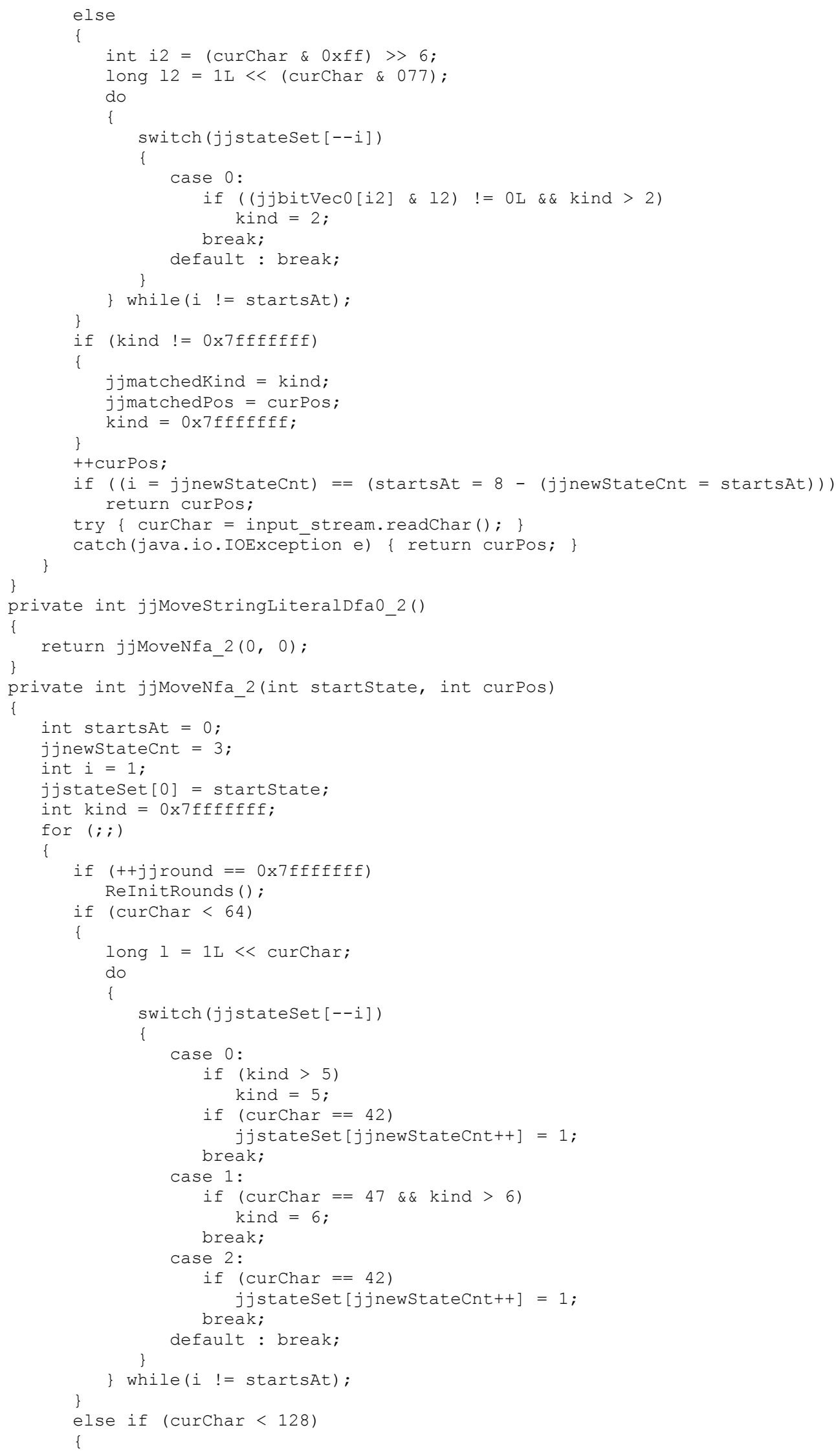




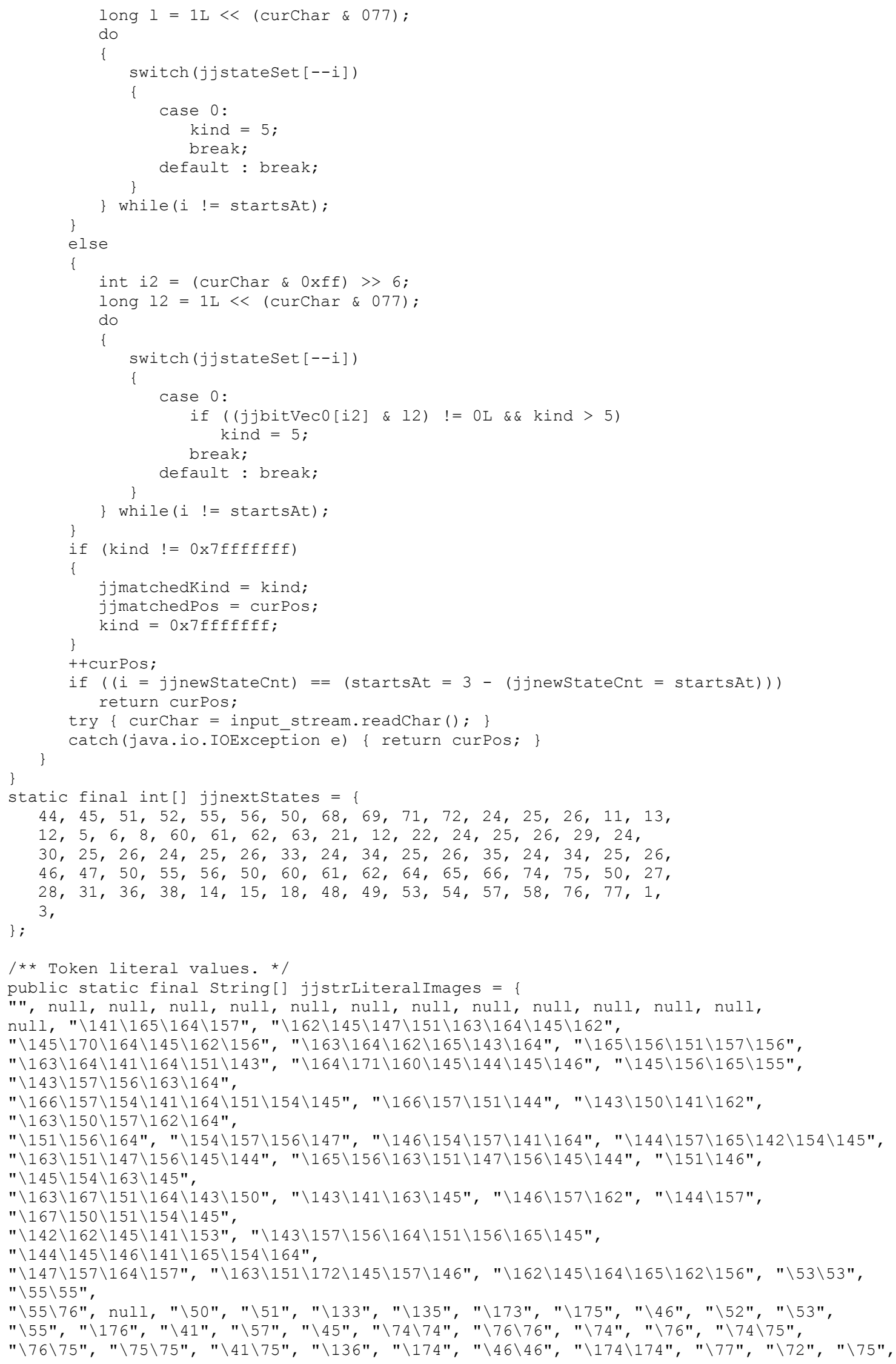




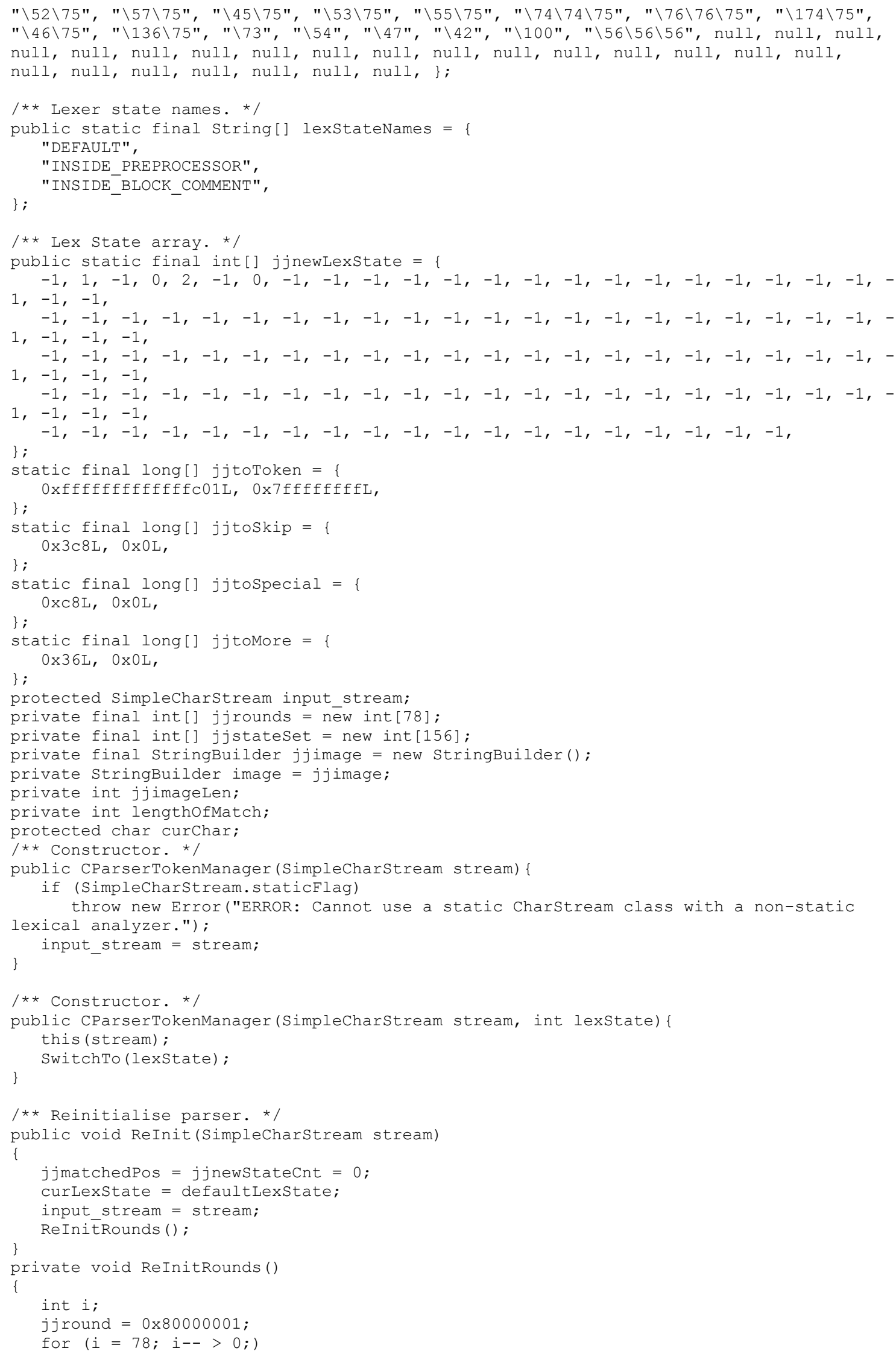




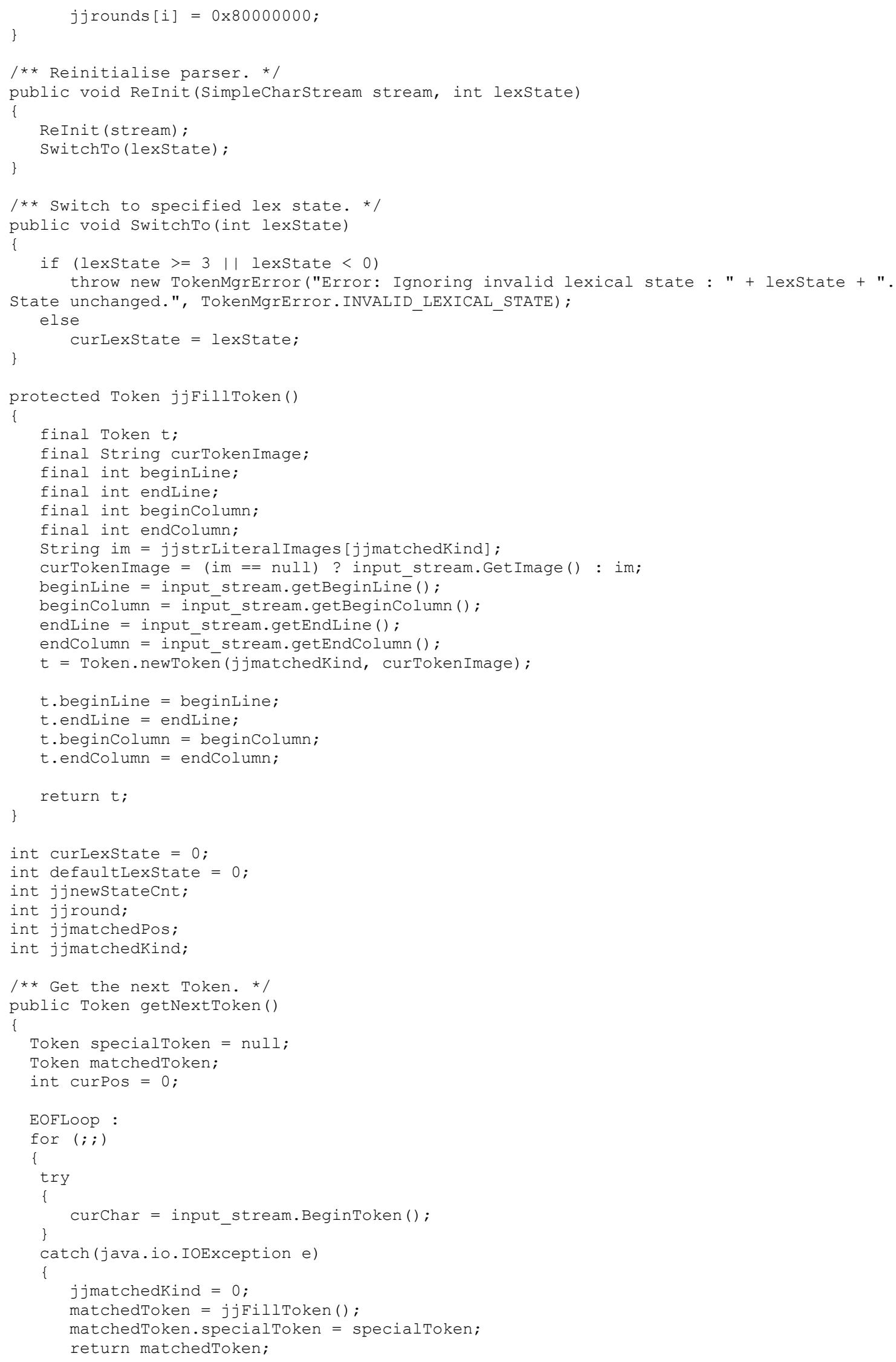




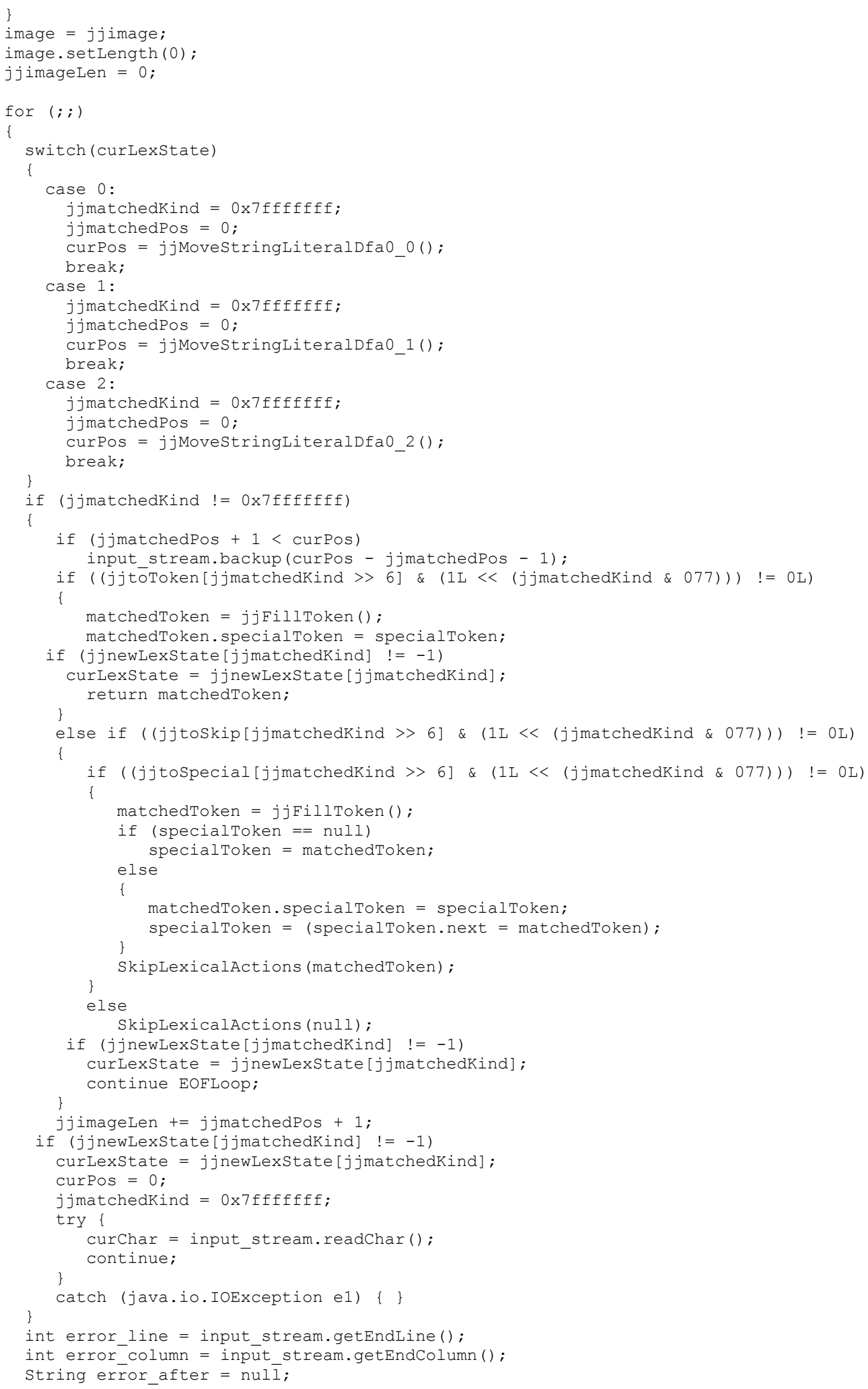




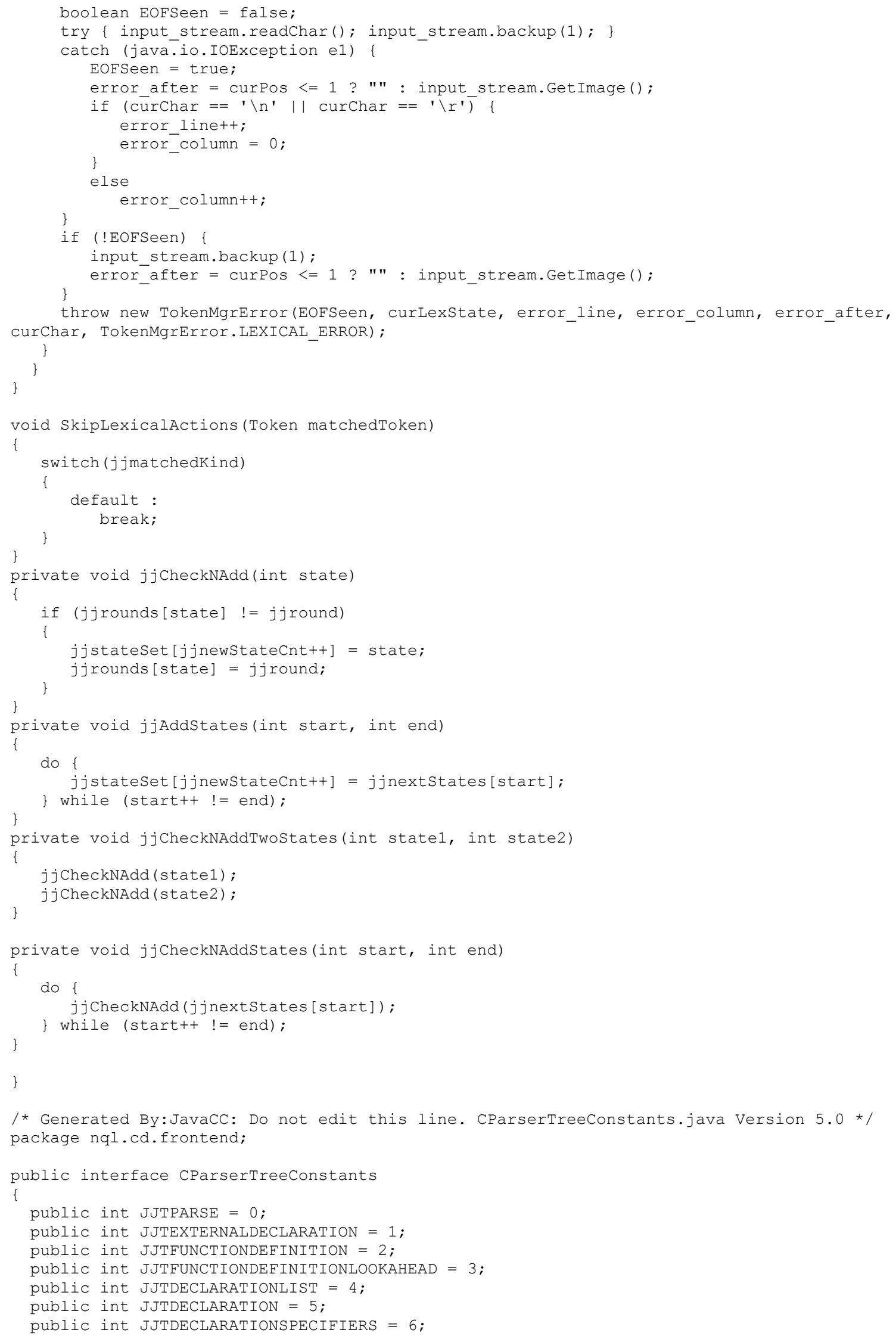




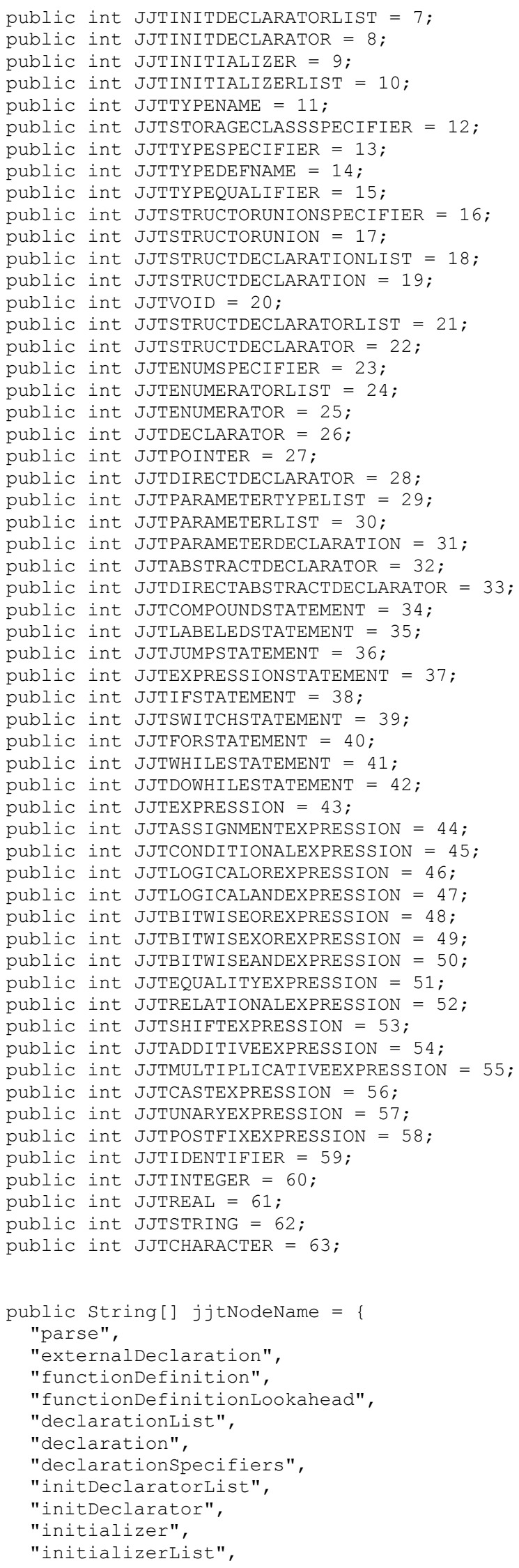




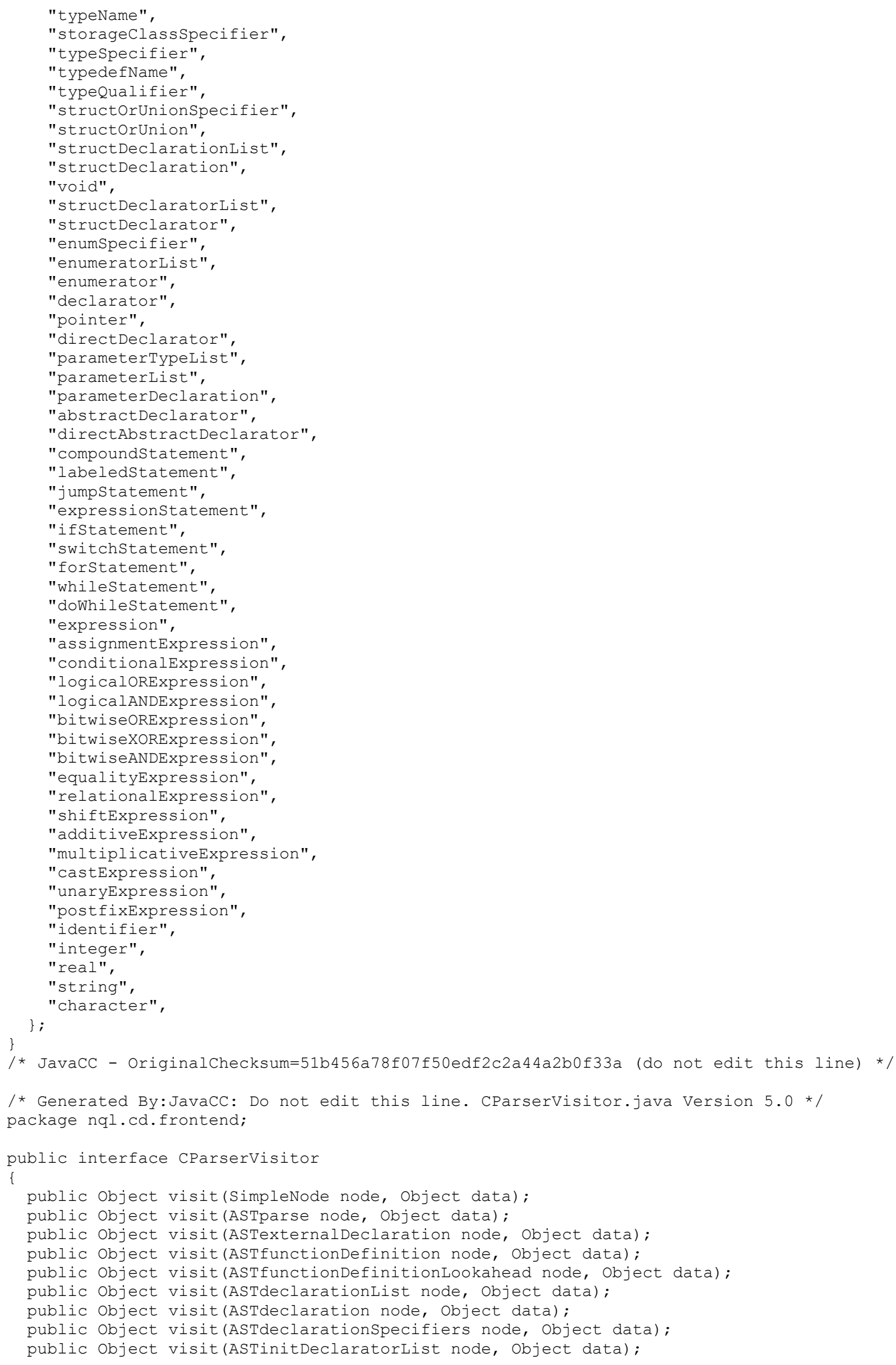


public Object visit(ASTinitDeclarator node, Object data); public Object visit(ASTinitializer node, Object data); public Object visit (ASTinitializerList node, Object data); public Object visit (ASTtypeName node, Object data); public object visit(ASTstorageClassSpecifier node, object data); public Object visit(ASTtypeSpecifier node, Object data); public Object visit(ASTtypedefName node, Object data); public Object visit(ASTtypeQualifier node, Object data); public Object visit(ASTstructorUnionspecifier node, object data); public Object visit(ASTstructorUnion node, object data); public Object visit(ASTstructDeclarationList node, object data); public Object visit(ASTstructDeclaration node, Object data); public Object visit(ASTstructDeclaratorList node, Object data); public object visit(ASTstructDeclarator node, Object data); public Object visit(ASTenumSpecifier node, Object data); public object visit(ASTenumeratorList node, object data); public Object visit(ASTenumerator node, Object data); public object visit(ASTdeclarator node, Object data); public Object visit(ASTpointer node, Object data); public object visit(ASTdirectDeclarator node, Object data); public Object visit (ASTparameterTypeList node, Object data); public Object visit(ASTparameterList node, Object data); public Object visit(ASTparameterDeclaration node, object data); public Object visit(ASTabstractDeclarator node, Object data); public object visit(ASTdirectAbstractDeclarator node, object data); public Object visit (ASTcompoundStatement node, Object data); public Object visit(ASTlabeledStatement node, Object data); public Object visit(ASTjumpstatement node, Object data); public Object visit(ASTexpressionstatement node, object data); public Object visit(ASTifStatement node, Object data); public Object visit(ASTswitchstatement node, Object data); public object visit(ASTforStatement node, Object data); public Object visit(ASTwhilestatement node, Object data); public Object visit(ASTdoWhilestatement node, Object data); public Object visit(ASTexpression node, Object data); public Object visit(ASTassignmentExpression node, object data); public object visit(ASTconditionalExpression node, object data); public Object visit(ASTlogicaloRExpression node, Object data); public Object visit(ASTlogicalANDExpression node, object data); public Object visit(ASTbitwiseoRExpression node, object data); public Object visit (ASTbitwiseXORExpression node, Object data); public Object visit(ASTbitwiseANDExpression node, Object data); public Object visit(ASTequalityExpression node, Object data); public Object visit(ASTrelationalExpression node, Object data); public Object visit(ASTshiftExpression node, Object data); public Object visit(ASTadditiveExpression node, Object data); public object visit(ASTmultiplicativeExpression node, object data); public Object visit(ASTcastExpression node, Object data); public Object visit(ASTunaryExpression node, Object data); public Object visit (ASTpostfixExpression node, Object data); public Object visit(ASTidentifier node, Object data); public Object visit(ASTinteger node, Object data); public Object visit(ASTreal node, object data); public Object visit(ASTstring node, object data); \} public Object visit(ASTcharacter node, Object data);

/* JavaCC - OriginalChecksum=7da48192cbde959bde3cddf30a62a9ad (do not edit this line) */

/* Generated By:JavaCC: Do not edit this line. JJTCParserState.java Version 5.0 */ package nql.cd.frontend;

public class JJTCParserstate private java.util.List $<$ Node $>$ nodes; private java.util.List<Integer> marks;

private int sp; // number of nodes on stack private int mk; // current mark

private boolean node_created;

public JJTCParserstate() \{ nodes = new java.util. ArrayList $<$ Node $>()$; 


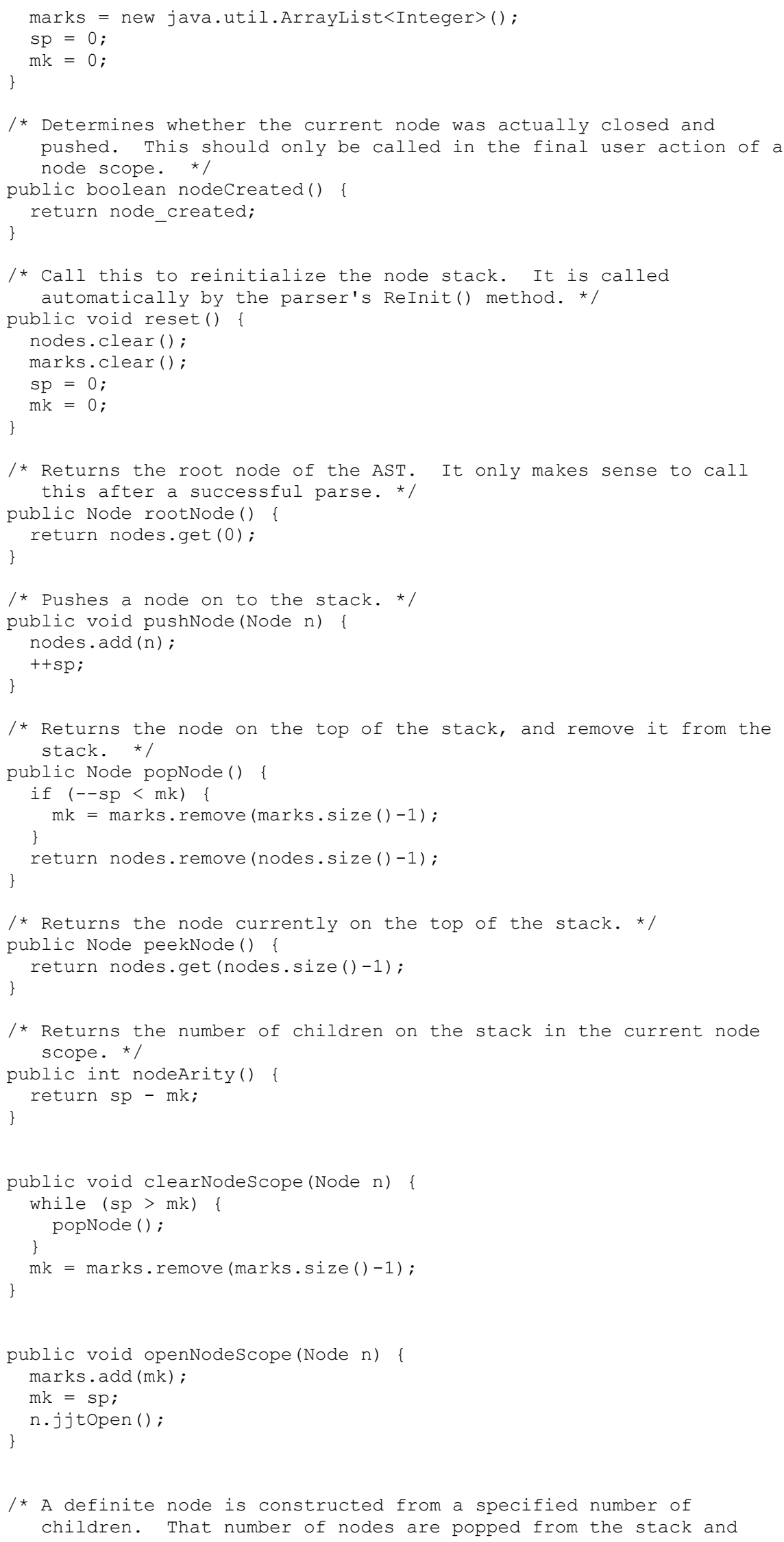




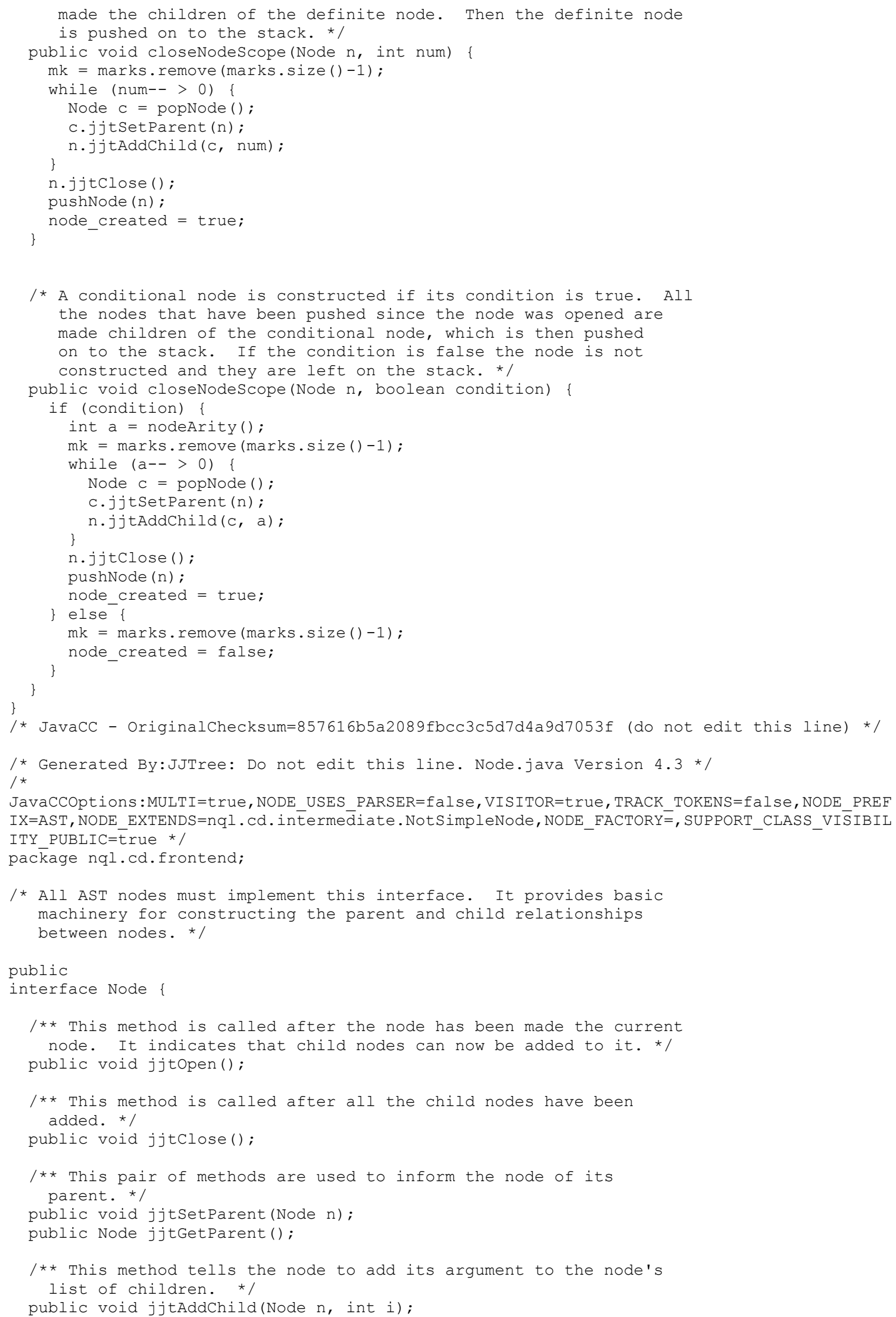




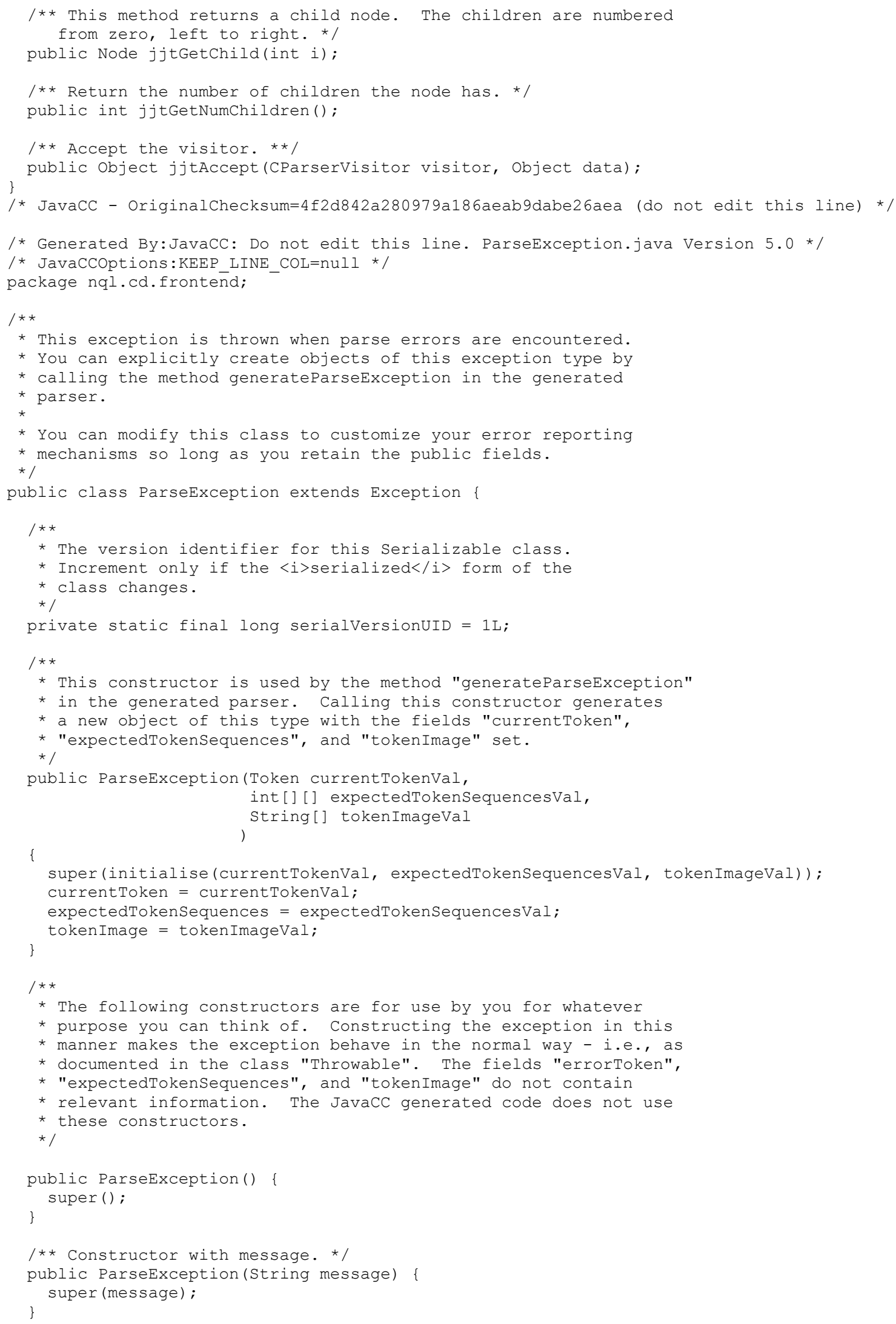




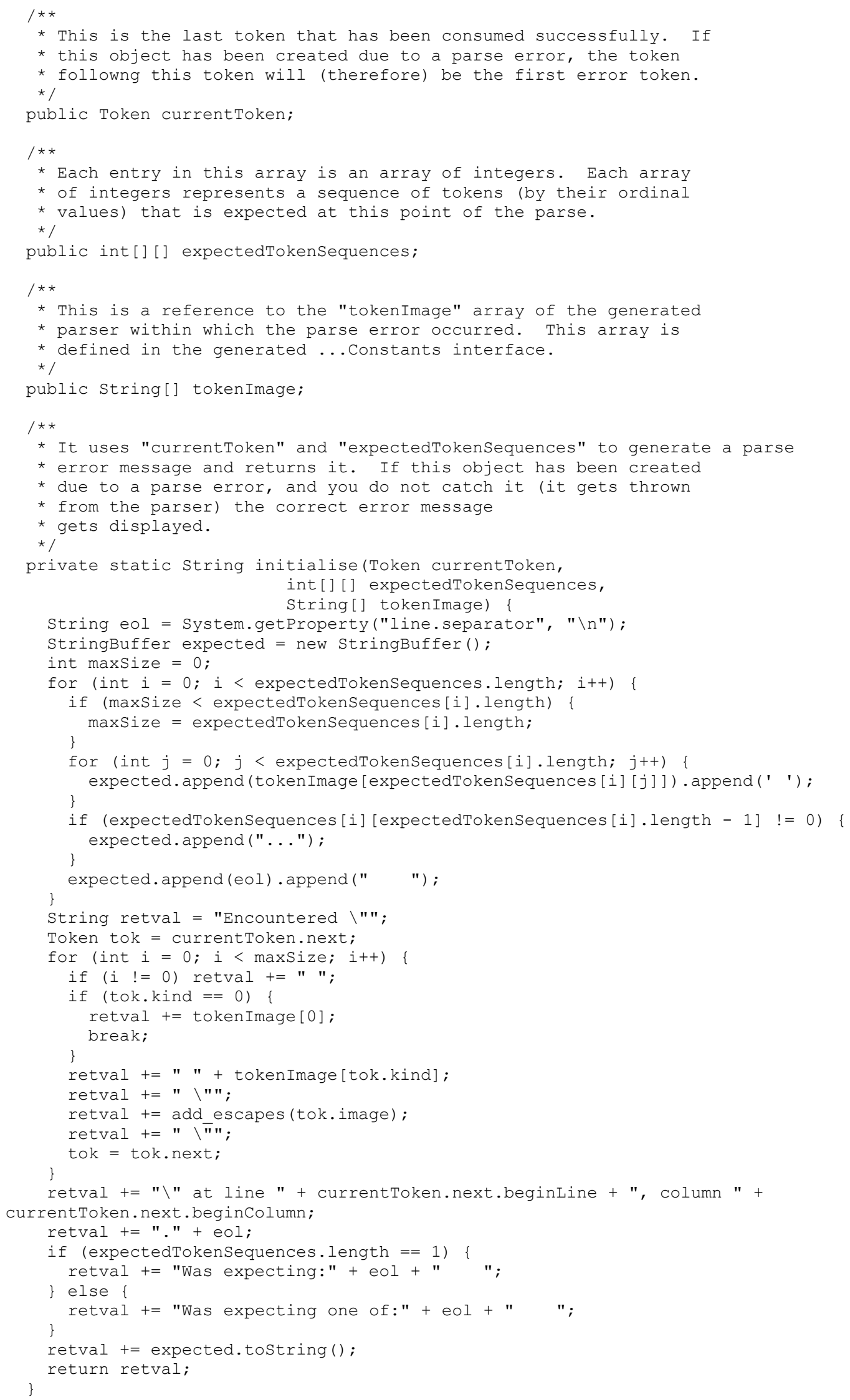




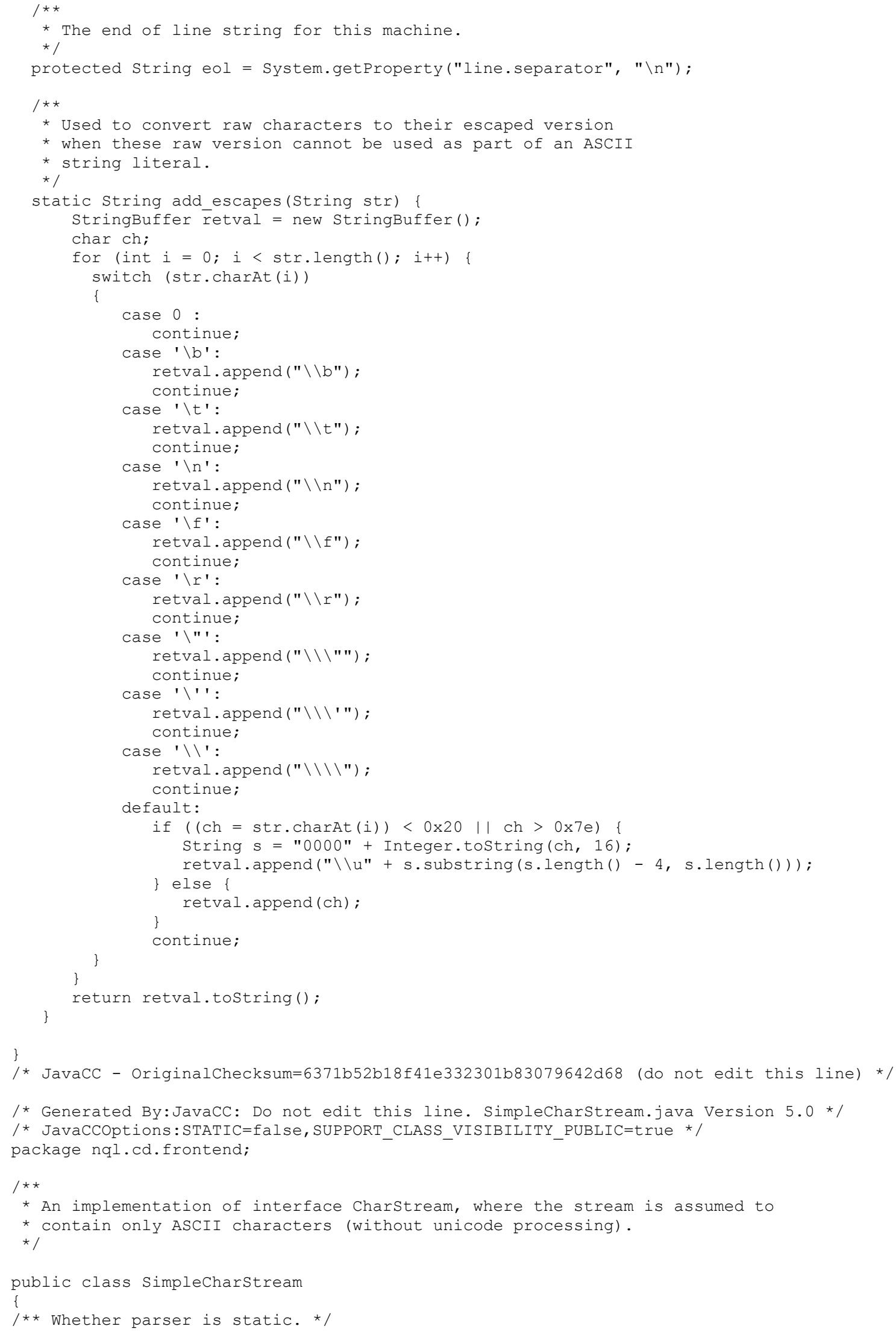




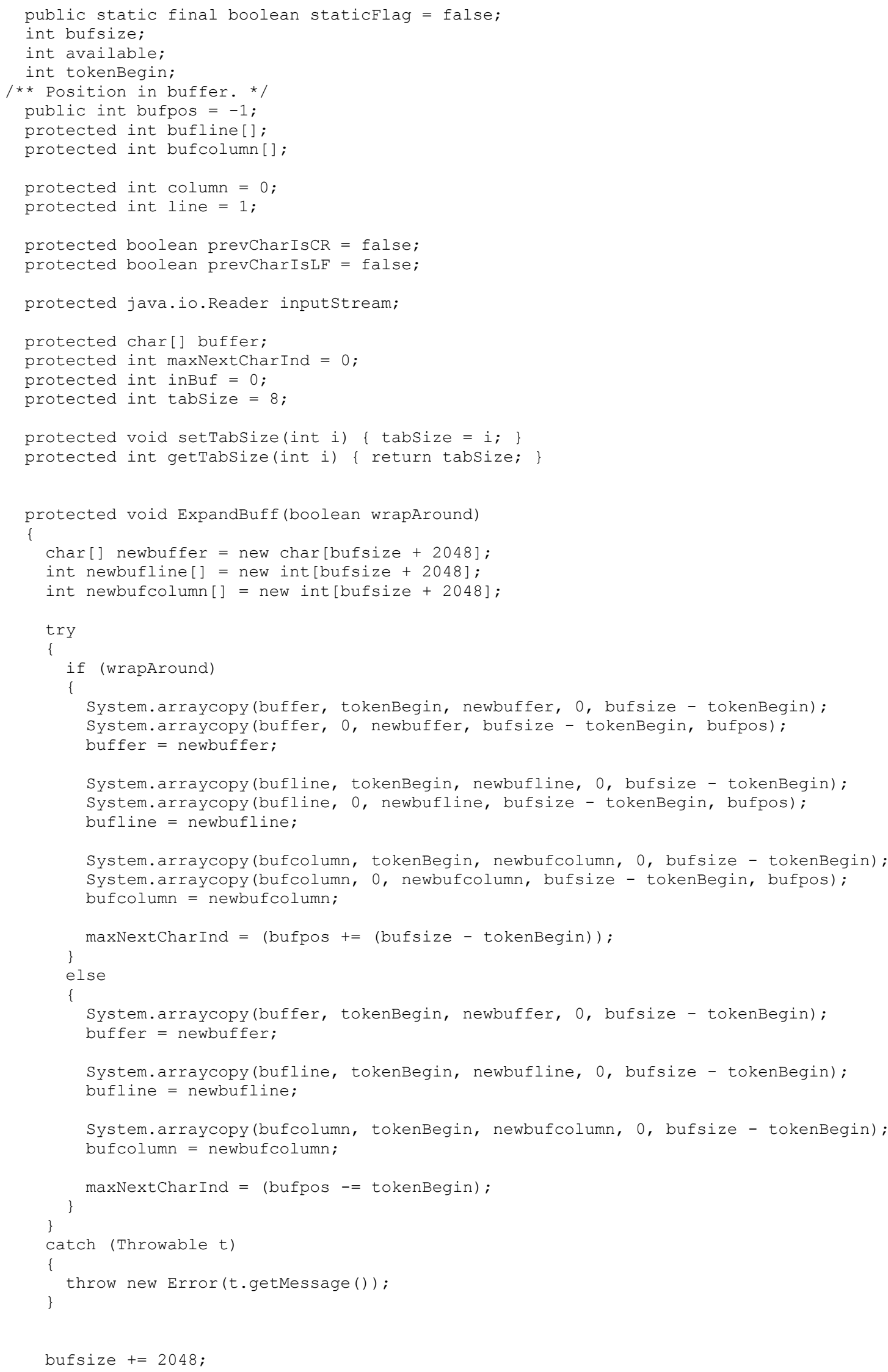




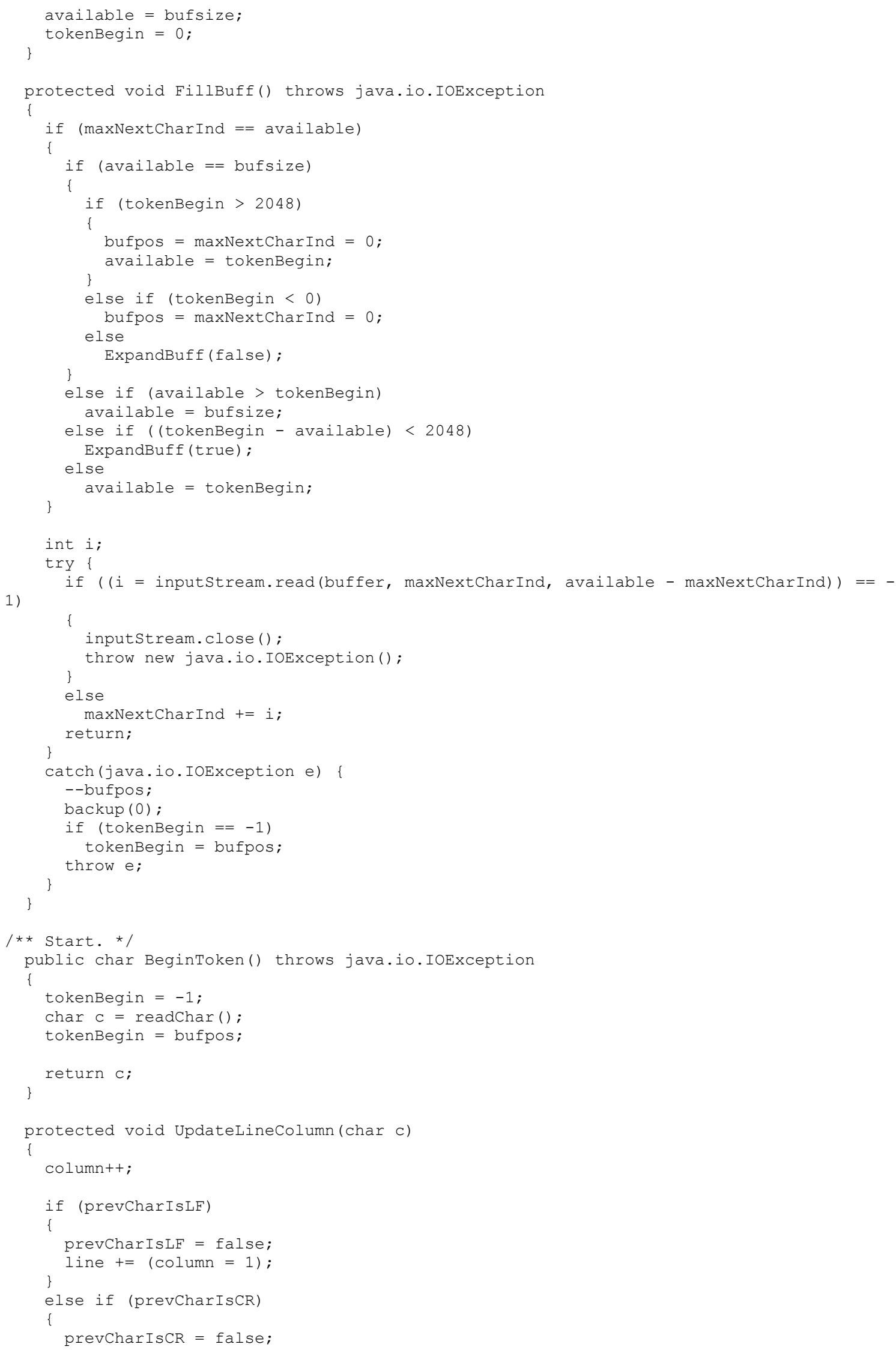




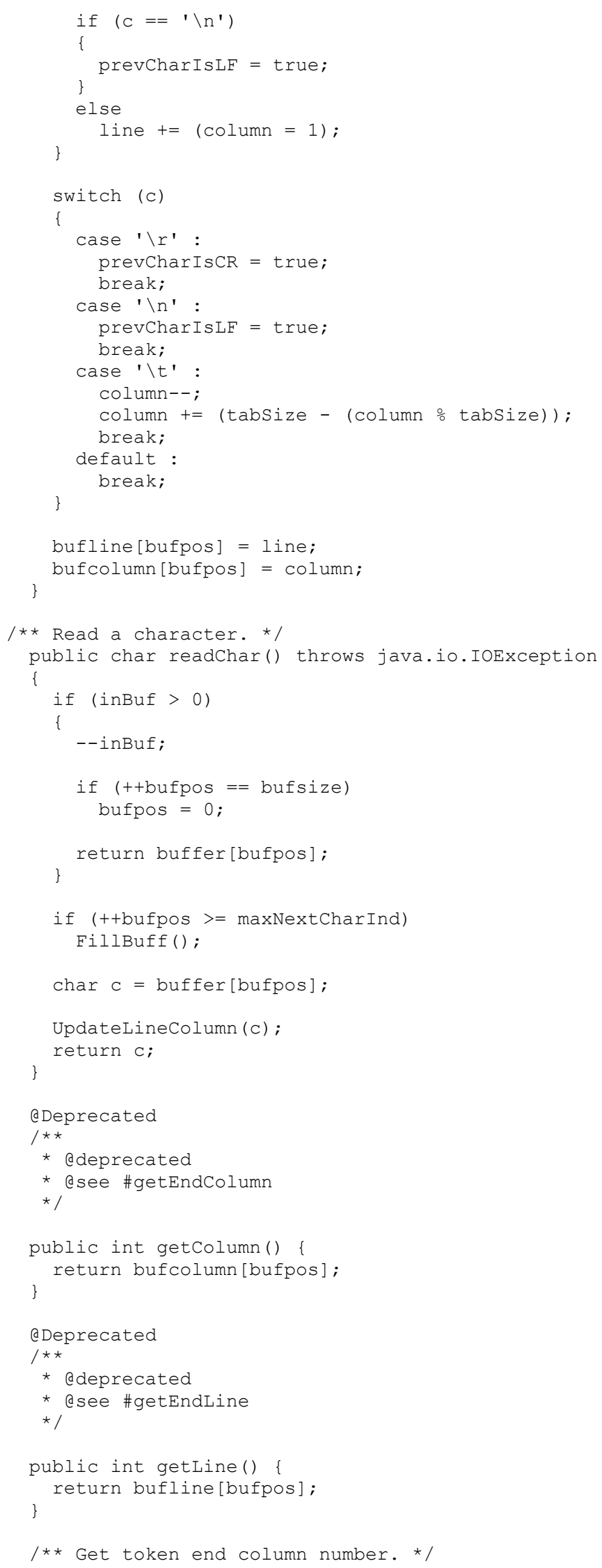




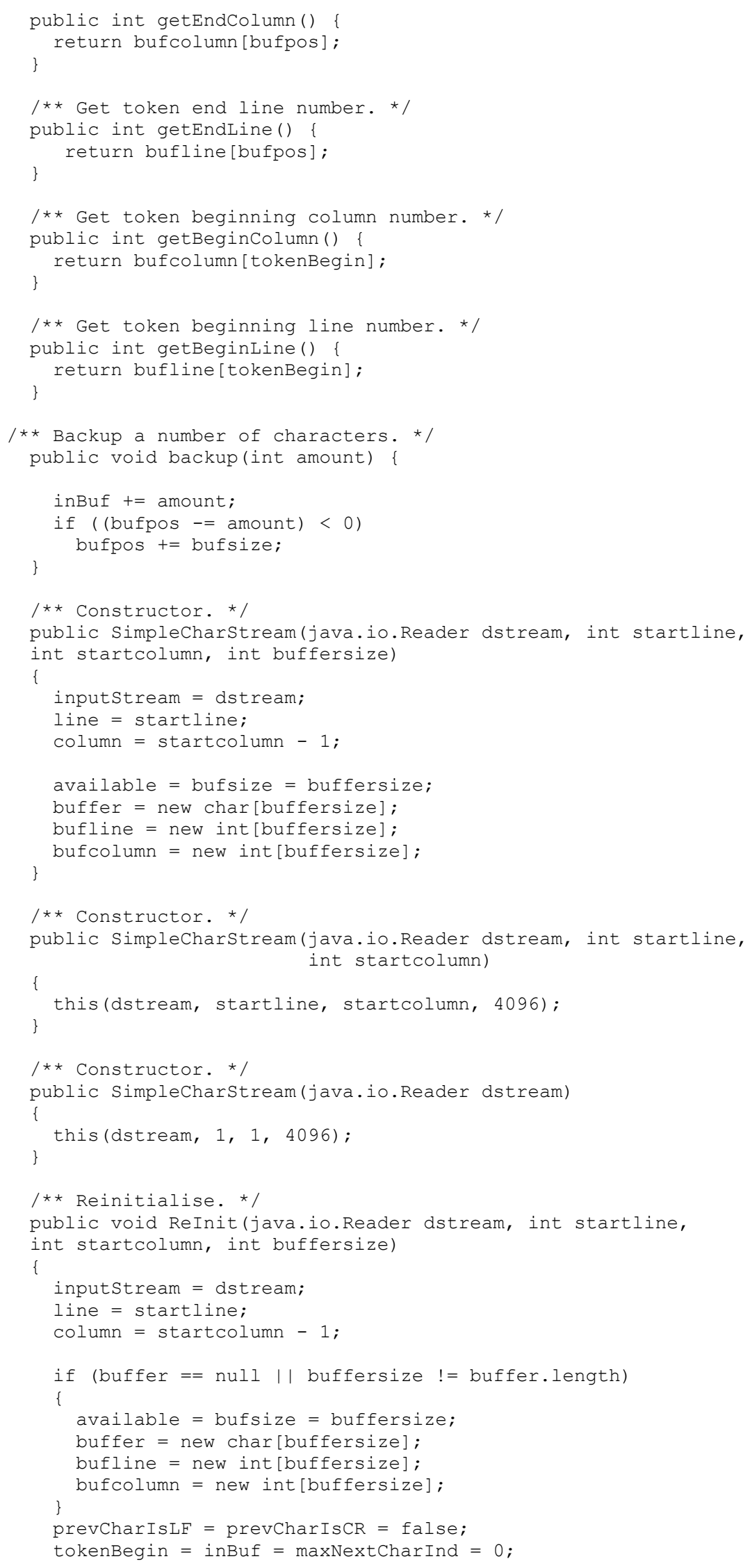




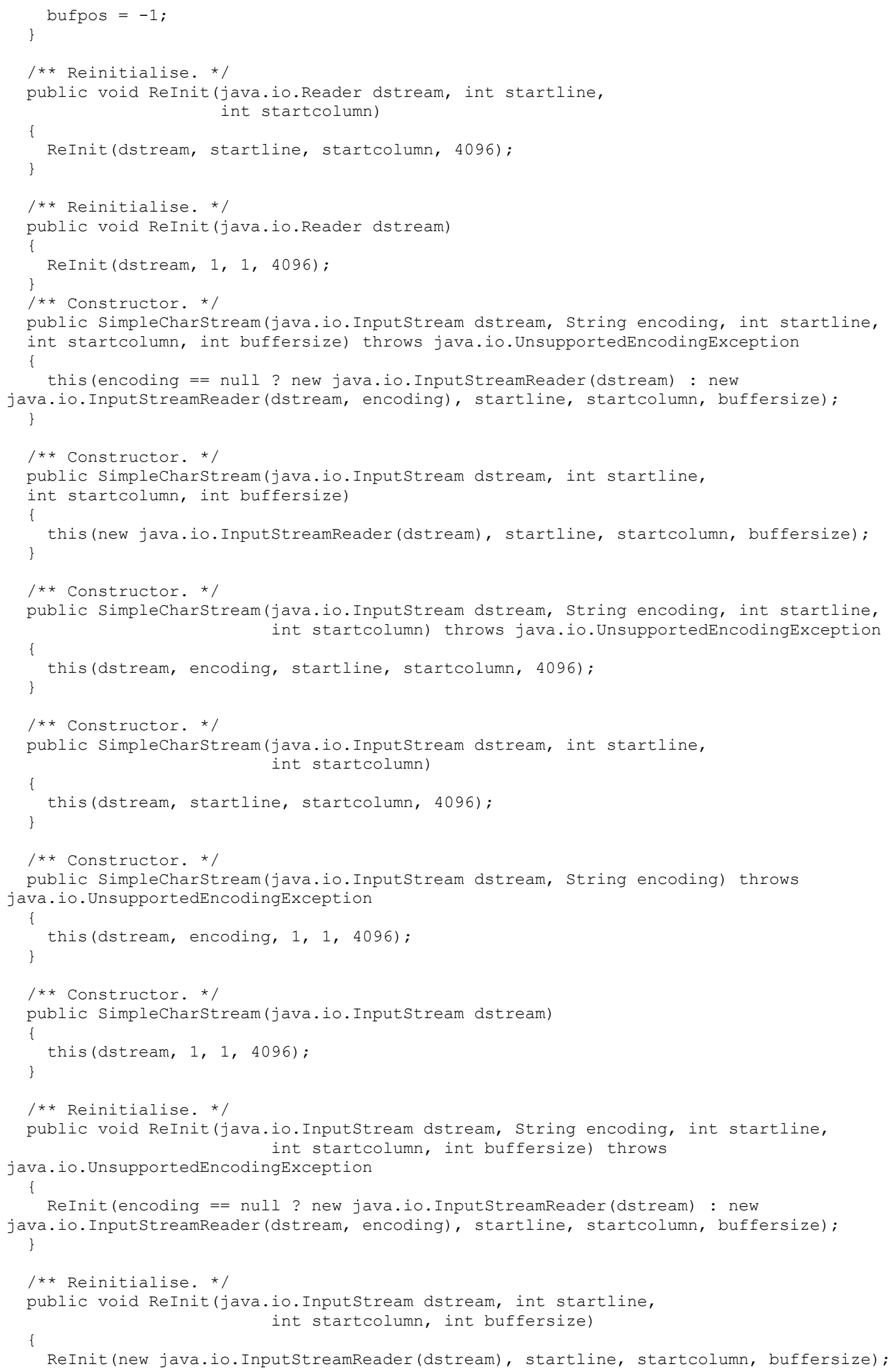


/** Reinitialise. */

public void ReInit(java.io.Inputstream dstream, string encoding) throws java.io.UnsupportedEncodingException \} 


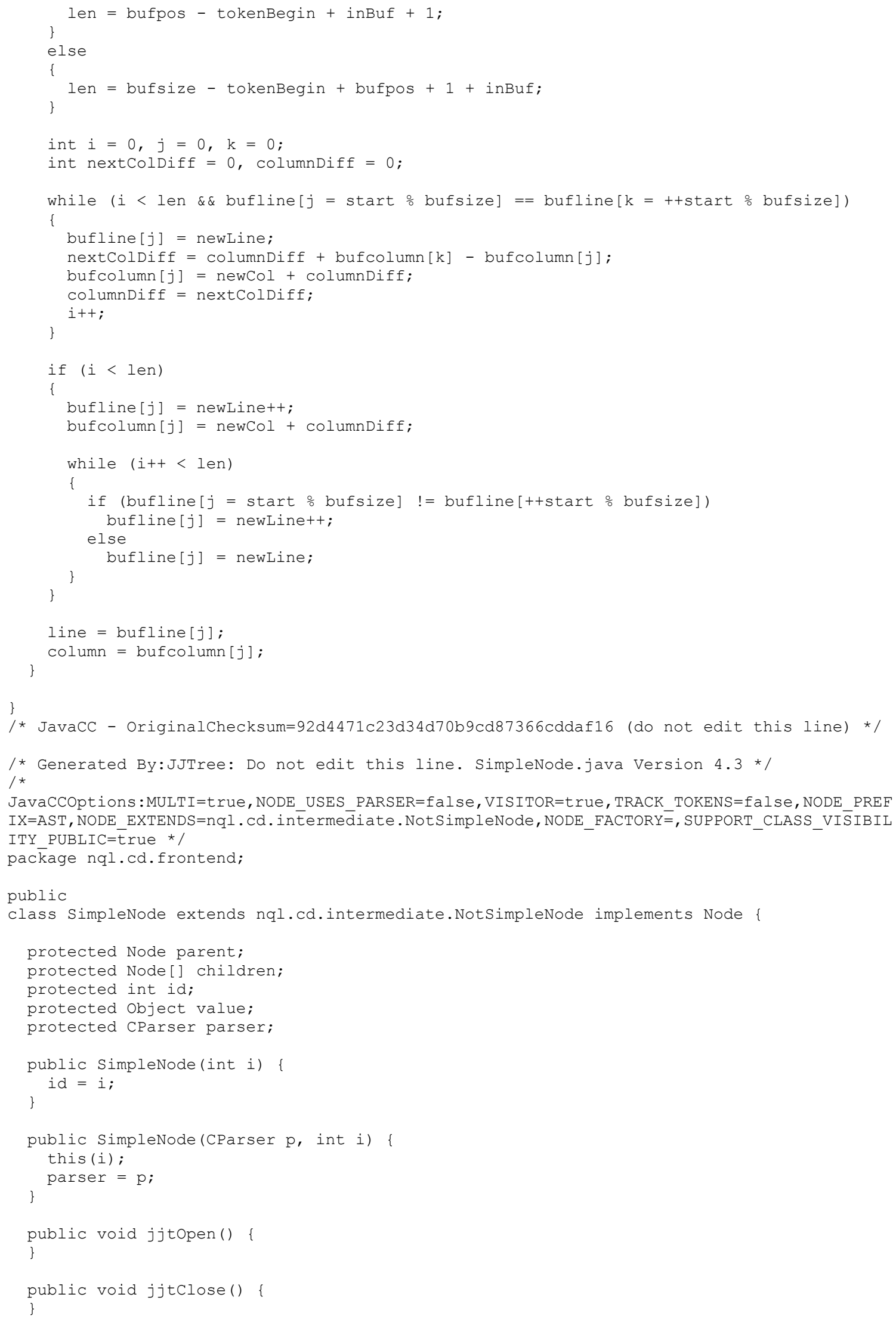




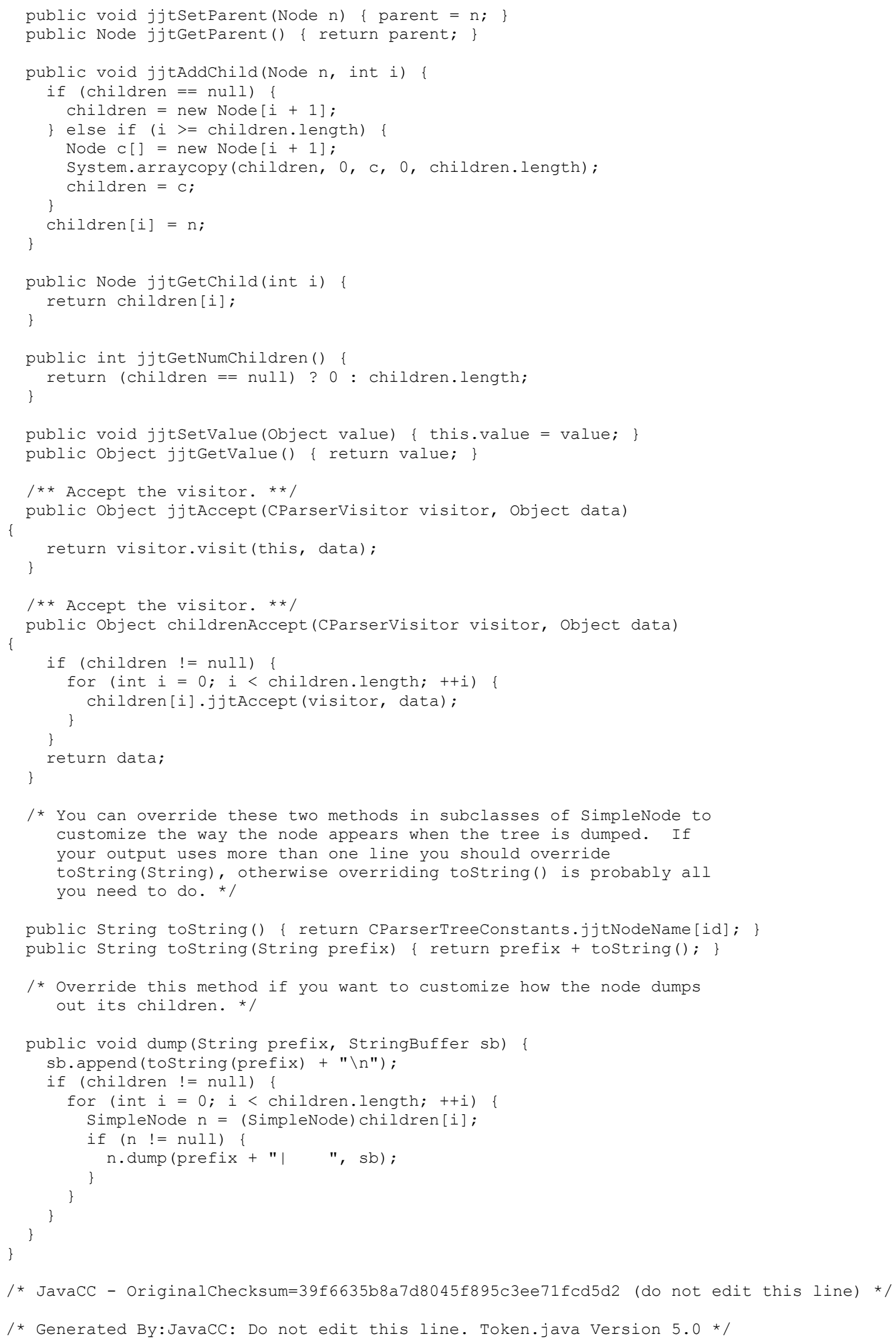




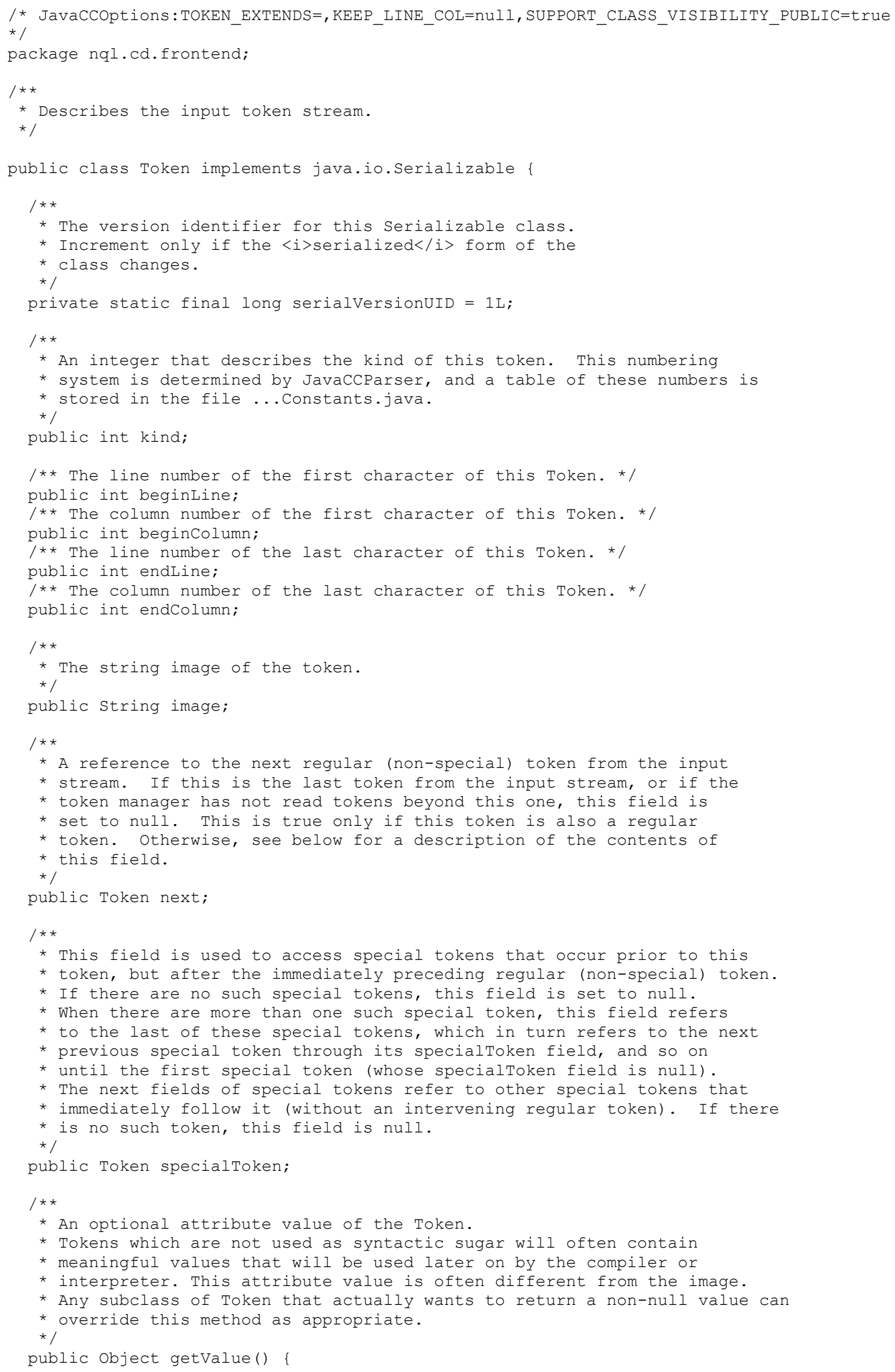




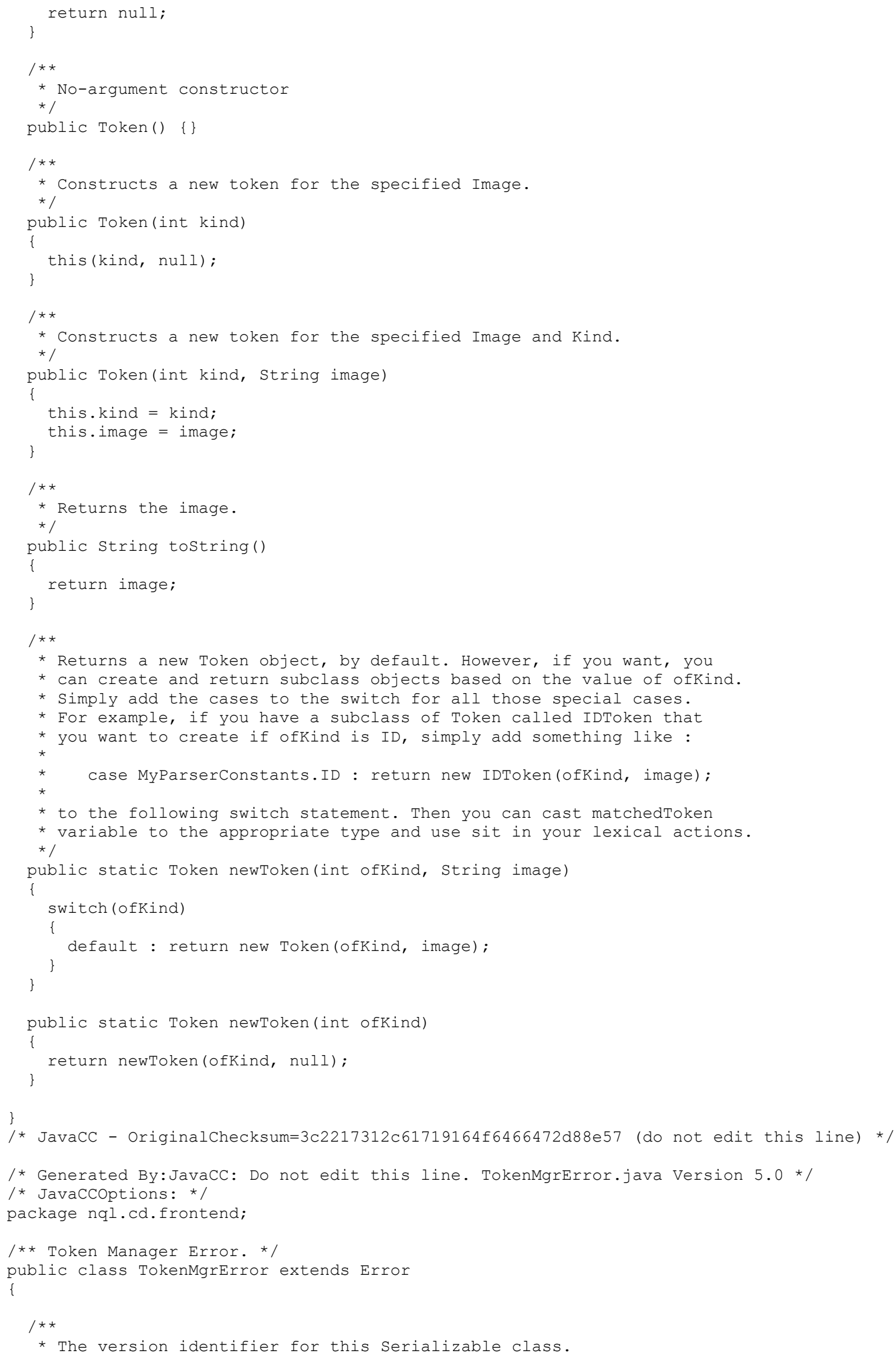




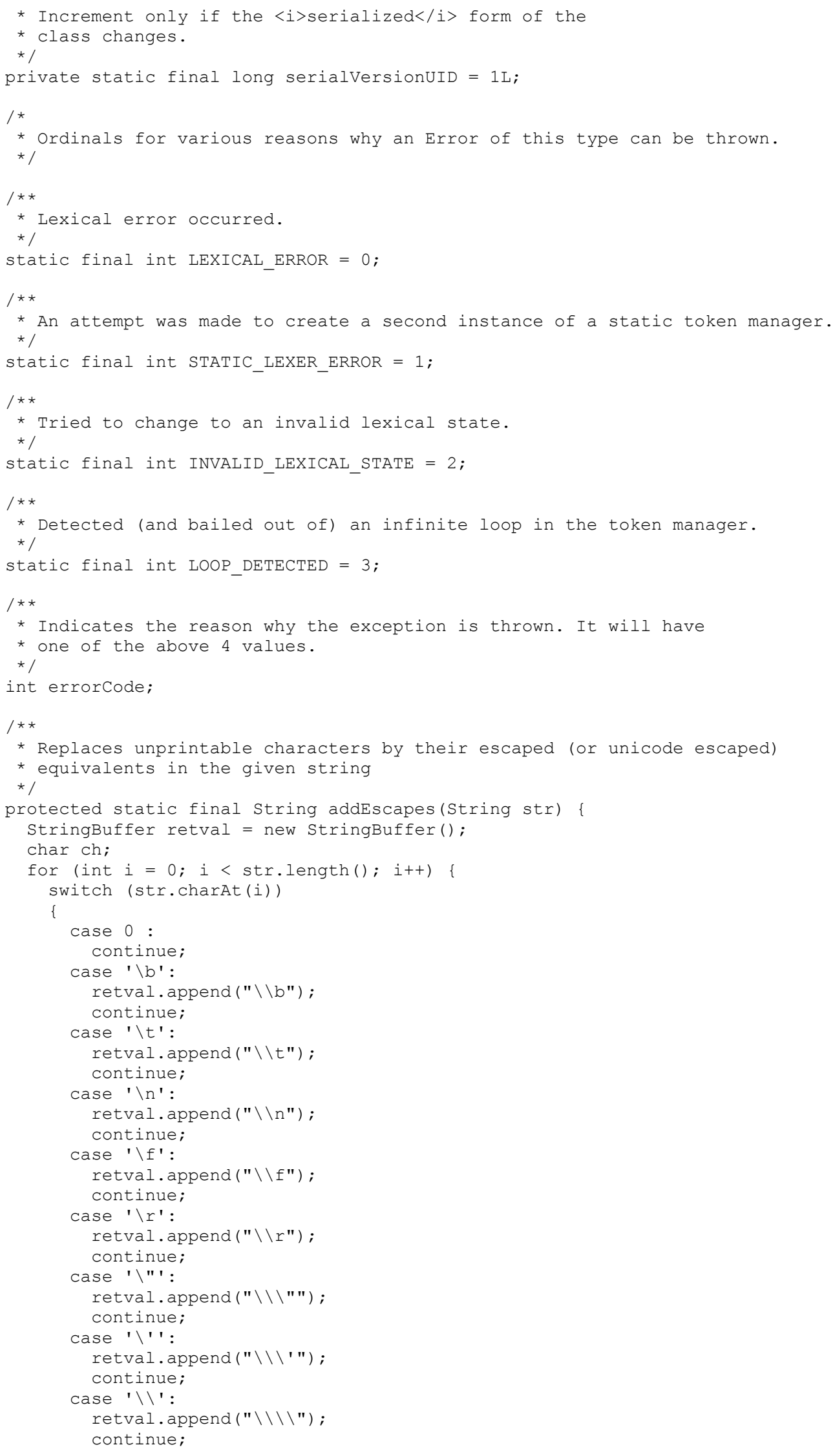




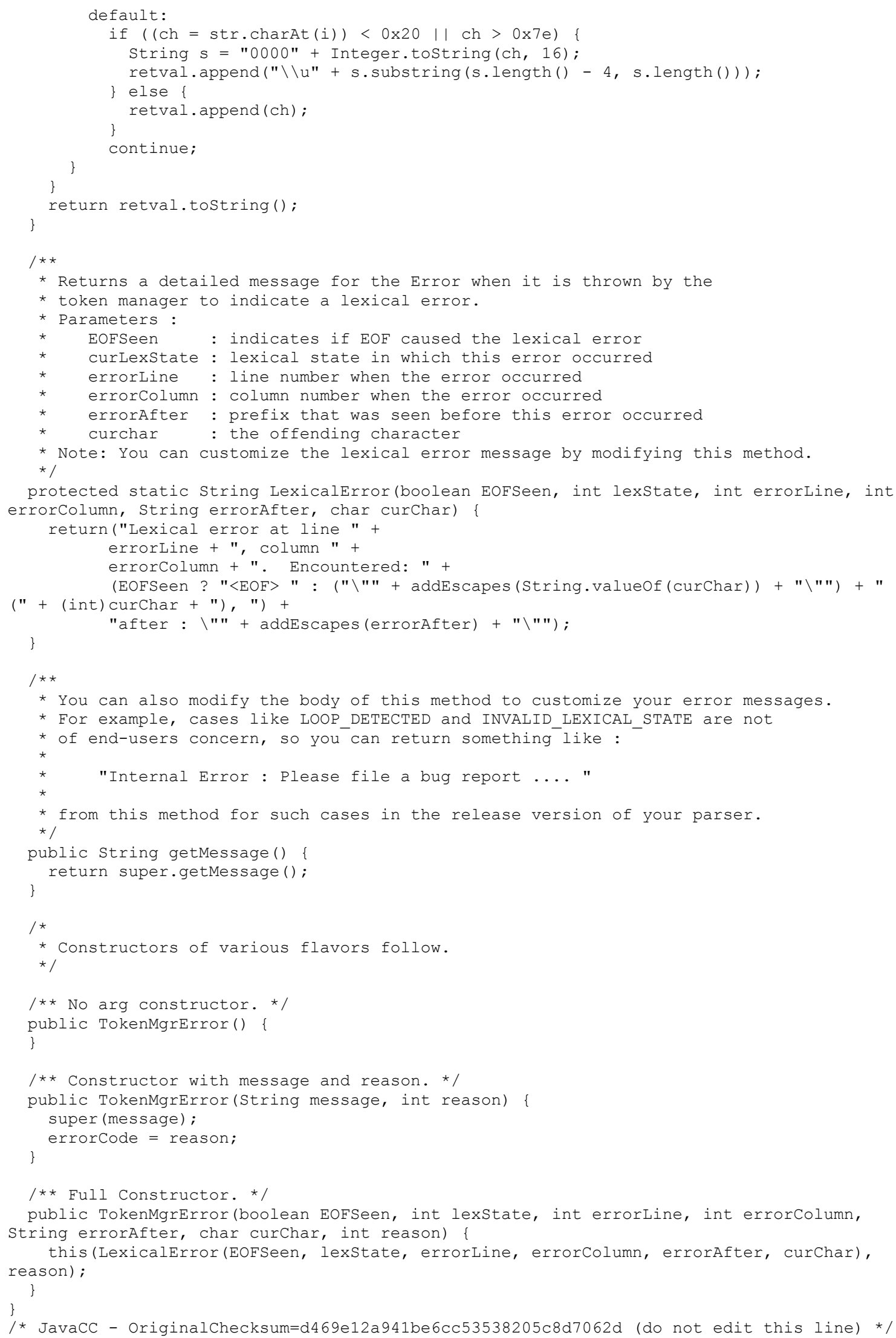




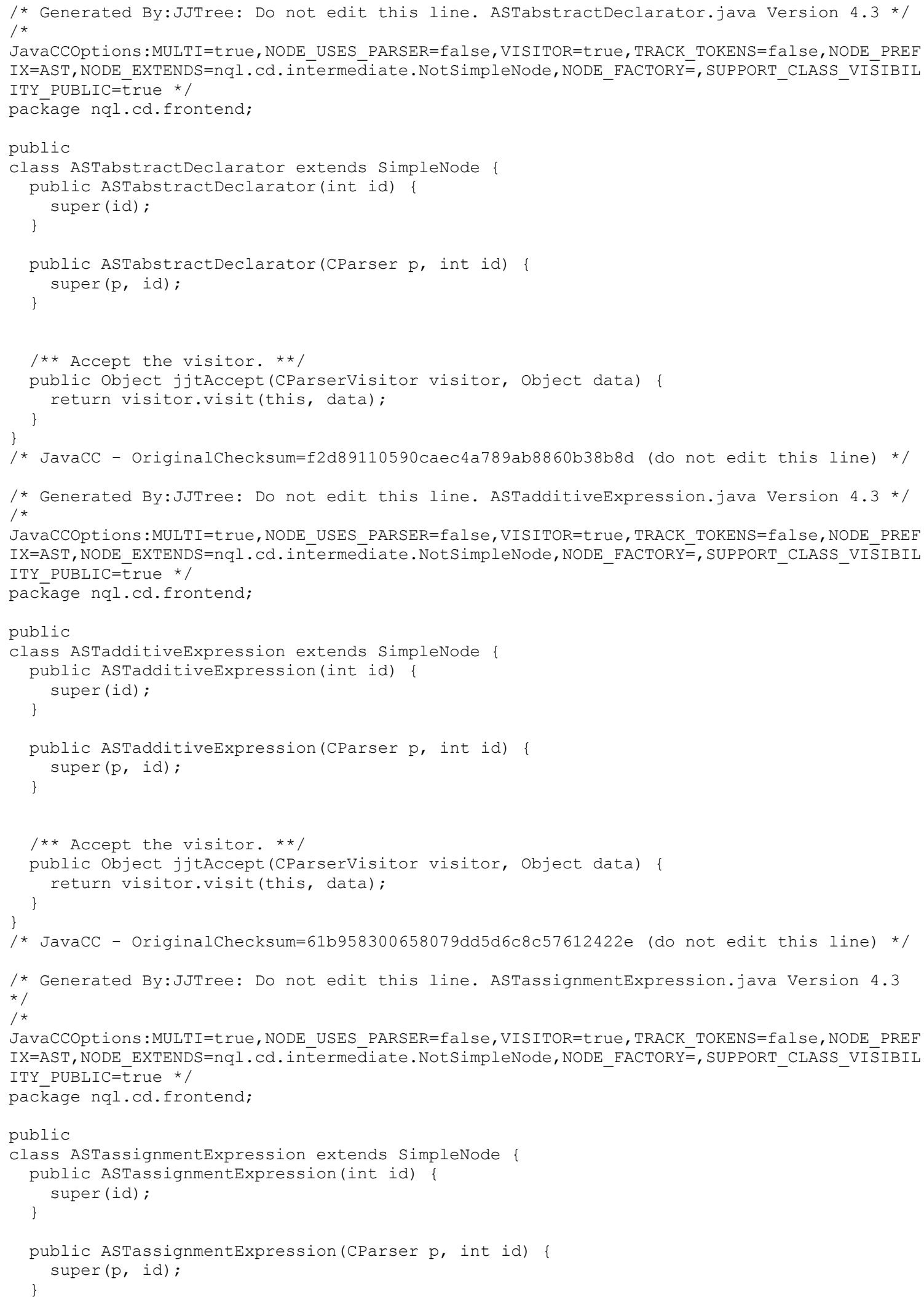




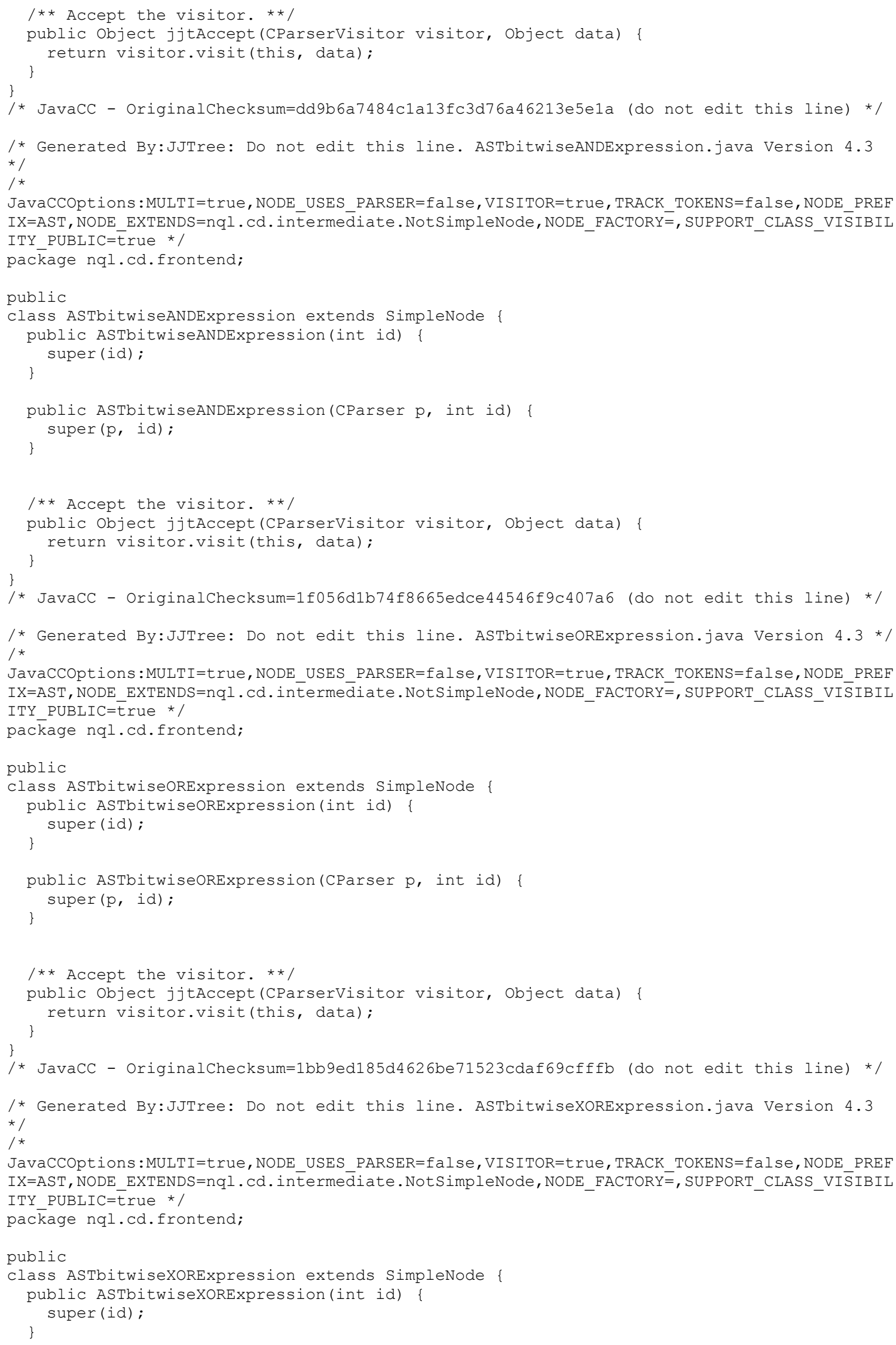




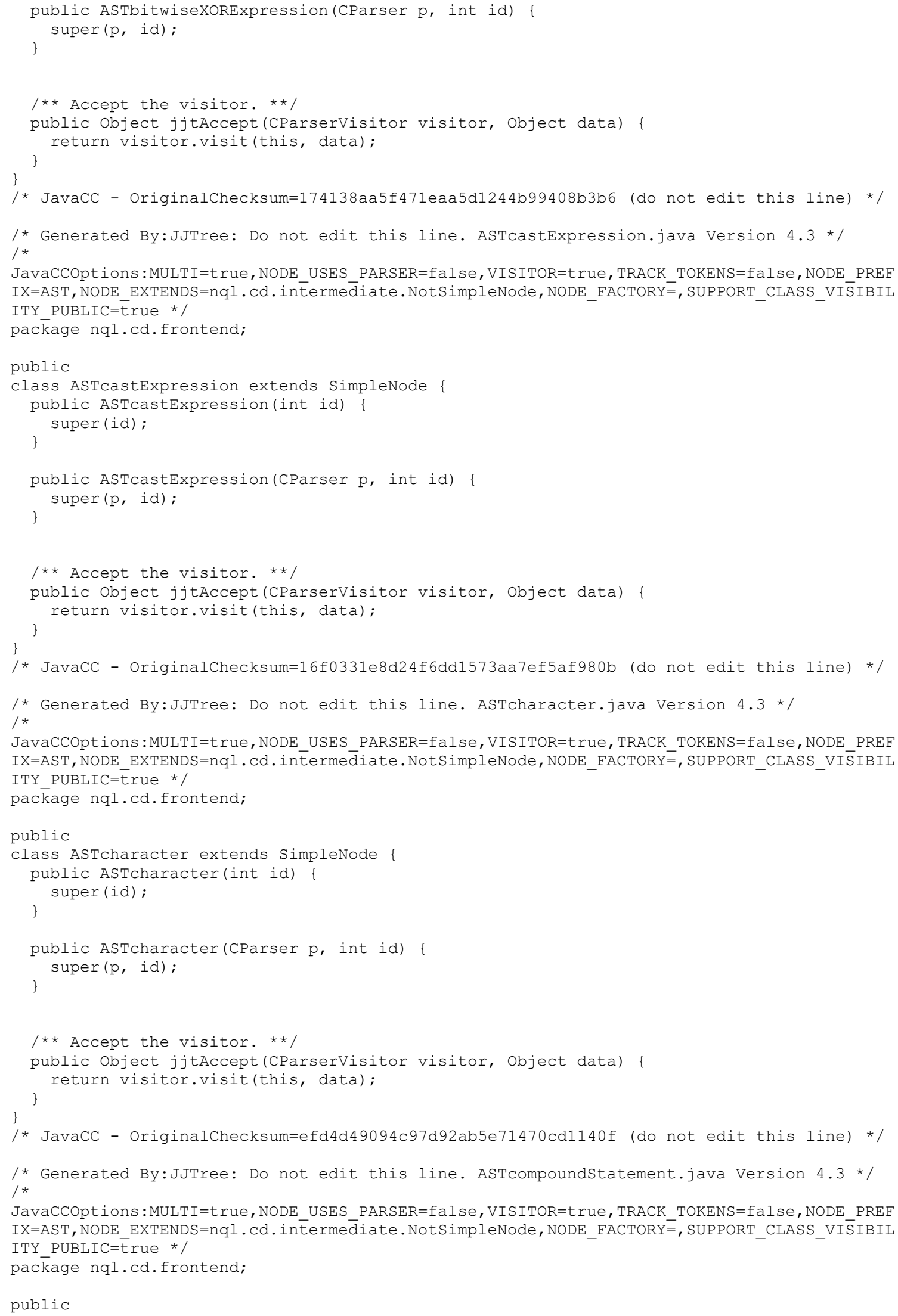




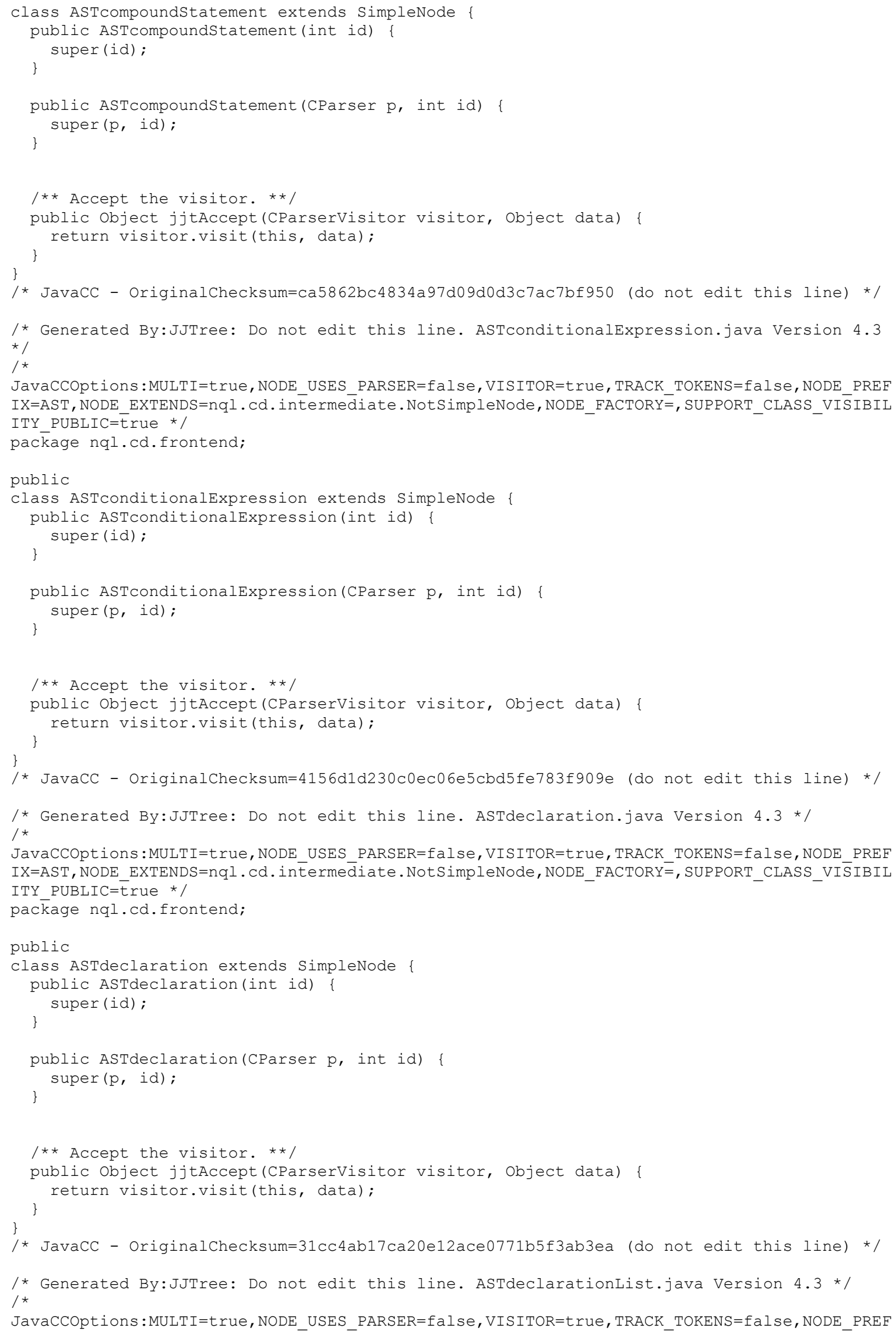




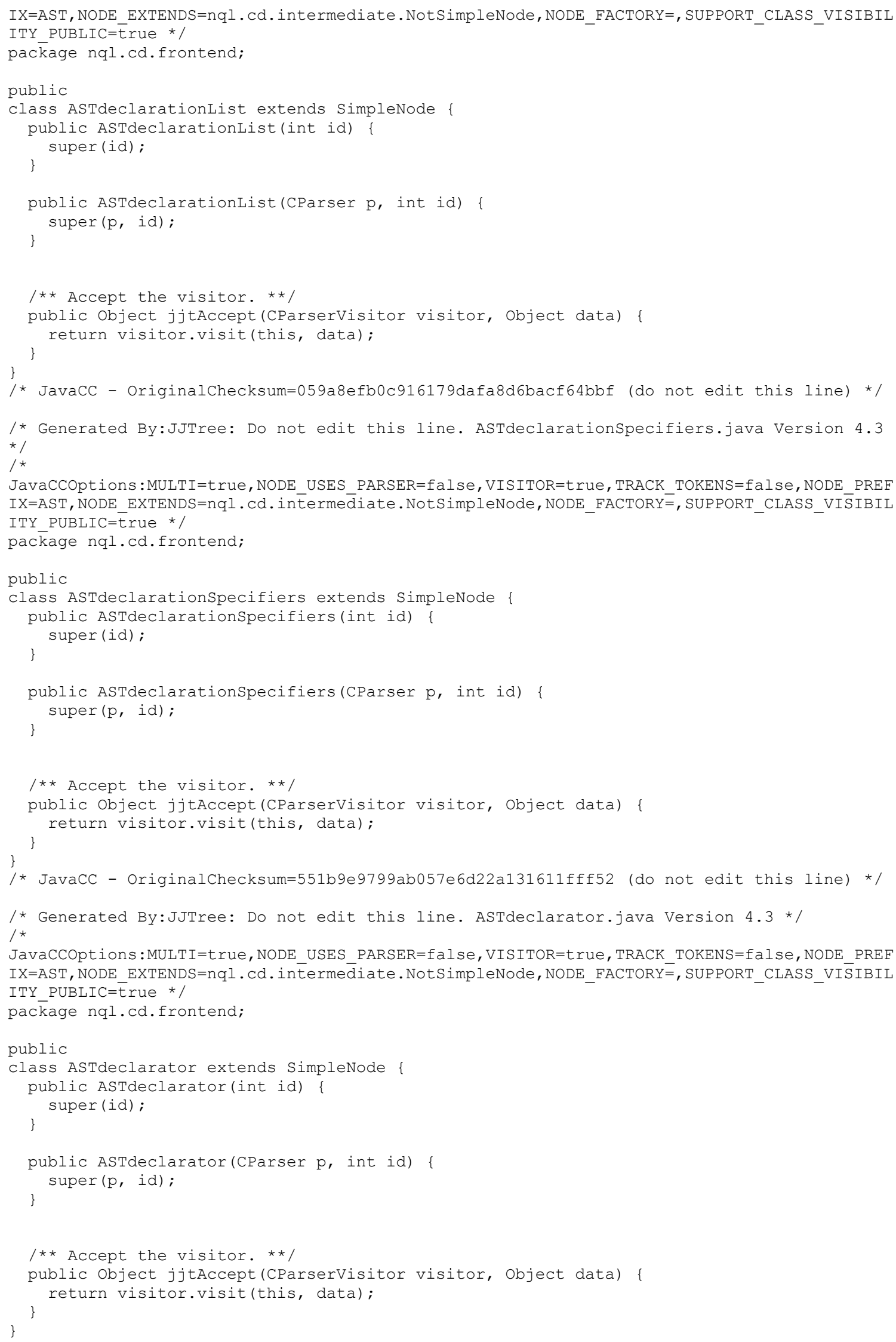




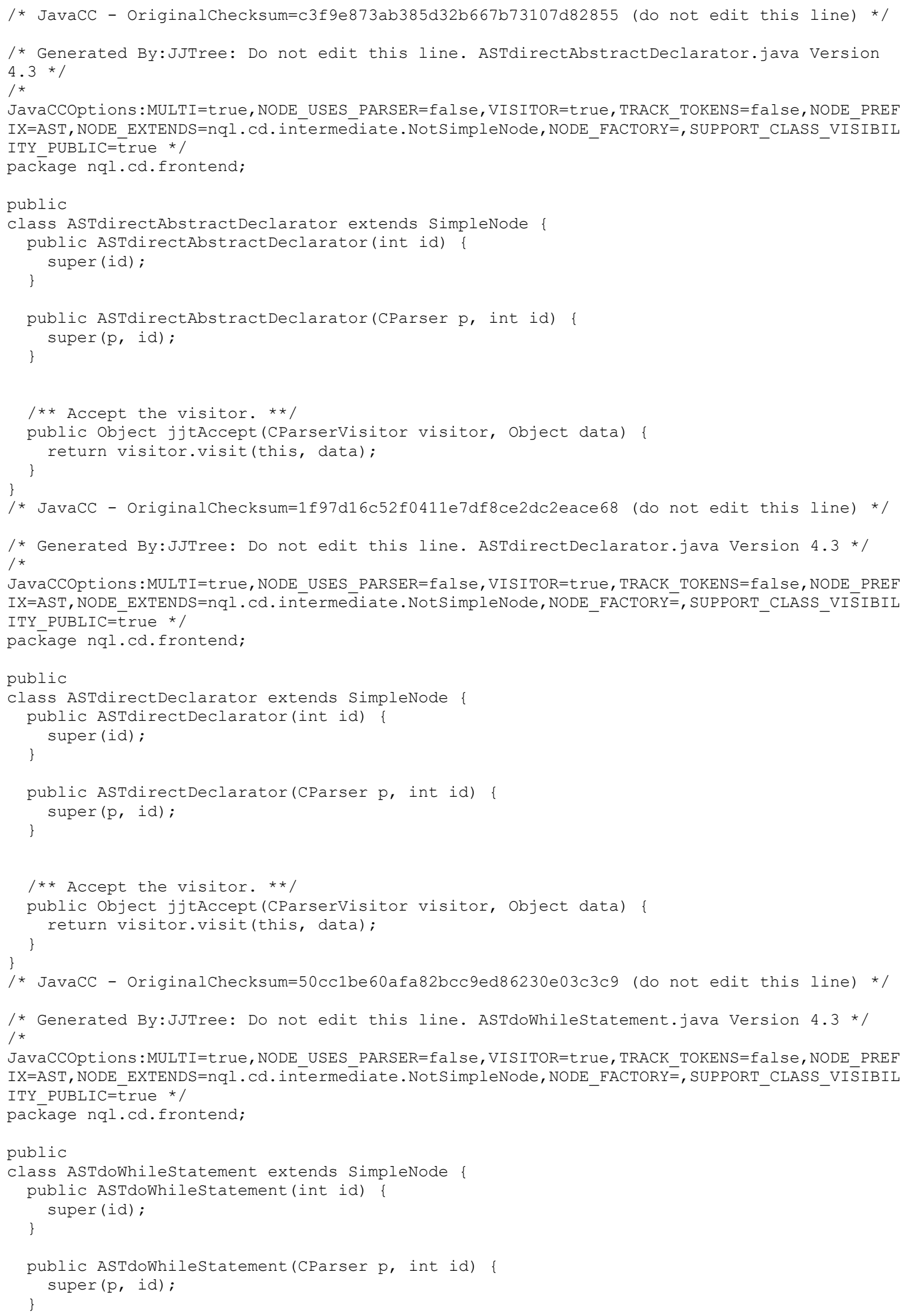




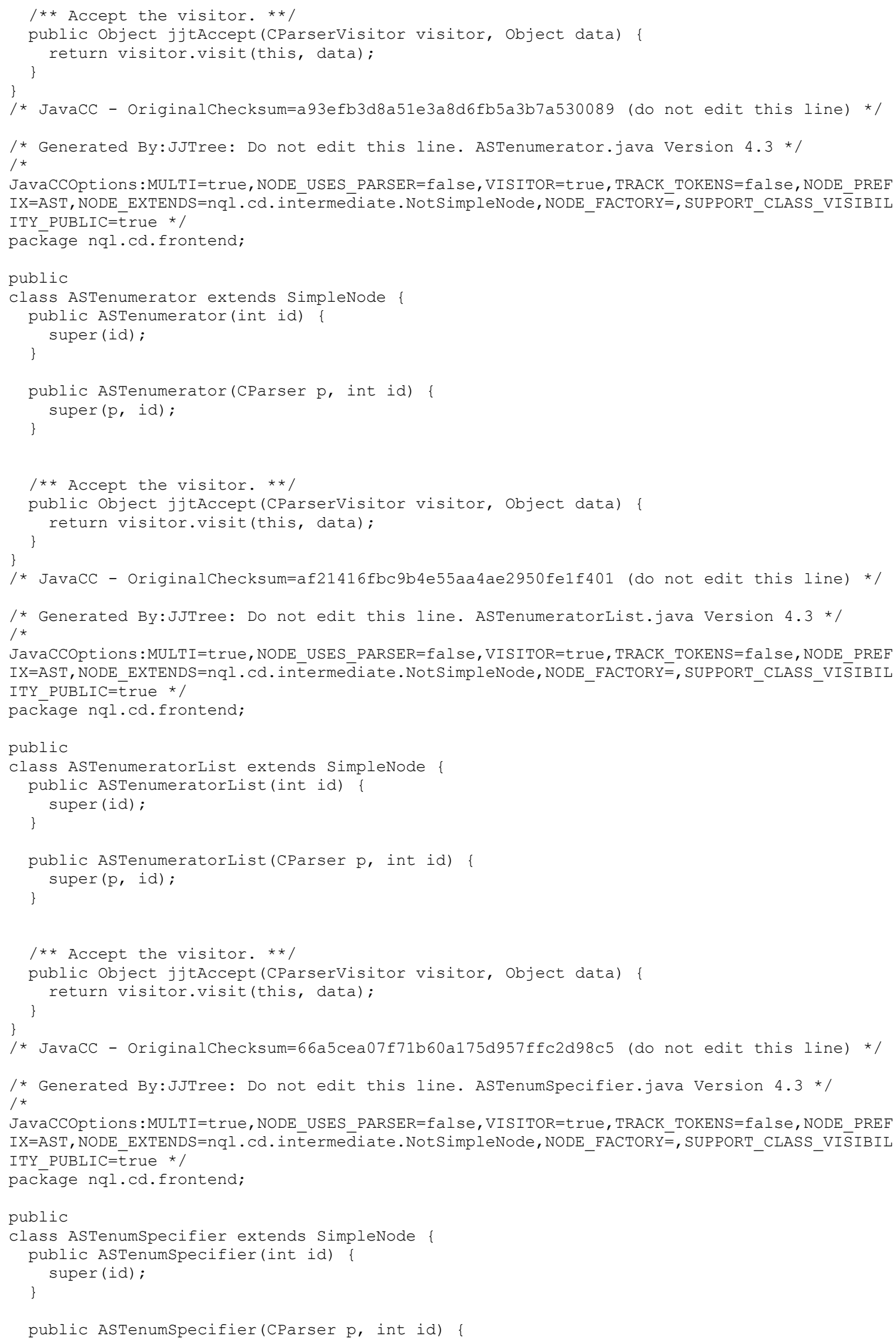




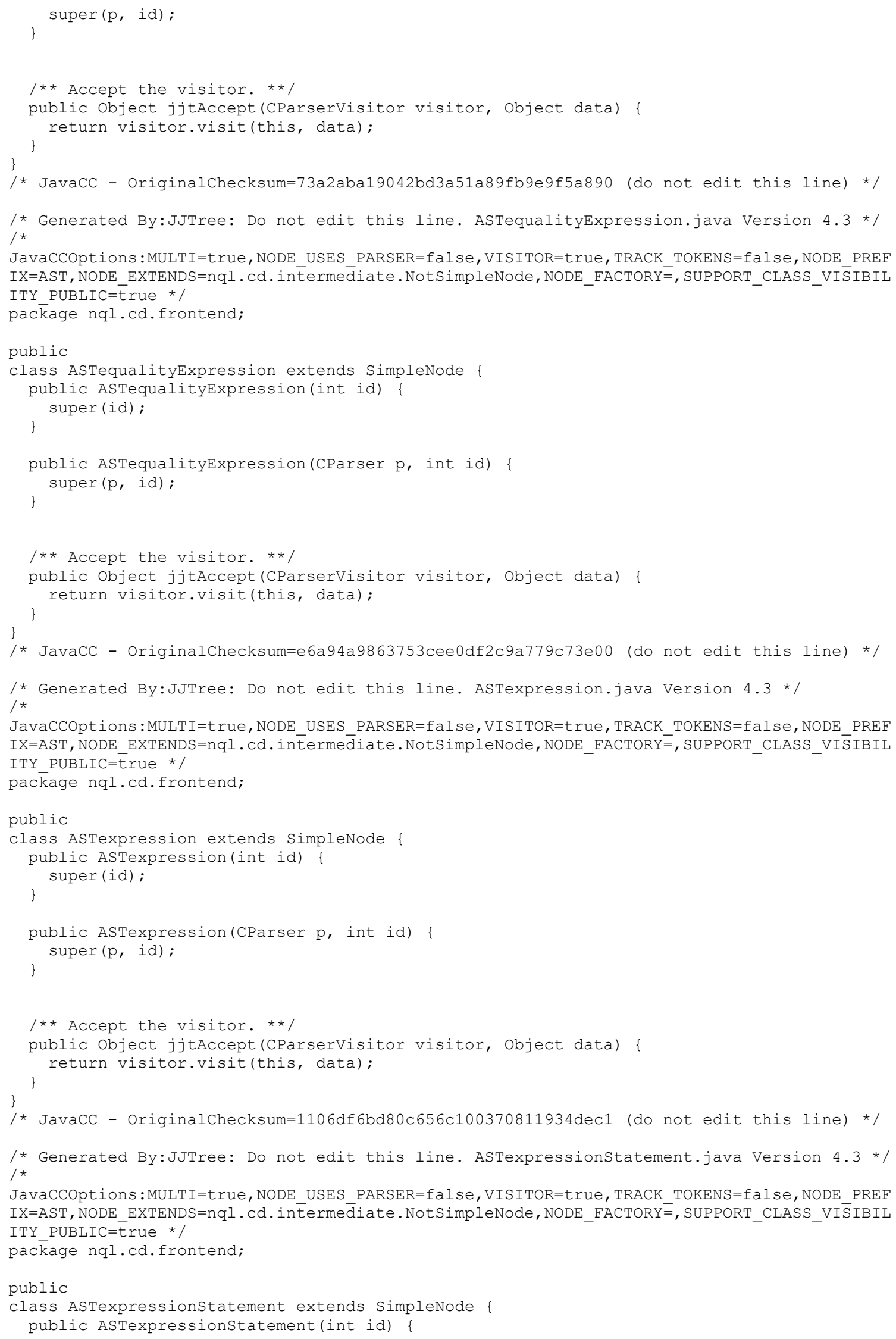




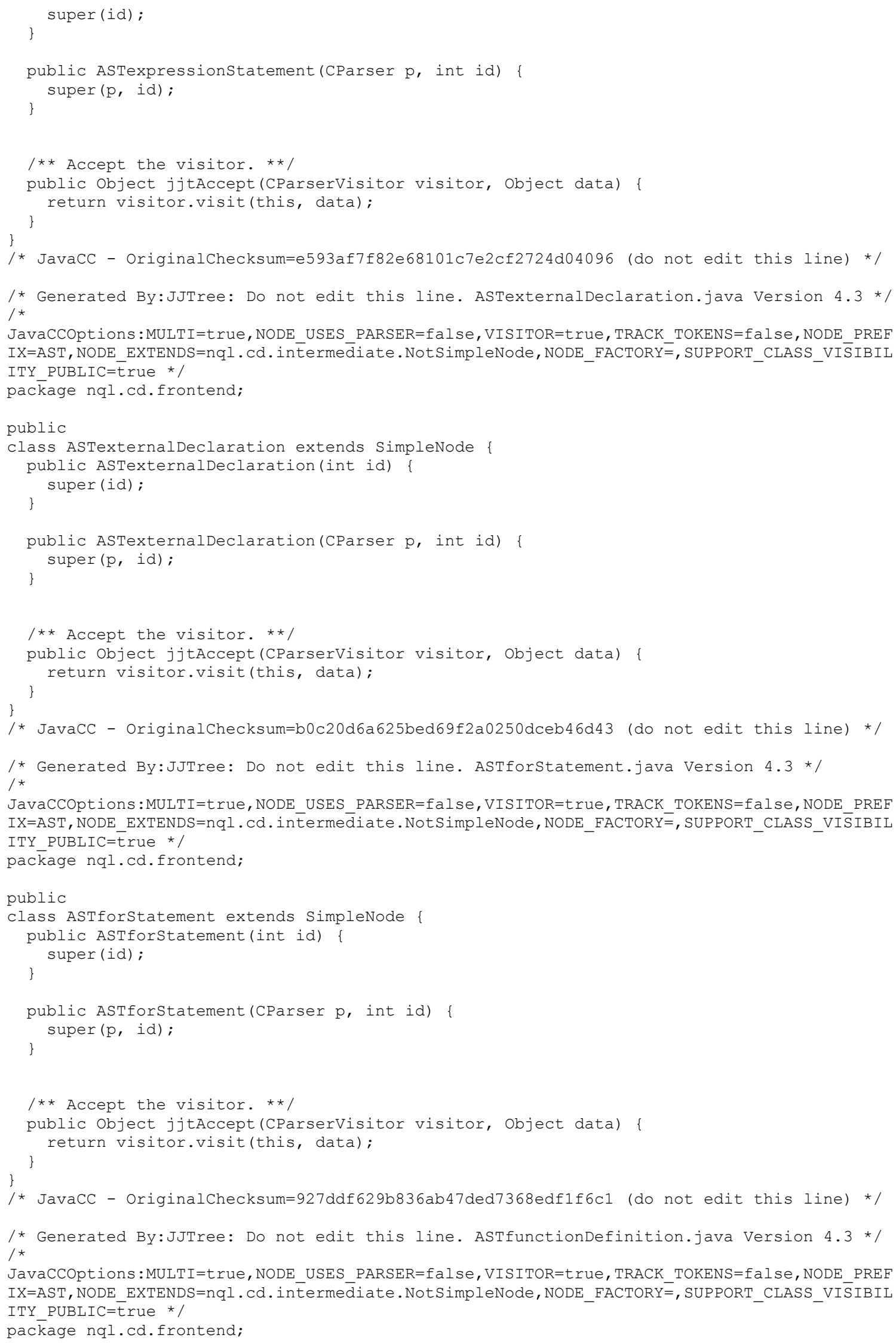




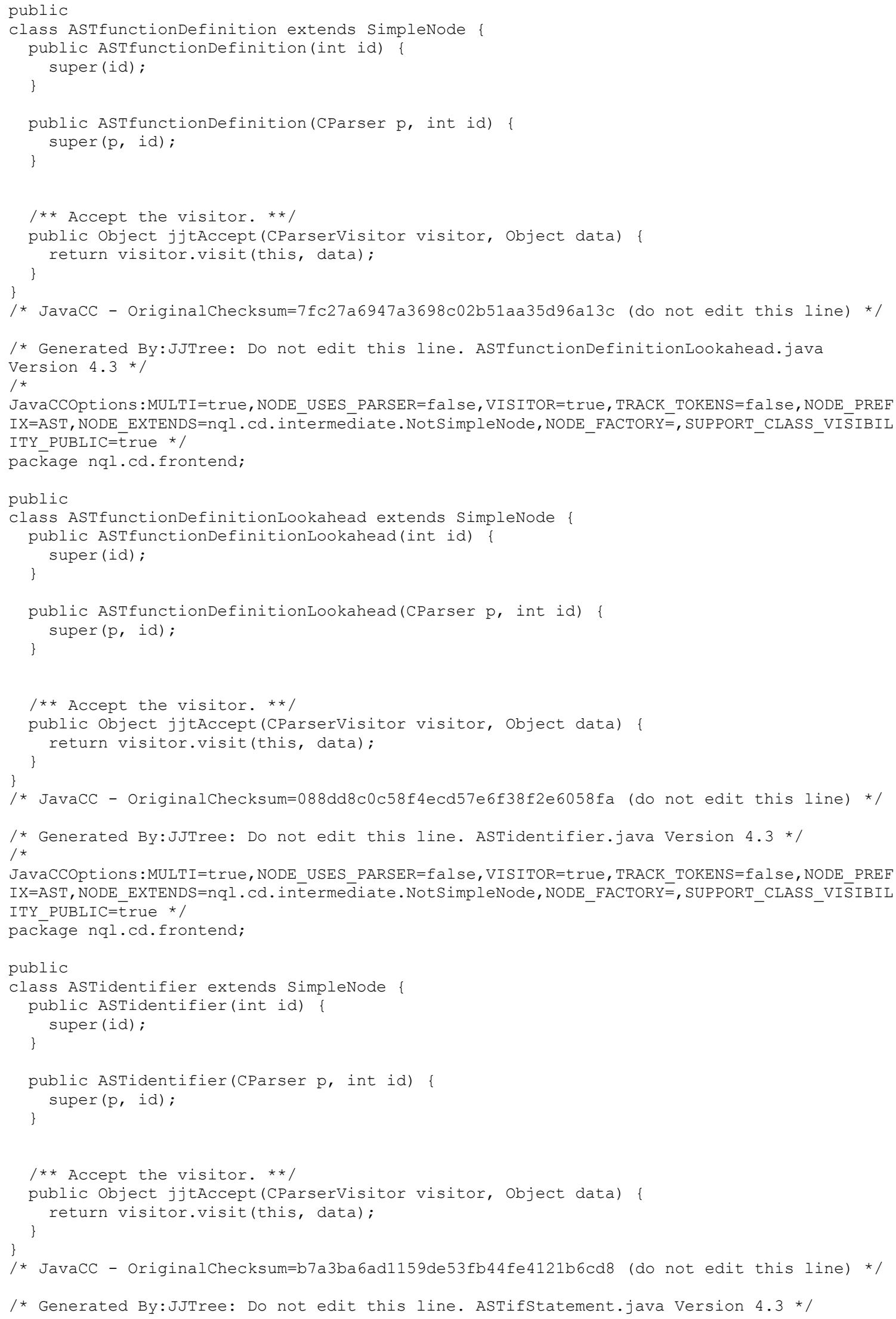




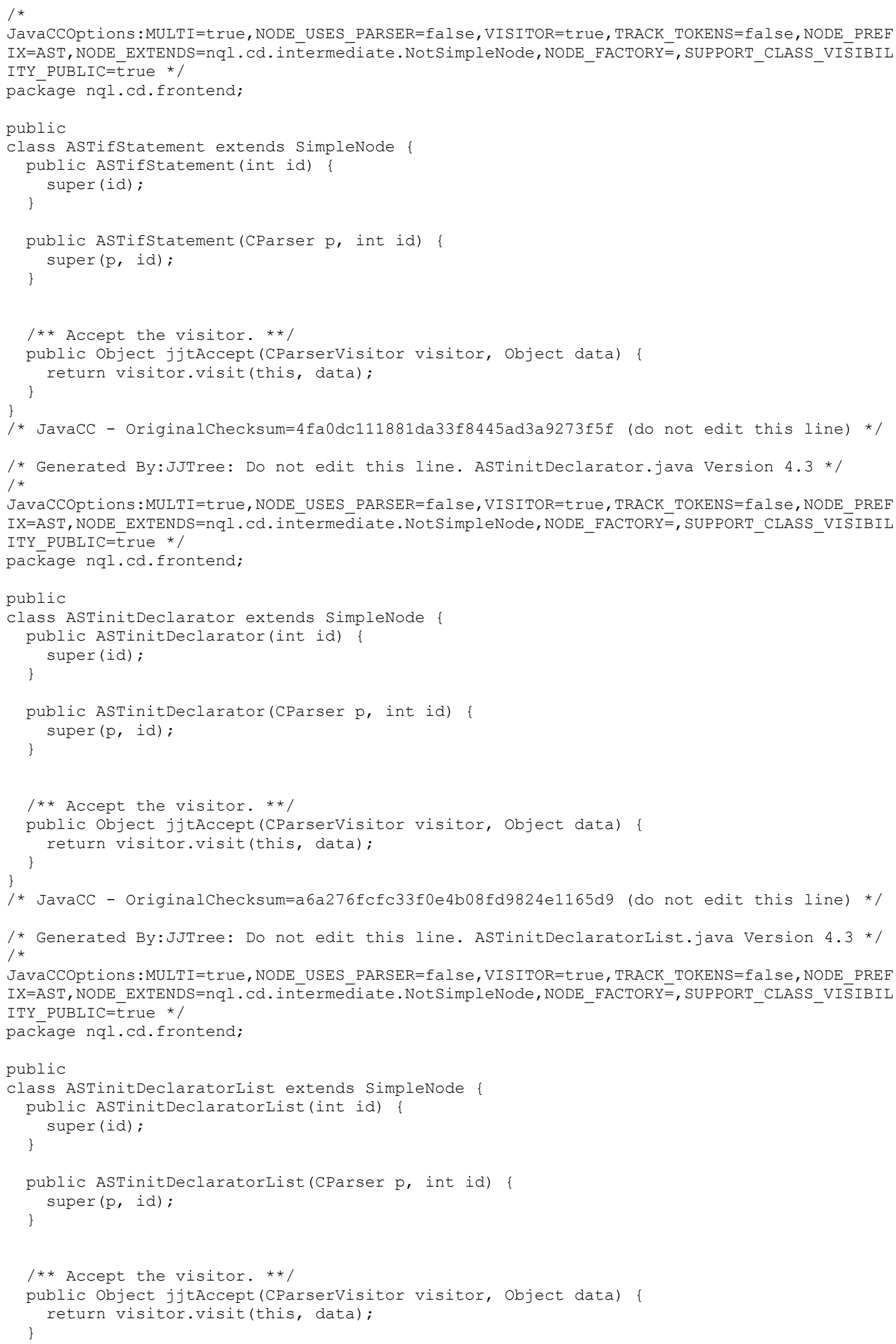




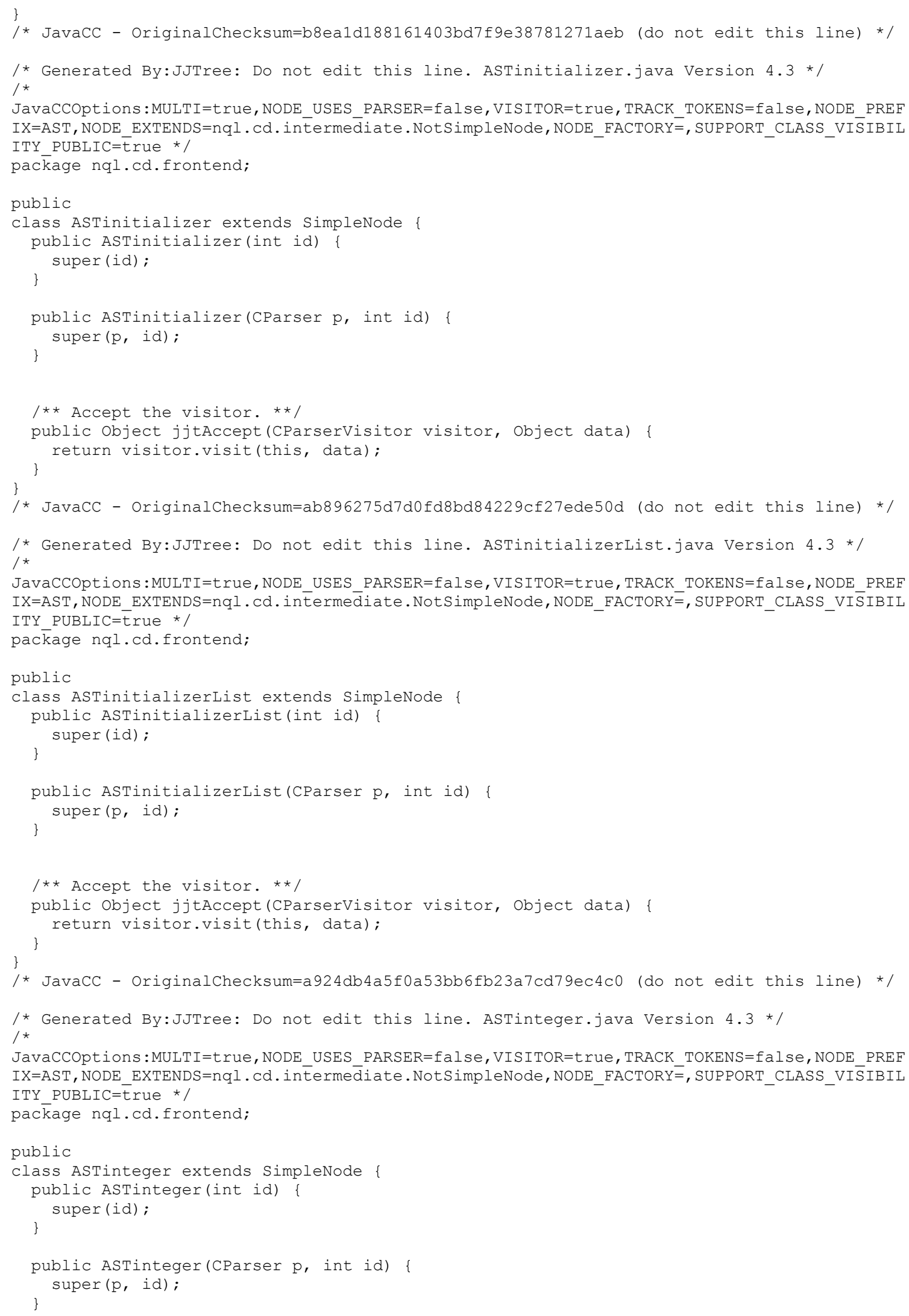




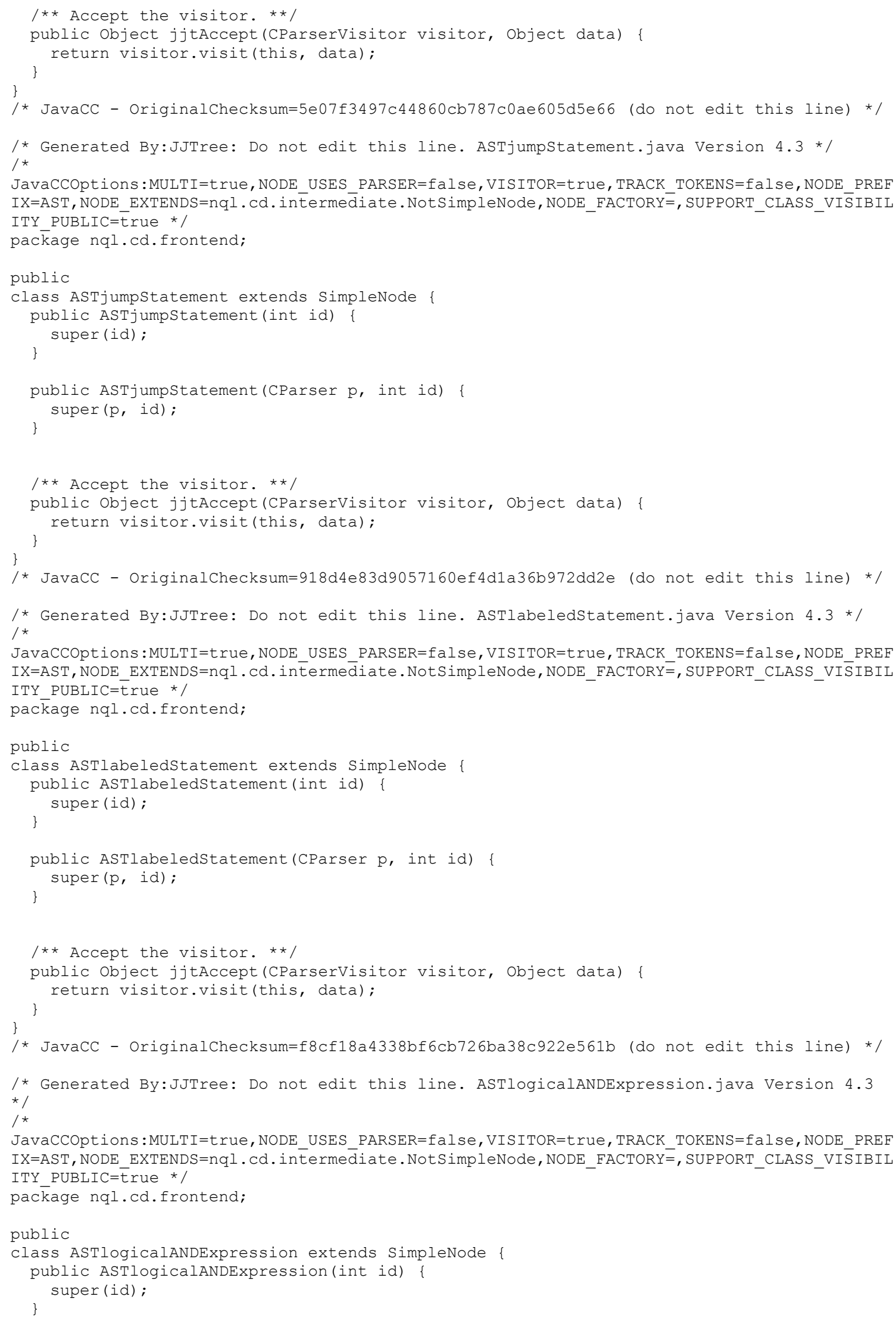




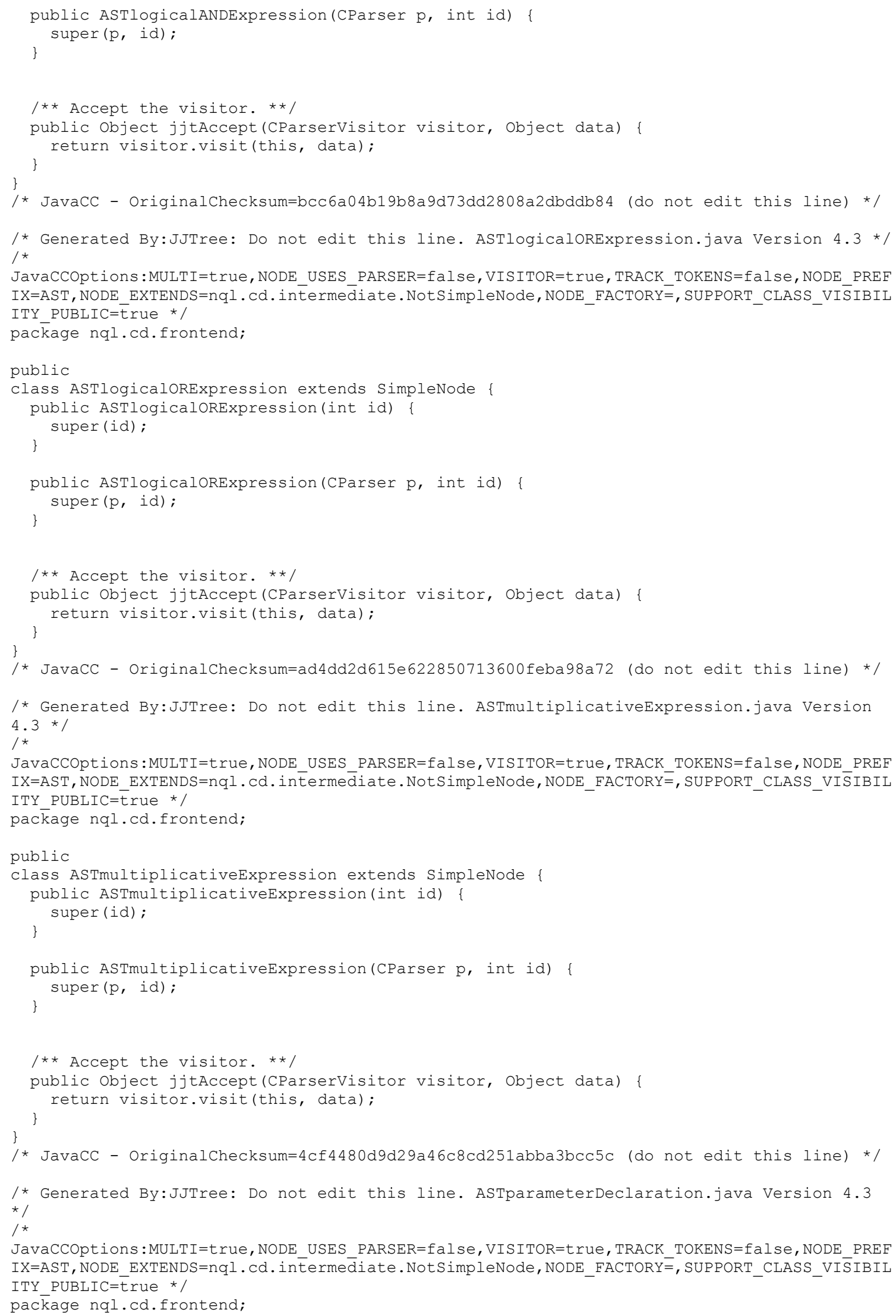




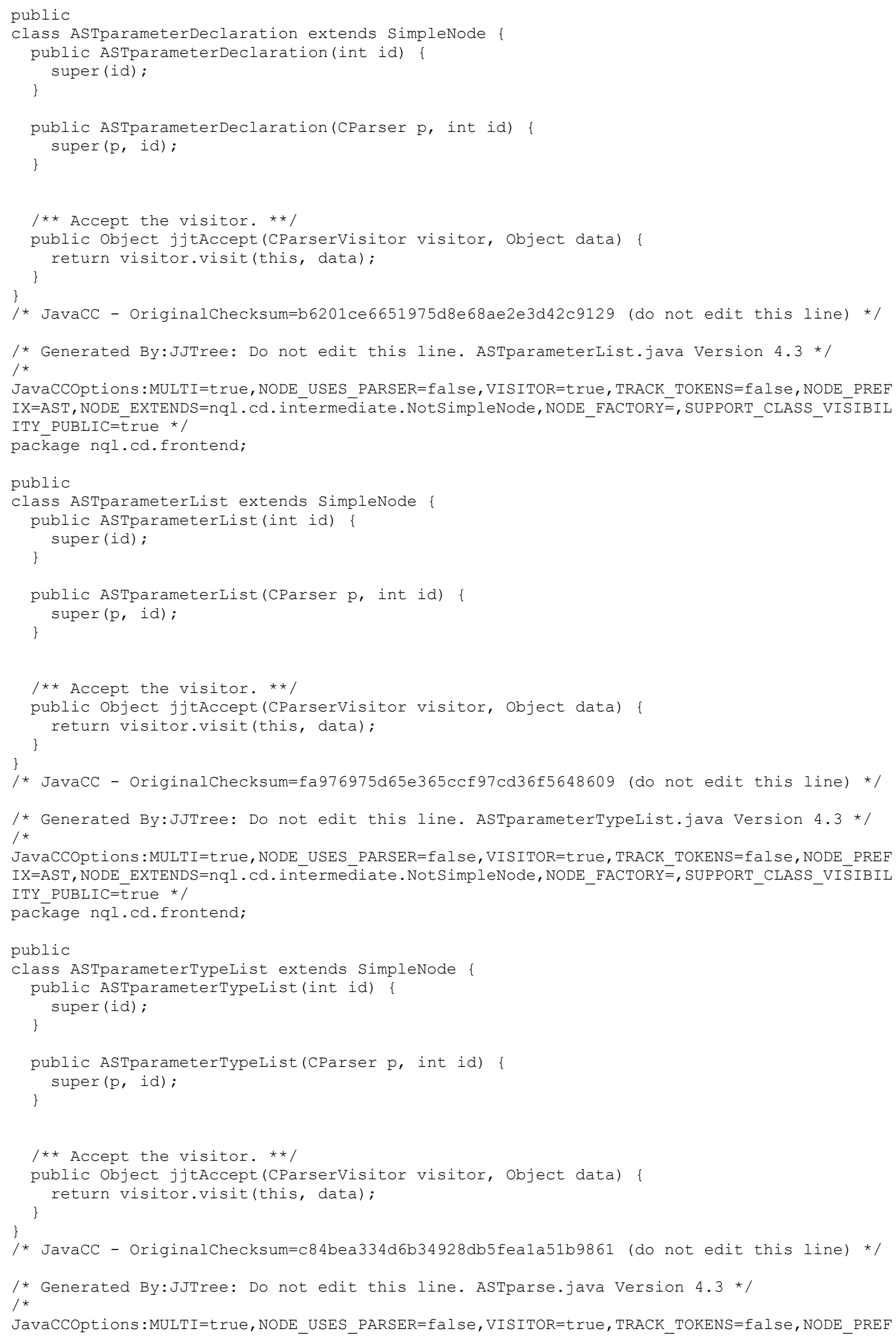




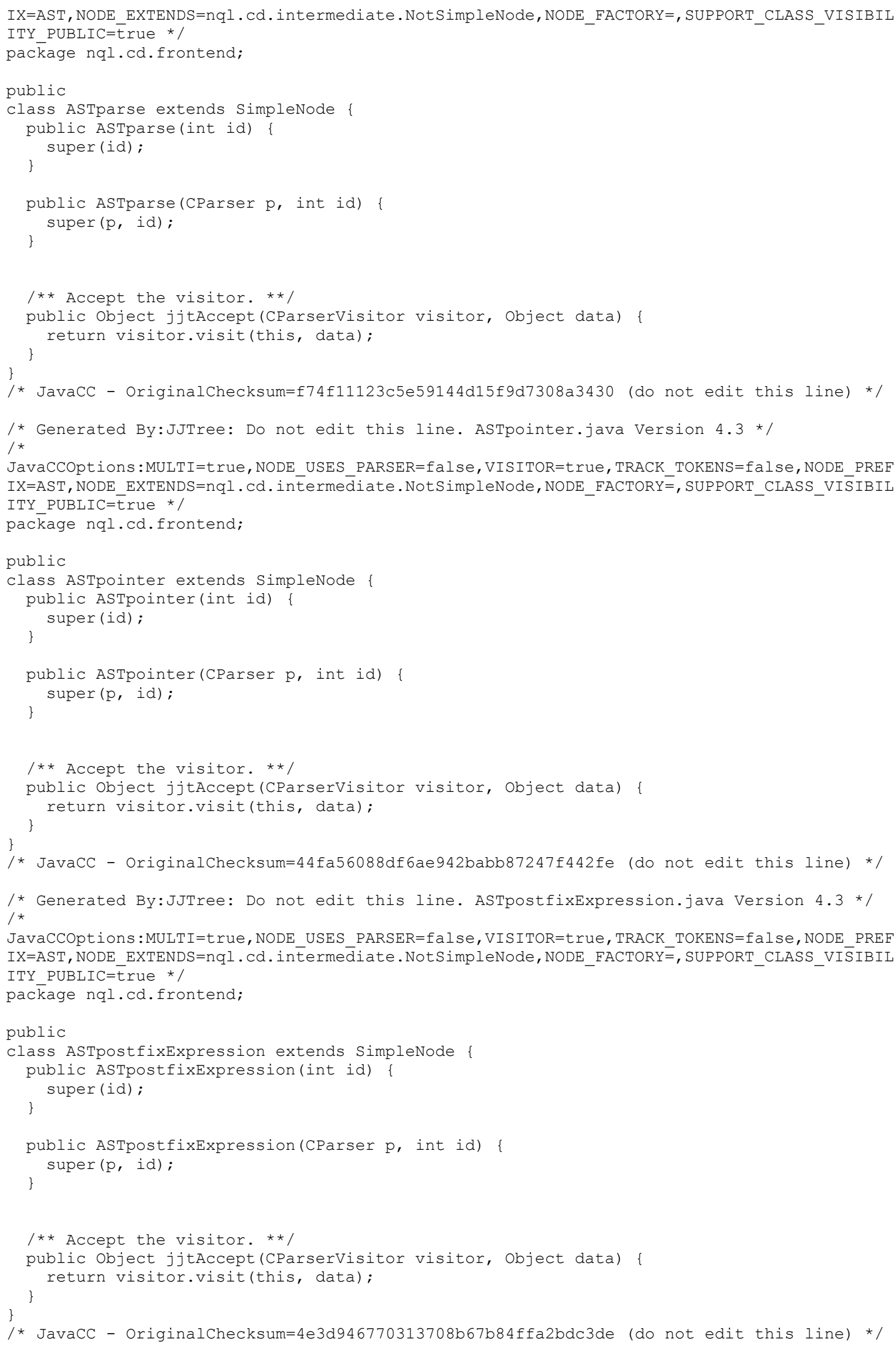




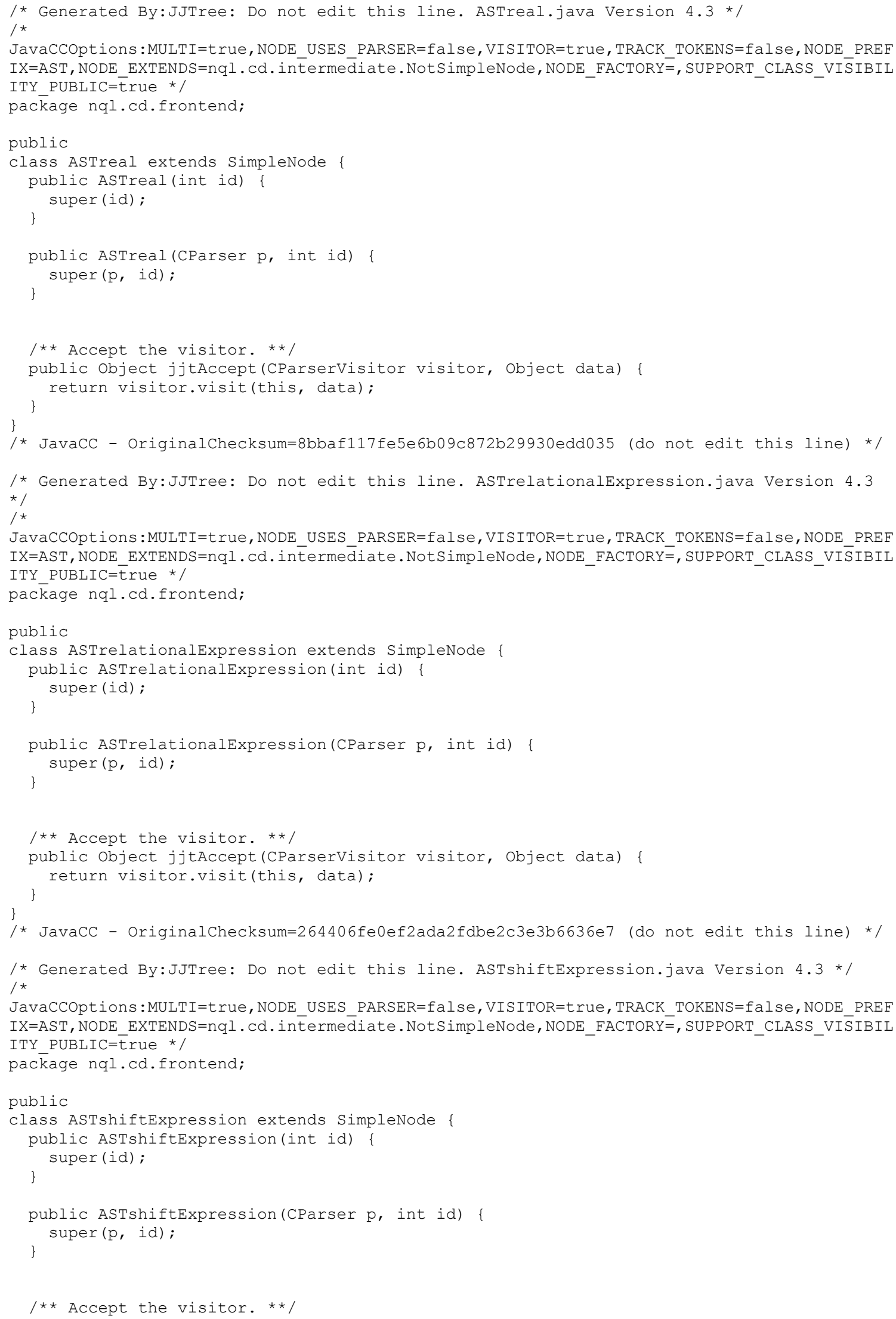




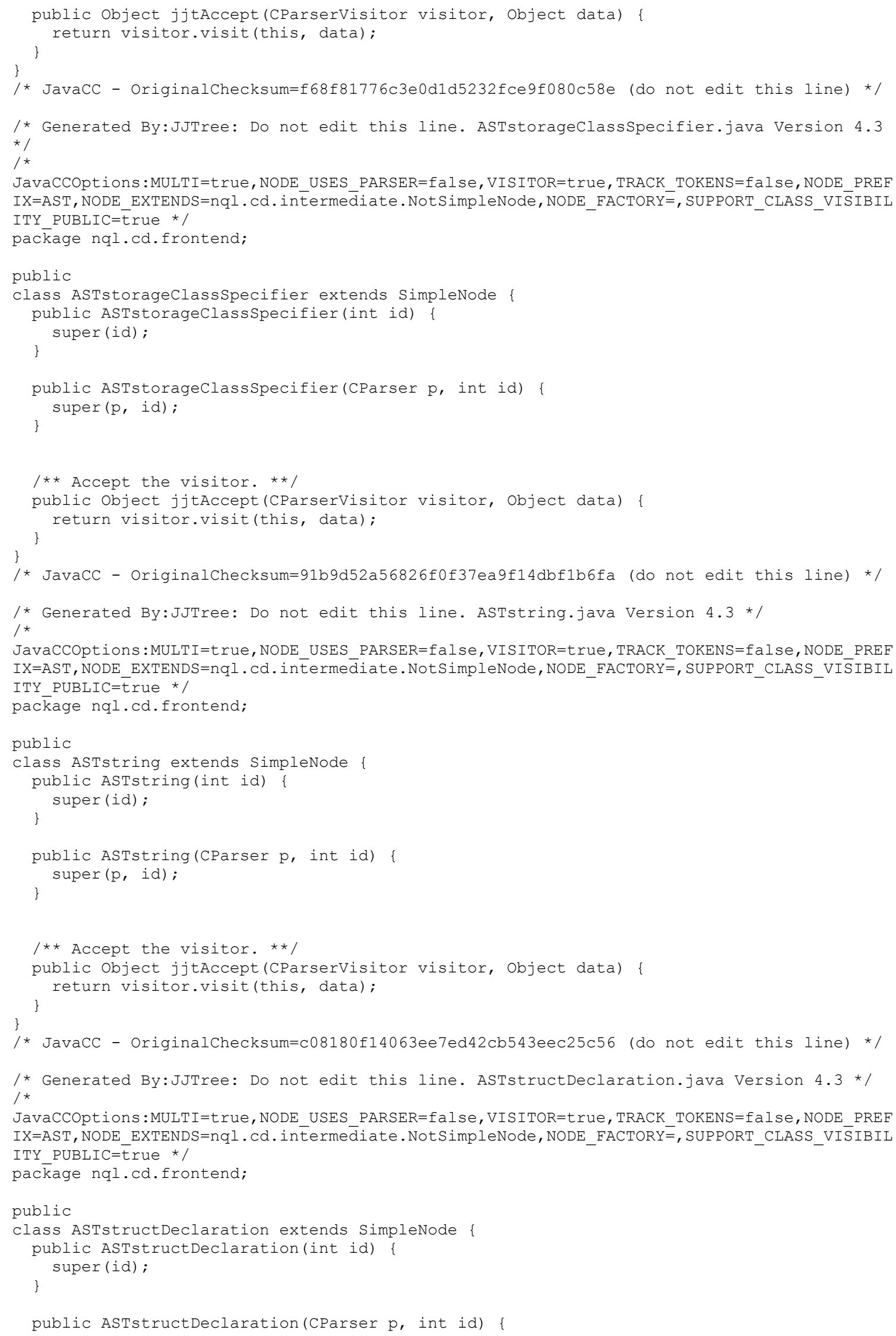




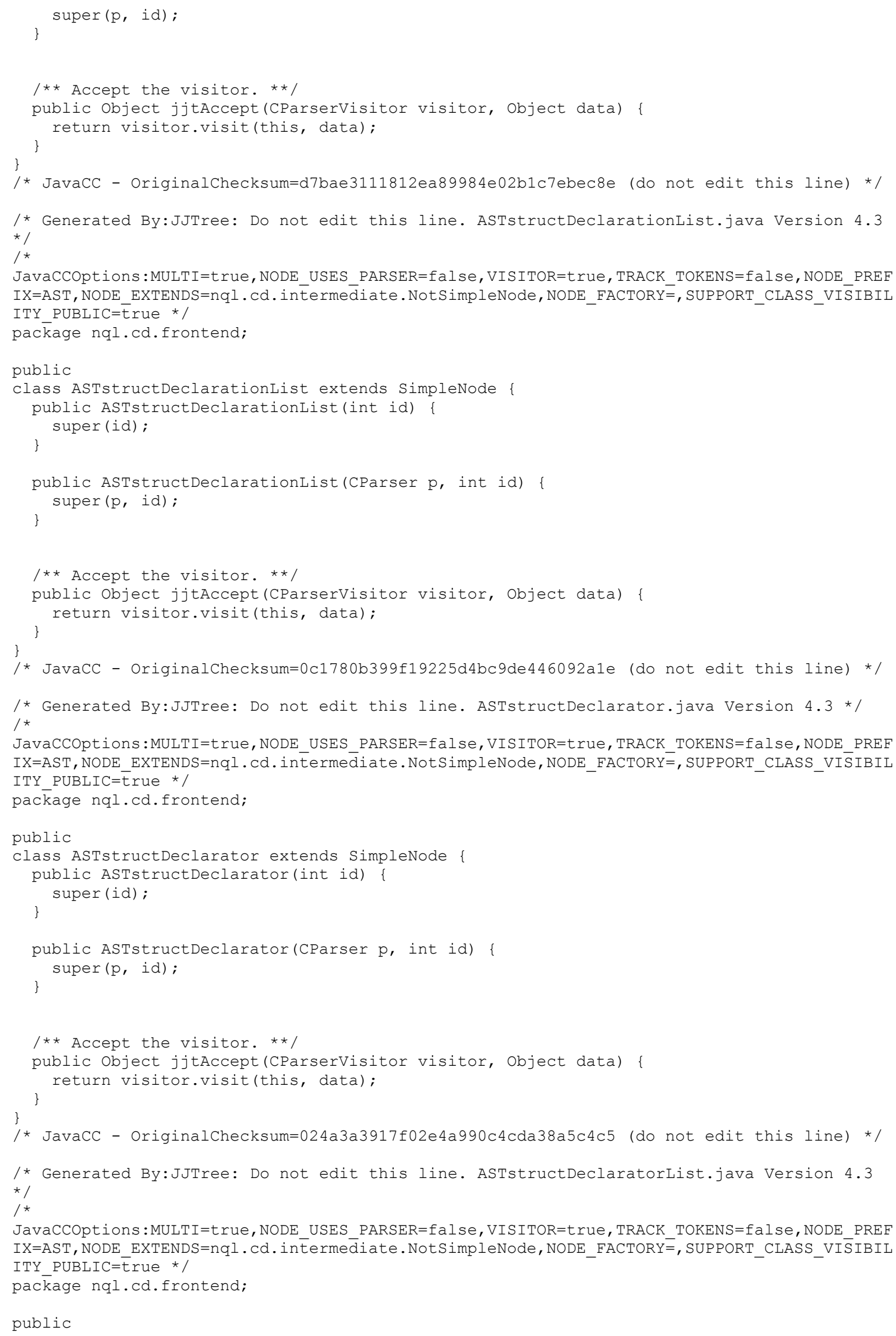




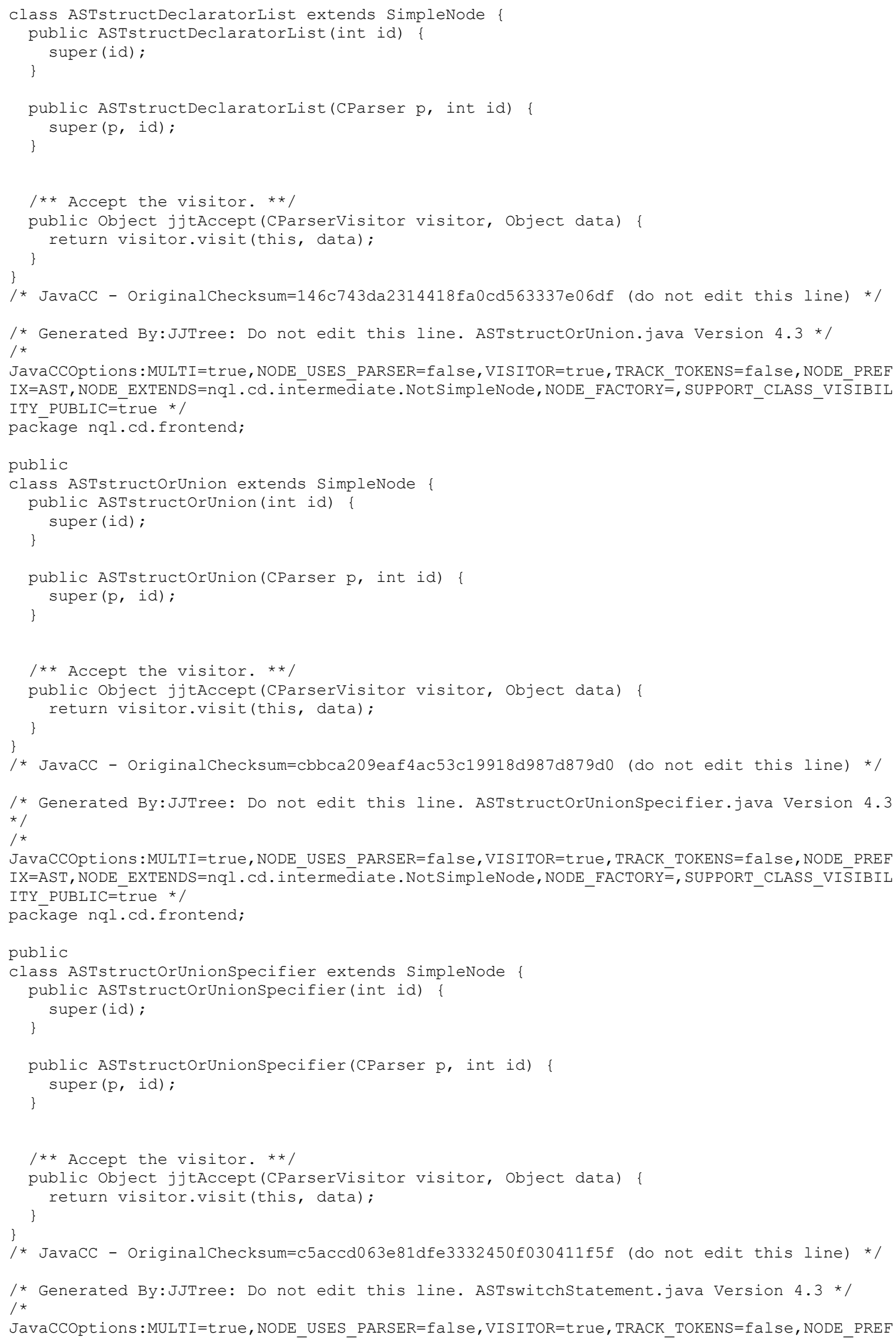




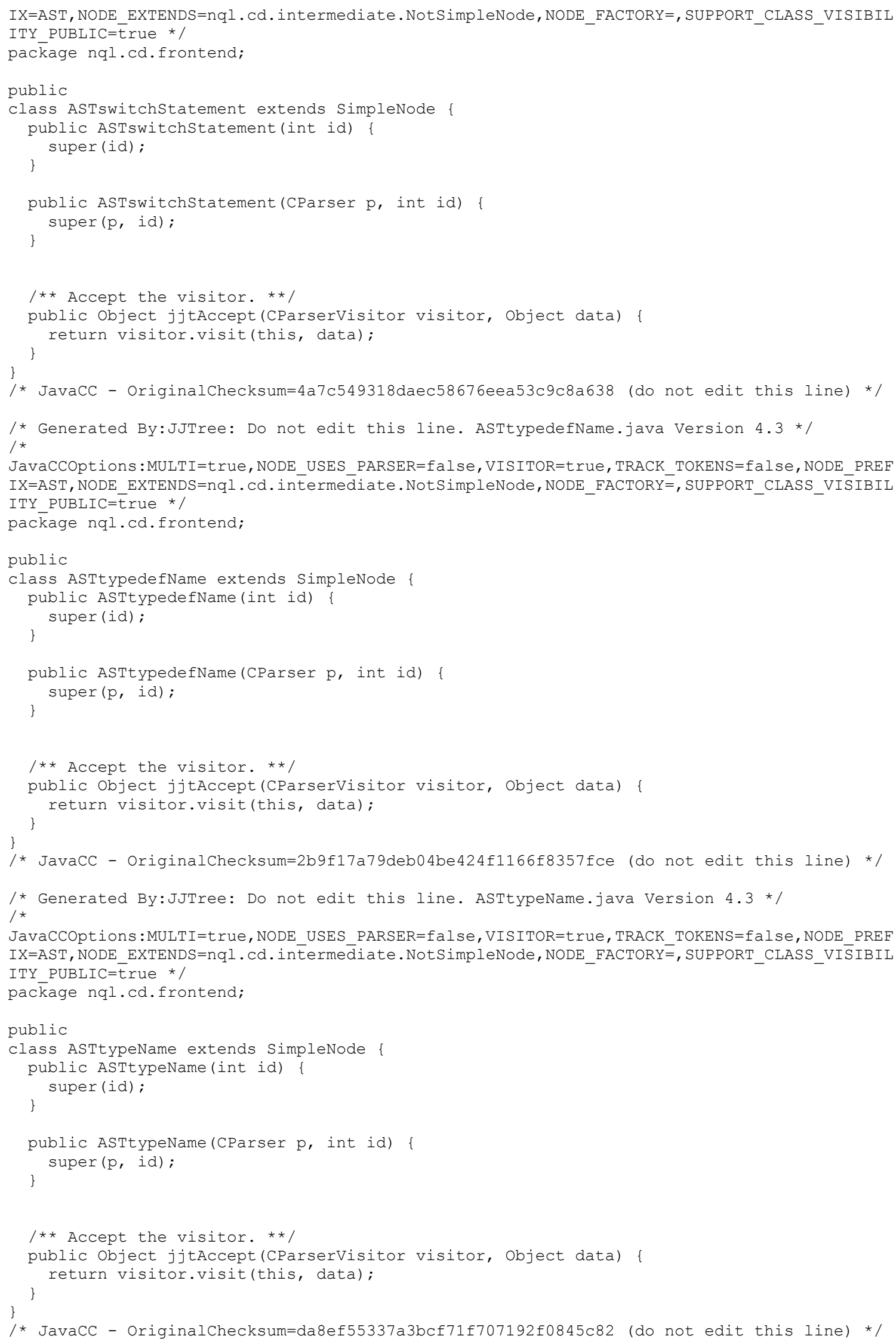




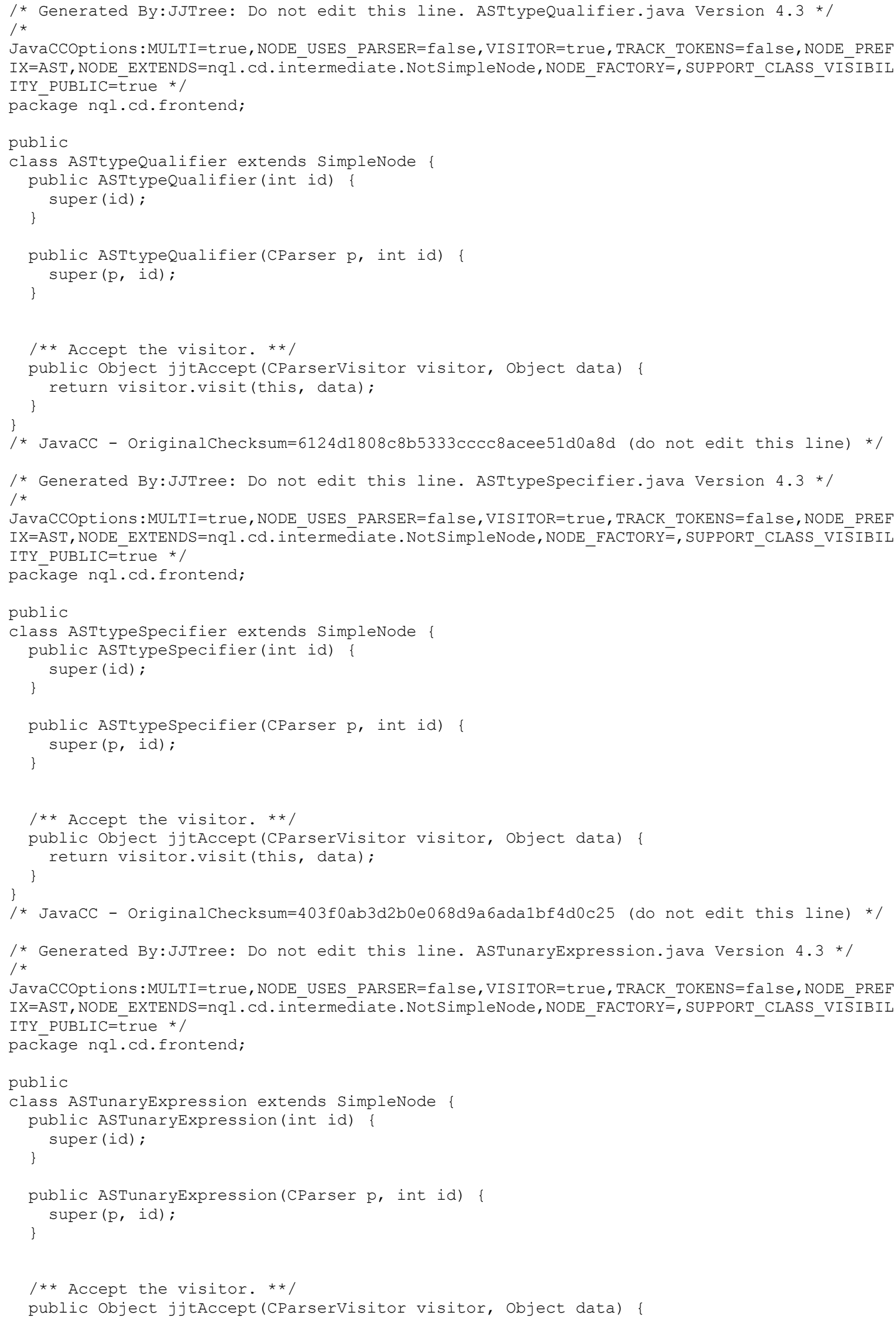




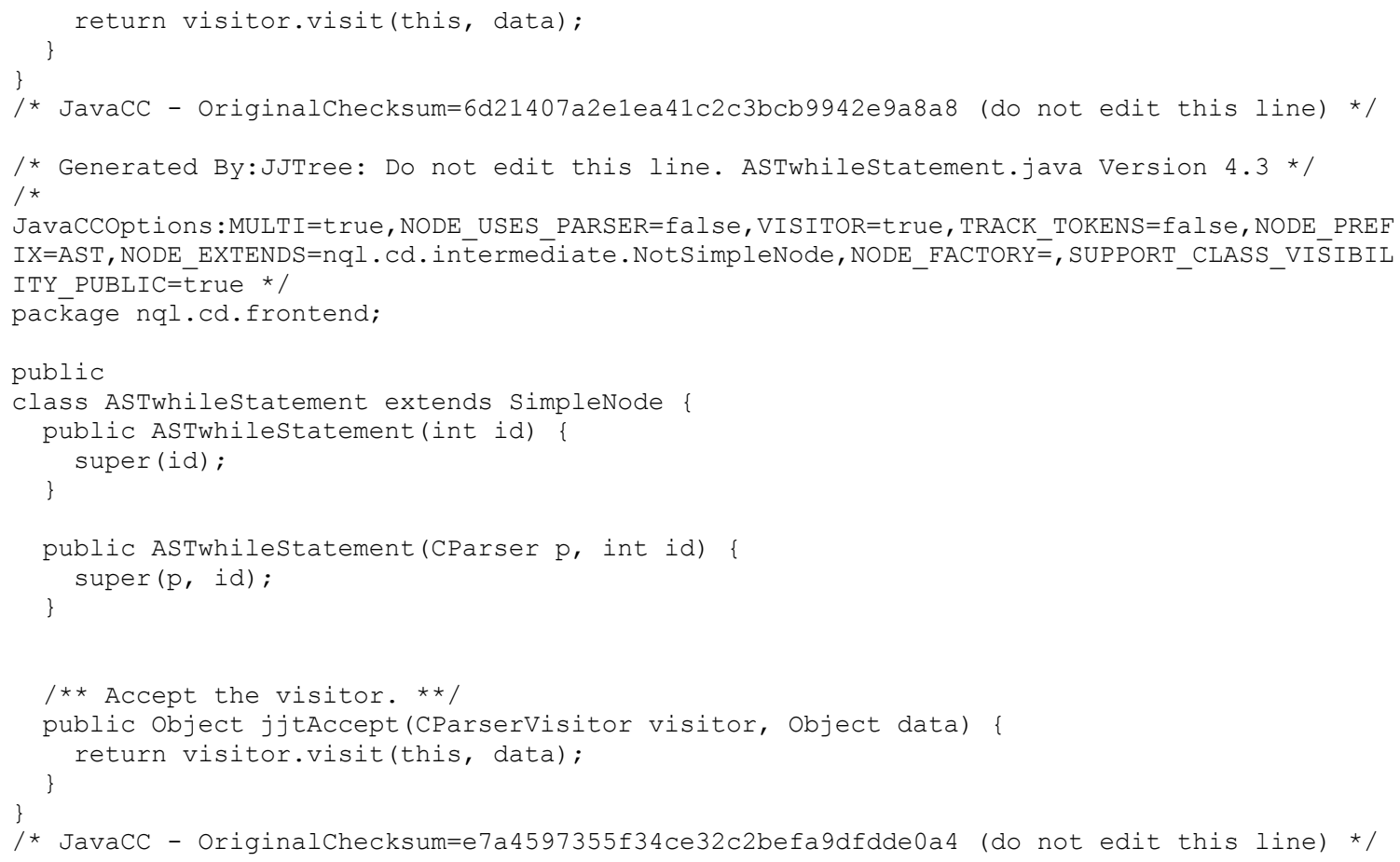

